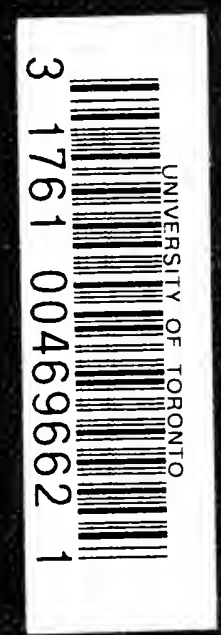


Digitized by the Internet Archive in 2007 with funding from Microsoft Corporation 

B. G. TEUBNER'S SAMMLUNG VON LEHRBÜCHERN

AUF DEM GEBIETE DER

MATHEMATISCHEN WISSENSCHAFTEN MIT EINSCHLUSS IHRER ANWENDUNGEN.

BAND VI.

\section{LINEAR GROUPS}

WITH AN EXPOSITION

\section{OF THE GALOIS FIELD THEORY}

LEONARD EUGENE DICKSON, PH. D., ASSISTANT PROFESSOR OF MATHEMATICS IN THE UNIVERSITY OF CHICAGO.
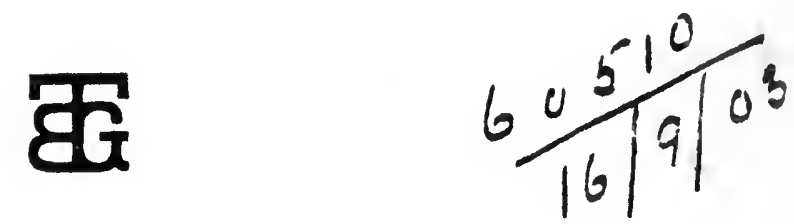

LEIPZIG

PUBLISHED BY B. G. TEUBNER

1901. 
QA
171
$D 53$

PRINTED BY B. G. TEUBNER, DRESDEN.

ELECTOBUC VEXSION

AVALABLE

No. 57000219 


\section{PREFACE.}

Since the appearance in 1870 of the great work of Camille Jordan on substitutions and their applications, there have been many important additions to the theory of finite groups. The books of Netto, Weber and Burnside have brought up to date the theory of abstract and substitution groups. On the analytic side, the theory of linear groups has received much attention in view of their frequent occurrence in mathematical problems both of theory and of application. The theory of collineation groups will be treated in a forthcoming volume by Loewy. There remains the subject of linear groups in a finite field (including linear congruence groups) having immediate application in many problems of geometry and function-theory and furnishing a natural method for the investigation of extensive classes of important 'groups. The present volume is intended as an introduction to this subject. While the exposition is restricted to groups in a finite field (endliche Körper), the method of investigation is applicable to groups in an infinite field; corresponding theorems for continuous and collineation groups may often be enunciated without modification of the text.

The earlier chapters of the text are devoted to an elementary. exposition of the theory of Galois Fields chiefly in their abstract form. The conception of an abstract field is introduced by means of the simplest example, that of the classes of residues with respect to a prime modulus. For any prime number $p$ and positive integer $n$, there exists one and but one Galois Field of order $p^{n}$. In view of the theorem of Moore that every finite field may be represented as a Galois Field, our investigations acquire complete generality when we take as basis the general Galois Field. It was found to be impracticable to attempt to indicate the sources of the individual theorems and conceptions of the theory. Aside from the independent discovery of theorems by different writers and a general lack of reference to earlier papers, the later writers have given wide generalizations of the results of earlier investigators. It will suffice to give the following list of references on Galois Fields and higher irreducible congruences:

Galois, "Sur la théorie des nombres", Bulletin des sciences mathématiques de M. Férussac, 1830; Journ. de mathématiques, 1846. Schönemann, Crelle, vol. 31 (1846), pp. 269-325. 
Dedekind, Crelle, vol. 54 (1857), pp. 1-26.

Serret, Journ. de math., 1873, p. 301, p. 437; Algèbre supérieure. Jordan, Traité des substitutions, pp. $14-18$, pp. 156-161.

Pellet, Comptes Rendus, vol. 70, p. 328, vol. 86, p.1071, vol.90, p.1339, vol. 93, p. 1065; Bull. Soc. Math. de France, vol. 17, p. 156.

Moore, Bull. Amer. Math. Soc., Dec., 1893; Congress Mathematical Papers.

Dickson, Bull. Amer. Math. Soc., vol. 3, pp.381-389; vol.6, pp.203-204. Annals of Math., vol. 11, pp. 65-120; Chicago Univ. Record, 1896, p. 318. Borel et Drach, Théorie des nombres et algèbre supérieure, 1895.

The second part of the book is intended to give an elementary exposition of the more important results concerning linear groups in a Galois Field. The linear groups investigated by Galois; Jordan and Serret were defined for the field of integers taken modulo $p$; the general Galois Field enters only incidentally in their investigations. The linear fractional group in a general Galois Field was partially investigated by Mathieu, and exhaustively by Moore, Burnside and Wiman. The work of Moore first emphasized the importance of employing in group problems the general Galois Field in place of the special field of integers, the results being almost as simple and the investigations no more complicated. In this way the systems of linear groups studied by Jordan have all be generalized by the author and in the investigation of new systems the Galois Field has been employed ab initio.

The method of presentation employed in the text often differs greatly from that of the original papers; the new proofs are believed to be much simpler than the old. For example, the structure of all linear homogeneous groups on six or fewer indices which are defined by a quadratic invariant is determined by setting up their isomorphism with groups of known structure. Then the structure of the corresponding groups on $m$ indices, $m>6$, follows without the difficult calculations of the published investigations. In view of the importance thus placed upon the isomorphisms holding between various linear groups, the theory of the compounds of a linear group has been developed at length and applied to the question of isomorphisms. Again, it was found practicable to treat together the two (generalized) hypoabelian groups. The identity from the group standpoint of the problem of the trisection of the periods of a hyperelliptic function of four periods and the problem of the determination of the 27 straight lines on a general cubic surface is developed in Chapter XIV by an analysis involving far less calculation than the proof by Jordan.

Chicago, November, 1900. 


\section{TABLE OF CONTENTS.}

\section{FIRST PART. \\ INTRODUCTION TO THE GALOIS FIELD THEORY.}

\section{CHAPTER I.}

Section Definition and properties of finite fields.

1-3. Classes of residues with respect to a prime modulus . . . 3-4

4. Fermat's theorem . . . . . . . . . . . . . . . . 4

5. Definition of a field . . . . . . . . . . . . . . . 5

6-7. Definition of a Galois Field . . . . . . . . . . . . 6-8

8-10. Order of a finite field is a power of a prime . . . . . . 9-10

11-17. Period of a mark of a field; primitive roots . . . . . . 11-12

18. Every finite field may be represented as a Galois Field . . 13-14

\section{CHAPTER II. \\ Proof of the existence of the $G F\left[p^{m}\right]$ for every prime $p$ and integer $m$.}

19-22. Decomposition of functions belonging to the $G F\left[p^{n}\right]$. . . 14-15

23-25. Irreducible factors of $x^{p^{n m}}-x$. . . . . . . . . . . 15-16

26-27. Expression for product of all irreducible quantics of degree $m$ in the $G F\left[p^{n}\right]$. Their number . . . . . . . . . . . 17-18

Exercices . . . . . . . . . . . . . . . . . . . 19

\section{CHAPTER III.}

Classification and determination of irreducible quantics.

29-30. Exponent to which an irreducible quantic belongs . . . . 19-20

31-32. Roots of an irreducible quantic; their exponents . . . . . 21

33. When $x^{t}+x^{t-1}+\cdots+x+1$ is irreducible . . . . . . 21

$34-38$. Determination of irreducible quantics in the $G F\left[p^{n}\right]$ whose degree contains no prime factor other than those of $p^{n-1} \cdot 22-27$

39-46. Irreducible quantics of degree $p^{s}$ in the $G F\left[p^{n}\right]$. . . . . $\quad 28-32$

47-49. Miscellaneous theorems on irreducible quantics . . . . . 32-34

50-58. Primitive roots and primitive irreducible quantics . . . . 35-42

59. Exercises . . . . . . . . . . . . . . . . . . . 42-44

60. Table of primitive irreducible quantics . . . . . . . . . . 44 
CHAPTER IV.

Section Miscellaneous properties of Galois Fields.

61-62. Squares and not-squares

Page

$49 \div 51$

52

52

$53-54$

\section{CHAPTER V. \\ Analytic representation of substitutions on the marks of a Galois Field.}

76-78. Definitions. Representation of a given substitution

$54-55$

79-83. Special functions suitable to represent substitutions. .

$56-59$

84. Necessary and sufficient conditions for a substitution quantic

$59-60$

85-89. Applications of preceding theorem. Reduced form... .

$61-63$

90. Table of all substitution quantics of degree $<6 .$. . .

63-64

91-94. Betti-Mathieu Group. Certain of its subgroups . . . . 64-68

95. Identity of Betti-Mathieu Group in the $G F\left[p^{n m}\right]$ with Jordan's linear homogeneous group in the $G F\left[p^{n}\right]$ on $m$ indices

96. Exercises

$69-70$

$70-71$

\section{SECOND PART.}

\section{THEORY OF LINEAR GROUPS IN A GALOIS FIELD.}

\section{CHAPTER I.}

General linear homogeneous group.

97- 98. Two definitions of the group . . . . . . . . . . . $75-77$

99-100. Order and generators . . . . . . . . . . . . . . 77-79

101-102. Transformation of indices. Invariance of characteristic determinant . . . . . . . . . . . . . . . . . 80-81

103-107. Factors of composition of the linear homogeneous group . 81-86 108-109. Linear fractional group. Isomorphic permutation group . 87-88

\section{CHAPTER II.}

\section{The Abelian linear group.}

110-112. Conditions for Abelian substitutions. Inverse substitution . $89-91$ 114-115. Generators and order of Abelian group . . . . . . . 92-94 116-119. Factors of composition of the Abelian linear group . . . 94-100 120-121. Conjugacy of operators of period two of the Abelian group 100-105 122-123. Operators of period two in the quotient-group $A\left(2 m, p^{n}\right)$ 105-109 
CHAPTER III.

Section

A generalization of the Abelian linear group.

124-125. Definition of the substitutions; their inverse.

\section{CHAPTER IV.}

\section{The hyperabetian group.}

129-130. Conditions on its substitutions; their inverse

131. Largest subgroup containing the Abelian group selfconjugately .

132-133. Corresponding theorems for their quotient-groups

134-136. Binary linear homogeneous subgroups of the quaternary hyperabelian group. Application to their quotient-groups

137. Identity of binary hyperabelian and binary linear group .

$122-125$

\section{CHAPTER V.}

The hyperorthogonal and related linear groups.

139-142. Definition. Structure in the general case . . . . . . 126-131 143-151. Order, generators and structure in the hyperorthogonal case 131-144

\section{CHAPTER VI.}

\section{The compounds of a linear homogeneous group.}

153. Isomorphism of linear group with its compounds . . . . 145-146

154. Multiplicity of isomorphism for general linear group . . 146-147

156. Pfaffian invariant of the second compound . . . . . . 147-148

157-158. Group induced upon certain Pfaffians by the second compound 148-150

159-162. The second compound of the general and special Abelian groups . . . . . . . . . . . . . . 151-153

163-165. The second compound of the quaternary linear group . . 153-155

CHAPTER VII.

\section{Linear homogeneous group in the $G F\left[p^{n}\right], p>2$,} defined by a quadratic invariant.

166-169. Canonical forms of the quadratic invariant

170-171. Orthogonal substitutions; the first and second orthogonal groups

$156-158$

172-180. Order and generators of the orthogonal groups

178. The ternary first orthogonal group and the linear fractional group .

181-198. The structure of the orthogonal groups

186-188. Senary orthogonal groups isomorphic with quaternary linear groups . . . . . . . . . . . . . . . .

189. Quinary orthogonal group isomorphic with quaternary Abelian group

190. Senary orthogonal groups isomorphic with hyperabelian groups 


\section{CHAPTER VIII.}

Linear homogeneous group in the GF $\left[2^{n}\right\rfloor$ defined

Section by a quadratic invariant.

199. Canonical forms of the quadratic invariant

200. Structure of group on an odd number of indices. . .

201-204. Definition, order and generators of the hypoabelian groups

205. Invariant defining the subgroup $J_{\lambda}$. . . . . . . . . . .

206-208. Isomorphism of senary group $J_{\lambda}$ with certain quaternary groups

209. Simplicity of $\dot{J}_{\lambda}$ on more than six indices . . . . .

210. Miscellaneous exercises on chapters I-VIII .

\section{CHAPTER IX.}

Linear groups with certain invariants of degree $q>2$.

211-213. Definition, generators and structure of group

$218-221$

\section{CHAPTER X.}

Canonical form and classification of linear substitutions.

214-216. Canonical form of linear homogeneous substitutions .

221-229

217-220. Substitutions commutative with a given linear substitution

221-223. Distribution of the substitutions of the general ternary and quaternary linear groups into sets of conjugate substitutions

\section{CHAPTER XI.}

Operators and cyclic subgroups of the simple groip

$$
L F\left(3, p^{n}\right) \text {. }
$$

224-225. Notations. The seven distinct canonical forms

238. $\operatorname{LF}\left(3,2^{2}\right)$ not isomorphic with the alternating group on 8 letters, each group being simple and of equal order.

$259-260$

\section{CHAPTER XII.}

Subgroups of the linear fractional group $L F\left(2, p^{n}\right)$.

239. Doubly transitive substitution group on $p^{n}+1$ letters . . 260-261

240-244. Commutative subgroups of order $p^{n}$; cyclic subgroups . . 261-265

245. Concerning dihedron groups and their subgroups . . . . 265-266

246-248. Subgroups of dihedron and four-group types . . . . . 267-268

249-255. Subgroups containing operators of period $p$. . . . . . 268-280

256. Subgroups containing no operators of period $p$. . . . . 280-282

257-259. Subgroups of tetrahedral, octahedral and icosahedral types. . . . . . . . . . . . . . . . . . . 282-285

260-261. Summary of subgroups. Simplicity theorem . . . . . 285-286

262. Galois' theorem on the minimum index of a subgroup . . 286

263. Lowest degree of isomorphic substitution group . . . . $\quad 287$ 
CHAPTER XIII.

Auxiliary theorems on abstract groups. Abstract forms

Section of various linear groups.

264-267. Abstract groups isomorphic with the symmetric and alternating groups. . . . . . . . . . . . . . . . .

268-269. Quaternary linear group modulo 2 isomorphic with the alternating group on 8 letters . . . . . . . . . . . 290-292

270-274. Abstract form of quinary orthogonal group modulo 3 . . 292-298

275-276. Its isomorphism with a hyperabelian group . . . . . . 298-299

278-282. Abstract group isomorphic with $\operatorname{LF}\left(2, p^{n}\right)$. . . . . . 300-303

\section{CHAPTER XIV.}

Group of the equation for the 27 straight lines on a general surface of the third order.

283. Notation for the configuration of the 27 lines . . . . . 303-305

284-285. Group of the equation. Isomorphism with linear groups . 305-306

286. Subgroups of indices $27,36,40,45$. . . . . . . . 306-307

\section{CHAPTER XV.}

Summary of the known systems of simple groups.

287. The ten known infinite systems . . . . . . . . . . 307

288. Isomorphisms between certain groups of the systems . . 308

289. Two triply infinite systems of non-isomorphic simple groups of equal order . . . . . . . . . . . . . . 309

290. Table of simple groups of orders less than a million . . 309-310 


\section{DEFINITION OF SYMBOLS.}

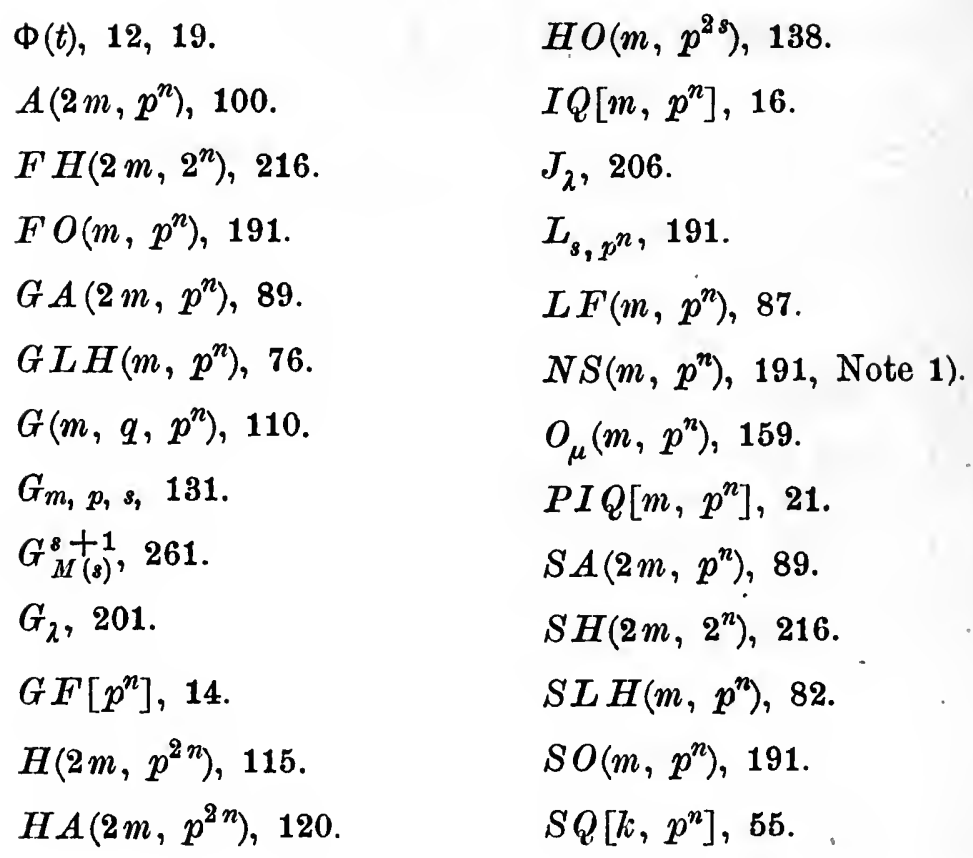




\section{F I R S T PA R T.}

\section{INTRODUCTION}

\section{TO THE GALOIS FIELD THEORY.}




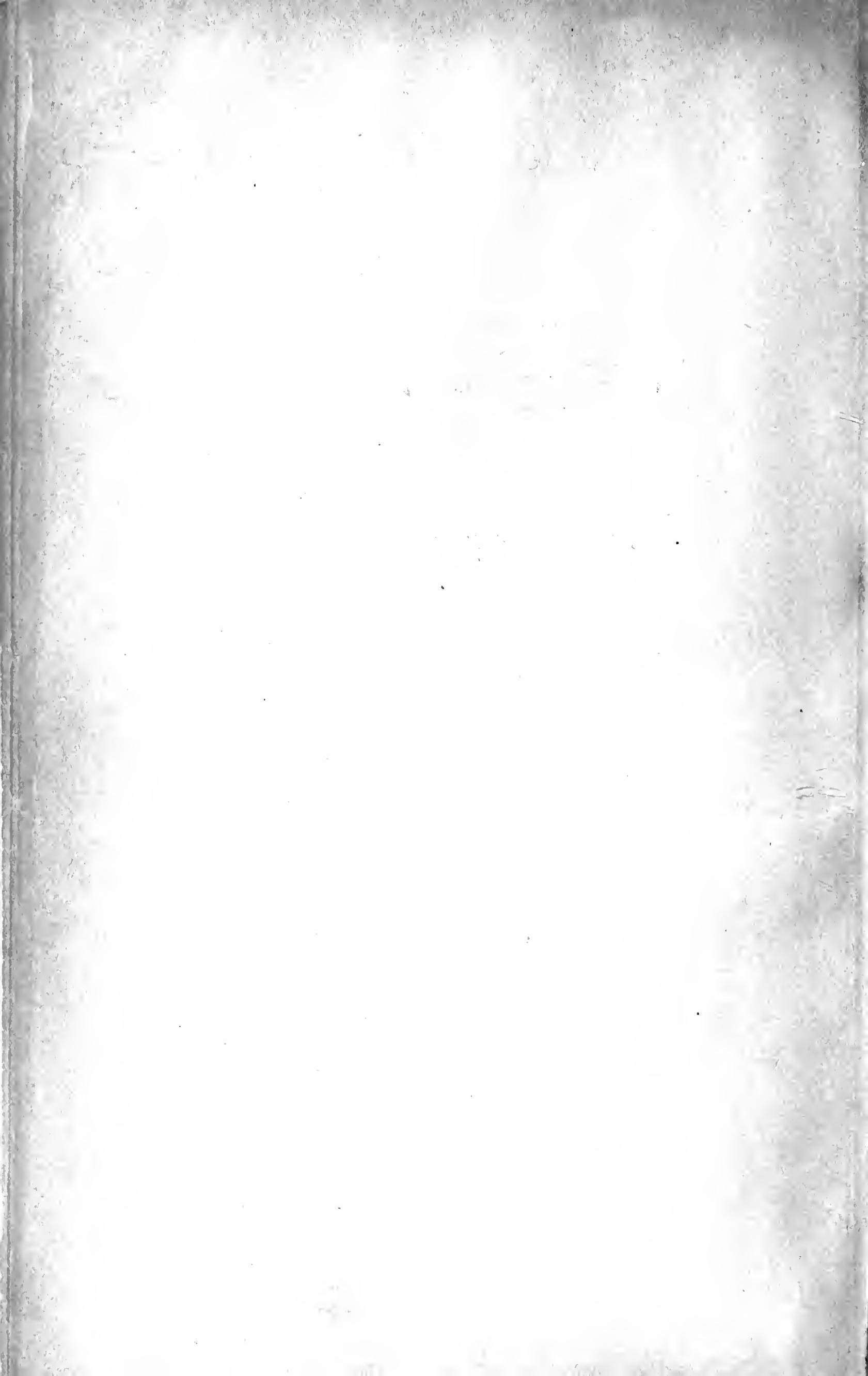




\section{CHAPTER I.}

\section{DEFINITION AND PROPERTIES OF FINITE FIELDS.}

1. If the difference of two integers $t$ and $r$ be divisible by a third integer $p$, then $t$ and $r$ are said to be congruent modulo $p$, or according to the modulus $p$. This property is expressed by the following notation due to Gauss:

$$
t \equiv r(\bmod p) \text {. }
$$

For example, $7 \equiv 1(\bmod 3), 7 \equiv 2(\bmod 5)$.

The totality of integers congruent modulo $p$ with a given positive integer $r<p$ is given by the formula

$$
l p+r \quad(l=0, \pm 1, \pm 2, \ldots) .
$$

This totality, which will be designated $C_{r}$, is said to form a class of residues modulo $p$; it includes every integer which gives the residue $r$ when divided by $p$. It follows that the $p$ classes $C_{0}, C_{1}, C_{2}, \ldots$, $C_{p-1}$ include every integer, positive or negative. They are therefore said to form a complete system of classes of residues modulo $p$.

Example. - The three classes $C_{0}, C_{1}, C_{2}$ form a complete system of classes of residues modulo 3 ; indeed, every integer falls under one of the three forms $3 l, 3 l+1,3 l+2$.

2. An instructive diagram is furnished by the regular polygon of $p$ sides inscribed in a circle. Denote the vertices taken in positive order (counter-clockwise) by $C_{0}, C_{1}, \ldots, C_{p-1}$. Regarding $C_{0}$ to be the origin, we take as the plot of any given integer $\pm m$ that vertex which is obtained by counting off from the origin $m$ of the divisions on the circle in the positive or the negative direction according to the sign of $\pm m$. All integers of the form $l p+r(l=0, \pm 1$, $\pm 2, \ldots)$ are evidently plotted by the one point $C_{r}$, so that congruent integers give rise to the same point. The $p$ classes of residues modulo $p$ are represented unambiguously by the $p$ vertices of the polygon. 
3. From the numerical identities

$$
\begin{aligned}
(l p+r) \pm(t p+s) & =(l \pm t) p+(r \pm s), \\
(l p+r)(t p+s) & =(l t p+l s+r t) p+r s,
\end{aligned}
$$

we obtain the following formulae for the addition, subtraction and multiplication of classes of residues:

$$
C_{r} \pm C_{s}=C_{r \pm s}, \quad C_{r} \cdot C_{s}=C_{r s} .
$$

If two given classes $C_{r}$ and $C_{s}, C_{s} \neq C_{0}$, lead uniquely to a third class $C_{x}$ such that $C_{r}=C_{s} C_{x}$, then $C_{x}$ is said to be the quotient of $C_{r}$ by $C_{s}$ and the following notation employed

$$
\mathcal{C}_{x}=C_{r} / C_{s}
$$

The condition for the quotient is evidently identical with the condition that there exist a solution $x$ of the equation

$$
r=s x+m p \text {. }
$$

In order that a solution $x$ shall exist for $r$ and $s$ arbitrary integers. such that $s$ is not divisible by $p$, it is necessary and sufficient that $p$ be a prime number. To prove the condition necessary, let $p=p_{1} p_{2}$, where $p_{1}>1, p_{2}>1$. Then 1) can not always be satisfied; for example, when $s=p_{1}$ and $r$ is not divisible by $p_{1}$. The condition that $p$ be a prime is, moreover, a sufficient one by the corollary of $\S 4$. Hence the division of classes of residues, the divisor being other than the class $C_{0}$, is always possible if, and only if, the modulus $p$ be a prime number.

In particular, these remarks show that the classes of residues with respect to a prime modulus may be combined by the rational operations of algebra and that each result is itself one of the classes of residues. For example, let $p=3$. Then

$C_{1}+C_{2}=C_{0}, C_{2}+C_{2}=C_{1}, C_{2} \cdot C_{2}=C_{1}, C_{2} / C_{1}=C_{2}, C_{2} / C_{2}=C_{1}, C_{1} / C_{2}=C_{2}$.

4. Fermat's Theorem. - If an integer a be not divisible by a prime number $p$, then $a^{p-1} \equiv 1(\bmod p)$.

Since the integers $a, 2 a, 3 a, \ldots,(p-1) a$ are all distinct modulo $p$, their residues must be identical, apart from their order, with the integers $1,2,3, \ldots, p-1$.

Forming the product of the integers in each set, we have

$$
a^{p-1} \cdot 1 \cdot 2 \cdot 3 \ldots(p-1) \equiv 1 \cdot 2 \cdot 3 \ldots(p-1) \quad(\bmod p) .
$$

Corollary. - If a be not divisible by the prime number $p$, there exists an unique solution of the congruence $a x \equiv b(\bmod p)$.

Applying the theorem just proven, the solution is evidently

$$
x \equiv a^{p-2 b}(\bmod p) .
$$


5. Definition of a field. - A set of elements $u_{1}, u_{2}, \ldots, u_{\sigma}$, which may be combined by addition subject to the formal laws

$$
u_{i}+u_{j}=u_{j}+u_{i} ; \quad u_{i}+\left(u_{j}+u_{k}\right)=\left(u_{i}+u_{j}\right)+u_{k},
$$

such that the sum of any two elements is likewise an element of the set is called an additive-field. If two elements $u_{i}$ and $u_{k}$ are given, there may or may not exist a third element $u_{j}$ in the set such that $u_{i}+u_{j}=u_{k}$. If existent, $u_{j}$ is said to be determined by subtraction, $u_{j} \equiv u_{k}-u_{i}$. Assume ${ }^{1}$ ) that subtraction is always possible in the given additive-field. The set will contain the differences $u_{1}-u_{1}, u_{2}-u_{2}, \ldots, u_{\sigma}-u_{\sigma}$. Each has the additive property of zero, since $u_{j}+\left(u_{i}-u_{i}\right) \equiv u_{j}$. From the latter, $u_{i}-u_{i} \equiv u_{j}-u_{j}$ follows by the definition of subtraction. Hence the above differences all have a common value $u$. There exists no new zero element $u$, since $u_{j}+u^{\prime}=u_{j}$ requires $u^{\prime}=u_{j}-u_{j} \equiv u$. Two elements are called equal or distinct according as their difference is or is not the zero element $u$. Select from the original set all the distinct elements and denote them by $u_{0}, u_{1}, u_{2}, \ldots, u_{s-1}$, where $u_{0}$ denotes the unique zero element:

Assume next that the $s$ elements $u_{0}, u_{1}, \ldots, u_{s-1}$ may be combined by multiplication subject to the formal laws

$$
u_{i} u_{j}=u_{j} u_{i}, \quad u_{i}\left(u_{j} u_{k}\right)=\left(u_{i} u_{j}\right) u_{k}, \quad u_{i}\left(u_{j} \pm u_{k}\right)=u_{i} u_{j} \pm u_{i} u_{k},
$$

such that the product of any two elements is itself an element of the set. Then the element $u_{0}$ will have the multiplicative properties of zero, viz., for any element $u_{j}$ of the set,

$$
u_{j} u_{0}=u_{0} u_{j}=u_{0} .
$$

Indeed, since every product $u_{j} u_{i}$ is an element of the set,

$$
u_{j}\left(u_{i}-u_{i}\right)=u_{j} u_{i}-u_{j} u_{i} \equiv u_{0}, \quad\left(u_{i}-u_{i}\right) u_{j}=u_{0} .
$$

Given two elements $u_{i}$ and $u_{k}, u_{i} \neq u_{0}$, there may or may not exist a third element $u_{j}$ in the set such that $u_{i} u_{j}=u_{k}$. If existent, $u_{j}$ is said to be determined by division, $u_{j}=u_{k} / u_{i}$. Assume ${ }^{2}$ ) lastly that division is always possible in the set, and in a single way, the divisor being other than the zero element. A set of $s$ distinct elements satisfying the above four conditions is said to form a field of order $s$.

To obtain a field of finite order, the assumption concerning division may be replaced by the postulate that a product of two

1) In the additive-field of all positive integers, not every difference of two elements belongs to the field.

2) The set of all positive and negative integers satisfies the assumptions as to addition, subtraction and multiplication, but not that for division. 
elements shall be the zero element $u_{0}$ only when one of the factors is $u_{0}$. Under the latter hypothesis, the series of products

$$
u_{0} u_{i}, \quad u_{1} u_{i}, \quad u_{2} u_{i}, \ldots, u_{s-1} u_{i} \quad\left(u_{i} \neq u_{0}\right)
$$

are all distinct and therefore (their number $s$ being finite) are identical in some order with the series $u_{0}, u_{1}, u_{2}, \ldots, u_{s-1}$. Hence if $u_{j}$ be any element of the set, the equation

$$
x u_{i}=u_{j} \quad\left(u_{i} \neq u_{0}\right)
$$

is satisfied by one and but one element $x$ of the given set. Hence division by any element except $\iota_{0}$ is always possible within the set and gives an unique result.

For a field of infinite order, the assumption that division is not possible in more than one way may be replaced by the above postulate that a product vanishes only when one factor vanishes. Indeed, if 2) be satisfied by two distinct values $x_{1}$ and $x_{2}$ of $x$, then $u_{i}\left(x_{1}-x_{2}\right)=u_{0}$, whereas each factor differs from $u_{0}$.

After the above explanations, we make the formal definition:

$A$ set of $s$ distinct elements forms a field of order $s$ if the elements can be combined by addition, subtraction, multiplication and division, the divisor not being the element zero (necessarily in the set); these operations being subject to the laws of elementary algebra, and if the resulting sum, difference, product or quotient be uniquely determined as an element of the set. 1 )

A field may therefore be defined by the property that the rational operations of algebra can be performed within the field.

The results of $\S 3$ may now be stated in the form: The complete system of classes of residues modulo $p$ forms a field it, and only if, $p$ be a prime number.

6. Definition of a Galois Field. - Let $P(x)$ be a rational integral function of degree $n$ having integral coefficients not all divisible by a given integer $p$. If we divide an arbitrary integral function $F(x)$ having integral coefficients by the function $P(x)$, we obtain a quotient $Q(x)$ and a remainder which can be written in the form $f(x)+p \cdot q(x)$, where $f(x)$ is of the form

$$
f(x) \equiv a_{0}+a_{1} x+a_{2} x^{2}+\cdots+a_{n-1} x^{n-1},
$$

each $\dot{a}_{i}$ belonging to the series $0,1,2, \ldots, p-1$. Then

$$
F(x)=f(x)+p \cdot q(x)+P(x) \cdot Q(x) .
$$

We say that $f(x)$ is the residue of $F(x)$ moduli $p$ and $P(x)$ and write

$4_{1}$ )

$$
F(x) \equiv f(x) \quad \text { [modd } p, P(x)] \text {. }
$$

1) Moore, Mathematical Papers, Chicago Congress of 1893, pp. 208-242; Bull. Amer. Math. Soc., December, 1893. 
The totality of functions $F(x)$ obtained by giving to the polynomials $Q(x)$ and $q(x)$ in 4) all possible forms is said to constitute a class of residues; two functions are called congruent if, and only if, they belong to the same class of residues. From the form of 3) there are evidently $p^{n}$ distinct classes.

Consider two integral functions having integral coefficients

$$
F_{i}(x)=f_{i}(x)+p \cdot q_{i}(x)+P(x) \cdot Q_{i}(x) \quad[i=1,2] .
$$

It is evident that the class to which $F_{1} \pm F_{2}$ or $F_{1} F_{2}$ belongs depends merely upon the functions $f_{1} \pm f_{2}$ or $f_{1} f_{2}$ respectively, being independent of the functions $q_{i}, Q_{i}$. Hence classes of residues combine unambiguously under addition, subtraction and multiplication. In order that the division of an arbitrary class by any class $C$, not the class zero $C_{0}$, shall lead uniquely to a third class, it is necessary that the equation $C_{i} C=C_{0}$. shall require $C_{i}=C_{0}$. Evidently this will not be the case if $p$ be composite, $p=p_{1} p_{2}$, or if $P(x)$ be reducible modulo $p$, viz.,

$$
P(x)=P_{1}(x) P_{2}(x)+p P_{3}(x)
$$

where the $P_{i}(x)$ are integral functions having integral coefficients, the degrees of $P_{1}(x)$ and $P_{2}(x)$ being less than the degree of $P(x)$. Hence $p$ must be prime and $P(x)$ irreducible modulo $p$.

Inversely, if $p$ be prime and $P(x)$ irreducible modulo $p$, it follows from $\S 7$ that to any class $C_{F_{1}}$ other than the class $C_{0}$ there corresponds an unique class $C_{F_{1}^{\prime}}$ such that $C_{F_{1}^{\prime}} C_{F_{1}}$ is the class unity. Hence there exists the quotient class

$$
\frac{C_{F_{2}}}{C_{F_{1}}}=\frac{C_{F_{2}} C_{F_{1}^{\prime}}}{C_{F_{1}} C_{F_{1}^{\prime}}}=C_{F_{2}} C_{F_{1}^{\prime}}=C_{F_{2} F_{1}^{\prime}}
$$

The $p^{n}$ classes of residues therefore form a field called a Galois Field of order $p^{n}$. Moreover, the $p^{n}$ classes of residues moduli $p$ and $P(x)$ form a field if, and only if, $p$ be prime and $P(x)$ be irreducible modulo $p$.

As an example, let $p=3$ and $P(x)=x^{2}-x-1$. The $3^{2}$ residues are

$$
0,1,-1, \quad x, \quad x+1, \quad x-1,-x,-x+1,-x-1 \text {. }
$$

The sum, difference or product of any two of these may evidently be reduced moduli 3 and $x^{2}-x-1$ to. one of the nine residues. Moreover, the quotient of any one by any residue except 0 may be reduced to one of the set. For example,

$$
\frac{1}{x} \equiv \frac{x-1}{x(x-1)} \equiv x-1, \frac{1}{x+1} \equiv \frac{x}{-x+1} \equiv \frac{-x^{2}}{x^{2}-x} \equiv-x^{2} \equiv-x-1 \text {. }
$$

The nine residues thus form a Galois Field of order $3^{2}$. 
7. Theorem. - If two integral functions $F(x)$ and $P(x)$ having integral coefficients admit of no common divisor containing $x$ modulo $p$, $p$ being prime, we can determine two integral functions $F^{\prime}(x)$ and $P^{\prime}(x)$ having integral coefficients such that

$$
F^{\prime}(x) \cdot F(x)-P^{\prime}(x) \cdot P(x) \equiv 1 \quad(\bmod p) .
$$

Applying \& 4, we can set

$$
F(x) \equiv \dot{a} \cdot A(x), \quad P(x) \equiv b \cdot B(x) \quad(\bmod p)
$$

the coefficients of the highest power of $x$ in $A(x)$ and $B(x)$ being unity and the remaining coefficients integers. We perform the usual process to determine the greatest common divisor of $A$ and $B$, neglecting however, multiples of $p$. Each remainder is congruent modulo $p$ to a product of an integer $r$ and an integral function $R(x)$ with integral coefficients, that of the highest power of $x$ being unity. Supposing for definiteness that the degree of $A$ is not less than that of $B$, we obtain the congruences $(\bmod p)$ :

$$
\begin{gathered}
A \equiv B Q_{1}+r_{1} R_{1} \\
B \equiv R_{1} Q_{2}+r_{2} R_{2} \\
R_{1} \equiv R_{2} Q_{3}+r_{3} R_{3} \\
\cdot \cdot \cdot \cdot \cdot \cdot \cdot \cdot \cdot \cdot \cdot \cdot \cdot \cdot \cdot \cdot \cdot \cdot \cdot \cdot \cdot \cdot \cdot \cdot R_{m-1} Q_{m}+r_{m} .
\end{gathered}
$$

We derive at once the following congruences modulo $p$ :

$$
\begin{aligned}
& r_{1} R_{1} \equiv A-Q_{1} B \\
& r_{1} r_{2} R_{2} \equiv-Q_{2} A+\left(r_{1}+Q_{1} Q_{2}\right) B \\
& r_{1} r_{2} r_{3} R_{3} \equiv\left(r_{2}+Q_{2} Q_{3}\right) A-\left(r_{2} Q_{1}+r_{1} Q_{3}+Q_{1} Q_{2} Q_{3}\right) B \\
& r_{1} r_{2} \ldots r_{m} \equiv M A-N B,
\end{aligned}
$$

where $M$ and $N$ are integral functions of $x$ having integral coefficients.

None of the integers $r_{1} \ldots, r_{m}$ are divisible by $p$; for, $A$ and $B$ would then have a common divisor containing $x$. Hence, by $\S 4$, there exists an integer $r$ such that

$$
r \cdot a b r_{1} r_{2} \ldots r_{m} \equiv 1 \quad(\bmod p)
$$

From the last congruence in the above set, we therefore find

$$
1 \equiv \operatorname{rab}(M A-N B) \equiv F(x) \cdot r b M-P(x) \cdot r a N(\bmod p) .
$$

Corollary. - If $F(x) \equiv \equiv 0$ [modd $p, P(x)], p$ being prime and $P(x)$ irreducible modulo $p$, we can determine an integral function $F^{\prime}(x)$ such that

$$
F^{\prime \prime}(x) \cdot F(x) \equiv 1 \quad[\operatorname{modd} p, P(x)] .
$$

Note. - By an analogous use of the process for finding the greatest common divisor, we obtain the following theorem: 
If two integers $f$ and $p$ be relatively prime, we can determine two integers $f^{\prime}$ and $p^{\prime}$ such that $f^{\prime} f-p^{\prime} p=1$.

8. The proof of the existence of a function of degree $n$ irreducible modulo $p$ and hence of the existence of a Galois Field of order $p^{n}$, for every prime $p$ and integer $n$, will be given in $\S \S 19-27$. We will first prove that no other finite fields exist and that not more than one Galois Field of a given order $p^{n}$ exists.

9. Consider an abstract field $F[s]$ composed of a finite number $s>1$ of elements or marks $u_{0}, u_{1}, \ldots, u_{s-1}$. Having every difference $u_{i}-u_{i}$; the field contains a mark, denoted by $u_{(0)}$, which has the properties of zero viz., for every $u_{i}$,

$$
u_{i}+u_{(0)}=u_{\text {舟, } i} u_{(0)} u_{i}=u_{i} u_{(0)}=u_{(0)} .
$$

Having every quotient

$$
u_{i} / u_{i} \quad\left(u_{i} \neq u_{(0)}\right),
$$

the field contains a mark $u_{(1)}$ having the properties of unity; viz., for every $u_{i}$,

$$
u_{i} u_{(1)}=u_{(1)} u_{i}=u_{i} \text {. }
$$

The field thus contains every integral mark

$$
\begin{aligned}
u_{(c)} & =u_{(1)}+u_{(1)}+\cdots+u_{(1)} \quad(c \text { terms }), \\
u_{(-c)} & =u_{(0)}-u_{(c)} .
\end{aligned}
$$

Since there exists only a finite number of marks in the $F[s]$, there must arise equalities in the series

If $u_{(r)}=u_{(s)}$, we have

$$
\ldots, u_{(-2)}, \quad u_{(-1)}, \quad u_{(0)}, \quad u_{(1)}, \quad u_{(2)}, \ldots
$$

$$
u_{(0)}=u_{(r)}-u_{(s)}=u_{(r-s)} .
$$

Denoting by $p$ the least positive integer such that $u_{(p)}=u_{(0)}$, the $p$ marks

are all distinct, while

$$
u_{(0)}, \quad u_{(1)}, \quad u_{(2)}, \ldots, u_{(p-1)}
$$

$$
u_{(r)}=u_{(s)} \text { if, and only if, } r \equiv s \quad(\bmod p) .
$$

This integer $p$ is a prime number. For, if

$$
p=p_{1} p_{2}, \quad p>p_{1},
$$

we have, by hypothesis, $u_{\left(p_{1}\right)} \neq u_{(0)}$. Hence, from

$$
u_{\left(p_{1}\right)} \cdot u_{\left(p_{2}\right)}=u_{(p)}=u_{(0)},
$$

we derive $u_{\left(p_{2}\right)}=u_{(0)}$ and hence $p_{2}>p$. Hence the integral marks of the $F[s]$ form a field $F[p]$ which is the abstract form of the field of the classes of residues with respect to a prime modulus $p$. When there is no ambiguity, we denote by $c$ the integral mark $u_{(c)}$. 
10. Theorem. - The order of $F[s]$ is a power of $p$.

If $u_{1}$ be a fixed mark $\neq u_{0}$ of the $F[s]$, the products

$$
c_{1} u_{1} \quad\left(c_{1}=0,1, \ldots, p-1\right)
$$

give $p$ distinct marks of the field. If $s>p$, there exists a mark $u_{2}$ not of the form $c_{1} u_{1}$. Then

$$
c_{1} u_{1}+c_{2} u_{2} \quad\left(c_{1}, c_{2}=0,1, \ldots, p-1\right)
$$

gives $p^{2}$ distinct marks. If $s>p^{2}$, there exists a mark $u_{3}$ not of the form $c_{1} u_{1}+c_{2} u_{2}$, so that

$$
c_{1} u_{1}+c_{2} u_{2}+c_{3} u_{3} \quad\left(c_{1}, c_{2}, c_{3}=0,1, \ldots, p-1\right)
$$

gives $p^{3}$ distinct marks. Proceeding similarly, we must ultimately obtain all the marks of the $F[s]$ expressed by the formula

$$
\left.c_{1} u_{1}+c_{2} u_{2}+\cdots+c_{n} u_{n} \text { (every } c_{i}=0,1, \ldots, p-1\right),
$$

not two of these $p^{n}$ expressions being equal. Hence $s=p^{n}$.

Definition. - A set of marks $u_{1}, u_{2}, \ldots, u_{k}$ are said to be linearly independent with respect to the included field $F[p]$, if the equation

$$
c_{1} u_{1}+c_{2} u_{2}+\cdots+c_{k} u_{k}=0,
$$

where the $c$ 's are marks of the $F[p]$, can be satisfied only when every $c_{i}=0$.

Definition. - A rational integral function of any number of indeterminates $X_{1}, X_{2}, \ldots, X_{k}$ is said to belong to a field if its coefficients are marks of that field. It is irreducible in the field if it is not identically the product of two or more functions belonging to the field, each function involving some of the indeterminates $X_{i}$. An equation between functions belonging to a field is said to belong to the field.

11. Theorem. - Any mark $u$ of the $F\left[s=p^{n}\right]$ satisfies an equation of degree $k \overline{<} n$,

$$
\sum_{i=0}^{k} c_{i} X^{i}=0, \quad\left(c_{k} \neq 0\right)
$$

belonging to and irreducible in the $F[p]$.

Indeed, a linear relation with coefficients belonging to the $F[p]$ certainly holds between any $n+1$ marks of the $F\left[p^{n}\right]$ and hence between

$$
u^{0}, u^{1}, u^{2}, \ldots, u^{n} \text {. }
$$

If such a relation holds between the first $k+1$ of these powers of $u$, $u$ satisfies an equation of degree $k$. 
12. Let $u$ be any mark $\neq 0$ of the $F\left[s=p^{n}\right]$. The marks

$$
u^{t} \quad(t=0, \pm 1, \pm 2, \ldots)
$$

belonging to our finite field are not all distinct. From $u^{r}=u^{s}$, we derive $u^{r-s}=1$. The least positive integer $e$ for which $u^{e}=1$ is called the period of the mark $u$, while $u$ is said to belong to the exponent $e$. The marks $1, u, u^{2}, \ldots, u^{e-1}$ are all distinct.

We may form a rectangular array of the marks $\neq 0$ of the field as follows:

$$
\begin{aligned}
& 1 u \quad u^{2} \quad \ldots u^{e-1} \\
& u_{1} u u_{1} u^{2} u_{1} \ldots u^{e-1} u_{1}
\end{aligned}
$$

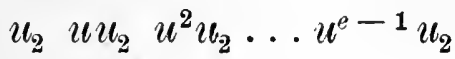

where $u_{1}$ is any mark $\neq 0$ not occurring in the first line, $u_{2}$ any mark $\neq 0$ not in the first or second lines, etc. Evidently the marks in any line are different from each other and from those in the preceding lines. Since each new mark $u_{i}$ gives rise to a set of $e$ new marks, the number $p^{n}-1$ of the marks $\neq 0$ in the $F\left[p^{n}\right]$ is a multiple of $e$.

Theorem. - The period of any mark $\neq 0$ of the $F\left[p^{n}\right]$ is a divisor of $p^{n}-1$.

13. Raising $u^{\circ}$ to the power $\left(p^{n}-1\right) / e$, we have

$$
u^{p^{n}-1}=1, \quad \text { if } u \neq 0 .
$$

We have thus the following generalization of Fermat's Theorem:

Every mark of the $F\left[p^{n}\right]$ satisfies the equation

$$
X^{p^{n}}-X=0 .
$$

We have therefore the following decomposition in the $F\left[p^{n}\right]$ :

$$
X^{p^{n}}-X=\prod_{i=0}^{p^{n}-1}\left(X-u_{i}\right),
$$

$u_{i}$ running over the $p^{n}$ marks of the $F\left[p^{n}\right]$.

14. Theorem. - If two marks $u_{1}, u_{2}$ belong respectively to exponents $e_{1} e_{2}$ which are relatively prime, their product $u_{1} u_{2}$ belongs to the exponent $e_{1} e_{2}$ and the $e_{1} e_{2}$ marks

are all distinct.

$$
u_{1}^{d_{1}} u_{2}^{d_{2}} \quad\left(\begin{array}{l}
d_{1}=0,1, \ldots, e_{1}-1 \\
d_{2}=0,1, \ldots, e_{2}-1
\end{array}\right)
$$


If $u_{1} u_{2}$ has the period $t$, we have

$$
\left(u_{1} u_{2}\right)^{t e_{1}}=u_{2}^{t e_{1}}=1 \text {, }
$$

whence $t$ is divisible by $e_{2}$; similarly, $t$ is divisible by $e_{1}$. But

Hence $t=e_{1} e_{2}$.

$$
\left(u_{1} u_{2}\right)^{e_{1} e_{2}}=1 \text {. }
$$

15. We prove as in algebra the theorem:

An equation of degree $k$ belonging to a field has in the field at most $k$ roots, unless it be an identity, when every mark of the field is a root.

16. Theorem. - For every divisor $d$ of $s-1$, the equation

$$
X^{d}-1=0
$$

has in the $F\left[s=p^{n}\right]$ exactly d roots.

Setting $s-1=d q$, we have the identity

$$
X^{s-1}-1 \equiv\left(X^{d}-1\right)\left(X^{d(q-1)}+X^{d(q-2)}+\cdots+X^{d}+1\right) \text {. }
$$

Since the last factor belongs to the $F[s]$ and does not vanish for the mark zero, it vanishes for at most $d(q-1)$ marks of the field. But the left side of the identity vanishes for $s-1$ marks of the field. Hence the factor $X^{d}-1$ must vanish for at least $d$ marks.

17. Decompose $p^{n}-1$ into its prime factors,

$$
p^{n}-1=p_{1}^{h_{1}} p_{2}^{h_{2}} \ldots p_{k}^{h_{k}} .
$$

For each integer $i$ of the series $1,2, \ldots, k$, the equation

$$
X^{p_{i}^{h_{i}}}-1=0
$$

has by $\S 16$ exactly $p_{i}^{h_{i}}$ roots belonging to the $F\left[s=p^{n}\right]$. Of these roots $p_{i}^{h_{i}-1}$ are also roots of the equation

$$
X^{p_{i} i_{i}^{-1}}-1=0
$$

and thus belong to exponents less than $p_{i}^{h_{i}}$. The remaining roots $u_{i}$, in number

$$
p_{i}^{h_{i}}-p_{i}^{h_{i}-1}=p_{i}^{h_{i}}\left(1-\frac{1}{p_{i}}\right)
$$

belong to the exponent $p_{i}^{h_{i}}$ itself. Any product of the form

$$
w=u_{1} u_{2} \ldots u_{k}
$$

will by $\S 14$ belong to the exponent $p^{n}-1$. Forming in every possible way the product $w$, we obtain ${ }^{1}$ )

1) This number equals $\Phi\left(p^{n}-1\right)$, where $\Phi(t)$ denotes the number of integers less than and relatively prime to the positive integer $t$. See Dirichlet, Vorlesungen über Zahlentheorie, § 11. 


$$
\prod_{i=1}^{k} p_{i}^{h_{i}}\left(1-\frac{1}{p_{i}}\right)
$$

such marks. Each mark $w$ belonging to the exponent $s-1$ is called a primitive root of the equation

$$
X^{s-1}-1=0
$$

and also a primitive root of the $F[s]$. Since the powers $w^{1}, w^{2}, \ldots, w^{s-1}$ are all distinct, we may state the theorem:

The $p^{n}-1$ marks $\neq 0$ of the $F\left[s=p^{n}\right]$ are the $p^{n}-1$ successive powers of a primitive root of that field.

Corollary. If $d$ be any divisor of $p^{n}-1$, the mark $w^{\left(p^{n}-1\right) / d}$ belongs to the exponent $d$.

18. We may now recognize in our $F[s]$ the abstract form of a Galois Field of order $s=p^{n}$. Indeed, by $\S 11$, the primitive root $w$ satisfies an equation of degree $k \overline{<} n$.

$$
W_{k}(x)=0,
$$

belonging to and irreducible in the $F[p]$. Every mark $\neq 0$ of the $F[s]$, being a power of $w$, can be reduced by the identity $W_{k}(w) \equiv 0$ to the form

$$
c_{1} w^{k-1}+c_{2} w^{k-2}+\cdots+c_{k-1} w+c_{k},
$$

where the $c$ 's belong to the $F[p]$. The mark zero evidently falls under this form. Since, inversely, every one of these $p^{k}$ expressions is a mark of the $F[s]$, we must have $k=n$. Hence every mark of the $F\left[s=p^{n}\right]$ represents a class of residues moduli $p$, a prime, and $W_{n}(x)$, a function with integral coefficients irreducible modulo $p$. Every existent field is therefore the abstract form of a Galois Field.

Suppose there could exist a second field $F^{\prime \prime}\left[p^{n}\right]$ of order equal to that of $F\left[p^{n}\right]$. The field $F\left[p^{n}\right]$ possesses a primitive root $w$ satisfying an equation $W_{n}(x)=0$, of degree $n$, belonging to and irreducible in the $F[p]$. The function $W_{n}(x)$ divides $x^{p^{n}}-x$ in the $\left.F[p]^{1}\right)$. We may, indeed, apply in the $F[p]$ Euclid's process for finding the greatest common divisor of these functions. If there were no common factor, we would ultimately reach as a remainder a constant, whereas the process may be interpreted in the $G F\left[p^{n}\right]$, in which field the common factor $x-w$ exists. Hence $W_{n}$ and $x^{p^{n}}-x$ have a common factor in the $F[p]$. Moreover, $W_{n}$ is irreducible in that field.

Since $F[p]$ is contained in $F^{\prime}\left[p^{n}\right]$, the division of $x^{p^{n}}-x$ by $W_{n}$ is, à fortiori, possible in the $F^{\prime}\left[p^{n}\right]$. It follows from $\S 13$ that

1) Another proof is given in $\S 23$. 
the equation $W_{n}(x)=0$ completely decomposes in the $F^{\prime}\left[p^{n}\right]$. Any one of its roots $w^{\prime}$ is a primitive root in the $F^{\prime}\left[p^{n}\right]$. Indeed, by its definition, $W_{n}(x)$ does not divide $x^{e}-x$ in the $F[p]$ for $e<p^{n}$. The powers of $w^{\prime}$ therefore give all the marks of the $F^{\prime}\left[p^{n}\right]$. Hence $F\left[p^{n}\right]$ and $F^{\prime}\left[p^{n}\right]$ are abstract forms of the same Galois Field. These results, first proven by Moore (loc. cit.), may be stated as follows:

Theorem. - Every existent field of finite order s may be represented as a Galois Field of order $s=p^{n}$. The $G F\left[p^{n}\right]$ is defined uniquely by its order; in particular, it is independent of the special irreducible congruence used in its construction.

\section{CHAPTER II.}

\section{PROOF OF THE EXISTENCE OF THE GF[ $\left.p^{n}\right]$ FOR EVERY}

\section{PRIME $p$ AND INTEGER $m$.}

19. The next step is to prove the existence, for every prime number $p$ and positive integer $m$, of a congruence of degree $m$ irreducible modulo $p$, from which will follow the existence of the $G F\left[p^{m}\right]$. We will, however, make a more general investigation, taking as our basis a fixed $G F\left[p^{n}\right]$ (in its abstract form), whose existence is supposed known. We will prove that functions belonging to and irreducible in the $G F\left[p^{n}\right]$ exist for every integer $m$ and will determine their number. Since the $G F[p]$, the field of integers taken modulo $p$, is known to exist, we shall have proven (taking $n=1$ ) the existence, for every value of $m$, of functions belonging to and irreducible in the $G F[p]$, i. e., irreducible modulo $p$.

At the same time, we shall have deduced some important properties of the $G F\left[p^{n m}\right]$ with respect to the included field, the $G F\left[p^{n}\right]$.

20. Theorem. - If two functions $F(x)$ and $P(x)$ belonging to the $G F\left[p^{n}\right]$ have in the field no common divisor containing $x$, we can determine two functions $F^{\prime}(x)$ and $P^{\prime}(x)$, belonging to the $G F\left[p^{n}\right]$ such that

$$
F^{\prime}(x) \cdot F(x)-P^{\prime}(x) \cdot P(x)=1 .
$$

The proof is quite analogous to that of $\S 7$.

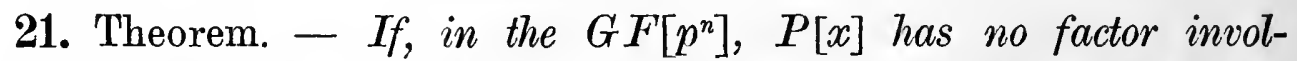
ving $x$ in common with $F(x)$ but divides the product $E(x) \cdot F(x)$, then $P(x)$ divides $E(x)$ in the $G F\left[p^{n}\right]$.

Indeed, by multiplying the given equation

$$
E(x) \cdot F(x)=P(x) \cdot S(x)
$$

by $F^{\prime}(x)$, determined as in $\S 20$, we find

$$
E(x)=P(x)\left[S(x) \cdot F^{\prime}(x)-E(x) \cdot P^{\prime}(x)\right] .
$$


22. Theorem. - A function $E(x)$ belonging to the $G F\left[p^{n}\right]$ can be decomposed into factors belonging to and irreducible in the $G F\left[p^{n}\right]$ in a single way.

For if

$$
E(x)=f_{1} f_{2} \ldots f_{h}=F_{1} F_{2} \ldots F_{k},
$$

where $f_{i}(x)$ and $F_{i}(x)$ are irreducible, $F_{1}$ must by $\S 21$ divide one of the factors $f_{i}$, and, since the latter are irreducible, be identical (apart from a factor independent of $x$ ) with one of them, say $f_{1}$. Proceeding similarly with the equality

$$
f_{2} f_{3} \ldots f_{h}=F_{2} F_{3} \ldots F_{k},
$$

we may suppose $f_{2}=F_{2}$, etc. In particular, $h=k$.

23. Theorem. - Every function $F(x)$ of degree $m$ belonging to and irreducible in the $G F\left[p^{n}\right]$ divides

$$
x^{p^{n m}}-x \text {. }
$$

Upon dividing any function $E(x)$ belonging to the $G F\left[p^{n}\right]$ by $F(x)$, we obtain a residue of the form

$$
a_{0}+a_{1} x+a_{2} x^{2}+\cdots+a_{m-1} x^{m-1}
$$

the $a$ 's being marks of the $G F\left[p^{n}\right]$. We denote the $p^{n m}$ distinct residues of the above form by

$$
X_{i} \quad\left(i=0,1, \ldots, p^{n m}-1\right),
$$

and, in particular, by $X_{0}$ the residue zero. Consider the products by a fixed residue $X_{j} \neq X_{0}$,

$$
X_{j} X_{i} \quad\left(i=0,1, \ldots, p^{n m}-1\right) .
$$

By the theorem of $\S 21$, the products 6 ) are all distinct and different from $X_{0}$. Hence the residues obtained on dividing them by $F(x)$ must coincide apart from their order with the residues 5). Forming the products of the residues not zero in each series,

$$
\prod_{i=1}^{n m}\left(X_{j} X_{i}\right) \equiv \prod_{i=1}^{p n} X_{i} \quad[\bmod F(x)]
$$

Since $\Pi X_{i} \equiv \equiv$, we have by $\S 21$,

$$
X_{j}^{p^{n m}-1}-1 \equiv 0 \quad[\bmod F(x)] .
$$

Taking for $X_{j}$ the particular residue $x$, the proof of the theorem follows.

24. Theorem. - If $f(x)$ belongs to the $G F\left[p^{n}\right]$, we have, for every integer $t$, the following identity in the field:

$$
f\left(x^{p^{n t}}\right)=[f(x)]^{p^{n t}}
$$


Let

$$
f(x)=c_{0}+c_{1} x+c_{2} x^{2}+\cdots+c_{k} x^{k}
$$

where the $c$ 's belong to the $G F\left[p^{n}\right]$, so that

$$
c_{i}^{p^{n}}=c_{i} \quad(i=0, \dot{1}, \ldots, l i) .
$$

Raising $f(x)$ to the power $p$ and noting that the multinomial coefficients of the product terms (viz., those not $p^{\text {th }}$ powers) are multiples of $p$, we have the algebraic identity,

$$
[f(x)]^{p}=c_{0}^{p}+c_{1}^{p} x^{p}+\cdots+c_{k}^{p} x^{k p}+p \cdot Q_{1}(x) .
$$

We obtain by induction the formula

$$
[f(x)]^{p^{s}}=c_{0}^{p^{s}}+c_{1}^{p^{s}} x^{p^{s}}+\cdots+c_{k}^{p^{s}} x^{k p^{s}}+p \cdot Q_{s}(x) .
$$

Applying 7), we obtain in the $G F\left[p^{n}\right]$ the identity:

$$
[f(x)]^{p^{n}}=c_{0}+c_{1} x^{p^{n}}+\cdots+c_{k} x^{k p^{n}}=f\left(x^{p^{n}}\right) .
$$

Our theorem now follows by a simple induction.

25. Theorem. - A finction $\boldsymbol{F}(x)$ of degree $m$ belonging to and irreducible in the $G F\left[p^{n}\right]$ divides (in the field) the function

$$
x^{p^{n \iota}}-x
$$

only when the integer $t$ is a multiple of $m$.

Let $t=s m+r$, where $0 \overline{<} r<m$. By the theorem of $\S 23$, we have

$$
x^{p^{n t}}-x=\left(x^{p^{n s m}}\right) p^{n r}-x \equiv x^{p^{n r}}-x \quad[\bmod F(x)] .
$$

Hence, if $x^{p^{n t}}-x$ be divisible by $F(x)$ in the $G F\left[p^{n}\right]$, we have

$$
x^{p^{n r}} \equiv x \quad[\bmod F(x)]
$$

Denote by $f(x)$ any one of the $p^{n m}$ expressions

$$
c_{0}+c_{1} x+c_{2} x^{2}+\cdots+c_{m-1} x^{m-1}
$$

in which the $c$ 's are marks of the $G F\left[p^{n}\right]$. By $\$ 24$, we derive from 8)

$$
[f(x)]^{p^{n r}}=f\left(x^{p^{n r}}\right) \equiv f(x) \quad[\bmod F(x)] .
$$

Hence the congruence

$$
\xi p^{n r} \equiv \xi \quad[\bmod F(x)]
$$

is satisfied by the $p^{n m}$ expressions $f(x)$, which are distinct modulo $F(x)$, the latter being an irreducible function of degree $m$. Since $r<m$, it follows from $\S 15$ that the congruence must be an identity, whence $r=0$.

26. The number $N_{m, y^{n}}$ of functions $F(x)$ of degree $m$ belonging to and irreducible in the $G F\left[p^{n}\right]$ may now be readily determined. For brevity, such an irreducible quantic will be designated an $I Q\left[m, p^{n}\right]$. 
It is to be understood throughout the investigation that all our operations upon quantics are performed in the $G F\left[p^{n}\right]$. We may therefore state the results of $\S \S 23$ and 25 as follows:

An IQ $\left[m_{1}, p^{n}\right]$ is a divisor of $x^{p^{n m}}-x$ if, and only if, $m_{1}$ be a divisor of $m$.

It follows that an irreducible factor of $x^{p^{n n t}}-x$ will be of degree $m$ if, and only if, it is a factor of none of the functions

$$
x^{p^{n m_{1}}}-x \quad\left(m_{1}<m, m_{1} \text { a divisor of } m\right) .
$$

After showing that the irreducible factors of any such function are all distinct, it will follow that, if we divide $x^{p^{n m_{t}}}-x$ by the product of all the distinct irreducible factors of the expressions 9), we obtain a quotient $V_{m, p^{n}}$ which equals the product of all the $I Q\left[m, p^{n}\right]$.

For example, if $m$ be prime, the irreducible factors of $x^{p^{n n}}-x$ are of degree $m$ or 1 . By $\S 13$, the product of the distinct linear factors is $x^{p^{n}}-x$. Hence, if $m$ be prime,

$$
V_{m, p^{n}}=\frac{x p^{n m}-x}{x p^{n}-x}, \quad N_{m, p^{n}}=\frac{p^{n m}-p^{n}}{m} .
$$

We next prove that the irreducible factors of $x^{p^{n m}}-x$ are all distinct. If such a factor be of degree $m$, it can be used to define the $\left.G F\left[p^{n m}\right]^{1}\right)$. In this field the equation

$$
x^{p^{n m}}-x=0
$$

has $p^{n n_{t}}$ distinct roots; viz., the marks of the field. Hence no factor can be a multiple factor in this field and therefore not in the included field the $G F\left[p^{n}\right]$. If an irreducible factor $f$ be of degree $m_{1}<m$, it cannot be a multiple factor. Indeed, $m_{1}$ must be a divisor of $m$, and $f$ must divide $x^{p^{n m_{1}}}-x$ in the $G F\left[p^{n}\right]$. By the former case, $f$ is a simple factor of the expression just given. It remains to prove that $f$ cannot divide the quotient

$$
Q \equiv\left(x^{p^{n m}}-x\right) /\left(x^{x^{n n_{1}}}-x\right)
$$

It suffices to show that $Q$ and $x^{p^{n m_{1}}}-x$ have no common factor in the $G F\left[p^{n}\right]$. Setting

$$
y \equiv x^{p^{n m}-1} y \equiv x^{n m_{1}}[1], r \equiv \frac{p^{n m}-1}{p^{n m_{1}}-1}, \text { not in prover. } \Omega \equiv
$$

it suffices to prove that $y-1$ and

$$
\frac{y^{r}-1}{y-1} \equiv y^{r-1}+\cdots+y+1
$$

have no common factor. The condition for a common divisor is that $r$ be the mark zero in the field. But $r \equiv 1(\bmod p)$.

1) See $\S 28$.

DICKsoN, Linear Groups. 
2\%. Continuing the investigation, let

$$
m \equiv q_{1}^{r_{1}} q_{2}^{r_{2}} \ldots \dot{q}_{s}^{r_{s}}
$$

$q_{1}, q_{2}, \ldots, q_{s}$ being the distinct prime factors of $m$. For brevity, we use the symbol

$$
[t] \equiv x^{p^{n t}}-x \text {. }
$$

We proceed to prove the formula, due to Dedekind for $n=1$,

In this expression, the term

$$
V_{m, p^{n}}=\frac{[m] \prod\left[\frac{m}{q_{i} q_{j}}\right] \prod\left[\frac{m}{q_{i} q_{j} q_{k} q_{l}}\right] \ldots}{\prod\left[\frac{m}{q_{i}}\right] \prod\left[\frac{m}{q_{i} q_{j} q_{k}}\right] \ldots}
$$

$$
\Pi_{k} \equiv \Pi\left[\frac{m}{q_{i_{1}} q_{i_{2}} \ldots q_{i_{k}}}\right]
$$

in which the product extends over the $C_{s, k}$ combinations $q_{i_{1}}, \ldots, \ddot{q}_{i_{k}}$ of the integers $q_{1}, \ldots, q_{s}$ taken $k$ together, occurs in the numerator or in the denominator according as $k$ is even or odd. Each $I Q\left[m, p^{n}\right]$ occurs once as a factor in $\Pi_{0} \equiv[m]$ but divides no other $\Pi_{k}$; it is therefore a simple factor of the fraction. If there be any factor of the fraction having the degree $m_{1}<m$, we denote it by $F(x)$. By $\S 25, m_{1}$ must be a divisor of $m$. Denote by $q_{1}, q_{2}, \ldots, q_{s_{1}}$ the prime factors entering in $m$ to a higher power than in $m_{1}$. Then $m_{1}$ divides $\frac{m}{q_{1} q_{2} \ldots q_{s_{1}}}$ but not $\frac{m}{q_{j}}\left(j=s_{1}+1, s_{1}+2, \ldots, s\right)$. It follows that, if $k>s_{1}, \Pi_{k}$ does not contain $F(x)$ of degree $m_{1}$; while, for $k \overline{<}$, $\Pi_{k}$ contains $F(x)$ as often as $k$ integers can be selected from $q_{1}, q_{2}, \ldots, q_{s_{1}}$; viz., $C_{s_{1}, k}$ times. Hence $F(x)$ occurs in the numerator and denominator of our fraction to the respective degrees,

$$
1+C_{s_{1}, 2}+C_{s_{1}, 4}+\cdots, \quad C_{s_{1}, 1}+C_{s_{1}, 3}+C_{s_{1}, 5}+\cdots
$$

These numbers are equal, since their difference equals $(1-1)^{s_{1}} \equiv 0$. It follows that every irreducible factor of our expression is an $I Q\left[m, p^{n}\right]$. The number of the latter multiplied by the degree $m$ of must equal the degree of the fraction, so that

$$
N_{m, p^{n}}=\frac{1}{m}\left[p^{n m}-\sum p^{\frac{n m}{q_{1}}}+\sum p^{\frac{n m}{\eta_{1} q_{2}}}-\cdots+(-1)^{s} p^{\frac{n m}{q_{1} q_{2} \cdots q_{s}}}\right] .
$$

This number cannot be zero; for, upon dividing by the last term, which is the lowest power of $p$ entering into the expression, we would then obtain unity expressed as the algebraic sum of a series of powers of the prime number $p$ with exponents $>1$. It follows that the number of $I Q\left[m, p^{n}\right]$ is $>1$. [See Ex. 2 below].

28. Let $F(x)$ be an $I Q\left[m, p^{n}\right]$. As in $\S 6$, the totality of rational functions of $x$ belonging to the $G F\left[p^{n}\right]$ can be separated into $p^{n m}$ 
distinct classes of residues modulo $F(x)$, each being represented by one of the $p^{n m}$ residues

$$
\left.a_{0}+a_{1} x+a_{2} x^{2}+\cdots+a_{m-1} x^{m-1} \quad\left(a \text { 's in the GF[ } p^{n}\right]\right) \text {. }
$$

Proceeding as in $\S 6$, we find that these classes of residues form the $G F\left[p^{n m}\right]$. We can therefore construct the $G F\left[p^{r}\right]$ in as many ways as we can express $r$ as the product of two positive integers $n, m$; viz., by using an $I Q\left[m, p^{n}\right]$. From the theorem at the beginning of $\S 26$ it follows that the $G F\left[p^{n m_{1}}\right]$ is contained in the $G F\left[p^{n m}\right]$ if, and only if, $m_{1}$ divides $m$.

\section{EXERCISES.}

Ex. 1. Granting the existence of the $G F\left[p^{n}\right]$, the existence of the $G F\left[p^{n q}\right], q$ being prime, follows by $\S 26$. By induction, the $G F\left[p^{r}\right]$ exists for $r$ arbitrary.

Ex. 2. Obtain for the number of $I Q\left[m, p^{n}\right]$ given in $\S 27$ the following limits:

$$
\frac{p^{n m}-p^{n}}{m}>N_{m, p^{n}}>\frac{\Phi(m)}{m-1} \cdot \frac{p^{n m}-p^{n}}{m} .
$$

Hint: Expand each power of $p^{n}$ into a series in $\log p^{n}$ and apply

$$
\Phi(m) \equiv m\left(1-\frac{1}{q_{1}}\right)\left(1-\frac{1}{q_{2}}\right) \cdots\left(1-\frac{1}{q_{3}}\right) .
$$

Ex. 3. By decomposing modulo 2 the expression $\left(x^{2^{4}}-x\right) /\left(x^{2^{2}}-x\right)$, obtain the three $I Q[4,2]$ given in the left members below. Defining the $G F\left[2^{2}\right]$ by means of the irreducible congruence

$$
i^{2}+i+1 \equiv 0 \quad(\bmod 2),
$$

obtain the six $I Q\left[2,2^{2}\right]$ by means of the following decompositions:

$$
\begin{aligned}
& x^{4}+x+1 \equiv\left(x^{2}+x+i\right)\left(x^{2}+x+i^{2}\right), \\
& x^{4}+x^{3}+1 \equiv\left(x^{2}+i x+i\right)\left(x^{2}+i^{2} x+i^{2}\right), \\
& x^{4}+x^{3}+x^{2}+x+1 \equiv\left(x^{2}+i x+1\right)\left(x^{2}+i^{2} x+1\right) .
\end{aligned}
$$

\section{CHAPTER III.}

\section{CLASSIFICATION AND DETERMINATION OF IRREDUCIBLE QUANTICS.}

29. Definition. - An $I Q\left[m, p^{n}\right]$, as $F(x)$, is said to belong to an exponent $e$ if $e$ be the least positive integer for which $F(x)$ divides $x^{s}-1$ in the $G F\left[p^{n}\right]$. [Compare $\left.\S 32.\right]$ 
The exponent e to which $F(x)$ belongs must divide $p^{n m}-1$.

For, if

$$
p^{n m}-1=k e+r
$$

where $0 \overline{<} r<e$, then $F(x)$, dividing $x^{e}-1$, must divide $x^{k e}-1$ and, by $\S 23$, also $x^{k e+r}-1$. It must therefore divide their difference,

Hence must $r$ be zero.

$$
x^{k e}\left(x^{r}-1\right) \text {. }
$$

Furthermore, $e$ must not divide $p^{n t}-1$, for $t<m$; for, if so, $x^{s}-1$ and hence also $F(x)$ would divide $x^{p^{n t}}-x$, so that the degree of $F(x)$ would be a divisor of $t$.

An integer which divides $a^{m}-1$ but not $a^{t}-1, t<m$, is said to be a proper divisor of $a^{m}-1$. We may state the result:

The exponent to which an $I Q\left[m, p^{n}\right]$ belongs is a proper divisor of $\left(p^{n}\right)^{m}-1$.

30. Theorem. - The number $N_{m, p^{n}}^{(e)}$ of $I Q\left[m, p^{n}\right]$ which belong to an exponent $e$, a proper divisor of $\left(p^{n}\right)^{m}-1$, is $\Phi(e) / m$.

Let $q_{1}, q_{2}, \ldots, q_{s}$ be the distinct prime factors of $e$. Proceeding as in $\S 26$, we rid $x^{e}-1$ of those of its factors which are irreducible in the $G F\left[p^{n}\right]$ and belong to an exponent $<e$. We obtain the expression

$$
\frac{\left(x^{e}-1\right) \Pi\left(x^{\frac{e}{q_{i} q_{j}}}-1\right.}{\Pi\left(x^{\frac{e}{q i}}-1\right) \Pi\left(x^{\frac{e}{q_{i} q_{j} q_{k}}}-1\right) \ldots}
$$

which is therefore the product of the irreducible factors of $x^{s}-1$ belonging to the exponent $e$. Each of them is an irreducible factor of

$$
x^{p^{n m}}-x
$$

and hence of degree $m$ or a divisor of $m$. Since each belongs to an exponent which is a proper divisor of $\left(p^{n}\right)^{m}-1$, the degree must be $m$.

The degree of the above function is clearly

$$
\begin{gathered}
\quad e-\sum \frac{e}{q_{i}}+\sum \frac{e}{q_{i} q_{j}}-\sum \frac{e}{q_{i} q_{j} q_{k}}+\cdots+(-1)^{s} \frac{e}{q_{1} q_{2} \ldots q_{s}} \\
=e\left(1-\frac{1}{q_{1}}\right)\left(1-\frac{1}{q_{2}}\right) \cdots\left(1-\frac{1}{q_{s}}\right)=\Phi(e) . \\
\text { Hence } \quad m \cdot N_{m, p^{n}}^{(e)}=\Phi(e) .
\end{gathered}
$$

31. Theorem. - If $\boldsymbol{F}(x)$ and $\varphi(x)$ belong to and are irreducible in the $G F\left[p^{n}\right]$ and are of the respective degrees $m$ and $t$, a divisor of $m$, the roots of the congruence

$$
\text { are }
$$

$$
\begin{aligned}
& \varphi(X) \equiv 0 \quad[\bmod F(x)] \\
& X_{1}, \quad X_{1}^{p^{n}}, \quad X_{1}^{p^{2 n}}, \ldots, X_{1}^{p^{n(t-1)}}
\end{aligned}
$$

if $X_{1}$ be one root of 10 ) necessarily belonging to the $G F\left[p^{n m}\right]$. 
By $\S 24$ we have in the $G F\left[p^{n}\right]$ the identity

$$
\varphi\left(X^{p^{n r}}\right)=\{\varphi(X)\}^{n r} \text {. }
$$

Hence, if $X_{1}$ be a root of 10$)$, so is every $X_{1}^{p^{n r}}$. Since $\varphi(X)$ is an $I Q\left[t, p^{n}\right]$, we have $(\S 23)$ in the $G F\left[p^{n}\right]$,

$$
X_{1}^{p^{n t}}-X_{1} \equiv \varphi\left(X_{1}\right) \cdot \psi\left(X_{1}\right) \equiv 0 \quad[\bmod F(x)]
$$

Hence, $m$ being a multiple of $t$,

$$
X_{1}^{p^{n m}} \equiv X_{1} \quad[\bmod F(x)]
$$

We next prove that the above $t$ powers of $X_{1}$ are distinct modulo $F(x)$. Indeed, if

$$
X_{1}^{p^{n a}} \equiv X_{1}^{p^{n b}} \quad[\bmod F(x)]
$$

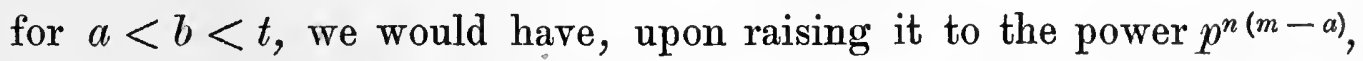

$$
X_{1}^{p^{n m}} \equiv X_{1} \equiv X_{1}^{p^{n(m+b-a)}} \quad[\bmod F(x)],
$$

so that, by $\S 25, m+b-a$ would be divisible by $m$. Hence $b=a$.

Corollary. - We have in the $G F\left[p^{n m}\right]$ the decomposition

$$
\varphi(X) \equiv\left(X-X_{1}\right)\left(X-X_{1}^{p^{n}}\right) \ldots\left(X-X_{1}^{p^{n(t-1)}}\right) .
$$

In particular, $F(x)=0$ has in the $G F\left[p^{n m}\right]$ the distinct roots

$$
x, x^{p^{n}}, \ldots, x^{p^{n(m-1)}} \text {. }
$$

32. Theorem. - If $F(x)$ be an $I Q\left[m, p^{n}\right]$ belonging to the exponent $e$, every root of $F(x)=0$ in the $G F\left[p^{n m}\right]$ belongs to the exponent $e$, and inversely.

We may define the $G F\left[p^{n m}\right]$ by means of $F(x)$. In it, all the roots of $F(x)=0$ satisfy the equation $x^{e}-1=0$, but do not all satisfy $x^{f}-1=0$ for $f<e$. But, $p^{n}$ being relatively prime to $e, a$ divisor of $p^{n m}-1$, it follows from the corollary of $\S 31$ that the roots of $F(x)=0$ in the $G F\left[p^{n m}\right]$ all belong te the same exponent. This common exponent is therefore $e$.

In particular, for $e=p^{n m}-1$, the roots of $F(x)=0$ are primitive roots in the $G F\left[p^{n m}\right]$. Such a quantic $F(x)$ will be called a primitive irreducible quantic of degree $m$ in the $G F\left[p^{n}\right]$ and will be referred to as a $P I Q\left[m, p^{n}\right]$.

33. Theorem. - If e be a prime number, the function

$$
V \equiv \frac{x^{e}-1}{x-1} \equiv x^{-1}+\cdots+x+1
$$

is irreducible with respect to every prime modulus $p$ which is a primitive root of $e$.

By hypothesis, $p$ belongs to the exponent $e-1$ modulo $e$, so that $e$ is a proper divisor of $p^{e-1}-1$. Hence, by $\S 30$ for $n=1$, $m=e-1$, the number of irreducible factors of $V$ is $\frac{\Phi(e)}{e-1}=1$. 
Note. - If $a$ be a primitive root of $e$, then $a+k e(k=0, \pm 1, \pm 2, \ldots)$ are also primitive roots of $e$. By the theorem of Dirichlet, this arithmetical progression contains an infinity of prime numbers. With respect to any such prime $p, V$ is irreducible modulo $p$. $\grave{\mathbf{A}}$ fortiori, $V$ is algebraically irreducible.

Determination of $I Q\left[m, p^{n}\right]$ whose degree $m$ contains no prime factors other than those of $p^{n}-1, \S \S 34-38$.

34. Theorem. - Let $F_{1}(x), F_{2}(x), \ldots, F_{N}(x)$ denote the $I Q\left[m, p^{n}\right]$ which belong to an exponent

$$
e \equiv\left(p^{n m}-1\right) / d,
$$

and let $\lambda$ be an integer relatively prime to $d$ and containing no prime factors other than those occurring in $p^{n m}-1$. With the exception of the case in which $\lambda$ is a multiple of 4 while $p^{n m}$ is of the form $4 l-1$,

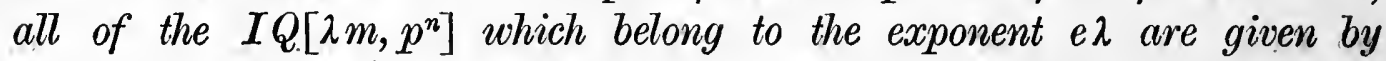
the $N$ quantics $F_{1}\left(x^{\lambda}\right), \ldots, F_{N}\left(x^{\lambda}\right)$.

By definition, $\lambda$ contains no prime factor other than those occurring in $\dot{e}$. Hence $e \lambda$ and $e$ contain exactly the same prime factors; so that

By $\S 30$, we have

$$
\frac{\Phi(e \lambda)}{e \lambda}=\frac{\Phi(e)}{e} \text {. }
$$

$$
N \equiv N_{m, p^{n}}^{(e)}=\frac{\Phi(e)}{m} \text {. }
$$

If we suppose satisfied the conditions (obtained below) under which $e \lambda$ shall be a proper divisor of $\left(p^{n}\right)^{m \lambda}-1$, we will have

$$
N_{m \lambda, p^{n}}^{(e \lambda)} \equiv \frac{\Phi(e \lambda)}{m \lambda}=\frac{\Phi(e)}{m}=N
$$

Since $e$ divides $p^{n m}-1$, the irreducible factors of $x^{e}-1$ are of degree $\overline{<} m(\S 25)$. Hence, in the notation of the theorem,

$$
x^{e}-1=F_{1}(x) F_{2}(x) \ldots F_{N}(x) \cdot Q(x)
$$

where the irreducible factors of $Q(x)$ either belong to an exponent $<e$ or else are of degree $<m$. Therefore

$$
x^{\imath}-1=F_{1}\left(x^{\lambda}\right) F_{2}\left(x^{\lambda}\right) \ldots F_{N}\left(x^{\lambda}\right) \cdot Q\left(x^{\lambda}\right),
$$

where every irreducible factor of $Q\left(x^{\lambda}\right)$ is of degree $<\lambda m$ or else belongs to an exponent $<e \lambda$. Since there are exactly $N$ irreducible factors of degree $m \lambda$ which belong to the exponent $e \lambda$, they must be identical with $F_{1}\left(x^{\lambda}\right), \ldots, F_{N}\left(x^{\lambda}\right)$.

Calling $v$ the least integer such that $p^{n v}-1$ is divisible by $e \lambda$, we seek the conditions under which $\nu=m \lambda$. Since $m$ is by hypothesis the least integer for which $p^{n m}-1$ is divisible by $e, v$ must be a multiple of $m$. For, if $v=q m+r, 0 \overline{\overline{<}} r<m$, then $e$ divides $p^{n(q m+r)}-1$ and $p^{n m q}-1$ and hence also their difference $p^{n m q}\left(p^{n r}-1\right)$, 
which requires $r=0$. Having $\nu=q m$, we inquire under what conditions does $q=\lambda$ ? Since

$$
\frac{p^{n v-1}}{e \lambda} \equiv \frac{d}{\lambda} \cdot \frac{p^{n m q}-1}{p^{n m}-1}
$$

it follows that $\lambda$ divides $\left(p^{n m q}-1\right) /\left(p^{n m}-1\right)$. Raising to the power $q$ the identity $p^{n m} \equiv 1+\left(p^{n m}-1\right)$, we find

12) $\frac{p^{n m q-1}}{p^{n m-1}} \equiv q+\frac{q(q-1)}{1 \cdot 2}\left(p^{n m}-1\right)+\cdots+C_{q, k}\left(p^{n m}-1\right)^{k-1}+\cdots\left(p^{n m}-1\right)^{1-1}$.

Let $\theta$ be a prime factor of $\lambda$ and $\theta^{a}$ the highest power of $\theta$ contained in $\lambda$. Since $\theta$ divides $p^{n m}-1$ and the left member of 12), it must divide $q$. Further, if $\theta>2, \theta^{a}$ divides $q$. Indeed, the ratio of the $k^{\text {th }}$ term of 12) to the first term $q$ can be written

$$
\frac{(q-1)(q-2) \ldots(q-k+1)}{1 \cdot 2 \ldots(k-1)} \cdot\left(\frac{p^{n m}-1}{\theta}\right)^{k-1} \cdot \frac{\theta^{k}-1}{k},
$$

of which the first two factors are integers, while the third factor

$$
{\frac{\{1+(\theta-1)\}^{k-1}}{k}}^{k} \frac{1+(k-1)(\theta-1)}{k} \equiv 1+\frac{(k-1)(\theta-2)}{k}
$$

is $>1$ if $k>2$. Hence the irreducible fraction equal to $\theta^{k-1} / k$ has the factor $\theta$ in its numerator. Hence the terms of 12) beginning with the second contain $\theta$ to a higher power than the first term $q$. Since $\theta^{a}$ divides $\lambda$, which divides the left member of 12), it follows that $\theta^{a}$ divides the first term $q$ on the right. Hence, if $\lambda$ be odd or the double of an odd number, $q$ is divisible by $\lambda$. Inversely, if $q$ be divisible by $\lambda, \lambda$ being odd or the double of an odd number, the above argument shows that the right member of 12) will contain the factor $\lambda$ and therefore that the left member of 11) will be an integer. In order that $v$ be the least integer for which this can happen, we must have $q=\lambda$.

If $\lambda$ be a multiple of $4, p^{n m}-1$ is even by hypothesis. Then $\theta=2$ will be a factor of $q$ as before. The ratio of the second term of 12) to the first term will be divisible by 2 if, and only if, $p^{n m}-1$ be a multiple of 4 ; the ratio of the $k^{\text {th }}$ term to the first will, for $k \bar{\sum} 3$, contain the factor 2. Hence, if $p^{n m}$ be of the form $4 l+1$, we can conclude that $q=\lambda$. [The case $p^{n m}=4 l-1$ leads to the entirely different theorem of $\S 36$.]

35. Let $\varrho$ be a primitive root in the $G F\left[p^{n}\right]$. The function $x-\varrho^{t}$ belongs to the exponent $\left(p^{n}-1\right) / d$ where $d$ is the greatest common divisor of $t$ and $p^{n}-1$. Applying the theorem $\S 34$ for $m=1$, we have the result:

If $\lambda$ be any integer containing no prime factor not occurring in $p^{n}-1$ and if $t$ be an integer prime to $\lambda$, the $I Q\left[\lambda, p^{n}\right]$ belonging 
to the exponent $\lambda\left(p^{n}-1\right) / d$, $d$ being the greatest common divisor of $t$ and $p^{n}-1$, are the binomials $x^{\lambda}-\rho^{t}$, the case $p^{n}=4 l-1, \lambda=4 \lambda_{1}$ being excluded.

Inversely, we obtain by this theorem every binomial irreducible in the $G F\left[p^{n}\right]$. In the first place, $\lambda$ and $t$ must have no factor in common, since otherwise $x^{\lambda}-\varrho^{t}$ would be algebraically reducible. On the other hand, if $\lambda$ contains a prime factor $\theta$, not a factor of $p^{n}-1$, we can determine $\left(\S 7\right.$, Note) an integer $\theta_{1}$, such that

$$
\theta \theta_{1} \equiv 1 \quad\left[\bmod p^{n}-1\right] \text {. }
$$

Since $\varrho^{\theta \theta_{1}}=\varrho$, it follows that $\varrho^{\theta_{1} t} \equiv \alpha$ is a root of

$$
x^{\theta}-\rho^{t}=0 \text {. }
$$

Hence $x-\alpha$ is a factor of $x^{\theta}-\varrho^{t}$, so that $x^{\lambda / \theta}-\alpha$ divides $x^{\lambda}-\varrho^{t}$.

Example. - For $p^{n}=7$, we may take $\varrho=5$. Then for $\lambda=2$ and $t=1,3,5$, we obtain the irreducible binomials $x^{2}-5, x^{2}+1$, $x^{2}-3$ belonging to the exponents $12,4,12$ respectively. For $\lambda=3$ and $t=1,2,4,5$ respectively, we obtain the binomials

$$
x^{3}-5, \quad x^{3}-4, \quad x^{3}-2, \quad x^{3}-3
$$

irreducible modulo 7 and belonging to the respective exponents 18 , $9,9,18$.

36. Theorem. - Let $p^{n}=2^{i} t-1, i \overline{>} 2, t$ odd $; \lambda=2^{j} s, j \overline{>}$, $s$ odd; let $k$ be the smaller of the integers $i$ and $j$; finally, let $m$ be odd. Then if, in the $N$ quantics $I Q\left[m, p^{n}\right]$ belonging to the exponent

$$
e=\left(p^{n m}-1\right) / d,
$$

we replace $x$ by $x^{\lambda}$, where $\lambda \equiv 2^{j}$ s is prime to $d$ and contains no prime factors other than those occurring in $p^{n m}-1$, we obtain $N$ quantics of degree $m \lambda$ each decomposing into $2^{k-1}$ quantics irreducible in the $G F\left[p^{n}\right]$, so that we obtain all of the $2^{-1} N$ quantics

belonging to the exponent $e \lambda$.

$$
\text { - } I Q\left[\frac{m \lambda}{2^{k-1}}, p^{n}\right]
$$

If $v$ denote the least integer such that $p^{n v}-1$ is divisible by $e \lambda$, we find as in $\S 34$ that $v=q m$. In the present case, $q$ is even; for, if $q$ be odd, $v$ would be odd and $p^{n v}-1$ the double of an odd number, whereas $\lambda$ is divisible by 4 . By the restrictions on $p^{n}$ and $m$,

$$
p^{n m}=2^{i} \tau-1 \quad(\tau \text { odd }) .
$$

Raising this identity to the power $q$, we find

14) $\frac{p^{n m q}-1}{p^{n m}-1}=\frac{2^{i} \tau}{2^{i} \tau-2}\left[-q+\frac{q(q-1)}{1 \cdot 2} 2^{i} \tau-\cdots \pm C_{q, b} 2^{i(l-1)} \tau^{l-1} \mp \ldots\right]$. 
The ratio of the $l^{\text {th }}$ term within the parenthesis to the first term is

$$
\mp \frac{(q-1)(q-2) \ldots(q-l+1)}{1 \cdot 2 \ldots(l-1)} \tau^{l-1} \cdot \frac{2^{i(l-1)}}{l},
$$

where the first and second factors are integers, while the third factor, being $>1$ for $l>2$, equals an irreducible fraction with an even numerator. Hence the first term contains 2 to a lower power than the remaining terms in the above parenthesis. In order that $p^{n v}-1$ shall be divisible by $e \lambda$, formula 11) requires that $\lambda$ shall divide the left member of 14). Hence $2^{j}$ must divide the first term of the right member and consequently also $2^{i-1} q$. Hence the even integer $q$ must contain 2 to the power 1 or $j-i+1$ according as $j \overline{\overline{<}} i$ or $j>i$. Furthermore, by $\S 34, q$ must contain every odd factor of $\lambda$. Hence, if $\nu$ be the least possible integer,

$$
q=\frac{\lambda}{2^{j-1}} \text { or } q=\frac{\lambda}{2^{i-1}}
$$

according as $j \overline{\overline{<}} i$ or $j>i$, i. e., according as $k=j$ or $k=i$. Hence

$$
q=\frac{\lambda}{2^{k-1}}, \quad v=\frac{m \lambda}{2^{k-1}} .
$$

As at the beginning of $\S 34$, we have

$$
N \equiv N_{m, p^{n}}^{(e)}=\frac{\Phi(e)}{m}=\frac{\Phi(e \lambda)}{m \lambda}=\frac{1}{2^{k-1}} \frac{\Phi(e \lambda)}{\nu},
$$

so that the number of $I Q\left[v, p^{n}\right]$ belonging to the exponent $e \lambda$ is $2^{k-1} N$.

By hypothesis,

$$
x^{e}-1 \equiv F_{1}(x) F_{2}(x) \ldots F_{N}(x) \cdot Q(x),
$$

where the irreducible factors of $Q(x)$ in the $G F\left[p^{n}\right]$ belong to exponents $<e$ or are of degree $<m$. The irreducible factors of $Q\left(x^{\lambda}\right)$ are therefore of degree $<\lambda m$ or else belong to exponents $<\lambda e$. Hence the irreducible factors of degree $\lambda m$ of the expression $x^{e \lambda}-1$ must, if they belong to the exponent $e \lambda$, be factors of $F_{1}\left(x^{\lambda}\right), \ldots, F_{N}\left(x^{\lambda}\right)$. Since the combined degree of the latter is $N m \lambda \equiv 2^{k-1} \nu N$, and since there are exactly $2^{k-1} N$ irreducible quantics of degree $v$ belonging to the exponent $e \lambda$, it follows that each $F_{i}\left(x^{\lambda}\right)$ is the product of $2^{k-1}$ irreducible quantics of degree $\nu$.

Corollary. - Since the distinct functions of degree $m=1$ which belong to the exponent $e=\left(p^{n}-1\right) / d$ are given by the formula

$$
x-\varrho^{a d} \text {, }
$$

o being a fixed primitive root in the $G F\left[p^{n}\right]$ and $a$ being any integer prime to $e$, it follows that $x^{\lambda}-\varrho^{a d}$ decomposes in the $G F\left[p^{n}\right]$ into $2^{k-1}$ irreducible factors of degree $\lambda / 2^{k-1}$ belonging to the exponent 
$e \lambda$, provided $p^{n}$ and $\lambda$ are subject to the conditions given in the main theorem.

3\%. Since irreducible binomials are lacking in the case treated in the last section, we proceed to set up trinomial $I Q\left[\lambda, p^{n}\right]$. It is, however, not necessary to suppose that $\lambda$ is a multiple of 4 . We suppose merely that

$$
p^{n}=2^{i} t-1 \quad(t \text { odd }, i>2)
$$

and that $\lambda$ is an even integer containing no prime factor not occurring in $p^{n}-1$. Set

$$
v=2^{i-1} \lambda,
$$

so that $v$ is divisible by $2^{i}$. If $\varrho$ be a primitive root in the $G F\left[p^{n}\right]$ and if $s$ be any integer prime to $\lambda$ and hence also to $v$, then $x-\rho^{s}$ belongs to the exponent $\left(p^{n}-1\right) / d$, where $d$ is the greatest common divisor of $s$ and $p^{n}-1$, and $v$ is prime to $d$. Hence ( $\left.\S 36\right)$, the binomial $x^{v}-\rho^{s}$ decomposes into $2^{i-1}$ irreducible quantics of degree $\lambda$. We proceed to determine them.

Since $2^{i-1}$ and $\left(p^{n}-1\right) / 2$ are relatively prime, we can determine (§ 7, Note) two integers $l_{1}$ and $h_{1}$ such that

$$
l_{1} 2^{i-1}-h_{1}\left(p^{n}-1\right) / 2=1 \text {. }
$$

Multiplying this equation by the even integer $s+\left(p^{n}-1\right) / 2$, we obtain two integers $l$ and $h$ for which

$$
l 2^{i}-h\left(p^{n}-1\right)=s+\left(p^{n}-1\right) / 2 \text {. }
$$

Since the $\left(p^{n}-1\right) / 2$ power of the primitive root $\rho$ is -1 , we have

$$
x^{\nu}-\varrho^{s} \equiv x^{22^{i-1}}+\varrho^{l 2^{i}} .
$$

In the GF[ $\left[p^{n}\right]$ we have the decomposition

$$
x^{\lambda 2^{i-1}}+\varrho^{l 2^{i}} \equiv \prod_{j=1}^{2^{i-1}}\left(x^{\lambda}-\xi_{j} \varrho^{l} x^{\lambda / 2}-\varrho^{2 l}\right),
$$

where the $\xi_{j}$ are marks of the GF[ $\left.p^{n}\right]$ determined as the roots of the equation

$$
E(\xi) \equiv \xi^{i-1}+2^{i-1} \sum_{k=1}^{2^{i}-2-1} \frac{\left(2^{i-1}-k-1\right) !}{k !\left(2^{i-1}-2 k\right) !} \xi^{2^{i-1}-2 k}+2=0 .
$$

In fact, by Waring's formula $\left.{ }^{1}\right)$, the sum of the $\left(2^{i-1}\right)^{\text {st }}$ powers of the roots $u$ and $\frac{-1}{u}$ of the quadratic

$$
X^{2}-\xi X-1=0
$$

is found to be $E(\xi)$. Expressed otherwise, if $\xi \equiv u-\frac{1}{u}$, then

$$
u^{2^{i-1}}+u^{-2^{i-1}}=E(\xi) \text {. }
$$

1) Serret, Cours d’Algèbre Supérieure, I, p. 449. 
Hence, if $\xi_{j} \equiv u_{j}-\frac{1}{u_{j}}$ is a root of $E(\xi)=0$, we have

$$
u_{j}^{2^{i}}+1=0 \text {. }
$$

Then, since $p^{n}+1=2^{i} t, t$ odd, we have

$$
u_{j}^{p^{n}+1}+1=0, \quad u_{j}^{p^{n}}=-1 / u_{j} .
$$

Applying $\S 24$, we have modulo $p$,

$$
\xi_{j}^{n} \equiv\left(u_{j}-\frac{1}{u_{j}}\right)^{p^{n}} \equiv u^{p^{n}}-1 / u_{j}^{p^{n}}=-\frac{1}{u_{j}}+u_{j} \equiv \xi_{j},
$$

so that every $\operatorname{root}^{1}$ ) of $E(\xi)=0$ belongs to the $G F\left[p^{n}\right]$. Hence

$$
u^{2^{i-1}}+u^{-2^{i}-1} \equiv \prod_{j=1}^{2^{i-1}}\left(\xi-\xi_{j}\right)
$$

Substituting in this identity

$$
u=\frac{x^{2 / 2}}{\rho^{\prime}}, \quad \xi \equiv u-\frac{1}{u}=\frac{x^{2}-\rho^{2 l}}{\varrho^{l} x^{\lambda / 2}},
$$

and clearing the equation of fractions, we obtain formula 15).

38. As a simple example, let $p^{n}=7=2^{3}-1, \lambda=4$. The binomials

$$
x^{16}-\check{5}^{s} \quad(s=1,3,5)
$$

can be readily decomposed into irreducible quartics. The congruence

$$
E(\xi) \equiv \xi^{4}+4 \xi^{2}+2 \equiv 0 \quad(\bmod 7)
$$

has the roots \pm 1 and \pm 3 . Further

$$
x^{16}-5^{s} \equiv x^{16}+5^{s+3}=x^{16}+5^{2 l} \quad(s+3=2 l=4,6,8) .
$$

Since $\tilde{5}^{l \cdot 2^{3}} \equiv \tilde{5}^{2 l}(\bmod 7)$, equation 15$)$ becomes

$$
x^{16}+5^{2 l} \equiv \prod_{j=1}^{4}\left(x^{4}-\xi_{j} 5^{l} x^{2}-\tilde{5}^{2 l} \quad(\bmod 7),\right.
$$

holding for $l=4,2,3$. Taking each in turn, we have modulo 7:

$$
\begin{aligned}
& x^{16}+4 \equiv\left(x^{4}-x^{2}-4\right)\left(x^{4}+x^{2}-4\right)\left(x^{4}-2 x^{2}-4\right)\left(x^{4}+2 x^{2}-4\right), \\
& x^{16}+2=\left(x^{4}-2 x^{2}-2\right)\left(x^{4}+2 x^{2}-2\right)\left(x^{4}-4 x^{2}-2\right)\left(x^{4}+4 x^{2}-2\right), \\
& x^{16}+1=\left(x^{4}-x^{2}-1\right)\left(x^{4}+x^{2}-1\right)\left(x^{4}-4 x^{2}-1\right)\left(x^{4}+4 x^{2}-1\right) .
\end{aligned}
$$

1) For another proof see Serret, Cours d'Algèbre supérieure, II, pp. 160-3. Compare \& 82 below. 
Determination and classification $\left.{ }^{1}\right)$ of the $I Q\left[p^{s}, p^{n}\right]$, $\S 39-46$.

39. Consider for positive integers $\mu$ the auxiliary quantics

16) $X_{\mu} \equiv x^{p^{n \mu}}-\mu x^{p^{n(\mu-1)}}+\cdots+(-1)^{k} C_{\mu, k} x^{p^{n(\mu-k)}}+\cdots+(-1)^{\mu} x$, where $C_{\mu, k}$ denotes the number of combinations of $\mu$ things $k$ at a time. Since $C_{p^{r}, k}$ is a multiple of $p$, if $0<k<p^{r}$, we have

$$
X_{p} \equiv x^{p^{n} p^{r}}-x \quad(\bmod p) .
$$

Hence, by $\S 26$, the product of all the $I Q\left[p^{s}, p^{n}\right]$ is given by

$$
V_{p^{s}, p^{n}} \equiv X_{p^{s}} / X_{p^{s-1}}
$$
From

We derive a simple expression for the quotient 18) as follows.

$$
C_{\mu, k}+C_{\mu, k-1}=C_{\mu+1, k},
$$

we deduce at once the congruence

$$
X_{\mu+1} \equiv X_{\mu}^{p^{n}}-X_{\mu} \quad(\bmod p) .
$$

Multiplying together the congruences (for $i=1,2, \ldots, v$ )

$$
X_{u+i} \equiv X_{u+i-1}^{p^{n}}-X_{u+i-1}(\bmod p),
$$

and dividing the resulting formula by the product

we find

$$
X_{u+1} X_{u+2} \ldots X_{u+v-1}
$$

$$
X_{u+v} \equiv X_{u} \prod_{i=u}^{+v-1}\left(X_{i}^{p^{n}-1}-1\right) \quad(\bmod p)
$$

Taking $u=p^{s-1}, u+v=p^{s}$, we find from 18) and 20) the result

$$
V p^{s}, p^{n} \equiv \prod_{i=p^{s}-1}^{p^{s}-1}\left(X_{i}^{p^{n}-1}-1\right)
$$

Further, if $\nu_{1}, \nu_{2}, \ldots, v_{p^{n}-1}$ denote the marks $\neq 0$ of the $G F\left[p^{n}\right]$. we have

$$
X_{i}^{p^{n}-1}-1=\prod_{j=1}^{p^{n}-1}\left(X_{i}-v_{j}\right) .
$$

Since $X_{i}-v_{j}$ is of degree $p^{n i}$ in $x$, it must decompose in the $G F\left[p^{n}\right]$ into $p^{n i-s}$ factors each an $I Q\left[p^{s}, p^{n}\right]$.

1) For the case $n=1$, Serret, Journal de Mathématiques, 1873, p. 301; Algèbre, II, ch. IV. For general n, Dickson, Bull. Amer. Math. Soc., 1897, pp. $384-389$. 
40. For $s=1$, there are $p-1$ factors in the product 21), given by $i=1,2, \ldots, p-1$. The irreducible factors of $X_{i}^{p^{n}-1}-1$ are then said to form together the $i^{\text {th }}$ class of $I Q\left[p, p^{n}\right]$. Consider first

$$
X_{1}-v \equiv x^{p^{n}}-x-v,
$$

which is the product of $p^{n-1} I Q\left[p, p^{n}\right]$ of the first class. To decompose it, consider the equation

$$
\eta^{p^{n-1}}+\eta^{p^{n-2}}+\cdots+\eta^{p}+\eta=c .
$$

It follows at once that

$$
c^{p}-c \equiv \eta^{p^{n}}-\eta \quad(\bmod p) .
$$

Hence every root $\eta$ of 22 ) belongs to the $G F\left[p^{n}\right]$ if, and only if, $c$ be an integer. Setting in 22)

$$
c=\lambda \nu, \quad \eta=(\lambda x)^{p}-\lambda x,
$$

where $\lambda$ belongs to the $G F\left[p^{n}\right]$, we find

$$
\lambda\left(x^{p^{n}}-x-v\right) \equiv 0 \quad(\bmod p) .
$$

We have therefore in the $G F\left[p^{n}\right]$ the decomposition ${ }^{1}$ )

where the $\beta_{j}$ are the roots of

$$
\lambda\left(x^{p^{n}}-x \perp \nu\right) \equiv \prod_{j=1}^{p^{n-1}}\left(\lambda^{p} x^{p}-\lambda x-\beta_{j}\right),
$$

$$
\eta^{p^{n-1}}+\eta^{p^{n-2}}+\cdots+\eta^{p}+\eta=\lambda \nu
$$

$\lambda$ or $\nu$ being determined so that $\lambda \nu$ is an integer. We have therefore the theorem: The quantic $\lambda^{p} x^{p}-\lambda x-\beta$ is an $I Q\left[p, p^{n}\right]$ if, and only if,

$$
B \equiv \beta^{p^{n-1}}+\beta^{p^{n-2}}+\cdots+\beta^{p}+\beta \neq 0 .
$$

Corollary. - If $b$ is an integer not divisible by the prime $p$, $x^{p}-x-b$ is irreducible in the $G F\left[p^{n}\right]$ if, and only if, $n$ is not divisible by $p$; in particular, it is always irreducible modulo $p$.

In fact, the condition becomes in this case

$$
B \equiv n b \equiv \equiv \quad(\bmod p) \text {. }
$$

41. The decomposition 23) may be given a more explicit form useful below. If $\beta$ be one root of 24 ), then is also

$$
\beta_{j} \equiv \alpha^{p}-\alpha+\beta,
$$

for every mark $\alpha$ in the $G F\left[p^{n}\right]$. Indeed, we have

$$
\beta_{j}^{p^{n-1}}+\cdots+\beta_{j}^{p}+\beta_{j}=\alpha^{p^{n}}-\alpha+\beta^{p^{n-1}}+\cdots+\beta^{p}+\beta=\lambda \nu .
$$

1) For the case $\nu=0$, this decomposition was given without proof by Mathieu, Journal de Mathématiques, (2) vol. 6, 1861, p. 280. 
Further, the formula 25) furnishes all the roots of 24). For, if

then

$$
\begin{gathered}
\alpha^{p}-\alpha+\beta=\alpha_{1}^{p}-\alpha_{1}+\beta, \\
\left(\alpha-\alpha_{1}\right)^{p} \equiv\left(\alpha-\alpha_{1}\right) \quad[\bmod p],
\end{gathered}
$$

so that $\alpha=\alpha_{1}+$ an integer. Hence there are $p^{n} / p \equiv p^{n-1}$ distinct expressions $\alpha^{j}-\alpha$ and hence as many roots $\beta_{j}$. Hence

$$
\left.23_{1}\right)(\lambda x)^{p^{n}}-\lambda x-\left(\beta^{p^{n-1}}+\cdots+\beta^{p}+\beta\right) \equiv \Pi\left[\left(\lambda x-\alpha_{i}\right)^{p}-\left(\lambda x-\alpha_{i}\right)-\beta\right] \text {, }
$$

the product extending over $p^{n-1}$ marks $\alpha_{i}$ of the $G F\left[p^{n}\right]$ no two of which differ by an integer.

42. Consider an irreducible factor $x^{p}-x-\beta$ of $x^{p^{n}}-x-1$, where therefore

$$
\beta^{p^{n-1}}+\beta^{p^{n-2}}+\cdots+\beta^{p}+\beta=1 .
$$

Denote by $I$ one root of the equation

$$
x^{p}-x-\beta=0 \text {. }
$$

Its remaining roots are $I+1, I+2, \ldots, I+p-1$.

Then by $23_{1}$ ) every root of every $I Q\left[p, p^{n}\right]$ of the first class is a linear function of $I$, viz., $\lambda x-\alpha_{i}=I+i, i=$ integer:

$$
x=\left(I+i+\alpha_{i}\right) / \lambda,
$$

the coefficients $1 / \lambda$ and $\left(i+\alpha_{i}\right) / \lambda$ being marks of the $G F\left[p^{n}\right]$.

Inversely, every such linear function containing $I$ is the root of an $I Q\left[p, p^{n}\right]$.

43. Consider an $I Q\left[p, p^{n}\right]$ of class $\mu$. Its roots belong to the $G F\left[p^{n p}\right]$ and are therefore functions of $I$ of the form

$$
f(I) \equiv \sum_{j=0}^{p-1} \alpha_{j} I^{j},
$$

where the $\alpha_{j}$ belong to the $Q F\left[p^{n}\right]$. By $\S 39, f(I)$ will be a root of

$$
X_{\mu}=\sigma \text {, }
$$

if $\sigma$ be suitable chosen in the $G F\left[p^{n}\right]$. But, by $\S 42$,

$$
I^{p^{n}}=I+1 \text {. }
$$

Hence, by $\S 24$, we have for any integer $m$,

$$
[f(I)]^{p^{n m}}=f\left(I^{p^{n m}}\right)=f(I+m) .
$$

Substituting $f(I)$ in equation 27), $X_{\mu}$ being given by 16), we find $f(I+\mu)-\mu f(I+\mu-1)+\cdots+(-1)^{k} C_{\mu, k} f(I+\mu-k)+\cdots+(-1)^{\mu} f(I)=\sigma$. The degree of this equation in $I$ being less than $p$, it must be an identity. But its first member is the $\mu^{\text {th }}$ difference of the polynomial $f(I)$ with respect to the constant difference unity attributed to $I$. 
Since it reduces to the constant $\sigma \neq 0$, the degree of $f(I)$ is exactly $\mu^{1}$ ). Hence

$$
\alpha_{\mu}=\frac{\sigma}{\mu !}, \quad \alpha_{\mu+1}=\alpha_{\mu+2}=\cdots=\alpha_{p-1}=0 .
$$

We have therefore proved that the roots of every $I Q\left[p, p^{n}\right]$ of class $\mu$ are integral functions of $I$ of degree $\mu$.

44. We can readily obtain a formula including all $I Q\left[p, p^{n}\right]$. In the above expression $f(I)$, let the $\alpha_{j}$ be arbitrary such, however, that $f(I)$ does not reduce to $\alpha_{0}$. To set up the equation of which $f(I)$ is a root, consider the $p$ equations

$$
I^{\lambda}[f(I)-\xi]=0 \quad(\lambda=0,1, \ldots, p-1) .
$$

Reducing the exponents of $I$ below $p$ by using the identity

$$
I^{p}=I+\beta,
$$

we obtain the series of equations

$$
\begin{aligned}
& \left(\alpha_{0}-\xi\right)+\alpha_{1} I+\alpha_{2} I^{2}+\cdots+\alpha_{p-1} I^{p-1}=0, \\
& \beta \alpha_{p-1}+\left(\alpha_{0}-\xi+\alpha_{p-1}\right) I+\alpha_{1} I^{2}+\cdots+\alpha_{p-2} I^{p-1}=0 \text {, } \\
& \beta \alpha_{1}+\left(\beta \alpha_{2}+\alpha_{1}\right) I+\left(\beta \alpha_{3}+\alpha_{2}\right) I^{2}+\cdots+\left(\alpha_{0}-\xi+\alpha_{p-1}\right) I^{p-1}=0 .
\end{aligned}
$$

Eliminating $I^{0}, I^{1}, \ldots, I^{p-1}$ from these $p$ equations, we reach the required irreducible quantic $F(\xi)$,

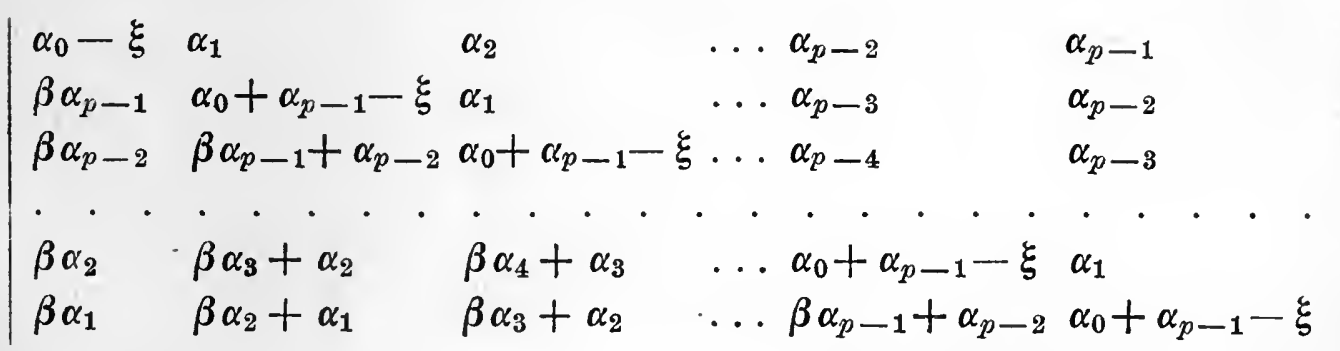

Setting $\alpha_{\mu+1}=\alpha_{\mu+2}=\cdots=\alpha_{p-1}=0$ and giving to $\alpha_{0}, \alpha_{1}, \ldots, \alpha_{\mu-1}$ all possible values in the $G F\left[p^{n}\right]$ and to $\alpha_{\mu}$ every value $\neq 0$, we obtain $p^{n} \mu\left(p^{n}-1\right)$ irreducible quantics of class $\mu$. Since $f(I+m)$ leads to the same determinant as $f(I)$, if $m$ be an integer, the number of distinct $I Q\left[p, p^{n}\right]$ of class $\mu$ is $p^{n \mu-1}\left(p^{n}-1\right)$, a result also following from $\S 39$.

For $\mu=1$, we find that

$$
\frac{-F(\xi)}{\alpha_{1} p} \equiv\left(\frac{\xi}{\alpha_{1}}-\frac{\alpha_{0}}{\alpha_{1}}\right)^{p}-\left(\frac{\xi}{\alpha_{1}}-\frac{\alpha_{0}}{\alpha_{1}}\right)-\beta,
$$

so that we may derive a new proof of formula $23_{1}$ ).

1) Boole, Calculus of Finite Differences, p. 5 and p. 19, formula 3). 
An interesting type of $I Q\left[p, p^{n}\right]$ of class $p-1$ is given by setting every $\alpha_{j}=0$ except $\alpha_{0}$ and $\alpha_{p-1}$; viz.,

$$
F(\xi) \equiv\left(\xi-\alpha_{0}-\alpha_{p-1}\right)^{p}+\alpha_{p-1}\left(\xi-\alpha_{0}-\alpha_{p-1}\right)^{p-1}-\beta^{p-1} \alpha_{p-1}^{p} .
$$

Multiplying this by $\xi-\alpha_{0}-\alpha_{p-1}$ and setting $F(\xi)=0$, we find that $\xi^{p}$ is a linear fractional function of $\xi$. But, by $\S 31$, the roots of $F(\xi)=0$ may be expressed in the form

$$
\xi, \xi^{p^{n}}, \xi^{p^{2}}, \ldots, \xi^{p^{n(p-1)}} .
$$

Hence its roots are all linear fractional functions of one of them.

This result also follows from the fact that

$$
f(I) \equiv \alpha_{0}+\alpha_{p-1}(I+\beta) / I, \quad I^{p}=I+\beta,
$$

so that each root is a linear fractional function of $I$.

45. Formula 19) expresses the fact that $X_{\mu}$ becomes $X_{\mu+1}$ when $x$ is changed into $x^{p^{n}}-x$. Further, if we set $\left.X_{0} \equiv x, 19\right)$ holds true for $\mu=0$; viz.,

$$
X_{1} \equiv X_{0}^{p^{n}}-X_{0}
$$

Hence in order to change $x$ into $x^{p^{n}}-x$ in any formula involving the $X_{\mu}$, we have merely to advance the subscripts of each $X_{\mu}$ by unity. Applying this operation to formula 21), we have the theorem:

If $F(x)$, an $I Q\left[p^{s}, p^{n}\right]$, divides $X_{i}^{p^{n}-1}-1$ for $i<p^{s}-1$, then $F\left(x^{p^{n}}-x\right)$ decomposes into $p^{n} I Q\left[p^{s}, p^{n}\right]$, cach one being a factor of $X_{i+1}^{p^{n}-1}-1$; but if $F(x)$ divides $X_{p^{s}-1}^{p^{n}-1}-1$, then $F\left(x^{p^{n}}-x\right)$ decomposes into $p^{n-1}$ factors each an $I Q\left[p^{s+1}, p^{n}\right]$ which divides $X_{p^{p}}^{p^{n}-1}-1$.

46. As an example under the second part of the last theorem, consider the $I Q\left[p, p^{n}\right]$ of class $p-1$ given at the end of $\S 44$. From it we obtain the $I Q\left[p^{2}, p^{n}\right]$, $F\left(x^{p}-x\right) \equiv\left(x^{p}-x-\alpha_{0}-\alpha_{p-1}\right)^{p}+\alpha_{p-1}\left(x^{p}-x-\alpha_{0}-\alpha_{p-1}\right)^{p-1}-\beta^{p-1} \alpha_{p-1}^{p}$, where $\alpha_{0}, \alpha_{p-1}, \beta$ are arbitrary marks of the $G F\left[p^{n}\right]$ such that

$$
\alpha_{p-1} \neq 0, \beta^{p^{n-1}}+\beta^{p^{n-2}}+\cdots+\beta^{p}+\beta \neq 0 .
$$

For an $I Q\left[p^{3}, p\right]$ see Serret, Cours d'Algèbre supérieure, II, p. 209.

Miscellaneous theorems on irreducible quantics, $\S 547-49$.

4\%. Theorem. - An IQ $\left[m, p^{d}\right]$ is irreducible in the $G F\left[p^{n d}\right]$ if $n$ be prime to $m$. 
The given quantic being $F(x)$, the roots of $F(x)=0$ are

$$
x, x^{p^{d}}, x^{p^{2 d}}, \ldots, x^{p^{d(m-1)}}
$$

all belonging to the $G F\left[p^{d m}\right]$. If $F(x)$ be reducible in the $G F\left[p^{d n}\right]$, the root $x$ will satisfy an $I Q\left[t, p^{d n}\right], t<m$, of the form

$$
(X-x)\left(X-x^{p^{d n}}\right)\left(X-x^{p^{2 d n}}\right) \ldots\left(X-x^{p^{d n(t-1)}}\right)=0 .
$$

Its constant term must be a mark of the $G F\left[p^{d n}\right]$, so that

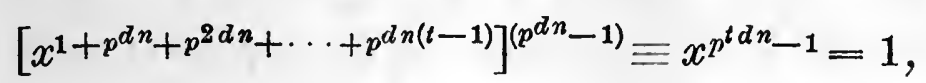

in virtue of the single relation $F(x)=0$. But this requires that $t n$ shall be a multiple of $m$; and therefore that $t$ be a multiple of $m$, in contradiction with $t<m$. In fact, by $\S 23, F(x)$ divides in the $G F\left[p^{d}\right]$ the function $x^{p^{k d}}-x$ if, and only if, $k$ be a multiple of $m$.

48. Theorem. $\left.{ }^{1}\right)$ - An IQ $\left[\mu, p^{n}\right]$ decomposes in the $G F\left[p^{n v}\right]$ into $\delta$ factors each an $I Q\left[\frac{\mu}{\delta}, p^{n \nu}\right], \delta$ being the greatest common divisor of $\mu$ and $\nu$.

The given quantic being $F(x)$, the roots of $F(x)=0$ in the $G F\left[p^{n \mu}\right]$ are

$$
x, \quad x^{p^{n}}, \quad x^{p^{2 n}}, \ldots, x^{p^{n(\mu-1)}} \quad\left[x^{p^{n \mu}} \equiv x\right] .
$$

They may be separated into $\delta$ sets each of $\mu / \delta$ roots,

$$
x^{p^{n i}}, x^{p^{n(\delta+i)}}, x^{p^{n(2 \delta+i)}}, \ldots, x^{p^{n}\left[\left(\frac{\mu}{\delta}-1\right) \delta+i\right]}
$$

for $i=0,1, \ldots, \delta-1$. A symmetric function of the roots in one set is unaltered upon being raised to the power $p^{n \delta}$ and therefore belongs to the $G F\left[p^{n j}\right]$. The roots of the general set therefore satisfy an equation

$$
\dot{F}_{i}(X) \equiv\left(X-x^{p^{n i}}\right)\left(X-x^{p^{n(\delta+i)}}\right) \cdots=0,
$$

with coefficients belonging to the $G F\left[p^{n \delta}\right]$ and à fortiori to the $G F\left[p^{n \nu}\right]$. If

then

$$
F_{0}(X) \equiv X^{\mu / \delta}+A_{1} X^{\mu / \delta-1}+A_{2} X^{\mu / \delta-2}+\cdots
$$

$$
F_{i}(X) \equiv X^{\mu / \delta}+A_{1}^{p^{n i}} X^{\mu / \delta-1}+A_{2}^{p^{n i}} X^{\mu / \delta-2}+\cdots
$$

We next prove that the $F_{i}(X)$ are irreducible in the $G F\left[p^{n}\right.$ o $]$. Suppose, on the contrary, that in the latter field,

Then

$$
F_{0}(X) \equiv f_{0}(X) \cdot \dot{\varphi}_{0}(X) \text {. }
$$

$$
F_{i}(X) \equiv f_{i}(X) \cdot \varphi_{i}(X),
$$

1) For the case $n=1$, this theorem and the corollary of $\S 49$ were stated without proof by Pellet, Comptes Rendus, vol. 70 (1870), pp. 328-330.

DrCKsoN, Linear Groups. 
the coefficients of $f_{i+1}(X)$ being the power $p^{n}$ of the corresponding ones of $f_{i}(X)$, those of $f_{0}$ being the power $p^{n}$ of those of $f_{\delta-1}$. The coefficients of the product $f_{0} f_{1} \ldots f_{\delta-1}$ are consequently unchanged when we replace the coefficients of $f_{0}$ by their $\left(p^{n}\right)^{\text {th }}$ powers and are therefore unaltered upon being raised to the power $p^{n}$. Hence that product belongs to the $G F\left[p^{n}\right]$, so that $F(x)$ would be reducible in that field, contrary to hypothesis.

Since the degree $\mu / \delta$ of $F_{i}(X)$, an $I Q\left[\mu / \delta, p^{n \delta}\right]$, is relatively prime to $v / \delta, F_{i}(X)$ is irreducible in the $G F\left[p^{n v}\right]$ by $\S 47$.

49. Theorem. - If $F(\xi)$ be an $I Q\left[m, p^{n}\right]$ in which the coefficient a of $\xi^{m-1}$ is such that in the $G F\left[p^{n}\right]$

$$
\alpha+\alpha^{p}+\alpha^{p^{2}}+\cdots+\alpha^{p^{n-1}} \neq 0,
$$

then $F\left(\xi^{p}-\xi\right)$ is an $\operatorname{IQ}\left[m p, p^{n}\right]$.

If $x$ be one root of $F(\xi)=0$, its roots are

$$
x, \quad x^{p^{n}}, x^{p^{2 n}}, \ldots, x^{p^{n(m-1)}} .
$$

By the hypothesis concerning the coefficient

we have

$$
\alpha \equiv-x-x^{p^{n}}-\cdots-x^{p^{n(m-1)}},
$$

$$
x+x^{p}+x^{p^{2}}+-\cdots+x^{p^{n} m-1} \neq 0 .
$$

Hence, by $\S 40, \xi p-\xi-x$ is irreducible in the $G F\left[p^{n m}\right]$. The same holds for each of the quantics

$$
X_{i} \equiv \xi^{p}-\xi-x^{p^{n i}} \quad(i=0,1, \ldots, m-1) .
$$

Consider the function belonging to the $G F\left[p^{n}\right]$,

$$
F\left(\xi^{p}-\xi\right) \equiv \prod_{i=0}^{m-1} X_{i}
$$

By $\S 22$, it has in the $G F\left[p^{n m}\right]$ no irreducible factors other than the $X_{i}$. Hence if $F(\xi p-\xi)$ have a factor $f(\xi)$ belonging to and irreducible in the $G F\left[p^{n}\right], f(\xi)$ must be in the $G F\left[p^{n m}\right]$ a product of the $X_{i}$,

$$
f(\xi) \equiv X_{r} X_{s} X_{t} \ldots,
$$

an identity in virtue of $F(x)=0$. Replacing $x$ by $x^{p^{n}}$, another root of $F(x)=0$, and therefore $X_{i}$ by $X_{i+1}(i<m)$ and $X_{m}$ by $X_{0}$, we obtain from the above identity,

$$
f(\xi) \equiv X_{r+1} X_{s+1} X_{t+1} \cdots
$$

Hence $f(\xi)$ contains every factor $X_{i}$ and therefore coincides with $F\left(\xi^{p}-\xi\right)$. The latter function is therefore irreducible in the $G F\left[p^{n}\right]$. 
Corollary. - If $F(\xi)$ be an $I Q[m, p]$ in which the coefficient of $\xi^{m-1}$ is not zero, $F\left(\xi^{p}-\xi\right)$ is an IQ[mp, $\left.p\right]$.

Examples. - The following congruences are irreducible:

$$
\begin{aligned}
& \left(x^{2}-x\right)^{2}+\left(x^{2}-x\right)+1 \equiv x^{4}+x+1 \equiv 0 \quad(\bmod 2), \\
& \left(x^{3}-x\right)^{2}+\left(x^{3}-x\right)-1 \equiv x^{6}+x^{4}+x^{3}+x^{2}-x-1 \equiv 0 \quad(\bmod 3) .
\end{aligned}
$$

Primitive roots and primitive irreducible quantics, $\$ \$ 50-58$.

50. Theorem. - If $R$ be a primitive root of the GF[ $\left[p^{n m}\right]$ and $m_{1}$ a divisor of $m$, any $I Q\left[m_{1}, p^{n}\right]$ belonging to an exponent e may be exhibited as a product

$$
\varphi(X) \equiv \prod_{i=0}^{m_{1}-1}\left(X-R^{t p^{n i}}\right)
$$

where $t$ is a multiple of $d \equiv\left(p^{n m}-1\right) / e$ such that $\frac{t}{d}$ is prime to e. Inversely, if $e$ be a proper divisor of $\left(p^{n}\right)^{m_{1}}-1$ and $t$ be a multiple of $d$ and $\frac{t}{d}$ be prime to $e$, the above product gives an $I Q\left[m_{1}, p^{n}\right]$ belonging to the exponent $e$.

Suppose first that $\varphi(X)$ is an $I Q\left[m_{1}, p^{n}\right]$ belonging to the exponent $e$, where $m_{1}$ is a divisor of $m$. By \& 23, $\varphi(X)$ divides $X^{p^{n m}}-X$ in the $G F\left[p^{n}\right]$, so that any $\operatorname{root} X_{1}$ of $\varphi(X)=0$ belongs to the $G F\left[p^{n m}\right]$. We may therefore set $X_{1}=R^{t}$. Then, by $\S 31$, we have the decomposition 28). Since $\varphi(X)$ belongs to the exponent $e$, $X_{1} \equiv R^{t}$ must belong to the exponent $e(\S 32)$. Hence $t$ must be a multiple of $d \equiv\left(p^{n m}-1\right) / e$ and $\frac{t}{d}$ be prime to $e$.

To establish the inverse, we first prove that $R^{t}$ belongs to the exponent $e$. Since $e t$ is assumed to be a multiple of $p^{n m}-1$, we have $R^{e t}=1$. If $R^{t j}=1, t j$ is divisible by $p^{n m}-1$. Set $t=d d^{\prime}$, so that $d^{\prime}$ is prime to $e$. Then must

$$
j l^{\prime} \cdot\left(p^{n m}-1\right) / e \equiv 0 \quad\left(\bmod p^{n m}-1\right) .
$$

Hence must $j d^{\prime}$, and therefore, $j$ be divisible by $e$. Hence

$$
R^{t}, R^{t p^{n}}, R^{t p^{2 n}}, \ldots, R^{t p^{n\left(m_{1}-1\right)}}
$$

all belong to the exponent $e$. Upon raising these marks to the power $p^{n}$, they are merely permuted. Hence any symmetric function of them, and consequently $\varphi(X)$ defined by 28$)$, belongs to the $G F\left[p^{n}\right]$. Furthermore, $\varphi(X)$ is irreducible in the $G F\left[p^{n}\right]$; for, if $\varphi^{1}(X)$ be an irreducible factor of degree $m^{1}>1$, it belongs to the exponent $e$. Then by $\S 29, e$ would be a proper divisor of $\left(p^{n}\right)^{m^{1}}-1$, so that $m^{1}=m_{1}$. 
Corollary. - Every $P I Q\left[m, p^{n}\right]$ is given by the formula

$$
F_{t}(x) \equiv\left(x-R^{t}\right)\left(x-R^{t p^{n}}\right) \ldots\left(x-R^{t p^{n(m-1)}}\right),
$$

where $t$ is an integer relatively prime to $p^{n m}-1$.

Evidently $F_{t} \equiv F_{t p^{n}} \equiv F_{t p^{2 n}} \equiv \cdots$

51. The determination of a primitive root in the $G F\left[p^{n m}\right]$ is one of the most important as well as most difficult problems in the theory. Special methods of procedure are illustrated in $\$ \S 54-57$. We may determine simultaneously all the $P I Q\left[m, p^{n}\right]$ and therefore all the primitive roots of the $G F\left[p^{n m}\right]$ by the following method of undetermined coefficients.

The roots of $F_{t}(x)=0$ are the $t^{\text {th }}$ powers of the roots of $F_{1}(x)=0$. Hence the equations

$$
F_{t}(x)=0, \quad F_{1}\left(x^{\frac{1}{t}}\right)=0
$$

are equivalent in the $G F\left[p^{n m}\right]$. Since $t$ is prime to $p^{n m}-1$, we may determine $t^{\prime}$ by the congruence

$$
t t^{\prime} \equiv 1 \quad\left(\bmod p^{n m}-1\right) .
$$

Hence $F_{1}\left(x^{\frac{1}{t}}\right)=0$ and $F_{1}\left(x^{t^{\prime}}\right)=0$ are equivalent equations in virtue of $x^{p^{n m}}=x$. By $\S 30$, the product of all the $P I Q\left[m, p^{n}\right]$ is given thus:

$$
\prod_{t} F_{t}(x) \equiv \frac{\left(x^{p^{n m}-1}-1\right) \Pi\left(x^{\frac{p^{n m}-1}{q_{i} q_{j}}}-1\right) \ldots}{\Pi\left(x^{\left(p^{n m}-1\right) / q_{j}}-1\right) \ldots}
$$

where $q_{1}, q_{2}, \ldots$ denote the distinct prime factors of $p^{n}-1$.

But

$$
\prod_{t} F_{t}(x)=0, \quad \prod_{t^{\prime}} F_{1}\left(x^{t^{\prime}}\right)=0
$$

are equivalent if $t$ and $t^{\prime}$ each run through the integers less than and relatively prime to $p^{n m}-1$, which give distinct functions $F(x)$. Giving $F_{1}(x)$ the undetermined form

$$
F_{1}(x) \equiv x^{m}+a x^{m-1}+b x^{m-2}+\cdots,
$$

and forming the product of the $\frac{1}{m} \Phi\left(p^{n m}-1\right)$ distinct quantics $F_{1}\left(x^{t^{\prime}}\right)$, the result may be identified with the above fractional expression in $x$, giving a series of conditions for the coefficients $a, b, \ldots$ The examples which follow will serve to make clear the method.

52. For $p^{n}=3, m=2$, we have $p^{n m}-1=2^{3}$. The integers less than and prime to $2^{3}$ are $1,3,5,7$. But

$$
F_{3}(x)=F_{1}(x), \quad F_{7}(x)=F_{3 \cdot 5}(x)=F_{5}(x) .
$$


Hence

$$
F_{1}(x) F_{5}(x) \equiv \frac{x^{8}-1}{x^{4}-1}=x^{4}+1
$$

Since $5 \cdot 5 \equiv 1\left(\bmod 2^{3}\right), F_{5}(x)=0$ and $F_{1}\left(x^{5}\right)=0$ are equivalent in the $G F\left[3^{2}\right]$. Let

$$
F_{1}(x) \equiv x^{2}+a x+b .
$$

If $x$ be a primitive root in the $G F\left[3^{2}\right], x^{8}=1, x^{4}=-1$. Hence

\section{Hence}

$$
F_{1}\left(x^{5}\right) \equiv x^{10}+a x^{5}+b=x^{2}-a x+b .
$$

giving

$$
\left(x^{2}+a x+b\right)\left(x^{2}-a x+b\right) \equiv x^{4}+1 \text {, }
$$$$
a^{2} \equiv 2 b, \quad b^{2} \equiv 1 \quad(\bmod 3) \text {. }
$$

Hence $b \equiv-1, a \equiv \pm 1(\bmod 3)$, so that the two $P I Q[2,3]$ are $x^{2} \pm x-1$.

53. For $p^{n}=5, m=2$, we have

$$
\prod_{t} F_{t}(x) \equiv \frac{\left(x^{24}-1\right)\left(x^{4}-1\right)}{\left(x^{12}-1\right)\left(x^{8}-1\right)}=\frac{x^{12}+1}{x^{4}+1}=x^{8}-x^{4}+1 .
$$

The eight integers $\tau$ less than and prime to 24 are

$$
1,5 ; \quad 7,11 \equiv 5 \cdot 7 ; \quad 13,17 \equiv 5 \cdot 13 ; \quad 19,23 \equiv 5 \cdot 19 \quad(\bmod 24)
$$

Each pair of integers furnishes a single $F_{t}(x)$. For each of the eight values of $\tau$, we have $\tau^{2} \equiv 1(\bmod 24)$. Hence $F_{\tau}(x)=0$ is identical with $F_{1}\left(x^{\tau}\right)=0$ in the $G F\left[5^{2}\right]$. For a primitive root $x$, we have $x^{12} \equiv-1$. We have therefore in the field,

$$
\begin{aligned}
F_{1}(x) & \equiv x^{2}+a x+b, \quad F_{1}\left(x^{13}\right) \\
x^{2} F_{1}\left(x^{11}\right) & \equiv b x^{2}-a x+1, \quad x^{2}-a x+b, \\
F_{1}\left(x^{23}\right) & \equiv b x^{2}+a x+1 .
\end{aligned}
$$

The product of these four quadratics is therefore identical modulo 5 with

$$
b^{2}\left(x^{8}-x^{4}+1\right) \text {. }
$$

It follows that $b^{2} \equiv-1(\bmod 5)$ and, by subsequent expansion,

$$
2 a^{2} \equiv b \quad(\bmod 5) \text {. }
$$

Hence the four $P I Q[2,5]$ are $x^{2}+a x+2 a^{2}$, viz.,

$$
x^{2} \pm x+2, x^{2} \pm 2 x-2 .
$$

Another method of solving this example is to require that $x^{2}+a x+b$ shall divide $x^{8}-x^{4}+1$ modulo 5 . We reduce the latter function by means of the relation

$$
x^{2}=-a x-b \quad\left[a^{4} \equiv b^{4} \equiv 1(\bmod 5)\right],
$$

and find, modulo 5, that

$$
x^{8}-x^{4}+1 \equiv\left(-a^{5} b-a b^{3}\right) x-a^{4} b^{2}-a^{2} b^{3}+2 .
$$

Hence

$$
b^{2} \equiv-1,2 a^{2} \equiv b \quad(\bmod 5) .
$$


54. The eight $P I Q[4,3]$ are the factors of

$$
\frac{\left(x^{80}-1\right)\left(x^{8}-1\right)}{\left(x^{40}-1\right)\left(x^{16}-1\right)} \equiv x^{32}-x^{24}+x^{16}-x^{8}+1 \text {. }
$$

It suffices, however, in view of $\S 50$, to determine a primitive root $\varrho$ of the $G F\left[3^{4}\right]$. To get an $I Q[4,3]$, we employ the theorem of $\S 37$ for $\lambda=4, i=2, \varrho=2, l=1$, giving the decomposition

$$
x^{8}+1 \equiv \Pi\left(x^{4} \pm x^{2}-1\right) \quad(\bmod 3) .
$$

Hence a root $i$ of the irreducible congruence

$$
x^{4}-x^{2}-1 \equiv 0 \quad(\bmod 3)
$$

belongs to the exponent 16 . If then we find a mark $\sigma$ belonging to the exponent $5, \varrho \equiv i \sigma$ will, by $\S 14$, be a primitive root of $x^{80}=1$. We readily verify that the fifth power of $i^{2} \pm i$ is congruent to unity modulo 3. To find the irreducible congruence satisfied by the primitive root $\varrho=i\left(i^{2} \pm i\right)$, we form its powers,

$$
\varrho^{2}=\mp i^{3} \mp i-1, \quad \varrho^{3}=\mp i^{2}-i \pm 1, \quad \varrho^{4}=\mp i^{3}-i^{2} \mp i+1 .
$$

Eliminating the powers of $i$, we have

$$
\varrho^{4} \pm \varrho^{3}+\varrho^{2} \mp \varrho-1 \equiv 0 \quad(\bmod 3) .
$$

The product of the two $P I Q[4,3]$ thus reached is $\varrho^{8}+\varrho^{6}+\varrho^{4}+1$. Since the expression 31) contains only exponents which are multiples of 4 , we would expect the new factor $\varrho^{8}-\varrho^{6}+\varrho^{4}+1$. In fact, the product of these two quantics of degree 8 gives $\rho^{16}+\varrho^{12}-\rho^{4}+1$, which divides 31) giving the quotient

$$
\varrho^{16}-\varrho^{12}+\varrho^{4}+1 \equiv\left(\varrho^{8}+\varrho^{4}+\varrho^{2}+1\right)\left(\rho^{8}+\varrho^{4}-\varrho^{2}+1\right) .
$$

We therefore have two new $P I Q[4,3]$ given by the decomposition

$$
\rho^{8}-\rho^{6}+\varrho^{4}+1 \equiv\left(\varrho^{4}+\varrho^{3}-1\right)\left(\rho^{4}-\rho^{3}-1\right) \text {. }
$$

Since $\varrho^{8}+\varrho^{4} \pm \varrho^{2}+1$ is derived from $\varrho^{8} \pm \varrho^{6}+\varrho^{4}+1$ upon replacing $\rho$ by $\frac{1}{\rho}$ in the latter and multiplying by $\rho^{8}$, we find

$$
\begin{aligned}
& \varrho^{8}+\varrho^{4}+\varrho^{2}+1=\Pi\left(\varrho^{4} \pm \varrho^{3}-\varrho^{2} \mp \varrho-1\right), \\
& \varrho^{8}+\varrho^{4}-\varrho^{2}+1=I I\left(\varrho^{4} \mp \varrho-1\right) .
\end{aligned}
$$

Hence the eight $P I Q[4,3]$ are

$$
\varrho^{4} \pm \varrho^{3}-1, \quad \varrho^{4} \pm \rho-1, \quad \varrho^{4}+\varepsilon \varrho^{3} \pm \varrho^{2}-\varepsilon \rho-1 \quad(\varepsilon= \pm 1) .
$$

55. To obtain a primitive root $\varrho$ of the $G F\left[5^{4}\right]$, we define the latter by means of a root $i$ of the irreducible congruence

$$
x^{4} \equiv 2 \quad(\bmod 5) \text {. }
$$

Indeed, by $\S 35, x^{4}-3^{3}$ is an $I Q[4,5]$ belonging to the exponent 16 . Since $5^{4}-1=16 \cdot 3 \cdot 13$, we seek marks belonging to the exponents 3 
and 13. We verify at once that $2 i^{2}+2$ belongs to the exponent 3 . To find the most general mark $\eta$ which belongs to the exponent 13 , we simplify the calculations by first determining the marks

$$
\eta_{1} \equiv a i^{3}+b i^{2}+c i+d
$$

of the $G F\left[5^{4}\right]$ for which $\eta_{1}^{26}=1$. Then either $\left(+\eta_{1}\right)^{13}$ or $\left(-\eta_{1}\right)^{13}$ equals unity. Now

$$
\begin{aligned}
\eta_{1}^{25} & \equiv a i^{75}+b i^{50}+c i^{25}+d \\
& \equiv a 2^{18} i^{3}+b 2^{12} i^{2}+c 2^{6} i+d \\
& \equiv-a i^{3}+b i^{2}-c i+d .
\end{aligned}
$$

The condition $\eta_{1}^{26}=1$ thus gives

$$
\left(b i^{2}+d\right)^{2}-\left(a i^{3}+c i\right)^{2} \equiv 1 \text {. }
$$

Reducing by $i^{4} \equiv 2$, we obtain the conditions, modulo 5 ,

$$
-2 a^{2}-c^{2}+2 b d \equiv 0,-4 a c+2 b^{2}+d^{2} \equiv 1 \text {. }
$$

For $a \equiv 0$, the only solutions are seen to be

$$
b^{2} \equiv 1, \quad d^{2} \equiv-1, \quad c^{2} \equiv \pm 1 ; \quad b \equiv c \equiv 0, \quad d \equiv \pm 1 .
$$

Hence $\pm i^{2}+c i \pm 2(c=1,2,3$ or 4$)$, or else the negative of this expression, belongs to the exponent 13 . We may verify that $i^{2}+i+3$ belongs to the exponent 13 . We may therefore take

Then

$$
\varrho=i\left(2 i^{2}+2\right)\left(i^{2}+i+3\right) \equiv 3 i^{3}+2 i^{2}+4 .
$$

$$
\varrho^{2} \equiv-i^{3}-i^{2}-i-1, \quad \varrho^{3} \equiv i^{3}-2 i^{2}+i+1, \quad \varrho^{4} \equiv-i^{3}+i+2 .
$$

Hence we obtain the following $P I Q[4,5]$ satisfied by the primitive root $\varrho$,

$$
\varrho^{4}-\varrho^{3}-\varrho-2 \equiv 0 \quad(\bmod 5) .
$$

This quartic can be decomposed into the two $P I Q\left[2,5^{2}\right]$,

$$
(x-\varrho)\left(x-\varrho^{25}\right), \quad\left(x-\varrho^{5}\right)\left(x-\varrho^{125}\right) .
$$

But

$$
\varrho^{5} \equiv 4 i^{3}+3 i^{2}+4, \quad \varrho^{25} \equiv 2 i^{3}+2 i^{2}+4, \quad \varrho^{125} \equiv i^{3}+3 i^{2}+4 .
$$

Hence

$$
\begin{aligned}
& (x-\varrho)\left(x-\varrho^{25}\right) \equiv x^{2}-x\left(-i^{2}+3\right)+3 i^{2}+4, \\
& \left(x-\varrho^{5}\right)\left(x-\varrho^{125}\right) \equiv x^{2}-x\left(i^{2}+3\right)-3 i^{2}+4 .
\end{aligned}
$$

56. The determination of primitive roots in the $G F\left[5^{6}\right]$ and in the $G F\left[5^{3}\right]$ may be made to depend upon the congruence

$$
x^{6}+x^{5}+x^{4}+x^{3}+x+x^{2}+1 \equiv 0 \quad(\bmod 5),
$$

which, by $\S 33$, is irreducible. The root $x$ belongs to the exponent 7 . The general mark of the $G F\left[5^{6}\right]$ may be expressed in the form

$$
\sigma \equiv \sum_{i=0}^{5} c_{i} x^{i} \quad \text { (each } c_{i} \text { an integer). }
$$


It will belong to the included field $G F\left[5^{3}\right]$ if, and only if, $\sigma^{125}=\sigma$. Applying $x^{7} \equiv 1$, we have $(\bmod 5)$

$$
\sigma^{125} \equiv \sum_{i=0}^{5} c_{i} x^{125 i} \equiv \sum_{i=1}^{5} c_{i} x^{7-i}+c_{0} .
$$

Applying 32), this becomes

$$
\left(c_{0}-c_{1}\right)-c_{1} x+\left(c_{5}-c_{1}\right) x^{2}+\left(c_{4}-c_{1}\right) x^{3}+\left(c_{3}-c_{1}\right) x^{4}+\left(c_{2}-c_{1}\right) x^{5} .
$$

The conditions that this shall be identical with $\sigma$ are

$$
c_{1} \equiv 0, \quad c_{2} \equiv c_{5}, \quad c_{3} \equiv c_{4}(\bmod 5)
$$

Hence the $5^{3}$ marks of the $G F\left[5^{3}\right]$ are given by

$$
\text { 33) } c_{0}+c_{2}\left(x^{2}+x^{5}\right)+c_{3}\left(x^{3}+x^{4}\right) \quad\left[c_{0}, c_{2}, c_{3}=0,1,2,3,4\right] \text {. }
$$

Since

$$
\left(x^{2}+x^{5}\right)^{5} \equiv x^{3}+x^{4}
$$

we infer that $\tau \equiv x^{2}+x^{5}$ defines the $G F\left[5^{3}\right]$. In fact, we find

$$
\tau^{5} \equiv x^{3}+x^{4}, \quad \tau^{25} \equiv x+x^{6}, \quad \tau^{30} \equiv x^{5}+x^{4}+x^{3}+x^{2},
$$

and finally that $\tau^{31}=1$. Hence $\lambda \equiv 2\left(x^{2}+x^{5}\right)$ belongs to the exponent 4.31 and is therefore a primitive root in the $G F\left[5^{3}\right]$. We derive at once the $P I Q[3,5]$ satisfied by $\lambda$, viz.,

$$
2 \lambda^{3}=\lambda^{2}+\lambda+1 \quad(\bmod 5) .
$$

We next verify that $x-2$ belongs to the exponent $2^{3} \cdot 3^{2} \cdot 31$, so that $\varrho \equiv x(x-2)$ belongs to the exponent

$$
5^{6}-1 \equiv 2^{3} \cdot 3^{2} \cdot 7 \cdot 31 \text {, }
$$

so that $\varrho$ is a primitive root in the $G F\left[5^{6}\right]$. We have

$$
(x-2)^{126} \equiv\left(x^{6}-2\right)(x-2) \equiv-2\left(x+x^{6}\right) \equiv-2 t^{25}
$$

But $\tau^{25}$ belongs to the exponent 31. Hence the exponent of $x-2$ contains the factor 31 and, moreover, the factor $2^{3}$, since

$$
(x-2)^{\frac{1}{2}\left(5^{6}-1\right)}=(x-2)^{126 \cdot 31 \cdot 2} \equiv(-2)^{62} \equiv-1 \quad(\bmod 5) .
$$

We next prove that the power $2^{3} \cdot 3^{2} \cdot 31$ of $x-2$ gives unity. Indeed,

$$
(x-2)^{15} \equiv\left(x^{5}-2\right)^{3} \equiv 2 x^{5}-x^{3}+x+2 \quad(\bmod 5),
$$

and, by a slight calculation,

$$
(x-2)^{18}=2 x^{5}+x^{4}+x^{3}+2 x^{2}+4 .
$$

This being of the form 33), we have

$$
(x-2)^{2^{3} \cdot 3^{2} \cdot 31} \equiv\left[(x-2)^{18}\right]^{124} \equiv 1 \quad(\bmod 5) .
$$

For the same reason,

$$
(x-2)^{2^{3} \cdot 3 \cdot 31} \equiv\left[(x-2)^{6}\right]^{124} \equiv\left(2 x^{5}-x^{4}-x^{3}-x^{2}+2 \dot{x}+3\right)^{124} \neq 1 .
$$


To determine the $P I Q[6,5]$ satisfied by the primitive root $\varrho \equiv x^{2}-2 x$, we form the powers,

$\varrho^{3} \equiv-2 x^{5}+x^{4}+x^{3}-x^{2}-x-1 ; \quad \rho^{5} \equiv x^{3}-2 x^{5}$, $\varrho^{4} \equiv-x^{5}+2 x^{4}+x^{3}+x^{2}+2 x+3, \quad \varrho^{6} \equiv 2 x^{5}-x^{4}+x^{3}+x^{2}+x-1$.

We derive at once the required congruence

$$
\varrho^{6}-\varrho^{5}+\varrho^{4}-\varrho^{3}+2 \varrho+2 \equiv 0 \quad(\bmod 5) .
$$

5\%. We can set up the $P I Q\left[2,2^{3}\right]$ and $P I Q[6,2]$ by means of the theorem:

34)

$$
\lambda^{2} x^{2}+\lambda x+\beta
$$

is a PIQ $\left[2,2^{3}\right]$ if, and only if, $\beta$ is a root of

$$
j^{3} \equiv j^{2}+1 \quad(\bmod 2)
$$

and $\lambda$ is any mark except zero and $\beta^{4}$.

By $\S 40$, the quadratic 34 ) is an $I Q\left[2,2^{3}\right]$ for every mark $\lambda \neq 0$ in the $G F\left[2^{3}\right]$ and for every root $\beta$ of the congruence

$$
\beta^{4}+\beta^{2}+\beta+1 \equiv(\beta+1)\left(\beta^{3}+\beta^{2}+1\right) \equiv 0 \quad(\bmod 2) .
$$

Defining the $G F\left[2^{3}\right]$ by means of the irreducible congruence 35), we may take $\beta=1, j, j^{2}$ or $j^{4}$. We first find the exponent $e_{\beta}$ to which belongs a root $\xi$ of the congruence

$$
\xi^{2} \equiv \xi+\beta \quad(\bmod 2) .
$$

Since $\xi$ belongs to the $G F\left[2^{2 \cdot 3}\right], e_{\beta}$ is a divisor of $2^{6}-1 \equiv 3^{2} \cdot 7$. But $\xi^{3}=\xi(\beta+1)+\beta, \quad \xi^{7}=\xi\left(\beta^{3}+\beta+1\right)+\beta^{3}+\beta, \quad \xi^{9}=\xi(\beta+1)\left(\beta^{3}+\beta^{2}+1\right)+\beta$. Hence for $\beta=1, e_{\beta}=3$; for a $\operatorname{root} \beta$ of 35 ), we find

$$
\xi^{9}=\beta, \quad \xi^{21}=\xi^{18} \cdot \xi^{3}=\xi+\beta^{3}, \quad \xi^{7}=\xi\left(\beta^{2}+\beta\right)+\beta^{2}+\beta+1,
$$

so that $e_{\beta}=2^{6}-1$. The theorem is therefore proven for the case $\lambda=1$.

Setting $\xi=\lambda x$, it follows that, for $\beta \neq 1, x$ belongs to the exponent $2^{6}-1$ unless $x^{9}=1$, which occurs only when $\lambda^{2}=\beta$, i. e., $\lambda=\beta^{4}$. We therefore reach all $\frac{1}{2} \Phi\left(2^{6}-1\right) \equiv 18$ PIQ $\left[2,2^{3}\right]$. Half of them are given in the left members of the identities below. To pick out a set of three whose product gives a $P I Q[6,2]$, we select three which are like functions of respectively $j, j^{2}, j^{4}$, the latter being the roots of 35). We thus find

$$
\begin{aligned}
& \left(x^{2}+x+j\right)\left(x^{2}+x+j^{2}\right)\left(x^{2}+x+j^{4}\right)=x^{6}+x^{5}+x^{3}+x^{2}+1, \\
& \left(j^{6} x^{2}+j^{3} x+j\right)\left(j^{5} x^{2}+j^{6} x+j^{2}\right)\left(j^{3} x^{2}+j^{5} x+j^{4}\right)=x^{6}+x^{5}+x^{4}+x+1, \\
& \left(j^{6} x^{2}+j^{3} x+j^{4}\right)\left(j^{5} x^{2}+j^{6} x+j\right)\left(j^{3} x^{2}+j^{5} x+j^{2}\right)=x^{6}+x^{5}+1 .
\end{aligned}
$$


Replacing $x$ by $\frac{1}{x}$ and multiplying by $x^{6}$, we find

$$
x^{6}+x^{4}+x^{3}+x+1, \quad x^{6}+x^{5}+x^{2}+x+1, \quad x^{6}+x+1,
$$

which with the above three sextics give the six existing PIQ[6,2].

58. Theorem. - The necessary and sufficient conditions that $x^{p}-x-\alpha$ shall be a $P I Q[p, p]$ are that $\alpha$ be a primitive root modulo $p$ and that a root of $y^{p} \equiv y+1(\bmod p)$ belong to the exponent $\left(p^{p}-1\right) /(p-1)$.

If $\alpha$ be an integer not divisible by $p$, the congruence

$$
x^{p} \equiv x+\alpha \quad(\bmod p)
$$

is irreducible by $\S 40$. The product of its roots is

$$
x x^{p} x^{p^{2}} \ldots x^{p^{p-1}} \equiv x^{\frac{p^{p}-1}{p-1}}=\alpha .
$$

Setting $x=\alpha y$, we find that

$$
y^{p} \equiv y+1 \quad(\bmod p) .
$$

Hence if $x$ belong to the exponent $p^{p}-1$, then $\alpha$ is a primitive root modulo $p$ and $y$ belongs to the exponent $\left(p^{p}-1\right) /(p-1)$. The inverse is true by $\S 14$, since $p-1$ and $\left(p^{p}-1\right) /(p-1)$ are relatively prime.

59.

\section{EXERCISES ON CHAPTER III.}

Ex. 1. If $\varrho$ be a root of one of the $P I Q[2,5]$ of $\S 53$, then $x^{3}-\varrho$ is an $I Q\left[3,5^{2}\right]$. Eliminate $\varrho$ and derive the following $I Q[6,5]$ :

$$
x^{6} \pm x^{3}+2, \quad x^{6} \pm 2 x^{3}-2 .
$$

Ex. 2. (Moore). If $x$ be a root of the irreducible congruence

$$
x^{6}-2 x^{3}-2 \equiv 0 \quad(\bmod 5) \text {, }
$$

a mark $c_{0}+c_{1} x+c_{2} x^{2}+c_{3} x^{3}+c_{4} x^{4}+c_{5} x^{5}$ of the $G F\left[5^{6}\right]$ will belong to the included field $G F\left[5^{3}\right]$ if and only if

$$
c_{3} \equiv 0, \quad c_{4} \equiv 3 c_{1}+4 c_{2}, \quad c_{5} \equiv 2 c_{1}+3 c_{2} \quad(\bmod 5) .
$$

Show that $\varphi \equiv x+x^{2}+2 x^{4}$ is a primitive root of the $G F\left[5^{3}\right]$ and that it satisfies the congruence $\varphi^{3} \equiv 2 \varphi+3(\bmod 5)$.

Ex. 3. (Pellet). If $\gamma$ belong to the $G \boldsymbol{F}\left[p^{n}\right]$, and $m$ be the least integer for which $\gamma^{p^{m}}=\gamma$, then $x^{p}-x-\gamma$ is irreducible in the field if neither $n / m$ nor $\gamma+\gamma^{p}+\gamma^{p^{2}}+\cdots+\gamma^{p^{m-1}}$ be divisible by $p$; in the contrary case it decomposes in the field into linear factors. Prove this theorem equivalent to that of $\S 40$ for $\lambda=1$.

Ex. 4. (Pellet). If $p$ be a prime number which is a primitive root of the prime number $n, \frac{\left(x^{p}-x\right)^{n}-1}{x^{p}-x-1}$ is irreducible modulo $p$.

Ex. 5. Show that the theorems of $\S 34$ and $\S 36$ may be combined into the theorem stated without proof by Pellet: 
If in an $I Q\left[v_{1}, p^{v}\right]$ belonging to the exponent $n$, we replace $x$ by $x^{\lambda}$, where $\lambda$ contains only the prime factors of $n$, the resulting quantic decomposes into $\frac{1}{n} D 2^{k-1}$ quantics $I Q\left[\frac{\lambda n v_{1}}{D 2^{k-1}}, p^{v}\right]$ belonging to the exponent $\lambda n$, where $D$ is the greatest common divisor of $\lambda n$ and $p^{\nu \nu_{1}}-1$ and where $2^{k-1}$ is the highest power of 2 dividing the numerators of each of the fractions $\frac{p^{v v_{1}+1}}{2}$ and $\frac{\lambda n}{2 D}$ when reduced to their simplest form.

Ex. 6. (Schönemann). If $F(x, \alpha)$ be an $I Q\left[m, p^{n}\right]$ in which the coefficient of at least one power of $x$ satisfies the equation $c^{p^{2}-1} \equiv 1$ if, and only if, $v=n$ or a multiple of $n$, the product

$$
F(x, \alpha) \cdot F\left(x, \alpha^{p}\right) \ldots F\left(x, \alpha^{p^{n-1}}\right)
$$

gives an $I Q[m n, p]$.

Ex. 7. (Schönemann). Generalize the theorem of $\S 33$ as follows:

If $p$ belong to the exponent $t$ modulo $e, e$ being prime, $\left(x^{e}-1\right) /(x-1)$ decomposes modulo $p$ into $(e-1) / t$ quantics irreducible modulo $p$.

Ex. 8. Prove that $x^{5}-x+1$ is a PIQ[5, 3].

Ex. 9. (Pellet). If $e$ be the exponent to which belongs

$$
i^{1+p+p^{2}}+\cdots+p^{\nu-1}
$$

the product of the roots of an irreducible congruence of degree $\nu, F(x) \equiv 0$ $(\bmod p)$, and if $\lambda$ be a prime divisor of $e$, then

1) $F\left(x^{\lambda}\right)$ is irreducible modulo $p$ if $\lambda$ does not divide $(p-1) / e$;

2) $F\left(x^{\lambda}\right)$ decomposes into $\lambda$ irreducible factors of degree $\nu$ if $\lambda$ divides $(p-1) / e$. According as $\lambda$ divides or does not divide $e$, all of these factors belong or do not belong to the same exponent.

Ex. 10. Using Jordan's irreducible congruence

$$
x^{9} \equiv x+1 \quad(\bmod 2),
$$

show that $x$ belongs to the exponent 73 and $x+x^{4}+x^{6}+x^{7}+x^{8}$ to the exponent 7. The product $y \equiv x\left(x+x^{4}+x^{6}+x^{7}+x^{8}\right)$ belongs to the exponent $2^{9}-1$ and is therefore a primitive root of the $G F\left[2^{9}\right]$. Verify that it satisfies the congruence

$$
y^{9}+y^{8}+y^{4}+y^{3}+y^{2}+y+1 \equiv 0 \quad(\bmod 2) .
$$

Ex. 11. If the $G F\left[3^{2}\right]$ be defined by $i^{2} \equiv i+1(\bmod 3)$, the 16 $P I Q\left[2,3^{2}\right]$ are given by the decomposition of the PIQ $[4,3]$ of $\S 54$; for example,

$$
\begin{aligned}
& x^{4} \pm x-1 \equiv\left\{x^{2} \pm(i+1) x-i\right\}\left\{x^{2} \mp(i+1) x+i-1\right\} \\
& x^{4} \mp x^{3}-1 \equiv\left\{x^{2} \pm(i-1) x+i\right\}\left\{x^{2} \mp i x-i+1\right\} .
\end{aligned}
$$


Ex. 12. (Mathieu). If $H$ belong to the $G H\left[p^{n m}\right]$, we have the decomposition

$$
\begin{aligned}
H\left(Z^{p^{n}}-Z\right)=\Pi\left\{(H Z)^{p^{n(m-1)}}\right. & +(H Z)^{p^{n(m-2)}}+\cdots \\
& \left.+(H Z)^{p^{n}}+H Z+\mu\right\},
\end{aligned}
$$

where $\mu$ runs through the series of marks of the $G F\left[p^{n}\right]$.

60. Table of primitive irreducible quantics ${ }^{1}$ ). When more than one $P I Q[m, p]$ is known, we choose that one $x^{m} \equiv \alpha x^{r}+\beta x^{r-1}+\ldots$ $(\bmod p)$ in which the exponent $r$ is as small as possible.

Modulo 2: $x^{2} \equiv x+1, x^{3} \equiv x+1, x^{4} \equiv x+1, x^{5} \equiv x^{2}+1, x^{6} \equiv x+1$,

$$
x^{7} \equiv x+1, x^{8} \equiv x^{4}+x^{3}+x^{2}+1, x^{9} \equiv x^{8}+x^{4}+x^{3}+x^{2}+x+1 \text {. }
$$

Modulo 3: $x^{2} \equiv 2 x+1, x^{3} \equiv x+2, x^{4} \equiv 2 x^{3}+2 x^{2}+x+1, x^{5} \equiv x+2$.

Modulo 5: $x^{2} \equiv 2 x+2, x^{3} \equiv 2 x+3, x^{4} \equiv x^{2}+x+2, x^{5} \equiv x+2$,

$$
x^{6} \equiv x^{5}-x^{4}+x^{3}-2 x-2
$$

Modulo $\left.^{2}\right) 7: x^{2} \equiv x-3, x^{3} \equiv x-2, x^{4} \equiv 2 x^{3}+2 x+2, x^{5} \equiv 6 x+3$, $x^{6} \equiv-x^{5}-x^{4}-x^{3}-x^{2}-x-3, x^{7} \equiv x+3$.

Modulo 11: $x^{2} \equiv 4 x-2$.

\section{CHAPTER IV.}

\section{MISCELLANEOUS PROPERTIES OF GALOIS FIELDS.}

Squares, not-squares, $m^{\text {th }}$ powers in a Galois Field, §§ 61-63.

61. Every mark of the $G F\left[2^{n}\right]$ satisfies the equation $x^{2^{n}}=x$, so that $x$ is the square of the mark $x^{2^{n-1}}$. Every mark has one and only one square root, since $-1=+1$ in the $G F\left[2^{n}\right]$.

In the $G F\left[p^{n}\right], p>2$, a mark may or may not be the square of a mark belonging to the field, and is called a square or a notsquare respectively. If $\varrho$ be a primitive root of the $G F\left[p^{n}\right]$, so that

$$
\varrho^{p^{n}-1}=1, \quad \varrho^{\left(p^{n}-1\right) / 2}=-1,
$$

the even powers of $\rho$ are squares, $\varrho^{2 h}=\left( \pm \varrho^{h}\right)^{2}$; while the odd powers are not-squares. In fact, $\rho^{2 h+1}=x^{2}$ would require

$$
\varrho^{(2 h+1)\left(p^{n}-1\right) / 2} \equiv \varrho^{h\left(p^{n}-1\right)} \cdot \varrho^{\left(p^{n}-1\right) / 2}=-1=x^{p^{n}-1}=+1 .
$$

Hence there are $\left(p^{n}-1\right) / 2$ squares and as many not-squares in the $G F\left[p^{n}\right]$. Furthermore, the product or quotient of two squares or of two not-squares is again a square; but the product or quotient of a square by a not-square, or vice versa, is a not-square.

1) A table of irreducible quantics (not all primitive) is given by Jordan, Comptes Rendus, 72 (1871), pp. 283-290. His quantic $x^{8}+x^{3}+x^{2}+x+1$ is divisible by $x^{3}+x^{2}+1$ modulo 2 , while $x^{3}+x+2$ is divisible by $x-5 \bmod 11$.

2) Serret, Cours d'Algèbre supérieure, II, pp. 181-189. 
62. Theorem. - The not-squares of any $G F\left[p^{n}\right], p>2$, are not-squares or squares in the $G F\left[p^{n m}\right]$ according as $m$ is odd or even.

If $\sigma$ be a primitive root of the $G F\left[p^{n m}\right]$, then $\rho \equiv \sigma^{u}$, where $u \equiv\left(p^{n m}-1\right) /\left(p^{n}-1\right)$, is a primitive root of the $G F\left[p^{n}\right]$. Hence the marks $\neq 0$ of the $G F\left[p^{n}\right]$ are given by the formula

$$
\rho^{v} \equiv \sigma^{u v} \quad\left(v=1,2, \ldots, p^{n}-1\right) .
$$

Let $\varrho^{v}$ be a not-square in the $G F\left[p^{n}\right]$, so that $v$ is odd. It will be a not-square or a square in the $G F\left[p^{n m}\right]$ according as $u v$ is odd or even, i. e., according as $u$ is odd or even. But

$$
u \equiv\left(p^{n m}-1\right) /\left(p^{n}-1\right)=\sum_{i=0}^{m-1} p^{n i}=\text { sum of } m \text { odd terms. }
$$

Hence $u$ is odd or even according as $m$ is odd or even.

63. Theorem. - If $d$ be the greatest common divisor of $m$ and $p^{n}-1$, there exist exactly $\left(p^{n}-1\right) / d$ marks $\neq 0$ in the $G F\left[p^{n}\right]$ which are $m^{\text {th }}$ powers in the field.

If $\mu \neq 0$ be the $m^{\text {th }}$ power of some mark $\nu$ of the field, we find, upon raising $\mu=v^{m}$ to the power $\left(p^{n}-1\right) / d$ and noting that the power $p^{n}-1$ of the mark $v^{m / d} \neq 0$ is 1 , the equation

$$
\mu^{\left(x^{n}-1\right) / d}=1 \text {. }
$$

Inversely, there are $\left(p^{n}-1\right) / d$ roots of 37$)$ in the $G F\left[p^{n}\right]$ by $\S 16$ and each root is an $m^{\text {th }}$ power in the $G F\left[p^{n}\right]$. To prove the last statement, we note first that such a root $\mu$ is a $d^{\text {th }}$ power. In fact, the roots of 37) may be exhibited as follows:

$$
\varrho^{d i}\left(i=0,1, \ldots, \frac{p^{n}-1}{d}-1\right),
$$

where $\varrho$ is a primitive root of the $G F\left[p^{n}\right]$. That these roots are distinct is shown by supposing

Then

$$
\varrho^{d i}=\varrho^{d j} \quad\left(j \overline{<} i<\left(p^{n}-1\right) / d\right) .
$$

$$
\varrho^{d j}\left(\varrho^{d(i-j)}-1\right)=0 \quad\left[d(i-j)<p^{n}-1\right] .
$$

Hence $i-j=0$. We next prove that $\mu \equiv \varrho^{d i}$ is an $m^{\text {th }}$ power. Since $m / d$ is relatively prime to $p^{n}-1$, we can determine integers $l$ and $t$ satisfying the equation

Hence

$$
t\left(p^{n}-1\right)+l m / d=1 \text {. }
$$

Therefore

$$
\begin{aligned}
& \varrho=\left(\varrho^{l}\right)^{m / d} \cdot\left(\varrho^{p^{n}-1}\right)^{t}=\varrho^{l m / d} . \\
& \mu=\varrho^{d i}=\left(\varrho^{l i}\right)^{m} .
\end{aligned}
$$

Corollary. - Every mark of the $G F\left[p^{n}\right]$ will be an $m^{\text {th }}$ power in the field if, and only if, $d=1$. Extraction of the $m^{\text {th }}$ root of an 
arbitrary mark of the $G F\left[p^{n}\right]$ is possible if, and only if, $m$ be relatively prime to $p^{n}-1$. With this condition satisfied, there exists but one $m^{\text {th }}$ root of each mark.

Number of solutions of certain quadratic equations in a Galois Field, §§ 64-67.

64. Theorem. $\left.{ }^{1}\right)-$ If $v=+1$ or -1 according as $-\alpha_{1} \alpha_{2}$ is a square or a not-square in the $G F\left[p^{n}\right], p>2$, the equation belonging to the field,

$$
\alpha_{1} \xi_{1}^{2}+\alpha_{2} \xi_{2}^{2}=* \quad\left(\alpha_{1} \neq 0 ; \quad \alpha_{2} \neq 0\right),
$$

has $p^{n}-v$ or $p^{n}+\left(p^{n}-1\right) v$ sets of solutions according as $x \neq 0$ or $\varkappa=0$.

Setting $\alpha_{1} \xi_{1} \equiv \dot{\eta}$, the equation becomes

$$
\eta^{2}+\alpha_{1} \alpha_{2} \xi_{2}^{2}=\alpha_{1} \varkappa
$$

$1^{0}$. If $-\alpha_{1} \alpha_{2}=\lambda^{2}$, a square $\neq 0$ in the $G F\left[p^{n}\right]$, we set

whence

$$
\eta+\lambda \xi_{2}=\varrho, \quad \eta-\lambda \xi_{2}=\sigma,
$$

The equation becomes

$$
\eta=\frac{1}{2}(\varrho+\sigma), \quad \xi_{2}=\frac{1}{2 \lambda}(\varrho-\sigma) .
$$

If $\varkappa \neq 0$, we can give to $\sigma$ any one of the $p^{n}-1$ marks $==0$ in the $G F\left[p^{n}\right]$, when the corresponding value of $\varrho$ is determined by the equation. There are in this case $p^{n}-1$ sets of solutions $\xi_{1}, \xi_{2}$ in the field of the given equation.

If $x=0$, there are evidently $1+2\left(p^{n}-1\right)$ sets of solutions. $2^{0}$. If $-\alpha_{1} \alpha_{2}$ be a not-square in the $G F\left[p^{n}\right]$, the equation

$$
\varphi^{2}=-\alpha_{1} \alpha_{2}
$$

is irreducible in the field. If one root be $i$, the other is $i^{p^{n}} \equiv-i$ by the corollary of $\S 31$. We therefore have the identity

$$
\eta^{2}+\alpha_{1} \alpha_{2} \xi_{2}^{2}=\left(\eta+i \xi_{2}\right)\left(\eta+i^{p^{n}} \xi_{2}\right)=\left(\eta+i \xi_{2}\right)^{p^{n}+1} \text {. }
$$

We are thus led to determine the number of roots in the $G F\left[p^{2 n}\right]$ of the equation in the unknown $Z \equiv \eta+i \xi_{2}$,

$$
Z^{p^{n}+1}=\alpha_{1} x \text {. }
$$

If $x=0$, we have $Z=0$ and hence a single set of solutions $\xi_{1}=0, \xi_{2}=0$.

If $\varkappa \neq 0$, let $R$ be a primitive root of the $G F\left[p^{2 n}\right]$. We may set $\alpha_{1} x \equiv R^{k}$, whence

$$
R^{k\left(p^{n}-1\right)}=\left(\alpha_{1} x\right)^{p^{n}-1}=1
$$

so that $k\left(p^{n}-1\right)$ is divisible by $p^{2 n}-1$, the exponent to which $R$ belongs. We may therefore set $k=l\left(p^{n}+1\right), l$ being an integer.

1) The theorems of $\$ \S 64-67$ are immediate generalizations of Nos. 197-200 of Jordan's Traité des substitutions. 
Since $Z$ belongs to the $G F\left[p^{2 n}\right]$, we may set $Z \equiv R^{t}$. The equation 38) becomes

Hence

$$
R^{t\left(p^{n}+1\right)}=R^{l\left(p^{n}+1\right)} .
$$

$$
t\left(p^{n}+1\right) \equiv l\left(p^{n}+1\right) \quad\left[\bmod p^{2 n}-1\right] .
$$

This congruence has $p^{n}+1$ distinct solutions for $t$, viz.,

$$
t \equiv l, \quad l+\left(p^{n}-1\right), \quad l+2\left(p^{n}-1\right), \ldots, l+p^{n}\left(p^{n}-1\right) .
$$

The corresponding values of $R^{t} \equiv Z \equiv \eta+i \xi_{2}$ give $p^{n}+1$ distinct sets of solutions $\xi_{1}$, $\xi_{2}$ of the given equation.

65. Theorem. - The number of sets of solutions $\left(\xi_{1}, \xi_{2}, \ldots, \xi_{2 m}\right)$

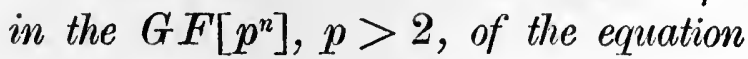

$$
\alpha_{1} \xi_{1}^{2}+\alpha_{2} \xi_{2}^{2}+\cdots+\alpha_{2 m} \xi_{2 m}^{2}=\varkappa,
$$

where every $\alpha_{j}$ is a mark $\neq 0$ in the field, is

$$
\begin{array}{ll}
p^{n(2 m-1)}-v p^{n(m-1)} & (\text { if } \varkappa \neq 0) \\
p^{n(2 m-1)}+\nu\left(p^{n n}-p^{n(m-1)}\right) & (\text { if } \varkappa=0),
\end{array}
$$

where $\nu$ is +1 or -1 according as $(-1)^{m} \alpha_{1} \alpha_{2} \ldots \alpha_{2 m}$ is a square or a not-square in the field.

By $\S 64$, the theorem is true if $m=1$. To prove the theorem by induction, we suppose it true for equations in $2(m-1)$ variables. The proposed equation is equivalent to the system of two equations

$$
\alpha_{1} \xi_{1}^{2}+\alpha_{2} \xi_{2}^{2}=\eta, \quad \alpha_{3} \xi_{3}^{2}+\cdots+\alpha_{2 m} \xi_{2 m}^{2}=\varkappa-\eta .
$$

$1^{0}$. Let $x \neq 0$. For each of the $p^{n}-2$ values of $\eta$ different from $x$ and 0 , the first equation has $p^{n}-\lambda$ sets of solutions, while by hypothesis the second has $p^{n(2 m-3)}-\mu p^{n(m-2)}$, where $\lambda= \pm 1$ according as $-\alpha_{1} \alpha_{2}$ is a square or a not-square, and $\mu= \pm 1$ according as $(-1)^{m-1} \alpha_{3} \alpha_{4} \ldots \alpha_{2 m}$ is a square or a not-square. For the value $\eta=0$, they have respectively $p^{n}+\left(p^{n}-1\right) \lambda$ and $p^{n(2 m-3)}-\mu p^{n(m-2)}$ sets of solutions. Finally, for $\eta=\varkappa$, they have respectively $p^{n}-\lambda$ and $\dot{p}^{n(2 n-3)}+\mu\left(p^{n(n-1)}-p^{n(n-2)}\right)$ sets of solutions. The total number of sets of solutions is therefore

$$
\begin{aligned}
& \left(p^{n}-2\right)\left(p^{n}-\lambda\right)\left(p^{n(2 m-3)}-\mu p^{n(m-2)}\right)+\left[p^{n}+\left(p^{n}-1\right) \lambda\right]\left[p^{n(2 m-3)}-\mu p^{n(m-2)}\right] \\
& \quad+\left(p^{n}-\lambda\right)\left[p^{n(2 m-3)}+\mu\left(p^{n(m-1)}-p^{n(m-2)}\right]\right. \\
& \quad \equiv p^{n(m-1)}\left(p^{n n}-\lambda \mu\right) .
\end{aligned}
$$

By $\S 61, \lambda \mu=\nu$. Hence the induction is complete.

$2^{0}$. Let $x=0$. Separating the two cases $\eta \neq 0$ and $\eta=0$, we find the total number of solutions to be

$$
\begin{aligned}
& \left(p^{n}-1\right)\left(p^{n}-\lambda\right)\left(p^{n(2 m-3)}-\mu p^{n(m-2)}\right) \\
+ & {\left[p^{n}+\lambda\left(p^{n}-1\right)\right]\left[p^{n(2 m-3)}+\mu\left(p^{n(m-1)}-p^{n(m-2)}\right)\right] } \\
\equiv & p^{n(2 m-1}+\lambda \mu\left(p^{n m}-p^{n(m-1)}\right) .
\end{aligned}
$$


66. Theorem. - The number of sets of solutions in the $G F\left[p^{n}\right], p>2$, of the equation

$$
\alpha_{1} \xi_{1}^{2}+\alpha_{2} \xi_{2}^{2}+\cdots+\alpha_{2 m+1} \xi_{2 m+1}^{2}=x
$$

where each $\alpha_{j}$ is a mark $\neq 0$ in the field and $x$ belongs to the field, is $p^{2 n m}+\omega p^{n m}$, where $\omega=+1,-1$ or 0 according as $(-1)^{m} x \alpha_{1} \alpha_{2} \ldots \alpha_{2 m+1}$ is a square, a not-square or zero in the field.

Consider the equivalent system of equations

$$
\alpha_{1} \xi_{1}^{2}=\eta, \quad \alpha_{2} \xi_{2}^{2}+\cdots+\alpha_{2 m+1} \xi_{2 m+1}^{2}=x-\eta .
$$

The first equation has one solution if $\eta=0$. If $\eta \neq 0$, it has two or no solutions according as $\alpha_{1} \eta$ is a square or a not-square. Let $\mu=0$ if $x=0$, and $\mu= \pm 1$ according as $\alpha_{1} x$ is a square or a notsquare. We may express the number of solutions of the second equation by $\S 65$, if we set $\nu= \pm 1$ according as $(-1)^{m} \alpha_{2} \ldots \alpha_{2 m+1}$ is a square or a not-square. Evidently we have $\mu \nu=\omega$.

According as $\mu=0,+1$, or -1 , the total number of sets of solutions of the pair of equations is respectively

$$
\begin{aligned}
& 1\left[p^{n(2 m-1)}+v\left(p^{n m}-p^{n(m-1)}\right]+2\left(\frac{p^{n}-1}{2}\right)\left[p^{n(2 m-1)}-\nu p^{n(m-1)}\right] \equiv p^{2 n m},\right. \\
& 1\left[p^{n(2 m-1)}-v p^{n(m-1)}\right]+2\left[p^{n(2 m-1)}+v\left(p^{n m}-p^{n(m-1)}\right]\right. \\
& +2\left(\frac{p^{n}-3}{2}\right)\left[p^{n(2 m-1)}-v p^{n(m-1)}\right] \equiv p^{2 n m}+\nu p^{n m},
\end{aligned}
$$

$1\left[p^{n(2 m-1)}-\nu p^{n(m-1)}\right]+2\left(\frac{p^{n}-1}{2}\right)\left[p^{n(2 m-1)}-\nu p^{n(m-1)}\right] \equiv p^{2 n m}-\nu p^{n m}$.

In each of the three cases, we have enumerated separately the number of solutions arising when $\eta=0$, when $\eta=x$ and when $\eta$ is one of the values $\neq 0$ for which the first equation has solutions (viz., two).

6\%. Theorem. - If $S$ denote the number of squares $\left.^{1}\right) \sigma^{2}$ in the $G F\left[p^{n}\right]$ for which $\sigma^{2}+1$ is a square and $N$ the number of square $\tau^{2}$ for which $\tau^{2}+1$ is a not-square, we have

$$
\begin{array}{ll}
S=\frac{1}{4}\left(p^{n}-5\right), \quad N=\frac{1}{4}\left(p^{n}-1\right), \quad \text { if }-1=\text { square } \\
S=\frac{1}{4}\left(p^{n}-3\right), \quad N=\frac{1}{4}\left(p^{n}+1\right), \quad \text { if }-1=\text { not-square. }
\end{array}
$$

Indeed, the number of sets of solutions $\xi, \eta$ in the $G F\left[p^{n}\right]$ of the equation

$$
\eta^{2}=\xi^{2}+1
$$

is always $p^{n}-1$ (by $\S 64$ ). These solutions are of three kinds:

$$
\begin{aligned}
& 1^{0} . \xi=0, \quad \eta= \pm 1 \text {; } \\
& 2^{0} \text {. } \xi^{2}=-1, \quad \eta=\overline{0} \text {, }
\end{aligned}
$$

occurring when -1 is a square;

$$
3^{0} . \xi^{2}=\alpha \neq 0, \quad \eta^{2}=\alpha+1 \neq 0,
$$

giving $4 S$ sets of solutions $\xi, \eta$.

1) The mark zero is not reckoned as a square. 
Hence, if -1 be a square, we have

$$
p^{n}-1=2+2+4 S, \quad N+S+1=\frac{1}{2}\left(p^{n}-1\right) \text {. }
$$

If -1 be a not-square, we have

$$
p^{n}-1=2+4 S, \quad N+S=\frac{1}{2}\left(p^{n}-1\right) .
$$

Additive-groups in the $G F\left[p^{n}\right]$ and their multiplier Galois Fields ${ }^{1}$ ), $\S \S 68-71$.

68. A set of $m$ marks $\lambda_{1}, \lambda_{2}, \ldots, \lambda_{m}$ belonging to the $G F\left[p^{n}\right]$ and linearly independent with respect to the $G F[p]$ give rise to $p^{m}$ distinct marks of the larger field,

$$
\text { 39) } \left.c_{1} \lambda_{1}+c_{2} \lambda_{2}+\cdots+c_{m} \lambda_{m} \quad \text { (every } c_{i}=0,1, \ldots, p-1\right)
$$

Indeed, an identity between two of the marks 39) would contradict the linear independence of $\lambda_{1}, \lambda_{2}, \ldots, \lambda_{m}$. Since the sum of any two of these $p^{m}$ marks 39) may be expressed as one of the set, they are said to form an additive-group $\left[\lambda_{1}, \lambda_{2}, \ldots, \lambda_{m}\right]$ of rank $m$ with respect to the $G F[p]$ and the marks $\lambda_{1}, \lambda_{2}, \ldots, \lambda_{m}$ are said to form its basis-system. In particular, the $G F\left[p^{n}\right]$ may be exhibited as an additive-group of rank $n(\S 10)$.

These conceptions are capable of the following direct generalization. Any $m$ marks $\lambda_{1}, \lambda_{2}, \ldots, \lambda_{m}$ of the $G F\left[p^{n r}\right]$ are called linearly independent with respect to the $G F\left[p^{r}\right]$ if the equation

$$
\gamma_{1} \lambda_{1}+\gamma_{2} \lambda_{2}+\cdots+\gamma_{m} \lambda_{m}=0
$$

in which the $\gamma_{i}$ are marks of the $G F\left[p^{r}\right]$, can be satisfied only in case every $\gamma_{i}=0$. [See $\S 72$ ]. A system of $m$ linearly independent marks gives rise to $p^{r m}$ distinct marks of the $G F\left[p^{n r}\right]$

$$
\gamma_{1} \lambda_{1}+\gamma_{2} \lambda_{2}+\cdots+\gamma_{m} \lambda_{m}
$$

by letting th $\gamma_{i}$ 's run independently through the series of the marks of the $G F\left[p^{r}\right]$. These $p^{r m}$ marks are said to form an additive-group $\left[\lambda_{1}, \lambda_{2}, \ldots, \lambda_{m}\right]$ of rank $m$ with respect to the $G F\left[p^{r}\right]$, the marks $\lambda_{1}, \ldots, \lambda_{m}$ forming its basis-system.

If $\lambda_{m+1}$ be any mark of $G F\left[p^{n r}\right]$ not in the additive-group $\left[\lambda_{1}, \ldots, \lambda_{m}\right]$ of rank $m$ with respect to the $G F\left[p^{r}\right]$, then the $m+1$ marks $\lambda_{1}, \ldots, \lambda_{m}, \lambda_{m+1}$ are linearly independent with respect to the $G F^{\prime}\left[p^{r}\right]$ and therefore define an additive-group $\left[\lambda_{1}, \ldots, \lambda_{m}, \lambda_{m+1}\right]$ of rank $m+1$ with respect to the $G F\left[p^{r}\right]$.

69. Theorem. - Within the $G F\left[p^{n r}\right]$ the number of additivegroups $\left[\lambda_{1}, \ldots, \lambda_{m}\right]$ of rank $m$ with respect to the $G F\left[p^{r}\right]$ is

$$
\frac{\left(p^{n r}-1\right)\left(p^{n r}-p^{r}\right) \ldots\left(p^{n r}-p^{(m-1) r}\right)}{\left(p^{m r}-1\right)\left(p^{m r}-p^{r}\right) \cdots\left(p^{m r}-p^{(m-1) r}\right)} \text {. }
$$

1) Moore, Mathematical Papers, Congress of 1893, p. 214, p. 216; Math. Ann. vol. 55, § 12.

DrCksoN, Linear Groups. 
We first prove that the numerator expresses the number of sets of $m$ marks $\lambda_{1}, \lambda_{2}, \ldots, \lambda_{m}$ of the $G F\left[p^{n} r\right]$ linearly independent with respect to the $G F\left[p^{r}\right]$. For $\lambda_{1}$ we may take any one of the $p^{n r}-1$ marks $\neq 0$ of the $G F\left[p^{n r}\right]$; for $\lambda_{2}$ any one of the $p^{n r}-p^{r}$ marks not of the form $\varrho_{1} \lambda_{1}$, where $\varrho_{1}$ belongs to the $G F\left[p^{r}\right]$; for $\lambda_{3}$ any one of the $p^{n r}-p^{2 r}$ marks not of the form $\varrho_{1} \lambda_{1}+\varrho_{2} \lambda_{2}$, where $\varrho_{1}$ and $\varrho_{2}$ belong to the $G F\left[p^{r}\right]$; etc.

We next show that the denominator expresses the number of these sets of $m$ independent marks which generate the same additivegroup $\left[\lambda_{1}, \lambda_{2}, \ldots, \lambda_{m}\right]$. In fact, we may use as a basis-system for the latter any set of $m$ marks $\lambda_{1}^{\prime}, \lambda_{2}^{\prime}, \ldots, \lambda_{m}^{\prime}$ chosen as follows. $\lambda_{1}^{\prime}$ may be chosen in $p^{m r}-1$ ways:

$$
\lambda_{1}^{\prime}=\sum_{i=1}^{m} \gamma_{1 i} \lambda_{i}
$$

each $\gamma_{1 i}$ being arbitrary in the $G F\left[p^{r}\right]$ provided not all are simultaneously zero. $\lambda_{2}^{\prime}$ may be chosen in $p^{m r}-p^{r}$ ways, viz.,

$$
\lambda_{2}^{\prime}=\sum_{i=1}^{n} \gamma_{2 i} \lambda_{i}
$$

the $\gamma_{2 i}$ being taken arbitrarily in the $G F\left[p^{r}\right]$ but so as to exclude the $p^{r}$ sets of values which make $\lambda_{2}^{\prime}=\varrho \lambda_{1}^{\prime}$, viz.,

$$
\gamma_{2 i}=\varrho \gamma_{1 i} \quad(i=1,2, \ldots, m),
$$

where $\varrho$ runs through the series of marks of the $G F\left[p^{r}\right]$; etc.

70. If the $p^{m}$ marks $c_{1} \lambda_{1}+\cdots+c_{m} \lambda_{m}$ of the additive-group $\left[\lambda_{1}, \ldots, \lambda_{m}\right]$ of rank $m$ with respect to the $G F[p]$ are multiplied by any particular mark $\mu \neq 0$ of the $G F\left[p^{n}\right]$, the resulting $p^{m}$ marks constitute the additive-group

$$
\mu\left[\lambda_{1}, \ldots, \lambda_{m}\right] \equiv\left[\mu \lambda_{1}, \ldots, \mu \lambda_{m}\right]
$$

likewise of rank $m$ with respect to the $G F[p]$. We will say that $\left[\mu \lambda_{1}, \ldots, \mu \lambda_{m}\right]$ is derived from $\left[\lambda_{1}, \ldots, \lambda_{m}\right]$ by multiplication by $\mu$. In particular, we seek those multipliers $\mu=x$ which do not alter $\left[\lambda_{1}, \ldots, \lambda_{m}\right]$, such a mark being called a multiplier of the additivegroup $\left[\lambda_{1}, \ldots, \lambda_{m}\right]$. If $x_{1}$ and $x_{2}$ be multipliers, then will evidently the product $x_{1} x_{2}$ be a multiplier. To prove that $\mu \equiv x_{1}+x_{2}$ will also be a' multiplier, we observe first that $\left[\mu \lambda_{1}, \ldots, \mu \lambda_{m}\right]$ is an additivegroup included within $\left[\lambda_{1}, \ldots, \lambda_{m}\right]$, since $x_{1}$ and $x_{2}$ are multipliers of the latter, and further that it is of rank $m$ if $\mu \neq 0$. Hence $x_{1}+x_{2}$ is a multiplier unless it be zero. Hence the multipliers $x$ together with the mark zero constitute an additive, as well as a multiplicative, group and therefore constitute a Galois Field $G F\left[p^{k}\right]$ included within 
the fundamental $G F\left[p^{n}\right]$. It is called the multiplier Galois Field of the additive-group $\left[\lambda_{1}, \ldots, \lambda_{m}\right]$. Every $G F\left[p^{k^{\prime}}\right]$ included within the $G F\left[p^{k}\right]$ is called a multiplier Galois Field of the additive-group. By $\$ 23, k^{\prime}$ is a divisor of $k$ and $k_{\text {a }}$ a divisor of $n$.

The additive-group $\left[\lambda_{1}, \ldots, \lambda_{m}\right]$ of rank $m$ with respect to the $G F[p]$ may be exhibited as an additive-group $\left[\lambda_{1}^{\prime}, \ldots, \lambda_{m^{\prime}}^{\prime}\right]$ of rank $m^{\prime}=m / k^{\prime}$ with respect to any multiplier $G F\left[p^{k^{\prime}}\right]$.

In proof, let $\gamma_{1}, \gamma_{2}, \ldots, \gamma_{m^{\prime}}$ run independently through the series of marks of the $G F\left[p^{k^{\prime}}\right]$. Taking $\lambda_{1}^{\prime}$ to be any particular mark $\lambda \neq 0$ in $\left[\lambda_{1}, \ldots, \lambda_{n}\right]$, the $p^{k^{\prime}}$ marks $\gamma_{1} \lambda_{1}^{\prime}$ are all distinct and all belong to $\left[\lambda_{1}, \ldots, \lambda_{m}\right]$. Taking $\lambda_{2}^{\prime}$ any mark in $\left[\lambda_{1}, \ldots, \lambda_{m}\right]$ different from the $\gamma_{1} \lambda_{1}^{\prime}$, the $p^{2 k^{\prime}}$ marks $\gamma_{1} \lambda_{1}^{\prime}+\gamma_{2} \lambda_{2}^{\prime}$ are all distinct and all belong to $\left[\lambda_{1}, \ldots, \lambda_{m}\right]$. Proceeding similarly, we obtain ultimately a set of $p^{m^{\prime} k^{\prime}}$ distinct marks $\gamma_{1} \lambda_{1}^{\prime}+\gamma_{2} \lambda_{2}^{\prime}+\cdots+\gamma_{m^{\prime}} \lambda_{m^{\prime}}^{\prime}$ giving all the marks of $\left[\lambda_{1}, \ldots, \lambda_{m}\right]$. In particular, $p^{m}=p^{k^{\prime} m^{\prime}}$, so that $k^{\prime}$ divides $m$.

Corollary I. Since $k_{i}$ is a particular $k^{\prime}, k^{\prime}$ divides $m$.

Corollary II. Within the GF[p $p^{n}$ the number $A(p, n, m, 7)$ of additive-groups of rank $m$ with respect to the $G F[p]$ which have the $G \cdot F\left[p^{k}\right]$ as a multiplier Galois Field equals the total number of additive-groups of rank $m / k$ with respect to the $G F\left[p^{k}\right]$ :

$$
A(p, n, m, k)=\frac{\left(p^{n}-1\right)\left(p^{n}-p^{k}\right)\left(p^{n}-p^{2 k}\right) \ldots\left(p^{n}-p^{m}-k\right)}{\left(p^{m}-1\right)\left(p^{m}-p^{k}\right)\left(p^{m}-p^{2 k}\right) \ldots\left(p^{m}-p^{m-k}\right)} .
$$

91. If $k$ be a divisor of $m$ and $n$ and if $h_{1}, h_{2}, \ldots, h_{t}$ are the prime factors occurring in both $m$ and $n$ to a higher power than in $k$, there are in the $G F\left[p^{n}\right]$ exactly

$$
\begin{aligned}
A\left(p, n, m, k_{i}\right)-\sum_{i=1}^{t} A\left(p, n, m, k h_{i}\right) & +\sum_{i, j}^{1, \ldots, t} A\left(p, n, m, k_{i} h_{i} h_{j}\right)-\cdots \\
& +(-1)^{t} A\left(p, n, m, k_{1} h_{1} \ldots h_{t}\right)
\end{aligned}
$$

additive-groups of rank $m$ with respect to the $G F[p]$ which have the $G F\left[p^{k}\right]$ as the multiplier Galois Field.

Indeed, from the $A(p, n, m, k)$ additive-groups having the $G F\left[p^{k}\right]$ as a multiplier Galois Field, we must eliminate those having a larger multiplier Galois Field. It suffices to eliminate those having the $G F\left[p^{k h_{i}}\right]$, for $i=1,2, \ldots$, or $t$, as $a$ multiplier Galois Field. But the $A\left(p, n, m, k h_{1}\right)$ additive-groups with the $G F\left[p^{k h_{1}}\right]$ and the $A\left(p, n, m, l_{i} h_{2}\right)$ additive-groups with the $G F\left[p^{k h_{2}}\right]$ are not distinct but have in common $A\left(p, n, m, k h_{1} h_{2}\right)$ additive-groups each with the $G F\left[p^{k h_{1} l_{2}}\right]$ as a multiplier Galois Field. By this principle, we readily determine the number of distinct additive-groups among the sets of $A\left(p, n, m, l_{i} h_{i}\right)$ with the $G F\left[p^{k h_{i}}\right]$ as multiplier Galois Fields. Subtracting this number from $A(p, n, m, k)$, we obtain the required number. 
72. Theorem. The marks $\lambda_{1}, \lambda_{2}, \ldots, \lambda_{m}$ of the $G F\left[p^{n m}\right]$ are linearly independent with respect to the $G F\left[p^{n}\right]$ if and only if the following determinant $^{1}$ ) is not zero in the $G F\left[p^{n m}\right]$ :

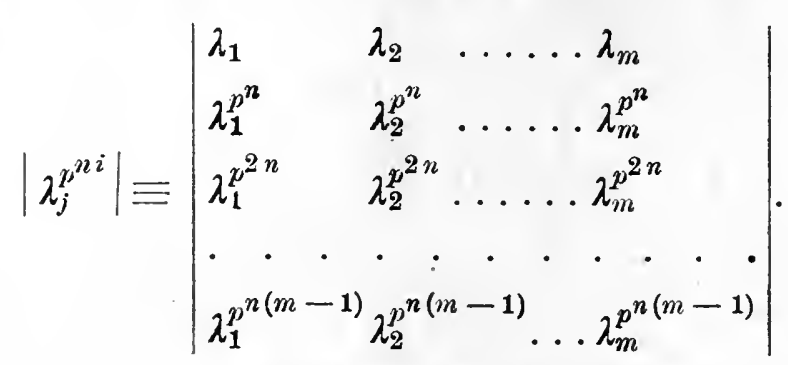

First, if $\lambda_{1}, \lambda_{2}, \ldots, \lambda_{m}$ be linearly dependent, i. e., if a relation $\gamma_{1} \lambda_{1}+\gamma_{2} \lambda_{2}+\cdots+\gamma_{m} \lambda_{m}=0$

holds, the coefficients $\gamma_{j}$ being marks of the $G F\left[p^{n}\right]$ not all zero, then will the determinant $|\lambda|$ vanish.

Secondly, if the determinant vanish, set

$$
\lambda_{i} \equiv \sum_{j=0}^{m-1} \mu_{i j} R^{j} \quad(i=1,2, \ldots, m),
$$

where $R$ is a primitive root of the $G F\left[p^{n m}\right]$ and therefore satisfies an equation of degree $m$ belonging to and irreducible in the $G F\left[p^{n}\right]$, and where the $\mu_{i j}$ belong to the latter field. Then

$$
\left|\lambda_{j}^{p^{n i}}\right|=\left|\mu_{i j}\right| \cdot\left|R^{j p^{n i}}\right| \quad(j=1, \ldots, m ; i=0, \ldots, m-1),
$$

where the determinant in $R$, when written in full, is ${ }^{2}$ )

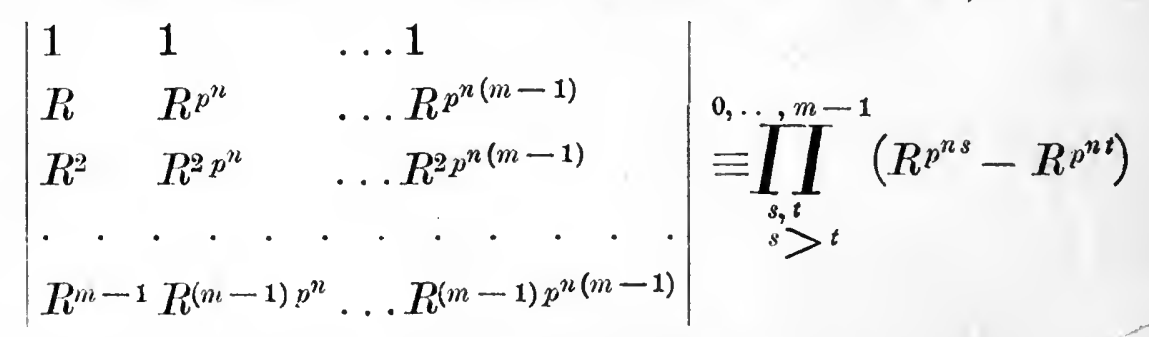

and therefore is not zero in the $G F\left[p^{n m}\right]$. Hence, if $|\lambda|=0$, then must $|\boldsymbol{\mu}|=0$, so that between the $\lambda_{i}$ exists a linear relation with coefficients belonging to the $G F\left[p^{n}\right]$ and not all zero.

73. If $\lambda$ be a mark of the $G F\left[p^{n m}\right]$, the marks

$$
\lambda, \quad \lambda^{p^{n}}, \quad \lambda p^{2 n}, \ldots, \lambda^{p^{n(m-1)}} \quad\left(\lambda p^{n m}=\lambda\right)
$$

are said to be conjugate with respect to the $G F\left[p^{n}\right]$. Any symmetric function of them is unaltered upon being raised to the power $p^{n}$ and

1) Its decomposition into linear factors is given by Moore, "A two-fold generalization of Fermat's theorem", Bull. Amer. Math. Soc., vol. 2 (1896).

2) Baltzer, Determinanten, p. 85 . 
hence belongs to the $G F\left[p^{n}\right]$. Hence the $m$ conjugate marks are the roots of an equation of degree $m$ with coefficients in the $G F\left[p^{n}\right]$.

By $\S 31$, the roots of an equation of degree $m$ belonging to and irreducible in the $G F\left[p^{n}\right]$ are conjugate with respect to the $G F\left[p^{n}\right]$.

In particular, the marks $A, A^{p^{n}}$ of the $G F\left[p^{2 n}\right]$ are conjugate with respect to the $G F\left[p^{n}\right]$. The conjugate $A^{p^{n}}$ of $A$ will be designated by $\bar{A}$. Evidently $\bar{A}=A$ if, and only if, $A$ belongs to the $G F\left[p^{n}\right]$. The following relations are proven at once:

$$
\overline{A B}=\bar{A} \cdot \bar{B}=\overline{B A}, \quad \overline{A^{t}}=(\bar{A})^{t}, \quad \overline{A+B}=\bar{A}+\bar{B}, \quad \overline{(A / B)}=\bar{A} / \bar{B} .
$$

74. Newton's identities. If $S_{t}$ denote the sum of the $t^{\text {th }}$ powers of the roots of the equation

$$
f(x) \equiv x^{n}+a_{1} x^{m-1}+a_{2} x^{m-2}+\cdots+a_{m-1} x+a_{m}=0,
$$

in which the coefficients $a_{i}$ belong to the $G F\left[p^{n}\right]$, then

$$
\begin{aligned}
& 0=S_{1}+a_{1} \\
& 0=S_{2}+a_{1} S_{1}+2 a_{2} \\
& \text { 40) } 0=S_{3}+a_{1} S_{2}+a_{2} S_{1}+3 a_{3} \\
& 0=S_{m-1}+a_{1} S_{m-2}+a_{2} S_{m-3}+\cdots+a_{m-2} S_{1}+(m-1) a_{m-1} .
\end{aligned}
$$

These identities follow as in algebra upon equating the coefficients of like powers of $x$ in the following identity, in which $\alpha_{1}, \ldots, \alpha_{m}$ are the roots of $f(x)=0$ :

$$
\begin{aligned}
f^{\prime}(x) \equiv \sum_{i=1}^{m} \frac{f(x)}{x-\alpha_{i}}=m x^{m-1} & +(m-1) a_{1} x^{m-2} \\
& +(m-2) a_{2} x^{m-3}+\cdots+a_{m-1}
\end{aligned}
$$

This identity, evidently true for $m=1$, may be proven by simple induction ${ }^{1}$ ). Supposing it true for a particular $m$, we have proven it true for the value $m+1$. Let

$$
\begin{gathered}
F(x) \equiv f(x) \cdot\left(x-\alpha_{m+1}\right) \equiv x^{m+1}+\left(a_{1}-\alpha_{m+1}\right) x^{m}+\left(a_{2}-a_{1} \alpha_{m+1}\right) x^{m-1}+\cdots \\
+\left(a_{m}-a_{m-1} \alpha_{m+1}\right) x-a_{m} \alpha_{m+1}
\end{gathered}
$$

Multiplying 41) by $x-\alpha_{m+1}$ and adding $f(x)$ to the left member and $x^{m}+\cdots+a_{m}$ to te right member, we find

$$
\begin{aligned}
\sum_{i=1}^{m} \frac{F(x)}{x-\alpha_{i}} & +\frac{F(x)}{x-\alpha_{m}+1}=(m+1) x^{m}+m\left(a_{1}-\alpha_{m+1}\right) x^{m-1} \\
& +(m-1)\left(a_{2}-a_{1} \alpha_{m+1}\right) x^{m-2}+\cdots+2\left(a_{m-1}-a_{m-2} \alpha_{m+1}\right) x \\
& +\left(a_{m}-a_{m-1} \alpha_{m+1}\right) .
\end{aligned}
$$

1) Since equations in the $G F\left[p^{n}\right]$ are not algebraic identities, we avoid the consideration of derivatives. We might, however, employ Weber's definition (Algebra, I, § 13) of the derivatives of a polynomial in $x$ for the derivatives up to the $p^{\text {th }}$, but not for the higher derivatives on account of the denominators $\Pi(m)$. 
Hence if 41) be true for $f(x)=0$ with the roots $\alpha_{1}, \ldots, \alpha_{m}$, a like formula is true for the equation $F(x)=0$ with the roots $\alpha_{i}, \ldots, \alpha_{m}$, $\alpha_{m+1}$.

Forming the sums

$$
\sum_{i=1}^{m} f\left(\alpha_{i}\right), \quad \sum_{i=1}^{m} \alpha_{i} f\left(\alpha_{i}\right), \quad \sum_{i=1}^{m} \alpha_{i}^{2} f\left(\alpha_{i}\right), \ldots
$$

we derive the new identities,

$$
\begin{aligned}
& 0=S_{m}+a_{1} S_{m-1}+a_{2} S_{m-2}+\cdots+m a_{m}, \\
& 0=S_{m+1}+a_{1} S_{m}+a_{2} S_{m-1}+\cdots+a_{m} S_{1}, \\
& 0=S_{m+2}+a_{1} S_{m+1}+a_{2} S_{m}+\cdots+a_{m} S_{2},
\end{aligned}
$$

Corollary. - If $f(x)=0$ have a double root $\alpha$, the right member of 41) must vanish for $x=\alpha$.

75. Theorem. - If $t$ be a positive integer and $u_{0}, u_{1}, \ldots, u_{p}^{n}-1$ denote the marks of the $G F\left[p^{n}\right]$, then

$$
\sum_{i=0}^{p^{n}-1} u u_{i}^{t}=\left\{\begin{array}{cc}
0 & \text { for } t<p^{n}-1 \\
-1 & \text { for } t=p^{n}-1
\end{array}\right.
$$

In fact, the marks $u_{i}$ are the roots in the $G F\left[p^{n}\right]$ of the equation

$$
x^{p^{n}}-x=0 \text {. }
$$

Applying to the latter the identities 40), we find

$$
S_{i}=0 \quad\left(i=1,2, \ldots, p^{n}-2\right), \quad S_{p^{n}-1}-\left(p^{n}-1\right)=0 .
$$

\section{CHAPTER V.}

\section{ANALYTIC REPRESENTATION OF SUBSTITUTIONS}

\section{ON THE MARKS OF A GALOIS FIELD.}

76. Consider the problem to find every quantic $\Phi(\xi)$ belonging to the $G F\left[p^{n}\right]$ such that the equation $\Phi(\xi)=\beta$ has a root in the field whatever mark of the field $\beta$ may be. For example,

$$
\xi^{3} \equiv \beta \quad(\bmod 5)
$$

is solvable for every integer $\beta$, since we have

$$
0^{3} \equiv 0, \quad 1^{3} \equiv 1, \quad 3^{3} \equiv 2, \quad 2^{3} \equiv 3, \quad 4^{3} \equiv 4 \quad(\bmod 5)
$$


If we denote the marks of the $G F\left[p^{n}\right]$ as follows,

$$
\mu_{0}, \quad \mu_{1}, \mu_{2}, \ldots, \mu_{p^{n}-1},
$$

the necessary and sufficient conditions that $\Phi(\xi)=\beta$ be solvable in the field for arbitrary $\beta$ are that the marks

$$
\Phi\left(\mu_{0}\right), \quad \Phi\left(\mu_{1}\right), \quad \Phi\left(\mu_{2}\right), \ldots, \Phi\left(\mu_{p^{n}-1}\right)
$$

be identical with the series 44 ) apart from their order. In fact, the $p^{n}$ values which $\Phi(\xi)$ takes must all be distinct, since $\beta$ is to have $p^{n}$ distinct values. When the conditions named are satisfied, the series 45) forms a permutation of the series 44), and the quantic $\Phi(\xi)$ is said to represent the substitution

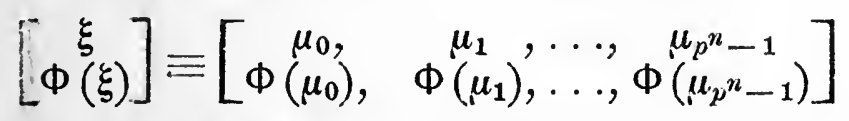

on the marks of the $G F\left[p^{n}\right]$. For example, $\xi^{3}$ represents the substitution

$$
\left[\begin{array}{c}
\xi \\
\xi
\end{array}\right] \equiv\left[\begin{array}{lllll}
0, & 1, & 2, & 3, & 4 \\
0, & 1, & 3, & 2, & 4
\end{array}\right]
$$

on the marks of the $G F[5]$, i. e., the field of integers taken modulo 5. A quantic of degree $k$ with coefficients belonging to the $G F\left[p^{n}\right]$ will be called a substitution quantic $S Q\left[k, p^{n}\right]$ if it satisfy the above conditions. Its degree $k$ will be supposed $<p^{n}$ in view of the equation $\xi p^{n}=\xi$ satisfied by every mark of the field.

9\%. An arbitrary substitution on the marks of the GF[ $\left.p^{n}\right]$,

$$
\left[\begin{array}{ll}
\mu_{0}, & \mu_{1}, \ldots, \mu_{p^{n}-1} \\
\mu_{a_{0}}, & \mu_{a_{1}}, \ldots, \mu_{a_{p}{ }^{n}}
\end{array}\right]
$$

can be represented by the quantic $\Phi(\xi)$ given by Lagrange's interpolation formula,

where

$$
\Phi(\xi) \equiv \sum_{i=0}^{p^{n}-1} \frac{\mu_{a_{i}} F^{\prime}(\xi)}{\left(\xi-\mu_{i}\right) F^{\prime}\left(\mu_{i}\right)},
$$

$$
F(\xi) \equiv \prod_{i=0}^{p^{n}-1}\left(\xi-\mu_{i}\right),
$$

and $F^{\prime}(\xi)$ denotes the function derived from $F(\xi)$ by formula 41). Evidently $\Phi(\xi)$ is an integral function of $\xi$ of degree $<p^{n}$.

78. Theorem. Two distinct quantics $\Phi(\xi)$ and $\Psi(\xi)$ belonging to the $G F\left[p^{n}\right]$ can not represent the same substitution on its marks.

For, if

$$
\Phi\left(\mu_{i}\right)=\Psi\left(\mu_{i}\right) \quad\left(i=0,1, \ldots, p^{n}-1\right),
$$

the equation $\Phi(\xi)-\Psi(\xi)=0$ would have in the field $p^{u}$ distinct roots $\mu_{i}$, whereas its degree is less than $p^{n}$. By $\S 15$, it must be an identity in $\xi$. 
79. Theorem. $\xi^{m}$ is a $S Q\left[m, p^{n}\right]$ if, and only if, $m$ be prime to $p^{n}-1$.

The theorem follows immediately from the corollary of $\S 63$. However, to illustrate a method of proof used below, we will verify that, if $m$ be relatively prime to $p^{n}-1$, $\xi^{m}$ takes $p^{n}$ distinct values in the $G F\left[p^{n}\right]$ when $\xi$ does. It is sufficient to prove that from

$$
\xi_{1}^{m}=\xi_{2}^{m}
$$

follows $\xi_{1}=\xi_{2}$, provided $\xi_{1}$ and $\xi_{2}$ are marks of the field. This being evident if either be the mark zero, we suppose $\xi_{1} \neq 0, \xi_{2} \neq 0$, so that

$$
\xi_{1}^{p^{n}-1}=\xi_{2}^{p^{n}-1}=1
$$

Raising the members of equations 46) and 47) to the respective powers $t$ and $\tau$, chosen ( $\S 7$, note) so that $t m+\tau\left(p^{n}-1\right)=1$, and forming the product of the resulting equations, we find that $\xi_{1}=\xi_{2}$.

80. Theorem. - For an arbitrary mark $\alpha$ of the GF[p $\left.p^{n}\right]$,

$$
\Phi(\xi) \equiv 5 \xi^{5}+5 \alpha \xi^{3}+a^{2} \xi
$$

is a $S Q\left[5, p^{n}\right]$, if $p$ is a prime of the form $5 m \pm 2$ and $n$ is odd. To prove that, in the $G F\left[p^{n}\right], \xi_{1}=\xi_{2}$ is the only solution of

or

$$
\Phi\left(\xi_{1}\right)=\Phi\left(\xi_{2}\right)
$$

$\left(\xi_{1}-\xi_{2}\right)\left\{5\left(\xi_{1}^{4}+\xi_{1}^{3} \xi_{2}+\xi_{1}^{2} \xi_{2}^{2}+\xi_{1} \xi_{2}^{3}+\xi_{2}^{4}\right)+5 \alpha\left(\xi_{1}^{2}+\xi_{1} \xi_{2}+\xi_{2}^{2}\right)+\alpha^{2}\right\}=0$ we set

$$
\xi_{1}=\lambda+\mu, \quad \xi_{2}=\lambda-\mu,
$$

here $^{1}$ ) limiting our proof to the case $p>2$. Then 16 times the quantity within the braces becomes

$$
\begin{aligned}
& 16\left\{5\left(5 \lambda^{4}+3 \alpha \lambda^{2}+10 \lambda^{2} \mu^{2}+\alpha \mu^{2}+\mu^{4}\right)+\alpha^{2}\right\} \\
& \equiv\left\{\left(20 \lambda^{2}+\alpha\right)+5\left(4 \mu^{2}+\alpha\right)\right\}^{2}-5\left\{2\left(4 \mu^{2}+\alpha\right)\right\}^{2} .
\end{aligned}
$$

But $\left.^{2}\right)+5$ is a quadratic residue of no odd number of the form $5 m+2$ or $5 m-2$. Hence $(\S 62) 5$ is a not-square in the $G F\left[5^{n}\right]$, $n$ being odd and $p=5 m \pm 2$. Hence, if the above expression vanishes, we must have

$$
4 \mu^{2}+\alpha=0,20 \lambda^{2}+\alpha=0,
$$

whence, for $p>2, \mu^{2}=5 \lambda^{2}$, so that $\lambda=\mu=0, \xi_{1}=\xi_{2}$.

1) An analogous proof for $p=2$ is given in Annals of Math., 1897, pp. 84-85. For an arbitrary prime $p$, the theorem is a special case of that of $\S 82$.

2) Gauss, Disquisitiones Arithmeticae, Art. 121. 
81. Theorem. - The quantic belonging to the $G F\left[p^{n m}\right]$,

$$
\Psi(X) \equiv \sum_{i=1}^{m} A_{i} X^{p^{n(m-i)}}
$$

will represent a substitution on its marks if, and only if, $X=0$ is the only solution in the field of $\Psi(X)=0$.

Indeed, the necessary and sufficient condition is that

$$
\Psi\left(X_{1}-X_{2}\right) \equiv \Psi\left(X_{1}\right)-\Psi\left(X_{2}\right)=0
$$

shall require $X_{1}=X_{2}$, or $X_{1}-X_{2}=0$.

Corollary. $X^{p^{n r}}-A X^{p^{n s}}$ represents a substitution on the marks of the $G F\left[p^{n m}\right]$ if, and only if, either $A=0$ or else $A$ is not the power $p^{n r}-p^{n s}$ of a mark of the field.

82. Theorem. - If 7 be an odd integer relatively prime to $p^{2 n}-1$, and if $\alpha$ be an arbitrary mark of the $G F\left[p^{n}\right]$, the quantic

$$
\Phi_{k}(\xi, \alpha) \equiv \xi^{k}+k \alpha \xi^{k-2}+7 \sum_{l=2}^{\frac{1}{2}(k-1)} \frac{(k-l-1)(k-l-2) \ldots(k-2 l+1)}{2 \cdot 3 \ldots l} \alpha^{l} \xi^{k-2 l}
$$

represents a substitution on the marks of the $G F\left[p^{n}\right]$.

We are to prove that the equation

$$
\Phi_{k}(\xi, \alpha)=\beta
$$

has a solution $\xi$ in the $G F\left[p^{n}\right], \quad \beta$ being an arbitrary mark of the field. By Waring's formula, $\Phi_{k}(\xi, \alpha)$ is the sum of the $k^{\text {th }}$ powers of the roots of the quadratic

$$
\eta^{2}-\xi \eta-\alpha=0
$$

Hence, in virtue of the equation

we have the identity

$$
\begin{gathered}
\xi=\eta-\alpha / \eta, \\
\Phi_{k}(\xi, \alpha) \equiv \eta^{k}-(\alpha / \eta)^{k} .
\end{gathered}
$$

The equation 48) thus becomes

$$
\eta^{2 k}-\beta \eta^{k}-\alpha^{k}=0
$$

Setting $Y \equiv \eta^{k}$, this becomes

$$
Y^{2}-\beta Y-\alpha^{k}=0 .
$$

According as 49) is reducible or irreducible in the $G F\left[p^{n}\right]$, it is solvable in the $G F\left[p^{n}\right]$ or in the $G F\left[p^{2 n}\right]$, and therefore always solvable in the larger field. Call its roots $Y_{1}$ and $Y_{2}$. Since $k$ is prime to $p^{2 n}-1$, we can determine uniquely ( 879 or $\S 63$ ) two marks $\eta_{1}$ and $\eta_{2}$ belonging to the $G F\left[p^{2}\right]$ such that

$$
\eta_{1}^{k}=Y_{1}, \quad \eta_{2}^{k}=Y_{2}
$$


Likewise, it follows from $Y_{1} Y_{2}=-\alpha^{k}$ that

$$
\eta_{1} \eta_{2}=-\alpha
$$

If 49 ) be irreducible in the $G F\left[p^{n}\right]$, we have ( $\$ 31$, corollary)

and therefore

$$
Y_{2}^{p^{n}}=Y_{1}, \quad Y_{1}^{p^{n}}=Y_{2},
$$

Hence

$$
\eta_{2}^{p^{n}}=\eta_{1}, \quad \eta_{1}^{p^{n}}=\eta_{2}
$$

$$
\left(\eta_{1}+\eta_{2}\right)^{p^{n}}=\eta_{2}+\eta_{1}
$$

It follows that 48) has the solution in the $G F\left[p^{n}\right]$

$$
\xi=\eta_{1}-\alpha / \eta_{1}=\eta_{2}-\alpha / \eta_{2}=\eta_{1}+\eta_{2} \text {. }
$$

If 49) be reducible, $Y_{1}$ and $Y_{2}$ belong to the $G F\left[p^{n}\right]$. Since $\%$ is prime to $p^{n}-1$, it follows that $\eta_{1}$ and $\eta_{2}$ belong to the $G F\left[p^{n}\right]$.

Remark 1. - We have shown in $\S 37$ that $\Phi_{2} i-1(\xi, 1)$ completely decomposes in the $G F\left[p^{n}\right]$ into linear factors, if $p^{n}=2^{i} t-1$, $t$ odd and $i>1$.

Remark 2. - If $k$ be a prime number, $\Phi_{k}(\xi, \alpha)$ is the only quantic of degree $k$ suitable to represent a substitution on the marks of every $G F\left[p^{n}\right]$ for which $p^{2 n}-1$ is not divisible by 7 (Annals of Mathematics, 1897, pp. 89-91).

Remark 3. - The equation 48) is algebraically solvable, having as roots

where

$$
\varrho \varepsilon^{m}+\sigma \varepsilon^{k-m} \quad(m=0,1, \ldots, k-1)
$$

$$
\varrho, \sigma=\sqrt[k]{\beta / 2 \pm \sqrt{\beta^{2} / 4+\alpha^{k}}}
$$

and $\varepsilon$ denotes a primitive $k^{\text {th }}$ root of unity. This result is a direct generalization of Cardan's formula for the roots of the reduced cubic and of Vallès' solution of the quintic ${ }^{1}$ ) .

$$
\xi^{5}+\alpha \xi^{3}+\frac{\alpha^{2}}{5} \xi=\beta .
$$

83. Theorem. $\left.{ }^{2}\right)$ - If $d$ be a divisor of $p^{r}-1$ and $v$ be not a $d^{\text {th }}$ power in the GF $\left[p^{n}\right]$, the quantic

is a $S Q\left[p^{r}, p^{n}\right]$.

$$
\Phi(\xi) \equiv \xi\left(\xi^{d}-\nu\right)^{\left(p^{r}-1\right) / d}
$$

We are to prove that $\Phi(\xi)=\beta$ has a solution $\xi$ in the $G F\left[p^{n}\right]$ for $\beta$ chosen arbitrarily in the field. This being evident if $\beta=0$, we will suppose that $\beta=1=0$. Writing

$$
\eta \equiv \xi^{d}-v,
$$

1) Formes imaginaires en Algèbre, 1869, vol. 1, pp. 90-92.

2) For the case $r=n$, this theorem is included in the theorem of $\S 85$. 
we are to prove that

50)

$$
[\Phi(\xi)]^{d}=(\eta+v) \eta^{p^{r}-1} \equiv \eta^{p^{r}}+v \eta^{p^{r}-1}=\beta^{d}
$$

has a solution $\eta$ in the $G F\left[p^{n}\right]$; for, if $\eta$ be such a solution (necessarily $=1=0$ ), then

$$
\xi=(\eta+\nu)^{1 / d}=\beta / \eta^{\left(p^{r}-1\right) / d}
$$

will belong to the $G F\left[p^{n}\right]$ and will satisfy $\Phi(\xi)=\beta$.

Setting $\eta=1 / \omega$ in 50) and multiplying by $\omega^{p^{r}}$, we find

$$
1+\boldsymbol{\nu} \boldsymbol{\omega}=\boldsymbol{\beta}^{d} \boldsymbol{\omega}^{p^{r}} \text {. }
$$

This has a solution $\omega$ in the $G F\left[p^{n}\right]$ for $\beta$ arbitrary in the field. Indeed, by $\S 81$, corollary,

$$
\omega^{p^{r}}-\left(\frac{\nu}{\beta^{d}}\right) \omega
$$

represents a substitution on the marks of the $G F\left[p^{n}\right]$, since $\nu / \beta^{d}$ is not a $d^{\text {th }}$ power and hence not a $\left(p^{r}-1\right)^{s t}$ power in the field.

Note. - For $p=3,5,7$ and partially for $p=11$, the author has shown ${ }^{1}$ ) that the only $S Q\left[p, p^{n}\right]$ which exist are reducible to the form

$$
\xi(\xi-\nu)^{(p-1) / d},
$$

where $d$ is a divisor of $p-1$ and $\nu$ is not a $d^{\text {th }}$ power in the $G F\left[p^{n}\right]$.

84. Theorem. $\left.{ }^{2}\right)$ - The necessary and sufficient conditions that $\Phi(\xi)$ shall represent a substitution on the marks of the $G F\left[p^{n}\right]$ are:

$1^{0}$. Every $t^{\text {th }}$ power of $\Phi(\xi)$, for $t \overline{<} p^{n}-2$ and prime to $p$, shall reduce to a degree $\overline{<} p^{n}-2$ on applying the equation $\xi^{n}=\xi$;

$2^{0}$. There shall be one and only one root in the $G F\left[p^{n}\right]$ of $\Phi(\xi)=0$.

After the exponents of $\xi$ are reduced below $p^{n}$, let

$$
[\Phi(\xi)]^{t} \equiv \sum_{i=0}^{p^{n}-1} \alpha_{i}^{(t)} \xi^{i}
$$

Put for $\xi$ the $p^{n}$ marks $\mu_{j}$ of the $G F\left[p^{n}\right]$ and add the resulting indentities. We find, on applying $\S 75$,

$$
\sum_{j=0}^{p^{n}-1}\left[\Phi\left(\mu_{j}\right)\right]^{t}=p^{n} \alpha_{0}^{(t)}+\alpha_{1}^{(t)} \sum_{j=0}^{p^{n}-1} \mu_{j}+\cdots+\alpha_{p^{n}-1}^{(t)} \sum_{j=0}^{p^{n}-1} \mu_{j}^{p^{n}-1}=-\alpha_{p^{n}-1}^{(t)} .
$$

If $\Phi(\xi)$ represent a substitution, we must have

$$
\sum_{j=0}^{p^{n}-1}\left[\Phi\left(\mu_{j}\right)\right]^{t}=\sum_{j=0}^{p^{n}-1} \mu_{j}^{t}=0 \quad\left(t<p^{n}-1\right) .
$$

1) Dissertation, Annals of Mathematics, 1897, pp. 101-108.

2) For the case $n=1$, this theorem is due to Hermite, Comptes Rendus, vol. 57 (1863), pp. $750-757$. 
Hence a necessary condition is that

$$
\alpha_{p^{n}-1}^{(t)}=0 \quad\left(t=1,2, \ldots, p^{n}-2\right) .
$$

The condition $2^{0}$ is evidently a necessary condition.

Suppose, inversely, that $1^{0}$ and $2^{0}$ are satisfied. Consider the equation satisfied by the marks $\Phi\left(\mu_{j}\right)$,

$$
\prod_{j=0}^{p^{n}-1}\left[\eta-\Phi\left(\mu_{j}\right)\right] \equiv \sum_{i=0}^{p^{n}} \gamma_{i} \eta^{\boldsymbol{x}^{n}-i}=0
$$

the sum of the $m^{\text {th }}$ powers of whose roots is denoted by $\sigma_{m}$. Then

$$
\begin{aligned}
\sigma_{t} & \equiv \sum_{j=0}^{p^{n}-1}\left[\Phi\left(\mu_{j}\right)\right]^{t}=-\alpha_{p^{n}-1}^{(t)}=0 \quad\left\{\begin{array}{l}
t=1,2, \ldots, p^{n-2} \\
t \equiv 0(\bmod p)
\end{array}\right\} \\
\sigma_{p^{n}-1} & \equiv \sum_{j=0}^{p^{n}-1}\left[\Phi\left(\mu_{j}\right)\right]^{p^{n}-1}=p^{n}-1 \equiv-1,
\end{aligned}
$$

since all but one of the $\Phi\left(\mu_{j}\right)$ are $==0$ by $2^{0}$ and hence have unity for their $\left(p^{n}-1\right)^{s t}$ powers. Applying Newton's identities 40), we readily find

$$
\begin{aligned}
& \gamma_{i}=0 \quad\left[i=1,2, \ldots, p^{n}-2 ; i \equiv \equiv(\bmod p)\right] \\
& \sigma_{p}=\sigma_{2 p}=\cdots=\sigma_{p^{n}-p}=\sigma_{p^{n}}=0, \quad \gamma_{p^{n}-1}=-1 .
\end{aligned}
$$

To determine $\gamma_{p}, \gamma_{2 p}, \ldots$, we apply the identities 42), viz.,

$$
\sigma_{k}+\gamma_{1} \sigma_{k-1}+\gamma_{2} \sigma_{k-2}+\cdots+\gamma_{p^{n}} \sigma_{k-p^{n}}=0 \quad\left(k>p^{n}\right),
$$

which here reduce to the form

51) $\sigma_{k}+\gamma_{p} \sigma_{k-p}+\gamma_{2 p} \sigma_{k-2 p}+\cdots+\gamma_{p^{n}-p} \sigma_{k-p^{n}+p}+\gamma_{p^{n}-1} \sigma_{k-p^{n}+1}=0$, since by $2^{0}$,

$$
\gamma_{p^{n}} \equiv-\prod_{j=0}^{y^{n}-1} \Phi\left(\boldsymbol{\mu}_{j}\right)=0 .
$$

Furthermore, since any mark equals its $\left(p^{n}\right)^{\text {th }}$ power, we have

$$
\sigma_{s+p^{n}-1}=\sigma_{s} \quad(s=1,2, \ldots)
$$

Applying 51) for $k=p^{n}+p-1$, we find

$$
\gamma_{p} \sigma_{p^{n}-1}=0 \text {. }
$$

More generally, for $k=p^{n}+l p-1, l=p^{n-1}-1$, we get

$$
\gamma_{t p} \sigma_{p^{n}-1}=0 \text {. }
$$

Hence $\gamma_{l p}=0$. We have therefore the result

$$
\prod_{j=0}^{p^{n}-1}\left[\eta-\Phi\left(\mu_{j}\right)\right]=\eta^{z^{n}}-\eta
$$

so that the marks $\Phi\left(\mu_{j}\right)$ form a permutation of the marks $\mu_{j}$ of the $G F\left[p^{n}\right]$. 
85. Theorem. ${ }^{1}$ ) - If $r$ be less than and prime to $p^{n}-1$ and $s$ be a divisor of $p^{n}-1$, and if $f(\xi)$ be a rational integral function of $\xi^{s}$ with coefficients belonging to the $G F\left[p^{n}\right]$ such that $f\left(\xi^{s}\right)=0$ has no root in the field, then

$$
\xi\left[f\left(\xi^{s}\right)\right]^{\left(p^{n}-1\right) / s}
$$

represents a substitution on the $p^{n}$ marls of the field.

The conditions of the theorem of $\S 84$ for a substitution quantic are all satisfied by the given quantic. In fact, upon raising it to any power $l$, not divisible by $s$, we obtain a set of terms whose exponents are of the form $m s+l r$ and therefore not divisible by $s$ and consequently not by $p^{n}-1$. If, however, we take the power

$$
l=t s<p^{n}-1,
$$

we get the result $\xi^{l r}$, since the power $p^{n}-1$ of $f\left(\xi^{s}\right) \neq 0$ is unity in the field. But $l r$ is not divisible by $p^{n}-1$.

Condition $2^{0}$ is satisfied by our quantic, since it vanishes in the field only when $\xi=0$.

86. As examples under the preceding theorem, we note first $\xi$ if $r$ be prime to $p^{n}-1$ [Compare $\S 79$ ]. Next, if $p>2$,

$$
\xi^{r}\left(\xi^{\left(p^{n}-1\right) / 2}-\tau\right)^{2} \equiv-2 \tau\left\{\xi^{r}+\left(p^{n}-1\right) / 2-\frac{1}{2}\left(\tau+\frac{1}{\tau}\right) \xi^{r}\right\}
$$

represents a substitution on the marks of the $G F\left[p^{n}\right]$ if $\tau$ be any mark in the field except $+1,-1,0$. For the remaining $p^{n}-3$ marks $\tau$, the quantics 52) coincide in pairs. We note the following special substitution quantics 52):

$$
\begin{array}{lll}
n=1, & p=5: & \xi^{3}, \xi, \\
n=1, & p=7: & \xi^{4} \pm 3 \xi \text { and } \xi^{5} \pm 2 \xi^{2} \text { (Hermite), } \\
n=1, & p=11: & \xi^{6} \pm 2 \xi, \quad \xi^{6} \pm 4 \xi \\
n=2, & p=3: & \xi, \quad \xi^{3}, \quad \xi^{5}, \quad \xi^{5} \pm 2^{1 / 2} \xi .
\end{array}
$$

For $n=1, p=7, v^{3}=-1$, the theorem of $\S 85$ gives the quantics

$$
\begin{aligned}
& \xi\left(\xi^{2}-\nu\right)^{3} \equiv-3 \nu\left(\xi^{5}-\nu \xi^{3}+3 \nu^{2} \xi\right), \\
& \xi^{5}\left(\xi^{2}-\nu\right)^{3} \equiv 2\left(\xi^{5}+2 \nu \xi^{3}+3(2 \nu)^{2} \xi\right),
\end{aligned}
$$

which together give the following $S Q[5,7]$ of $\$ 80$ :

$$
\xi^{5}+\alpha \xi^{3}+3 \alpha^{2} \xi \quad(\alpha=\text { arbitrary }) .
$$

1) For $n=1$, this theorem is due to Rogers, Proc. Lond. Math. Soc., vol. $22(1890)$, pp. $210-218$. 
87. If $\Phi(\xi) \equiv \alpha_{0} \xi^{k}+\alpha_{1} \xi^{k-1}+\cdots$ be a $S Q\left[k, p^{n}\right]$, then will also $\Phi_{1}(\xi)$, obtained by forming the compound substitution,

$$
\left(\begin{array}{c}
\xi \xi \\
\alpha \xi+\beta
\end{array}\right)\left(\begin{array}{c}
\xi \\
\Phi(\xi)
\end{array}\right)\left(\begin{array}{c}
\xi \\
\gamma \xi+\delta
\end{array}\right) \equiv\left(\begin{array}{c}
\xi \\
\Phi_{1}(\xi)
\end{array}\right),
$$

$\Phi_{1}(\xi) \equiv \gamma \Phi(\alpha \xi+\beta)+\delta=\gamma \alpha_{0} \xi^{k}+\gamma\left(k \beta \alpha_{0}+\alpha_{1}\right) \xi^{k-1}+\cdots+(\gamma \Phi(\beta)+\delta)$, if $\alpha=1$. We may dispose of $\gamma, \beta, \delta$ to simplify $\Phi_{1}(\xi)$. We take $\gamma=\alpha_{0}^{-1}$, and, in case $k$ is prime to $p$, we choose $\beta$ so that

$$
l_{k} \beta \alpha_{0}+\alpha_{1}=0 \text {. }
$$

Finally, we take $\delta=-\gamma \Phi(\beta)$. The quantic $\Phi_{1}(\xi)$, in which the coefficient of $\xi^{k}$ is unity, the constant term zero, and, when $k$ is not a multiple of $p$, the coefficient of $\xi^{k-1}$ is zero, will be called the reduced form of $\Phi(\xi)$ for the $G F\left[p^{n}\right]$.

88. To illustrate the use of the theorem of $\S 84$, we apply it to determine all reduced $S Q\left[3, p^{n}\right]$. For $p \neq 3$, the reduced cubic in the $G F\left[p^{n}\right]$ is $\xi^{3}+\alpha \xi$. The sub-case $p^{n}=3 m+1$ must be rejected, since the $m^{\text {th }}$ power of $\xi^{3}+\alpha \xi$ contains the power $\xi^{3 m} \equiv \xi^{p^{n}-1}$ with coefficient unity and hence $\neq 0$. For the sub-case $p^{n}=3 m+2$, the condition given by the power $m+1$ is $(m+1) \alpha=0$. But if $m+1$ be divisible by $p$, then would also $3 m+3 \equiv p^{n}+1$. Hence must $\alpha=0$. The resulting form $\xi^{3}$ is a $S Q\left[3, p^{n} \equiv 3 m+2\right]$ by $\S 79$.

There remains the case $p^{n}=3^{n}$, when the reduced cubic is

$$
\Phi(\xi) \equiv \xi^{3}+\alpha_{1} \xi^{2}+\alpha_{2} \xi \text {. }
$$

Raising it to the power $3^{n-1}+3^{n-2}+\cdots+3+1 \equiv\left(3^{n}-1\right) / 2$, we find $(\bmod 3)$,

$\left(\xi^{3^{n}}+\alpha_{1}^{3^{n-1}} \xi^{2 \cdot 3^{n-1}}+\alpha_{2}^{3^{n-1}} \xi^{3^{n-1}}\right)\left(\xi^{3^{n-1}}+\alpha_{1}^{3^{n-2}} \xi^{2 \cdot 3^{n-2}}+\alpha_{2}^{3^{n-2}} \xi^{3^{n-2}}\right) \ldots\left(\xi^{3}+\alpha_{1} \xi^{2}+\alpha_{2}\right.$

The highest exponent of $\xi$ in this product is $<2\left(3^{n}-1\right)$. The coefficient of

$$
\xi^{3^{n}-1} \equiv \xi^{2\left(3^{n-1}+3^{n-2}+\cdots+3+1\right)}
$$

is evidently $\alpha_{1}^{3^{n-1}+3^{n-2}+\cdots+1}$. Hence must $\alpha_{1}=0$. Applying then the corollary of $\S 81$, the resulting form $\xi^{3}+\alpha_{2} \xi$ is a $S Q\left[3,3^{n}\right]$ if, and only if, either $\alpha_{2}=0$ or else $-\alpha_{2}$ is a not-square in the $G F\left[3^{n}\right]$.

89. To treat a more characteristic example, we seek the $S Q\left[5, p^{n}\right]$, when $p^{n}$ is of the form $5 m+3$. The reduced quintic is

$$
\xi^{5}+\alpha \xi^{3}+\beta \xi^{2}+\gamma \xi \text {. }
$$

The power $m+1$ gives $(m+1) \beta$ as the coefficient of $\xi^{5 m+2} \equiv \xi^{p^{n}-1}$. If $m+1 \equiv 0(\bmod p)$, then $5 m+5 \equiv p^{n}+2 \equiv 0$ and therefore $p=2$. 
Hence, for $p \neq 2$, we must have $\beta=0$. The power $m+2$ of 53)

$$
\xi^{5}+\alpha \xi^{3}+\gamma \xi
$$

requires

$$
C_{m+2,2} \gamma^{2}+C_{m+2,3} \cdot 3 \alpha^{2} \gamma+C_{m+2.4} \alpha^{4}=0 .
$$

If $p$ is neither 2 nor $7 ; C_{m+2,2} \equiv \equiv 0(\bmod p)$ and may be divided out; for, if $m+2$ be divisible by $p$, then is also $5(m+2)=p^{n}+7$. Multiplying the resulting equation by $5^{2}$ and replacing $5 m$ and $5(m-1)$ by $p^{n}-3$ and $p^{n}-8$, respectively, we have for $p \neq 2, p \neq 7$,

$$
25 \gamma^{2}-15 \alpha^{2} \gamma+2 \alpha^{4} \equiv\left(5 \gamma-\alpha^{2}\right)\left(5 \gamma-2 \alpha^{2}\right)=0 \text {. }
$$

The power $m+4$ of 53) requires, if $\left.{ }^{1}\right) p^{n}>13$,

$C_{m+4,5} 5 \alpha \gamma^{4}+C_{m+4,6} 20 \alpha^{3} \gamma^{3}+C_{m+4,7} 21 \alpha^{5} \gamma^{2}+C_{m+4,8} 8 \alpha^{7} \gamma+C_{m+4,9} \alpha^{9}=0$.

If $p$ is not $2,3,7$ or 17 , we may divide out the factor

$$
(m+4)(m+3)(m+2)(m+1) m \text {. }
$$

Multiplying afterwards by $5^{4} \cdot 7 !$ and replacing $5(m-1)$ by $-8(\bmod p)$, etc., we find

$210 \alpha(5 \gamma)^{4}-8 \cdot 140 \alpha^{3}(5 \gamma)^{3}+8 \cdot 13 \cdot 21 \alpha^{5}(5 \gamma)^{2}-8 \cdot 13 \cdot 18 \alpha^{7}(5 \gamma)+23 \cdot 26 \alpha^{9}=0$.

This equation is an identity for $5 \gamma=\alpha^{2}$, but reduces to $-10 \alpha^{9}$ for $5 \gamma=2 \alpha^{2}$. In the latter case, $\alpha=\gamma=0$, if $p \neq 2$. Hence, for $p^{n} \neq 13,2^{n}, 3^{n}, 7^{n}$ or $17^{n}$, the only possible quintic which represents a substitution on the marks of the $G F\left[p^{n} \equiv 5 m+3\right]$ is reducible to

$$
5 \xi^{5}+5 \alpha \xi^{3}+\alpha^{2} \xi \text {. }
$$

We have shown in $\S 80$ that this quintic is indeed a $S Q\left[5, p^{n} \equiv 5 m+3\right]$. The special cases above excluded require separate treatment.

90. The foregoing methods may be employed ${ }^{2}$ ) to show that the following table gives every reduced $S Q\left[k, p^{n}\right]$ for $k<6$ :

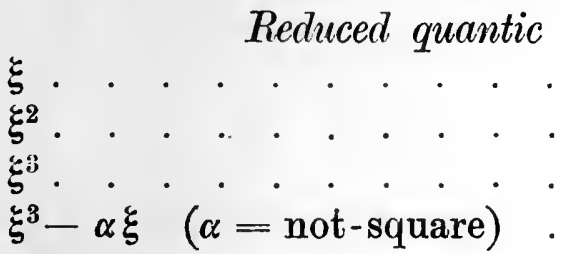

$\xi^{4} \pm 3 \xi$.

$\xi^{4}+\alpha_{2} \xi^{2}+\alpha_{3} \xi$ (if it vanishes only for $\xi=0$ ) $2^{n}$

$\xi^{5}$

$\xi^{5}-\alpha \xi \quad(\alpha$ not a fourth power $)$

$\xi^{5} \pm 2^{1 / 2} \xi$

$\xi^{5} \pm 2 \xi^{2}$

$\xi^{5}+\alpha \xi^{3} \pm \xi^{2}+3 \alpha^{2} \xi \quad(\alpha=$ not-square $)$
Suitable for $p^{n}=$ any $p^{n}$

$2^{n}$

$3^{n}, 3 m+2$

$3^{n}$

7

$5^{n}, 5 m \pm 2,5 m+4$ $5^{n}$

$3^{2}$

7

7

1) If $p^{n}=13$, the power $m+4 \equiv 6$ brings in terms $\xi^{24} \equiv \xi^{2(p n-1)}$.

2) Compare the author's Dissertation, l. c. pp. 77-86 and 101-102. 
Reduced quantic

$$
\begin{aligned}
& \xi^{5}+\alpha \xi^{3}+\frac{\alpha^{2}}{5} \xi \quad(\alpha \text { arbitrary }) \\
& \xi^{5}+\alpha \xi^{3}+3 \alpha^{2} \xi \quad(\alpha=\text { not-square }) \quad \text {. . . } 13 \\
& \xi^{5}-2 \alpha \xi^{3}+\alpha^{2} \xi \quad(\alpha=\text { not-square }) \quad . \quad . \quad .5^{n}
\end{aligned}
$$

That in fact these quantics do represent substitutions on the marks of the corresponding $G F\left[p^{n}\right]$ follows from $\S \S 79,80,81,83$ and 86 , with the exception possibly of the eleventh and thirteenth forms. To verify ${ }^{1}$ ) that the latter two are substitution -quantics, set

Then

$$
\Phi(\xi) \equiv \xi^{5}+\alpha \xi^{3}+3 \alpha^{2} \xi, \quad \Psi(\xi) \equiv \Phi(\xi) \pm \xi^{2} .
$$

$$
\mu^{-5} \Phi(\mu \xi) \equiv \xi^{5}+\left(\mu^{-2} \alpha\right) \xi^{3}+3\left(\mu^{-2} \alpha\right)^{2} \xi \text {. }
$$

Since $\alpha$ is a not-square, we can choose an integer $\mu$ so that $\mu^{-2} \alpha$ shall be a particular not-square $\nu$. But

$$
\mu^{-5} \Psi(\mu \xi)=\xi^{5}+\left(\mu^{-2} \alpha\right) \xi^{3}+3\left(\mu^{-2} \alpha\right)^{2} \xi \pm \mu^{-3} \xi^{2} .
$$

Since $\mu^{3} \equiv \pm 1(\bmod 7)$, we can choose the sign of $\mu= \pm(\alpha / \nu)^{1 / 2}$ to make the coefficient of $\xi^{2}$ unity. It follows, therefore, from $\$ .87$ that $\Phi(\xi)$ and $\psi(\xi)$ will be substitution-quantics modulo 13 and 7, respectively, for $\alpha$ an arbitrary not-square, if they be such for $\alpha$ a particular not-square $\nu$ and for the $+\operatorname{sign}$ in $\Psi(\xi)$. We take $\nu=5$, a non-residue of both 7 and 13 . In the notation of $\S 76$, these reduced forms represent the substitutions,

$$
\begin{aligned}
& \left(\begin{array}{l}
\xi \\
\xi^{5}+5 \xi^{3}+\xi^{2}+5 \xi
\end{array}\right) \equiv\left(\begin{array}{l}
0,1,2,3,4,5,6 \\
0,5,2,3,1,6,4
\end{array}\right), \\
& \left(\begin{array}{c}
\xi \\
\xi^{5}+5 \xi^{3}+10 \xi
\end{array}\right) \equiv\left(\begin{array}{l}
0,1,2,3,4,5,6,-6,-5,-4,-3,-2,-1 \\
0,3,1,5,6,4,-2,2,-4,-6,-5,-1,-3
\end{array}\right),
\end{aligned}
$$

modulo 7 and 13 respectively.

\section{The Betti-Mathieu Group, §§ 91-94.}

91. It was shown in $\S 81$ that the quantic belonging to the $G F\left[p^{n m}\right]$,

$$
\Psi_{A}(X) \equiv \sum_{i=1}^{m} A_{i} X^{p^{n(m-i)}},
$$

represents a substitution upon the $p^{n m}$ marks of the field if, and only if, $X=0$ is the only solution in the field of the equation

$$
\Psi_{A}(X)=0
$$

1) For a verification by means of the theorem of $\S 84$, see the author's paper, American Journal, vol. 18, pp. 210-218; in particular, §§ 7 and 9. 
Suppose that this condition is satisfied by two functions $\Psi_{A}(X)$ and $\Psi_{B}(X)$ and consider the effect of applying first the substitution ${ }^{1}$ )

$$
A: \quad X^{\prime}=\Psi_{A}(X) \equiv \sum_{i=1}^{m} A_{i} X^{p^{n(m-i)}}
$$

and afterwards the substitution

$$
B: \quad X^{\prime \prime}=\Psi_{B}\left(X^{\prime}\right) \equiv \sum_{j=1}^{m} B_{j} X^{\prime p^{n(m-j)}} .
$$

The result is equivalent to that produced by the single substitution

$$
X^{\prime \prime}=\Psi_{B}\left[\Psi_{A}(X)\right] \equiv \sum_{i, j}^{1, \ldots, m} B_{j} A_{i}^{p^{n(m-j)}} X^{p^{n(2 m-i-j)}}
$$

After reduction by means of $X^{p^{n m}}=X$, this equation may be written

$$
C: \quad X^{\prime \prime}=\Psi_{C}(X) \equiv \sum_{i=1}^{m} C_{i} X^{p^{n(m-i)}},
$$

each $C_{i}$ being a definite function of the $A_{j}$ 's and $B_{j}$ 's. By hypothesis,

$$
\Psi_{B}\left[\Psi_{A}(X)\right]=0
$$

requires $\Psi_{A}(X)=0$, which in turn requires $X=0$. Hence, $X=0$ is the only solution in the field of $\Psi_{C}(X)=0$. It follows that the transformation $C$ represents a substitution upon the marks of the $G F\left[p^{n m}\right] . \quad C$ is called the compound, or product, of $A$ and $B$, and the above relation is expressed in the symbolic form,

$$
C=A B \text {. }
$$

Giving to the coefficients $A_{i}$ every possible combination of values in the $G F\left[p^{n m}\right]$ such that

$$
X^{\prime}=\sum_{i=1}^{m} A_{i} X^{p^{n(m-i)}}
$$

represents a substitution on its marks, we obtain a set of substitutions having the property that the result of applying first any one of the set and afterwards any one of the set is identical with the result of applying a single substitution of the set, called the product of the two. Such a set of substitutions is said to form a group. In the present case, the group will be called the Betti-Mathieu Group. ${ }^{2}$ )

1) The present notation is used in place of and as equivalent to

$$
A: \quad\left[\begin{array}{c}
X \\
\Psi_{A}(X)
\end{array}\right] .
$$

2) For $n=1$, this group was studied by Betti, Annali di Scienze Mat. $e$ Fisiche, vol. 3 (1852), pp. 49-115, vol. 6 (1855), pp. 5-34; for general $n$, by Mathieu, Journal de Math., (2) vol. 5 (1860), pp. 9-42, vol. 6, pp. 241-323. The theorems of $\S \S 92-94$ are due to the author, Annals of Math., (1897), pp. $94-96,178-183$.

DiCkson, Linear Groups. 
92. Theorem. - The necessary and sufficient condition that the transformation

$$
X^{\prime}=\sum_{i=1}^{m} A_{i} X^{p^{n(m-i)}} \quad\left(X, A_{i} \text { in the } G F\left[p^{n m}\right]\right)
$$

shall represent a substitution on the marks of the $G F\left[p^{n m}\right]$ is

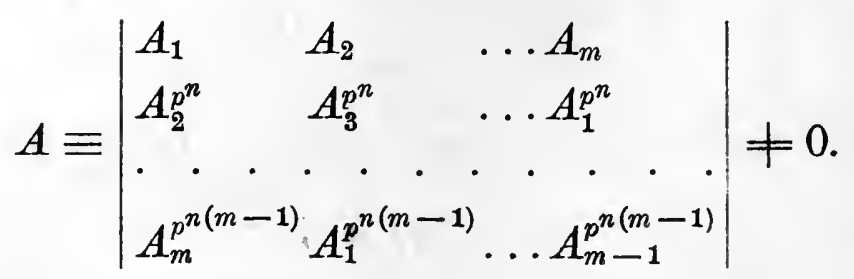

We seek the condition under which 54) is solvable for $X$. Raising 54) to the powers $1, p^{n}, p^{2 n}, \ldots, p^{n(m-1)}$ and reducing the powers of $X$ by

$$
X^{p^{n m}}=X
$$

we obtain the following $m$ equations (written with detached coefficients):

$$
\begin{aligned}
& X^{p^{n(m-1)}} X^{p^{n(m-2)}} \ldots X^{p^{n}} \quad X
\end{aligned}
$$

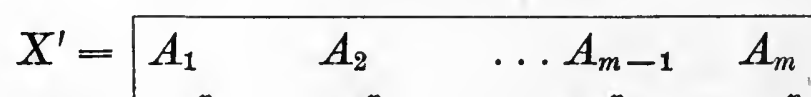

$$
\begin{aligned}
& X^{\prime p^{n}}=\begin{array}{llll}
A_{2}^{p^{n}} & A_{3}^{p^{n}} & \ldots A_{m}^{p^{n}} & A_{1}^{p^{n}}
\end{array} \\
& X^{\prime p^{n(m-1)}}=A_{m}^{p^{n(m-1)}} A_{1}^{p^{n(m-1)}} \ldots A_{m-2}^{p^{n(m-1)}} A_{m-1}^{p^{n(m-1)}} .
\end{aligned}
$$

The solution of this system of equations in $X, X^{p^{n}}, \ldots$ gives

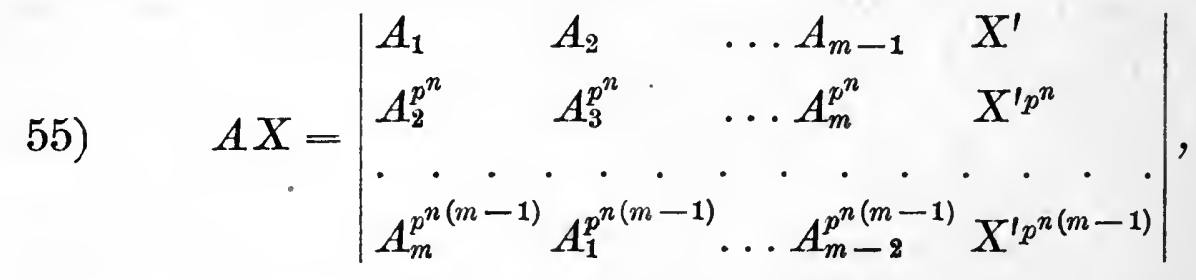

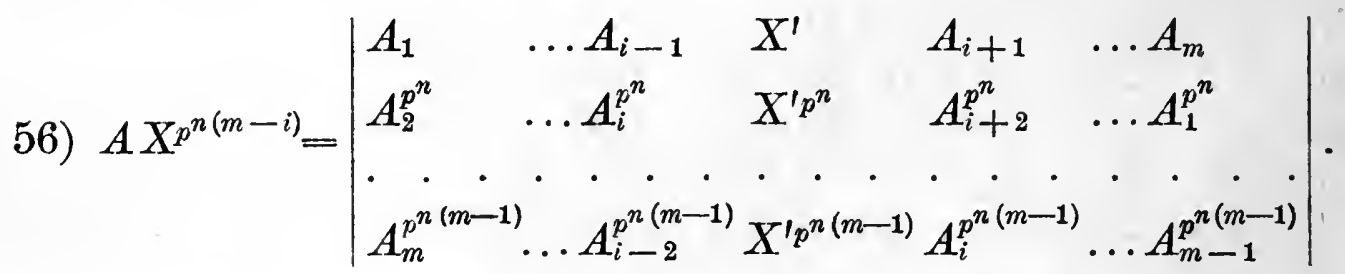

The condition $A \neq 0$ is necessary, since otherwise there would exist a relation between the powers of $X^{\prime}$ with exponents $<p^{n m}$. To prove that the condition $A \neq 0$ is sufficient, we need only verify that the $X$ given by 55) satisfies the relations 56) for $i=1,2, \ldots, m-1$. Observing that $A^{p^{n}}=A$ in the field, we find the following relations upon raising 55) to the power $p^{n(m-i)}$ and moving the first $i$ rows below the last $m-i$ rows: 
ANALYTIC REPRESENTATION OF SUBSTITUTIONS, etc.

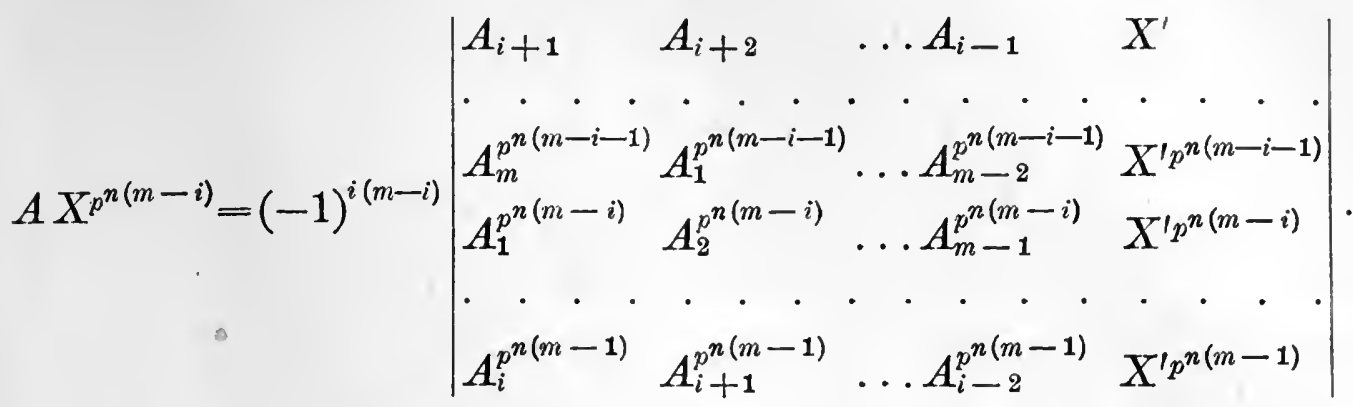

Moving the last $i$ columns before the $m-i$ preceding columns, which brings in an additional factor $(-1)^{i(m-i)}$, we obtain the determinant of formula 56).

It follows as a corollary that formula 55) gives the reciprocal of 54).

A second proof may be given, based on the theorem of $\S 72$. The condition that 54) shall represent a substitution on the marks of the $G F\left[p^{n m}\right]$ is identical with the condition under which $X_{1}^{\prime}, X_{2}^{\prime}, \ldots, X_{m}^{\prime}$ shall be linearly independent with respect to the $G F\left[p^{n}\right]$ when it is given that $X_{1}, X_{2}, \ldots, X_{m}$ are similarly independent. We seek the condition under which

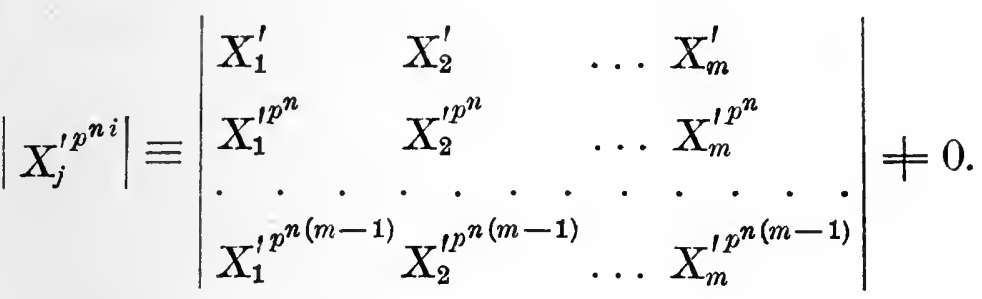

Substituting the values of $X_{j}^{\prime}, X_{j}^{\prime p^{n}}, \ldots, X_{j}^{\prime p^{n(m-1)}}$ in terms of $X_{j}$, $X_{j}^{p^{n}}, \ldots, X_{j}^{p^{n(m-1)}}$ and the $A_{i}$, as given by the above table, we find that

$$
\left|X_{j}^{i p^{n i}}\right|=\left|X_{j}^{p^{n i}}\right| \cdot A \text {. }
$$

The required condition is therefore that $A \neq 0$.

93. To illustrate a general method ${ }^{1}$ ) of obtaining sub-groups of the Betti-Mathieu Group, we take $m=3$ and consider the totality of substitutions in the $G F\left[p^{3 n}\right]$ on a variable $X$ of that field,

$$
X^{\prime}=A_{1} X^{p^{2 n}}+A_{2} X^{p^{n}}+A_{3} X,
$$

which multiply by a factor $\varrho$ the function

$$
Z \equiv(B X)^{p^{2 n}}+(B X)^{p^{n}}+B X \quad\left(B \text { in the } G F\left[p^{3 n}\right]\right) .
$$

1) See the author's paper in the American Journal, vol. 22, pp. 49-54. 
The conditions for the identity $Z^{\prime} \equiv \varrho Z$ are readily seen to be

$$
\begin{aligned}
& B A_{3}+B^{p^{n}} A_{1}^{p^{n}}+B^{p^{2 n}} A_{2}^{p^{2 n}}=\varrho B, \\
& B A_{2}+B^{p^{n}} A_{3}^{p^{n}}+B^{p^{2 n}} A_{1}^{p^{2 n}}=\varrho B^{p^{n}}, \\
& B A_{1}+B^{p^{n}} A_{2}^{p^{n}}+\dot{B}^{p^{2 n}} A_{3}^{p^{2 n}}=\varrho B^{p^{2 n}} .
\end{aligned}
$$

Since the left members of 59) and 60) are the powers $\dot{p}^{n}$ and $p^{2 n}$, respectively, of the left member of 58), we must have $\varrho^{p^{n}}=\varrho$. Hence the totality of substitutions 57) for which the expression

$$
\varrho \equiv A_{3}+B^{p^{n}-1} A_{1}^{p^{n}}+B^{p^{2 n}-1} A_{2}^{p^{2 n}}
$$

is a mark of the $G F\left[p^{n}\right]$ form a group leaving $Z$ relatively invariant.

94. Consider next the substitutions 57) which multiply the function

$$
Y \equiv D X^{p^{n}+1}+D^{p^{n}} X^{p^{2 n}+p^{n}}+D^{p^{2 n}} X^{p^{2 n}+1}
$$

by a parameter $\varrho$, where $D$ is a mark $\neq 0$ in the $G F\left[p^{3 n}\right]$.

To form the function $Y^{\prime}$ into which 57) transforms $Y$, we note that

$$
\begin{aligned}
& X^{\prime} p^{n}+1=A_{3} A_{1}^{p^{n}} X^{2}+A_{2} A_{3}^{p^{n}} X^{2 p^{n}}+A_{1} A_{2}^{p^{n}} X^{2 p^{2 n}}+\left(A_{3}^{p^{n}+1}+A_{2} A_{1}^{p^{n}}\right) X^{p^{n}+1} \\
& +\left(A_{1}^{p^{n}+1}+A_{3} A_{2}^{p^{n}}\right) X^{p^{2 n}+1}+\left(A_{2}^{p^{n}+1}+A_{1} A_{3}^{p^{n}}\right) X^{p^{2 n}+p^{n}} \text {. }
\end{aligned}
$$

Denoting by $W$ the product of the expression on the right by $D$ and forming the sum $Y^{\prime} \equiv W+W^{p^{n}}+W^{p^{2 n}}$, we find that the conditions for the identity $Y^{\prime}=\varrho Y$ are the following six relations:

$$
\tau=0, \quad \tau^{p^{n}}=0, \quad \tau^{p^{2 n}}=0, \quad f=\varrho D, \quad f^{p^{n}}=\varrho D^{p^{n}}, \quad f^{p^{2 n}}=\varrho D^{p^{2 n}},
$$

where, for brevity,

$$
\begin{gathered}
\tau \equiv D A_{3} A_{1}^{p^{n}}+D^{p^{n}} A_{1}^{p^{n}} A_{2}^{p^{2 n}}+D^{p^{2 n}} A_{3} A_{2}^{p^{2 n}} \\
f \equiv D\left(A_{3}^{p^{n}+1}+A_{2} A_{1}^{p^{n}}\right)+D^{p^{n}}\left(A_{1}^{p^{2} n}+p^{\dot{n}}+A_{3}^{p^{n}} A_{2}^{p^{2 n}}\right) \\
+D^{p^{2 n}}\left(A_{2}^{p^{2}+1}+A_{3} A_{1}^{p^{2} n}\right) .
\end{gathered}
$$

In particular, it follows that $\varrho^{p^{n}}=\varrho$. Hence those substitutions 57) whose coefficients $A_{1}, A_{2}, A_{3}$ make $\tau=0$ and give to the function $f / D$ a value belonging to the $G F\left[p^{n}\right]$ form a group with the relative invariant $Y$.

The method may be readily extended to determine for general $m$ the substitutions 54) which leave relatively invariant the following function

$$
Y_{s} \equiv \sum_{j=0}^{m-1} D^{p^{n j}} X^{p^{n j}+p^{n(s+j)}} \quad\left(D \text { in the } G F\left[p^{n m}\right]\right),
$$

where $s$ may be any integer $<m$, except perhaps $m / 2$. 
It is found that the number of independent conditions upon the coefficients $A_{i}$, in order that 54) shall leave $Y_{s}$ relatively invariant, is at most $\frac{1}{2}(m+1)$ or $\frac{1}{2}(m+2)$ according as $m$ is odd or even. One of these conditions merely requires that a certain function of $D$ and the $A_{i}$ shall belong to the $G F\left[p^{n}\right]$.

95. We proceed to identify the Betti-Mathieu Group in the $G F\left[p^{n m}\right]$ with Jordan's linear homogeneous group on $m$ indices with coefficients in the $G F\left[p^{n}\right]$. Let $R$ be a primitive root of the $G F\left[p^{n m}\right]$, so that any mark of that field can be expressed in the form $\gamma_{0}+\gamma_{1} R+\gamma_{2} R^{2}+\cdots+\gamma_{m-1} R^{m-1}$, where each $\gamma_{i}$ is a mark of the $G F\left[p^{n}\right]$. Consider the general substitution 54) of the BettiMathieu Group. We may set

$$
X \equiv \sum_{i=0}^{m-1} \xi_{i} R^{i}, \quad X^{\prime} \equiv \sum_{i=0}^{m-1} \xi_{i}^{\prime} R^{i}, \quad A_{k} \equiv \sum_{i=0}^{m-1} a_{i}^{(k)} R^{i},
$$

where each $\xi_{i}, \xi_{i}^{\prime}$ and $a_{i}^{(k)}$ belong to the $G F\left[p^{n}\right]$.

Substituting these values in the identity 54) and reducing the powers of $R$ to a degree $\overline{\bar{z}} m-1$ by means of the equation of degree $m$ satisfied by the primitive root $R$, we may equate the coefficients of like powers of $R$ in the resulting identity. Since

$$
\xi_{i}^{p^{n}}=\xi_{i}, \quad \xi_{i}^{\prime p^{n}}=\xi_{i}^{\prime}
$$

we evidently reach a set of $m$ equations of the form

$$
\xi_{i}^{\prime}=\sum_{i=0}^{m-1} \alpha_{i j} \xi_{j} \quad(i=0,1, \ldots, m-1)
$$

in which the coefficients $\alpha_{i j}$ belong to the $G F\left[p^{n}\right]$. By hypothesis, equation 54) is solvable for $X$ in terms of $X^{\prime}$. Starting from this solved form, our process evidently yields the $\xi_{j}$ as functions of the $\xi_{j}^{\prime}$, so that equations 63 ) are solvable in the field $G F\left[p^{n}\right]$. Hence $\left|\alpha_{i j}\right| \neq 0$. According to the definition given in $\S 97$, the transformation 63) belongs to Jordan's linear homogeneous group.

Inversely, every linear substitution 63), with coefficients in the $G F\left[p^{n}\right]$ such that the determinant $\left|\alpha_{i j}\right| \neq 0$, can be represented in the form 54). We note first that 63 ) transforms $X \equiv \sum_{i=0}^{m-1} \xi_{i} R^{i}$ into

where

$$
X^{\prime} \equiv \sum_{i=0}^{m-1}\left(\sum_{j=0}^{m-1} \alpha_{i j} \xi_{j}\right) R^{i}=\sum_{j=0}^{m-1} \xi_{j}\left(\sum_{i=0}^{m-1} \alpha_{i j} R^{i}\right) \equiv \sum_{j=0}^{m-1} \xi_{j} \tau_{j}
$$

$$
\tau_{j} \equiv \sum_{i=0}^{m-1} \alpha_{i j} R
$$


Furthermore, $\tau_{0}, \tau_{1}, \ldots, \tau_{m-1}$ are linearly independent with respect to the $G F\left[p^{n}\right]$; for, if $\varkappa_{0}, \ldots, x_{m-1}$ be marks of the latter field such that

then

$$
\varkappa_{0} \tau_{0}+\varkappa_{1} \tau_{1}+\cdots+x_{m-1} \tau_{m-1}=0
$$

$$
\sum_{j=0}^{m-1} x_{j} \alpha_{i j}=0 \quad(i=0,1, \ldots, m-1)
$$

and therefore, since $\left|\alpha_{i j}\right| \neq 0$, each $x_{j}=0$. Hence, when each $\xi_{i}$ runs independently through the series of $p^{n}$ marks of the $G F\left[p^{n}\right]$, the expressions $X$ and $X^{\prime}$ both run through the $p^{n m}$ marks of the $G F\left[p^{n m}\right]$. Every substitution 63) therefore gives rise to a permutation on the marks of that field.

But we can always find a set of marks $A_{1}, A_{2}, \ldots, A_{m}$ of the $G F\left[p^{n m}\right]$ such that 54) will transform the set of marks $1, R, R^{2}, \ldots$, $R^{m-1}$, linearly independent with respect to the $G F\left[p^{n}\right]$, into an arbitrary set of $m$ marks of the $G F\left[p^{n m}\right]$,

$$
B_{i} \equiv \sum_{j=0}^{m-1} \beta_{i j} R^{j} \quad(i=0, \ldots, m-1)
$$

linearly independent with respect to the $G F\left[p^{n}\right]$. The conditions are

$$
\sum_{i=1}^{m} A_{i}\left(R^{k}\right)^{p^{n(m-i)}}=B_{k} \quad(k=0,1, \ldots, m-1)
$$

which can be solved for $A_{1}, A_{2}, \ldots, A_{m}$, since the determinant in $R$ is not zero by $\S 72$. The resulting substitution 54) will transform the $p^{n m}$ marks $\sum_{i=0}^{m-1} \lambda_{i} R^{i}$ of the $G F\left[p^{n m}\right]$ into the marks $\sum_{i=0}^{m-1} \lambda_{i} B_{i}$ all distinct; indeed, we have the identity

$$
\sum_{i=1}^{m} A_{i}\left(\lambda_{0} X_{0}+\lambda_{1} X_{1}+\cdots+\lambda_{t} X_{t}\right)^{n(m-i)} \equiv \sum_{j=0}^{t}\left\{\lambda_{j} \sum_{i=1}^{m} A_{i} X_{j}^{p^{n(m-i)}}\right\}
$$

96. EXERCISES ON THE TEXT OF CHAPTER V.

Ex. 1. Verify that $\xi^{6}+\alpha \xi^{5}-\alpha^{4} \xi^{2}(\alpha$ arbitrary) represents a substitution on the marks of either the $G F\left[3^{3}\right]$ or of the $G F\left[2^{5}\right]$.

Ex. 2. (Hermite). A group of order 168 is generated by the substitutions

$$
x^{\prime} \equiv a x+b, \quad x^{\prime} \equiv a \Theta(x+b)+c \quad(\bmod 7),
$$

where $\Theta(x) \equiv-x^{5}-2 x^{2}$ and $a$ is a quadratic residue of 7 .

Ex. 3. (Rogers.) In applying Hermite's conditions ( $\$ 84$ ) for a substitution quantic, it suffices, when $n=1$, to test only the first $\frac{1}{2}\left(p^{\prime}-1\right)$ powers. This result of Rogers does not generalize immediately 
to the case $n>1$; for $S Q\left[6,3^{2}\right]$ it is necessary to consider, besides the $2^{d}$ and $4^{\text {th }}$ powers, also the power $5>\frac{1}{2}\left(3^{2}-1\right)$.

Ex. 3. By the theorem given by Weber, Algebra, II, p. 299, every substitution on $p^{n}$ letters, each affected with $n$ indices $z_{1}, z_{2}, \ldots, z_{n}$ taken modulo $p$, may be represented by the transformation $(\bmod p)$,

$$
z_{i}^{\prime}=\Phi_{i}\left(z_{1}, z_{2}, \ldots, z_{n}\right) \quad(i=1,2, \ldots, n)
$$

where each $\Phi_{i}$ is a rational integral function with integral coefficients. Apply the method of $\S 84$ and show that, on raising each $\Phi_{i}$ to the powers $1,2, \ldots, p-2$ and reducing by means of $z_{i}^{p} \equiv 2_{i}(\bmod p)$, the coefficient of $z_{1}^{p-1} z_{2}^{p-1} \ldots z_{n}^{p-1}$ in each power must be congruent to zero.

Ex. 4. The following substitution in the $G F\left[p^{2 n}\right]$

a) $\quad X^{\prime}=A_{1} X^{p^{n}}+A_{2} X \quad\left(A_{1} \neq 0, A_{1}^{p^{n}+1}-A_{2}^{p^{n}+1} \neq 0\right)$

can be reduced to the form $Y^{\prime}=R Y$ by introducing a new index

b)

$$
Y \equiv B_{1} X^{p^{n}}+B_{2} X \quad\left(B_{1}^{p^{n}+1}-B_{2}^{p^{n}+1} \neq 0\right)
$$

if and only if there exists no root in the $G F\left[p^{n}\right]$ of the equation

$$
\left|\begin{array}{ll}
A_{2}-R & A_{1} \\
A_{1}^{p^{n}} & A_{2}^{p^{n}}-R
\end{array}\right| \equiv 0 .
$$

If $A_{2}+A_{2}^{p^{n}} \neq 0$, it is not possible to reduce a) to the form

$$
Y^{\prime}=K Y^{p^{n}} \quad\left(K \text { in the } G F\left[p^{2 n}\right]\right)
$$

by a transformation of indices of the form $b$ ).

[The first result is in marked contrast to that of $\S 214$ for $m=2$ ].

Ex. 5. By the method of $\S 95$, show that the sub-groups of the Betti-Mathieu Group defined in $\$ \S 93-94$ by means of the invariants $Z$ and $Y$ are identical with certain linear homogeneous groups on $m$ indices in the $G F\left[p^{n}\right]$ defined by a linear and a quadratic invariant respectively.

Ex. 6. (Moore.) The multiplier-GF[ $\left[p^{k}\right]$ of the additive-group $\left[\lambda_{1}, \ldots, \lambda_{m}\right]$ is the (largest) additive-group common to the additive-groups

$$
\left[\lambda_{i}^{-1} \lambda_{1}, \ldots, \lambda_{i}^{-1} \lambda_{m}\right] \quad(i=1, \ldots, m)
$$

and is contained in the $p^{m}-1$ additive-groups

$$
\left[\lambda^{-1} \lambda, \ldots, \lambda^{-1} \lambda_{m}\right] \quad\left(\lambda \neq 0 \text { of }\left[\lambda_{1}, \ldots, \lambda_{m}\right]\right) \text {. }
$$





\section{SEC0ND PART.}

\section{THE0RY}

\section{OF LINEAR GROUPS IN A GALOIS FIELD.}





\section{CHAPTER I.}

\section{GENERAL LINEAR HOMOGENEOUS GROUP. ${ }^{1}$ )}

97. First definition. - Consider the $p^{n m}$ letters, or symbols,

$$
l_{\xi_{1}}, \xi_{2}, \ldots, \xi_{m}
$$

characterized by $m$ indices, each running through the series of marks of the $G F\left[p^{n}\right]$. The general linear homogeneous substitution $A$ on the $m$ indices $\xi_{i}$ with coefficients in the $G F\left[p^{n}\right]$ replaces the letter $l_{\xi_{1}}, \xi_{2}, \ldots, \xi_{m}$ by $l_{\xi^{\prime}}{ }_{1}^{\prime}, \xi^{\prime}{ }_{2}^{\prime}, \ldots, \xi_{m}^{\prime}$ where

$$
A: \quad \xi_{i}^{\prime}=\sum_{j=1}^{m} \alpha_{i j} \xi_{j} \quad(i=1, \ldots, m)
$$

the coefficients $\alpha_{i j}$ being marks of the field. But $A$ will indeed permute the $p^{n m}$ letters if, and only if, the determinant of $A$ is not zero,

$$
|A| \equiv\left|\alpha_{i j}\right| \neq 0 \text {. }
$$

In fact, there must be one and only one system of $m$ indices which $A$ replaces by a given system $\xi_{i}^{\prime}$ and hence an unique set of values $\xi_{j}$ satisfying the equations

$$
\sum_{j=1}^{m} \alpha_{i j} \xi_{j}=\xi_{i}^{\prime} \quad(i=1,2, \ldots, m) .
$$

Let $B$ denote a second substitution with coefficients in the $G F\left[p^{u}\right]$,

$B:$

$$
\xi_{k}^{\prime}=\sum_{i=1}^{m} \beta_{k i} \xi_{i} \quad(k=1,2, \ldots, m)
$$

where

$$
|B| \equiv\left|\beta_{k i}\right| \neq 0 \text {. }
$$

1) Jordan, Traité des substitutions, Nos. 119, 169; author's dissertation, Part II. Cf. $\$ 95$ above. 
The result of applying first the substitution $A$, which replaces $l_{\xi_{1}}, \ldots, \xi_{m}$ by $l_{\xi^{\prime}}, \ldots, \xi_{m}^{\prime}$ where

$$
\xi_{i}^{\prime}=\sum_{j=1}^{m} \alpha_{i j} \xi_{j} \quad(i=1, \ldots, m),
$$

and afterwards the substitution $B$, which replaces the general letter $l_{\xi_{1}^{\prime}}, \ldots, \xi_{m}^{\prime}$ by $l_{\xi^{\prime \prime}}{ }_{1}^{\prime}, \ldots, \xi_{m}^{\prime \prime}{ }_{m}$ where

$$
\xi_{k}^{\prime \prime}=\sum_{i=1}^{m} \beta_{k i} \xi_{i}^{\prime} \quad(k=1,2, \ldots, m),
$$

is identical with the result of applying a single linear substitution, called their compound or product $A B$, which replaces $l_{\xi_{1}}, \ldots, \xi_{m}$ by $l_{\xi^{\prime \prime}}, \ldots, \xi^{\prime \prime}{ }_{m}$, where, by eliminating the $\xi_{i}^{\prime}$ between 64) and 65), we have

Setting

$$
\xi_{k}^{\prime \prime}=\sum_{j=1}^{m}\left(\sum_{i=1}^{m} \beta_{k i} \alpha_{i j}\right) \xi_{j} \quad(k=1, \ldots, m) .
$$

$$
\gamma_{k j} \equiv \sum_{i=1}^{m} \beta_{k i} \alpha_{i j} \quad(k, j=1, \ldots, m),
$$

we may write the product of $A$ and $B$ in the form

$A B$ :

$$
\xi_{k}^{\prime}=\sum_{j=1}^{m} \gamma_{k j} \xi_{j} \quad(k=1, \ldots, m)
$$

By the theorem for the multiplication of determinants.

$$
A B|\equiv| \gamma_{k j}|=| \beta_{k i}|\cdot| \alpha_{i j}|\equiv| B|\cdot| A \mid \neq 0 \text {. }
$$

Moreover, the coefficients $\gamma_{k j}$ belong to the $G F\left[p^{n}\right]$. Hence the compound $A B$ is indeed a substitution and has its coefficients in the same field as those of $A$ and $B$. If therefore we let the coefficients of $A$ run through all the sets of values in the $G F\left[p^{n}\right]$ for which the determinant $\left|\alpha_{i j}\right| \neq 0$, we obtain a set of substitutions forming a group called the general linear homogeneous group on $m$ indices with coefficients in the $G F\left[p^{n}\right]$ and denoted by the symbol $G L H\left(m, p^{n}\right)$.

Remark. - If the substitution $A$ be identical with the substitution.

$$
\xi_{i}^{\prime}=\sum_{j=1}^{m} \bar{\alpha}_{i j} \xi_{j} \quad(i=1, \ldots, m)
$$

then must $\bar{\alpha}_{i j}=\alpha_{i j}(i, j=1, \ldots, m)$. This follows by taking in turn for $j=1,2, \ldots, m$ the particular set of values

$$
\xi_{j}=1, \quad \xi_{k}=0 \quad(k=1,2, \ldots, m ; k \neq j) .
$$


98. Second definition of $G L H\left(m, p^{n}\right)$. - The essential thing in the substitution $A$ is the matrix of its coefficients $\left(\alpha_{i j}\right)$. Taking the indices $\xi_{1}, \ldots, \xi_{m}$ to be variable marks of the $G F\left[p^{n}\right]$, we obtained an immediate interpretation of $A$ as a permutation of certain $p^{n m}$ letters, so that the linear group was recognized as a permutation-group. We may, however, let the indices $\xi_{1}, \ldots, \xi_{m}$ be arbitrary variables and consider the linear transformations

$$
A: \quad \xi_{i}^{\prime}=\sum_{j=1}^{m} \alpha_{i j} \xi_{j} \quad(i=1, \ldots, m), \quad\left|\alpha_{i j}\right| \neq 0,
$$

where each coefficient belongs to the $G F\left[p^{n}\right]$. As in $\S 97$, the compound of two such transformations will be a linear transformation of determinant not zero and with all its coefficients in the $G F\left[p^{n}\right]$. Since $\left|\alpha_{i j}\right| \neq 0$, the inverse of $A$ exists and has similar properties. Hence the totality of transformations $A$ form a group, evidently the $G L H\left(m, p^{n}\right)$.

Employing this second definition, we may represent the transformation group as a group of permutations on $p^{n m}$ letters. Consider, indeed, the $p^{n m}$ linear functions $\lambda_{1} \xi_{1}+\lambda_{2} \xi_{2}+\cdots+\lambda_{m} \xi_{m}$ where each $\lambda$ runs through the marks of the $G F\left[p^{n}\right]$. These functions are merely permuted by the linear transformations $A$.

99. Theorem. - The order $G L H\left[m, p^{n}\right]$ of the group $G L H\left(m, p^{n}\right)$ is

$$
\left(p^{n m}-1\right)\left(p^{n m}-p^{n}\right)\left(p^{n m}-p^{2 n}\right) \ldots\left(p^{n m}-p^{n(m-1)}\right) .
$$

The number of distinct linear functions

$$
f_{1}=\sum_{j=1}^{m} \alpha_{1 j} \xi_{j}
$$

by which the substitutions of the group can replace the index $\xi_{1}$ is $p^{n m}-1$, since the marks $\alpha_{1 j}$ may be chosen arbitrarily in the $G F\left[p^{n}\right]$ provided not all are zero. Let $T$ be one of the substitutions which replace $\xi_{1}$ by a definite linear function $f_{1}$. If then

$$
R_{1} \equiv I \text { (identity) }, R_{2}, R_{3}, \ldots, R_{N}
$$

denote all the substitutions of the group which leave $\xi_{1}$ fixed, the $N$ products,

$$
T, T R_{2}, T R_{3}, \ldots, T R_{N}
$$

will replace $\xi_{1}$ by $f_{1}$. No other substitution of the group has this property; for, if $U$ replace $\xi_{1}$ by $f_{1}, T^{-1} U$ will leave $\xi_{1}$ fixed and hence be a certain $R_{i}$, so that $U=T R_{i}$. To each of the $p^{n m}-1$ distinct functions $f_{1}$ there corresponds a set of $N$ substitutions. Hence

$$
G L H\left[m, p^{n}\right]=N\left(p^{n m}-1\right) \text {. }
$$


The substitutions $R_{i}$ are of the form

$$
\xi_{1}^{\prime}=\xi_{1}, \quad \xi_{k}^{\prime}=\sum_{j=1}^{m} \alpha_{k j} \xi_{j} \quad(k=2, \ldots, m)
$$

where the $m-1$ coefficients $\alpha_{k 1}$ are arbitrary and the coefficients $\alpha_{k j}(k, j=2, \ldots, m)$ are such that their determinant $\neq 0$ in the field. The latter set of coefficients can be chosen in $G L H\left[m-1, p^{n}\right]$ ways. Hence

$$
N=p^{n(m-1)} G L H\left[m-1, p^{n}\right],
$$

$$
G L H\left[m, p^{n}\right]=p^{n(m-1)}\left(p^{n m}-1\right) G L H\left[m-1, p^{n}\right] .
$$

This recursion formula gives, since $G L H\left[1, p^{n}\right]=p^{n}-1$, the result

$$
\begin{aligned}
G L H\left[m, p^{n}\right] & =p^{n(m-1)}\left(p^{n m}-1\right) p^{n(m-2)}\left(p^{n(m-1)}-1\right) \ldots p^{n}\left(p^{2 n}-1\right)\left(p^{n}-1\right) \\
& =\left(p^{n m}-1\right)\left(p^{n m}-p^{n}\right) \ldots\left(p^{n m}-p^{n(m-1)}\right) .
\end{aligned}
$$

100. Theorem. - Every linear homogeneous substitution $A$ on $m$ indices with coefficients in the $G F\left[p^{n}\right]$ can be expressed as a product $B D_{m}$, where $B$ is derived from the totality of substitutions of the form

$$
B_{r, s \lambda}: \quad \xi_{r}^{\prime}=\xi_{r}+\lambda \xi_{s}, \quad \xi_{i}^{\prime}=\xi_{i} \quad(i=1, \ldots, \dot{m} ; i \neq r ; r \neq s)
$$

$\lambda$ being an arbitrary mark of the $G F\left[p^{n}\right]$, and where $D_{m}$ denotes the substitution altering only the index $\xi_{m}$ which it multiplies by the determinant of $A$.

Let the given substitution $A$ be the following:

$A$ :

$$
\xi_{i}^{\prime}=\sum_{j=1}^{m} \alpha_{i j} \xi_{j} \quad(i=1, \ldots, m) .
$$

The product $A B_{1,2,2}$ has the form

$$
\xi_{1}^{\prime}=\sum_{j=1}^{m}\left(\alpha_{1 j}+\lambda \alpha_{2 j}\right) \xi_{j}, \quad \xi_{i}^{\prime}=\sum_{j=1}^{m} \alpha_{i j} \xi_{j} \quad(i=2, \ldots, m),
$$

the matrix of its coefficients being

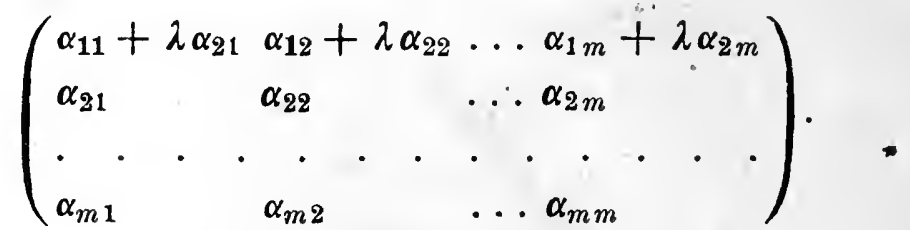

Similarly, the matrix for the product $B_{1,2, \lambda} A$ is

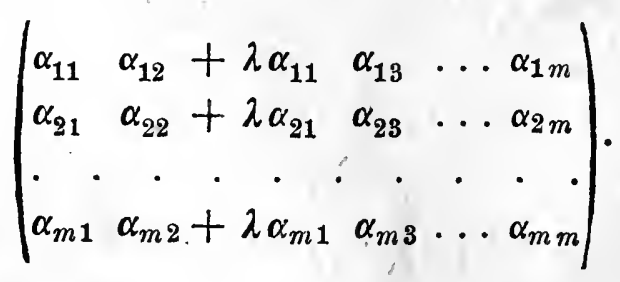


To multiply $A$ on the right by $B_{r, s, \lambda}$, we therefore multiply the $s^{\text {th }}$ row of the matrix $\left(\alpha_{i j}\right)$ by $\lambda$ and add to the $r^{\text {th }}$ row; to multiply $A$ on the left by the same substitution, we multiply the $r^{\text {th }}$ column by $\lambda$ and add to the $s^{\text {th }}$ column of the matrix $\left(\alpha_{i j}\right)$. We make use of these operations, which are recognized to be identical with the elementary operations permissible in reducing a determinant, to simplify the form of the matrix $A$. It is shown below that, if $m>1$, we can set $\alpha_{11}=1$. Then by multiplying $A$ on the right and left by suitable generators $B_{i, j, \lambda}$, we can reach a new matrix $A^{\prime}$ having the elements of the first row and first column all zero, except $\alpha_{11}$ which $=1$. After $m-1$ such steps, we would reach a matrix $A^{(m-1)}$ having every element zero except those in the main diagonal and the latter all unity except that lying in the last row. The resulting substitution would be $D_{m}$. From the identity thus established, $B_{1} A B_{2} \equiv D_{m}$, where $B_{1}$ and $B_{2}$ are products derived from the $B_{i, j, \lambda}$, we find

$$
A=B_{1}^{-1} D_{m} B_{2}^{-1}=B_{1}^{-1} B_{3} D_{m}=B D_{m} \text {. }
$$

It remains to be shown that, if $m>1$, a matrix can be obtained from $A$ having $\alpha_{11}=1$. From the given generators we derive the substitution

$$
B_{i, j, \lambda} B_{j, i,-\lambda}-1 B_{i, j, \lambda}: \quad \xi_{i}^{\prime}=\lambda \xi_{j}, \quad \xi_{j}^{\prime}=-\lambda-1 \xi_{i},
$$

affecting only the indices $\xi_{i}$ and $\xi_{j}$. In particular, for $\lambda=1, i=1$, we get

$$
J:
$$$$
\xi_{1}^{\prime}=\xi_{j}, \quad \xi_{j}^{\prime}=-\xi_{1} .
$$

We determine a substitution $K$ derived from the $B_{i, j, 2}$ such that the product $A^{\prime} \equiv K A$ will have the coefficient $\alpha_{21}^{\prime} \neq 0$. If $\alpha_{21} \neq 0$, we take $K=I$, the identity; if $\alpha_{21}=0$, but $\alpha_{2 j} \neq 0$, we take $K=J$. The product

$$
A^{\prime \prime} \equiv A^{\prime} B_{1,2, \lambda}
$$

has the coefficient $\alpha_{11}^{\prime \prime} \equiv \alpha_{11}^{\prime}+\lambda \alpha_{21}^{\prime}$, which can be made equal to unity by choice of $\lambda$ in the $G F\left[p^{n}\right]$.

Corollary I. The only linear homogeneous substitutions commutative with every $B_{r, s, \lambda}(r, s=1, \ldots, m, r \neq s)$, where $\lambda$ is a fixed mark $\neq 0$ of the $G F\left[p^{n}\right]$, are those of the form

$$
\xi_{i}^{\prime}=\varrho \xi_{i} \quad(i=1, \ldots, m) .
$$

It follows by inspection of the above two matrices for $A B_{1,2,2}$. and $B_{1,2, \lambda} A$ that they are identical only when

$$
\alpha_{11}=\alpha_{22}, \quad \alpha_{i 1}=0 \quad(i=2,3, \ldots, m), \quad \alpha_{2 j}=0 \quad(j=3, \ldots, m) .
$$

Since the indices 1,2 can be replaced by any pair $r, s$ of distinct integers $\bar{\Sigma} m$, it follows that every element of the matrix $\left(\alpha_{i j}\right)$ must be zero except those in the main diagonal, which must all be equal. 
Corollary II. The group of binary linear homogeneous substitutions of determinant unity is generated by the substitutions $B_{1,2, \lambda}$ and

$$
T: \quad \xi_{1}^{\prime}=-\xi_{2}, \quad \xi_{2}^{\prime}=\xi_{1} \text {. }
$$

Indeed, $T$ transforms $B_{1,2,-\lambda}$ into $B_{2,1, \lambda}$.

101. Transformation of indices. - We can introduce in place of $\xi_{1}, \xi_{2}, \ldots, \xi_{m}$ the $m$ new indices

$$
\eta_{i} \equiv \sum_{k=1}^{m} \beta_{i k} \xi_{k} \quad(i=1,2, \ldots, m)
$$

provided the determinant $\left|\beta_{i k}\right| \neq 0$. In fact, the substitution

$$
A: \quad \xi_{i}=\sum_{j=1}^{m} \alpha_{i j} \xi_{j} \quad(i=1,2, \ldots, m)
$$

will replace $\eta_{i}$ by $\sum_{j, k}^{1} \ldots m \beta_{i k} \alpha_{k j} \xi_{j}$, which, by solving 67 ), can be put into the form $\sum_{j=1}^{m} \gamma_{i j} \eta_{j}$. The substitution $A$ becomes

$$
B^{-1} A B: \quad \eta_{i}^{\prime}=\sum_{j=1}^{m} \gamma_{i j} \eta_{j} \quad(i=1,2, \ldots, m)
$$

where $B$ denotes the substitution 67 ) replacing the $\xi_{i}$ by the $\eta_{i}$. In fact

$$
B^{-1} A B=\left(\begin{array}{l}
\eta_{i} \\
\xi_{i}
\end{array}\right)\left(\begin{array}{c}
\xi_{i} \\
\xi_{i}^{\prime}
\end{array}\right)\left(\begin{array}{c}
\xi_{i}^{\prime} \\
\eta_{i}^{\prime}
\end{array}\right) \equiv\left(\begin{array}{c}
\eta_{i} \\
\eta_{i}^{\prime}
\end{array}\right)
$$

The determinant of the transformed substitution equals that of $A$,

$$
\left|B^{-1} A B\right|=\left|B^{-1}\right| \cdot|A| \cdot|B|=|A| \text {. }
$$

This result is, however, a special case $(\varrho=0)$ of the next theorem.

102. Theorem. - The characteristic determinant (with parameter $\varrho$ ) of a linear homogeneous substitution $A$,

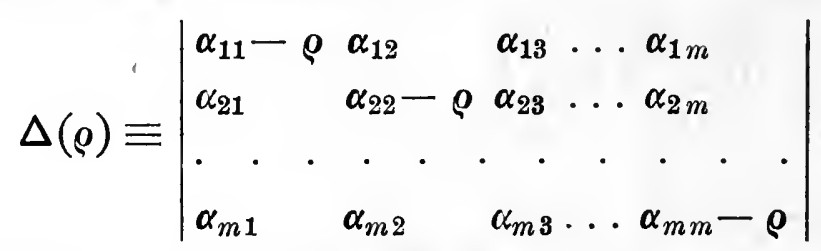

is unchanged under every linear transformation of indices.

It is only necessary to prove the theorem for the following types of transformations of indices, since by $\S 100$ every linear transformation can be derived from them:

$$
\begin{aligned}
D_{1}: & \eta_{1}=D \xi_{1}, \quad \eta_{2}=\xi_{2}, \ldots, \eta_{m}=\xi_{m} \\
B_{1,2, \lambda}: & \eta_{1}=\xi_{1}+\lambda \xi_{2}, \quad \eta_{2}=\xi_{2}, \ldots, \eta_{m}=\xi_{m} .
\end{aligned}
$$


Under the transformation of indices $D_{1}, A$ takes the form

$$
\begin{aligned}
& \eta_{1}^{\prime}=D \sum_{j=1}^{m} \alpha_{1 j} \xi_{j}=\alpha_{11} \eta_{1}+D \sum_{j=2}^{m} \alpha_{1 j} \eta_{j}, \\
& \eta_{i}^{\prime}=\sum_{j=1}^{m} \alpha_{i j} \xi_{j}=\frac{1}{D} \alpha_{i 1} \eta_{1}+\sum_{j=2}^{m} \alpha_{i j} \eta_{j} \quad(i=2,3, \ldots, m) .
\end{aligned}
$$

The characteristic determinant of the transformed substitution is

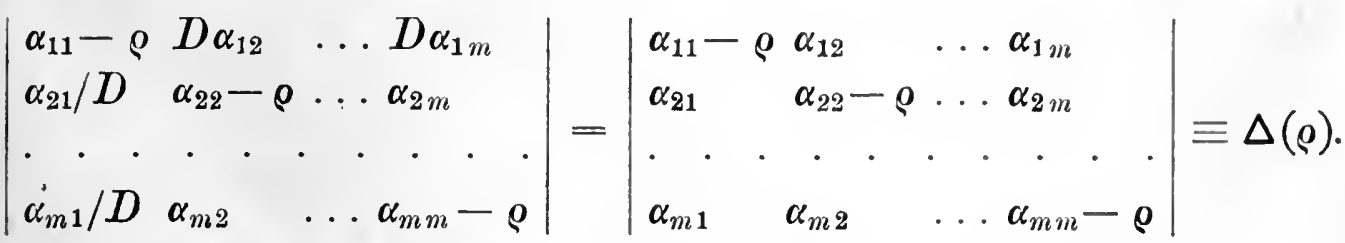

Under the transformation of indices $B_{1,2, \lambda}, A$ becomes

$$
\begin{aligned}
\eta_{1}^{\prime}=\sum_{j=1}^{m}\left(\alpha_{1 j}+\lambda \alpha_{2 j}\right) \xi_{j}=\left(\alpha_{11}+\lambda \alpha_{21}\right) \eta_{1} & +\left(\alpha_{12}+\lambda \alpha_{22}-\lambda \alpha_{11}-\lambda^{2} \alpha_{21}\right) \eta_{2} \\
& +\sum_{j=3}^{m}\left(\alpha_{1 j}+\lambda \alpha_{2 j}\right) \eta_{j}
\end{aligned}
$$$$
\eta_{i}^{\prime}=\sum_{j=1}^{m} \alpha_{i j} \xi_{j}=\alpha_{i 1} \eta_{1}+\left(\alpha_{i 2}-\lambda \alpha_{i 1}\right) \eta_{2}+\sum_{j=3}^{m} \alpha_{i j} \eta_{j} \quad(i=2, \ldots, m) .
$$

The characteristic determinant of this substitution is

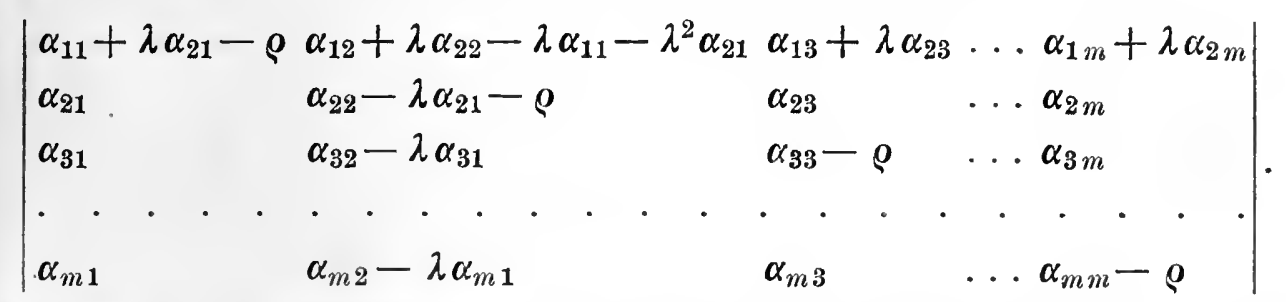

Multiplying the second row by $\lambda$ and subtracting from the first row, and afterwards adding the first column multiplied by $\lambda$ to the second column, we reach the original determinant $\Delta(\varrho)$.

Corollary. - The transformed of $A$ by any linear substitution $B$ has the same characteristic determinant as $A$. Indeed, by $\S 101, A$ is converted into $B^{-1} A B$ by the transformation of indices indicated by the substitution $B$.

Factors of composition $\left.{ }^{1}\right)$ of $G L H\left(m, p^{n}\right), \S \S 103-107$.

103. Let $\varrho$ be a primitive root of the $G F\left[p^{n}\right]$. If two linear substitutions have as determinants $\varrho^{r l}$ and $\varrho^{s l}$, their compound has

1) For the case $n=1$, Jordan, Traité, pp. $106-110$; for general $n$, author's dissertation, Annals of Mathematics, vol. 11 (1897), pp. 168-175; also Burnside, The theory of groups, pp. $340-341$.

DICKsoN, Linear Groups. 
the determinant $\varrho^{(r+s) l}$. Hence the totality of substitutions in the group $G \equiv G L H\left(m, p^{n}\right)$ having as determinants powers of $\varrho^{l}$ forms a subgroup $G_{l}$. Suppose that

$$
p^{n}-1=p_{1} p_{2} \ldots p_{k},
$$

where $p_{1}, p_{2}, \ldots, p_{k}$ are all primes. Denote by $G_{p_{1}}, G_{p_{1} p_{2}}, \ldots, G_{p^{n}-1} \equiv \Gamma$ the subgroups of $G$ formed of those of its substitutions whose determinants are respectively powers of $\varrho^{p_{1}}, \varrho^{p_{1} p_{2}}, \ldots, \varrho^{p^{n}-1} \equiv 1$. By $\S 63$, the orders of these groups are respectively

$$
\Omega / p_{1}, \quad \Omega / p_{1} p_{2}, \ldots, \Omega / p^{n}-1 \quad \text { (where } \Omega \equiv G L H\left[m, p^{n}\right] \text { ). }
$$

In fact, by $\S 100, G$ contains substitutions of every determinant $\neq 0$ in the $G F\left[p^{n}\right]$ and contains the same number of one determinant as of another.

If $S$ and $T$ be linear substitutions, $S$ and $T^{-1} S T$ have the same determinant ( $\S 101)$. Hence the groups $G_{p_{1}}, G_{p_{1} p_{2}}, \ldots, \Gamma$ are selfconjugate under $G$, i. e., each is transformed into itself by any substitution of $G$. Since $p_{1}, \ldots, p_{k}$ are primes, there is no group lying between $G$ and $G_{p_{1}}$, no one between $G_{p_{1}}$ and $G_{p_{1} p_{2}}$, etc. Hence we may descend from $G$ to $\Gamma$ by the composition-series

$$
G, \quad G_{p_{1}}, \quad G_{p_{1} p_{2}}, \ldots, G_{p^{n}-1} \equiv \Gamma .
$$

The group $\Gamma$ of all substitutions of determinant unity is called the special linear homogeneous group $S L H\left(m, p^{n}\right)$. It has a self-conjugate subgroup $H$ formed of those of its substitutions which are of the form

$$
M_{\mu}: \quad \xi_{i}^{\prime}=\mu \xi_{i} \quad\left[\mu^{m}=1\right] \quad(i=1,2, \ldots, m) .
$$

The mark $\mu$ must also satisfy the equation

$$
\mu^{p^{n}-1}=1 \text {. }
$$

Hence, if $d$ be the greatest common divisor of $m$ and $p^{n}-1$, we find (by the method of proof used in $\S 79$ ) that

$$
\mu^{d}=1 \text {. }
$$

Inversely, each of the $d$ distinct solutions in the $G F\left[p^{n}\right]$ of 68 ) [see $\S 16$ ], leads to a substitution $M_{\mu}$ belonging to the group $H$. The order of $H$ is therefore $d$.

If $\delta$ be a mark of the $G F\left[p^{n}\right]$ which belongs to the exponent $d$ (§ 17, Corollary), then $\mu$ is a power of $\delta$. Suppose that

$$
d=q_{1} q_{2} \ldots q_{\iota} \quad \text { (each } q_{i} \text { a prime). }
$$

Denote by $H_{q_{1}}, H_{q_{1} g_{2}}, \ldots, H_{d} \equiv I$ the groups-formed of those substitutions of $H$ which multiply every index ty a like power of $\delta^{q_{1}}$, by 
a like power of $\delta^{q_{1} q_{2}}, \ldots$, by a like power of $\delta^{d} \equiv 1$, respectively. Since we have, for any mark $v$,

$$
M_{v}^{-1} M_{\mu} M_{v} \equiv M_{\mu}
$$

a composition-series of $H$ is given by

$$
H, \quad H_{q_{1}}, \quad H_{q_{1} q_{2}}, \ldots, \quad H_{q_{\mathrm{x}} q_{2}} \ldots q_{l} \equiv I .
$$

In view of the theorem proven in $\S \S 104-107$, we may state the complete

Theorem. - The factors of composition of $G L H\left(m, p^{n}\right)$ are

$$
p_{1}, \quad p_{2}, \ldots, p_{k}, \Omega / d\left(p^{n}-1\right), \quad q_{1}, q_{2}, \ldots, q_{b},
$$

except in the two cases $\left(m, p^{n}\right)=(2,2)$ and $(2,3)$, when the factors of composition are 2,3 and $2,3,2,2,2$ respectively.

104. Theorem. - Excluding the above two cases, the group $H$ is a maximal self-conjugate subgroup of $\Gamma$.

Suppose that $\Gamma$ contains a self-conjugate subgroup $J$ which contains all the substitutions of $H$ and still further substitutions. We will prove that, aside from the two exceptional cases mentioned, $J$ coincides with $\Gamma$.

By hypothesis, $J$ contains a substitution

$$
S: \quad \xi_{i}^{\prime}=\sum_{j=1}^{m} \alpha_{i j} \xi_{j} \quad(i=1, \ldots, m)
$$

which is not in $H$ and therefore does not multiply all the indices by the same factor. Hence, by Corollary I of $\S 100, S$ is not commutative with every $B_{r, s, \lambda}(r, s=1,2, \ldots, m ; r \neq s)$. Changing the notation if necessary, we may suppose that $S$ is not commutative with $B_{1,2, \lambda}$, a substitution of determinant unity and therefore in the group $\Gamma$. It therefore transforms the substitution $S$ of the self-conjugate subgroup $J$ into a substitution belonging to $J$. Hence $J$ contains the product

$$
T \equiv S^{-1} \cdot B_{1,2,2}^{-1} S B_{1,2, \lambda}
$$

which does not reduce to the identity $I$. In calculating this product, let $\Phi$ be the linear function by which $S^{-1}$ replaces $\xi_{2}$. Then $T$ is seen to have the form, in which the values of the $\beta_{1 j}$ need not be determined:

$$
T: \quad \xi_{1}^{\prime}=\sum_{j=1}^{m} \beta_{1 j} \xi_{j} ; \quad \xi_{i}^{\prime}=\xi_{i}-\lambda \alpha_{i 1} \Phi \quad(i=2,3, \ldots, m) .
$$

Suppose first that the $\alpha_{i 1}$ are not all zero, say $\alpha_{21} \neq 0$. For $m>2$, we introduce new indices $\eta_{i}$ defined by the substitution $V$ of determinant unity,

$$
\eta_{1} \equiv \xi_{1}, \quad \eta_{2} \equiv \xi_{2}, \eta_{i} \equiv \xi_{i}-\frac{\alpha_{i 1}}{\alpha_{21}} \xi_{2} \quad(i=3,4, \ldots, m) .
$$


The resulting substitution $V^{-1} T V$ belongs to $J$ and leaves $\eta_{i}(i>2)$ unaltered:

$$
\eta_{i}^{\prime}=\left(\xi_{i}-\lambda \alpha_{i 1} \Phi\right)-\frac{\alpha_{i 1}}{\alpha_{21}}\left(\xi_{2}-\lambda \alpha_{21} \Phi\right)=\xi_{i}-\frac{\alpha_{i 1}}{\alpha_{21}} \xi_{2} \equiv \eta_{i}
$$

If, however, every $\alpha_{i 1}=0, T$ itself leaves fixed $m-1$ indices. In either case, $J$ contains a substitution $\neq I$ of the form ${ }^{1}$ )

$$
R: \quad \eta_{1}^{\prime}=\sum_{j=1}^{m} \gamma_{1 j} \eta_{j}, \quad \eta_{2}^{\prime}=\sum_{j=1}^{m} \gamma_{2 j} \eta_{j}, \quad \eta_{i}^{\prime}=\eta_{i} \quad(i=3, \ldots, m) .
$$

Then $J$ contains the two substitutions leaving $\eta_{3}, \ldots, \eta_{m}$ fixed:

$$
\begin{aligned}
& T_{2} \equiv R^{-1} B_{1,3, \mu}^{-1} R B_{1,3, \mu}:\left\{\begin{array}{l}
\eta_{1}^{\prime}=\eta_{1}-\mu\left(\gamma_{11}-1\right) \eta_{3}, \\
\eta_{2}^{\prime}=\eta_{2}-\mu \gamma_{21} \eta_{3} .
\end{array}\right. \\
& T_{3} \equiv R^{-1} B_{2,3, \mu}^{-1} R B_{2,3, \mu}:\left\{\begin{array}{l}
\eta_{1}^{\prime}=\eta_{1}-\mu \gamma_{12} \eta_{3}, \\
\eta_{2}^{\prime}=\eta_{2}-\mu\left(\gamma_{22}-1\right) \eta_{3} .
\end{array}\right.
\end{aligned}
$$

These substitutions are both of the form

$$
U: \quad \eta_{1}^{\prime}=\eta_{1}+\sigma_{1} \eta_{3}, \quad \eta_{2}^{\prime}=\eta_{2}+\sigma_{2} \eta_{3}, \quad \eta_{i}^{\prime}=\eta_{i} \quad(i=3, \ldots, m) .
$$

If $T_{2}$ and $T_{3}$ reduce to the identity, $R$ itself becomes

$$
\begin{aligned}
R_{1}: \quad \eta_{1}^{\prime}=\eta_{1}+\gamma_{13} \eta_{3}+\cdots+\gamma_{1 m} \eta_{m}, & \eta_{2}^{\prime}=\eta_{2}+\gamma_{23} \eta_{3}+\cdots+\gamma_{2 m} \eta_{m}, \\
& \eta_{i}^{\prime}=\eta_{i} \quad(i>2) .
\end{aligned}
$$

If $\gamma_{1 j}=\gamma_{2 j}=0(j=4, \ldots, m)$, this substitution $\neq I$ is of the form $U$. In the contrary case, we may suppose that $\gamma_{14}$ and $\gamma_{24}$ are not both zero. Then

$$
R_{1}^{-1} B_{4,3, \mu}^{-1} R_{1} B_{4,3, \mu}:\left\{\begin{array}{l}
\eta_{1}^{\prime}=\eta_{1}-\mu \gamma_{14} \eta_{3}, \\
\eta_{2}^{\prime}=\eta_{2}-\mu \gamma_{24} \eta_{3}, \quad \eta_{i}^{\prime}=\eta_{i} .(i>2)
\end{array}\right.
$$

is a substitution $\neq I$ of the form $U$ and belonging to $J$. Hence, in every case $J$ contains a substitution $U$ not the identity. For definiteness, let $\sigma_{1} \neq 0$ and introduce the new indices

$$
\xi_{1} \equiv \eta_{1}, \quad \xi_{2} \equiv \eta_{2}-\frac{\sigma_{2}}{\sigma_{1}} \eta_{1}, \quad \xi_{i}=\eta_{i} \quad(i>2) .
$$

Then $U$ becomes $B_{1,3, \sigma_{1}}$. Transforming the latter by the substitution

$$
\xi_{1}^{\prime}=\lambda \xi_{1}, \quad \xi_{2}^{\prime}=\lambda^{-1} \xi_{2}, \quad \xi_{i}^{\prime}=\xi_{i} \quad(i=3, \ldots, m),
$$

where $\lambda$ is an arbitrary mark $\neq 0$ of the $G F\left[p^{n}\right]$, we reach in $J$ the substitution $B_{1,3, \lambda \sigma}$, and therefore every $B_{1,3, \lambda}$. The latter is transformed into $B_{k, 3, \lambda}(k \neq 1,3)$ by the following substitution of $\Gamma$ :

$$
\xi_{1}^{\prime}=-\xi_{k}, \quad \xi_{k}^{\prime}=\xi_{1}, \quad \xi_{i}^{\prime}=\xi_{i} \quad(i=2, \ldots, k-1, k+1, \ldots, m) .
$$

1) From this point, the proofs by Burnside and Jordan (1. c.) are incomplete. The specific errors were made in the Traité, p. 108, $1^{\circ}$ and in The theory of groups; p. 316, "This process may now be repeated", etc. 
Finally, for $j \neq k, B_{k, 3, \lambda}$ is transformed into $B_{k, j, \lambda}$ by the substitution $\xi_{3}^{\prime}=-\xi_{j}, \quad \xi_{j}^{\prime}=\xi_{3}, \quad \xi_{i}^{\prime}=\xi_{i} \quad(i=1, \ldots, m ; i \neq 3, i \neq j)$.

It follows from $\S 100$ that, if $m>2, J$ is identical with $\Gamma$.

105. For $m=2$, we are given that $J$ contains a substitution $S: \quad \xi_{1}^{\prime}=\alpha \xi_{1}+\beta \xi_{2}, \quad \xi_{2}^{\prime}=\alpha^{\prime} \xi_{1}+\beta^{\prime} \xi_{2} \quad\left(\alpha \beta^{\prime}-\alpha^{\prime} \beta=1\right)$, which is neither the identity $I$ nor

$E$ :

$$
\xi_{1}^{\prime}=-\xi_{1}, \quad \xi_{2}^{\prime}=-\xi_{2} \text {. }
$$

We proceed to prove that, for $p^{n}>3, J$ contains a substitution of the form $B_{2,1, \lambda}$ in which $\lambda \neq 0$.

a) Suppose first that $\beta=0$, so that $J$ contains

$$
S_{1}: \quad \xi_{1}^{\prime}=\alpha \xi_{1}, \quad \xi_{2}^{\prime}=\alpha^{\prime} \xi_{1}+\alpha^{-1} \xi_{2},
$$

where $\alpha^{\prime} \neq 0$ if $\alpha=\alpha^{-1}$, since $S_{1} \neq I$ or $E$.

$a_{1}$ ) If $\alpha=\alpha^{-1}$, whence $\alpha= \pm 1$, the group $J$ contains both $S_{1}$ and $S_{1} E$, one of which has the form

$$
\xi_{1}^{\prime}=\xi_{1}, \quad \xi_{2}^{\prime}=\xi_{2}+\lambda \xi_{1} \quad\left(\lambda \equiv \pm \alpha^{\prime} \neq 0\right) .
$$

$a_{2}$ ) If $\alpha \neq \alpha^{-1}, J$ contairs the substitution $\neq I$,

$$
S_{1} B_{2,1,1}^{-1} S_{1}^{-1} B_{2,1,1}: \quad \xi_{1}^{\prime}=\xi_{1}, \xi_{2}^{\prime}=\xi_{2}+\left(1-\alpha^{2}\right) \xi_{1} .
$$

b) Suppose next that $\beta \neq 0$. The following substitution

$$
\Sigma: \quad \xi_{1}^{\prime}=x \alpha \xi_{1}+x \beta \xi_{2}, \quad \xi_{2}^{\prime}=-\frac{1+x^{2} \alpha^{2}}{x \beta} \xi_{1}-x \alpha \xi_{2}
$$

has determinant unity and therefore belongs to $\Gamma$. Hence $J$ contains $S_{2} \equiv \Sigma^{-1} S \Sigma S ;$ viz.,

$$
\xi_{1}^{\prime}=-x^{-2} \xi_{1}, \quad \xi_{2}^{\prime}=\frac{-\left(1+x^{2}\right)\left(\beta^{\prime}+\alpha x^{2}\right)}{\beta x^{2}} \xi_{1}-x^{2} \xi_{2} .
$$

If $p^{n}=4$ or if $p^{n}>5, \varkappa$ can be chosen in the $G F\left[p^{n}\right]$ so that

$$
x^{4} \neq 1, \quad x^{-2} \neq x^{2}
$$

Proceeding with $S_{2}$ as in case $a_{2}$ ), we obtain in $J$ a substitution $B_{2,1, \lambda}$, where $\lambda \neq 0$.

If $p^{n}=5$, we take $\varkappa=1$, when $S_{2} E$ becomes

$$
\xi_{1}^{\prime}=\xi_{1}, \quad \xi_{2}^{\prime}=\frac{2\left(\beta^{\prime}+\alpha\right)}{\beta} \xi_{1}+\xi_{2} .
$$

Our result follows unless $\beta^{\prime}+\alpha \equiv 0(\bmod 5)$. But $J$ contains the product $S B_{2,1,1}^{-1} S^{-1} B_{2,1,1}$, viz.,

$$
\xi_{1}^{\prime}=(1+\alpha \beta) \xi_{1}+\beta^{2} \xi_{2}, \quad \xi_{2}^{\prime}=\left(1+\alpha \beta-\alpha^{2}\right) \xi_{1}+\left(1-\alpha \beta+\beta^{2}\right) \xi_{2},
$$

for which the sum corresponding to the above $\beta^{\prime}+\alpha$ is

$$
(1+\alpha \beta)+\left(1-\alpha \beta+\beta^{2}\right) \equiv \beta^{2}+2 \equiv \equiv 0 \quad(\bmod 5) \text {. }
$$


We have now proved that, if $p^{n}>3, J$ contains a substitution $B_{2,1, \lambda}(\lambda \neq 0)$. It is transformed into $B_{2,1, \lambda \rho^{2}}$ by the substitution

Also

$$
\xi_{1}^{\prime}=\varrho^{-1} \xi_{1}, \quad \xi_{2}^{\prime}=\varrho \xi_{2} .
$$

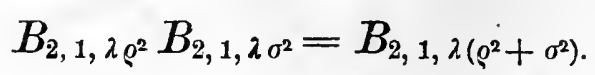

By $\S 64$, there exist solutions in the $G F\left[p^{n}\right]$ of $\varrho^{2}+\sigma^{2}=x / \lambda$ for $x$ arbitrary in the field. Hence $J$ contains $B_{2,1, x}$. Transforming the latter by $\left(\xi_{1}^{\prime}=\xi_{2}, \xi_{2}^{\prime}=-\xi_{1}\right)$ we get $B_{1,2,-\%}$. It follows from $\S 100$ that $J \equiv \Gamma$. By $\S \S 99$ and 103, the order of the group $\Gamma$ of binary linear homogeneous substitutions of determinant unity is $p^{n}\left(p^{2 n}-1\right)$.

106. For $p^{n}=2, m=2$, the group $\Gamma$ is of order 6 and is identical with $G L H(2,2)$. It contains a subgroup of order 3 generated by the substitution

$$
\xi_{1}^{\prime}=\xi_{2}, \quad \xi_{2}^{\prime}=\xi_{1}+\xi_{2} .
$$

The index of this subgroup being 2 , it is self-conjugate. The factors of composition are therefore 2 and 3 .

10\%. For $p^{n}=3, m=2$, the group $G \equiv G L H\left(m, p^{n}\right)$ is of order $48 \equiv\left(3^{2}-1\right)\left(3^{2}-3\right)$ and contains the following substitutions

$$
\begin{array}{lll}
A: & \xi_{1}^{\prime}=-\xi_{1}, & \xi_{2}^{\prime}=\xi_{1}+\xi_{2} ; \\
B: & \xi_{1}^{\prime}=\xi_{2}, & \xi_{2}^{\prime}=-\xi_{1}+\xi_{2} ; \\
C: & \xi_{1}^{\prime}=-\xi_{2}, & \xi_{2}^{\prime}=\xi_{1} ; \\
D: & \xi_{1}^{\prime}=\xi_{1}+\xi_{2}, & \xi_{2}^{\prime}=\xi_{1}-\xi_{2} ; \\
E: & \xi_{1}^{\prime}=-\xi_{1}, \quad \xi_{2}^{\prime}=-\xi_{2},
\end{array}
$$

of which $A$ has determinant -1 and the others determinant +1 modulo 3. In virtue of the relations

$$
\begin{aligned}
& E^{2}=1 ; \\
& D^{2}=E, \quad D E=E D ; \\
& C^{2}=E, \quad C E=E C, \quad C D=E D C ; \\
& B^{3}=E, \quad B E=E B, \quad B D=C D B, \quad B C=D B ; \\
& A^{2}=1, \quad A E=E A, \quad A D=C A, \quad A C=D A, \quad A B=E C B^{2} A ;
\end{aligned}
$$

it results that the groups generated as follows:

$$
\{E\} ; \quad\{E, D\} ; \quad\{E, D, C\} ; \quad\{E, D, C, B\}, \quad\{E, D, C, B, A\}
$$

have the orders $2,4,8,24,48$ respectively and that each group is self-conjugate under the following group. The last group is identical with $G$, whose factors of composition are therefore $2,3,2,2,2$. 
108. From the linear homogeneous substitution $A$ of $\S 98$ on the arbitrary variables $\xi_{1}, \xi_{2}, \ldots, \xi_{m}$, we obtain the linear fractional substitution

$$
A^{\prime}: \quad x_{i}^{\prime}=\frac{\alpha_{i 1} x_{1}+\alpha_{i 2} x_{2}+\cdots+\alpha_{i m-1} x_{m-1}+\alpha_{i m}}{\alpha_{m 1} x_{1}+\alpha_{m 2} x_{2}+\cdots+\alpha_{m m-1} x_{m-1}+\alpha_{m m}} \quad(i=1, \ldots, m-1)
$$

upon setting $x_{i} \equiv \xi_{i} / \xi_{m}$ for $i=1, \ldots, m-1$. It being only a question of the ratios of the coefficients $\alpha_{i j}$ in $A^{\prime}$, its determinant $\left|\alpha_{i j}\right|$ is determined only up to a factor $\mu^{m}, \mu$ being a mark $\neq 0$. Also, $A^{\prime}$ is the identity if, and only if, $A$ be one of the $p^{n}-1$ substitutions

$$
M_{\mu}: \quad \xi_{i}^{\prime}=\mu \xi_{i} \quad(i=1, \ldots, m) .
$$

The products $M_{\mu} A$ and no other linear homogeneous substitutions correspond to the same linear fractional substitution $A^{\prime}$. Hence the group $G \equiv G L H\left(m, p^{n}\right)$ has $\left(p^{n}-1,1\right)$ isomorphism with the group $L$ of the substitutions $A^{\prime}$. If $\Omega$ denote the order of $G$, the order of $L$ is $\Omega \div\left(\dot{p}^{n}-1\right)$. To the subgroup $\Gamma$ formed of the substitutions of $G$ having determinant unity there corresponds a subgroup $\wedge$ of $L$ composed of those of its substitutions whose determinant is an $m^{\text {th }}$ power in the field. If $d$ be the greatest common divisor of $m$ and $p^{n}-1$, there are exactly $d$ substitutions of the form $M_{\mu}$ in $\Gamma$ and they form the group $H(\S 103)$. Hence $\Gamma$ has $(d, 1)$ isomorphism with $\Lambda$. The order of $\Lambda$ is therefore $\Omega \div d\left(p^{n}-1\right)$. Aside from the cases $\left(m, p^{n}\right)=(2,2)$ and $(2,3), H$ was shown to be the maximal self-conjugate subgroup of $\Gamma$; hence $\Lambda$ has no self-conjugate subgroup other than itself and the identity and is therefore simple.

The group $L F\left(m, p^{n}\right)$ of all linear fractional substitutions in the $G F\left[p^{n}\right]$ on $m-1$ variables and having determinant unity or some $m^{\text {th }}$ power in the field has the order

$$
\frac{1}{d}\left(p^{n m}-1\right) p^{n(m-1)}\left(p^{n(m-1)}-1\right) p^{n(m-2)} \ldots\left(p^{2 n}-1\right) p^{n}
$$

$d$ being the greatest common divisor of $m$ and $p^{n}-1$. It is a simple group except in the two cases $\left(m, p^{n}\right)=(2,2)$ and $(2,3)$. The group of all linear fractional substitutions of determinants not zero has d times the order of $L F\left(m, p^{n}\right)$.

The notation $L F\left(m, p^{n}\right)$ emphasizes the point that the essential quality of the linear fractional substitution lies in the matrix $\left(\alpha_{i j}\right)$ of degree $m$ and not in the $m-1$ variables $x_{1}, \ldots, x_{m-1}$ which play the rô le of indeterminates. For $m=2$, we use the suggestive notation

$$
\left(\frac{\alpha, \beta}{\gamma, \delta}\right): \quad x^{\prime}=\frac{\alpha x+\beta}{\gamma x+\delta} \quad(\Delta \equiv \alpha \delta-\beta \gamma \neq 0) .
$$

In virtue of the identity of the two substitutions

$$
\left(\frac{\alpha, \beta}{\gamma, \delta}\right), \quad\left(\frac{\alpha \mu, \beta \mu}{\gamma \mu, \delta \mu}\right) \quad(\mu \text { any mark } \neq 0)
$$

of determinants $\Delta$ and $\mu^{2} \Delta$, we may choose $\mu$ so that the substitution takes its normal form, viz., of determinant unity if $p=2$, but of 
determinant unity or a particular not-square' $\nu$ if $p>2$. In fact, if $\Delta$ is a square, $\mu^{2} \Delta$ may be made equal to unity by choice of $\mu$ in the field; while for $\Delta$ a not-square, $\mu^{2} \Delta$ may be made equal to $\nu$.

If $p^{n}>3$, the group $L F\left(2, p^{n}\right)$ of all linear fractional substitutions in the $G F\left[p^{n}\right]$ of determinant unity (when in their normal forms) is a simple ${ }^{1}$ group of order.

$$
M\left(p^{n}\right) \equiv \frac{p^{n}\left(p^{2 n}-1\right)}{2 ; 1} \quad(2 ; 1 \text { according as } p>2 ; p=2) .
$$

There are $p^{n}\left(p^{2 n}-1\right)$ linear fractional substitutions of determinant $\neq 0$.

From the formula of composition of binary linear homogeneous substitutions ( $\$ 97$ ), we derive the product $S S_{1}$ of linear fractional substitutions $S \equiv\left(\frac{a, \beta}{\gamma, \delta}\right), \quad S_{1} \equiv\left(\frac{\alpha_{1}, \beta_{1}}{\gamma_{1}, \delta_{1}}\right)$ :

$$
z^{\prime}=\frac{\alpha z+\beta}{\gamma z+\delta}, \quad z^{\prime \prime}=\frac{\alpha_{1} z^{\prime}+\beta_{1}}{\gamma_{1} z^{\prime}+\delta_{1}}, \quad z^{\prime \prime}=\frac{\left(\alpha_{1} \alpha+\beta_{1} \gamma\right) z+\left(\alpha_{1} \beta+\beta_{1} \delta\right)}{\left(\gamma_{1} \alpha+\delta_{1} \gamma\right) z+\left(\gamma_{1} \beta+\delta_{1} \delta\right)} .
$$

Hence if $S$ operate first and $S_{1}$ afterwards, the product $S S_{1}$ is ${ }^{2}$ )

$$
\left(\frac{\alpha, \beta}{\gamma, \delta}\right)\left(\frac{\alpha_{1}, \beta_{1}}{\gamma_{1}, \delta_{1}}\right)=\left(\frac{\alpha_{1} \alpha+\beta_{1} \gamma, \alpha_{1} \beta+\beta_{1} \delta}{\gamma_{1} \alpha+\delta_{1} \gamma, \gamma_{1} \beta+\delta_{1} \delta}\right)
$$

109. The quotient-group $\Gamma / H$ may be readily represented as a permutation-group on $q \equiv\left(p^{n m}-1\right) \div\left(p^{n}-1\right)$ letters $\left.^{3}\right)$. Of the $p^{n m}-1$ letters $l_{\xi_{1}}, \xi_{2}, \ldots, \xi_{m}$ in which $\xi_{1}, \xi_{2}, \ldots, \xi_{m}$ denote marks of the $G F\left[p^{n}\right]$ not all zero, we combine into a single system the $p^{n}-1$ letters $l_{\mu} \xi_{1}, \mu \xi_{2}, \ldots, \mu \xi_{m}$ in which $\mu$ runs through the series of marks $\neq 0$ while $\xi_{1}, \xi_{2}, \ldots, \xi_{m}$ denotes a set of fixed marks not all zero. Any linear homogeneous substitution on $\xi_{1}, \ldots, \xi_{m}$ with $\dot{c}$ efficients in the field replaces the letters of any one system by letters all of some one system and therefore permutes the $q$ systems amongst themselves. In particular, the substitutions $M_{\mu}$ do not displace any system. Hence the group $\Gamma$ of substitutions of determinant unity corresponds to a permutation-group on the $q$ systems, which represents concretely the quotient-group $\Gamma / H$.

1) Cf. Moore, Congress Mathematical Papers, pp. 208-242, Bull. Amer. Math. Soc., Dec. 1893; Burnside, Proc. Lond. Math. Soc., vol. 25, pp. 113-139 (Feb., 1894); also see $\$ 261$ below.

2) For the same product of matrices, the notation $S_{1} S$ is sometimes used, $S$ operating first.

3) Compare the method of $\S 228,224$; also, for $m=2$, that of $\S 239$. 


\section{CHAPTER II.}

\section{THE ABELIAN LINEAR GROUP.')}

110. A linear homogeneous substitution on $2 m$ indices with coefficients belonging to the $G F\left[p^{n}\right]$ is called Abelian if, when operating simultaneously upon two sets of $2 m$ indices,

$$
\xi_{i 1}, \quad \eta_{i 1} ; \quad \xi_{i 2}, \quad \eta_{i 2} \quad(i=1,2, \ldots, m),
$$

it leaves formally invariant up to a factor (belonging to the field) the bilinear function

$$
\varphi \equiv \sum_{i=1}^{m}\left|\begin{array}{ll}
\xi_{i 1} & \eta_{i 1} \\
\xi_{i 2} & \eta_{i 2}
\end{array}\right| .
$$

The totality of such substitutions constitutes a group called the general Abelian linear group $\left.{ }^{2}\right) G A\left(2 m, p^{n}\right)$. These of its substitutions which leave $\varphi$ absolutely invariant form the special Abelian linear group $S A\left(2 m, p^{n}\right)$. For other definitions of these groups see $\S 160$ below and the author's article, Transactions of the American Mathematical Society, vol. 1, pp. $30-38$.

The conditions that the linear substitution

75)

$$
S:\left\{\begin{aligned}
\xi_{i}^{\prime} & =\sum_{j=1}^{m}\left(\alpha_{i j} \xi_{j}+\gamma_{i j} \eta_{j}\right) \\
\eta_{i}^{\prime} & =\sum_{j=1}^{m}\left(\beta_{i j} \xi_{j}+\delta_{i j} \eta_{j}\right)
\end{aligned}(i=1,2, \ldots, m)\right.
$$

shall leave $\varphi$ formally $^{3}$ ) invariant up to the factor $\mu$ are

$$
\text { 76) } \begin{aligned}
\sum_{i=1}^{m}\left|\begin{array}{ll}
\alpha_{i j} & \gamma_{i j} \\
\beta_{i j} & \delta_{i j}
\end{array}\right| & =\mu, \quad \sum_{i=1}^{m}\left|\begin{array}{cc}
\alpha_{i j} & \gamma_{i k} \\
\beta_{i j} & \delta_{i k}
\end{array}\right|=0, \\
\sum_{i=1}^{m}\left|\begin{array}{ll}
\alpha_{i j} & \alpha_{i k} \\
\beta_{i j} & \beta_{i k}
\end{array}\right| & =0, \quad \sum_{i=1}^{m}\left|\begin{array}{ll}
\gamma_{i j} & \gamma_{i k} \\
\delta_{i j} & \delta_{i k}
\end{array}\right|=0 .
\end{aligned}
$$

For $m=1$, the Abelian group $G A\left(2, p^{n}\right)$ is evidently identical with the general binary linear homogeneous group $G L H\left(2, p^{n}\right)$. In

1) Investigated by Jordan, Traité, pp. $171-186$, for the case $n=1$; by the author, Quar. Jour. of Math., 1897, pp. 169-178, ibid., 1899, pp. 383-4, for general $n$.

2) To distinguish these groups from the ordinary Abelian, i. e. commutative, groups, we prefix the adjective linear. The Abelian linear group is not commutative in general.

3) The indice's $\xi_{i}$ and $\eta_{i}$ are treated as arbitrary quantities. Formal invariance is used in antithesis to numerical invariance. 
determining the structure of the Abelian group, we may therefore suppose $m>1$.

111. We proceed to determine the substitution reciprocal to $S$,

$$
S^{-1}: \quad\left\{\begin{array}{rl}
\xi_{i}^{\prime} & =\sum_{j=1}^{m}\left(\alpha_{i j}^{\prime} \xi_{j}+\gamma_{i j}^{\prime} \eta_{j}\right) \\
\eta_{i}^{\prime} & =\sum_{j=1}^{m}\left(\beta_{i j}^{\prime} \xi_{j}+\delta_{i j}^{\prime} \eta_{j}\right)
\end{array}(i=1,2, \ldots, m) .\right.
$$

Supposing $S$ to be Abelian, we obtain the same result upon multiplying $\varphi$ by $\mu$ that we obtain upon operating the substitution $S$ upon the two sets of indices. The identity of the two results is not destroyed by operating the substitution $S^{-1}$ upon the indices $\xi_{i 1}, \eta_{i 1}$ $(i=1, \ldots, m)$ of one set. The result obtained upon multiplying $\varphi$ by $\mu$ and then applying the substitution $S^{-1}$ upon the indices $\xi_{i 1}, \eta_{i 1}$ is therefore identical with the result obtained by applying the substitution $S$ upon the indices $\xi_{i 2}, \eta_{i 2}$ alone. Equating the two results, we find

$$
\begin{aligned}
& \mu \sum_{i, j}^{1, \ldots, m}\left\{\left(\alpha_{i j}^{\prime} \xi_{j 1}+\gamma_{i j}^{\prime} \eta_{j 1}\right) \eta_{i 2}-\left(\beta_{i j}^{\prime} \xi_{j 1}+\delta_{i j}^{\prime} \eta_{j 1}\right) \xi_{i 2}\right\} \\
& \equiv \sum_{i, j}^{1 \cdots \ldots m}\left\{\xi_{i 1}\left(\beta_{i j} \xi_{j 2}+\delta_{i j} \eta_{j 2}\right)-\eta_{i 1}\left(\alpha_{i j} \xi_{j 2}+\gamma_{i j} \eta_{j 2}\right)\right\} .
\end{aligned}
$$

From this identity in the indices $\xi_{i j}, \eta_{i j}$, we find

$$
\mu \alpha_{i j}^{\prime}=\delta_{j i}, \quad \mu \gamma_{i j}^{\prime}=-\gamma_{j i}, \quad \mu \beta_{i j}^{\prime}=-\beta_{j i}, \quad \mu \delta_{i j}^{\prime}=\alpha_{j i} .
$$

Hence the reciprocal of the Abelian substitution 75) is

77)

$$
S^{-1}:\left\{\begin{array}{l}
\xi_{i}^{\prime}=\frac{1}{\mu} \sum_{j=1}^{m}\left(\delta_{j i} \xi_{j}-\gamma_{j i} \eta_{j}\right) \\
\eta_{i}^{\prime}=\frac{1}{\mu} \sum_{j=1}^{m}\left(-\beta_{j i} \xi_{j}+\alpha_{j i} \eta_{j}\right)
\end{array}(i=1,2, \ldots, m) .\right.
$$

When $S^{-1}$ is operated upon the two sets of indices, $\varphi$ must be multiplied by $1 / \mu$. Forming the relations expressing this fact, we obtain the following conditions, together entirely equivalent to the set of conditions 76 ):

$$
\text { 78) } \begin{aligned}
\sum_{i=1}^{m}\left|\begin{array}{ll}
\alpha_{k i} & \gamma_{k i} \\
\beta_{k i} & \delta_{k i}
\end{array}\right|=\mu, \quad \sum_{i=1}^{m}\left|\begin{array}{ll}
\alpha_{k i} & \gamma_{k i} \\
\beta_{j i} & \delta_{j i}
\end{array}\right|=0, \\
\sum_{i=1}^{m}\left|\begin{array}{ll}
\alpha_{k i} & \gamma_{k i} \\
\alpha_{j i} & \gamma_{j i}
\end{array}\right|=0, \quad \sum_{i=1}^{m}\left|\begin{array}{ll}
\beta_{k i} & \delta_{k i} \\
\beta_{j i} & \delta_{j i}
\end{array}\right|=0 .
\end{aligned}
$$


112. Since the conditions 76 ) and 78) will be used repeatedly in this and the succeeding chapters, it will be found to be of great assistance to apply the following scheme by which these conditions can be read off by inspection from the matrix of the coefficients of $S$ :

\begin{tabular}{|cc|cc|ccc|cc|}
\hline$\alpha_{11}$ & $\gamma_{11}$ & $\alpha_{12}$ & $\gamma_{12}$ &. &. &. & $\alpha_{1 m}$ & $\gamma_{1 m}$ \\
$\beta_{11}$ & $\delta_{11}$ & $\beta_{12}$ & $\delta_{12}$ &. &. &. & $\beta_{1 m}$ & $\delta_{1 m}$ \\
\hline$\alpha_{21}$ & $\gamma_{21}$ & $\alpha_{22}$ & $\gamma_{22}$ & $\cdot$ &. & $\cdot$ & $\alpha_{2 m}$ & $\gamma_{2 m}$ \\
$\beta_{21}$ & $\delta_{21}$ & $\beta_{22}$ & $\delta_{22}$ & $\cdot$ & $\cdot$ & $\cdot$ & $\beta_{2 m}$ & $\delta_{2 m}$ \\
\hline$\cdot$ & $\cdot$ & $\cdot$ & $\cdot$ & $\cdot$ & $\cdot$ & $\cdot$ & $\cdot$ \\
$\cdot$ & $\cdot$ & $\cdot$ & $\cdot$ & $\cdot$ & $\cdot$ & $\cdot$ &. \\
$\cdot$ & $\cdot$ & $\cdot$ & $\cdot$ & $\cdot$ & $\cdot$ & $\cdot$ & $\cdot$ \\
\hline$\alpha_{m 1}$ & $\gamma_{m 1}$ & $\alpha_{m 2}$ & $\gamma_{m 2}$ & $\cdot$ & $\cdot$ & $\cdot$ & $\alpha_{m m}$ & $\gamma_{m m}$ \\
$\beta_{m 1}$ & $\delta_{m 1}$ & $\beta_{m 2}$ & $\delta_{m 2}$ & $\cdot$ & $\cdot$ & $\cdot$ & $\beta_{m m}$ & $\delta_{m m}$ \\
\hline
\end{tabular}

The $1^{\text {st }}$ and $2^{\text {nd }}$ rows of this matrix will be called complementary, likewise the $3^{\text {rd }}$ and $4^{\text {th }}$ rows,$\ldots$, finally the $2 m-1^{\text {st }}$ and the $2 m^{\text {th }}$ rows. Similarly, the $1^{\text {st }}$ and $2^{\text {nd }}$ columns will be called complementary, also the $3^{\text {rd }}$ and $4^{\text {th }}, \ldots$, finally, the $2 m-1^{\text {st }}$ and $2 m^{\text {th }}$ columns.

The left member of each of the relations 78) is a sum of determinants built from the coefficients of two rows, the elements of each individual determinant belonging to complementary columns. If the two rows be the $s^{\text {th }}$ and $t^{\text {th }}$, we denote this sum by $R_{3 t}$. The relations 78) may then be written (taking $s<t$ )

$$
R_{2 l-1,2 l}=\mu, \quad R_{s t}=0 \quad \text { (unless } t=s+1=\text { even). }
$$

Similarly, if we denote by $C_{s t}$ the sum of the determinants built from the coefficients of the $s^{\text {th }}$ and $t^{\text {th }}$ columns, the elements of each individual determinant belonging to complementary rows; we may write the relations 76 ) in the compact form

$$
C_{2 l-12 l}=\mu, \quad C_{s t}=0 \quad \text { (unless } t=s+1=\text { even). }
$$

113. Theorem. - The factors of composition of $G A\left(2 m, p^{n}\right)$ are the prime factors of $p^{n}-1$ together with the factors of composition of $S A\left(2 m, p^{n}\right)$.

Let $\varrho$ be a primitive root of the $G F\left[p^{n}\right]$. The general Abelian group contains the substitution

$$
U: \quad \xi_{i}^{\prime}=\varrho \xi_{i}, \quad \eta_{i}^{\prime}=\eta_{i} \quad(i=1,2, \ldots, m)
$$

which multiplies $\varphi$ by $\varrho$. Let $S$ be any Abelian substitution and $\boldsymbol{\mu} \equiv \boldsymbol{\rho}^{r}$ the factor by which it multiplies $\boldsymbol{\varphi}$. We have 


$$
S=U^{r} T
$$

where $T$ is a new Abelian substitution not altering $\varphi$ and hence in the special Abelian group. Since $r$ may be any one of the integers $1,2, \ldots, p^{n}-1$, the order of $G A\left(2 m, p^{n}\right)$ is $p^{n}-1$ times the order $S A\left[2 m, p^{n}\right]$ of the group $S A\left(2 m, p^{n}\right)$.

Let $\alpha, \beta, \ldots$ be the prime factors whose product gives $p^{n}-1$. Let $A, A_{\alpha}, A_{\alpha \beta}, \ldots, A_{p^{n}-1} \equiv S A\left(2 m, p^{n}\right)$ be the groups formed by the combination of the substitutions of $S A\left(2 m, p^{n}\right)$ with

$$
U, \quad U^{\alpha}, \quad U^{\alpha \beta}, \ldots, U^{p^{n}-1} \equiv I
$$

respectively. Evidently these groups have the respective orders

$$
\begin{gathered}
\left(p^{n}-1\right) S A\left[2 m, p^{n}\right], \frac{1}{\alpha}\left(p^{n}-1\right) S A\left[2 m, p^{n}\right], \\
\frac{1}{\alpha \beta}\left(p^{n}-1\right) S A\left[2 m, p^{n}\right], \ldots, S A\left[2 m, p^{n}\right],
\end{gathered}
$$

while each is self-conjugate under $A \equiv G A\left(2 m, p^{n}\right)$.

114. Theorem. - The group $S A\left(2 m, p^{n}\right)$ is generated by the substitutions ${ }^{1}$ )

$$
\begin{array}{rll}
M_{i}: & \xi_{i}^{\prime}=\eta_{i}, & \eta_{i}^{\prime}=-\xi_{i} ; \\
L_{i, \lambda}: & \xi_{i}^{\prime}=\xi_{i}+\lambda \eta_{i} ; & \\
N_{i, j, \lambda}: & \xi_{i}^{\prime}=\xi_{i}+\lambda \eta_{j}, & \xi_{j}^{\prime}=\xi_{j}+\lambda \eta_{i},
\end{array}
$$

where $i, j=1,2, \ldots, m ; i \neq j$; and where $\lambda$ is an arbitrary mark of the $G F\left[p^{n}\right]$. Every substitution of the group has determinant unity.

From these substitutions leaving $\varphi$ absolutely invariant, we obtain other simple substitutions of $S A\left(2 m, p^{n}\right) \equiv G$ as follows:

$$
\begin{array}{rlrl}
L_{i, \lambda}^{\prime} & \equiv M_{i}^{-1} L_{i,-\lambda} M_{i}: & & \eta_{i}^{\prime}=\eta_{i}+\lambda \xi_{i} ; \\
Q_{i, j, \lambda} & \equiv M_{j} N_{i, j,-\lambda} M_{j}^{-1}: & & \xi_{i}^{\prime}=\xi_{i}+\lambda \xi_{j}, \quad \eta_{j}^{\prime}=\eta_{j}-\lambda \eta_{i} ; \\
R_{i, j, \lambda} & \equiv M_{j}^{-1} Q_{j, i, \lambda} M_{j}: & & \eta_{i}^{\prime}=\eta_{i}-\lambda \xi_{j}, \quad \eta_{j}^{\prime}=\eta_{j}-\lambda \xi_{i} ; \\
T_{i, \lambda} & \equiv L_{i, \lambda}^{\prime} L_{i,-\lambda-1} L_{i, \lambda}^{\prime} M_{i}: & \xi_{i}^{\prime}=\lambda \xi_{i}, \quad \eta_{i}^{\prime}=\lambda-1 \eta_{i} ; \\
P_{i j} \equiv Q_{j, i, 1}^{-1} Q_{i, j, 1} Q_{j, i, 1}=\left(\xi_{i} \xi_{j}\right)\left(\eta_{i} \eta_{j}\right) . &
\end{array}
$$

Let $S$ be any substitution of $G$ and let it replace $\xi_{1}$ by

$$
\omega_{1} \equiv \sum_{j=1}^{m}\left(\alpha_{1 j} \xi_{j}+\gamma_{1 j} \eta_{j}\right) \quad\left[\alpha_{1 j}, \gamma_{1 j} \text { not all zero }\right] \text {. }
$$

We can set $S=V S^{\prime}$, where $V$ is derived from the above substitutions and $S^{\prime}$ is a substitution of $G$ in which the coefficient corresponding

1) In the expression for each substitution we omit the indices not altered. For example, $M_{i}$ alters only the two indices $\eta_{i}$ and $\xi_{i}$. 
to $\alpha_{11}$ in $S$ is not zero. Indeed, according as $\alpha_{1 j} \neq 0$ or $\gamma_{1 j} \neq 0$, we may take $V=P_{1 j}$ or $P_{1 j} M_{j}$. Let $S^{\prime}$ replace $\xi_{1}$ by

$$
\boldsymbol{\omega}_{1}^{\prime} \equiv \sum_{j=1}^{m}\left(\alpha_{1 j}^{\prime} \xi_{j}+\gamma_{1 j}^{\prime} \eta_{j}\right) \quad\left[\alpha_{11}^{\prime} \neq 0\right] .
$$

We can determine a substitution $S_{1}$ derived from the above types which shall replace $\xi_{1}$ by $\omega_{1}^{\prime}$, viz.,

$$
S_{1}=L_{1, \beta} M_{1} L_{1, \alpha} Q_{1,2, \alpha_{12}^{\prime}} N_{1,2, \gamma_{12}} \ldots Q_{1, m, \alpha} N_{1 m} N_{1, m, \gamma_{1 m}^{\prime},}
$$

where $\alpha$ and $\beta$ are determined by the conditions

$$
\alpha=-\alpha_{11}^{\prime}+\alpha_{12}^{\prime} \gamma_{12}^{\prime}+\cdots+\alpha_{1 m}^{\prime} \gamma_{1 m}^{\prime}, \quad 1+\beta \alpha_{11}^{\prime}=\gamma_{11}^{\prime} \text {. }
$$

Hence $S^{\prime}=S_{1} \dot{S}^{\prime \prime}$, where $S^{\prime \prime}$ is a new substitution of $G$ which leaves $\xi_{1}$ fixed. Let $S^{\prime \prime}$ replace $\eta_{1}$ by

$$
\omega_{2} \equiv \sum_{j=1}^{m}\left(\beta_{1 j} \xi_{j}+\delta_{1 j} \eta_{j}\right)
$$

For $\mu=1, \alpha_{11}=1, \gamma_{11}=\alpha_{12}=\gamma_{12}=\cdots=\alpha_{1 m}=\gamma_{1 m}=0$, the relation $R_{12}=\mu$ of 79 ) gives $\delta_{11}=1$ in the substitution $S^{\prime \prime}$. The substitution

$$
S_{2} \equiv L_{1, \tau}^{\prime} R_{1,2,-\beta_{12}} Q_{2,1,-\delta_{12}} \ldots R_{1, m_{1}-\beta_{1 m}} Q_{m, 1,-\delta_{1 m}}
$$

will replace $\eta_{1}$ by $\omega_{2}$ if we take

$$
\tau=\beta_{11}-\beta_{12} \delta_{12}-\beta_{13} \delta_{13}-\cdots-\beta_{1 m} \delta_{1 m} .
$$

Hence $S^{\prime \prime}=S_{2} S^{\prime \prime \prime}$, where $S^{\prime \prime \prime}$ is a new substitution of $G$ which leaves $\xi_{1}$ and $\eta_{1}$ unaltered and thus has the form

$$
S^{\prime \prime \prime}:\left\{\begin{array}{l}
\xi_{1}^{\prime}=\xi_{1}, \quad \xi_{i}^{\prime}=\sum_{j=1}^{m}\left(\alpha_{i j} \xi_{j}+\gamma_{i j} \eta_{j}\right) \\
\eta_{1}^{\prime}=\eta_{1}, \quad \eta_{i}^{\prime}=\sum_{j=1}^{m}\left(\beta_{i j} \xi_{j}+\delta_{i j} \eta_{j}\right)
\end{array} \quad(i=2,3, \ldots, m) .\right.
$$

Applying the following relations of set 79),

we find

$$
R_{1 t}=0, \quad R_{2 t}=0 \quad(t=3,4, \ldots, 2 m),
$$

$$
\alpha_{i 1}=\beta_{i 1}=\gamma_{i 1}=\delta_{i 1}=0 \quad(i=2,3, \ldots, m) .
$$

The relations between the coefficients $\alpha_{i j}, \gamma_{i j}, \beta_{i j}, \delta_{i j}(i, j=2, \ldots, m)$ of $S^{\prime \prime \prime}$ are seen to be precisely those holding for a special Abelian substitution on $m-1$ pairs of indices. Furthermore,

$$
S=V S^{\prime}=V S_{1} S^{\prime \prime}=V S_{1} S_{2} S^{\prime \prime \prime},
$$

where $V, S_{1}, S_{2}$ were derived from the types of substitutions given in the theorem.

After $m$ operations similar to that by which $S^{\prime \prime \prime}$ was derived from $S$, we reach a substitution which leaves fixed all the indices 
and is therefore the identity. Hence $S$ is a product of substitutions of the given types. Since the latter are all of determinant unity, so is also the general substitution $S$ of the group.

115. Theorem. - The order $S A\left[2 m, p^{n}\right]$ of the special Abelian group equals

$$
\left(p^{n(2 m)}-1\right) p^{n(2 m-1)}\left(p^{n(2 m-2)}-1\right) p^{n(2 m-3)} \ldots\left(p^{2 n}-1\right) p^{n} .
$$

There are $\left(p^{n}\right)^{2 m}-1$ sets of values of $\alpha_{1 j}, \gamma_{1 j}(j=1, \ldots, m)$, not all zero, which give distinct functions $\omega_{1}$. In the function $\omega_{2}, \delta_{11}=1$ while $\beta_{11}, \beta_{1 j}, \delta_{1 j}(j=2, \ldots, m)$ are arbitrary in the field. Hence $\omega_{2}$ may be chosen in $\left(p^{n}\right)^{2 m-1}$ ways. We have therefore the recursion formula

$$
S A\left[2 m, p^{n}\right]=\left(p^{n(2 m)}-1\right) p^{n(2 m-1)} \cdot S A\left[2 m-2, p^{n}\right] .
$$

116. Theorem. - For $p>2$, the factors of composition of $S A\left(2 m, p^{n}\right)$ are $\frac{1}{2} S A\left[2 m, p^{n}\right]$ and 2 , the case $p^{n}=3, m=1$ being exceptional. ${ }^{1}$ )

Every substitution of $G \equiv S A\left(2 m, p^{n}\right)$ is commutative with

$$
T \equiv T_{1,-1} T_{2,-1} \ldots T_{m,-1}: \xi_{i}^{\prime}=-\xi_{i}, \quad \eta_{i}^{\prime}=-\eta_{i} \quad(i=1, \ldots, m) .
$$

The group $K \equiv\{I, T\}$ of order 2 is therefore self-conjugate under $G$. In order to show that $K$ is the maximal self-conjugate subgroup of $G$, we prove that a self-conjugate subgroup $J$ of $G$, which contains $K$ without being identical with $K$, must coincide with $G$.

Let $S$, given by 75 ), be a substitution of $J$ not in $K$. Then $J$ contains the products

$$
S^{-1} \cdot L_{i, \lambda}^{-1} S L_{i, \lambda}, \quad S^{-1} \cdot L_{i, \lambda}^{\prime-1} S L_{i, \lambda}^{\prime} \quad(i=1, \ldots, m)
$$

where $\lambda$ is a fixed mark $\neq 0$. Suppose first that all of these products reduce to the identity. Then, for example, $S$ is commutative with both $L_{1, \lambda}$ and $L_{1, \lambda}^{\prime}$, so that, by the proof of Corollary I of $\S 100, S$ has the form

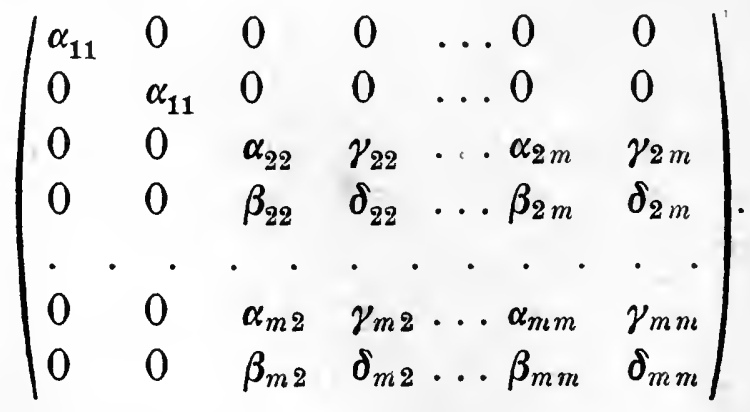

1) For $m=1, S A\left(2 m, p^{n}\right)$ is identical with the group of all binary linear homogeneous substitutions of determinant unity. Its factors of composition are therefore given by the theorem of $\S 103$. 
But $S$ is to be commutative with every pair $L_{i, \lambda}$ and $L_{i, \lambda}^{\prime}$. It follows that $S$ reduces to the form

$$
\bar{S}: \quad \xi_{i}^{\prime}=\alpha_{i i} \xi_{i}, \quad \eta_{i}^{\prime}=\alpha_{i i} \eta_{i} \quad(i=1,2, \ldots, m) .
$$

By the first type of Abelian conditions given under 79), we have $\alpha_{i i}= \pm 1$. Since $S$ is not in $K$, the $\alpha_{i i}$ are not all +1 and not all -1 . Transforming $\bar{S}$ by a suitable product of the form $P_{1 r} P_{2 s}$, we may suppose that $\alpha_{11}=1, \alpha_{22}=-1$ in $\bar{S}$. Then $J$ contains $N_{12, \mu}^{-1} \bar{S} N_{1,2, \mu}$, which replaces $\xi_{1}$ by $\xi_{1}-2 \mu \eta_{2}$ and is therefore (since $p \neq 2$ ) not of the form $\bar{S}$. Taking it in place of our initial substitution $S$, we are led to the case next considered.

Suppose that not all of the above products reduce to the identity $I$; for example, let

$$
S_{1} \equiv S^{-1} L_{1, \lambda}^{-1} S L_{1, \lambda} \neq I .
$$

If $S^{-1}$ replaces $\eta_{1}$ by the linear function $\omega / \lambda$, the product denoted by $S_{1}$ has the following form, in which the coefficients of $\xi_{1}$ have not been calculated:

$$
S_{1}:\left\{\begin{array}{lll}
\xi_{1}^{\prime}=\sum_{j=1}^{m}\left(\alpha_{j} \xi_{j}+\gamma_{j} \eta_{j}\right), & \xi_{i}^{\prime}=\xi_{i}-\alpha_{i 1} \omega & (i=2, \ldots, m), \\
\eta_{i}^{\prime}=\eta_{i}-\beta_{i 1} \omega & (i=1, \ldots, m) .
\end{array}\right.
$$

From $S_{1}$ we proceed to determine a substitution $\neq I$ belonging to $J$ and leaving $2 m-3$ indices unaltered. $S_{1}$ itself is such a substitution if $\alpha_{i 1}=\beta_{i 1}=0(i=2, \ldots, m)$. In the contrary case, the transformed of $S$ by a suitable $P_{2 j}$ or $P_{2 j} M_{2}$ will have $\alpha_{21} \neq 0$. Consider therefore $S_{1}$ when $\alpha_{21} \neq 0$, and introduce the new indices

$$
\begin{aligned}
& \bar{\xi}_{1}=\xi_{1}, \quad \bar{\eta}_{1}=\eta_{1}, \quad \bar{\xi}_{2}=\xi_{2}, \quad \bar{\eta}_{2}=-\frac{1}{\alpha_{21}} \sum_{j=2}^{m}\left(\beta_{j 1} \xi_{j}-\alpha_{j 1} \eta_{j}\right), \\
& \bar{\xi}_{i}=\xi_{i}-\frac{\alpha_{i 1}}{\alpha_{21}} \xi_{2}, \quad \bar{\eta}_{i}=\eta_{i}-\frac{\beta_{i 1}}{\alpha_{21}} \xi_{2} \quad(i=3, \ldots, m),
\end{aligned}
$$

an operation equivalent to the transformation of $S_{1}$ by the following product $T$ belonging to the group $G$ :

where

$$
Q_{3,2,-\alpha_{31} / \alpha_{21}} R_{3,2, \beta_{31} / \alpha_{21}} \ldots Q_{m, 2,-\alpha_{n 1} / \alpha_{21}} R_{m, 2, \beta_{m 1} / \alpha_{21}} L_{2, \sigma}^{\prime}
$$

$$
\sigma \equiv-\sum_{j=2}^{m} \frac{\alpha_{j 1}}{a_{21}} \frac{\beta_{j 1}}{\alpha_{21}}
$$

We obtain the substitution $S_{2} \equiv T^{-1} S_{1} T$, leaving fixed $2 m-3$ indices, viz.,

$$
\begin{aligned}
& \bar{\xi}_{i}^{\prime}=\left(\xi_{i}-\alpha_{i 1} \omega\right)-\frac{\alpha_{i 1}}{\alpha_{21}}\left(\xi_{2}-\alpha_{21} \omega\right) \equiv \bar{\xi}_{i}, \quad \bar{\eta}_{i}^{\prime}=\bar{\eta}_{i} \quad(i=3, \ldots, m) \\
& \vec{\eta}_{2}^{\prime}=-\frac{1}{\alpha_{21}} \sum_{j=2}^{m}\left\{\beta_{j 1}\left(\xi_{j}-\alpha_{j 1} \omega\right)-\alpha_{j 1}\left(\eta_{j}-\beta_{j 1} \omega\right)\right] \equiv \bar{\eta}_{2} .
\end{aligned}
$$


Writing $\xi_{i}, \eta_{i}$ for $\bar{\xi}_{i}, \bar{\eta}_{i}$ in $S_{2}$, and applying conditions 79$)$, viz., $R_{34}=1, \quad R_{14}=R_{24}=0, \quad R_{1 j}=R_{2 j}=R_{3 j}=0 \quad(j=5,6, \ldots, 2 m)$, we find that $S_{2}$ takes the form

$$
S_{2}:\left\{\begin{array}{l}
\xi_{1}^{\prime}=\alpha_{11} \xi_{1}+\gamma_{11} \eta_{1}+\gamma_{12} \eta_{2}, \quad \eta_{1}^{\prime}=\beta_{11} \xi_{1}+\delta_{11} \eta_{1}+\delta_{12} \eta_{2}, \\
\xi_{2}^{\prime}=\alpha_{21} \xi_{1}+\gamma_{21} \eta_{1}+\xi_{2}+\gamma_{22} \eta_{2}, \quad \eta_{2}^{\prime}=\eta_{2},
\end{array}\right.
$$

the indices $\xi_{i}, \eta_{i}(i=3, \ldots, m)$, not being altered by $S_{2}$ and the substitutions below, are not written in the formulae.

The group $J$ contains the product

$$
S_{3} \equiv S_{2}^{-1} \cdot N_{1,2, \mu}^{-1} S_{2} N_{1,2, \mu}: \begin{cases}\xi_{1}^{\prime}=\xi_{1}+\mu\left(1-\alpha_{11}\right) \eta_{2}, & \xi_{2}^{\prime}=\xi_{2}+\Phi, \\ \eta_{1}^{\prime}=\eta_{1}-\mu \beta_{11} \eta_{2}, & \eta_{2}^{\prime}=\eta_{2},\end{cases}
$$

where $\Phi$ is a linear function of $\xi_{1}, \eta_{1}, \eta_{2}$.

a) Suppose first that $S_{3}$ is not the identity. If $1-\alpha_{11} \neq 0$, we may define $\tau$ by the equation

$$
\left(1-\alpha_{11}\right) \tau .=\beta_{11} \text {. }
$$

Then $J$ contains $S_{4} \equiv L_{1, \tau}^{\prime}{ }^{-1} S_{3} L_{1, \tau}^{\prime}$, which has the form

$$
\begin{array}{ll}
\xi_{1}^{\prime}=\xi_{1}+\gamma_{12} \eta_{2}, & \eta_{1}^{\prime}=\eta_{1}, \\
\xi_{2}^{\prime}=\xi_{2}+\alpha_{21} \xi_{1}+\gamma_{21} \eta_{1}+\gamma_{22} \eta_{2}, & \eta_{2}^{\prime}=\eta_{2} .
\end{array}
$$

Applying the conditions $R_{13}=R_{23}=0$ of 79), we find that $\gamma_{12}=\gamma_{21}$, $\alpha_{21}=0$, so that $S_{4}$ has the following form (with $\alpha \neq 0$ ):

$$
\begin{cases}\xi_{1}^{\prime}=\xi_{1}+\alpha \eta_{2}, & \eta_{1}^{\prime}=\eta_{1}, \\ \xi_{2}^{\prime}=\xi_{2}+\alpha \eta_{1}+\beta \eta_{2}, & \eta_{2}^{\prime}=\eta_{2} .\end{cases}
$$

If, on the contrary, $1-\alpha_{11}=0, J$ will contain $M_{1}^{-1} S_{3} M_{1}$, which is not the identity and has the form 81 ). In either case, $J$ contains a substitution 81) in which $\alpha$ and $\beta$ are not both zero.

If $\alpha=0, \beta \neq 0,81$ ) is of the form $L_{2, \beta} \neq I$. If $\alpha \neq 0$, $J$ contains the transforned of 81 ) by $Q_{2,1, \lambda}$, giving the substitution

$$
\begin{array}{ll}
\xi_{1}^{\prime}=\xi_{1}+\alpha \eta_{2}, & \eta_{1}^{\prime}=\eta_{1}, \\
\xi_{2}^{\prime}=\xi_{2}+\alpha \eta_{1}+(\beta+2 \alpha \lambda) \eta_{2}, & \eta_{2}^{\prime}=\eta_{2} .
\end{array}
$$

Taking $\lambda=-\beta / 2 \alpha$, this becomes $N_{1,2, \alpha}$. Then $J$ contains

82) $L_{1,-\alpha^{2}} \equiv N_{1,2, \alpha} \cdot M_{2}^{-1} N_{1,2, \alpha}^{-1} M_{2}\left(M_{2} L_{2,1}\right)^{-1} N_{1,2, \alpha}\left(M_{2} L_{2,1}\right)$.

Transforming by $P_{12}$, we reach $L_{2,-\alpha^{2}}$. In either case, $J$ contains a substitution of the form $L_{2, \lambda}(\lambda \neq 0)$.

We next prove that $J$ contains all the generators $L_{i, \mu}, M_{i}$ and $N_{i, j, \mu}$ of the group $G$. Having $L_{2, \lambda}, J$ contains the product

$$
T_{2, \tau}^{-1} L_{2, \lambda} T_{2, \tau} \equiv L_{2, \lambda \tau^{2}} \quad(\tau \text { any mark } \neq 0) \text {. }
$$




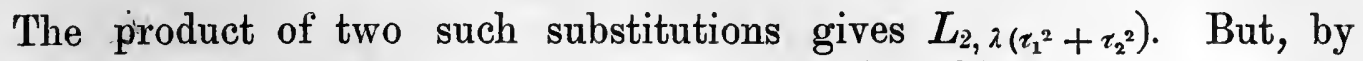
$\S 64$, marks $\tau_{1}$ and $\tau_{2}$ can be found in the $G F\left[p^{n}\right], p>2$, such that $\tau_{1}^{2}+\tau_{2}^{2}$ has an arbitrary value $\mu$ in the field. Hence $J$ contains $L_{2, \mu}$. Then $I$ contains the product

$$
M_{2} \equiv L_{2,1} \cdot M_{2}^{-1} L_{-, 1} M_{2} \cdot L_{2,1} .
$$

Hence $J$ contains $L_{i, \mu}$ and $M_{i}$, the transformed of $L_{2, \mu}$ and $M_{2}$ respectively by $P_{2 i}$. Finally, $J$ contains ${ }^{1}$ )

$$
N_{i, j, \mu} \equiv Q_{i, j, 1}^{-1} L_{i, \mu} L_{j, \mu} Q_{i, j, 1} L_{i, \mu}^{-2} L_{j, \mu}^{-1} .
$$

b) Suppose, however, that $S_{3} \equiv I$. Then $S_{2}$ is commutative with $N_{1,2, \mu}$, so that

$$
\alpha_{11}=1, \quad \beta_{11}=0, \quad \delta_{11}=1, \quad \delta_{12}=\alpha_{21} .
$$

Applying the Abelian conditions $R_{13}=R_{23}=0$, we find that $\delta_{12}=0$, $\gamma_{12}=\gamma_{21}$, so that $S_{2}$ becomes

$$
S_{2}: \quad \begin{cases}\xi_{1}^{\prime}=\xi_{1}+\gamma_{11} \eta_{1}+\gamma_{12} \eta_{2}, & \eta_{1}^{\prime}=\eta_{1}, \\ \xi_{2}^{\prime}=\xi_{2}+\gamma_{12} \eta_{1}+\gamma_{22} \eta_{2}, & \eta_{2}^{\prime}=\eta_{2} .\end{cases}
$$

$S_{2}$ is not the identity since $S_{1}$ is not. If $\gamma_{11}=0, S_{2}$ is of the form 81) considered under case a). If $\gamma_{11} \neq 0, J$ contains $S_{2}^{\prime}$, the transformed of $S_{2}$ by $Q_{2,1, \lambda}$, where $\lambda \equiv-\gamma_{12} / \gamma_{11}$, viz.,

$$
S_{2}^{\prime}:\left\{\begin{array}{ll}
\xi_{1}^{\prime}=\xi_{1}+\gamma_{11} \eta_{1}, & \eta_{1}^{\prime}=\eta_{1}, \\
\xi_{2}^{\prime}=\xi_{2}+\delta \eta_{2}, & \eta_{2}^{\prime}=\eta_{2} .
\end{array} \quad\left[\delta \equiv \gamma_{22}+\lambda \gamma_{12}\right]\right.
$$

For $\delta=0, S_{2}^{\prime} \equiv L_{1, \gamma_{11}}$. For $\delta \neq 0, J$ contains the transformed of $S_{2}^{\prime}$ by $T_{1, \lambda} T_{2, \mu}, \lambda$ and $\mu$ being arbitrary marks $\neq 0$,' giving the substitution

$$
\begin{array}{ll}
\xi_{1}^{\prime}=\xi_{1}+\lambda^{2} \gamma_{11} \eta_{1}, & \eta_{1}^{\prime}=\eta_{1}, \\
\xi_{2}^{\prime}=\xi_{2}+\mu^{2} \delta \eta_{2}, & \eta_{2}^{\prime}=\eta_{2} .
\end{array}
$$

Forming the product of two such substitutions and noting that, for $p>2$, the equation $\lambda_{1}^{2}+\lambda_{2}^{2}=\varkappa$ has solutions in the $G F\left[p^{n}\right]$ for $\varkappa$ an arbitrary mark $\neq 0$ of the field, we find that $J$ contains

$$
L_{1, \alpha} L_{2, \beta}: \quad \xi_{1}^{\prime}=\xi_{1}+\alpha \eta_{1}, \quad \xi_{2}^{\prime}=\xi_{2}+\beta \eta_{2},
$$

where $\alpha$ and $\beta$ are arbitrary marks $\neq 0$. A suitable product of two such substitutions gives

$$
L_{1, \alpha} L_{2, \beta} \cdot L_{1, \alpha} L_{2,-\beta} \equiv L_{1,2 \alpha} .
$$

In every case we reach in $J$ a substitution $L_{1, \lambda}$, where $\lambda \neq 0$, and therefore also $L_{2, \lambda}$. It follows as in case a) that $J \equiv G$.

11\%. Theorem. - For $p=2, S A\left(2 m, p^{n}\right)$ is simple except when $m=2, p^{n}=2$, and when $m=1, p^{n}=2$.

1) We might reach $N_{1,2, \alpha}$ by 82 ) and then obtain $N_{i, j, \mu}$ in the group $J$. 
For $p=2$, a substitution $S$ of $G \equiv S A\left(2 m, p^{n}\right)$ is commutative with every $L_{i, \lambda}$ and every $\dot{L}_{i, \lambda}^{\prime}$ only when $S$ is the identity. Proceeding as in $\S 116$, we find that a self-conjugate subgroup $J$ of $G$, which contains a substitution $S \neq I$, will contain either a substitution of the form 81) with $\alpha$ and $\beta$ not both zero or else a substitution $S_{2}^{\prime}$ of the form 84) in which $\gamma_{11} \neq 0$.

We next prove that $J$ contains either $L_{1, \lambda}(\lambda \neq 0)$ or else $N_{1,2,1} L_{2,1}$. For $\delta=0, S_{2}^{\prime}=L_{1, \gamma_{11}}$. For $\delta \neq 0$, we transform $S_{2}^{\prime}$ by a suitable $T_{1, \lambda} T_{2, \mu}$ and obtain the substitution $L_{1,1} L_{2,1}$. Hence $J$ contains $^{1}$ )

$$
Q_{1,2,1}^{-1} L_{1,1} L_{2,1}^{\prime} Q_{1,2,1} \equiv \dot{N}_{1,2,1} L_{2,1} \text {. }
$$

For $\alpha=0,81$ ) becomes $L_{2, \beta}$, so that we reach $L_{1, \beta}$ in $J$. If $\beta=0,81$ ) becomes $N_{1,2, \alpha}$, so that, by 82 ), $J$ contains $L_{1,-\alpha^{2}}$. Finally, if $\alpha \neq 0, \beta \neq 0$, the transformed of 81 ) by $T_{1, \lambda} T_{2, \mu}$ gives the substitution

$$
\xi_{1}^{\prime}=\xi_{1}+\alpha \lambda \mu \eta_{2}, \quad \xi_{2}^{\prime}=\xi_{2}+\alpha \lambda \mu \eta_{1}+\beta \mu^{2} \eta_{2} .
$$

In the $G F\left[2^{n}\right]$, we may take

$$
\mu=\beta^{-1 / 2}, \quad \lambda=\alpha^{-1} \mu^{-1},
$$

when the last substitution becomes $N_{1,2,1} L_{2,1}$.

Having a substitution $L_{1, \lambda}(\lambda \neq 0), J$ will coincide with $G$.

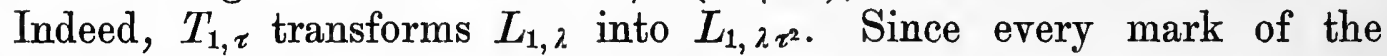
field is a square, we reach $L_{1, \sigma}$, $\sigma$ arbitrary. Then, as at the end of case a) of $\S 116, J$ contains every $L_{i, \sigma}, M_{i}, N_{i, j, \sigma}$ and hence coincides with $G$.

There remains the case in which $J$ contains $N_{1,2,1} L_{2,1}$. Then $J$ will contain all the products, two at a time, of the substitutions

$$
L_{i, 1}, \quad M_{i}, \quad N_{i, j, 1} \quad(i, j=1,2, \ldots, m ; i \neq j) .
$$

Indeed, if $i$ and $j$ be any two distinct integers $\overline{\overline{<}} m, J$ contains

$$
\begin{aligned}
& \left(P_{1 i} P_{2 j}\right)^{-1} N_{1,2,1} L_{2,1}\left(P_{1 i} P_{2 j}\right)=N_{i, j, 1} L_{i, 1}=L_{i, 1} N_{i, j, 1}, \\
& L_{i, 1} N_{i, j, 1} \cdot L_{j, 1} N_{i, j, 1}=L_{i, 1} L_{j, 1}, \\
& M_{i}^{-1} L_{i, 1} L_{j, 1} M_{i} \cdot L_{i, 1} L_{j, 1}=L_{i, 1} M_{i}, \quad\left(L_{i, 1} M_{i}\right)^{2}=M_{i} L_{i, 1}, \\
& L_{i, 1} L_{j, 1} \cdot L_{i, 1} M_{i}=L_{j, 1} M_{i}=M_{i} L_{j, 1}, \quad M_{i} L_{j, 1} \cdot L_{j, 1} M_{j}=M_{i} M_{j} .
\end{aligned}
$$

Our statement is therefore proved if $m=2$. If $m>2$, let $i, j, k$ be any three distinct integers $\overline{\overline{<}} m$. Then $J$. contains

$$
\begin{aligned}
& N_{i, j, 1} L_{i, 1} \cdot L_{i, 1} L_{k, 1}=N_{i, j, 1} L_{k, 1}=L_{k, 1} N_{i, j, 1}, \\
& N_{i, j, 1} L_{i, 1} \cdot L_{i, 1} M_{k}=N_{i, j, 1} M_{k}=M_{k} N_{i, j, 1} .
\end{aligned}
$$

1) This relation follows from 83 , if $p=2$, by taking $i=1, j=2, \mu=1$. 
We next prove that, for $m>2, J$ contains $L_{1,1}$. Since, for $p=2$,

$$
L_{i, 1}^{\prime}=M_{i} L_{i, 1} M_{i}, \quad R_{i, j, 1}=M_{i} M_{j} N_{i, j, 1} M_{i} M_{j},
$$

it follows that $J$ will contain the substitution

$$
D \equiv L_{1,1}^{\prime} L_{2,1}^{\prime} L_{3,1}^{\prime} R_{1,2,1} R_{2,3,1} R_{3,1,1}
$$

the latter being the product of an even number 24 of the substitutions 85). This product is seen to be

$$
D: \quad \xi_{i}^{\prime}=\xi_{i}, \quad \eta_{i}^{\prime}=\eta_{i}+\xi_{1}+\xi_{2}+\xi_{3} \quad(i=1,2,3) .
$$

But $D$ is transformed into $L_{1,1}$ by the following Abelian substitution of period two:

$$
\begin{array}{ll}
\xi_{1}^{\prime}=\eta_{1}+\xi_{2}+\xi_{3}, & \eta_{1}^{\prime}=\xi_{1}+\xi_{2}+\xi_{3}, \\
\xi_{2}^{\prime}=\xi_{2}, & \eta_{2}^{\prime}=\xi_{1}+\eta_{1}+\xi_{2}+\eta_{2}+\xi_{3}, \\
\xi_{3}^{\prime}=\xi_{3}, & \eta_{3}^{\prime}=\xi_{1}+\eta_{1}+\xi_{2}+\xi_{3}+\eta_{3} .
\end{array}
$$

Hence $J$ contains $L_{1,1}$ and therefore also

$$
\begin{aligned}
& L_{i, 1} L_{1,1} \cdot L_{1,1}=L_{i, 1} \quad T_{i, \tau}^{-1} L_{i, 1} T_{i, \tau}=L_{i, \tau^{2}}, \quad M_{i} L_{1,1} \cdot L_{1,1}=M_{i}, \\
& N_{i, j, 1} L_{1,1} \cdot L_{1,1}=N_{i, j, 1}, \quad T_{i, \tau}^{-1} N_{i, j, 1} T_{i, \tau}=N_{i, j, \tau} .
\end{aligned}
$$

Hence, for $p=2, m>2, J$ is identical with $G$, so that $G$ is simple.

For $p=2, m=2, J$ contains $M_{1} M_{2}$ as above, and therefore also

$$
M_{1} M_{2} \cdot T_{1, \tau}^{-1} M_{1} M_{2} T_{1, \tau} \equiv T_{1, \tau^{2}} \text {. }
$$

Hence $J$ contains every $T_{1, \alpha}$. But $R_{1,2, \lambda}$ transforms $T_{1, \alpha}$ into $R_{1,2, \lambda(1+\alpha)} T_{1, \alpha}$. If $n>1$, the $G F\left[2^{n}\right]$ contains a mark $\alpha$ neither zero nor unity, so that $1+\alpha \neq 0, \alpha \neq 0$. Hence, for $n>1$, the

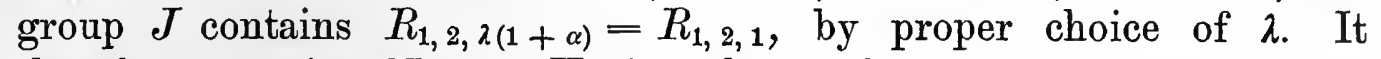
therefore contains $N_{1,2,1}$. Having the products in pairs of the substitutions 85 ), $J$ contains $M_{i}$ and $L_{i, 1}$. Thus $J \equiv G$.

The fact that the case $m=2, p=2, n=1$ is exceptional is shown in the following section.

118. Theorem. - The Abelian group $S A(4,2)$ on four indices modulo 2 is holoedrically isomorphic with the symmetric group on six letters..$\left.^{1}\right)$

By $\S 264$ of Chapter XIII, the symmetric group on 6 letters is holoedrically isomorphic with the abstract group $G_{61}$ generated by $B_{1}, B_{2}, B_{3}, B_{4}, B_{5}$ subject to the generational relations

$$
\begin{aligned}
B_{1}^{2} & =B_{2}^{2}=B_{3}^{2}=B_{4}^{2}=B_{5}^{2}=I, \\
\left(B_{1} B_{2}\right)^{3} & =\left(B_{2} B_{3}\right)^{3}=\left(B_{3} B_{4}\right)^{3}=\left(B_{4} B_{5}\right)^{3}=I, \\
\left(B_{1} B_{3}\right)^{2} & =\left(B_{1} B_{4}\right)^{2}=\left(B_{1} B_{5}\right)^{2}=\left(B_{2} B_{4}\right)^{2}=\left(B_{2} B_{5}\right)^{2}=\left(B_{3} B_{5}\right)^{2}=I .
\end{aligned}
$$

1) This theorem was first proved by Jordan by means of the groups of Steiner, Traité, No. 335. The proof given in the text is due to the author, Proc. Lond. Math. Soc., vol. 31, pp. 40-41. 
To the operators $B_{i}$ we make correspond the following substitutions of $S A(4,2)$ :

86) $\quad B_{1} \sim M_{1}, \quad B_{2} \sim L_{1,1}, \quad B_{3} \sim S, \quad B_{1} \sim L_{2,1}, \quad B_{5} \sim M_{2}$, where $S$ denotes the Abelian substitution of period two:

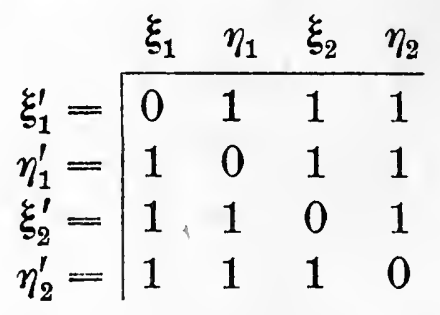

We readily verify that the relations corresponding to the above generational relations are satisfied in virtue of the correspondences 86 ). Since $S A(4,2)$ has the order

$$
\left(2^{4}-1\right) 2^{3}\left(2^{2}-1\right) 2 \equiv 6 !
$$

the isomorphism between $S A(4,2)$ and $G_{6 !}$ is holoedric.

119. In determining the factors of composition of the general and special Abelian groups on $2 m$ indices with coefficients in the $G F\left[p^{n}\right]$, we have been led to a quotient-group, $S A\left(2 m, p^{n}\right) / K$, where $K \equiv\{I, T\}$ is of order 1 or 2 according as $p=2$ or $p>2$. Owing to the great importance of simple groups, we will designate this quotient-group as $A\left(2 m, p^{n}\right)$, it being a simple group except in the three cases $m=1, p^{n}=2 ; m=1, p^{n}=3 ; m=2, p^{n}=2$, when its factors of composition are 2,$3 ; 2,2,3 ; 2, \frac{1}{2} 6$ !, respectively. The order $A\left[2 m, p^{n}\right]$ of $A\left(2 m, p^{n}\right)$ is

$$
\frac{1}{a}\left(p^{n(2 m)}-1\right) p^{n(2 m-1)}\left(p^{n(2 m-2)}-1\right) p^{n(2 m-3)} \ldots\left(p^{2 n}-1\right) p^{n},
$$

where $a=1$ or 2 according as $p=2$ or $p>2$.

Conjugacy of operators of period two $\left.{ }^{1}\right)$ in $S A\left(2 m, p^{n}\right)$ and $A\left(2 m, p^{n}\right)$.

120. Theorem. - Within the special Abelian group $S A\left(2 m, p^{n}\right)$ any substitution $S$ defined by 75 ) is conjugate with a substitution $\Sigma$ which replaces $\xi_{1}$ and $\eta_{1}$ by the respective functions

$$
\alpha_{11} \xi_{1}+\gamma_{11} \eta_{1}+\alpha_{1 m} \xi_{m}, \quad \beta_{11} \xi_{1}+\delta_{11} \eta_{1}+\delta_{1 m-1} \eta_{m-1}+\delta_{1 m} \dot{\eta}_{m},
$$

where either $\delta_{1 m-1}=0$ or else $\delta_{1 m}=0$.

The theorem is evident if $\alpha_{1 i}=\gamma_{1 i}=\beta_{1 i}=\delta_{1 i}=0(i=2, \ldots, m)$. In the contrary case, we may suppose that $\alpha_{1 m}, \gamma_{1 m}, \beta_{1 m}, \delta_{1 m}$ are not all zero, first transforming $S$ by $P_{i m}$, where $i$ is a certain one

1) Taken from the author's article, Quarterly Journal, vol. 32, pp. 42-63. 
of the integers $2,3, \ldots, m$. According as $\alpha_{1 m} \neq 0, \gamma_{1 m} \neq 0, \beta_{1 m} \neq 0$ or $\delta_{1 m} \neq 0$, we transform $S$ by $I, M_{m}, M_{1}$, or $M_{1} M_{m}$ respectively and obtain a substitution $S^{\prime}$ in which $\alpha_{1 m} \neq 0$. Transforming $S^{\prime}$ by $L_{m, \lambda}$, we obtain a substitution $S^{\prime \prime}$ which replaces $\xi_{1}$ by

$$
\alpha_{11} \xi_{1}+\gamma_{11} \eta_{1}+\cdots+\alpha_{1 m} \xi_{m}+\left(\gamma_{1 m}-\lambda \alpha_{1 m}\right) \eta_{m} \text {. }
$$

Since $\alpha_{1 m} \neq 0$, we can choose $\lambda$ in the field to make the coefficient of $\eta_{m}$ vanish. Transforming $S^{\prime \prime}$ (in which now $\alpha_{1 m} \neq 0, \gamma_{1 m}=0$ ) by $L_{1, \varrho}^{\prime}$, we reach, a substitution $S_{1}$ which replaces $\xi_{1}, \eta_{1}$ by respectively

$$
\begin{gathered}
\left(\alpha_{11}-\varrho \gamma_{11}\right) \xi_{1}+\gamma_{11} \eta_{1}+\cdots+\alpha_{1 m} \xi_{m}, \\
() \xi_{1}+() \eta_{1}+\cdots+\left(\beta_{1 m}+\varrho \alpha_{1 m}\right) \xi_{m}+\delta_{1 m} \eta_{m} .
\end{gathered}
$$

We choose $\varrho$ to make $\beta_{1 m}+\varrho \alpha_{1 m}=0$. Hence $S_{1}$ has $\alpha_{1 m} \neq 0$, $\gamma_{1 m}=\beta_{1 m}=0$.

We next determine an Abelian substitution which affects only the indices $\xi_{2}, \eta_{2}, \xi_{m}, \eta_{m}$ and which transforms $S_{1}$ into a substitution $S_{2}$ having $\alpha_{1 m} \neq 0, \gamma_{1 m}=\beta_{1 m}=\gamma_{12}=\beta_{12}=0$.

a) Let $\alpha_{12}=\gamma_{12}=0$. If $\delta_{12}=0$, the transformed of $S_{1}$ by $M_{2}$ gives $S_{2}$. If $\beta_{12}$ and $\delta_{12}$ are both not zero, we transform $S_{1}$ by $L_{2, \varrho}^{\prime}$, where $\beta_{12}-\varrho \delta_{12}=0$, and obtain $S_{2}$.

b) Let $\alpha_{12}$ and $\gamma_{12}$ be not both zero. Transforming by $M_{2}$ when $\gamma_{12} \neq 0$, we may suppose that $\alpha_{12} \neq 0$ in $S_{1}$. Transforming it by $L_{2, \varrho}$; we can make $\gamma_{12}=0$. If then $\delta_{12} \neq 0$, we transform by $L_{2, \varrho}^{\prime}$ and make $\beta_{12}=0$. Suppose, however, that $\delta_{12}=0$. If $\delta_{1 m} \neq 0$, we transform by $R_{2, m, \lambda}$, where $\beta_{12}+\lambda \delta_{1 n}=0$, and reach $S_{2}$. But if $\delta_{1 m}=0$, we have $S_{2}$ if $\beta_{12}=0$; while for $\beta_{12} \neq 0$, we transform. by $Q_{m, 2, \varrho} M_{2}$, where $\alpha_{12}-\varrho \alpha_{1 m}=0$, and reach $S_{2}$.

In an analogous manner, we can determine an Abelian substitution which affects only $\xi_{3}, \eta_{3}, \xi_{m}, \eta_{m}$ and which transforms $S_{2}$ into a substitution $S_{3}$ having

$$
\alpha_{1 m} \neq 0, \quad \gamma_{12}=\beta_{12}=\gamma_{13}=\beta_{13}=\gamma_{1 m}=\beta_{1 m}=0 .
$$

Repeating the process, we may also make

$$
\gamma_{14}=\beta_{14}=\cdots=\gamma_{1 m-1}=\beta_{1 m-1}=0 .
$$

We therefore reach a substitution $\bar{S}$ conjugate with $S$ within the special Abelian group and replacing $\xi_{1}, \eta_{1}$ by respectively

$$
\alpha_{11} \xi_{1}+\gamma_{11} \eta_{1}+\sum_{j=2}^{m} \alpha_{1 j} \xi_{j}, \quad \beta_{11} \xi_{1}+\sum_{j=1}^{m} \delta_{1 j} \eta_{j} \quad\left[\alpha_{1 m} \neq 0\right] .
$$

Transforming $\bar{S}$ by $Q_{m, 2, \sigma}$, where $\alpha_{12}-\sigma \alpha_{1 m}=0$, we obtain a substitution of the form $\bar{S}$ but having $\alpha_{12}=0$. Similarly, we may make $\alpha_{13}=\cdots=\alpha_{1 m-1}=0$. If, in the resulting substitution $\bar{S}_{1}$, 
$\delta_{12}=\cdots=\delta_{1 m}=0$, we have reached $\Sigma$. If $\delta_{1 m} \neq 0$, we transform $\bar{S}_{1}$ by $Q_{2, m, \sigma}$, where $\delta_{12}+\sigma \delta_{1 m}=0$, and reach a substitution of the form $\overline{S_{1}}$ but having also $\delta_{12}=0$. In a similar manner we make $\delta_{13}=\cdots=\delta_{1 m-1}=0$ and reach $\Sigma$. Finally, if $\delta_{1 m}=0$ but $\delta_{12}$, $\delta_{13}, \ldots, \delta_{1 m-1}$ are not all zero, we may suppose that $\delta_{1 m-1} \neq 0$, first transforming by some $P_{i m-1}$. We then transform it by $Q_{i, m-1, \varrho}$, for $i=2,3, \ldots, m-2$ in succession, and make

so that we reach $\Sigma$.

$$
\delta_{12}=\delta_{13}=\cdots=\delta_{1 m-2}=0,
$$

Corollary. - If $\alpha_{1 i}, \gamma_{1 i}, \beta_{1 i}, \delta_{1 i}(i=2, \ldots, m)$ are not all zero in $S$, it is conjugate within $S A\left(2 m, p^{n}\right)$ with one of the two types of substitutions:

$$
\begin{array}{lll}
\Sigma_{1}: & \xi_{1}^{\prime}=\alpha_{11} \xi_{1}+\gamma_{11} \eta_{1}+\xi_{m}, & \eta_{1}^{\prime}=\beta_{11} \xi_{1}+\delta_{11} \eta_{1}+\delta \eta_{m}, \ldots \\
\Sigma_{2}: & \xi_{1}^{\prime}=\alpha_{11} \xi_{1}+\gamma_{11} \eta_{1}+\xi_{m}, & \eta_{1}^{\prime}=\beta_{11} \xi_{1}+\delta_{11} \eta_{1}+\eta_{m-1}, \ldots
\end{array}
$$

Since the conjugate substitution $\Sigma$ then has $\alpha_{1 m} \neq 0$, we may transform it by $T_{m}, \alpha_{1 m}$. Then if $\delta_{1 m-1}=0$, we have $\Sigma_{1}$. In the contrary case, we transform also by $I_{m-1}^{-1}, \delta_{1 m-1}$ and get $\Sigma_{2}$.

121. Theorem. - The special Abelian group $S A\left(2 m, p^{n}\right), p>2$, contains exactly $m$ sets of conjugate substitutions of period 2 . The $r^{\text {th }}$ set includes

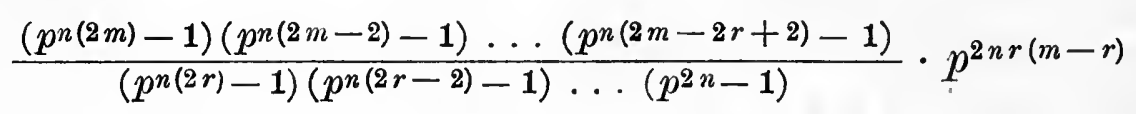

substitutions all conjugate with $T_{r} \equiv T_{1,-1} T_{2,-1} \ldots T_{r,-1}$.

In order that the special Abelian substitution 75) shall be identical with its reciprocal 77), for $\mu=1$, it is necessary and sufficient that

$$
\alpha_{i j}=\delta_{j i}, \quad \gamma_{i j}=-\gamma_{j i}, \quad \beta_{i j}=-\beta_{j i}, \quad(i, j=1, \ldots, m) .
$$

Every substitution of period 2 of $S A\left(2 m, p^{n}\right), p>2$, has therefore the form

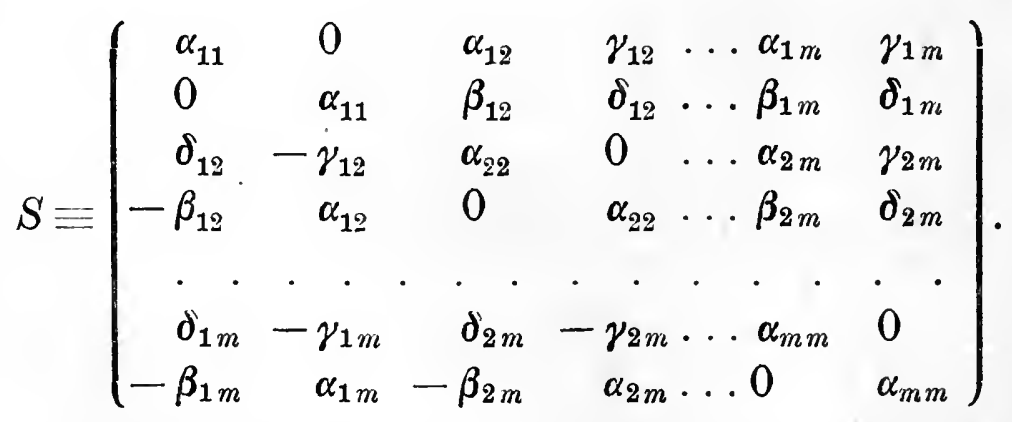

For $m=1$, we have $\alpha_{11}^{2}=1$, so that $T_{1,-1}$ is the only substitution $S$. In order to prove the first part of our theorem by induction, we assume that every special Abelian substitution in the 
$G F\left[p^{n}\right], p>2$, on $t<m$ pairs of indices is conjugate within the group $S A\left(2 t, p^{n}\right)$ with one of the substitutions $T_{r}(r \overline{\overline{<}} t)$ and proceed to prove that a like result holds for $m$ pairs of indices. In view of $\S 120$, we may suppose that $S$ has one of the three forms $\Sigma_{1}, \Sigma_{2}$ or $S_{1}$, the latter having $\alpha_{1 i}=\gamma_{1 i}=\beta_{1 i}=\delta_{1 i}=0 \quad(i=2, \ldots, m)$. An $S$ of the form $S_{1}$ is evidently a product $T_{1, \pm 1} S_{2}$, where $S_{2}$ affects only the $m-1$ sets of indices $\xi_{2}, \eta_{2}, \ldots, \xi_{m}, \eta_{m}$. By hypothesis, $S_{2}$ is conjugate with one of the products, $I, T_{2,-1}, T_{2,-1} T_{3,-1}, \ldots$, $T_{2,-1} T_{3,-1} \ldots T_{m,-1}$. Hence an $S$ of the form $S_{1}$ is conjugate with some $I_{r}(r=1,2, \ldots, m)$. We proceed to consider $\Sigma_{1}$ and $\Sigma_{2}$ in the following three cases.

Case a). $\delta \neq 0$ in $\Sigma_{1}$. Then $S$ has the form

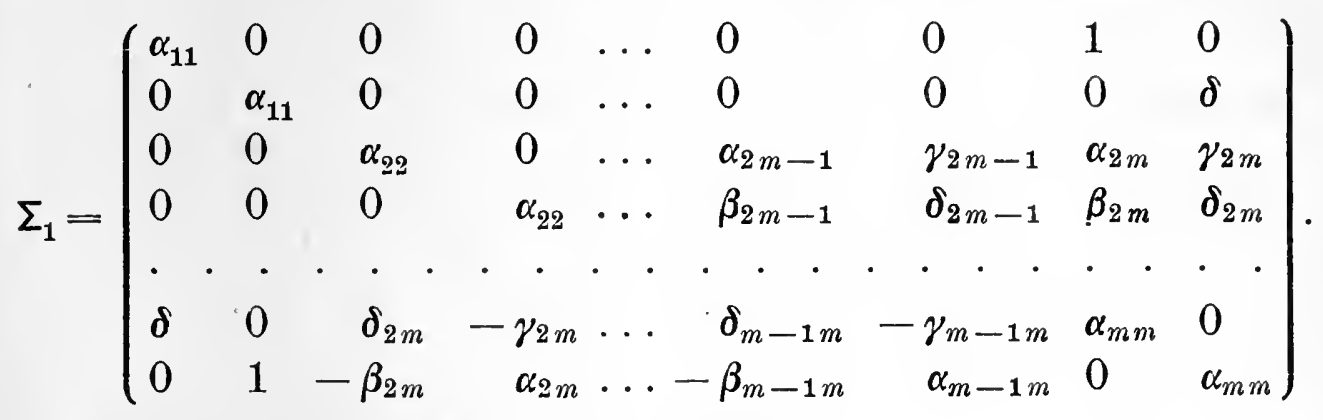

The Abelian conditions 79) give at once

$$
\alpha_{i m}=\beta_{i m}=\gamma_{i m}=\delta_{i m} \quad(i=2, \ldots, m-1), \quad \alpha_{11}+\alpha_{m m}=0 .
$$

Hence $\Sigma_{1} \equiv \Sigma_{1}^{\prime} \Sigma_{1}^{\prime \prime}$, where $\Sigma_{1}^{\prime \prime}$ affects only the indices

$$
\xi_{i}, \eta_{i} \quad(i=2, \ldots, m-1),
$$

while $\Sigma_{1}^{\prime}$ affects only $\xi_{1}, \eta_{1}, \xi_{m}, \eta_{m}$, viz.,

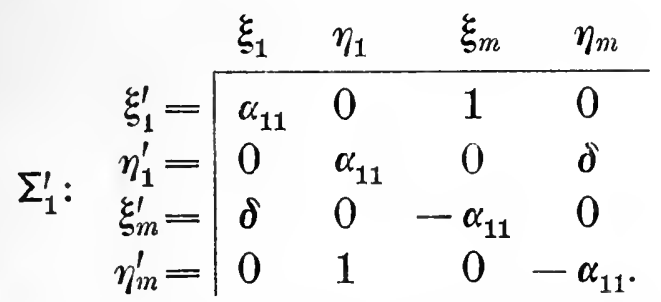

By hypothesis $\Sigma_{1}^{\prime \prime}$ is conjugate with some product of the

$$
T_{i,-1} \quad(i=2, \ldots, m-1) .
$$

In order to make the induction from one to two pairs of indices, we must prove that $\Sigma_{1}^{\prime}$ is conjugate with a product of $T_{1,-1}$ and $T_{m,-1}$. Transforming $\Sigma_{1}^{\prime}$ by $Q_{1, m, \sigma}$, we obtain the substitution

$$
\left(\begin{array}{llll}
\alpha_{11}+\sigma \delta & 0 & 1-2 \sigma \alpha_{11}-\sigma^{2} \delta & 0 \\
0 & \alpha_{11}+\sigma \delta & 0 & \delta \\
\delta & 0 & -\alpha_{11}-\sigma \delta & 0 \\
0 & 1-2 \sigma \alpha_{11}-\sigma^{2} \delta & 0 & -\alpha_{11}-\sigma \delta
\end{array}\right) .
$$


Taking $\alpha_{11}+\sigma \delta=0$ and transforming the resulting substitution by $T_{m, \delta}^{-1}$, we obtain $P_{1 m} \equiv\left(\xi_{1} \xi_{m}\right)\left(\eta_{1} \eta_{m}\right)$. The latter is transformed into $T_{1,-1}$ by the following Abelian substitutions (and by no others):

$$
\left(\begin{array}{rrrr}
\alpha_{11} & \gamma_{11} & -\alpha_{11} & -\gamma_{11} \\
\beta_{11} & \delta_{11} & -\beta_{11} & -\delta_{11} \\
\alpha_{m 1} & \gamma_{m 1} & \alpha_{m 1} & \gamma_{m 1} \\
\beta_{m 1} & \delta_{m 1}^{\prime} & \beta_{m 1} & \delta_{m 1}
\end{array}\right) \quad\left[\begin{array}{l}
2\left(\alpha_{11} \delta_{11}-\beta_{11} \gamma_{11}\right)=1 \\
2\left(\alpha_{m 1} \delta_{m 1}-\beta_{m 1} \gamma_{m 1}\right)=1
\end{array}\right]
$$

It follows that, if $\delta \neq 0, \Sigma_{1}$ is conjugate with some $T_{r}$.

Case b). $\delta=0$ in $\Sigma_{1}$. The Abelian conditions 79) now give

$$
\gamma_{i m}=\delta_{i m}=0 \quad(i=2, \ldots, m-1), \quad \alpha_{11}+\alpha_{m m}=0, \quad \alpha_{11}^{2}=1 .
$$

'Transforming $\Sigma_{1}$ by $Q_{1, m, \lambda}$, where $1-2 \lambda \alpha_{11}=0$, we obtain

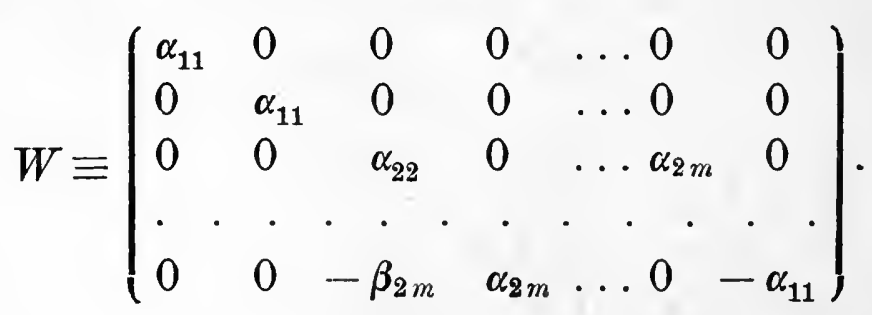

Hence $W=T_{1, \pm 1} W^{\prime}$, where $W^{\prime}$ affects only $\xi_{i}, \eta_{i}(i=2, \ldots, m)$ and may therefore, by hypothesis, be transformed into a product of the $T_{j,-1}$ by an Abelian substitution on the same indices. It will transform $W$ into a product of the $T_{j,-1}(j=1, \ldots, m)$, which is conjugate with some $T_{r}$.

Case c). In virtue of the Abelian conditions, $\Sigma_{2}$ becomes

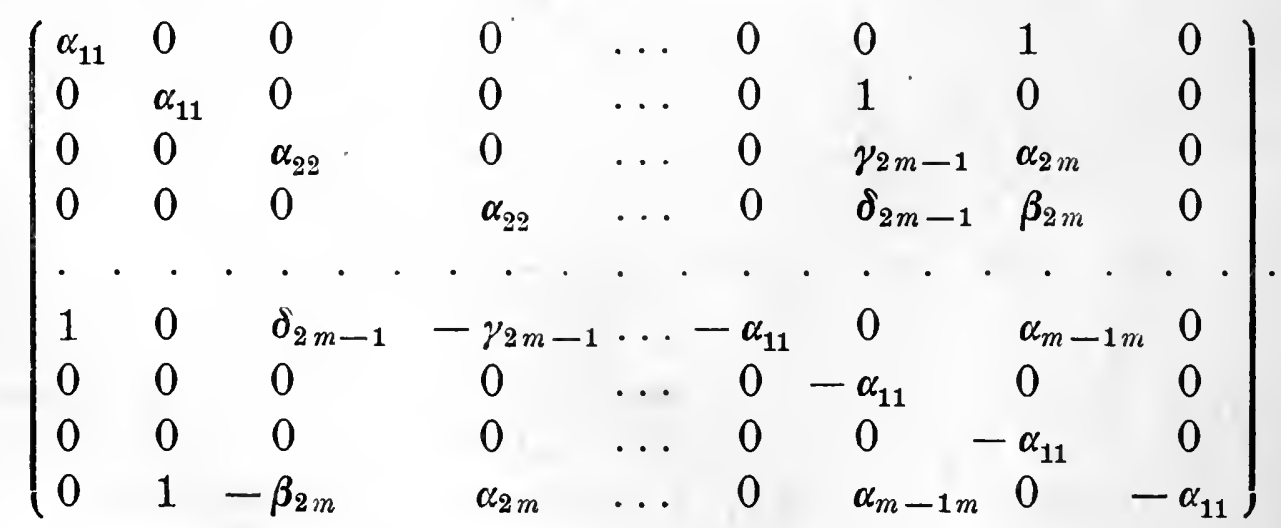

Transforming $\Sigma_{2}$ by the product $Q_{1, m, \lambda} Q_{m-1,1, \sigma}$, where $1-2 \lambda \alpha_{11}=0$, $1+2 \sigma \alpha_{11}=0$, we get a similar substitution but having zeros in place of the four elements 1 . Since it is of the form $W$, we may proceed as in case b). 
To complete the proof of our theorem, we note that

$$
T_{1} \equiv T_{1,-1}, \quad T_{2} \equiv T_{1,-1} T_{2,-1}, \ldots, T_{m} \equiv T_{1,-1} T_{2,-1} \ldots T_{m,-1}
$$

have the respective characteristic determinants (with parameter $K$ )

$$
(1+K)^{2}(1-K)^{2 m-2}, \quad(1+K)^{4}(1-K)^{2 m-4}, \ldots,(1+K)^{2 m} .
$$

Hence no two of them are conjugate under linear transformation.

The most general substitution of $S A\left(2 m, p^{n}\right)$ commutative with $T_{r}$ is seen to be $A \equiv A_{r} A_{m-r}$, where $A_{r}$ is an arbitrary special Abelian substitution on the indices $\xi_{i}, \eta_{i}(i=1, \ldots, r)$ and $A_{m-r}$ an arbitrary one on the indices $\xi_{i}, \eta_{i}(i=r+1, \ldots, m)$. By $\S 115$ the number of substitutions $A_{r}$ and $A_{m-r}$ is respectively $S A\left[2 r, p^{n}\right]$ and $S A\left[2 m-2 r, p^{n}\right]$. Dividing $S A\left[2 m, p^{n}\right]$ by the product of the foregoing numbers, we obtain the number of substitutions of $S A\left(2 m, p^{n}\right)$ conjugate with $T$, within the group.

Operators of period 2 of $A\left(2 m, p^{n}\right), \S \S 122-123$.

122. By $\S 119$, we obtain the quotient-group $A\left(2 m, p^{n}\right)$ by considering as identical $S$ and $S T \equiv T S$, where $S$ is an arbitrary substitution of $S A\left(2 m, p^{n}\right)$ and $T$ is the self-conjugate substitution $T_{1,-1} T_{2,-1} \ldots T_{m,-1}$. In particular, $T$, and $T_{r} T$ become identical in the quotient-group. But the latter is conjugate with $T_{m-r}$. Furthermore, if $s=m / 2$ or $(m-1) / 2$ according as $m$ is even or odd, no two of the operators $I_{1}, T_{2}, \ldots, T_{s}$ are conjugate within the quotient-group. The special Abelian substitutions of period 2 lead therefore to just $s$ distinct sets of conjugate operators of $A\left(2 m, p^{n}\right)$, $p>2$. To complete the study of the operators of period 2 of $A\left(2 m, p^{n}\right)$, it remains to determine the conjugacy of the special Abelian substitutions $S$ for which $S^{2}=T$. Being of period 4, such an $S$ is not conjugate to any $T_{r}$. Moreover, no two of the corresponding operators of the quotient-group are conjugate, since that would require one of the four relations

$$
A^{-1} S A=T_{r} \text { or } T T_{r}, \quad A^{-1}(S T) A=T_{r} \text { or } T T_{r},
$$

$A$ being Abelian. But any of these would require that $S$ be conjugate with some $T_{i}$ within the special Abelian group, whereas their periods are different. Making use of the result of $\S 123$, we may state the theorem:

According as $m$ is even or odd, the group $A\left(2 m, p^{n}\right), p>2$, has exactly $\frac{1}{2}(m+2)$ or $\frac{1}{2}(m+1)$ distinct sets of conjugate operators of period 2. 
123. Theorem. - Within the special Abelian group on $2 m$ indices in the $G F\left[p^{n}\right], p>2$, every substitution $S$, such that $S^{2}=T$, is conjugate with $M \equiv M_{1} M_{2} \ldots M_{m} \cdot{ }^{1}$ )

Taking as $S$ the general substitution 75), whose reciprocal is given by 77) for $\mu=1$, the condition $S=S^{-1} T$ is seen to require

$$
\alpha_{i j}=-\delta_{j i}, \quad \gamma_{i j}=\gamma_{j i}, \quad \beta_{i j}=\beta_{j i} \quad(i, j=1, \ldots, m) .
$$

The matrix of coefficients of the general $S$ is therefore

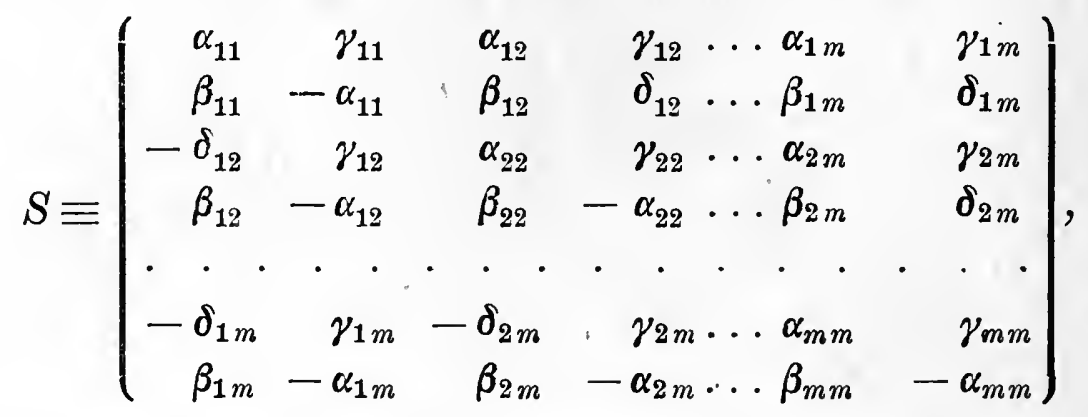

subject to the special Abelian conditions.

Take first $m=1$. Then $S$ has the form

$$
S \equiv\left(\begin{array}{cc}
\alpha_{11} & \gamma_{11} \\
\beta_{11} & -\alpha_{11}
\end{array}\right) \quad\left[-\alpha_{11}^{2}-\beta_{11} \gamma_{11}=1\right] .
$$

It is conjugate with a similar substitution in which $\alpha_{11}=0$. In fact, if $\beta_{11} \neq 0$, the transformed of $S$ by $L_{1, \lambda}$ replaces $\eta_{1}$ by

$$
\beta_{11} \xi_{1}-\left(\alpha_{11}+\lambda \beta_{11}\right) \eta_{1} \text {, }
$$

in which the coefficient of $\eta_{1}$ may be made zero by choice of $\lambda$. If $\beta_{11}=0, \gamma_{11} \neq 0$, we first transform $S$ by $M_{1}$ and then proceed as before. If $\beta_{11}=\gamma_{11}=0$, we first transform $S$ by $L_{1, \lambda}^{\prime}$ and obtain a substitution which replaces $\eta_{1}$ by $2 \lambda \alpha_{11} \xi_{1}-\alpha_{11} \eta_{1}$, so that the new $\beta_{11}+0$.

With $\alpha_{11}=0, S$ takes the form

$$
\left(\begin{array}{cc}
0 & \gamma \\
-\gamma^{-1} & 0
\end{array}\right)
$$

and is the transformed of $M_{1}$ by the special Abelian substitution

$$
\left(\begin{array}{cc}
\varrho \gamma & -\sigma \gamma \\
\sigma & \varrho
\end{array}\right) \quad\left[\gamma\left(\rho^{2}+\sigma^{2}\right)=1\right] .
$$

Indeed, by $\S 64$, there exist solutions in the $G F\left[p^{n}\right], p>2$, of

$$
\rho^{2}+\sigma^{2}=1 / \gamma \text {. }
$$

To prove the theorem by induction for $m$ pairs of indices, we assume it true for $t$ pairs of indices $t<m$.

1) For the number of conjugates see Ex. 8, end of Ch. VIII. 
If $\alpha_{1 i}=\beta_{1 i}=\gamma_{1 i}=\delta_{1 i}=0(i=2, \ldots, m)$, then $S=S_{1} S^{\prime}$, where $S_{1}$ affects only $\xi_{1}, \eta_{1}$ and is therefore conjugate with $M_{1}$, and where $S_{1}^{\prime}$ affects only $\xi_{i}, \eta_{i}(i=2, \ldots, m)$ and is, by assumption, conjugate with $M_{2} M_{3} \ldots M_{m}$. Hence $S$ is conjugate with $M_{1} M_{2} M_{3} \ldots M_{m}$ within $S A\left(2 m, p^{n}\right)$.

In the contrary case, $S$ is conjugate (by $\S 120$ ) with one of the two substitutions $\Sigma_{1}, \Sigma_{2}$. We consider the following three cases.

Case a). If $\Sigma_{1}$, with $\delta \neq 0$, be of the form $S$ above, the Abelian conditions give

$$
\begin{aligned}
& \alpha_{i m}=\beta_{i m}=\gamma_{i m}=\delta_{i m}=0 \quad(i=2, \ldots, m-1), \\
& \alpha_{m m}=-\alpha_{11}, \quad \gamma_{m m}=-\delta \gamma_{11}, \quad \delta \beta_{m m}=-\beta_{11} .
\end{aligned}
$$

Hence $\Sigma_{1} \equiv \Sigma_{1}^{\prime} \Sigma_{1}^{\prime \prime}$, where $\Sigma_{1}^{\prime}$ has the form

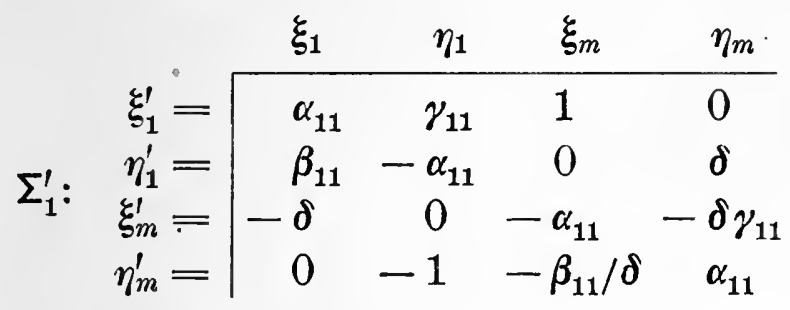

while $\Sigma_{1}^{\prime \prime}$ affects only $\xi_{i}, \eta_{i}(i=2, \ldots, m-1)$ and is, by assumption, transformed into $M_{2} M_{3} \ldots M_{m-1}$ by some special Abelian substitution affecting only the same indices. We proceed to prove that $\Sigma_{1}^{\prime}$ may be transformed into $M_{1} M_{m}$ by a special Abelian substitution on the indices $\xi_{1}, \eta_{1}, \xi_{m}, \eta_{m}$. The proposition that $\Sigma_{1}$ is conjugate with $M_{1} M_{2} \ldots M_{m}$ under $S A\left(2 m, p^{n}\right)$ will then follow.

If $\beta_{11}=\gamma_{11}=0$ in $\Sigma_{1}^{\prime}$, we transform it by $N_{1, m, \lambda}$ and get

$$
\left(\begin{array}{cccc}
\alpha_{11} & -2 \lambda & 1 & 0 \\
0 & -\alpha_{11} & 0 & \delta \\
-\delta & 0 & -\alpha_{11} & 2 \lambda \delta \\
0 & -1 & 0 & \alpha_{11}
\end{array}\right)
$$

This is of the form $\Sigma_{1}^{\prime}$, but has $\gamma_{11} \neq 0$. Next, if $\gamma_{11}=0, \beta_{11} \neq 0$, we transform $\Sigma_{1}^{\prime}$ by $M_{1} M_{m} I_{m, \delta}$ and get a substitution of the form $\Sigma_{1}^{\prime}$ in which, however, $\gamma_{11} \neq 0, \beta_{11}=0$. We may therefore assume that $\gamma_{11}=0$ in $\Sigma_{1}^{\prime}$. Transforming it by $L_{1, \lambda}^{\prime} L_{m, \lambda / \delta}^{\prime}$, where $\lambda \equiv \alpha_{11} / \gamma_{11}$, we get a substitution of the form

$$
V \equiv\left(\begin{array}{cccc}
0 & \gamma_{11} & 1 & 0 \\
\beta & 0 & 0 & \delta \\
-\delta & 0 & 0 & -\delta \gamma_{11} \\
0 & -1 & -\beta / \delta & 0
\end{array}\right) .
$$


If $\beta=0$, then $\delta=1$ and the transformed of $V$ by $R_{1, m},-\gamma_{11}^{-1}$ gives

$$
W \equiv\left(\begin{array}{clll}
0 & \gamma_{11} & 0 & 0 \\
-\gamma_{11}^{-1} & 0 & 0 & 0 \\
0 & 0 & 0 & -\delta \gamma_{11} \\
0 & 0 & \gamma_{11}^{-1} \delta^{-1} & 0
\end{array}\right)
$$

If $\beta \neq 0$, the transformed of $V$ by $N_{1, m, \delta / \beta}$ gives a substitution of the form $W$. Since $W$ is the product of a substitution on the indices $\xi_{1}, \eta_{1}$ and a substitution on the indices $\xi_{m}, \eta_{m}$, it is conjugate with $M_{1} M_{m}$.

Case b). If $\Sigma_{1}$, with $\delta=0$, be of the form $S$, the Abelian conditions give

$$
\beta_{11}=\gamma_{m m}=0, \quad \alpha_{11}+\alpha_{m m}=0, \quad \gamma_{i m}=\delta_{i m}=0 \quad(i=2, \ldots, m-1) .
$$

Transforming $\Sigma_{1}$ by $L_{1, \sigma}$, where $\gamma_{11}-2 \sigma \alpha_{11}=0$, we get

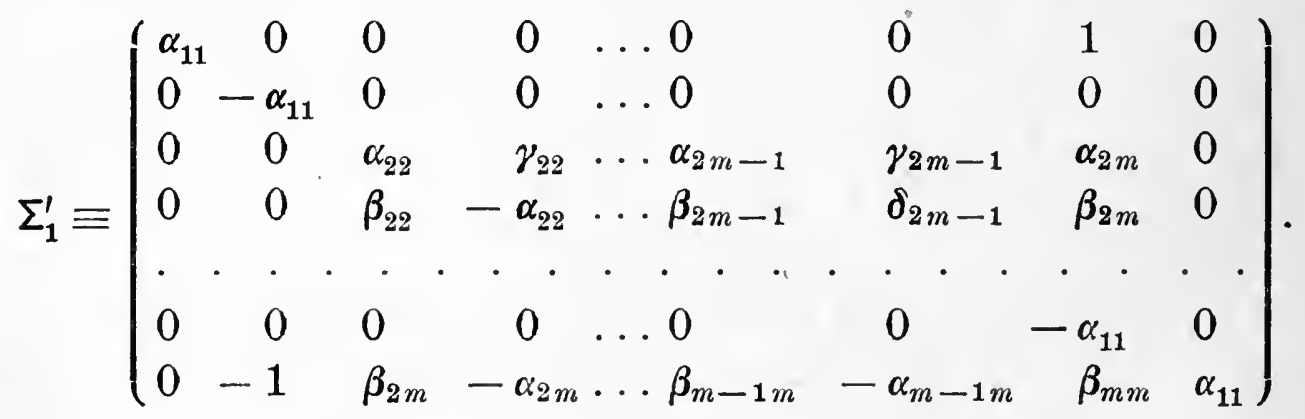

Transforming $\Sigma_{1}^{\prime}$ by $Q_{1, m, \sigma}$, we obtain a substitution $\Sigma_{1}^{\prime \prime}$ which differs from $\Sigma_{1}^{\prime}$ only in having the coefficients \pm 1 replaced by $\pm\left(1-2 \sigma \alpha_{11}\right)$. By choice of $\sigma$, the latter may be made zero. Hence $\Sigma_{1}^{\prime \prime} \equiv S_{1} S^{\prime}$, where $S_{1}$ affects only $\xi_{1}, \eta_{1}$ and is therefore conjugate with $M_{1}$, while $S^{\prime}$ affects only $\xi_{i}, \eta_{i}(i=2, \ldots, m)$ and is, by assumption, conjugate with $M_{2} M_{3} \ldots M_{m}$. Hence $\Sigma_{1}^{\prime \prime}$ is conjugate with

$$
M_{1} M_{2} \ldots M_{m} \text {. }
$$

Case c). If $\Sigma_{2}$ be an Abelian substitution of the form $S$, it becomes

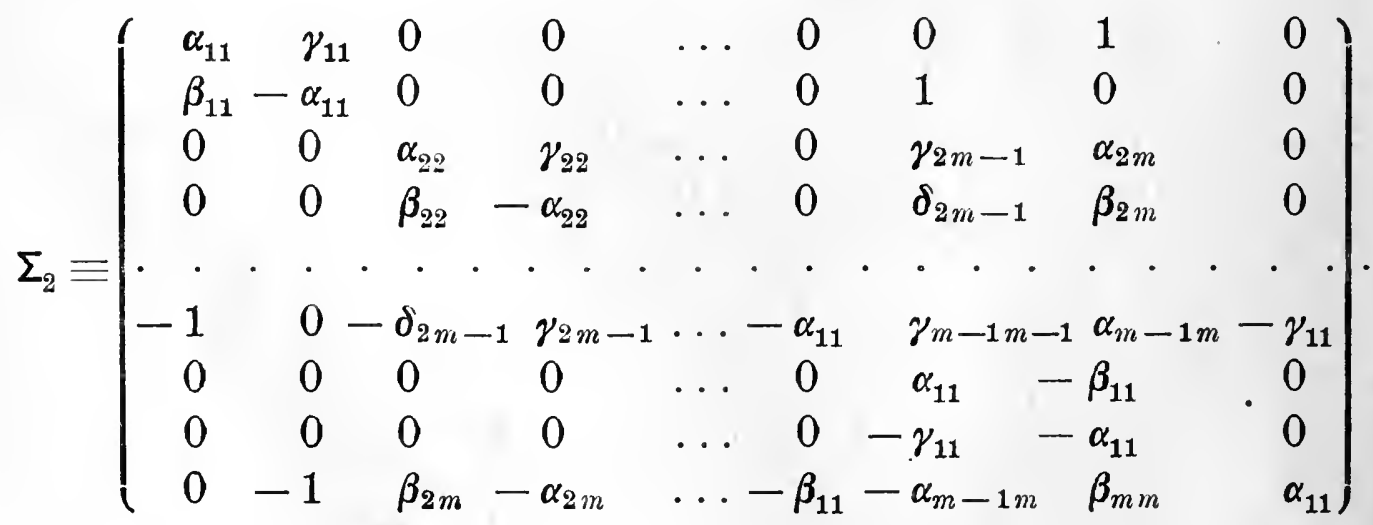


Suppose first that $\alpha_{11}=0$, so that $-\beta_{11} \gamma_{11}=1$. Transforming $\Sigma_{2}$ by $R_{1, m, \lambda}$, where $1+\lambda \gamma_{11}=0$, we reach a substitution equal to a product $S_{1} S^{\prime}$, where $S_{1}$ affects $\xi_{1}, \eta_{1}$ only and $S^{\prime}$ affects

only.

$$
\xi_{i}, \eta_{i} \quad(i=2, \ldots, m)
$$

Suppose, however, that $\alpha_{11} \neq 0$. Transforming $\Sigma_{2}$ by $L_{m-1, \varrho}$,

$$
\gamma_{m-1 m-1}+2 \varrho \alpha_{11}=0,
$$

we obtain a substitution $\Sigma_{2}^{\prime}$ of the form $\Sigma_{2}$ and having $\gamma_{m-1 n-1}=0$. Transforming $\Sigma_{2}^{\prime}$ by $L_{m, \varrho}^{\prime}$, where $\beta_{m m}-2 \varrho \alpha_{11}=0$, we obtain a substitution $\Sigma_{2}^{\prime \prime}$ of the form $\Sigma_{2}$, but having $\beta_{m m}=\gamma_{m-1 m-1}=0$.

If $\beta_{11}=\gamma_{11}=0$, we transform $\Sigma_{2}^{\prime \prime}$ by $Q_{m-1,1, \lambda}$, where $1-2 \lambda \alpha_{11}=0$, and afterwards by $Q_{1, m, \varrho}$, where $1-2 \varrho \alpha_{11}=0$, and obtain a product $S_{1} S^{\prime}$, where $S_{1}$ affects only $\xi_{1}, \eta_{1}$ and $S^{\prime}$ affects only $\xi_{i}, \eta_{i}(i>1)$. If $\gamma_{11}=0, \beta_{11} \neq 0$, we transform $\Sigma_{2}^{\prime \prime}$ by $P_{m-1 m} M_{1} M_{m-1} M_{m}$ and get

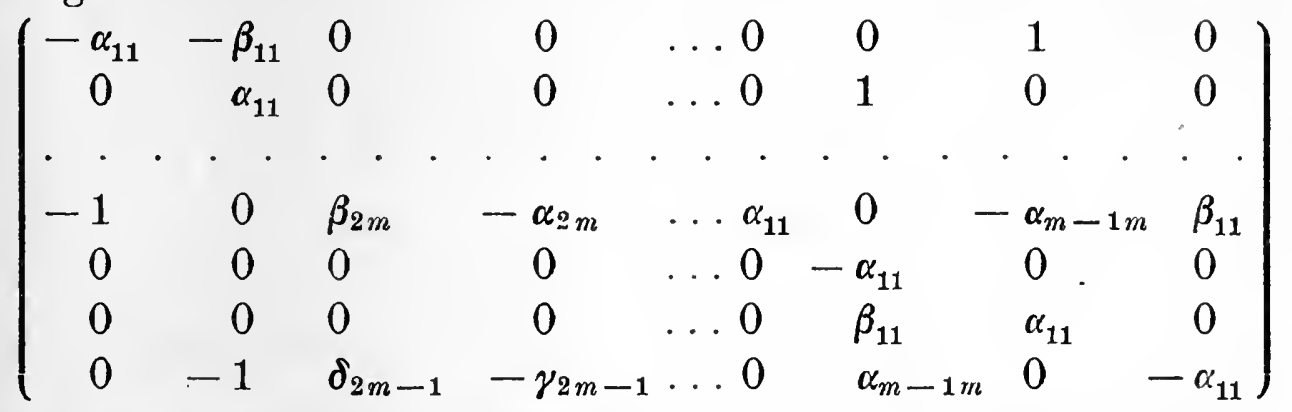

which has the form of $\Sigma_{2}^{\prime \prime}$ with $\gamma_{11} \neq 0$. We therefore treat the latter case only. Transforming . $\Sigma_{2}^{\prime \prime}$ by $L_{m, \varrho}^{\prime}$, where

$$
\alpha_{m-1 m}+\varrho \gamma_{11}=0
$$

we obtain a substitution $U$ of the form $\Sigma_{2}$, but having $\alpha_{m-1 m}$ and $\gamma_{m-1 m-1}$ both zero. Transforming $U$ by $R_{m-1, m, \lambda} L_{1,-\lambda}^{\prime}$, where $\alpha_{11}+\lambda \gamma_{11}=0$, we get a substitution of the form

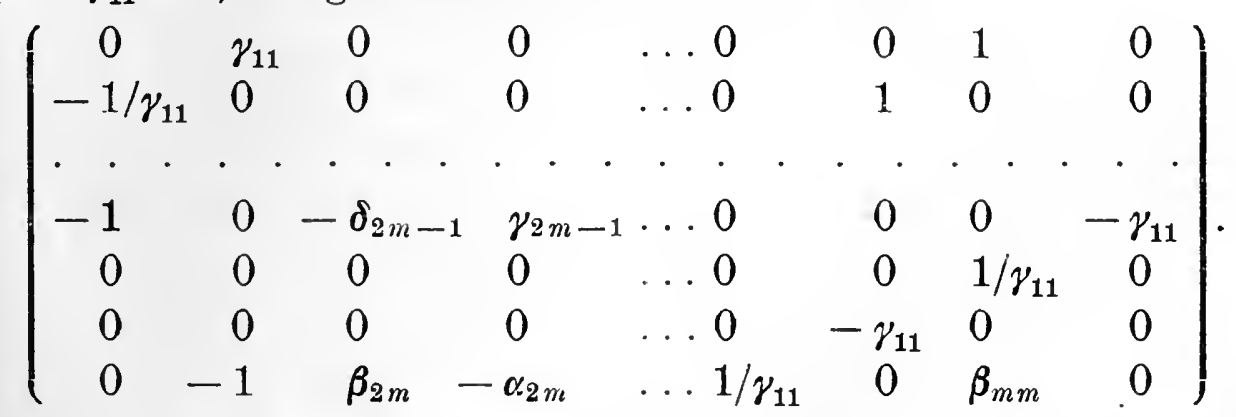

Transforming this by $R_{1, m, \lambda}$, where $1+\lambda \gamma_{11}=0$, we get a similar substitution with the elements \pm 1 replaced by zeros, and therefore the product of a substitution on $\xi_{1}, \eta_{1}$ by a substitution on the indices $\xi_{i}, \eta_{i}(i=2, \ldots, m)$. It is therefore conjugate with

$$
M_{1} M_{2} \ldots M_{m} \text {. }
$$




\section{CHAPTER III.}

\section{A GENERALIZATION OF THE ABELIAN LINEAR GROUP.')}

124. Those linear homogeneous substitutions in the $G F\left[p^{n}\right]$ on $m q$ indices,

87) $\quad S: \quad x_{i j}^{\prime}=\sum_{k=1}^{m}\left(\alpha_{k 1}^{i j} x_{k 1}+\alpha_{k 2}^{i j} x_{k 2}+\cdots+\alpha_{k q}^{i j} x_{k q}\right)$,

$$
(i=1, \ldots, m ; j=1, \ldots, q)
$$

which, if operating simultaneously upon $q$ independent sets of $m q$ variables, the $j^{\text {th }}$ set of which is given the notation

$$
x_{i 1}^{(j)}, x_{i 2}^{(j)}, \ldots, x_{i q}^{(j)} \quad(i=1,2, \ldots, m),
$$

leave formally invariant the function

$$
\Phi \equiv \sum_{i=1}^{m}\left|\begin{array}{cccc}
x_{i 1}^{(1)} & x_{i 2}^{(1)} & \ldots & x_{i q}^{(1)} \\
x_{i 1}^{(2)} & x_{i 2}^{(2)} & \ldots & x_{i q}^{(2)} \\
\cdot & \cdot & \cdot & \cdot \\
x_{i 1}^{(g)} & x_{i 2}^{(q)} & \ldots & x_{i q}^{(q)}
\end{array}\right|,
$$

form a group $G\left(m, q, p^{n}\right)$, which for $q=2$ is the Abelian group $S A\left(m, p^{n}\right)$.

The conditions upon $S$ for the absolute invariance of $\Phi$ are seen to be those given by formulae 88) and 89), viz.,

88)

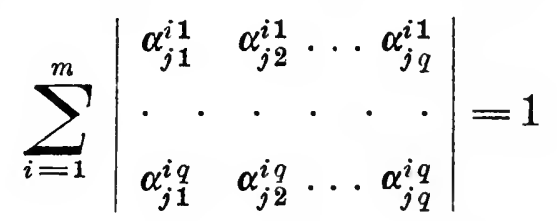

$$
\begin{aligned}
& (j=1, \ldots, m) \\
& \text { 89) } \sum_{i=1}^{m}\left|\begin{array}{cccc}
\alpha_{j_{1} k_{1}}^{i 1} & \alpha_{j_{2} k_{2}}^{i 1} & \ldots & \alpha_{j_{q} k_{q}}^{i 1} \\
\cdot & \cdot & \cdot & \cdot \\
\alpha_{j_{1} k_{1}}^{i q} & \alpha_{j_{2} k_{2}}^{i q} & \ldots & \alpha_{j_{q} k_{q}}^{i q}
\end{array}\right|=0\left(\begin{array}{l}
\text { each } k=1,2, \ldots, q \\
\text { each } j=1,2, \ldots, m, \\
j_{1}, j_{2}, \ldots, j_{q} \text { not all equal }
\end{array}\right) \text {. }
\end{aligned}
$$

125. The inverse of the general substitution 87) of $G\left(m, q, p^{n}\right)$ is

$$
\begin{aligned}
S^{-1}: x_{r s}^{\prime}=\sum_{i=1}^{m}\left(A_{r s}^{i 1} x_{i 1}+A_{r s}^{i 2} x_{i 2}+\cdots+A_{r s}^{i q} x_{i q}\right), & \\
( & (r=1, \ldots, m ; s=1, \ldots, q)
\end{aligned}
$$

1) Taken from the author's paper, "A class of linear groups including the Abelian group", Quarterly Journal, July, 1899. The group is mentioned, but not investigated, by Jordan, Traité, p. 219, No. 301. 
where $A_{r s}^{i j}$ denotes the adjoint of $\alpha_{r s}^{i j}$ in the determinant

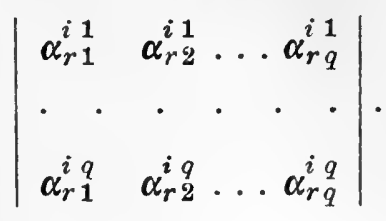

In fact, the product 87) 90) replaces $x_{r s}$ by

$$
\sum_{\substack{i=1, \ldots, m \\ j=1, \ldots, q}} A_{r s}^{i j}\left(\sum_{\substack{k=1, \ldots, m \\ l=1, \ldots, q}} \alpha_{k l}^{i j} x_{k l}\right) .
$$

Here the coefficient of $x_{k l}$ is

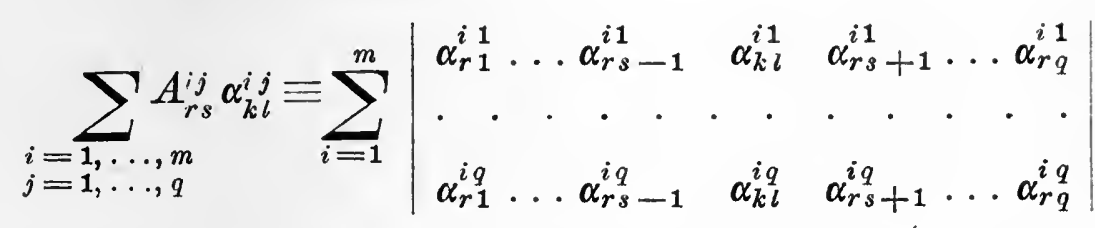

and therefore, by 88$)$ and 89$)$, equals unity if $(k, l)=(r, s)$, but equals zero if $(k, l) \neq(r, s)$. Hence the above product replaces $x_{r s}$ by $x_{r s}$. The reciprocal of $S$ is therefore obtained by replacing $\alpha_{k i}^{i j}$ by $A_{i j}^{k l}$ for $i, k=1, \ldots, m ; i, j=1, \ldots, q$.

Writing relations 88) for $S^{-1}$ given by 90 ), we find

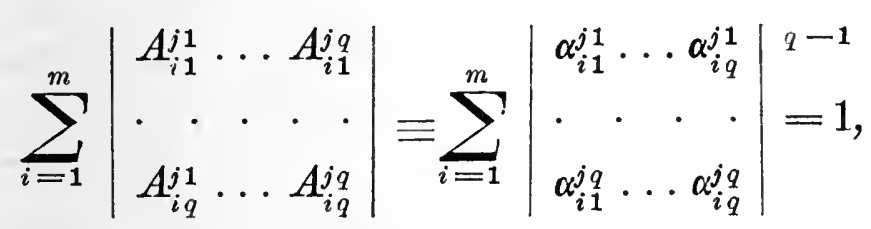

holding for $j=1,2, \ldots, m$.

Note. - For substitutions 87) which multiply $\Phi$ by a constant $\varrho$, the reciprocal is evidently obtained by replacing $\alpha_{k l}^{i j}$ by $\frac{1}{\varrho} A_{i j}^{k l}$.

126. The structure of the group $G\left(m, q, p^{n}\right)$ is essentially different in the two cases $q=2$ and $q>2$. The case $q=2$ has been investigated at length in Chapter II. In the following investigation we assume that $q>2$, a restriction necessary for the treatment given.

Let $j_{2}, j_{3}, \ldots, j_{q}$ have fixed values not all equal chosen arbitrarily from $1,2, \ldots, m$, and let $k_{2}, k_{3}, \ldots, k_{q}$ have fixed values chosen from $1,2, \ldots, q$. Then for $j_{1}=1, \ldots, m ; k_{1}=1, \ldots, q$, we obtain $m q$ equations 89). In fact, since $q>2, j_{1}, j_{2}, \ldots, j_{q}$ are not all equal and hence do not lead to conditions of the type 88). Expanding the determinants of 89) according to the elements in the first columns, our $m q$ equations may be written 
where

$$
\sum_{\substack{i=1, \ldots, m \\
l=1, \ldots, q}} \alpha_{j_{1} k_{1}}^{i l} B_{j_{1} k_{1}}^{i l}=0 \quad\left(\begin{array}{l}
j_{1}=1, \ldots, m \\
k_{1}=1, \ldots, q
\end{array}\right)
$$

$$
B_{j_{1} k_{1}}^{i l} \equiv\left|\begin{array}{ccc}
\alpha_{j_{2} k_{2}}^{i b_{2}} & \ldots & \alpha_{j_{q} k_{q}}^{i b_{2}} \\
\cdot & \cdot & \cdot \\
\alpha_{j_{2} k_{2}}^{i b_{2}} & \ldots & \alpha_{j_{q} k_{q}}^{i b_{q}}
\end{array}\right|,
$$

in which $b_{2}, b_{3}, \ldots, b_{q}$ denote the integers $1, \ldots, l-1, l+1, \ldots, q$. Since the determinant $\left|\alpha_{j_{1} k_{1}}^{i l}\right| \neq 0$, being the determinant of $S$, we have

$$
B_{j_{1} k_{1}}^{i l}=0 \quad\left(\begin{array}{l}
i=1, \ldots, m \\
l=1, \ldots, q
\end{array}\right) .
$$

Hence the determinant 92) vanishes for $i=1, \ldots, m$ and for $b_{2}, b_{3}, \ldots, b_{q}$ an arbitrary combination of $q-1$ distinct integers $\overline{<} q$.

If $q=3$, we have reached the relations 95) below. If $q>3$, we denote by $C_{j_{2} k_{2}}^{i b_{s}}$ the adjoint of $\alpha_{j_{2} k_{2}}^{i b_{s}}$ in the determinant 92) and consider the following expansions:

$$
\sum_{s=2}^{q} \alpha_{j_{2} k_{2}}^{i b_{s}} C_{j_{2} k_{2}}^{i b_{s}}=0
$$

Of these consider the $m q$ equations in which $i, j_{3}, \ldots, j_{q}$ have fixed values chosen arbitrarily from $1,2, \ldots, m$, but such that $j_{3}, j_{4}, \ldots, j_{q}$ are not all equal, and $k_{3}, \ldots, k_{q}$ fixed values chosen arbitrarily from $1,2, \ldots, q$, while lastly $j_{2}$ takes the values $1,2, \ldots, m$ and $k_{2}$ the values $1,2, \ldots, q$. Since the matrix

$$
\left(\begin{array}{l}
\alpha_{j_{2} k_{2}}^{i b_{s}}
\end{array}\right) \quad\left(\begin{array}{l}
j_{2}=1, \ldots, m ; k_{2}=1, \ldots, q \\
s=2, \ldots, q
\end{array}\right)
$$

comprises $q-1$ rows of the matrix of $S$, not all of its determinants of order $q-1$ are zero. Hence the $q-1$ determinants $C$, which are the same in each of the $m q$ equations 93), must be zero, viz.,

$$
C_{j_{2} k_{2}}^{i b_{s}} \equiv\left|\begin{array}{ccc}
\alpha_{j_{3} k_{3}}^{i c_{3}} & \ldots & \alpha_{j_{q} k_{q}}^{i c_{c_{3}}} \\
\cdot & \cdot & \cdot \\
\alpha_{j_{3} k_{3}}^{i c_{q}} & \ldots & \alpha_{j_{q} k_{q}}^{i c_{c_{q}}}
\end{array}\right|=0,
$$

where $c_{3}, \ldots, c_{q}$ denote any $q-2$ distinct integers $\overline{\overline{<}} q$.

If $q=4$, we have reached the relations 95) below. If $q>4$, we proceed as before. After $q-2$ such steps, we reach the set of relations

$$
\left|\begin{array}{cc}
\alpha_{j k}^{i r} & \alpha_{j^{\prime} k^{\prime}}^{i r} \\
\alpha_{j k}^{i s} & \alpha_{j^{\prime} k^{\prime}}^{i s}
\end{array}\right|=0 \quad\left(\begin{array}{l}
i, j, j^{\prime}=1, \ldots, m ; j^{\prime} \neq j \\
r, s, k, k^{\prime}=1, \ldots, q
\end{array}\right) .
$$


In virtue of the relations 95), the conditions 89) all reduce to identities. In fact, in each relation 89), at least two of the $j$ 's are distinct, say $j \neq j_{2}$, and therefore all minors formed from the first, and second columns vanish in virtue of 95).

$A$ substitution $S$ belongs to the group $G\left(m, q, p^{n}\right), q>2$, if and only if its coefficients satisfy the conditions 88) and 95).

12\%. Theorem. - Every substitution $S$ leaving $\Phi$ invariant can be derived from the totality of linear substitutions of determinant unity on $q$ indices

$$
\dot{x_{1 j}}=\sum_{k=1}^{q} \beta_{1 k}^{1 j} x_{1 k} \quad(j=1, \ldots, q)
$$

together with the linear substitutions, each on $2 q$ indices,

$$
P_{i j} \equiv\left(x_{i 1} x_{j 1}\right)\left(x_{i 2} x_{j 2}\right) \ldots\left(x_{i q} x_{j q}\right) \quad(i, j=1, \ldots, m) .
$$

We can evidently derive from these generators a substitution $T$ which belongs to $G\left(m, q, p^{n}\right)$ and replaces an arbitrary index $x_{k l}$ by any particular index as $x_{11}$. We may therefore suppose that in the product $S^{\prime} \equiv T S, S$ being defined by 87 ), the coefficient $\alpha_{11}^{11} \neq 0$. If then we set

$$
\alpha_{j k}^{11}=C_{j k} \alpha_{11}^{11} \quad(j=2, \ldots, m ; k=1, \ldots, q)
$$

it follows from 95), for $i=1, v=1, j^{\prime}=1, k^{\prime}=1, j>1$, that

$$
\alpha_{j k}^{1 s}=C_{j k} \alpha_{11}^{1 s} \quad(j=2, \ldots, m ; k, s=1, \ldots, q) .
$$

Substituting these values in the relation 91) for $j=1$, we find

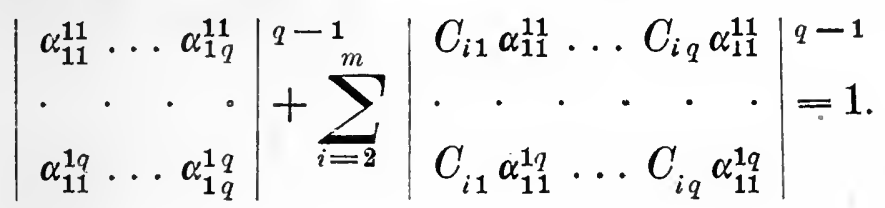

It follows that

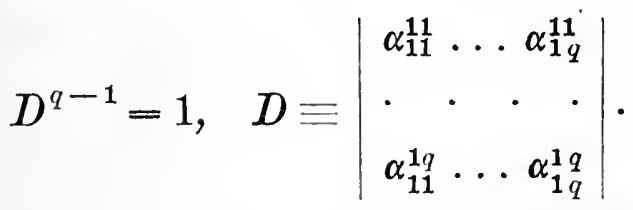

Hence the following substitution is of determinant $D \neq 0$.

$R:$

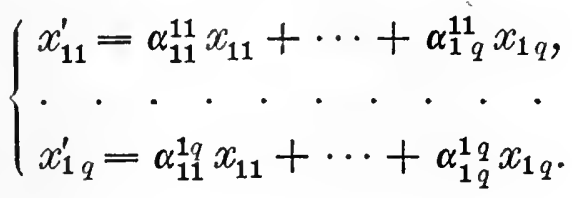


If we denote the determinants of $\Phi$ by $D_{i}$ so that $\Phi \equiv \sum_{i=1}^{m} D_{i}$, we readily see that $R$ multiplies $D_{1}$ by the factor $D$ but leaves unaltered $D_{i}(i=2, \ldots, m)$. Hence, if $W$ denote the substitution

$$
W: \quad x_{i 1}^{\prime}=D x_{i 1} \quad(i=2, \ldots, m),
$$

the product $W R$ multiplies $\Phi$ by the factor $D$. The product $S_{1} \equiv(W R)^{-1} S^{\prime}$ multiplies $\Phi$ by $D^{-1}$ and therefore satisfies the relations 89 ) and consequently also relations 95 ), derived from them. But $S_{1}$ affects the indices $x_{11}, x_{12}, \ldots, x_{1 q}$ as follows:

$$
x_{1 j}^{\prime}=x_{1 j}+\sum_{k=2}^{m}\left(\alpha_{k 1}^{1 j} x_{k 1}+\cdots+\alpha_{k q}^{1 j} x_{k q}\right),
$$

where $\alpha_{k 1}^{1 j}$ denotes $D^{-1}$ times the earlier $\alpha_{k 1}^{1 j}$, for $k=2, \ldots, m$. For the substitution $S_{1}$ we have $\alpha_{11}^{1 s}=0(s=2, \ldots, q)$. Hence by 96$)$,

$$
\alpha_{j k}^{1 s}=0 \quad(j=2, \ldots, m ; k=1, \ldots, q ; s=2, \ldots, q) .
$$

Also $\alpha_{1 s}^{11}=0(s=2, \ldots, q), \alpha_{1 s}^{1 s}=1$. Hence, by the following eases of 95$)$,

$$
\left|\begin{array}{ll}
\alpha_{j k}^{11} & \alpha_{1 s}^{11} \\
\alpha_{j k}^{1 s} & \alpha_{1 s}^{1 s}
\end{array}\right|=0, \quad\left(\begin{array}{l}
j=2, \ldots, m ; l=1, \ldots, q \\
s=2, \ldots, q
\end{array}\right)
$$

we find $\alpha_{j k}^{11}=0$. Hence every $\alpha_{j k}^{1 s}=0$, for $j>1$, so that $S_{1}$ leaves fixed $x_{11}, x_{12}, \ldots, x_{19}$.

Applying the Note of $\S 125$ to form the reciprocal of $S_{1}$, we find that the matrix of $S_{1}^{-1}$ has zeros throughout the first $q$ columns, except the diagonal terms $D$ in the first $q$ rows. By the above argument, the remaining elements of the first $q$ rows must be zeros. Reciprocating this matrix by the same rule, we find that $D=1$ and that $S_{1}$ reduces to a substitution on the indices

$$
x_{j 2}, \quad x_{j 3}, \ldots, x_{j q} \quad(j=2, \ldots, m) .
$$

Since $W$ is the identity, $S=T^{-1} S^{\prime}=T^{-1} R S_{1}$, where $I$ and $R$ are derived from the generators given in the theorem. Proceeding with $S_{1}$ as we did with $S$, we reach a substitution $S_{2}$ on the indices $x_{j 3}, \ldots, x_{j q}$. Finally, we reach the identity.

128. It follows from $\& 127$ that the group $G\left(m, q, p^{n}\right), q>2$, has an invariant subgroup $\Gamma$ composed of the substitutions

$$
x_{i j}^{\prime}=\sum_{k=1}^{q} \beta_{i k}^{i j} x_{i k} \quad(i=1, \ldots, m ; j=1, \ldots, q)
$$

where, for $i=1,2, \ldots, m$, the determinant

$$
\left|\beta_{i k}^{i j}\right|=1 \quad(j, k=1, \ldots, q) .
$$


The quotient-group is generated by the substitutions $\boldsymbol{P}_{i j}$ and is thus holoedrically isomorphic with the symmetric group on $m$ letters. The group $\Gamma$ is the direct product of $m$ groups each the special linear homogeneous group in the $G F\left[p^{n}\right]$ on $q$ indices ( $\$ 103$ ). The substitutions of the $i^{\text {th }}$ group are given as follows

$$
x_{i j}^{\prime}=\sum_{k=1}^{q} \beta_{i k}^{i j} x_{i k}, \quad x_{s j}^{\prime}=x_{s j} \quad(s=1, \ldots, m ; s \neq i ; j=1, \ldots, q) .
$$

The structure of the group $G\left(m, q, p^{n}\right)$ is therefore completely determined.

\section{CHAPTER IV.}

\section{THE HYPERABELIAN GROUP.}

129. The totality of linear homogeneous substitutions in the $G F\left[p^{2 n}\right]$

$$
S: \quad \xi_{i}^{\prime}=\sum_{j=1}^{2 m} \alpha_{i j} \xi_{j} \quad(i=1, \ldots, 2 m)
$$

which leave absolutely invariant the function

$$
\Psi \equiv \sum_{l=1}^{m}\left|\begin{array}{ll}
\xi_{2 l-1} & \xi_{2 l} \\
\xi_{2 l-1}^{p^{n}} & \xi_{2 l}^{p^{n}}
\end{array}\right|
$$

forms the hyperabelian group $\left.{ }^{1}\right) H\left(2 m, p^{2 n}\right)$. Its name is derived from the fact that the totality of its substitutions whose coefficients belong to the included field $G F\left[p^{n}\right]$ constitutes the Abelian group $S A\left(2 m, p^{n}\right)$, which is therefore a subgroup of the hyperabelian group.

A general substitution $S$ transforms $\Psi$ into

$$
\sum_{l=1}^{m}\left\{\sum_{j=1}^{2 m}\left|\begin{array}{ll}
\alpha_{2 l-1 j} & \alpha_{2 l j} \\
\alpha_{2 l-1 j}^{p^{n}} & \alpha_{2 l j}^{p^{n}}
\end{array}\right| \xi_{j}^{p^{n}+1}+\sum_{j=1=k}^{j, k=1, \ldots, 2 m}\left|\begin{array}{ll}
\alpha_{2 l-1 j} & \alpha_{2 l j} \\
\alpha_{2 l-1 k}^{p^{n}} & \alpha_{2 l k}^{p^{n}}
\end{array}\right| \xi_{j} \xi_{k}^{p^{n}}\right\} .
$$

The conditions upon $S$ for the absolute invariance of $\Psi$ are thus

$$
\sum_{l=1}^{m}\left|\begin{array}{ll}
\alpha_{2 l-1 j} & \alpha_{2 l j} \\
\alpha_{2 l-1 k}^{p^{n}} & \alpha_{2 l k}^{p^{n}}
\end{array}\right|=\varepsilon_{j k} \quad(j, k=1, \ldots, 2 m)
$$

where $\varepsilon_{j k}=0$, unless $j$ and $k$ differ by unity, when

$$
\varepsilon_{2 i-12 i}=1, \quad \varepsilon_{2 i 2 i-1}=-1 \quad(i=1, \ldots, m) .
$$

1) Introduced by the author, Proc. Lond. Math. Soc., vol. 31, pp. 30-68. It will hardly be confused with Picard's hyperabelian group of infinite order. 
The reciprocal of the hyperabelian substitution $S$ is

$$
S^{-1}:\left\{\begin{array}{l}
\xi_{2 l-1}^{\prime}=\sum_{j=1}^{m}\left(\alpha_{2 j 2 l}^{p^{n}} \xi_{2 j-1}-\alpha_{2 j-12 l}^{p^{n}} \xi_{2 j}\right) \\
\xi_{2 l}^{\prime}=\sum_{j=1}^{m}\left(-\alpha_{2 j 2 l-1}^{p^{n}} \xi_{2 j-1}+\alpha_{2 j-12 l-1}^{p^{n}} \xi_{2 j}\right)
\end{array}\right.
$$

Indeed, the product $S S^{-1}$ replaces $\xi_{2 l-1}$ by

$$
\begin{gathered}
\sum_{j, k}^{1, \ldots, m}\left|\begin{array}{cc}
\alpha_{2 j-12 k-1} & \alpha_{2 j 2 k-1} \\
\alpha_{2 j-12 l}^{p^{n}} & \alpha_{2 j 2 l}^{p^{n}}
\end{array}\right| \xi_{2 k-1}+\sum_{j, k}^{1, \ldots, m}\left|\begin{array}{cc}
\alpha_{2 j-12 k} & \alpha_{2 j 2 k} \\
\alpha_{2 j-12 l}^{p^{n}} & \alpha_{2 j 2 l}^{p^{n}}
\end{array}\right| \xi_{2 k} \\
\equiv \sum_{k=1}^{m}\left(\varepsilon_{2 k-12 l} \xi_{2 k-1}+\varepsilon_{2 k 2 l} \xi_{2 k}\right) \equiv \xi_{2 l-1} .
\end{gathered}
$$

Similarly, $S S^{-1}$ replaces $\xi_{2 \iota}$ by

$$
-\sum_{k=1}^{m}\left(\varepsilon_{2 k-12 l-1} \xi_{2 k-1}+\varepsilon_{2 k 2 l-1} \xi_{2 k}\right) \equiv \xi_{2 l} .
$$

The relations 97) in which $j>k$ are derived from those in which $j<k$ by raising the latter to the power $p^{n}$. We may therefore express the hyperabelian conditions in the convenient form

$$
\begin{aligned}
& \sum_{l=1}^{m}\left|\begin{array}{ll}
\alpha_{2 l-1 j} & \alpha_{2 l j} \\
\alpha_{2 l-1 k}^{j^{n}} & \alpha_{2 l k}^{\eta^{n}}
\end{array}\right|= \begin{cases}1 & (\text { if } k=j+1=\text { even }) \\
0 & \text { (unless } k=j+1=\text { even })\end{cases} \\
& (j, k=1, \ldots, 2 m ; j \overline{<} k) \text {. }
\end{aligned}
$$

The corresponding relations for $S^{-1}$ are found by replacing

by respectively $\alpha_{2 l-12 j-1}, \quad \alpha_{2 l-12 j}, \quad \alpha_{2 l 2 j-1}, \quad \alpha_{2 l 2 j}$

$$
\alpha_{2 j 2 l}^{p^{n}}, \quad-\alpha_{2 j-12 l}^{p^{n}}, \quad-\alpha_{2 j 2 l-1}^{p^{n}}, \quad \alpha_{2 j-12 l-1}^{p^{n}} .
$$

Writing out the four sets of relations 98) according to the evenness or oddness of $j$ and $k$, and making the replacement just indicated, we obtain four sets of relations for the invariance of $\psi$ by the substitution $S^{-1}$ and therefore together equivalent to the relations 98 ). We may combine the four sets into the single formula

$$
\begin{aligned}
& \sum_{l=1}^{m}\left|\begin{array}{cc}
\alpha_{j 2 l-1}^{p^{n}} & \alpha_{j 2 l}^{p^{n}} \\
\alpha_{k 2 l-1} & \alpha_{k 2 l}
\end{array}\right|= \begin{cases}1 & (\text { if } k=j+1=\text { even }) \\
0 & \text { (unless } k=j+1=\text { even })\end{cases} \\
& (j, k=1, \ldots, 2 m ; j \overline{\overline{<}} k) \text {. }
\end{aligned}
$$

130. The determinant $\Delta$ of the hyperabelian substitution $S$ must satisfy the relation

$$
\Delta^{p^{n}+1}=1
$$


For proof, we reflect on its main diagonal the determinant of $S^{-1}$, then change the signs of the $2 l-1^{\text {st }}$ row and column for $l=1, \ldots, m$, and finally interchange the $2 l-1^{\text {st }}$ row with the $2 l^{\text {th }}$ row for $l=1, \ldots, m$, and likewise interchange the corresponding columns. We obtain the determinant

$$
\left|\boldsymbol{\alpha}_{i j}^{p^{n}}\right| \equiv\left|\boldsymbol{\alpha}_{i j}\right|^{p^{n}} \equiv \Delta^{p^{n}} .
$$

Hence $\Delta \Delta^{n}=1$, being the determinant of the product $S S^{-1}$.

131. Theorem. - The maximal subgroup $M$ of the hyperabelian group $H\left(2 m, p^{2 n}\right)$ which transforms into itself the Abelian group $S A\left(2 m, p^{n}\right)$ is given by the extension of the latter by the substitution

$$
V_{\varrho}: \quad \xi_{2 l-1}^{\prime}=\varrho \xi_{2 l-1}, \quad \xi_{2 \iota}^{\prime}=\varrho^{-p^{n}} \xi_{2 l} \quad(l=1, \ldots, m),
$$

where $\varrho$ is a primitive root in the $G F\left[p^{2 n}\right]$. The index of $S A\left(2 m, p^{n}\right)$ under $M$ is $p^{n}+1$.

We determine all hyperabelian substitutions

$$
S: \quad \xi_{i}^{\prime}=\sum_{j=1}^{2 m} \alpha_{i j} \xi_{j} \quad(i=1, \ldots, 2 m)
$$

which transform the Abelian group into itself. Now $S$ transforms the Abelian substitution, affecting a single index,

into the substitution

$$
\xi_{2 r-1}^{\prime}=\xi_{2 r-1}+\xi_{2 r}
$$

$$
\begin{array}{r}
\xi_{i}^{\prime}=\xi_{i}+\alpha_{i 2 r-1} \sum_{j=1}^{m}\left(-\alpha_{2 j 2 r-1}^{p^{n}} \xi_{2 j-1}+\alpha_{2 j-12 r-1}^{p^{n}} \xi_{2 j}\right) \\
(i=1, \ldots, 2 m),
\end{array}
$$

whose coefficients must therefore belong to the $G F\left[p^{n}\right]$, viz.,

$$
\alpha_{i 2 r-1} \alpha_{j 2 r-1}^{p^{n}}(i, j=1, \ldots, 2 m ; r=1, \ldots, m) .
$$

Likewise, $S$ must transform the Abelian substitution

$$
\xi_{2 r}^{\prime}=\xi_{2 r}+\xi_{2 r-1}
$$

into a substitution belonging to the $G F\left[p^{n}\right]$. Hence the products

$$
\alpha_{i 2 r} \alpha_{j 2 r}^{p^{n}} \quad(i, j=1, \ldots, 2 m ; r=1, \ldots, m)
$$

must belong to the $G F\left[p^{n}\right]$. The reciprocal $S^{-1}$ must transform the Abelian group into itself. From the above results, it follows therefore that the products

$$
\alpha_{2 r s} \alpha_{2 r t}^{p^{n}}, \quad \alpha_{2 r-1 s} \alpha_{2 r-1 t}^{p^{n}} \quad(s, t=1, \ldots, 2 m ; r=1, \ldots, m)
$$

must belong to the $G F\left[p^{n}\right]$. Combining our results, every product 
must belong to the $G F\left[p^{n}\right]$.

But, if $\beta, \gamma$ be marks of the $G F\left[p^{2 n}\right]$ such that

$$
\beta \gamma^{p^{n}} \doteq \mu=\operatorname{mark} \text { of } G F\left[p^{n}\right],
$$

then, if $\gamma \neq 0, \beta / \gamma \equiv \mu \gamma^{-p^{n}-1}$ is a mark of the $G F\left[p^{n}\right]$. Hence by 101 ), the ratios of the non-vanishing coefficients in any row or any column of the matrix of $S$ must all belong to the $G F\left[p^{n}\right]$.

Suppose first that $m=1$. If $\alpha_{11} \neq 0$, we have

$$
\alpha_{21}=\lambda \alpha_{11}, \quad \alpha_{12}=\mu \alpha_{11} \quad\left(\lambda, \mu \text { in the } G F\left[p^{n}\right]\right) .
$$

Then if $\lambda$ and $\mu$ be not both zero, $\alpha_{22}=\nu \alpha_{11}, \nu$ being in the $G F\left[p^{n}\right]$. For $\lambda=\mu=0$, the hyperabelian condition gives $\alpha_{11} \alpha_{22}^{p^{n}}=1$, whence $\alpha_{22}=v \alpha_{11}$. If, however, $\alpha_{11}=0$, both $\alpha_{12}$ and $\alpha_{21}$ are not zero. Hence $\alpha_{22}=\varrho \alpha_{12}$, $\varrho$ in the $G F\left[p^{n}\right]$. By the hyperabelian condition, $-\alpha_{12} \alpha_{21}^{p^{n}}=1$, whence $\alpha_{21}=\sigma \alpha_{12}, \sigma$ in the $G F\left[p^{n}\right]$. In either case, we have reached a substitution of the form 103) below.

For $m>1, S$ transforms the Abelian substitution

into

$$
\xi_{2 r-1}^{\prime}=\xi_{2 r-1}+\xi_{2 s}, \quad \xi_{2 s-1}^{\prime}=\xi_{2 s-1}+\xi_{2 r} \quad(r \neq s)
$$

$$
\begin{aligned}
\xi_{i}^{\prime}=\xi_{i} & +\alpha_{i 2 r-1} \sum_{j=1}^{m}\left(-\alpha_{2 j 2 s-1}^{p^{n}} \xi_{2 j-1}+\alpha_{2 j-12 s-1}^{p^{n}} \xi_{2 j}\right) \\
& +\alpha_{i 2 s-1} \sum_{j=1}^{m}\left(-\alpha_{2 j 2 r-1}^{p^{n}} \xi_{2 j-1}+\alpha_{2 j-12 r-1}^{p^{n}} \xi_{2 j}\right) .
\end{aligned}
$$

Hence the sums

$$
\alpha_{i 2 r-1} \alpha_{j 2 s-1}^{p^{n}}+\alpha_{i 2 s-1} \alpha_{j 2 r-1}^{p^{n}} \quad(i, j=1, \ldots, 2 m ; r, s=1, \ldots, m)
$$

must all belong to the $G F\left[p^{n}\right]$. In like manner, if $S$ transform each of the following three Abelian substitutions (in which $\mathrm{r} \neq s$ ),

$$
\begin{aligned}
& \xi_{2 r-1}^{\prime}=\xi_{2 r-1}+\xi_{2 s-1}, \quad \xi_{2 s}^{\prime}=\xi_{2 s}-\xi_{2 r} ; \\
& \xi_{2 r}^{\prime}=\xi_{2 r}+\xi_{2 s}, \quad \xi_{2 s-1}^{\prime}=\xi_{2 s-1}-\xi_{2 r-1} \\
& \xi_{2 r}^{\prime}=\xi_{2 r}+\xi_{2 s-1}, \quad \xi_{2 s}^{\prime}=\xi_{2 s}+\xi_{2 r-1}
\end{aligned}
$$

into substitutions belonging to the $G F\left[p^{n}\right]$, then must the respective sums

$$
\left\{\begin{array}{ccc}
\alpha_{i 2 r-1} \alpha_{j 2 s}^{p^{n}}+\alpha_{i 2 s} & \alpha_{j 2 r-1}^{p^{n}} \\
\alpha_{i 2 r} & \alpha_{j 2 s-1}^{p^{n}}+\alpha_{i 2 s-1} \alpha_{j 2 r}^{p^{n}} \\
\alpha_{i 2 r} & \alpha_{j 2 s}^{p^{n}}+\alpha_{i 2 s} & \alpha_{j 2 r}^{p^{n}}
\end{array}\right\} \quad\left(\begin{array}{l}
i, j=1, \ldots, 2 m \\
r, s=1, \ldots, m
\end{array}\right)
$$

belong to the $G F\left[p^{n}\right]$. Combining our results, every sum

belongs to the $G F\left[p^{n}\right]$.

$$
\alpha_{i r} \alpha_{j s}^{p^{n}}+\alpha_{i s} \alpha_{j r}^{p^{n}} \quad(i, j, r, s=1, \ldots, 2 m ; r \neq s)
$$


Of the coefficients in the $i^{\text {th }}$ row of the matrix of $S$, we may suppose that $\alpha_{i r} \neq 0$, for example. If, then, $\alpha_{j r} \neq 0$, the ratios of the coefficients in the $i^{\text {th }}$ and $j^{\text {th }}$ rows must all belong to the $G F\left[p^{n}\right]$ [by the result following from 101)]. If, however, $\alpha_{j r}=0$, we may suppose that, for example, $\alpha_{j s} \neq 0(s \neq r)$. Then, by 102), the products $\alpha_{i r} \alpha_{j s}^{p^{n}}$ belong to the $G F\left[p^{n}\right]$. We have in either case the result that the ratios of the coefficients in the $i^{\text {th }}$ and $j^{\text {th }}$ rows belong to the $G F\left[p^{n}\right]$. Hence the ratios of all the coefficients in $S$ to any one non-vanishing coefficient belong to the $G F\left[p^{n}\right]$, so that $S$ may be written

103)

$$
\xi_{i}^{\prime}=\alpha \sum_{j=1}^{2 m} \lambda_{i j} \xi_{j}
$$$$
(i=1, \ldots, 2 m)
$$

where the $\lambda_{i j}$ belong to the $G F\left[p^{n}\right]$.

Inversely, every hyperabelian substitution of the form 103) transforms into itself the Abelian group defined for the $G F\left[p^{n}\right]$.

The conditions that 103) shall be hyperabelian are

$$
\begin{aligned}
& \sum_{l=1}^{m}\left|\begin{array}{ll}
\lambda_{2 l-1 j} & \lambda_{2 l j} \\
\lambda_{2 l-1 k} & \lambda_{2 l k}
\end{array}\right|= \begin{cases}\alpha^{-p^{n}-1} & (\text { if } k=j+1=\text { even }) \\
0 & (\text { unless } k=j+1=\text { even })\end{cases} \\
& (i, j=1, \ldots, 2 m ; i \overline{<} j)
\end{aligned}
$$

The substitution $\left(\lambda_{i j}\right)$, or 103$)$ with the factor $\alpha$ deleted, therefore belongs to the general Abelian group $G A\left(2 m, p^{n}\right)$ and multiplies $\psi$ by the mark $\alpha^{-p^{n}-1}$ of the $G F\left[p^{n}\right]$. If then we set

$$
\mu_{2 l-1 j} \equiv \lambda_{2 l-1 j}, \quad \mu_{2 l j} \equiv \alpha^{p^{n}+1} \lambda_{2 l j} \quad\left(\begin{array}{l}
l=1, \ldots, m \\
j=1, \ldots, 2 m
\end{array}\right)
$$

we find that $S=U V_{\alpha}$, where

$$
\begin{aligned}
& U \text { : } \\
& \xi_{i}^{\prime}=\sum_{j=1}^{2 m} \mu_{i j} \xi_{j} \\
& (i=1, \ldots, 2 m) \\
& V_{\alpha}: \\
& \xi_{2 l-1}^{\prime}=\alpha \xi_{2 l-1}, \quad \xi_{2 l}^{\prime}=\alpha-p^{n} \xi_{2 l} \\
& (l=1, \ldots, m),
\end{aligned}
$$

so that $V_{\alpha}$, and therefore also $U$, is a hyperabelian substitution. Moreover, in virtue of the relations 104) and 105), $U$ belongs to the special Abelian group $S A\left(2 m, p^{n}\right)$ and is therefore of determinant unity. The first part of our theorem is therefore proven.

If we form a rectangular array of the marks $\neq 0$ of the $G F\left[p^{2 n}\right]$ with those belonging to the $G F\left[p^{n}\right]$ as first row, the

$$
p^{n}+1 \equiv\left(p^{2 n}-1\right) /\left(p^{n}-1\right)
$$

"multipliers" form a set of marks $\alpha_{1}, \alpha_{2}, \ldots, \alpha_{p^{n}+1}$ such that none of their ratios belong to the $G F\left[p^{n}\right]$, while every mark of the $G F\left[p^{2 n}\right]$ not of this set has with some mark of the set a ratio belonging to the $G F\left[p^{n}\right]$. Furthermore, the product 


$$
V_{\alpha^{\prime}} V_{\alpha}^{-1}: \quad \xi_{2 l-1}^{\prime}=\alpha^{-1} \alpha^{\prime} \xi_{2 l-1}, \quad \xi_{2 l}^{\prime}=\alpha^{p^{n}} \alpha^{\prime-p^{n}} \xi_{2 l} \quad(l=1, \ldots, m)
$$

belongs to $S A\left(2 m, p^{n}\right)$ if and only if $\alpha^{-1} \alpha^{\prime}$ belongs to the $G F\left[p^{n}\right.$. It follows that the substitutions $V_{\alpha_{i}}\left(i=1,2, \ldots, p^{n}+1\right)$ give the totality of substitutions $V_{\alpha}$ such that $V_{\alpha^{\prime}} V_{\alpha}^{-1}$ does not belong to $S A\left(2 m, p^{n}\right)$. Hence an identity of the form

$$
U V_{\alpha_{i}} \equiv U^{\prime} V_{\alpha_{j}} \quad\left(i \text { and } j \overline{<} p^{n}+1 ; i \neq j\right)
$$

is impossible when $U$ and $U^{\prime}$ both belong to $S A\left(2 m, p^{n}\right)$. Every hyperabelian substitution 103) is therefore of the form $U V_{\alpha_{i}}, i$ being chosen from the series $1,2, \ldots, p^{n}+1$, while an identity $U V_{\alpha_{i}}=U^{\prime} V_{\alpha_{j}}$ requires $i=j, U=U^{\prime}$. Hence the number of distinct substitutions 103) is $\left(p^{n}+1\right) S A\left[2 m, p^{n}\right]$. The second part of our theorem is therefore proven.

132. Those substitutions of the hyperabelian group $H\left(2 m, p^{2 n}\right)$ which have determinant unity form a self-conjugate subgroup $H^{\prime}$ of index $p^{n}+1$. In fact, for $G$ any mark $\neq 0$ of the $G F\left[p^{2 n}\right]$, the substitution

$$
\xi_{1}^{\prime}=\sigma \xi_{1}, \quad \xi_{2}^{\prime}=\sigma^{-p^{n}} \xi_{2}, \quad \xi_{i}^{\prime}=\xi_{i} \quad(i=3, \ldots, 2 m)
$$

belongs to $H\left(2 m, p^{2 n}\right)$. Its determinant $\sigma^{-\left(p^{n}-1\right)}$ can, by choice of $\sigma$, be made equal to any one of the $p^{n}+1$ roots of $\Delta^{p^{n}+1}=1$. Hence there exist hyperabelian substitutions whose determinant $\Delta$ is any root of this equation. By $\S 130$, there are no other values of $\Delta$.

The group $H^{\prime}$ contains a self-conjugate subgroup formed by the substitutions

106) $\quad T_{\not x}: \quad \xi_{i}^{\prime}=x \xi_{i} \quad(i=1, \ldots, 2 m) \quad\left[x^{2 m}=1, x^{p^{n}+1}=1\right]$.

The quotient-group will be denoted by the symbol $H A\left(2 m, p^{2 n}\right)$. It will be proven simple except in the special cases $m=1, p^{n}=2$ or 3 (§§ 138, 145, 148). By the same references its order $H A\left[2 m, p^{2 n}\right]$ is

$$
\frac{1}{q}\left(p^{2 m n}-1\right) p^{n(2 m-1)}\left(p^{n(2 m-1)}+1\right) p^{n(2 m-2)} \ldots\left(p^{2 n}-1\right) p^{n},
$$

where $q$ denotes the greatest common divisor of $2 m$ and $p^{n}+1$. The order of $H\left(2 m, p^{2 n}\right)$ is

$$
\left(p^{2 m n}-1\right) p^{n(2 m-1)}\left(p^{n(2 m-1)}+1\right) p^{n(2 m-2)} \cdots\left(p^{2 n}-1\right) p^{n}\left(p^{n}+1\right) .
$$

The Abelian group $S A\left(2 m, p^{n}\right)$ has an invariant subgroup formed by the identity and $T_{-1}$. The quotient-group $A\left(2 m, p^{n}\right)$ is simple except in the three cases $m=1, p^{n}=2 ; m=1, p^{n}=3 ; m=2, p^{n}=2$ (§ 119). But $H\left(2 m, p^{2 n}\right)$ contains $S A\left(2 m, p^{n}\right)$ as a subgroup. In order that $T_{x}$ shall belong to the latter, the coefficient $x$ must belong to the $G F\left[p^{n}\right]$. But $x^{p^{n}}=x$ and $x^{p^{n}+1}=1$ require $x^{2}=1$. Hence 
would $T_{\%}$ be the identity or $T_{-1}$. It follows that $A\left(2 m, p^{n}\right)$ is a subgroup of $H A\left(2 m, p^{n}\right)$. We proceed to determine the number of conjugates to the former group within the latter group, using the result of $\S 131$.

133. Theorem. - The largest subgroup $M^{\prime}$ of $H A\left(2 m, p^{2 n}\right)$ which transforms $A\left(2 m, p^{n}\right)$ into itself is identical with $A\left(2 m, p^{n}\right)$ if $p=2$ or if $p>2$ and $p^{n}+1$ contains a higher power of 2 than $m$ contains; in the remaining case, the order of $M^{\prime}$ is double the order of $A\left(2 m, p^{n}\right)$.

The determinant of $S \equiv U V_{\alpha}$ being supposed to be unity and that of $U$ being unity, it follows that $V_{\alpha}$ has determinant

$$
\alpha^{-m\left(p^{n}-1\right)}=1 \text {. }
$$

Now $V_{\alpha}$ and $T_{\%} V_{\alpha}$ correspond in the quotient-group $H A\left(2 m, p^{2 n}\right)$ to the same operator. We investigate the conditions under which $T_{\psi} V_{\alpha}$ has its coefficients in the $G F\left[p^{n}\right]$. The necessary and sufficient condition is seen to be

$$
(\varkappa \alpha)^{p^{n}-1}=1 .
$$

Hence must $\varkappa^{2}=\alpha^{p^{n}-1}$ and therefore

$$
\alpha^{\frac{1}{2}\left(p^{2 n}-1\right)}=x^{p^{n}+1}=1,
$$

or $\alpha$ must be a square in the $G F\left[p^{2 n}\right]$. The remaining condition $x^{2 m}=1$ becomes an identity in virtue of 107). Hence, if the solutions of 107) are all squares in the $G F\left[p^{2 n}\right]$, the substitution $S=U V_{\alpha}$ will correspond in the quotient-group to an operator belonging to $A\left(2 m, p^{n}\right)$. But, if there occur not-squares as solutions of 107), the resulting substitutions $V_{\alpha}$ may be expressed as products $V_{\nu} V_{\beta^{2}}, \nu$, being a particular not-square. Then $V_{\beta^{2}}$ corresponds in the quotient-group to an operator of $A\left(2 m, p^{n}\right)$, while $V_{\imath}$ does not. In this case the group $A\left(2 m, p^{n}\right)$ is transformed into itself by a subgroup of $H A\left(2 m, p^{2 n}\right)$ of double the order of $A\left(2 m, p^{n}\right)$.

For $p=2$, the theorem follows at once since every mark of the $G F\left[2^{2 n}\right]$ is a square. For $p>2$, we are to determine in what cases 107) has as its solutions in the $G F\left[p^{2 n}\right]$ only squares. A common solution of the pair of equations

$$
\alpha^{m\left(p^{n}-1\right)}=1, \quad \alpha^{p^{2 n}-1}=1
$$

is required to be a solution of $a^{\frac{1}{2}\left(p^{2 n}-1\right)}=1$. A common solution of 108) satisfies $a^{d\left(p^{n}-1\right)}=1$, where $d$ is the greatest common divisor of $m$ and $p^{n}+1$. The condition is therefore that $d$ shall divide $\frac{1}{2}\left(p^{n}+1\right)$. It is satisfied if, and only if, $p^{n}+1$ contains 2 to a higher power than $m$ does. 
Corollary. - If $g=1$ or 2 according as the order of $M^{\prime}$ is equal or is double the order of $A\left(2 m, p^{n}\right)$, the number of subgroups of $H A\left(2 m, p^{2 n}\right)$ conjugate with $A\left(2 m, p^{n}\right)$ is

$$
\begin{gathered}
H A\left[2 m, p^{2 n}\right] \div g \cdot A\left[2 m, p^{n}\right] \equiv \\
\frac{a}{g q}\left(p^{n(2 m-1)}+1\right) p^{n(2 m-2)}\left(p^{n(2 m-3)}+1\right) p^{n(2 m-4)} \ldots\left(p^{3 n}+1\right) p^{2 n},
\end{gathered}
$$

where $a=1$ if $p=2, a=2$ if $p>2$, and $q$ denotes the greatest common divisor of $2 m$ and $p^{n}+1$.

134. The conditions that the quaternary substitution in the $G F\left[p^{2 n}\right]$

$$
\begin{array}{ll}
\xi_{1}^{\prime}=\alpha_{11} \xi_{1}+\alpha_{13} \xi_{3}, & \xi_{2}^{\prime}=\alpha_{22} \xi_{2}+\alpha_{24} \xi_{4}, \\
\xi_{3}^{\prime}=\alpha_{31} \xi_{1}+\alpha_{33} \xi_{3}, & \xi_{4}^{\prime}=\alpha_{42} \xi_{2}+\alpha_{44} \xi_{4},
\end{array}
$$

shall be hyperabelian include the following:

$$
\begin{aligned}
& \alpha_{11} \alpha_{22}^{p^{n}}+\alpha_{31} \alpha_{42}^{p^{n}}=1, \quad \alpha_{11} \alpha_{24}^{p^{n}}+\alpha_{31} \alpha_{44}^{p^{n}}=0, \\
& \alpha_{13} \alpha_{22}^{p^{n}}+\alpha_{33} \alpha_{42}^{p^{n}}=0, \quad \alpha_{13} \alpha_{24}^{p^{n}}+\alpha_{33} \alpha_{44}^{p^{n}}=1 .
\end{aligned}
$$

Setting $\Delta \equiv \alpha_{22} \alpha_{44}-\alpha_{24} \alpha_{42}$, we find from these conditions that

$$
\alpha_{11} \Delta^{p^{n}}=\alpha_{44}^{p^{n}}, \quad \alpha_{31} \Delta^{p^{n}}=-\alpha_{24}^{p^{n}}, \quad \alpha_{13} \Delta^{p^{n}}=-\alpha_{42}^{p^{n}}, \quad \alpha_{33} \Delta^{p^{n}}=\alpha_{22}^{p^{n}} .
$$

The above substitution then takes the form

$$
T:\left\{\begin{array}{l}
\xi_{1}^{\prime}=\left(\frac{\alpha_{44}}{\Delta}\right)^{p^{n}} \xi_{1}-\left(\frac{\alpha_{49}}{\Delta}\right)^{p^{n}} \xi_{3}, \quad \xi_{2}^{\prime}=\alpha_{22} \xi_{2}+\alpha_{24} \xi_{4}, \\
\xi_{3}^{\prime}=-\left(\frac{\alpha_{24}}{\Delta}\right)^{p^{n}} \xi_{1}+\left(\frac{\alpha_{22}}{\Delta}\right)^{p^{n}} \xi_{3}, \quad \xi_{4}^{\prime}=\alpha_{42} \xi_{2}+\alpha_{44} \xi_{4} .
\end{array}\right.
$$

Inversely, the substitution $T$ is seen to leave absolutely invariant

$$
\xi_{1} \xi_{2}^{p^{n}}-\xi_{2} \xi_{1}^{p^{n}}+\xi_{3} \xi_{4}^{p^{n}}-\xi_{4} \xi_{3}^{p^{n}}
$$

if $\alpha_{22}, \alpha_{24}, \alpha_{42}, \alpha_{44}$ belong to the $G F\left[p^{2 n}\right]$, so that $T$ belongs to $H\left(4, p^{2} n\right)$. The totality of the substitutions $T$ forms a group $G$ holoedrically isomorphic with the general binary linear group $G L H\left(2, p^{2 n}\right)$. Among the substitutions $T$ occur the simple ones of the form

$$
\begin{aligned}
T_{1}: & \xi_{1}^{\prime}=A^{-p^{n}} \xi_{1}, \quad \xi_{3}^{\prime}=-B^{p^{n}} \xi_{1}+A^{p^{n}} \xi_{3}, \\
& \xi_{2}^{\prime}=A \xi_{2}+B \xi_{4}, \quad \xi_{4}^{\prime}=A^{-1} \xi_{4},
\end{aligned}
$$

where $A$ and $B$ are arbitrary marks of the $G F\left[p^{2 n}\right]$ such that $A \neq 0$.

We proceed to determine every hyperabelian substitution

$S:$

$$
\xi_{i}^{\prime}=\sum_{j=1}^{4} \alpha_{i j} \xi_{j}
$$

which transforms the subgroup $G$ into itself. The product $S^{-1} T_{1} S$, 


$$
\begin{aligned}
\xi_{i}^{\prime}= & \left(\alpha_{i 1} A^{-p^{n}}-\alpha_{i 3} B^{p^{n}}\right) \sum_{j=1,2}\left(\alpha_{2 j 2}^{p^{n}} \xi_{2 j-1}-\alpha_{2 j-12}^{p^{n}} \xi_{2 j}\right) \\
& +\alpha_{i 2} A \sum_{j}\left(-\alpha_{2 j 1}^{p^{n}} \xi_{2 j-1}+\alpha_{2 j-11}^{p^{n}} \xi_{2 j}\right)+\alpha_{i 3} A^{p^{n}} \sum_{j}\left(\alpha_{2 j 4}^{p^{n}} \xi_{2 j-1}-\alpha_{2 j-14}^{p^{n}} \xi_{2 j}\right) \\
& +\left(\alpha_{i 2} B+\alpha_{i 4} A^{-1}\right) \sum_{j}\left(-\alpha_{2 j 3}^{p^{n}} \xi_{2 j-1}+\alpha_{2 j-13}^{p^{n}} \xi_{2 j}\right),
\end{aligned}
$$

must belong to $G$. Hence the coefficient of $\xi_{2 j-1}$ must vanish if $i$ be even and that of $\xi_{2 j}$ if $i$ be odd. Taking first $B=0$, we find, after dropping the common factor $(-1)^{k}$,

$$
\alpha_{i 1} \alpha_{k 2}^{p^{n}} A^{-p^{n}}-\alpha_{i 2} \alpha_{k 1}^{p^{n}} A+\alpha_{i 3} \alpha_{k 4}^{p^{n}} A^{p^{n}}-\alpha_{i 4} \alpha_{k 3}^{p^{n}} A^{-1}=0,
$$

where $i$ and $k$ are both even or both odd.

If $p^{n}>2$, this leads to an equation in $A$ of degree $2 p^{n}<p^{2 n}-1$. Being true for every $A \neq 0$, it is therefore an identity, so that

$$
\alpha_{i 1} \alpha_{k 2}=0, \quad \alpha_{i 3} \alpha_{k 4}=0 \quad(i, k \text { both even ox both odd }) .
$$

Taking next the terms in $B$, which can have two values $\neq 0$, we find

$$
\alpha_{i 3} \alpha_{k 2}=0 \quad(i, k \text { both even or both odd }) .
$$

Similarly, if $S$ transform the following substitution of the form $T$,

$$
\begin{array}{ll}
T_{2}: & \xi_{1}^{\prime}=D^{p^{n}} \xi_{1}-C^{p^{n}} \xi_{3}, \quad \xi_{3}^{\prime}=D^{-p^{n}} \xi_{3}, \\
& \xi_{2}^{\prime}=D^{-1} \xi_{2}, \quad \xi_{4}^{\prime}=C \xi_{2}+D \xi_{4},
\end{array}
$$

into a substitution of $G$, we find from the terms in $C$ that

$$
\alpha_{i 1} \alpha_{k 4}=0 \quad(i, k \text { both even or both odd). }
$$

If any $\alpha_{i j} \neq 0, i$ and $j$ being both even or both odd, the substitution $S$ reduces to the form 109) and must therefore belong to $G$. In fact, the relations 110$), 111$ ) and 112), holding if $p^{n}>2$, may be combined as follows:

$$
\text { 113) } \alpha_{2 i-12 l-1} \alpha_{2 k-12 \lambda}=0, \quad \alpha_{2 i 2 l-1} \alpha_{2 k 2 \lambda}=0 \quad(i, l, k, \lambda=1,2) \text {. }
$$

Hence, if $\alpha_{2 i_{1}-12 l_{1}-1} \neq 0$, we get $\alpha_{2 k-12 \lambda}=0(k, \lambda=1,2)$. Then, for fixed $\lambda, \alpha_{2 k 2 \lambda}$ is not zero for both $k=1$ and $k=2$, since otherwise all the coefficients in the $2 \lambda^{\text {th }}$ column would be zero and therefore the determinant of $S$ would vanish. It follows therefore from the second set of relations 113) that $\alpha_{2 i 2 l-1}=0(i, l=1,2)$. Hence $S$ has the form 109). Similarly, the hypothesis $\alpha_{2 k_{1} 2 \lambda_{1}} \neq 0$ requires, successively,

$$
\alpha_{2 i 2 l-1}=0 \quad(i, l=1,2) ; \quad \alpha_{2 k-12 \lambda}=0 \quad(k, \lambda=1,2) .
$$


If every $\alpha_{i j}=0$, when $i$ and $j$ are both even or both odd, for $p^{n}>2, S$ reduces at once to the form

$$
\begin{array}{ll}
\xi_{1}^{\prime}=\alpha_{12} \xi_{2}+\alpha_{14} \xi_{4}, & \xi_{2}^{\prime}=\alpha_{21} \xi_{1}+\alpha_{23} \xi_{3}, \\
\xi_{3}^{\prime}=\alpha_{32} \xi_{2}+\alpha_{34} \xi_{4}, & \xi_{4}^{\prime}=\alpha_{41} \xi_{1}+\alpha_{43} \xi_{3} .
\end{array}
$$

This is of the form $V g$, where $g$ is of the form 109) and $V$ denotes the hyperabelian substitution not in $G$,

$$
V: \quad \xi_{1}^{\prime}=-\xi_{2}, \quad \xi_{2}^{\prime}=\xi_{1}, \quad \xi_{3}^{\prime}=-\xi_{4}, \quad \xi_{4}^{\prime}=\xi_{3} .
$$

The theorem stated below has thus been proven for $p^{n}>2$.

For $p^{n}>2$, we consider the reciprocal of $S$ and find the conditions corresponding to 111) and 112) that $S^{-1}$ shall transform $T_{1}$ and $T_{2}$ into substitutions belonging to $G$, viz.,

$$
\alpha_{1 i} \alpha_{4 k}=0, \quad \alpha_{2 i} \alpha_{3 k}=0 \quad(i, k \text { both even or both odd }) .
$$

By 111), 112), 114), $S$ must be of the form $g$ or $V g, g$ being of the type 109). To illustrate the method of proof, let $\alpha_{13} \neq 0$. Then $\alpha_{41}=\alpha_{43}=0$ by 114). Since $\alpha_{42}$ and $\alpha_{44}$ can therefore not both vanish, $\alpha_{12}=\alpha_{14}=0$ by 114). Likewise from 111) $\alpha_{12}=\alpha_{32}=0$, $\alpha_{23}=\alpha_{43}=0$. The hyperabelian condition involving the coefficients of the first and third rows then gives $\alpha_{13} \alpha_{34}^{p^{n}}=0$, whence $\alpha_{34}=0$. Then $\alpha_{31}$ and $\alpha_{33}$ can not both vanish, so that $\alpha_{21}=0$ by 114). Hence $S$ has the form 109).

The order of $G$ is $\left(p^{4 n}-1\right)\left(p^{4 n}-p^{2 n}\right)$ by $\S 99$. The order of $H\left(4, p^{2 n}\right)$ is $\left(p^{4 n}-1\right) p^{3 n}\left(p^{3 n}+1\right) p^{2 n}\left(p^{2 n}-1\right) p^{n}\left(p^{n}+1\right)$ by $\S 132$.

Theorem. - The quaternary hyperabelian substitutions $T$ with coefficients in the $G F\left[p^{2 n}\right]$ form a group $G$ holoedrically isomorphic with GLH $\left(2, p^{2 n}\right)$. The only substitutions of $H\left(4, p^{2 n}\right)$ which transform the subgroup $G$ into itself are of the form $T$ or $V T$. $H\left(4, p^{n}\right)$ contains exactly $N \equiv \frac{1}{\mathbf{2}}\left(p^{3 n}+1\right) p^{3 n}\left(p^{n}+1\right) p^{n}$ subgroups conjugate with $G$.

135. Consider the subgroup $H^{\prime}$ formed of the substitutions of $H\left(4, p^{2 n}\right)$ of determinant unity. By $\S 132$, its index is $p^{n}+1$. The determinant of the substitution $T$ is seen to equal $\Delta^{-p^{n}+1}$. Those substitutions $T$ in the $G F\left[p^{2 n}\right]$ whose determinant is unity form a group $G^{\prime}$ of order $\left(p^{4 n}-1\right) p^{2 n}\left(p^{n}-1\right)$. Since $T_{1}$ and $T_{2}$ are of determinant unity, the proof in $\S 134$ leads to the following theorem:

Within the group $H^{\prime}$ of quaternary hyperabelian substitutions in the $G F\left[p^{2 n}\right]$ of determinant unity, the subgroup $G^{\prime}$ of the substitutions $T$ of determinant unity forms one of a complete set of $N$ conjugate subgroups, each being holoedrically isomorphic with the group of binary 
Tinear substitutions in the $G F\left[p^{2 n}\right]$ with determinant in the $\left.\left.G F\right] p^{n}\right]$. The only substitutions of $H^{\prime}$ which transform $G^{\prime}$ into itself are the substitutions $g^{\prime}$ of $G^{\prime}$ and the products $V g^{\prime}$.

136. The substitutions $T$ for which $\Delta=1$ form a group $G_{1}$ holoedrically isomorphic with the group of binary linear homogeneous substitutions of determinant unity in the $G F\left[p^{2 n}\right]$. Since $G_{1}$ contains $T_{1}$ and $T_{2}$, it follows from $\S 134$ that $g^{\prime}$ and $V g^{\prime}\left(g^{\prime}\right.$ in $\left.G^{\prime}\right)$ are the only substitutions of $H^{\prime}$ which transform $G_{1}$ into itself. Hence $G_{1}$ is one of a complete set of $N$ conjugate subgroups of $H^{\prime}$.

For $p=2, H^{\prime}$ is the simple group $H A\left(4,2^{2 n}\right)$ and $G_{1}$ is the simple group $L F\left(2,2^{2 n}\right)$. For $p>2$, we pass from $H^{\prime}$ to the simple quotient-group $H A\left(4, p^{2 n}\right)$ by making the substitutions $T_{*}$ 106) correspond to the identity. In particular, $T_{-1}$ corresponds to the identity, so that $G_{1}$ becomes $L F\left(2, p^{2 n}\right)$. The only $T_{\%}$ belonging to $G_{1}$ are $T_{-1}$ and the identity. We have therefore proven the

Theorem. - The simple group $H A\left(4, p^{2 n}\right)$ contains a complete set of $\frac{1}{2}\left(p^{3 n}+1\right) p^{3 n}\left(p^{n}+1\right) p^{n}$ simple conjugate subgroups $L F\left(2, p^{2 n}\right)$.

13\%. Theorem. - The group of hyperabelian substitutions $S$ of determinant unity on 2 indices with coefficients in the $G F\left[p^{2 n}\right]$ is identical with the group of binary linear substitutions of determinant unity with coefficients in the $G F\left[p^{n}\right]$.

For $m=1$, the conditions 98) and 99) that $S$ shall be hyperabelian are

$$
\begin{aligned}
& \alpha_{11} \alpha_{22}^{p^{n}}-\alpha_{21} \alpha_{12}^{p^{n}}=1, \quad \alpha_{11} \alpha_{21}^{p^{n}}-\alpha_{21} \alpha_{11}^{p^{n}}=0, \quad \alpha_{12} \alpha_{22}^{p^{n}}-\alpha_{22} \alpha_{12}^{p^{n}}=0, \\
& \alpha_{11}^{p^{n}} \alpha_{22}-\alpha_{12}^{p^{n}} \alpha_{21}=1, \quad \alpha_{11}^{p^{n}} \alpha_{12}-\alpha_{12}^{p^{n}} \alpha_{11}=0, \quad \alpha_{21}^{p^{n}} \alpha_{22}-\alpha_{22}^{p^{n}} \alpha_{21}=0 .
\end{aligned}
$$

Hence the products $\alpha_{11} \alpha_{22}^{p^{n}}, \alpha_{11} \alpha_{21}^{p^{n}}, \alpha_{11} \alpha_{12}^{p^{n}}$ belong to the $G F\left[p^{n}\right]$, being equal to their own $\left(p^{n}\right)^{\text {th }}$ powers. Hence if $\alpha_{11} \neq 0$, the ratios of $\alpha_{22}, \alpha_{21}, \alpha_{12}$ to $\alpha_{11}$ all belong to the $G F\left[p^{n}\right]$. Similarly, the products $\alpha_{22} \alpha_{11}^{p^{n}}, \alpha_{22} \alpha_{12}^{p^{n}}, \alpha_{22} \alpha_{21}^{p^{n}}$ all belong to the $G F\left[p^{n}\right]$ and therefore, if $\alpha_{22} \neq 0$, the ratios of $\alpha_{21}, \alpha_{12}, \alpha_{11}$ to $\alpha_{22}$ all belong to the $G F\left[p^{n}\right]$. Finally, if $\alpha_{11}=\alpha_{22}=0$, we have $\alpha_{21} \alpha_{12}^{p^{n}}=-1$, so that the ratio of $\alpha_{21}$ to $\alpha_{12}$ belongs to the $G F\left[p^{n}\right]$. In every case, $S$ has the form

$$
\xi_{1}^{\prime}=\alpha\left(a_{11} \xi_{1}+a_{12} \xi_{2}\right), \quad \xi_{2}^{\prime}=\alpha\left(a_{21} \xi_{1}+a_{22} \xi_{2}\right)
$$

where the $a_{i j}$ belong to the GF[p $\left.p^{n}\right]$. Since it is to be hyperabelian and since it is to have determinant unity, we have the respective relations

$$
\alpha^{p^{n}+1}\left(a_{11} a_{22}-a_{12} a_{21}\right)=1, \quad \alpha^{2}\left(a_{11} a_{22}-a_{12} a_{21}\right)=1 .
$$

Hence, by division, $\alpha^{p^{n}-1}=1$, or $\alpha$ belongs to the $G F\left[p^{n}\right]$. 
Corollary I. - $H A\left(2, p^{2 n}\right) \equiv A\left(2, p^{n}\right) \equiv L F\left(2, p^{n}\right)$.

Corollary II. - The group of all binary hyperabelian substitutions in the $G F\left[p^{2 n}\right]$ taken fractionally is the group of all linear fractional substitutions in the $G F\left[p^{n}\right]$.

138. In virtue of the transformation of indices,

$$
\eta_{1} \equiv J \xi_{1}+\xi_{2}, \quad \eta_{2} \equiv \varrho J^{p^{n}} \xi_{1}+\varrho \xi_{2},
$$

where $J$ and $\varrho$ are primitive roots of the respective equations

$$
J^{p^{n}+1}=1, \quad \varrho^{p^{n}+1}=-1,
$$

we have the following identity

$$
\eta_{1}^{p^{n}+1}+\eta_{2}^{p^{n}+1} \equiv\left(J-J^{p^{n}}\right)\left(\xi_{1} \xi_{2}^{p^{n}}-\xi_{2} \xi_{1}^{p^{n}}\right)
$$

Hence the hyperabelian group on $2 m$ indices with coefficients in the $G F\left[p^{2 n}\right]$ is holoedrically isomorphic with the group on $2 m$ indices in the $G F\left[p^{2 n}\right]$ defined by the invariant

$$
\eta_{1}^{p^{n}+1}+\eta_{2}^{p^{n}+1}+\cdots+\eta_{2 m}^{p^{n}+1}
$$

\section{CHAPTER V.}

\section{THE HYPERORTHOGONAL AND RELATED LINEAR GROUPS. ${ }^{1}$ )}

139. We first investigate the linear homogeneous group in the $G F\left[p^{n}\right]$ defined by an absolute invariant of the general type

$$
\Phi_{r} \equiv \lambda_{1} \xi_{1}^{r}+\lambda_{2} \xi_{2}^{r}+\cdots+\lambda_{m} \xi_{m}^{r},
$$

where each $\lambda$ is a mark $\neq 0$ of the $G F\left[p^{n}\right]$.

If $r=p^{\rho} r_{1}$, we have in the $G F\left[p^{n}\right]$ the identity

$$
\Phi_{r} \equiv \sum_{i=1}^{m} \lambda_{i} \xi_{i}^{r}=\left(\sum_{i=1}^{m} \lambda_{i}^{\prime} \xi_{i}^{r_{1}}\right)^{p^{\varphi}} \quad\left[\lambda_{i}^{\prime} \equiv \lambda_{i}^{p-\rho}\right]
$$

Hence a substitution which leaves $\Phi_{r}$ absolutely invariant will at most multiply the function

$$
\Phi_{r_{1}}^{\prime} \equiv \sum_{i=1}^{m} \lambda_{i}^{\prime} \xi_{i}^{r_{1}}
$$

by a mark $\eta$ which satisfies the equations

1) Dickson, Mathematische Annalen, vol. 52, pp. 561-581. 


$$
\eta^{p^{0}}=1, \quad \eta^{p^{n}-1}=1,
$$

from which $\eta=1$. We may therefore limit our discussion to the case in which $r$ is prime to $p$.

In order that the linear substitution on $m>1$ indices

$$
S: \quad \xi_{i}^{\prime}=\sum_{j=1}^{m} \alpha_{i j} \xi_{j} \quad(i=1, \ldots, m)
$$

shall leave $\Phi_{r}$ formally invariant, the following conditions upon its coefficients must be satisfied $\left.{ }^{1}\right)$ :

$$
\begin{gathered}
\sum_{i=1}^{m} \lambda_{i} \alpha_{i j}^{r}=\lambda_{j} \quad(j=1, \ldots, m) \\
\frac{r !}{r_{1} ! r_{2} ! \ldots r_{s} !} \sum_{i=1}^{m} \lambda_{i} \alpha_{i j_{1}}^{r_{1}} \alpha_{i j_{2}}^{r_{2}} \ldots \alpha_{i j_{s}}^{r_{s}}=0,
\end{gathered}
$$

holding for every partition of $r$ into $s$ integral parts

$$
r \equiv r_{1}+r_{2}+\cdots+r_{s}, \quad m>s>1,
$$

while for each partition $j_{1}, j_{2} \ldots, j_{s}$ may take every combination of $s$ distinct integers chosen from $1,2, \ldots, m$.

If $r$ be not divisible by $p$, the inverse of $S$ is

$$
S^{-1}: \quad \xi_{k}^{\prime}=\frac{1}{\lambda_{k}} \sum_{i=1}^{m} \lambda_{i} \alpha_{i k}^{r-1} \xi_{i} \quad(k=1, \ldots, m) .
$$

Indeed, the product $S S^{-1}$ replaces $\xi_{k}$ by

$$
\frac{1}{\lambda_{k}} \sum_{j=1}^{m}\left(\sum_{i=1}^{m} \lambda_{i} \alpha_{i k}^{r-1} \alpha_{i j}\right) \xi_{j} \equiv \xi_{k}
$$

upon applying 115) and 116) for $r_{1}=r-1, r_{2}=1$.

140. Theorem. - If $r>2$, if $r$ be not a multiple of $p$, and if $r-1$ be not a power of $p$, the only linear homogeneous substitutions in the $G F\left[p^{n}\right]$ which leave $\Phi_{r}$ invariant are those which merely permute the terms $\lambda_{1} \xi_{1}^{r}, \ldots, \lambda_{m} \xi_{m}^{r}$ amongst themselves.

Consider for $r>2$ the following equations of the set 116), in which $j_{1}$ and $j_{2}$ denote two arbitrarily fixed distinct integers $\overline{<} m$ :

1) If, as in $\S 97$, the indices are to belong to the $G F\left[p^{n}\right]$ so that the invariance of $\Phi_{r}$ is numerical and not formal, we must take $r<p^{n}$ in order that our results shall still hold true. Cf. § 152 . 


$$
\begin{aligned}
r \sum_{i=1}^{m}\left(\lambda_{i} \alpha_{i j_{1}}^{r-2} \alpha_{i j_{2}}\right) \alpha_{i j_{1}} & =0 \\
\frac{1}{2} r(r-1) \sum_{i=1}^{m}\left(\lambda_{i} \alpha_{i j_{2}}^{r-2} \alpha_{i j_{2}}\right) \alpha_{i j_{2}} & =0 \\
r(r-1) \sum_{i=1}^{m}\left(\lambda_{i} \alpha_{i j_{1}}^{r-2} \alpha_{i j_{2}}\right) \alpha_{i j_{3}} & =0 \quad\left(j_{3}=1, \ldots, m ; j_{3} \neq j_{1}, j_{2}\right) .
\end{aligned}
$$

If neither $r$ nor $r-1$ is divisible by $p$, we may drop the numerical factors from these $m$ equations. ${ }^{1}$ ) But

$$
\left|\alpha_{i j}\right| \neq 0 \text {. }
$$$$
(i, j=1, \ldots, m)
$$

being the determinant of $S$. Hence we have

$$
\lambda_{i} \alpha_{i j_{1}}^{r-2} \alpha_{i j_{2}}=0 \quad\left(i, j_{1}, j_{2}=1, \ldots, m ; j_{1} \neq j_{2}\right) .
$$

Hence only one element of each row of the matrix for $S$ is not zero. The determinant of $S$ being not zero, the non-vanishing coefficients lie in different columns as well as in different rows. Hence $S$ merely permutes the terms of the sum $\Phi_{r}$.

Suppose next that $r-1$ is divisible by $p$ and set

$$
r-1=g p^{s}
$$

where $g$ is not divisible by $p$. We now consider the case $g>1$. We make use of the following equations of the set 116):

$\frac{\left(g p^{s}+1\right) !}{\left[(g-1) p^{s}+1\right] ! p^{s !}} \sum_{i=1}^{m}\left(\lambda_{i} \alpha_{i j_{1}}^{(g-1) p^{s}} \alpha_{i j_{2}}^{p^{s}}\right) \alpha_{i j_{1}}=0$,

$\frac{\left(g p^{s}+1\right) !}{\left[(g-1) p^{s}\right] !\left(p^{s}+1\right) !} \sum_{i=1}^{m}\left(\lambda_{i} \alpha_{i j_{1}}^{(g-1)} p^{s} \alpha_{i j_{2}}^{p^{s}}\right) \alpha_{i j_{2}}=0$

$\frac{\left(g p^{s}+1\right) !}{\left[(g-1) p^{s}\right] ! p^{s ! 1 !}} \sum_{i=1}^{m}\left(\lambda_{i} \alpha_{i j_{1}}^{(g-1) p^{s}} \alpha_{i j_{2}}^{p^{s}}\right) \alpha_{i j_{3}}=0 \quad\left(j_{3}=1, \ldots, m ; j_{3} \neq j_{1}, j_{2}\right)$

of which the first two alone occur when $m=2$. We may verify that the numerical factors are not divisible by $\left.p .{ }^{2}\right)$ Then, since $\left|\alpha_{i j}\right| \neq 0$

$$
\lambda_{i} \alpha_{i j_{1}}^{(g-1)} p^{s} \alpha_{i j_{2}}^{p^{s}}=0 \quad\left(i, j_{1}, j_{2}=1, \ldots, m ; j_{1} \neq j_{2}\right) .
$$

It follows as before that $S$ at most permutes the terms of $\Phi_{r}$.

1) If $m=2$, only the first two equations occur. The same conclusion follows in this case that was derived for $m>2$.

2) This result follows by inspection from a general theorem on the residue of a multinomial coefficient taken modulo $p$ given in the author's Dissertation, Annals of Mathematics, 1897, § 14, p. 75. 
141. If $r$ is not divisible by $p$ and if $r \neq p^{s}+1$, the structure of the largest linear homogeneous group leaving $\Phi_{r}(r>2)$ invariant is now evident. Indeed, the group has as a self-conjugate subgroup the commutative group of the substitutions

$$
\xi_{i}^{\prime}=\alpha_{i i} \xi_{i} \quad(i=1, \ldots, m) \quad\left[\alpha_{i i}^{r}=1\right],
$$

the quotient-group being the symmetric group on the $m$ letters $\xi_{i}$.

142. Theorem. - The structure of the linear group in the $G F\left[p^{n}\right]$ which is defined by the absolute invariant $\Phi_{r}, r \equiv p^{s}+1>2$, results immediately from the structures of the groups in the $G F\left[p^{2 s}\right]$ defined by absolute invariants of the type

$$
\Phi \equiv \xi_{1}^{p^{8}+1}+\xi_{2}^{p^{8}+1}+\cdots+\xi_{m}^{p^{s}+1} .
$$

For the case $r=p^{s}+1$, the conditions that $S$ shall leave $\Phi_{r}$ invariant may be derived as special cases of 115) and 116), but are given by inspection from the identity,

$$
\sum_{i=1}^{m} \lambda_{i}\left(\sum_{j=1}^{m} \alpha_{i j} \xi_{j}\right)^{p^{8}+1} \equiv \sum_{i=1}^{m} \lambda_{i}\left(\sum_{i=1}^{m} \alpha_{i j}^{p^{s}} \xi_{j}^{x^{s}}\right)\left(\sum_{k=1}^{m} \alpha_{i k} \xi_{k}\right)=\sum_{i=1}^{m} \lambda_{i} \xi_{i}^{p^{8}+1} .
$$

By either method, the conditions in question are seen to be:

$$
\begin{aligned}
& \sum_{i=1}^{m} \lambda_{i} \alpha_{i j}^{p^{s}+1}=\lambda_{j} \quad(j=1, \ldots, m), \\
& \sum_{i=1}^{m} \lambda_{i} \alpha_{i j}^{p^{s}} \alpha_{i k}=0 \quad(j, k=1, \ldots, m ; j \neq k) .
\end{aligned}
$$

By $\S 139$, the inverse of $S$ has the form

$$
S^{-1}: \quad \xi_{i}^{\prime}=\sum_{j=1}^{m} \frac{\lambda_{j}}{\lambda_{i}} \alpha_{j i}^{p^{s}} \xi_{j} \quad(i=1, \ldots, m) .
$$

By the same rule, the inverse of the latter substitution is

$$
\xi_{i}^{\prime}=\sum_{j=1}^{m} \frac{\lambda_{j}}{\lambda_{i}}\left(\frac{\lambda_{i}}{\lambda_{j}} \alpha_{i j}^{p^{s}}\right)^{p^{s}} \xi_{j} \quad(i=1, \ldots, m) .
$$

Hence this substitution must be identical with $S$. Hence

$$
\left(\frac{\lambda_{i}}{\lambda_{j}}\right)^{p^{s}-1} \alpha_{i j}^{p^{2 s}}=\alpha_{i j} \quad(i, j=1, \ldots, m) .
$$

The determinant of $S^{-1}$ is

$$
\left|\frac{\lambda_{j}}{\lambda_{i}} \alpha_{j i}^{p^{s}}\right| \equiv\left|\alpha_{j i}^{p^{s}}\right|=\left|\alpha_{j i}\right| p^{s} \quad(i, j=1, \ldots, m) .
$$

DICKSON, Linear Groups. 
Hence, since the product $S S^{-1}=1$ has the determinant unity, we have

$$
\left|\alpha_{i j}\right| p^{s}+1=1 \text {. }
$$

From the form of the reciprocal $S^{-1}$, it follows that

$$
\frac{\lambda_{j}}{\lambda_{i}} \alpha_{j i}^{p^{s}}=\frac{\mathrm{A}_{j i}}{D} \quad(i, j=1, \ldots, m)
$$

where $A_{j i}$ denotes the adjoint of $\alpha_{j i}$ in the determinant

$$
D \equiv\left|\alpha_{i j}\right| \quad(i, j=1, \ldots, m) .
$$

The value of $n$, defining the $G F\left[p^{n}\right]$ to which the coefficients of our substitution $S$ and the quantities $\lambda_{i}$ were assumed to belong, has played no part in the above formulae. We proceed to prove that our problem can be reduced to a series of similar ones in which $n=2 s$. Consider the $G F\left[p^{2 n s}\right]$, which includes the $G F\left[p^{n}\right]$ and the $G F\left[p^{2 s}\right]$. Raising 119) to the power $\frac{p^{2 n s}-1}{p^{2 s}-1}$, we have

$$
\left(\frac{\lambda_{i}}{\lambda_{j}}\right)^{\frac{p^{2 n s}-1}{p^{s}+1}}=1
$$

if $\alpha_{i j} \neq 0$. Hence $\frac{\lambda_{i}}{\lambda_{j}}$ would be the power $p^{s}+1$ of some quantity. $\mu$ in the $G F\left[p^{2 n s}\right]$. The substitution $T_{j}$

$$
\xi_{k}^{\prime}=\xi_{k} \quad(k=1, \ldots, m ; k \neq j), \quad \xi_{j}^{\prime}=\mu \xi_{j}
$$

transforms $\varphi_{r}$ into

$$
\sum_{k=1=j}^{k=1 \ldots m} \lambda_{k} \xi_{k}^{p^{s}+1}+\lambda_{j}\left(\mu \xi_{j}\right)^{p^{s}+1} \equiv \sum_{k=1}^{m} \lambda_{k}^{\prime} \xi_{k}^{p^{s}+1}
$$

in which the coefficients $\lambda_{i}^{\prime}$ and $\lambda_{j}^{\prime}$ are equal. Evidently $T_{j}$ transforms $S$ into a substitution with coefficients in the $G F\left[p^{2 n s}\right]$.

Suppose that the coefficients $\alpha_{12}, \alpha_{13}, \ldots, \alpha_{1 m_{1}}$ do not vanish, while $\alpha_{1 j}=0$ for $j>m_{1}$, in all of the substitutions leaving $\varphi_{r}$ invariant. Then the group is isomorphic with a group of substitutions in the $G F\left[p^{2 n s}\right]$ leaving invariant

$$
\varphi_{r}^{\prime}=\sum_{k=1}^{m} \lambda_{k}^{\prime} \xi_{k}^{p^{s}+1} \quad\left(\lambda_{1}^{\prime}=\lambda_{2}^{\prime}=\cdots=\lambda_{m_{1}}^{\prime}\right) .
$$

In the latter substitutions the coefficients $\alpha_{1 j}\left(j>m_{1}\right)$ are all zero. If, among the coefficients $\alpha_{2 j}\left(j>m_{1}\right)$, any one as $\alpha_{2 j_{1}} \neq 0$, we transform the invariant $\varphi_{r}^{\prime}$ by $T_{j_{1}}$, giving the function

$$
\sum_{k=1}^{m} \lambda_{k}^{\prime \prime} \xi_{k}^{p^{s}+1} \quad\left(\lambda_{1}^{\prime \prime}=\lambda_{2}^{\prime \prime}=\cdots=\lambda_{m_{2}}^{\prime \prime}=\lambda_{j_{1}}^{\prime \prime}\right) .
$$


But this function is invariant under the transposition $\left(\xi_{1} \xi_{j_{1}}\right)$ and hence $\varphi_{r}$ must have been invariant under a substitution in which $\alpha_{1 j_{1}} \neq 0$. It follows that

$$
\alpha_{i j}=0 \quad\left(i=1, \ldots, m_{1} ; j=m_{1}+1, \ldots, m\right)
$$

in every substitution leaving $\varphi_{r}$ invariant. Considering the form of the reciprocal, we have

$$
\alpha_{j i}=0 \quad\left(i=1, \ldots, m_{1} ; j=m_{1}+1, \ldots, m\right) .
$$

Hence every substitution leaving $\varphi_{r}$ invariant is the product of two commutative substitutions, the one affecting the indices $\xi_{1}, \ldots, \xi_{m_{1}}$ only and leaving invariant

$$
\xi_{1}^{p^{s}+1}+\cdots+\xi_{m_{1}}^{p^{s}+1}
$$

and the other affecting only $\xi_{m_{1}+1}, \ldots, \xi_{m}$ and leaving invariant

$$
\sum_{i=m_{1}+1}^{m} \lambda_{i}^{\prime} \xi_{i}^{p^{s}+1}
$$

Proceeding with the latter substitutions in the same manner, it follows that the structure of the group in the $G F\left[p^{n}\right]$ leaving $\Phi_{r}$ invariant results immediately from the structures of various linear groups in the $G F\left[p^{2 n s}\right]$ defined by invariants of the type $\Phi$. But the relations 119) for substitutions of the latter groups become

$$
\alpha_{i j}^{p^{2 s}}=\alpha_{i j} \quad(i, j=1, \ldots, m) .
$$

Hence there is no limitation imposed in assuming that the field to which the substitutions belong is the $G F\left[p^{2 s}\right]$.

143. We designate by $G_{m, p, s}$ the group of all linear homogeneous $m$-ary substitutions in the $G F\left[p^{2 s}\right]$ which leave $\Phi$ invariant. For $p>2$, those of its substitutions whose coefficients belong to the $G F\left[p^{s}\right]$ constitute the first orthogonal group ${ }^{1}$ ) in the $G F\left[p^{s}\right]$ on $m$ indices. Indeed, relations 117) and 118), for $\lambda_{i}=1$, then become

$$
\sum_{i=1}^{m} \alpha_{i j}^{2}=1, \quad \sum_{i=1}^{m} \alpha_{i j} \alpha_{i k}=0 \quad(j, k=1, \ldots, m ; j \neq k) .
$$

The group $G_{m, p, s}$, having the orthogonal group as a subgroup, will be called the hyperorthogonal group in the $G F\left[p^{2 s}\right]$ on $m$ indices. We proceed to determine its structure, treating first the case $m=2$.

144. Theorem. - If $p^{s}>3$, the group of the substitutions of $G_{2, p, s}$ of determinant unity has a maximal invariant subgroup of order 1 or 2 according as $p=2$ or $p>2$; the quotient-group is $L F\left(2, p^{s}\right)$.

1) See Chapter VII, $\S 171$. For $p=2$, see Ex. 4 of $\S 210$. 
For $m=2$, we have by 117) and 121), when $\lambda_{1}=\lambda_{2}=1$,

$$
\alpha_{22}=D \alpha_{11}^{p^{s}}, \quad \alpha_{21}=-D \alpha_{12}^{p^{s}}, \quad \alpha_{11}^{p^{s}+1}+\alpha_{12}^{p^{s}+1}=1 .
$$

Inversely, every substitution satisfying these relations is seen to leave $\xi_{1}^{p^{s}+1}+\xi_{2}^{p^{s}+1}$ absolutely invariant. Every such substitution is the product of a substitution

$$
\left\{\begin{array}{l}
\xi_{1}^{\prime}=\alpha_{11} \xi_{1}+\alpha_{12} \xi_{2} \\
\xi_{2}^{\prime}=-\alpha_{12}^{p^{s}} \xi_{1}+\alpha_{11}^{p^{s}} \xi_{2}
\end{array} \quad\left(\alpha_{11}^{p^{s}+1}+\alpha_{12}^{p^{s}+1}=1\right)\right.
$$

by one of the $p^{3}+1$ distinct substitutions

$$
\xi_{1}^{\prime}=\xi_{1}, \quad \xi_{2}^{\prime}=D \xi_{2}
$$$$
\left(D^{p^{3}+1}=1\right) \text {. }
$$

The number of distinct substitutions 122$)$ is $\left(p^{2 s}-1\right) p^{s}$. Indeed, for the $p^{s}+1$ values of $\alpha_{12}$ for which $\alpha_{12}^{p^{s}+1}=1$, we must have $\alpha_{11}=0$; while for each of the remaining $\left(p^{2 s}-p^{3}-1\right)$ values of $\alpha_{12}$ in the $G F\left[p^{2 s}\right]$, there exist $p^{s}+1$ solutions in the field of

$$
\alpha_{11}^{p^{s}+1}=1-\alpha_{12}^{p^{s}+1}
$$

for, the second member belongs to the $G F\left[p^{s}\right]$ and is therefore the $p^{s}+1$ power of some mark in the $G F\left[p^{2 s}\right]$. But

$$
\left(p^{s}+1\right)+\left(p^{s}+1\right)\left(p^{2 s}-p^{s}-1\right)=\left(p^{s}+1\right)\left(p^{s}-1\right) p^{s} \text {. }
$$

The group of the substitutions 122) has an invariant subgroup of order 1 or 2 , according as $p=2$ or $p>2$, generated by the substitution

$$
C_{1} C_{2}: \quad \xi_{1}^{\prime}=-\xi_{1}, \quad \xi_{2}^{\prime}=-\xi_{2} .
$$

The quotient group (obtained concretely by taking the substitutions 122) fractionally) is, by $\$ \S 137-138$, simply isomorphic with the group of linear fractional substitutions of determinant unity in the $G F\left[p^{s}\right]$. By $\S 109$, it is a simple group when $p^{s}>3$.

Corollary. - Every binary hyperorthogonal substitution in the $G F\left[p^{2 s}\right]$ taken fractionally may be given the form

$$
\left(\frac{A}{-B^{p^{8}}}, \frac{B}{A^{p^{8}}}\right)
$$

of determinant a mark of the $G F\left[p^{8}\right]$, where $A, B$ belong to the $G F\left[p^{2 s}\right]$.

Indeed, since $D^{p^{s}+1}=1$, we may set $D=R^{p^{s}-1}, R$ belonging to the $G F\left[p^{2 s}\right]$. The fractional binary hyperabelian substitution becomes

$$
\left(\frac{\alpha_{11}}{-D \alpha_{12}^{p^{s}}}, \frac{\alpha_{12}}{D \alpha_{11}^{p^{s}}}\right) \equiv\left(\begin{array}{ll}
R \alpha_{11}, & R \alpha_{12} \\
\hline-R^{p^{s}} \alpha_{12}^{p^{s}}, & R^{p^{s}} \alpha_{11}^{p^{s}}
\end{array}\right)
$$


The group may be transformed into the group of all linear fractional substitutions in the $G F\left[p^{s}\right]$ (see $\S 138, \S 137$, corollary II).

145. For $m$ general, let $S$ be an arbitrary substitution of $G_{m, p, s}$, $S:$

$$
\xi_{i}^{\prime}=\sum_{j=1}^{m} \alpha_{i j} \xi_{j}
$$$$
(i=1, \ldots, m)
$$

By $\S 139$, its inverse is obtained by replacing $\alpha_{i j}$ by $\alpha_{j i}^{p^{8}}$. Hence the relations 117) and 118), for $\lambda_{i}=1$, when written for the inverse $S^{-1}$, give the equivalent set of conditions for the invariance of $\sum_{i=1}^{m} \xi_{l}^{p^{8}+1}$ :

$$
\begin{array}{ll}
\sum_{i=1}^{m} \alpha_{j i}^{p^{s}+1}=1 & (j=1, \ldots, m), \\
\sum_{i=1}^{m} \alpha_{j i} \alpha_{k i}^{p^{s}}=0 & \left(j, k=1, \ldots, m ; j \neq k_{i}\right) .
\end{array}
$$

By $\S 146$, the number of distinct linear functions

$$
f_{1} \equiv \sum_{j=1}^{m} \alpha_{1 j} \xi_{j}
$$

by which the substitutions of $G_{m, p, s}$ can replace $\xi_{1}$ is the number $\mathrm{P}_{m, p, s}$ of distinct sets of solutions in the $G F\left[p^{2 s}\right]$ of the equation

$$
\sum_{j=1}^{m} \alpha_{1 j}^{p^{s}+1}=1 .
$$

Let $T$ be a substitution of the group which replaces $\xi_{1}$ by a definite function $f_{1}$. Then, if $\Sigma, \Sigma^{\prime}, \ldots$ denote all of the $Q_{m, p, s}$ substitutions of the group which leave $\xi_{1}$ fixed, the products $T \Sigma, T \Sigma^{\prime}, \ldots$ and no other substitutions of the group will replace $\xi_{1}$ by $f_{1}$. Hence the order $\Omega_{m, p, s}$ of the group $G_{m, p, s}$ is

$$
\Omega_{m, p, s} \equiv Q_{m, p, s} P_{m, p, s} .
$$

But the substitutions $\Sigma, \Sigma^{\prime}, \ldots$, have

$$
\alpha_{11}=1, \quad \alpha_{1 i}=0 \quad(i=2, \ldots, m) .
$$

Hence by 124 ), for $j=1$, we have

$$
\alpha_{k 1}^{p^{s}}=0
$$$$
(k=2, \ldots, m)
$$

Hence $\Sigma, \Sigma^{\prime}, \ldots$ are substitutions of the group $G_{m-1, p, s}$ on the indices $\xi_{2}, \ldots, \xi_{m}$, so that $Q_{m, p, s} \equiv \Omega_{m-1, p, s}$. Hence, since 


$$
\Omega_{1, p, s}=p^{s}+1=\mathrm{P}_{1, p, s}
$$

is the number of substitutions affecting one index only, we have

$$
\Omega_{m, p, s}=\mathrm{P}_{m, p, s} \mathrm{P}_{m-1, p, s} \ldots \mathrm{P}_{1, p, s} .
$$

To evaluate $P_{n, p, s}$, the number of sets of solutions of

$$
\sum_{i=1}^{n} \eta_{i}^{p^{s}+1}=1
$$

we note that, for the $P_{n-1, p, s}$ sets of values of $\eta_{2}, \ldots, \eta_{n}$ which make $\sum_{i=2}^{n} \eta_{i}^{p^{s}+1}=1$, the corresponding value of $\eta_{1}$ is zero; while, for each of the $p^{2 s(n-1)}-\mathrm{P}_{n-1, p, s}$ sets of values in the $G F\left[p^{2 s}\right]$ for which that sum $\neq 1$, there exist $p^{s}+1$ values in the $G F\left[p^{2 s}\right]$ for $\eta_{1}$. Indeed,

$$
1-\sum_{i=2}^{n} \eta_{i}^{p^{s}+1}
$$

belongs to the $G F\left[p^{s}\right]$ and is therefore the power $p^{s}+1$ of a mark in the $G F\left[p^{2 s}\right]$. Hence we have

$$
\mathbf{P}_{n, p, s}=\mathbf{P}_{n-1, p, s}+\left(p^{s}+1\right)\left(p^{2 s(n-1)}-\mathbf{P}_{n-1, p, s}\right) .
$$

Since $\mathrm{P}_{1, p, s}=p^{s}+1$, we find by mathematical induction that

$$
\mathrm{P}_{n, p, s}=p^{s(2 n-1)}-(-1)^{n} p^{s(n-1)} \text {. }
$$

For another proof of this result, we consider only the case $p>2$. Then if $\nu$ be a not-square in the $G F\left[p^{s}\right]$, the $G F\left[p^{2 s}\right]$ may be defined by means of the irreducible equation

$$
I^{2}=\nu \quad\left(I^{p^{s}}=-I\right) .
$$

Setting

we have

$$
\eta_{i} \equiv \alpha_{i}+\beta_{i} I
$$$$
(i=1, \ldots, n)
$$

Hence

$$
\eta_{i}^{p^{\beta}} \equiv \alpha_{i}-\beta_{i} I \text {. }
$$

$$
\sum_{i=1}^{n} \eta_{i}^{p^{s}+1} \equiv \sum_{i=1}^{n}\left(\alpha_{\iota}^{2}-\nu \beta_{i}^{2}\right)=1 .
$$

By $\S 65$, this quadratic equation has $p^{s(2 n-1)}-(-1)^{n} p^{s(n-1)}$ sets of solutions $\alpha_{1}, \ldots, \alpha_{n}, \beta_{1}, \ldots, \boldsymbol{\beta}_{n}$ in the $G F\left[p^{s}\right]$. Hence $\Omega_{m, p, s}$ equals $\left[p^{s m}-(-1)^{m}\right] p^{s(m-1)}\left[p^{s(m-1)}-(-1)^{m-1}\right] p^{s(m-2)} \ldots\left[p^{2 s}-1\right] p^{s}\left[p^{s}+1\right]$.

146. Theorem. - If $\alpha_{11}, \alpha_{12}, \ldots, \alpha_{1 m}$ be any system of solutions in the GF[ $\left[p^{2 s}\right]$ of the equation 125), there exists a substitution $S$ in the group $G_{m, p, s}$ which replaces $\xi_{1}$ by 


$$
f_{1} \equiv \sum_{j=1}^{m} \alpha_{1 j} \xi_{j}
$$

and which is generated by the following substitutions [in which only the indices altered are written]:

$$
\begin{aligned}
& T_{i, \tau}: \quad \xi_{i}^{\prime}=\tau \xi_{i} \quad\left(\tau p^{s}+1=1\right), \\
& O_{i, j}^{\alpha, \beta}: \quad \quad\left\{\begin{array}{l}
\xi_{i}^{l}=\alpha \xi_{i}+\beta \xi_{j} \\
\xi_{j}^{\prime}=-\beta x^{s} \xi_{i}+\alpha x^{s} \xi_{j}
\end{array} \quad\left(\alpha^{p^{s}+1}+\beta x^{s}+1=1\right),\right.
\end{aligned}
$$

an additional generator being necessary if $p^{s}=2, m \geq 3$, viz.,

$$
W: \quad\left\{\begin{array}{l}
\xi_{1}^{\prime}=\xi_{1}+\xi_{2}+\xi_{3} \\
\xi_{2}^{\prime}=\xi_{1}+I \xi_{2}+I^{2} \xi_{3} \\
\xi_{3}^{\prime}=\xi_{1}+I^{2} \xi_{2}+I \xi_{3}
\end{array} \quad\left[I^{2} \equiv I+1(\bmod 2)\right] .\right.
$$

If $m=1$, we may take $S=T_{1, \alpha_{11}}$. If $m=2$, we take

$$
S=O_{1,2}^{\alpha_{11}, \alpha_{12}}
$$

If $m>2$, we prove the proposition by induction. Suppose first that the $\alpha_{1 i}^{p^{s}+1}(i=1, \ldots, m)$ are not all unity, for example,

$$
1-\alpha_{12}^{p^{s}+1} \neq 0 \text {. }
$$

The left member belongs to the $G F\left[p^{s}\right]$. Hence we may write

$$
\alpha_{12}^{p^{s}+1}+\mu^{p^{s}+1}=1
$$

$\mu$ being a mark $\neq 0$ in the $G F\left[p^{2 s}\right]$. The group therefore contains a substitution of the form $O_{1,2}^{\mu, \alpha_{12}}$. By 125) and 126), we have

$$
\left(\frac{\alpha_{11}}{\mu}\right)^{p^{s}+1}+\left(\frac{\alpha_{13}}{\mu}\right)^{p^{s}+1}+\cdots+\left(\frac{\alpha_{1 m}}{\mu}\right)^{p^{s}+1}=1
$$

Assuming our theorem to be true for $m-1$ indices, the group contains a substitution $S^{\prime}$ replacing $\xi_{1}$ by

$$
\frac{\alpha_{11}}{\mu} \xi_{1}+\frac{\alpha_{13}}{\mu} \xi_{3}+\cdots+\frac{\alpha_{1 m}}{\mu} \xi_{m} .
$$

Hence the product $S \equiv S^{\prime} O_{1,2}^{\mu, \alpha_{12}}$ will replace $\xi_{1}$ by $f_{1}$.

Suppose on the contrary that

$$
\alpha_{1 i}^{p^{s}}+1=1 \quad(i=1,2, \ldots, m) .
$$

If the group contains a substitution $S_{1}$ replacing $\xi_{1}$ by $\xi_{1}+\xi_{2}+\cdots+\xi_{m}$, the product

$$
S \equiv T_{1, \alpha_{11}} T_{2, \alpha_{22}} \ldots T_{m, \alpha_{1 m}} S_{1} \text {. }
$$

will replace $\xi_{1}$ by $f_{1}$. But the group will contain a substitution of the form $S_{1}$ if it contains $S_{2} \equiv O_{1,2}^{\alpha, \beta} S_{1}$, which replaces $\xi_{1}$ by

$$
\left(\alpha-\beta^{p^{s}}\right) \xi_{1}+\left(\beta+\alpha^{p^{s}}\right) \xi_{2}+\xi_{3}+\cdots+\xi_{m} .
$$


If $p \neq 2$, we can take $\alpha=\beta^{p^{s}}$, since the condition

$$
\alpha^{p^{s}+1}+\beta^{p^{s}+1} \equiv 2 \beta^{p^{s}+1}=1
$$

can be satisfied by a mark $\beta$ in the $G F\left[p^{2 s}\right]$. In this case, $S_{2} O_{2,1}^{0,1}$ replaces $\xi_{2}$ by the function

$$
2 \beta \xi_{2}+\xi_{3}+\cdots+\xi_{m},
$$

and therefore belongs to the group by our assumption on $m-1$ indices. If $p=2, s>1$, we can choose $\alpha$ and $\beta$ among the sets of solutions in the $G F\left[p^{2 s}\right]$ of

$$
\alpha^{p^{s}+1}+\beta^{p^{s}+1}=1
$$

in such a manner that

$$
\left(\alpha-\beta^{p^{s}}\right)^{p^{s}+1} \equiv \alpha^{p^{s}+1}+\beta^{p^{s}+1}-\alpha \beta-\alpha^{p^{s}} \beta^{p^{s}} \neq 1 .
$$

Indeed, the condition is (since $p=2$ ),

$$
\alpha^{p^{s}} \beta^{p^{s}} \neq \alpha \beta \text {. }
$$

Since $p^{s}>2$, we may take for $\alpha$ a mark neither zero nor unity in the $G F\left[p^{s}\right]$ and then determine a solution $\beta$ of 127) such that $\beta \neq \beta^{p^{s}}$. Then will $\alpha^{p^{s}} \beta^{p^{s}} \neq \alpha \beta$. To prove that such a choice for $\beta$ is possible, we note first that

$$
\alpha^{p^{s}}=\alpha, \quad \alpha^{2} \neq \alpha ; \quad \text { hence } \alpha^{p^{s}+1} \neq 1, \quad \beta \neq 0 .
$$

Further, if $\alpha^{\prime}, \beta^{\prime}$ be one set of solutions of 127), then is also $\alpha^{\prime}, \tau \beta^{\prime}$, where $\tau$ is any root of

$$
\tau x^{s}+1=1 \text {. }
$$

Not every root $\tau$ belongs to the $G F\left[p^{s}\right]$, and therefore not every solution $\beta$ corresponding to a given $\alpha$ belongs to the $\dot{G} F\left[p^{s}\right]$. Hence, if $p=2, p^{s}>2$, we may suppose that in the substitution $S_{2}$ the coefficient $\alpha_{11}$ is such that $\alpha_{11}^{p^{s}+1} \neq 1$, when the proposition follows as above.

For $p^{s}=2$, an additional generator $W$, for example, is necessary since the only substitutions of the form $O_{1,2}^{\alpha, \beta}$ are the products

$$
T_{1, \varrho} T_{2, \varrho}-1 \text { and }\left(\xi_{1} \xi_{2}\right) T_{1, \varrho} T_{2, \rho}-1 \quad\left(\varrho^{3}=1\right) \text {. }
$$

Indeed, there exists in the GF[2] only six sets of solutions of

$$
\alpha^{3}+\beta^{3}=1
$$

viz., $\alpha=\varrho, \beta=0$ and $\alpha=0, \beta=\varrho$, where $\rho^{3}=1$. Hence the substitutions $T_{i, \tau}$ and $O_{i, j}^{\alpha, \beta}$ can not combine to give a substitution replacing $\xi_{1}$ by $\xi_{1}+\xi_{2}+\xi_{3}$, for example. It follows readily that the additional generator $W$ is sufficient, together with the substitutions $T$ and $O$, to generate the group $G_{m, 2,1}$. 
14\%. Lemma. - If a substitution $S$ of the group $G_{m, p, s}$ be commutative with $O_{r, t}^{\alpha, \beta}$, for certain values of $\alpha$, then the following coefficients of $S$ must be zero, $\alpha_{r j}, \alpha_{t j}, \quad \alpha_{j r}, \alpha_{j t}$

$$
(j=1, \ldots, m ; j \neq r, t) .
$$

Among the conditions for the identity $S O_{r, t}^{\alpha, \beta}=O_{r, t}^{\alpha, \beta} S$ occur

$$
\left\{\begin{array} { c } 
{ ( \alpha - 1 ) \alpha _ { r j } + \beta \alpha _ { t j } = 0 , } \\
{ - \beta ^ { p ^ { s } } \alpha _ { r j } + ( \alpha ^ { p ^ { s } } - 1 ) \alpha _ { t j } = 0 , }
\end{array} \quad \left\{\begin{array}{r}
(\alpha-1) \alpha_{j r}-\beta^{p^{s}} \alpha_{j t}=0, \\
\beta \alpha_{j r}+\left(\alpha^{\gamma^{s}}-1\right) \alpha_{j t}=0 \\
(j=1, \ldots, m ; j \neq r, t) .
\end{array}\right.\right.
$$

Hence the theorem follows if the determinant

$$
(\alpha-1)\left(\alpha^{p^{s}}-1\right)+\beta^{p^{4}}+1 \equiv 2-\alpha-\alpha^{p^{4}} \neq 0 .
$$

The equation $2-\alpha-\alpha^{p^{s}}=0$ has $p^{s}$ solutions in the $G F\left[p^{2 s}\right]$; indeed,

$$
\alpha^{p^{2 s}}=(2-\alpha)^{p^{s}}=2-\alpha p^{s}=\alpha .
$$

But for $\alpha$ arbitrary there exists a mark $\beta$ in the $G F\left[p^{2 s}\right]$ such that

$$
\alpha^{p^{s}+1}+\beta^{p^{s}+1}=1 .
$$

Hence there are sets of solutions $\alpha, \beta$ for which the above determinant does not vanish, as well as sets for which it vanishes.

Note. Another statement of our result is that $S$ breaks up into the product of a substitution affecting only $\xi_{r}$ and $\xi_{t}$ by a substitution affecting only $\xi_{j}(j=1, \ldots, m ; j \neq r, t)$.

148. We proceed to determine the structure of the group $G_{m, p, s}$ of order $\Omega_{m, p, s}$. For $m=1$, the group is a commutative (cyclic) group of order $p^{s}+1$. For $m=2$, its structure was determined in $\S 144$.

The substitutions of $G_{m, p, s}$ of determinant $D=1$ form an invariant subgroup $\mathrm{H}_{m, p, s}$ of order $\Omega_{m, p, s} /\left(p^{s}+1\right)$. Indeed, we have shown that $D$ must be a root of

$$
D^{p^{s}+1}=1 .
$$

Inversely, substitutions do exist in the group $G_{m, p, s}$ having as determinants every root of 120); for example, $T_{1, \tau}$ and its powers, where $\tau$ is a primitive root of 120). Hence the factors of composition of $G_{m, p, s}$ are those of $H_{m, p, s}$ together with the prime factors of $p^{s}+1$.

Supposing $m \overline{>}$, let $I$ be an invariant subgroup of $\mathrm{H}_{m, p, s}$ containing a substitution

$S$ :

not of the form

$$
\xi_{i}^{\prime}=\sum_{j=1}^{m} \alpha_{i j} \xi_{j} \quad(i=1, \ldots, m)
$$

$T:$

$$
\xi_{i}^{\prime}=\tau \xi_{i} \quad(i=1, \ldots, m)
$$

$\left[\tau^{p^{s}+1}=1, \tau^{m}=1\right]$ 
With the single exception $m=3, p^{9}=2$, when $\mathrm{H}_{3,2,1}$ is of order 72 , we shall prove that $I$ coincides with $H$. Therefore the substitutions $T$ form a cyclic group of order $d$, the greatest common divisor of $m$ and $p^{s}+1$, which is the maximal invariant subgroup of $\mathrm{H}_{m, p, s}$. Hence the quotient-group gives a simple group of order $\frac{\Omega m, p, s}{d\left(p^{s}+1\right)}$. We shall designate it by the symbol $H O\left(m, p^{2 s}\right)$.

149. Theorem. - There exists in the group I a substitution replacing $\xi_{1}$ by $x \xi_{1}+\sigma \xi_{2}$ and not reducing to the identity.

Suppose that $\alpha_{13} \neq 0$, for example. Transforming $S$ by $O_{2,3}^{\lambda, \mu}$, we obtain a substitution $S^{\prime}$ replacing $\xi_{1}$ by

$$
\alpha_{11} \xi_{1}+\left(\alpha_{12} \lambda p^{s}+\alpha_{13} \mu p^{s}\right) \xi_{2}+\left(-\mu \alpha_{12}+\lambda \alpha_{13}\right) \xi_{3}+\sum_{j=4}^{m} \alpha_{1 j} \xi_{j} .
$$

To make the coefficient of $\xi_{3}$ zero, we have the conditions

$$
\lambda=\frac{\alpha_{12}}{\alpha_{13}} \mu, \quad \lambda^{p^{s}+1}+\mu^{p^{s}+1}=1 .
$$

The condition for $\mu$ is therefore

$$
\mu^{p^{s}+1}\left(\alpha_{12}^{p^{s}+1}+\alpha_{13}^{p^{s}+1}\right)=\alpha_{13}^{p^{s}+1} .
$$

Unless $\alpha_{1}^{p^{s}+1}+\alpha_{13}^{p^{s}+1}=0$, there exists a solution $\mu$ in the $G F\left[p^{2 s}\right]$ of this relation; indeed, the value of $\mu^{p^{s}+1}$ belongs to the $G F\left[p^{s}\right]$ and is therefore the $\left(p^{s}+1\right)^{s t}$ power of a quantity $\mu$ in the $G F\left[p^{2 s}\right]$.

It follows that we can assume that the only coefficients $\alpha_{1 j}(j>1)$ which do not vanish are $\alpha_{12}, \ldots, \alpha_{1 m_{1}}$ and that, if $m_{1}>2$, they have the property that

$$
\alpha_{1 j}^{p^{s}+1}+\alpha_{1 k}^{p^{s}+1}=0 \quad\left(j, k=2, \ldots, m_{1} ; j \neq k\right) .
$$

If $m_{1}=2$, the theorem is proven. If $m_{1}>3$, the terms in 128) must all be equal and therefore zero unless $p=2$. Supposing first that $p \neq 2$, our theorem is proven unless $m_{1}=3$, when we have

$$
\alpha_{11}^{p^{s}+1}=1, \quad \alpha_{12}^{p^{s}+1}+\alpha_{13}^{p^{s}+1}=0, \quad \alpha_{1 j}=0 \quad(j=4, \ldots, m) .
$$

In the latter case we may assume that not both

$$
\alpha_{11}^{p^{s}+1}+\alpha_{1 i}^{p^{s}+1}=0
$$$$
(i=2,3) \text {; }
$$

for, if so, $\alpha_{12}^{p^{s}+1}=\alpha_{13}^{p^{s}+1}$ and hence each is zero by 129), since $p \neq 2$. For definiteness, let

$$
\alpha_{11}^{p^{s}+1}+\alpha_{j 2}^{p^{8}+1} \neq 0 .
$$

If the left member be unity, then $\alpha_{12}=0$ by 129) and the theorem is proven. Suppose therefore that the left member is neither zero nor unity and consider the substitution 


$$
\bar{S} \equiv S^{-1} C_{1} C_{2} S C_{1} C_{2} \equiv S_{\alpha} C_{1} C_{2}
$$

where $S_{\alpha} \doteq S^{-1} C_{1} C_{2} S$ is seen to be the substitution

$$
\xi_{i}^{\prime}=\xi_{i}-2 \alpha_{i 1} \sum_{j=1}^{m} \alpha_{j 1}^{p^{\beta}} \xi_{j}-2 \alpha_{i 2} \sum_{j=1}^{m} \alpha_{j 2}^{p^{\beta}} \xi_{j} \quad(i=1, \ldots, m) .
$$

The coefficient $\bar{\alpha}_{11}$ in $\bar{S}$ is therefore

Hence

$$
\bar{\alpha}_{11} \equiv-\left(1-2 \alpha_{11}^{p^{s}+1}-2 \alpha_{12}^{p^{4}}+1\right) \text {. }
$$

$$
\bar{\alpha}_{11}^{p^{s}}+1 \equiv \bar{\alpha}_{11}^{p^{s}} \bar{\alpha}_{11}=\left(1-2 \alpha_{11}^{p^{s}}+1-2 \alpha_{12}^{p^{s}}+1\right)^{2} \text {, }
$$

which $\neq 1$ since $\alpha_{11}^{p^{s}+1}+\alpha_{12}^{p^{s}+1}$ is neither zero nor 1. Applying the above process to $\bar{S}$ in which $\bar{\alpha}_{11}^{p_{s}}+1 \neq 1$, we reach a substitution in the group $I$ in which all but one of the $\alpha_{1 j}(j=2, \ldots, m)$ are zero.

Suppose next that $p=2$. We have by 128)

$$
\alpha_{12}^{p^{s}+1}=\alpha_{13}^{p^{s}+1}=\cdots=\alpha_{1 m_{1}}^{p^{s}+1} \neq 0, \quad \alpha_{1 j}=0 \quad\left(j=m_{1}+1, \ldots, m\right) .
$$

The ratios of $\alpha_{12}, \alpha_{13}, \ldots, \alpha_{1 m_{1}}$ therefore satisfy the equation

$$
\tau^{p^{s}+1}=1 \text {. }
$$

Hence by transforming $S$ by suitable products of the form

$$
T_{1, \tau_{i}-1} T_{i, \tau_{i}} \quad(i=3, \ldots, m),
$$

where the $\tau_{i}$ are roots of 130 ), we reach a substitution $S^{\prime}$ belonging to $I$ in which $\alpha_{12}=\alpha_{13}=\cdots=\alpha_{1 m_{1}}$. Transforming $S^{\prime}$ by the reciprocal of $O_{2,3}^{\lambda, \mu}$, we obtain in $I$ a substitution $S^{\prime \prime}$ which replaces $\xi_{1}$ by

$$
\alpha_{11} \xi_{1}+\alpha_{12}\left\{\left(\lambda-\mu p^{s}\right) \xi_{2}+\left(\mu+\lambda p^{s}\right) \xi_{3}+\xi_{4}+\cdots+\xi_{m_{1}}\right\} .
$$

If $p^{s} \neq 2$, we can choose $\lambda$ and $\mu$ [see $\left.\S 146\right]$ such that

$$
\lambda^{p^{s}+1}+\mu^{p^{3}+1}=1, \quad\left(\lambda-\mu^{p^{s}}\right) p^{s}+1 \neq 1 .
$$

Hence in $S^{\prime \prime}$ the sum of the $\left(p^{s}+1\right)^{s t}$ powers of the coefficients $\alpha_{12}^{\prime \prime}$ and $\alpha_{14}^{\prime \prime}$ is not zero in the $G F\left[2^{2 s}\right]$. As above we can therefore make $\alpha_{14}^{\prime \prime}=0$. If $p^{s}=2$, we reach at once the same result by transforming $S^{\prime}$ by $\left(\xi_{1} \xi_{4}\right) W\left(\xi_{1} \xi_{4}\right), W$ being defined at the beginning of $\S 146$.

Repeating the process, we reach finally a substitution in $I$, not the identity, in which either

or else

$$
\alpha_{1 j}=0 \quad(j=3, \ldots, m)
$$

$$
\alpha_{12}^{p^{s}+1}=\alpha_{13}^{p^{s}+1}+0, \quad \alpha_{1 j}=0 \quad(j=4, \ldots, m) .
$$


In the latter case, the substitution $S$ thus obtained has (since $p=2$ )

$$
\alpha_{11}^{p^{s}}+1=1
$$

Transforming it by $T \equiv T_{1, \tau} T_{2, \tau}-1$, we obtain in $I$ the substitution

$$
S^{\prime} \equiv S^{-1} \cdot T^{-1} S T \equiv S_{1} T,
$$

where $S_{1}$ denotes the substitution

$$
\xi_{i}^{\prime}=\xi_{i}+\alpha_{i 1}\left(\tau^{-1}-1\right) \sum_{j=1}^{m} \alpha_{j 1}^{p^{s}} \xi_{j}+\alpha_{i 2}(\tau-1) \sum_{j=1}^{m} \alpha_{j 2}^{p^{s}} \xi_{j} .
$$

Hence for $S^{\prime} \equiv S_{1} T$ the coefficient of $\xi_{1}$ in $\xi_{1}^{\prime}$ is

$$
\bar{\alpha}_{11} \equiv \tau+(1-\tau) \alpha_{11}^{p^{s}}+1+\tau(\tau-1) \alpha_{12}^{p^{s}}+1=1+\tau(\tau-1) \alpha_{12}^{p^{s}}+1 .
$$

Setting for brevity $\alpha_{12}^{p^{s}+1} \equiv \alpha$, a mark $\neq 0$ in the $G F\left[p^{s}\right]$, we find, since $\tau^{p^{s}+1}=1$, that

$$
\bar{\alpha}_{11}^{p^{s}+1}=1+\alpha(\tau-1)\left(\tau^{p^{s}}-1\right)\left(\alpha-\tau^{p^{s}}-\tau-1\right) .
$$

Since the theorem follows as above if $\bar{\alpha}_{11}^{p^{s}+1} \neq 1$, we seek to prove that a value $\tau$ can be found for which

$$
\tau^{p^{s}+1}=1, \quad \tau \neq 1, \quad \tau^{p^{s}}-\tau-1 \neq \alpha .
$$

But a root of $\tau^{p^{s}+1}=1$ will satisfy

only when

$$
\begin{aligned}
& \tau^{p^{s}}-\tau-1=\alpha \\
& 1-\tau^{2}-\tau=\alpha \tau .
\end{aligned}
$$

The desired value of $\tau$ certainly exists if $p^{s}+1>3$. But if $p^{s}=2$, we have $\alpha=1$, whence the equation 131) has the single root $\tau=1$ in the $G F\left[2^{2}\right]$. The theorem has therefore been proven for all cases.

150. Theorem. - Excluding the case $m=3, p^{s}=2$, the group $I$ contains a substitution leaving one index fixed and not reducing to the identity.

By $\S 149, I$ contains a substitution $S \neq 1$ which replaces $\xi_{1}$ by a function of the form $x \xi_{1}+\sigma \xi_{2}$. Hence

$$
S=O_{1,2}^{*, \sigma} S_{1} \text {, }
$$

where $S_{1}$ is a substitution of $H_{m, p, s}$ of the form

$$
\xi_{1}^{\prime}=\xi_{1}, \xi_{i}^{\prime}=\sum_{j=2}^{m} \alpha_{i j}^{\prime} \xi_{j} \quad(i=2, \ldots, m) .
$$


Consider the substitution belonging to $\mathrm{H}$,

$$
T \equiv T_{1, \tau} T_{2, \tau} T_{i, \tau}-2
$$

where $i>2$. The group I will contain the product

$$
S^{\prime} \equiv S^{-1} T^{-1} S T \equiv S_{1}^{-1} T^{-1} S_{1} T,
$$

since $T$ and $O_{1,2}^{*, \sigma}$ are commutative. Since $S^{\prime}$ leaves $\xi_{1}$ fixed, our theorem is proven unless $S^{\prime}$ reduces to the identity. In the latter case, we find by comparing the values by which $S_{1} T$ und $T S_{1}$ replace $\xi_{2}$ that

$$
\alpha_{2 j}^{\prime}=0 \quad(j=3, \ldots, m ; j \neq i), \quad \tau \alpha_{2 i}^{\prime}=\tau^{-2} \alpha_{2 i}^{\prime} .
$$

If $m>3, i$ has at least two values and therefore

$$
\alpha_{2 j}^{\prime}=0 \quad(j=3, \ldots, m) .
$$

If $m=3$, the same result holds if $p^{s}>2$. For then a value of $\tau$ exists satisfying $\tau^{p^{3}+1}=1$ but not $\tau^{3}=1$. Hence must $\alpha_{2 i}^{\prime}=0$. Excluding the case $m=3, p^{s}=2$, it follows that $S_{1}$ (which was seen to leave $\xi_{1}$ fixed) alters $\xi_{2}$ at most by a constant factor $\lambda$. Hence

$$
S=O_{1,2}^{*, \pi} T_{2, \lambda} \Sigma \text {, }
$$

where $\Sigma$ leaves $\xi_{1}$ and $\xi_{2}$ fixed. Hence I contains

$$
S^{\prime} \equiv S^{-1}\left(T_{1 \tau} T_{2 \tau}-1\right)^{-1} S\left(T_{1 \tau} T_{2 \tau}-1\right) \quad\left[\tau^{p^{s}+1}=1\right]
$$

which leaves $\xi_{3}, \ldots, \xi_{m}$ fixed. If $S^{\prime} \neq 1$, the theorem is proven. If $S^{\prime}=1$, we find by comparing the values by which $S T_{1 \tau} T_{2 \tau}-1$ and $T_{1 \tau} T_{2 \tau}-1 S$ replace $\xi_{1}$ that

$$
\tau \sigma=\tau^{-1} \sigma \text {. }
$$

Hence, taking for $\tau$ a value for which $\tau^{2} \neq 1$, we have $\sigma=0$. The only case left for consideration is therefore that in which

$$
S=T_{1, x} T_{2, \varkappa-1} T_{2, \lambda} \Sigma \text {. }
$$

If $S^{\circ}$ be not commutative with every $O_{1,2}^{\alpha, \beta}$, we obtain at once a substitution $\neq 1$ in I which leaves $\xi_{3}, \ldots, \xi_{m}$ fixed. In the contrary case, $\lambda=x^{2}$, and therefore

$$
S=T_{1, \varkappa} T_{2, \varkappa}^{\prime} \Sigma
$$

If $m=3, \Sigma=T_{3, *}-2$, the determinant of $S$ being unity. Trans forming $S$ by $\left(\xi_{1} \xi_{3}\right) C_{1}$, we obtain the substitution

$$
S_{2}=T_{3, \varkappa} T_{2, \varkappa} T_{1, *}-2
$$

belonging to $\mathrm{I}$. Then I contains

$$
S S_{2}^{-1} \equiv T_{1, \varkappa^{3}} T_{3, *-3}
$$

leaving $\xi_{2}$ fixed and not reducing to the identity. For that requires $x^{3}=1$, when we should have

$$
S=T_{1, \varkappa} T_{2, \varkappa} T_{3, \varkappa}
$$

contrary to the hypothesis made in $\S 148$. 
Let $m \overline{>}$. If $\Sigma$ be not commutative with every

$$
O \equiv O_{i, j}^{\alpha, \beta} \quad\left(i, j=3, \ldots, m ; \alpha^{p^{s}+1}+\beta^{p^{s}+1}=1\right)
$$

then I contains the substitutions leaving $\xi_{1}$ and $\xi_{2}$ fixed,

$$
S^{-1} O^{-1} S O \equiv \Sigma^{-1} O^{-1} \Sigma O,
$$

not all of which reduce to the identity. In the contrary case, $\Sigma$ must, by $\S 147$, have the form

$$
\xi_{i}^{\prime}=\omega \xi_{i} \quad(i=3, \ldots, m) .
$$

Hence I contains the product

$$
S^{-1} C_{1}\left(\xi_{1} \xi_{3}\right) S\left(\xi_{1} \xi_{3}\right) C_{1}:\left\{\begin{array}{l}
\xi_{1}^{\prime}=\omega x^{-1} \xi_{1}, \quad \xi_{3}^{\prime}=\omega^{-1} \chi \xi_{3}, \\
\xi_{2}^{\prime}=\xi_{2}, \quad \xi_{i}^{\prime}=\xi_{i} \quad(i=4, \ldots, m),
\end{array}\right.
$$

which does not reduce to the identity; for, if so, $x=\omega$ and $S$ would, contrary to the hypothesis made in $\S 148$, have the form

$$
\xi_{i}^{\prime}=\omega \xi_{i} \quad(i=1,2, \ldots, m) .
$$

151. Theorem. Except in the case $m=3, p^{s}=2$, the group $I$ coincides with the group $\mathrm{H}_{m, p, s}$.

The proofs of the theorems of $\S \S 149-150$ hold for any value of $m \overline{>}$. Hence by a repeated application of these theorems, we finally reach in the group $I$ a substitution $S \neq 1$ leaving $m-2$ indices fixed and therefore of the form $O_{1,2}^{2, \sigma}$, we may assume. If it reduce to $C_{1} C_{2}$, when $p \neq 2$, its transformed by $O_{1,3}^{\alpha, \beta}$ gives the substitution

$$
O_{1,3}^{\alpha p^{\beta}+1}-\beta^{p^{\beta}+1},-2 \alpha \beta C_{1} C_{2},
$$

so that I will contain an $O_{1,3}$ neither the identity nor $C_{1} C_{3}$. Indeed, by $\S 144$, there exist solutions $\alpha \neq 0, \beta \neq 0$ in the $G F\left[p^{2 s}\right], p^{s}>2$, of the equation $\alpha^{p^{3}+1}+\beta^{p^{2}+1}=1$. Hence I contains a substitution $O_{1,2}^{*, \sigma}$ neither the identity nor $C_{1} C_{2}$. It follows then from $\S 144$ that, for $p^{s}>3$, I contains every substitution $O_{1,2}^{\alpha, \beta}$. Transforming by substitutions of the form $\left(\xi_{i} \xi_{j}\right) C_{i}$, we obtain in I every $O_{i, j}^{\alpha, \beta}$.

These substitutions suffice, except when $m \overline{>}, p^{s}=2$, to generate the group $\mathrm{H}_{m, p, s}$. Indeed, by applying the formula

$$
\left(O_{i, j}^{\alpha, \beta}\right)^{-1} T_{i, \tau} O_{i, j}^{\alpha, \beta} T_{i, \tau}^{-1} \equiv O_{i, j}^{\alpha^{\prime}, \beta^{\prime}}
$$

where

$$
\alpha^{\prime} \equiv \alpha^{p^{9}+1}+\tau^{-1} \beta^{p^{\natural}+1}, \quad \beta^{\prime} \equiv \alpha \beta\left(\tau^{-1}-1\right) ; \quad \tau^{p^{s}+1}=1,
$$

it follows from $\S 146$ that every substitution of $G_{m, p, s}$ has the form $h$ or $h T_{m, *}$ where $h$ is generated from the $O_{i, j}^{\alpha, \beta}$ and has determinant unity. Hence the substitutions of $\mathrm{H}_{m, p, s}$ (of determinant unity) are of the form $h$. 
For the case $p^{s}=3$, we first prove that I contains the substitution $C_{1} C_{2}$. We have shown that I contains an $O_{1,2}^{\alpha, \beta}$ not the identity and therefore $O_{1,2}^{\alpha^{\prime}, \beta^{\prime}}$ given by 132). If $\beta \neq 0$, we can make $\alpha^{\prime}=0$; indeed, if $\alpha$ be not itself zero, we have in the $G F\left[3^{2}\right]$

$$
\alpha^{4}=\beta^{4}=-1
$$

and we need only take $\tau=-1$. But the square of $O_{1,2}^{0, \beta^{\prime}}$ gives $C_{1} C_{2}$ since $\beta^{\prime 4}=1$ when $\alpha^{\prime}=0$. If, howerer, $\beta=0$, then $\alpha \neq 1$. If $\alpha=-1$, we have at once $O_{1,2}^{\alpha, 0}=C_{1} C_{2}$. If $\alpha \neq \pm 1$, then the square of $O_{1,2}^{\alpha, 0}$ gives $O_{1,2}^{\alpha, 0}=C_{1} C_{2}$.

Having $C_{1} C_{2}$, I contains (as above) the substitution

$$
O_{1,3}^{\lambda, \mu}, \quad \lambda \equiv \alpha^{p^{s}+1}-\beta^{p^{s}+1}, \quad \mu \equiv-2 \alpha \beta \equiv \alpha \beta(\bmod 3) .
$$

Taking for $\alpha$ and $\beta$ an arbitrary set of solutions of

$$
\alpha^{4}=-1, \quad \beta^{4}=-1, \quad \text { whence } \alpha^{4}+\beta^{4}=1,
$$

we have $O_{1,3}^{0, \mu}$ where $\mu=\alpha \beta$ is an arbitrary solution of $\mu^{4}=1$. Hence I contains

$$
O_{1,3}^{0, \mu}\left(O_{1,3}^{0,1}\right)^{-1} \equiv T_{1, u^{3}} T_{3, \mu} \text {. }
$$

Transforming the latter by $O_{1,2}^{\alpha, \beta}$, we obtain by 132 ),

$$
O_{1,2}^{\alpha^{\prime}, \beta^{\prime}} T_{1, \mu^{3}} T_{3, \mu} \quad\left(\begin{array}{c}
\alpha^{\prime} \equiv \alpha^{4}+\mu \beta^{4} \\
\beta^{\prime} \equiv \alpha \beta(\mu-1)
\end{array}\right) .
$$

Hence I contains every such $O_{1,2}^{\alpha^{\prime}, \beta^{\prime}}$. For $\alpha=0, \beta^{4}=1$, we have $\alpha^{\prime}=\mu, \beta^{\prime}=0 ;$ for $\alpha^{4}=-1, \beta^{4}=-1$, we have $\alpha^{\prime}=-1-\mu$. We have therefore reached in the group I every $O_{1,2}^{\prime, \sigma}$ in which $x=\mu$, $0, \pm 1+\mu$, where $\mu$ is an arbitrary one of the four roots of $\mu^{4}=1$. Defining the $G F\left[3^{2}\right]$ by the irreducible quadratic congruence,

$$
i^{2} \equiv-1 \quad(\bmod 3),
$$

we have $x=0, \pm 1, \pm i, \pm 1 \pm i$. Hence $x$ takes every value in the $G F\left[3^{2}\right]$. We thus reach all 24 substitutions $O_{1,2}^{\alpha, \sigma}$. It follows that I coincides with $\mathrm{H}_{m, 3,1}$.

For the case $p^{s}=2$, we have in I a substitution $O_{1,2}^{\alpha, \beta} \neq 1$. By the result at the end of $\S 146$, it must be one of the six substitutions

$$
T_{1, \varrho} T_{2, \rho}-1, \quad\left(\xi_{1} \xi_{2}\right) T_{1, \rho} T_{2, \rho}{ }^{-1} \quad\left(\varrho^{3}=1\right) .
$$

The transformed of the latter by $T_{1, \tau} T_{3, \tau}-1$ gives

$$
\left(\xi_{1} \xi_{2}\right) T_{1, \rho} T_{2, \rho}-1 \cdot T_{1, \tau} T_{2, \tau}-1 .
$$

Hence, in every case, I contains a substitution of the form

$$
\grave{T}_{1, \tau} T_{2, \tau}-1 \neq 1
$$


Its reciprocal gives $T_{1, \tau}-1 T_{2, \tau}$. If $m>3$, I contains

where

$$
\begin{aligned}
& W^{-1} T_{1, \tau} T_{4, \tau}-1 W \equiv\left(\begin{array}{ccc}
\tau & \tau+1 & \tau+1 \\
\tau+1 & \tau & \tau+1 \\
\tau+1 & \tau+1 & \tau
\end{array}\right) T_{4, \tau^{-1}} \\
& =T_{1, \tau} T_{2, \tau}-1 T_{3, \tau}-1 W T_{2, \tau} T_{3, \tau} T_{4, \tau}-1 \text {, }
\end{aligned}
$$

Hence I contains

$$
W \equiv\left(\begin{array}{ccc}
1 & 1 & 1 \\
1 & \tau & \tau^{2} \\
1 & \tau^{2} & \tau
\end{array}\right) \quad\left(\tau^{2}=\tau+1\right) .
$$

$$
T_{3, \tau}-1 W T_{2, \tau} \equiv T_{3, \tau}-1 T_{4, \tau} W T_{4, \tau}-1 T_{2, \tau}
$$

and therefore $W$. Hence I contains $W^{2}=\left(\xi_{2} \xi_{3}\right)$. Hence I coincides with $\mathrm{H}_{m, 2,1}$ if $m>3$.

152. Theorem. The group $G_{m, p, s}$ is isomorphic with a subgroup of the linear group $\left.{ }^{1}\right)$ on $2 m$ indices in the $G F\left[p^{i}\right]$ defined by a quadratic invariant

$$
\Psi \equiv \sum_{i=1}^{m}\left(x_{i}^{2}+y_{i}^{2}+\Theta x_{i} y_{i}\right)
$$

Indeed, we may define the $G F\left[p^{2 s}\right]$ by an equation of the form

$$
I^{2}-\Theta I+1=0
$$

belonging to and irreducible in the $G F\left[p^{\natural}\right]$. Its roots $I$ and $I^{p^{s}} \equiv I^{-1}$ belong to the $G F\left[p^{2 s}\right]$. Set

Then

$$
\xi_{i} \equiv x_{i}+I y_{i}, \quad \alpha_{i j}=a_{i j}+I c_{i j} \quad(i, j=1, \ldots, m) .
$$

$$
\xi_{i}^{p^{s}}=x_{i}+I^{-1} y_{i}, \quad \xi^{p^{s}+1}=x_{i}^{2}+y_{i}^{2}+\Theta x_{i} y_{i} .
$$

The invariant $\sum_{i=1}^{m} \dot{\xi}_{i}^{p^{s}+1}$ becomes the quadratic form $\psi$. The general substitution of $G_{m, p, s}$,

$S$ :

takes the following form

$$
\xi_{i}^{\prime}=\sum_{j=1}^{m} \alpha_{i j} \xi_{j} \quad(i=1, \ldots, m)
$$

$$
\left\{\begin{array}{l}
x_{i}^{\prime}=\sum_{j=1}^{m}\left(a_{i j} x_{j}-c_{i j} y_{j}\right) \\
y_{i}^{\prime}=\sum_{j=1}^{m}\left[c_{i j} x_{j}+\left(a_{i j}+\Theta c_{i j}\right) y_{j}\right]
\end{array} \quad(i=1, \ldots, m) .\right.
$$

1) Cf. Chapters VII and VIII. See also the note to $\$ 139$. 


\section{CHAPTER VI.}

\section{THE COMPOUNDS OF A LINEAR HOMOGENEOUS GROUP. ${ }^{1}$ )}

153. It was shown in $\S 98$ that the linear substitutions

$$
A: \quad \xi_{i}^{\prime}=\sum_{j=1}^{m} \alpha_{i j} \xi_{j} \quad(i=1, \ldots, m)
$$

combine according to the law

$$
A^{\prime \prime} \equiv A^{\prime} A: \quad \xi_{i}^{\prime}=\sum_{j=1}^{m} \alpha_{i j}^{\prime \prime} \xi_{j} \quad(i=1, \ldots, m)
$$

where

$$
\alpha_{i j}^{\prime \prime} \equiv \sum_{k=1}^{m} \alpha_{i k} \alpha_{k j}^{\prime} \quad(i, j=1, \ldots, m) .
$$

In Sylvester's umbral notation, the general $q^{\text {th }}$ minor of the determinant $\left|\alpha_{i j}\right|$ is as follows:

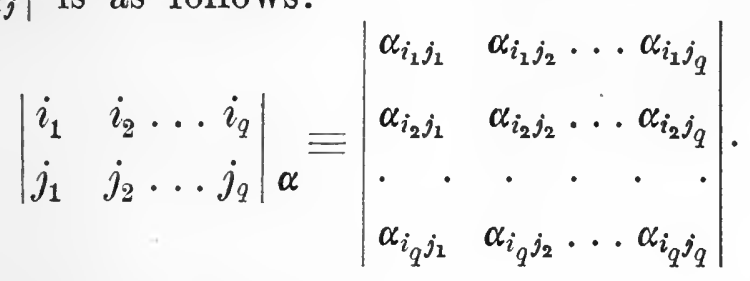

The formula expressing the $q^{\text {th }}$ minors of $\left|\alpha_{i j}^{\prime \prime}\right|$ in terms of the $q^{\text {th }}$ minors of $\left|\alpha_{i j}\right|$ and of $\left|\alpha_{i j}^{\prime}\right|$ is the following $\left.{ }^{2}\right)$ :

133)

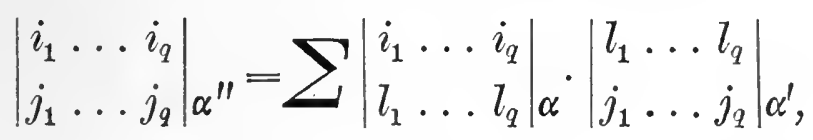

the summation extending over the $C_{m, q}$ combinations $l_{1}, l_{2}, \ldots, l_{q}$ of the $m$ integers $1,2, \ldots, m$ taken $q$ at a time.

Consider the linear homogeneous substitutions on $C_{m, q}$ variables $Y_{l_{1}, l_{2}, \ldots, l_{q}}$

$$
[\alpha]_{q}: \quad Y_{i_{1}, i_{2}, \ldots, i_{q}}^{\prime}=\sum_{l_{1}, \ldots, l_{q}}\left|\begin{array}{ll}
i_{1} & i_{2} \ldots i_{q} \\
l_{1} & l_{2} \ldots l_{q}
\end{array}\right|_{\alpha} Y_{l_{1}, l_{2}, \ldots, l_{q}}
$$

where the sets $\left(i_{1}, i_{2}, \ldots, i_{q}\right)$ and $\left(l_{1}, l_{2}, \ldots, l_{q}\right)$ take independently

1) This chapter gives a new exposition of results published by the author. in the following journals: Bulletin of the Amer. Math. Soc., vol. 5 (1898), pp. 120-135; Proceed. Lond. Math. Soc., vol. 30 (1898), pp. 70-98; Transactions of the Amer. Math. Soc., vol. 1 (1900), pp.91-96.

2) Scott, Theory of determinants, p. 53.

DICKsoN, Linear Groups. 
the $C_{m, q}$ combinations $q$ together of the integers $1,2, \ldots, m$ and where we suppose

$$
i_{1}<i_{2}<\ldots<i_{q}, \quad l_{1}<l_{2}<\ldots<l_{q} .
$$

The determinant of the substitution $[\alpha]_{7}$ is called the $q^{\text {th }}$ compound of the determinant $\left|\begin{array}{lll}1 & 2 \ldots m \\ 1 & 2 \ldots m\end{array}\right|_{\alpha}$ and equals ${ }^{1}$ ) the latter raised to the power $C_{m-1, q-1}$. In virtue of formula 133), we have the following formula of composition:

$$
\left[\alpha^{\prime \prime}\right]_{q}=\left[\alpha^{\prime}\right]_{q}[\alpha]_{q} .
$$

Hence if the substitutions $A \equiv\left(\alpha_{i j}\right)$ form a group $G_{m}$, the substitutions $[\alpha]_{q}$ form a group $G_{m, q}$ called "the $q^{\text {th }}$ compound of the $m$-ary group $G_{m}$ ". We may therefore state the theorem:

Any linear homogeneous group is isomorphic with each of its compounds.

154. Theorem. - The general linear homogeneous group $G L H\left(m, p^{n}\right)$ has $(d, 1)$ isomorphism with its $q^{\text {th }}$ compound, if $d$ be the greatest common divisor of $q$ and $p^{n}-1$.

We verify first that at least $d$ substitutions of $G_{m} \equiv G L H\left(m, p^{n}\right)$ correspond to the identical substitution in its $q^{\text {th }}$ compound $G_{m, 2}$. In fact, there exist in the $G F\left[p^{n}\right]$ exactly $d$ marks $\delta$ for which $\delta^{d}=1(\S 16)$. For every such mark $\delta$, the substitution belonging to $G_{m}$,

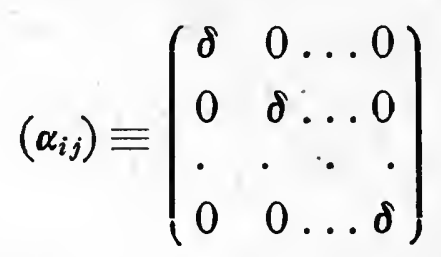

gives rise to the substitution $[\alpha]_{q} \equiv I$ in $G_{m, q}$.

To prove the inverse, consider the matrix $J$ formed of certain coefficients of the substitution $[\alpha]_{q}$, in which $q<j \overline{<} m$ :

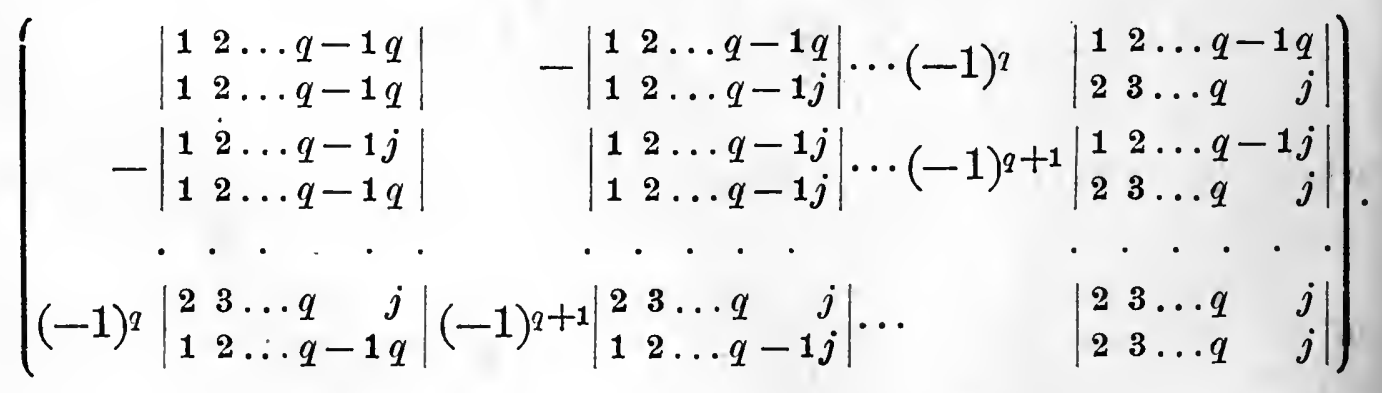

1) Muir, Theory of determinants, $\S 174$. 
Consider also the matrix $A$ of determinant $\Delta$,

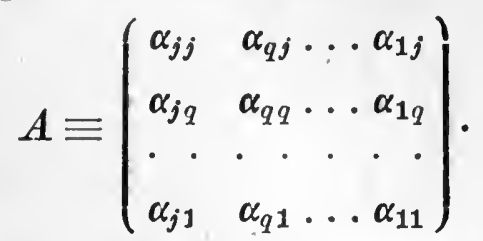

The composition of the matrices $J$ and $A$ gives the result

$$
J A \equiv\left(\begin{array}{cccc}
\Delta & 0 & \ldots & 0 \\
0 & \Delta & \ldots & 0 \\
\cdot & \cdot & \cdot & . \\
0 & 0 & \ldots & \Delta
\end{array}\right)
$$

We seek those substitutions of $G_{m}$ which correspond to the identity in $G_{m, q}$. Suppose, therefore, that $[\alpha]_{q}$ reduces to the identical substitution, so that the matrix $J$ is the identity. In this case we have

$$
\Delta^{q+1}=\Delta, \quad \alpha_{i k}=0, \quad \alpha_{i i}=\Delta \quad(i, k=1,2 \ldots, q, j ; i \neq k) .
$$

Taking in turn $j=q+1, q+2, \ldots, m$, we have the result

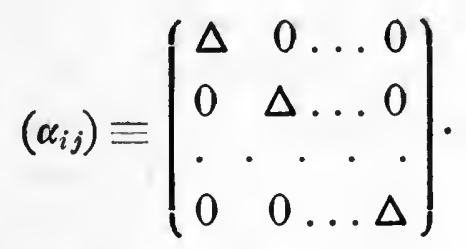

Hence $\Delta \neq 0$ and therefore $\Delta^{q}=1$.

155. Theorem. - The special linear homogeneous group $S L H\left(m, p^{n}\right)$ has $(g, 1)$ isomorphism with its $q^{\text {th }}$ compound, if $g$ denotes the greatest common divisor of $m, q, p^{n}-1$.

The proof is quite similar to that of the last section. The following $m$-ary substitution of determinant unity in the $G F\left[p^{n}\right]$,

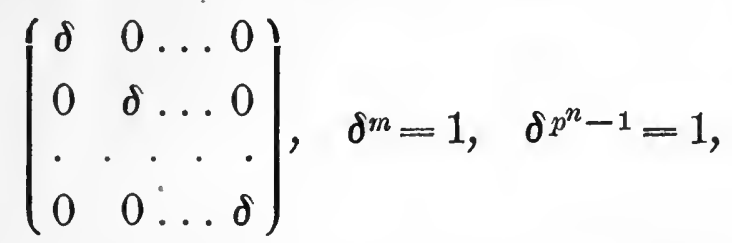

will give $[\alpha]_{q}=I$ only when $\delta^{q}=1$. Hence must $\delta^{g}=1$. The inverse is proven as above.

156. Theorem. - The second compound of the general linear homogeneous group $G L H\left(m, p^{n}\right)$ leaves invariant the Pfaffian

$$
\left[\begin{array}{lll}
1 & 2 \ldots m
\end{array}\right] \equiv\left|\begin{array}{ll}
Y_{12}, & Y_{13}, \ldots, Y_{1 m} \\
Y_{23}, \ldots, & Y_{2 m} \\
\cdot & \cdot \cdot \cdot \cdot \cdot \cdot \\
Y_{m-1 m}
\end{array}\right|
$$


The square of $\left[\begin{array}{llll}1 & 2 \ldots m\end{array}\right]$ is the skew-symmetric determinant

where $Y_{i j} \equiv-Y_{j i}$.

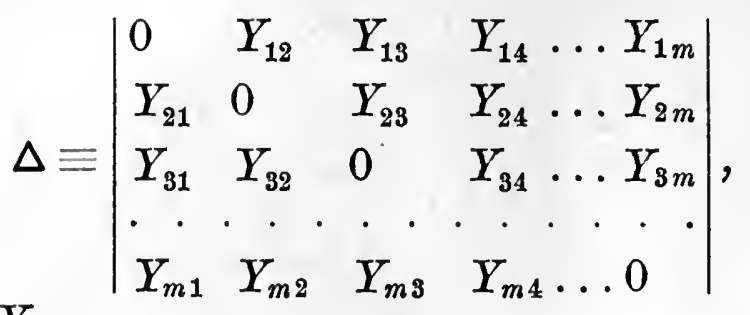

By $\S 100, G \equiv G L H\left(m, p^{n}\right)$ is generated by the substitutions $B_{r, s, \lambda}$ and $D_{m}$. The corresponding substitutions of the second compound $G_{m, 2}$ will therefore generate the latter group. To $B_{1,2, \lambda}$ and $D_{1}$ there correspond the respective substitutions of $G_{m, 2}$ :

$$
\begin{array}{rlr}
\bar{B}_{1,2, \lambda}: & \left\{\begin{array}{lr}
Y_{12}^{\prime}=Y_{12}, \quad Y_{1 i}^{\prime}=Y_{1 i}+\lambda Y_{2 i} & (i=3, \ldots, m) \\
Y_{i j}^{\prime}=Y_{i j} & (i, j=2, \ldots, m ; i<j) .
\end{array}\right. \\
\bar{D}_{1}: & Y_{1 j}^{\prime}=D Y_{1 j}(j=2, \ldots, m), & Y_{i j}=Y_{i j}(i, j=2, \ldots, m) .
\end{array}
$$

But $\Delta$ is unaltered by an interchange of any two subscripts as 1 with 3 ; for, the resulting determinant may be derived from $\Delta$ by interchanging the first and third rows and the first and third columns. It therefore suffices to prove that $\Delta$ remains invariant, up to a multiplicative constant, upon applying the substitutions $\bar{B}_{1,2,2}$ and $\bar{D}_{1}$. By inspection, $\bar{D}_{1}$ multiplies $\Delta$ by $D^{2}$. Also $\bar{B}_{1,2, \lambda}$ transforms $\Delta$ into the determinant

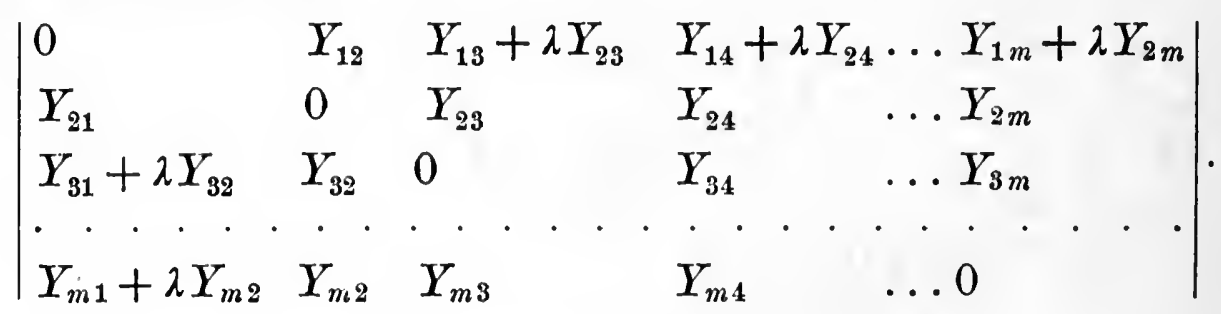

This reduces at once to $\Delta$ since $Y_{12}+Y_{21}=0$.

15\%. Theorem. - For $m$ odd, the substitution $[\alpha]_{2}$ of the second compound gives rise to the substitution

$$
F_{i}^{\prime}=\sum_{j=1}^{m} A_{i j} F_{j} \quad(i=1, \ldots, m)
$$

upon the Pfaffians $F_{j} \equiv\left[\begin{array}{lll}1 & 2 \ldots j-1 j+1 \ldots m\end{array}\right]$, if $A_{i j}$ denotes the minor ${ }^{1}$ ) complementary to $\alpha_{i j}$ in the determinant $\left|\alpha_{i j}\right|$.

1) Or the adjoint of $\alpha_{i j}$ without its prefixed sign. 
Consider the Pfaffian $F_{j}, j$ being a fixed integer $\overline{\overline{<}} m$. By the last section, it is unchanged by the substitutions

$$
\bar{B}_{r, j, \lambda} \quad(r, s=1,2, \ldots, j-1, j+1, \ldots, m) .
$$

Furthermore, $\bar{B}_{j, s, \lambda}$ alters no element of $F_{j}$ and hence leaves $F_{j}$ unchanged. Finally, we prove that $\bar{B}_{r, j, \lambda}$ replaces $F_{j}$ by

$$
F_{j}+(-1)^{j+r+1} \lambda F_{r}
$$

Indeed, $\bar{B}_{1, j, \lambda}$ replaces $F_{j}$ by

$$
\begin{aligned}
& Y_{12}+\lambda Y_{j 2}, \quad Y_{13}+\lambda Y_{j 3}, \ldots, Y_{1 j-1}+\lambda Y_{j j-1}, Y_{1 j+1}+\lambda Y_{j j+1}, \ldots, Y_{1 m}+\lambda Y_{j m} \mid \\
& Y_{23} \quad, \ldots, Y_{2 j-1} \quad, Y_{2 j+1} \quad, \ldots, Y_{2 m} \text {. } \\
& Y_{j-1 j+1} \quad, \ldots, Y_{j-1 m} \\
& \ldots, Y_{j+1 m} \\
& Y_{m-1 m}
\end{aligned}
$$

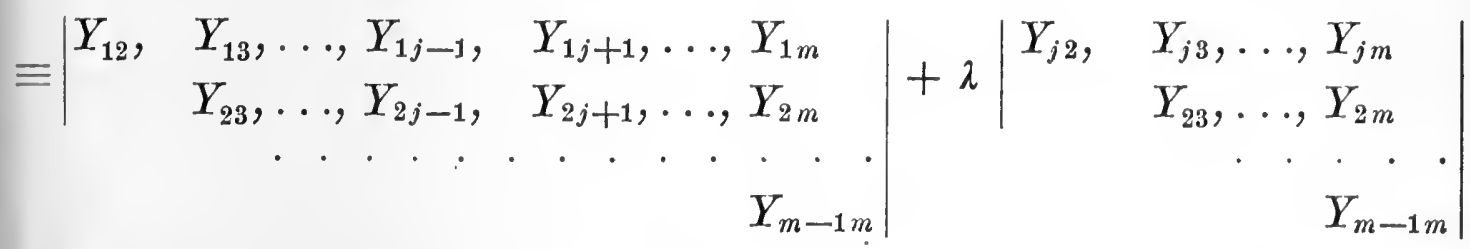

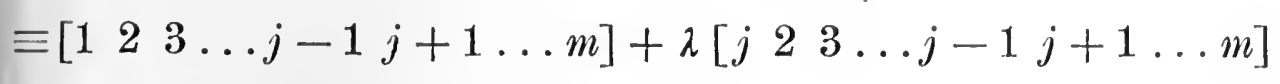$$
\equiv F_{j}+(-1)^{j-2} \lambda F_{1} \text {. }
$$

Interchanging 1 with $r$, we see that $\bar{B}_{r, j, \lambda}$ replaces by

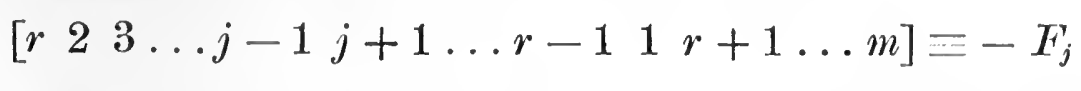$$
-F_{j}+(-1)^{j-2} \lambda\left[\begin{array}{llll}
2 & 3 \ldots r-1 & 1 & r+1 \ldots m
\end{array}\right] \equiv-F_{j}+(-1)^{j-2+r-2} \lambda F_{r} .
$$

Hence $B_{r, j, \lambda}$ induces upon the Pfaffians $F_{i}$ the substitution

$$
\begin{aligned}
S_{r j}: \quad F_{j}^{\prime}=F_{j}+(-1)^{j+r+1} \lambda F_{r}, \quad F_{i}^{\prime}=F_{i} \\
\\
(i=1, \ldots, j-1, j+1, \ldots, m) .
\end{aligned}
$$

By inspection, $\bar{D}_{j}$ gives rise to the substitution

$$
S_{j}: \quad F_{j}^{\prime}=F_{j}, \quad F_{i}^{\prime}=D F_{i} \quad(i=1, \ldots, j-1, j+1, \ldots, m) .
$$

Our theorem is therefore true for the particular substitutions $B_{r, s, \lambda}, D_{1}$ which generate the group $G_{m}$.

To complete the proof of the theorem, we show that, if $S \equiv\left(\alpha_{i j}\right)$ induces upon the $F_{i}$ the substitution 


$$
F_{i}^{\prime}=\sum_{j=1}^{m} A_{i j} F_{j}
$$

$$
(i=1, \ldots, m)
$$

where $A_{i j}$ is the minor of $\left|\alpha_{i j}\right|$ complementary to $\alpha_{i j}$, the products $D_{1} S$ and $B_{r, s, 2} S$ will induce upon the $F_{i}$ the substitutions called for by the theorem. First, the product $D_{1} S$ will induce upon the $F_{i}$ the substitution

$$
S_{1} \Sigma: \quad F_{i}^{\prime}=A_{i 1} F_{1}+\sum_{j=2}^{m} D A_{i j} F_{j} \quad(i=1 ; \ldots, m) .
$$

The matrices of the two products $D_{1} S$ and $S_{1} \Sigma$ are respectively

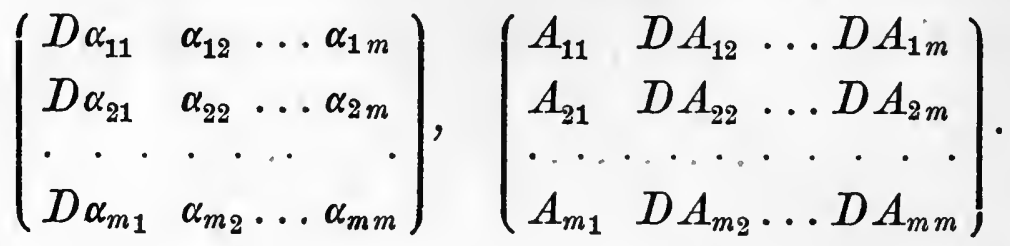

Here the second matrix is derived from the first by the law expressed by our theorem.

Next, the product $B_{r, s, \lambda} S$ induces upon the $F_{i}$ the substitution

$$
S_{r s} \Sigma: \quad F_{i}^{\prime}=\sum_{k=1}^{m} A_{i k} F_{k}+(-1)^{s+r+1} \lambda A_{i s} F_{r} \quad(i=1, \ldots, m) .
$$

The matrices of the two products $R_{r, s, \lambda} S$ and $S_{r, s} \Sigma$ are

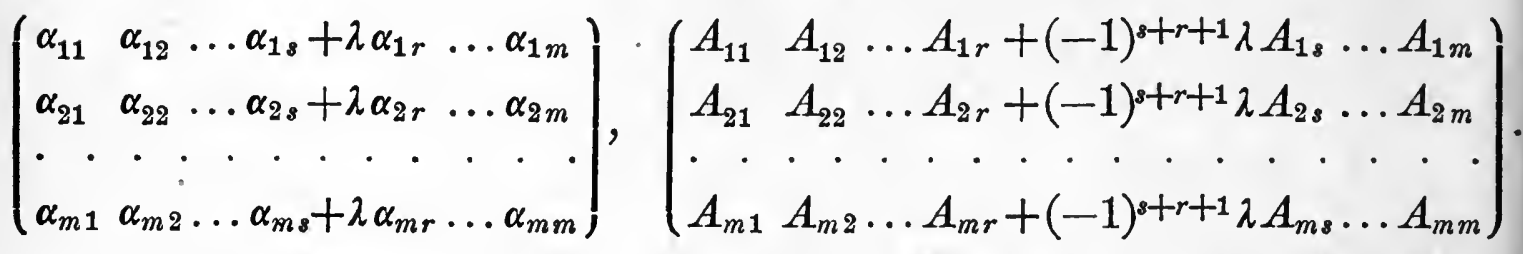

The second matrix is seen to be derived from the first according to the law expressed in the theorem.

Corollary. - The second compound of any linear homogeneous group $G_{m}$ gives rise to a linear group on the $m$ Pfaffians $F_{1}, \ldots, F_{m}$ which is identical with the $m-1^{s t}$ compound of $G_{m}$.

158. We can establish in an analogous manner the theorem: The linear substitution $[\alpha]_{2}$ of the second compound of any $m$-ary linear homogeneous group $G_{m}$, which corresponds to the substitution $\left(\alpha_{i j}\right)$ of $G_{m}$, effects upon the $C_{m, 2}$ Pfaffians

$$
\left[i_{1} i_{2} \ldots i_{m-2}\right] \quad\left(\begin{array}{l}
i_{1}, i_{2}, \ldots, i_{m-2}=1, \ldots, m \\
i_{1}<i_{2}<\ldots<i_{m-2}
\end{array}\right)
$$

a linear homogeneous substitution identical with the substitution $[\alpha]_{m-2}$ of the $(m-2)^{n d}$ compound of $G_{m}$.

The group induced by the second compound of $G_{m}$ upon these Pfaffians is therefore the $(m-2)^{n d}$ compound of $G_{m}$. 
159. Theorem. - The $q^{\text {th }}$ and $m-q^{\text {th }}$ compounds of the special linear group $S L H\left(m, p^{n}\right)$ are holoedrically isomorphic.

The theorem follows from $\S 155$ since the greatest common divisor of $m, q, p^{n}-1$ equals that of $m, m-q, p^{n}-1$.

We proceed to set up the correspondence between the individual substitutions of the two groups. We may express the $q^{\text {th }}$ minors of the determinant $\left|A_{i j}\right|$, adjungate to $D \equiv\left|\alpha_{i j}\right|$, in terms of the $m-q^{\text {th }}$ minors of the latter determinant by the formula,

$$
\left|\begin{array}{ll}
i_{1} & i_{2} \ldots i_{q} \\
j_{1} & j_{2} \ldots j_{q}
\end{array}\right| A=D^{q-1}\left|\begin{array}{lllll}
1 & 2 & \ldots i_{1}-1 & i_{1}+1 \ldots i_{q}-1 & i_{q}+1 \ldots m \\
1 & 2 \ldots j_{1}-1 & j_{1}+1 \ldots j_{q}-1 & j_{q}+1 \ldots m
\end{array}\right| .
$$

Hence, if we write (for every $i_{1}<i_{2}<\cdots<i_{q} \overline{<} m$ )

$$
Y_{1}{ }_{2} i_{1}-1 \quad i_{1}+1 \ldots i_{q}-1 \quad i_{q}+1 \ldots m \equiv Z_{i_{2}} i_{2} \ldots i_{q},
$$

the general substitution $[\alpha]_{m-q}$ of the $m-q^{\text {th }}$ compound of the general $m$-ary linear group takes the form

$$
Z_{i_{1}}^{\prime} \quad i_{2} \ldots i q=D^{1-q} \sum_{j_{1}<j_{2} \ldots<j_{q}}^{1 \ldots m}\left|\begin{array}{lll}
i_{1} & i_{2} \ldots i_{q} \\
j_{1} & j_{2} \ldots j_{q}
\end{array}\right| Z^{j_{1}} j_{2} \ldots j_{q} .
$$

If we take $D=1$, this substitution belongs to the $q^{\text {th }}$ compound, being derived from the substitution $\left(A_{i j}\right)$ of determinant

$$
\left|A_{i j}\right|=\left|\alpha_{i j}\right|^{m-1}=D^{m-1}=1 \text {. }
$$

Hence to $[\alpha]_{m-q}$, the $m-q^{\text {th }}$ compound of $\left(\alpha_{i j}\right)$ of determinant unity, corresponds $[A]_{1}$, the $q^{\text {th }}$ compound of $\left(A_{i j}\right)$.

160. Theorem. - The general Abelian group $G A\left(2 m, p^{n}\right)$ is the largest $2 m$-ary linear homogeneous group in the $G F\left[p^{n}\right]$ whose second compound has as a relative invariant the linear function of its $C_{m, 2}$ variables $Y_{i j}$,

$$
Z \equiv \sum_{l=1}^{m} Y_{2 l-12 l}
$$

It will be convenient to employ a notation for the general substitution $S$ of $G A\left(2 m, p^{n}\right)$ more compact than that of $\S 110$, viz.,

$$
S:
$$

$$
\xi_{i}^{\prime}=\sum_{j=1}^{2 m} \alpha_{i j} \xi_{j} \quad(i=1, \ldots, 2 m) .
$$

The Abelian conditions 76) then take the form (see $\S 112$ )

$$
\begin{aligned}
\sum_{l=1}^{m}\left|\begin{array}{ll}
\alpha_{2 l-1 j} & \alpha_{2 l-1 k} \\
\alpha_{2 l j} & \alpha_{2 l k}
\end{array}\right|=\mu \varepsilon_{j k} \equiv \begin{cases}\mu & (\text { if } k=j+1=\text { even }) \\
0 & (\text { unless } k=j+1=\text { even })\end{cases} \\
(j, k=1, \ldots, 2 m ; j \overline{<}) .
\end{aligned}
$$

These conditions may also be obtained by the method of $\S 129$. 
The corresponding substitution of the second compound is

$$
[\alpha]_{2}: \quad Y_{i_{1} i_{2}}^{\prime}=\sum_{j_{1}<j_{2}}^{j_{1}, j_{2}=1 \ldots 2 m}\left|\begin{array}{cc}
\alpha_{i_{1} j_{1}} & \alpha_{i_{1} j_{2}} \\
\alpha_{i_{2} j_{1}} & \alpha_{i_{2} j_{2}}
\end{array}\right| Y_{j_{1} j_{2}} \quad\left(\begin{array}{c}
i_{1}, i_{2}=1, \ldots, 2 m \\
i_{1}<i_{2}
\end{array}\right) .
$$

In virtue of 139), $[\alpha]_{2}$ transforms $Z$ into

$$
\sum_{j_{1}, j_{2}}\left\{\sum_{l=1}^{m}\left|\begin{array}{ll}
\alpha_{2 l-1 j_{1}} & \alpha_{2 l-1 j_{2}} \\
\alpha_{2 l j_{1}} & \alpha_{2 l j_{2}}
\end{array}\right|\right\} Y_{j_{1} j_{2}}=\mu \sum_{j_{1} j_{2}} \varepsilon_{j_{1} j_{2}} Y_{j_{1} j_{2}}=\mu Z
$$

Inversely, if $[\alpha]_{2}$ transforms $Z$ into $\mu Z$, the relations 139, follow.

161. Since the Abelian group $G A\left(2 m, p^{n}\right)$ contains the substitution

$$
T: \quad \xi_{i}^{\prime}=-\xi_{i} \quad(i=1, \ldots, 2 m),
$$

it is (by $\S 154$ ) holoedrically or hemiedrically isomorphic with its second compound according as $p=2$ or $p>2$.

If $S$ belong to the special Abelian group $S A\left(2 m, p^{n}\right)$, so that $\mu=1$, the corresponding substitution $[\alpha]_{2}$ of the second compound will leave $Z$ absolutely invariant. Since $S$ then has determinant unity $(\S 114),[\alpha]_{2}$ will leave absolutely invariant the Pfaffian $\left[\begin{array}{lll}1 & 2 \ldots 2 m\end{array}\right](\S 156)$. If in $S A\left(2 m, p^{n}\right)$ we consider $S$ and $T S$ to be identical, we obtain the quotient-group $A\left(2 m, p^{n}\right)$. The latter is therefore simply isomorphic with the second compound of $S A\left(2 m, p^{n}\right)$. Applying $\S 119$, we may state the theorem:

Except for $\left(2 m, p^{n}\right)=(2,2),(2,3)$ and $(4,2)$ the second compound of $S A\left(2 m, p^{n}\right)$ is a simple group which leaves absolutely invariant the Pfaffian $\left[\begin{array}{llll}1 & 2 & \ldots\end{array}\right]$ and the linear function $Z$.

162. For $2 m=4, p>2$, we introduce as new variables

$$
Y \equiv \frac{1}{2}\left(Y_{12}-Y_{34}\right), \quad Z_{1} \equiv \frac{1}{2}\left(Y_{12}+Y_{34}\right) \text {. }
$$

\begin{tabular}{|c|c|c|c|c|c|c|}
\hline & & $Y$ & $Y_{13}$ & $Y_{14}$ & $Y_{23}$ & $Y_{24}$ \\
\hline$Y^{\prime}=$ & 2 & \begin{tabular}{ll|l}
1 & 2 \\
12 & -1
\end{tabular} & \begin{tabular}{ll|}
1 & 2 \\
1 & 3
\end{tabular} \mid & $\left|\begin{array}{ll}1 & 2 \\
1 & 4\end{array}\right|$ & $\left|\begin{array}{ll}1 & 2 \\
2 & 3\end{array}\right|$ & $\left|\begin{array}{ll}1 & 2 \\
2 & 4\end{array}\right|$ \\
\hline$Y_{13}^{\prime}=$ & 2 & $\begin{array}{ll}1 & 3 \\
1 & 2\end{array} \mid$ & $\begin{array}{ll}1 & 3 \\
1 & 3\end{array} \mid$ & $\left|\begin{array}{ll}1 & 3 \\
1 & 4\end{array}\right|$ & $\left|\begin{array}{ll}1 & 3 \\
2 & 3\end{array}\right|$ & $\left|\begin{array}{ll}1 & 3 \\
2 & 4\end{array}\right|$ \\
\hline$Y_{14}^{\prime}=$ & 2 & \begin{tabular}{ll|}
14 & 4 \\
12
\end{tabular} \mid & $\left|\begin{array}{ll}1 & 4 \\
1 & 3\end{array}\right|$ & $\left|\begin{array}{ll}1 & 4 \\
1 & 4\end{array}\right|$ & $\left|\begin{array}{ll}1 & 4 \\
2 & 3\end{array}\right|$ & $\left|\begin{array}{ll}1 & 4 \\
2 & 4\end{array}\right|$ \\
\hline$Y_{23}^{\prime}=$ & 2 & $\left|\begin{array}{ll}2 & 3 \\
1 & 2\end{array}\right|$ & $\left|\begin{array}{ll}2 & 3 \\
1 & 3\end{array}\right|$ & $\left|\begin{array}{ll}2 & 3 \\
1 & 4\end{array}\right|$ & $\left|\begin{array}{ll}2 & 3 \\
2 & 3\end{array}\right|$ & $\begin{array}{l}23 \\
24\end{array} \mid$ \\
\hline$Y_{24}^{\prime}=$ & 2 & $\left|\begin{array}{ll}2 & 4 \\
1 & 2\end{array}\right|$ & \begin{tabular}{ll|}
2 & 4 \\
1 & 3
\end{tabular} \mid & $\left|\begin{array}{ll}2 & 4 \\
1 & 4\end{array}\right|$ & $\left|\begin{array}{ll}2 & 4 \\
2 & 3\end{array}\right|$ & $\left|\begin{array}{l}24 \\
24\end{array}\right|$ \\
\hline
\end{tabular}

The general substitution $[\alpha]_{2}$ of the second compound of $S A\left(4, p^{n}\right)$ takes the form, in which the unaltered index $Z_{1}$ does not appear ${ }^{1}$ ),

1) In $\S 164$ below, the second compound $[\alpha]_{2}$ of an arbitrary quaternary linear homogeneous substitution is written in matrix form. 
For example, $[\alpha]_{2}$ replaces $Y_{13}$ by the function

$$
\left|\begin{array}{ll}
1 & 3 \\
1 & 2
\end{array}\right|\left(Y+Z_{1}\right)+\left|\begin{array}{ll}
1 & 3 \\
1 & 3
\end{array}\right| Y_{13}+\left|\begin{array}{ll}
1 & 3 \\
1 & 4
\end{array}\right| Y_{14}+\left|\begin{array}{ll}
1 & 3 \\
2 & 3
\end{array}\right| Y_{23}+\left|\begin{array}{ll}
1 & 3 \\
2 & 4
\end{array}\right| Y_{24}+\left|\begin{array}{ll}
1 & 3 \\
3 & 4
\end{array}\right|\left(Z_{1}-Y\right),
$$

which becomes $Y_{13}^{\prime}$ of the table if we apply the Abelian relation

$$
\left|\begin{array}{ll}
1 & 3 \\
1 & 2
\end{array}\right|+\left|\begin{array}{ll}
1 & 3 \\
3 & 4
\end{array}\right| \equiv\left|\begin{array}{ll}
\alpha_{11} & \alpha_{12} \\
\alpha_{31} & \alpha_{32}
\end{array}\right|+\left|\begin{array}{ll}
\alpha_{13} & \alpha_{14} \\
\alpha_{33} & \alpha_{34}
\end{array}\right|=0 \text {. }
$$

Similarly, it replaces $Y$ by the function

$$
\begin{aligned}
& \frac{1}{2}\left\{\left|\begin{array}{ll}
1 & 2 \\
1 & 2
\end{array}\right|-\left|\begin{array}{ll}
3 & 4 \\
1 & 2
\end{array}\right|\right\}\left(Y+Z_{1}\right)+\frac{1}{2}\left\{\left|\begin{array}{ll}
1 & 2 \\
1 & 3
\end{array}\right|-\left|\begin{array}{ll}
3 & 4 \\
1 & 3
\end{array}\right|\right\} Y_{13}+\cdots \\
& \left.+\frac{1}{2}\left\{\left|\begin{array}{ll}
1 & 2 \\
3 & 4
\end{array}\right|-\left|\begin{array}{ll}
3 & 4 \\
3 & 4
\end{array}\right|\right\} Z_{1}-Y\right) \text {. }
\end{aligned}
$$

By means of the Abelian relations

$$
\left|\begin{array}{ll}
3 & 4 \\
1 & 2
\end{array}\right|=1-\left|\begin{array}{ll}
1 & 2 \\
1 & 2
\end{array}\right|, \quad\left|\begin{array}{ll}
1 & 2 \\
3 & 4
\end{array}\right|=1-\left|\begin{array}{ll}
1 & 2 \\
1 & 2
\end{array}\right|, \quad\left|\begin{array}{ll}
3 & 4 \\
3 & 4
\end{array}\right|=\left|\begin{array}{ll}
1 & 2 \\
1 & 2
\end{array}\right|, \quad\left|\begin{array}{ll}
3 & 4 \\
1 & 3
\end{array}\right|=-\left|\begin{array}{ll}
1 & 2 \\
1 & 3
\end{array}\right| .
$$

Hence $Y$ is replaced by the function $Y^{\prime}$ given by the above table.

It is therefore a substitution on five indices leaving absolutely invariant the function

$$
\Phi \equiv Z_{1}^{2}-\left[\begin{array}{llll}
1 & 2 & 3 & 4
\end{array}\right] \equiv Y^{2}+Y_{13} Y_{24}-Y_{14} Y_{23} .
$$

For $p>2$, the simple group $A\left(4, p^{n}\right)$ is holoedrically isomorphic with a subgroup of the quinary linear group leaving the quadratic function $\Phi$ absolutely invariant.

This theorem and the results of $\S 163-165$ find application in Chapters VII and VIII.

163. By $\S 155$, the quaternary linear group of determinant unity $S L H\left(4, p^{n}\right) \equiv G_{4}^{\prime}$ is holoedrically or hemiedrically isomorphic with its second compound $G_{4,2}^{\prime}$ according as $p=2$ or $p>2$. By $\S \S 103-104, G_{4}^{\prime}$ has as maximal invariant subgroup the group generated by the substitution

$$
M_{\mu}: \quad \xi_{i}^{\prime}=\mu \xi_{i} \quad(i=1,2,3,4),
$$

where $\mu$ is a primitive root of $\mu^{d}=1, d$ being the greatest common divisor of 4 and $p^{n}-1$. The quotient-group $L F\left(4, p^{n}\right)$ is a simple group of order

$$
\frac{1}{d}\left(p^{4 n}-1\right) p^{3 n}\left(p^{3 n}-1\right) p^{2 n}\left(p^{2 n}-1\right) p^{n} .
$$

To $M_{\mu}$ there corresponds in $G_{4,2}^{\prime}$ the substitution which multiplies every index by $\mu^{2}$ and therefore the identity if $p=2$ or $p^{n}=4 l+3$; while, for $p^{n}=4 l+1$, it is the substitution $T$ multiplying each of the six indices by -1 . We may state the theorem: 
For $p^{n}=2^{n}$ or $p^{n}=4 l+3, G_{4,2}^{\prime}$ is a simple group holoedrically isomorphic with $L F\left(4, p^{n}\right)$. For $p^{n}=4 l+1, G_{4,2}^{\prime}$ has a maximal self-conjugate subgroup $\{I, T\}$ of order two, the quotient-group being holoedrically isomorphic with $L F\left(4, p^{n}\right)$. If $e=1$ or 2 according as $p=2$ or $p>2$, the order of $G_{4,2}^{\prime}$ is

$$
\frac{1}{e}\left(p^{4 n}-1\right) p^{3 n}\left(p^{3 n}-1\right) p^{2 n}\left(p^{2 n}-1\right) p^{n} .
$$

164. Theorem. - The second compound $G_{4,2}$ of the general linear homogeneous group $G_{4}$ in the $G F\left[p^{n}\right]$ contains the substitution

$$
\text { 140) } Y_{12}^{\prime}=v Y_{12}, Y_{13}^{\prime}=Y_{13}, Y_{14}^{\prime}=Y_{14}, Y_{23}^{\prime}=Y_{23}, Y_{24}^{\prime}=Y_{24}, Y_{34}^{\prime}=v^{-1} Y_{34}
$$

if, and only if, $\nu$ be a square in the field.

To the substitution $\left(\alpha_{i j}\right)$ of $G_{4}$ corresponds in $G_{4,2}$ the sub-

\begin{tabular}{|c|c|c|c|c|c|c|}
\hline & $Y_{12}$ & $Y_{13}$ & $Y_{14}$ & $Y_{23}$ & $Y_{24}$ & $Y_{34}$ \\
\hline$Y_{12}^{\prime}=$ & $\left|\begin{array}{ll}1 & 2 \\
1 & 2\end{array}\right|$ & $\left|\begin{array}{ll}1 & 2 \\
1 & 3\end{array}\right|$ & $\left|\begin{array}{ll}1 & 2 \\
1 & 4\end{array}\right|$ & $\left|\begin{array}{ll}1 & 2 \\
2 & 3\end{array}\right|$ & $\left|\begin{array}{ll}1 & 2 \\
2 & 4\end{array}\right|$ & $\left|\begin{array}{ll}1 & 2 \\
3 & 4\end{array}\right|$ \\
\hline$Y_{13}^{\prime}=$ & $\left|\begin{array}{ll}1 & 3 \\
1 & 2\end{array}\right|$ & $\left|\begin{array}{ll}1 & 3 \\
1 & 3\end{array}\right|$ & $\left|\begin{array}{ll}1 & 3 \\
1 & 4\end{array}\right|$ & $\left|\begin{array}{ll}1 & 3 \\
2 & 3\end{array}\right|$ & $\left|\begin{array}{ll}1 & 3 \\
2 & 4\end{array}\right|$ & $\left|\begin{array}{ll}1 & 3 \\
3 & 4\end{array}\right|$ \\
\hline$Y_{14}^{\prime}=$ & $\left|\begin{array}{ll}1 & 4 \\
1 & 2\end{array}\right|$ & $\left|\begin{array}{ll}1 & 4 \\
1 & 3 .\end{array}\right|$ & $\left|\begin{array}{ll}1 & 4 \\
1 & 4\end{array}\right|$ & \begin{tabular}{ll|}
1 & 4 \\
2 & 3
\end{tabular} \mid & $\left|\begin{array}{ll}1 & 4 \\
2 & 4\end{array}\right|$ & $\left|\begin{array}{ll}1 & 4 \\
3 & 4\end{array}\right|$ \\
\hline$Y_{23}^{\prime}=$ & $\left|\begin{array}{ll}2 & 3 \\
1 & 2\end{array}\right|$ & $\left|\begin{array}{ll}2 & 3 \\
1 & 3\end{array}\right|$ & $\left|\begin{array}{ll}2 & 3 \\
1 & 4\end{array}\right|$ & $\begin{array}{l}23 \\
23\end{array}$ & $\left|\begin{array}{ll}2 & 3 \\
2 & 4\end{array}\right|$ & $\left|\begin{array}{ll}2 & 3 \\
3 & 4\end{array}\right|$ \\
\hline$Y_{24}^{\prime}=$ & $\left|\begin{array}{ll}2 & 4 \\
1 & 2\end{array}\right|$ & $\left|\begin{array}{ll}2 & 4 \\
1 & 3\end{array}\right|$ & $\left|\begin{array}{ll}2 & 4 \\
1 & 4\end{array}\right|$ & $\begin{array}{l}24 \\
23\end{array}$ & $\left|\begin{array}{ll}24 \\
24\end{array}\right|$ & $\left|\begin{array}{ll}2 & 4 \\
3 & 4\end{array}\right|$ \\
\hline$Y_{34}^{\prime}=$ & $\left|\begin{array}{ll}3 & 4 \\
1 & 2\end{array}\right|$ & $\left|\begin{array}{ll}3 & 4 \\
1 & 3\end{array}\right|$ & $\left|\begin{array}{ll}3 & 4 \\
1 & 4\end{array}\right|$ & $\begin{array}{l}34 \\
23\end{array}$ & $\left|\begin{array}{ll}3 & 4 \\
2 & 4\end{array}\right|$ & $\left|\begin{array}{ll}3 & 4 \\
3 & 4\end{array}\right|$ \\
\hline
\end{tabular}
stitution $[\alpha]_{2}$ :

Consider the "partial substitution", possibly of determinant zero,

\begin{tabular}{|c|c|c|c|}
\hline & $Y_{23}$ & $Y_{24}$ & $Y_{34}$ \\
\hline$Y_{23}^{\prime}=$ & $\left|\begin{array}{ll}2 & 3 \\
2 & 3\end{array}\right|$ & $\left|\begin{array}{ll}2 & 3 \\
2 & 4\end{array}\right|$ & $\mid \begin{array}{ll}2 & 3 \\
3 & 4\end{array}$ \\
\hline$Y_{24}^{\prime}=$ & $\left|\begin{array}{ll}2 & 4 \\
2 & 3\end{array}\right|$ & $\left|\begin{array}{ll}2 & 4 \\
2 & 4\end{array}\right|$ & $\mid \begin{array}{l}24 \\
34\end{array}$ \\
\hline$Y_{34}^{\prime}=$ & $\left|\begin{array}{ll}3 & 4 \\
2 & 3\end{array}\right|$ & $\left|\begin{array}{ll}3 & 4 \\
2 & 4\end{array}\right|$ & $\left|\begin{array}{l}34 \\
34\end{array}\right|$ \\
\hline
\end{tabular}

141)

Its determinant is readily seen to equal

$$
\left|\begin{array}{lll}
\alpha_{22} & \alpha_{23} & \alpha_{24} \\
\alpha_{32} & \alpha_{33} & \alpha_{34} \\
\alpha_{42} & \alpha_{43} & \alpha_{44}
\end{array}\right| .
$$


If $[\alpha]_{2}$ be the particular substitution 140$)$, the "partial substitution" 141) becomes

$$
\left(\begin{array}{lll}
1 & 0 & 0 \\
0 & 1 & 0 \\
0 & 0 & \nu^{-1}
\end{array}\right)
$$

of determinant $\nu^{-1}$. Hence if 140) belong to $G_{4,2}, \nu$ must be a square in the field.

Inversely, if $\nu$ be a square, 140) is the second compound of the following substitution of determinant unity:

$$
\left(\begin{array}{llll}
\boldsymbol{\nu}^{1 / 2} & 0 & 0 & 0 \\
0 & \boldsymbol{\nu}^{2 / 2} & 0 & 0 \\
0 & 0 & \boldsymbol{\nu}^{-1 / 2} & 0 \\
0 & 0 & 0 & \boldsymbol{\nu}^{-1 / 2}
\end{array}\right) .
$$

Note. - The second compound contains the substitution $Y_{12}^{\prime}=\nu Y_{12}, Y_{13}^{\prime}=\nu Y_{13}, Y_{14}^{\prime}=Y_{14}, Y_{23}^{\prime}=Y_{23}, Y_{24}^{\prime}=\nu^{-1} Y_{24}, Y_{34}^{\prime}=\nu^{-1} Y_{34}$. In fact, the latter is the second compound of the substitution

$$
\left(\begin{array}{llll}
\boldsymbol{\nu} & 0 & 0 & 0 \\
0 & 1 & 0 & 0 \\
0 & 0 & 1 & 0 \\
0 & 0 & 0 & \boldsymbol{\nu}^{-1}
\end{array}\right)
$$

165. Theorem. - For $p=2$, every substitution of $G_{4,2}^{\prime}$ satisfies the relation

$$
\begin{aligned}
\left|\begin{array}{ll}
1 & 2 \\
1 & 2
\end{array}\right|\left|\begin{array}{ll}
3 & 4 \\
3 & 4
\end{array}\right|+\left|\begin{array}{ll}
1 & 2 \\
1 & 3
\end{array}\right|\left|\begin{array}{ll}
3 & 4 \\
2 & 4
\end{array}\right|+\left|\begin{array}{ll}
1 & 2 \\
1 & 4
\end{array}\right|\left|\begin{array}{ll}
3 & 4 \\
2 & 3
\end{array}\right|+\left|\begin{array}{ll}
1 & 3 \\
1 & 2
\end{array}\right|\left|\begin{array}{ll}
2 & 4 \\
3 & 4
\end{array}\right|+\left|\begin{array}{ll}
1 & 3 \\
1 & 3
\end{array}\right|\left|\begin{array}{ll}
2 & 4 \\
2 & 4
\end{array}\right| \\
+\left|\begin{array}{ll}
1 & 3 \\
1 & 4
\end{array}\right|\left|\begin{array}{ll}
2 & 4 \\
2 & 3
\end{array}\right|+\left|\begin{array}{ll}
1 & 4 \\
1 & 2
\end{array}\right|\left|\begin{array}{ll}
2 & 3 \\
3 & 4
\end{array}\right|+\left|\begin{array}{ll}
1 & 4 \\
1 & 3
\end{array}\right|\left|\begin{array}{ll}
2 & 3 \\
2 & 4
\end{array}\right|+\left|\begin{array}{ll}
1 & 4 \\
1 & 4
\end{array}\right|\left|\begin{array}{ll}
2 & 3 \\
2 & 3
\end{array}\right| \equiv 1(\bmod 2)
\end{aligned}
$$

formed by multiplying each coefficient of the partial substitution 141) by that coefficient of the matrix $[\alpha]_{2}$ which lies symmetrical to it. $G_{4,2}^{\prime}$ does not contain the substitution $M_{1} \equiv\left(Y_{12} Y_{34}\right)$.

The left member of our relation is seen, to be the expansion of the expression

$$
\left|\begin{array}{llll}
\alpha_{11} & \alpha_{12} & \alpha_{13} & \alpha_{14} \\
\alpha_{21} & \alpha_{22} & \alpha_{23} & \alpha_{24} \\
\hline \alpha_{31} & \alpha_{32} & \alpha_{33} & \alpha_{34} \\
\alpha_{41} & \alpha_{42} & \alpha_{43} & \alpha_{44}
\end{array}\right|+2 \alpha_{44}\left|\begin{array}{lll}
\alpha_{11} & \alpha_{12} & \alpha_{13} \\
\alpha_{21} & \alpha_{22} & \alpha_{23} \\
\alpha_{31} & \alpha_{32} & \alpha_{33}
\end{array}\right|
$$

and is therefore $\equiv 1(\bmod 2)$, since $\left|\alpha_{i j}\right|=1$. The substitution $M_{1}$ does not satisfy the relation and so does not belong to the group $G_{4,2}^{\prime}$. 


\section{CHAPTER VII.}

\section{LINEAR HOMOGENEOUS GROUP IN THE $G F\left[p^{n}\right], p>2$,}

\section{DEFINED BY A QUADRATIC INVARIANT. $\left.{ }^{1}\right)$}

166. Any quadratic form with coefficients in the $G F\left[p^{n}\right], p>2$,

$$
f \equiv \alpha_{11} \xi_{1}^{2}+2 \alpha_{12} \xi_{1} \xi_{2}+\alpha_{22} \xi_{2}^{2}+2 \alpha_{13} \xi_{1} \xi_{3}+\cdots+\alpha_{m m} \xi_{m}^{2}
$$

may, by using the notation $\alpha_{j i} \equiv \alpha_{i j}$, be written in the form

$$
f \equiv \sum_{i, j}^{1, \ldots, m} \alpha_{i j} \xi_{i} \xi_{j}
$$

By the determinant (or discriminant) of $f$ we mean

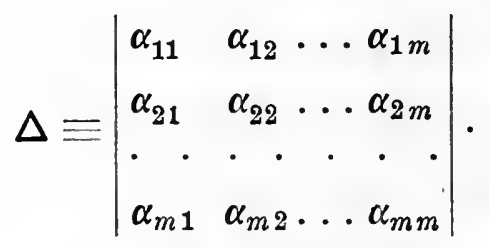

16\%. Theorem. - Upon applying to $f$ a linear m-ary transformation of determinant $D$, the determinant $\Delta$ of $f$ is multiplied by $D^{2}$.

In view of $\S 100$, it suffices to prove the theorem for the types of transformations considered in the cases $1^{0}$ and $2^{0}$ following.

$1^{0}$. Upon applying to $f$ the transformation

we obtain the function

$$
\xi_{1}^{\prime}=\xi_{1}+\lambda \xi_{2}, \quad \xi_{i}^{\prime}=\xi_{i} \quad(i=2, \ldots, m)
$$

Its determinant is

$$
f+2 \lambda \sum_{j=1}^{m} \alpha_{1 j} \xi_{2} \xi_{j}+\lambda^{2} \alpha_{11} \xi_{2}^{2} \text {. }
$$

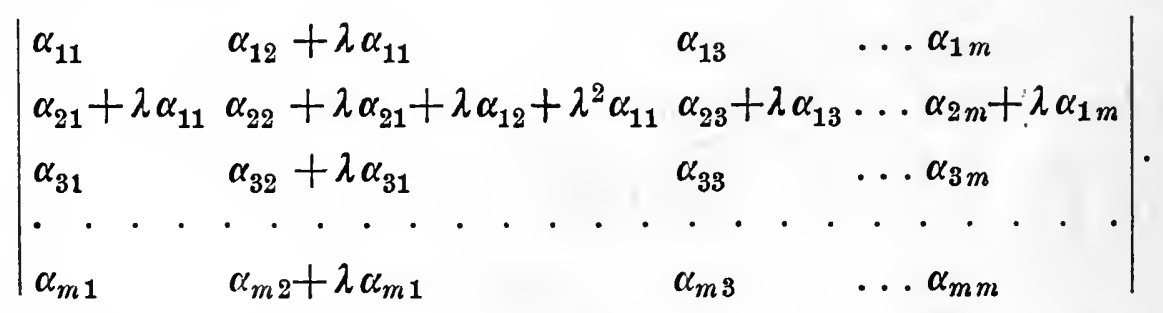

Multiply the first row by $\lambda$ and subtract from the second row; afterwards multiply the first column by $\lambda$ and subtract from the second. We obtain the original determinant $\Delta=\left|\alpha_{i j}\right|$.

1) The results of this chapter were given by the author in the American Journal of Mathematics, vol. 21 (1899), pp. 193-256, and partially in earlier papers there cited. For the case $n=1$, the order of the first orthogonal group was determined by Jordan, Traité, pp. 161-170. 
$2^{0}$. Upon applying to $f$ the transformation

we obtain the function

$$
\xi_{1}^{\prime}=D \xi_{1}, \quad \xi_{i}^{\prime}=\xi_{i}
$$

$$
D^{2} \alpha_{11} \xi_{1}^{2}+2 D \sum_{j=2}^{m} \alpha_{1 j} \xi_{1} \xi_{j}+\sum_{i, j}^{2, \ldots, m} \alpha_{i j} \xi_{i} \xi_{j} .
$$

Its determinant is

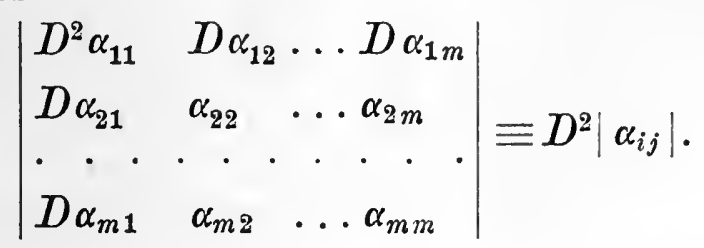

168. Theorem. - A quadratic form $f$ with coefficients in the $G F\left[p^{n}\right], p>2$, and of determinant $\Delta \neq 0$ can be reduced by a linear homogeneous substitution belonging to the field to the form

$$
\sum_{i=1}^{m} \alpha_{i} \xi_{i}^{2} \quad\left(\text { each } \alpha_{i} \neq 0\right) .
$$

Since $\Delta \neq 0$, the coefficients $\alpha_{11}, \alpha_{12}, \ldots, \alpha_{1 m}$ are not all zero. If $\alpha_{11}=0$, we may suppose that $\alpha_{12} \neq 0$, for example. Applying to $f$ the substitution of determinant $-2 \lambda \neq 0$,

$$
\xi_{1}^{\prime}=\lambda \xi_{1}+\lambda \xi_{2}, \quad \xi_{2}^{\prime}=\xi_{1}-\xi_{2}, \quad \xi_{i}^{\prime}=\xi_{i} \quad(i=3, \ldots, m)
$$

we obtain a form in which the coefficient of $\xi_{1}^{2}$ is $\alpha_{22}+2 \lambda \alpha_{12}$. Taking for $\lambda$ any one of the $p^{n}-2$ marks different from zero and from $-\alpha_{22} / 2 \alpha_{12}$, the coefficient of $\xi_{1}^{2}$ will be not zero. Whether $\alpha_{11}$ be zero or not, we thus obtain a form

$$
f^{\prime} \equiv \sum_{i, j}^{1, \ldots, m} \beta_{i j} \xi_{i} \xi_{j}
$$

whose determinant $\Delta^{\prime}$ is not zero by $\S 167$.

Applying to $f^{\prime}$ the substitution

$$
\xi_{1}^{\prime}=\xi_{1}-\frac{\beta_{12}}{\beta_{11}} \xi_{2}, \quad \xi_{i}^{\prime}=\xi_{i} \quad(i=2, \ldots, m)
$$

we obtain a form in which the coefficient of $\xi_{1} \xi_{2}$ is zero, while that $\xi_{1}^{2}$ remains $\beta_{11} \neq 0$. In a similar manner, we can make the coefficients of $\xi_{1} \xi_{3}, \ldots, \xi_{1} \xi_{m}$ all zero. In the resulting form

$$
\beta_{11} \xi_{1}^{2}+\sum_{i, j}^{2, \ldots m} \gamma_{i j} \xi_{i} \xi_{j}
$$

the coefficients $\gamma_{22}, \gamma_{23}, \ldots, \gamma_{2 m}$ are not all zero, since the determinant of the transformed form is not zero by $\S 167$. 
Proceeding with this form as we did with $f$, we reach a form

$$
\beta_{11} \xi_{1}^{2}+\delta_{11} \xi_{2}^{2}+\sum_{i, j}^{3, \ldots, m} \varepsilon_{i j} \xi_{i} \xi_{j}
$$

of determinant $\neq 0$. After $m-1$ such steps we reach the form 142).

169. Certain of the $\alpha_{i}$ in 142) are squares and the others are not-squares in the $G F\left[p^{n}\right]$. By applying a suitable substitution which interchanges the $\xi_{i}$, we may suppose that in the resulting form $\alpha_{1}, \ldots, \alpha_{s}$ are squares, say $a_{1}^{2}, \ldots, a_{s}^{2}$, while $\alpha_{s+1}, \ldots, \alpha_{m}$ are notsquares, say $v a_{s+1}^{2}, \ldots, v a_{m}^{2}, \nu$ being a particular not-square. Applying the substitution

our form is transformed into

$$
\xi_{i}^{\prime}=a_{i}^{-1} \xi_{i}
$$

$$
f_{s} \equiv \sum_{i=1}^{s} \xi_{i}^{2}+\nu \sum_{i=s+1}^{m} \xi_{i}^{2} .
$$

Furthermore, we can transform $f_{s}$ into $f_{s+2}$ and vice versa. In fact, the substitution of determinant $\alpha^{2}+\beta^{2}$

$$
\xi_{i}^{\prime}=\alpha \xi_{i}-\beta \xi_{j}, \quad \xi_{j}^{\prime}=\beta \xi_{i}+\alpha \xi_{j}
$$

transforms $\xi_{i}^{2}+\xi_{j}^{2}$ into $\left(\alpha^{2}+\beta^{2}\right)\left(\xi_{i}^{2}+\xi_{j}^{2}\right)$. By $\S 64$, $\alpha$ and $\beta$ may be chosen in the $G F\left[p^{n}\right], p>2$, such that

$$
\alpha^{2}+\beta^{2}=\nu \text {. }
$$

We have therefore only two canonical forms, $f_{m}$ and $f_{m-1}$. The latter form may be dropped if $m$ be odd. Indeed, $f_{m-1}$ can, for $m$ odd, be transformed into

$$
f_{0} \equiv \nu\left(\xi_{1}^{2}+\xi_{2}^{2}+\cdots+\xi_{m}^{2}\right) .
$$

But the linear group leaving $f_{0}$ invariant leaves also

$$
f_{m} \equiv \xi_{1}^{2}+\xi_{9}^{2}+\cdots+\xi_{m}^{2}
$$

invariant. We may therefore state the theorem:

The group of all linear homogeneous $m$-ary substitutions in the $G F\left[p^{n}\right], p>2$, which leave invariant a quadratic form $f$ belonging to the field and of determinant not zero, can be transformed by a linear homogeneous $m$-ary substitution belonging to the field into the group of all linear homogeneous $m$-ary substitutions in the $G F\left[p^{n}\right]$ which leave invariant

$$
F_{\mu} \equiv \sum_{i=1}^{m-1} \xi_{i}^{2}+\mu \xi_{m}^{2},
$$

where $\mu=1$ for $m$ odd, but $\mu=1$ or a particular not-square $\nu$ for $m$ even. 
170. The conditions that the substitution

$$
S: \quad \xi_{i}^{\prime}=\sum_{j=1}^{m} \alpha_{i j} \xi_{j} \quad(i=1, \ldots, m)
$$

shall leave $F_{\mu}$ invariant are the following:

$$
\begin{array}{r}
\alpha_{1 j}^{2}+\alpha_{2 j}^{2}+\cdots+\alpha_{m-1 j}^{2}+\mu \alpha_{m j}^{2}= \begin{cases}1 & (j=1, \ldots, m-1) \\
\mu & (j=m)\end{cases} \\
\alpha_{1 j} \alpha_{1 k}+\cdots+\alpha_{m-1 j} \alpha_{m-1 k}+\mu \alpha_{m j} \alpha_{m k}=0 \\
\\
(j, k=1, \ldots, m ; j \neq k) .
\end{array}
$$

It follows readily that the inverse of $S$ is

$$
S^{-1}: \quad\left\{\begin{array}{l}
\xi_{i}^{\prime}=\sum_{j=1}^{m-1} \alpha_{j i} \xi_{j}+\mu \alpha_{m i} \xi_{m} \quad(i=1, \ldots, m-1) \\
\xi_{m}^{\prime}=\frac{1}{\mu} \sum_{j=1}^{m-1} \alpha_{j m} \xi_{j}+\alpha_{m m} \xi_{m} .
\end{array}\right.
$$

The determinant of $S^{-1}$ is seen to equal the determinant $D$ of $S$. Hence $D^{2}=1$, being the determinant of $S^{-1} S \equiv I$.

Writing the relations 143 ) and 144) for the substitution $S^{-1}$, we obtain the following relations, which are evidently together equivalent to the set 143) and 144):

$$
\begin{aligned}
& \alpha_{j 1}^{2}+\alpha_{j 2}^{2}+\cdots+\alpha_{j m-1}^{2}+\frac{1}{\mu} \alpha_{j m}^{2}= \begin{cases}1 & (j=1, \ldots, m-1) \\
1 / \mu & (j=m)\end{cases} \\
& \alpha_{j 1} \alpha_{k 1}+\cdots+\alpha_{j m-1} \alpha_{k m-1}+\frac{1}{\mu} \alpha_{j m} \alpha_{k m}=0 \\
& \quad(j, k=1, \ldots, m ; j \neq k) .
\end{aligned}
$$

1\%1. The substitutions leaving $F_{\mu}$ invariant were proven to have determinant \pm 1 . Among them occur substitutions of determinant-1, as

$$
C_{i}: \quad \xi_{i}^{\prime}=-\xi_{i}, \quad \xi_{j}^{\prime}=\xi_{j} \quad(j=1, \ldots, m ; j \neq i) .
$$

The group $\bar{O}_{\mu}\left(m, p^{n}\right)$ of all linear substitutions leaving $F_{\mu}$ invariant has therefore a subgroup of index two $O_{\mu}\left(m, p^{n}\right)$ composed of all linear $m$-ary substitutions in the $G F\left[p^{n}\right]$ of determinant unity which leave $F_{\mu}$ invariant. The latter substitutions will be called orthogonal. ${ }^{1}$ ) For $\mu=1$, we have the first orthogonal group $O_{1}\left(m, p^{n}\right)$; for $m$ even and $\boldsymbol{\mu}=\boldsymbol{\nu}$, we have the second orthogonal group $O_{v}\left(m, p^{n}\right)$.

1) This unusual restriction of the term orthogonal to substitutions of determinant +1 is done in the interest of the later terminology and notation. We will be concerned with such substitutions alone. If it became necessary to consider substitutions of determinant -1 which leave $F_{\mu}$ invariant, they might be designated extended (erweiterte) orthogonal substitutions and the group $\overline{O_{\mu}}\left(m, p^{n}\right)$ designated the extended orthogonal group. 
172. Theorem. - The order $\Omega_{\mu}\left(m, p^{n}\right)$ of $O_{\mu}\left(m, p^{n}\right)$ is, for $m$ odd,

$$
\left(p^{n(m-1)}-1\right) p^{n(m-2)}\left(p^{n(m-3)}-1\right) p^{n(m-4)} \cdots\left(p^{2 n}-1\right) p^{n}
$$

and, for $m$ even,

$$
\left[p^{n(n-1)} \mp \varepsilon^{\frac{m}{2}} p^{n\left(\frac{m}{2}-1\right)}\right]\left(p^{n(m-2)}-1\right) p^{n(m-3)} \ldots\left(p^{2 n}-1\right) p^{n},
$$

where the sign $\mp$ is - or + according as $\mu=1$ or $\nu$, and where $\varepsilon= \pm 1$ according to the form $4 l \pm 1$ of $p^{n}$.

We notice first that the number of substitutions $S, S^{\prime}, \ldots$ of $O_{\mu}\left(m, p^{n}\right)$ which leave $\xi_{1}$ fixed is $\Omega_{\mu}\left(m-1, p^{n}\right)$. In fact, they have $\alpha_{11}=1, \alpha_{12}=\alpha_{13}=\cdots=\alpha_{1 m}=0$, and therefore by 146) for $j=1$,

$$
\alpha_{k 1}=0 \quad(k=2, \ldots, m) .
$$

Hence they belong to the group $O_{\mu}\left(m-1, p^{n}\right)$ leaving invariant

$$
\xi_{2}^{2}+\xi_{3}^{2}+\cdots+\xi_{m-1}^{2}+\mu \xi_{m}^{2} .
$$

Let $T$ be a general substitution of $O_{\mu}\left(m, p^{n}\right)$ and let it replace $\xi_{1}$ by

where, by 145) for $j=1$,

$$
\omega_{1} \equiv \sum_{k=1}^{m} \alpha_{1 k} \xi_{k},
$$

$$
\alpha_{11}^{2}+\alpha_{12}^{2}+\cdots+\alpha_{1 m-1}^{2}+\frac{1}{\mu} \alpha_{1 m}^{2}=1 .
$$

The $\Omega_{\mu}\left(m-1, p^{n}\right) \cdot$ substitutions $T S, T S^{\prime}, \ldots$ and no others of the group will replace $\xi_{1}$ by $\omega_{1}$. If, therefore, $P_{\mu}\left(m, p^{n}\right)$ denote the number of distinct linear functions $\omega_{1}$ by which the substitutions of $O_{\mu}\left(m, p^{n}\right)$ can replace $\xi_{1}$, we have for the order of the latter group,

$$
\Omega_{\mu}\left(m, p^{n}\right)=P_{\mu}\left(m, p^{n}\right) \Omega_{\mu}\left(m-1, p^{n}\right) .
$$

This recursion formula gives

$$
\Omega_{\mu}\left(m, p^{n}\right)=P_{\mu}\left(m, p^{n}\right) P_{\mu}\left(m-1, p^{n}\right) \ldots P_{\mu}\left(2, p^{n}\right),
$$

since the identity is the only substitution of determinant unity on one index which leaves $\mu \xi_{m}^{2}$ invariant, so that $\Omega_{\mu}\left(1, p^{n}\right)=1$.

It will be shown in $\S \S 174-180$ that $P_{\mu}\left(k, p^{n}\right)$ equals the number of sets of solutions in the $G F\left[p^{n}\right]$ of the equation

and hence, by $\S \S 65-66, P_{\mu}\left(k, p^{n}\right)=$

$$
\alpha_{1}^{2}+\alpha_{2}^{2}+\cdots+\alpha_{k-1}^{2}+\frac{1}{\mu} \alpha_{k}^{2}=1,
$$

$$
\begin{aligned}
& p^{n(k-1)} \mp \varepsilon^{\frac{k}{2}} p^{n\left(\frac{k}{2}-1\right)} \\
& p^{n(k-1)} \pm \varepsilon^{\frac{k-1}{2}} p^{n(k-1) / 2}
\end{aligned}
$$

the upper signs holding if $\mu=1$, the lower signs if $\mu=\nu$, and $\varepsilon$ denoting +1 or -1 according as -1 is a square or a not-square 
in the $G F\left[p^{n}\right]$. Whether the integer $t$ be even or odd, we find that the product

$$
\begin{aligned}
P_{\mu}\left(2 t+1, p^{n}\right) P_{\mu}\left(2 t, p^{n}\right) & =p^{n t}\left(p^{n t} \pm \varepsilon^{t}\right) \cdot p^{n(t-1)}\left(p^{n t} \mp \varepsilon^{t}\right) \\
& \equiv\left(p^{2 n t}-1\right) p^{n(2 t-1)} .
\end{aligned}
$$

We derive at once the expression for $\Omega_{\mu}\left(m, p^{n}\right)$ given in the theorem.

173. Theorem. - The orthogonal group $O_{\mu}\left(m, p^{n}\right)$ is generated by the substitutions ${ }^{1}$ )

$$
\begin{array}{ll}
O_{i, j}^{\alpha, \beta}: & \left\{\begin{array}{ll}
\xi_{i}^{\prime}=\alpha \xi_{i}+\beta \xi_{j} \\
\xi_{j}^{\prime}=-\beta \xi_{i}+\alpha \xi_{j}
\end{array}\left[\alpha^{2}+\beta^{2}=1\right] \quad(i, j<m)\right. \\
O_{i, m}^{\gamma, \delta}: & \left\{\begin{array}{l}
\xi_{i}^{\prime}=\gamma \xi_{i}+\delta \xi_{m} \\
\xi_{m}^{\prime}=-\frac{\delta}{\mu} \xi_{i}+\gamma \xi_{m}
\end{array}\left[\gamma^{2}+\frac{1}{\mu} \delta^{2}=1\right] \quad(i<m)\right.
\end{array}
$$

with the following exceptions:

10. For $p^{n}=5, m \overline{5}, \mu=1$, we may take as the necessary additional generator

$$
R_{123}: \quad\left\{\begin{array}{l}
\xi_{1}^{\prime}=\xi_{1}+\xi_{2}+2 \xi_{3}, \\
\xi_{2}^{\prime}=\xi_{1}+2 \xi_{2}+\xi_{3}, \\
\xi_{3}^{\prime}=2 \xi_{1}+\xi_{2}+\xi_{3} .
\end{array} \quad R_{123}^{2}=I .\right.
$$

$2^{0}$. For $p^{n}=3, m>4, \mu=1$, we may choose as the necessary additional generator

$$
W_{1234}: \quad\left\{\begin{array}{l}
\xi_{1}^{\prime}=\xi_{1}-\xi_{2}-\xi_{3}-\xi_{4}, \\
\xi_{2}^{\prime}=\xi_{1}-\xi_{2}+\xi_{3}+\xi_{4}, \\
\xi_{3}^{\prime}=\xi_{1}+\xi_{2}-\xi_{3}+\xi_{4}, \\
\xi_{4}^{\prime}=\xi_{1}+\xi_{2}+\xi_{3}-\xi_{4} .
\end{array} \quad W_{1234}^{3}=I .\right.
$$

$3^{0}$. For $p^{n}=3, m \overline{>} 3, \mu=v \equiv-1$, we may take as the necessary additional generator

$$
V_{12 m}: \quad\left\{\begin{array}{l}
\xi_{1}^{\prime}=\xi_{1}-\xi_{2}-\xi_{m}, \\
\xi_{2}^{\prime}=\xi_{1}-\xi_{2}+\xi_{m}, \\
\xi_{m}=-\xi_{1}-\xi_{2} .
\end{array} \quad V_{12 m}^{3}=I .\right.
$$

For $m=2$, the theorem is readily proven. If any orthogonal substitution replaces $\xi_{1}$ by $\gamma \xi_{1}+\delta \xi_{2}$, then $S \equiv O_{1,2}^{\gamma, \delta} S_{1}$, where $S_{1}$ leaves $\xi_{1}$ fixed and is therefore the identity.

For $m=3$, the theorem follows from $\S \S 174-179$. For $m>3$, it follows from $\S 180$.

1) For simplicity we write only the indices altered by the substitution. 
174. Theorem. - If $\alpha_{1}, \alpha_{2}, \alpha_{3}$ be any set of solutions in the $G F\left[p^{n}\right], p>2$, of the equation

$$
\alpha_{1}^{2}+\alpha_{2}^{2}+\frac{1}{\mu} \alpha_{3}^{2}=1
$$

there exists a substitution $S$, derived from the generators of $\& 173$ which leave $\xi_{1}^{2}+\xi_{2}^{2}+\mu \xi_{3}^{2}$ invariant, such that $S$ replaces $\xi_{1}$ by $\alpha_{1} \xi_{1}+\alpha_{2} \xi_{2}+\alpha_{3} \xi_{3}$.

The proposition follows if $1-\alpha_{1}^{2}$ or $1-\alpha_{2}^{2}$ be a square $\neq 0$ in the $G F\left[p^{n}\right]$. For example, if $1-\alpha_{2}^{2}=\tau^{2}$, then

so that we may take

$$
\left(\frac{\alpha_{1}}{\tau}\right)^{2}+\frac{1}{\mu}\left(\frac{\alpha_{3}}{\tau}\right)^{2}=1
$$

$$
S \equiv O_{1,3}^{\frac{\alpha_{1}}{\tau}, \frac{\alpha_{3}}{\tau}} O_{1,2}^{\tau, \alpha_{2}}
$$

The proposition will be true for $\alpha_{1}, \alpha_{2}, \alpha_{3}$ if true for the quantities where

$$
\alpha_{1}^{\prime} \equiv \alpha_{1} \varrho-\alpha_{2} \sigma, \quad \alpha_{2}^{\prime} \equiv \alpha_{1} \sigma+\alpha_{2} \varrho, \quad \alpha_{3}^{\prime} \equiv \alpha_{3},
$$

so that we have

$$
\varrho^{2}+\sigma^{2}=1 \text {, }
$$

$$
\alpha_{1}^{\prime 2}+\alpha_{2}^{\prime 2}+\frac{1}{\mu} \alpha_{3}^{\prime 2}=\alpha_{1}^{2}+\alpha_{2}^{2}+\frac{1}{\mu} \alpha_{3}^{2}=1 .
$$

In fact, if the group contains a substitution $S^{\prime}$ which replaces $\xi_{1}$ by $\alpha_{1}^{\prime} \xi_{1}+\alpha_{2}^{\prime} \xi_{2}+\alpha_{3}^{\prime} \xi_{3}$, it will contain the product $S \equiv O_{1,2}^{0,-\sigma} S^{\prime}$ which replaces $\xi_{1}$ by $\alpha_{1} \xi_{1}+\alpha_{2} \xi_{2}+\alpha_{3} \xi_{3}$.

175. Consider first the case in which -1 is a not-square in the $G F\left[p^{n}\right]$. By $\& 64$, there are $p^{n}+1$ sets of solutions $\varrho, \sigma$ in the field of the equation $\varrho^{2}+\sigma^{2}=1$. Not more than two of these sets of solutions give the same value to

$$
\alpha_{2}^{\prime} \equiv \alpha_{1} \sigma+\alpha_{2} \varrho .
$$

Indeed, upon eliminating $\sigma$, we obtain a quadratic for $\varrho$. Hence $\alpha_{2}^{\prime}$ takes at least $\frac{1}{2}\left(p^{n}+1\right)$ distinct values. But, by $\S 67$, there are exactly $\frac{1}{2}\left(p^{n}-3\right)$ distinct marks $\eta \neq 0$ for which $\eta^{2}-1$ is a square $\left.{ }^{1}\right)$, so that $1-\eta^{2}$ is a not-square. Hence there exist at least two values of $\alpha_{2}^{\prime}$ for which $1-\alpha_{2}^{\prime 2}$ is a square or zero. If it be a square, our theorem follows from the previous section. There remains the case $\alpha_{2}^{\prime 2}=1$, for which, by 148),

$$
\alpha_{1}^{\prime 2}=-\frac{1}{\mu} \alpha_{3}^{\prime 2}
$$

If $\mu=1$, we have $\alpha_{1}^{\prime}=\alpha_{3}^{\prime}=0$, since -1 is a not-square, and the required substitution is $S=O_{1,2}^{0, \alpha^{\prime} 2}$. If $\mu$ be a not-square, we may take $\mu=-1$, so that

$$
\alpha_{1}^{\prime}= \pm \alpha_{3}^{\prime}, \quad \alpha_{2}^{\prime 2}=1
$$

1) Zero is not reckoned as a square. 
But the theorem is true for $\alpha_{1}^{\prime}, \alpha_{2}^{\prime}, \alpha_{3}^{\prime}$ if true for the quantities

$$
\alpha_{1}^{\prime \prime} \equiv \alpha_{1}^{\prime} \beta-\alpha_{3}^{\prime} \gamma, \quad \alpha_{2}^{\prime \prime} \equiv \alpha_{2}^{\prime}, \quad \alpha_{3}^{\prime \prime} \equiv-\alpha_{1}^{\prime} \gamma+\alpha_{3}^{\prime} \beta,
$$

where $\beta^{2}-\gamma^{2}=1$. In fact, if $S^{\prime \prime}$ replaces $\xi_{1}$ by $\alpha_{1}^{\prime \prime} \xi_{1}+\alpha_{2}^{\prime \prime} \xi_{2}+\alpha_{3}^{\prime \prime} \xi_{3}$, then $O_{1,3}^{\beta, \gamma} S^{\prime \prime}$ will replace $\xi_{1}$ by $\alpha_{1}^{\prime} \xi_{1}+\alpha_{2}^{\prime} \xi_{2}+\alpha_{3}^{\prime} \xi_{3}$. The $p^{n}-1$ sets of solutions in the $G F\left[p^{n}\right]$ of the equation $\beta^{2}-\gamma^{2}=1$ are given by

$$
\beta=\frac{1}{2}(\tau+1 / \tau), \quad \mp \gamma=\frac{1}{2}(\tau-1 / \tau),
$$

where $\tau$ runs through the series of marks $\neq 0$ of the field. Hence $\beta \mp \gamma$ may be given an arbitrary value $\tau \neq 0$ in the field. The theorem being evident if $\alpha_{1}^{\prime}=0$, we exclude this case. Then $\alpha^{\prime \prime} \equiv$ $\alpha_{1}^{\prime}(\beta \mp \gamma)$ may be made to assume an arbitrary value except zero, and hence, if $p^{n}>3$, a value for which $1-\alpha_{1}^{\prime \prime 2}$ is a square in the field $(\S 64)$. For $p^{n}=3, \alpha_{1}^{\prime}, \alpha_{2}^{\prime}, \alpha_{3}^{\prime}$ are each \pm 1 , so that we may evidently take

$$
S=C V_{123} K
$$

where $C$ and $K$ are products formed from $C_{1}, C_{2}, C_{3}$. But, if $C$ be the product of an odd number of the $C_{i}$, we note that

$$
C_{1} V_{123}=C_{2} C_{3} V_{123} C_{1} C_{2} C_{3} \text {. }
$$

We may therefore assume that $C$ and $K$ are each products of an even number of the $C_{i}$ and therefore derived from the given generators.

176. Suppose next that -1 is the square of a mark $i$ of the $G F\left[p^{n}\right]$, while $\mu$ is a not-square. There exist $p^{n}+1$ sets of solutions in the field of the equation

$$
\beta^{2}+\frac{1}{\mu} \gamma^{2}=1
$$

But the theorem is true for $\alpha_{1}, \alpha_{2}, \alpha_{3}$ if true for

$$
\alpha_{1}^{\prime} \equiv \alpha_{1}, \quad \alpha_{2}^{\prime} \equiv \beta \alpha_{2}+\frac{\gamma}{\mu} \alpha_{3}, \quad \alpha_{3}^{\prime} \equiv-\gamma \alpha_{2}+\beta \alpha_{3} .
$$

Indeed, if $S^{\prime}$ replaces $\xi_{1}$ by $\alpha_{1}^{\prime} \xi_{1}+\alpha_{2}^{\prime} \xi_{2}+\alpha_{3}^{\prime} \xi_{3}$, then $O_{2,3}^{\beta, \gamma} S^{\prime}$ will replace $\xi_{1}$ by $\alpha_{1} \xi_{1}+\alpha_{2} \xi_{2}+\alpha_{3} \xi_{3}$.

There are at least $\frac{1}{2}\left(p^{n}+1\right)$ sets of solutions of 149) for which the values of $\alpha_{2}^{\prime}$ are distinct; for, upon eliminating $\beta$, we obtain a quadratic for $\gamma$. But, by $\S 67$, there exist only $\frac{1}{2}\left(p^{n}-1\right)$ marks $i \xi$, and hence as many distinct marks $\xi$, for which

$$
(i \xi)^{2}+1 \equiv 1-\xi^{2}=\text { not-square. }
$$


Hence at least one set of solutions of 149) will make $1-\alpha_{2}^{\prime 2}$ a square or zero. If it be a square, the theorem follows from $\S 174$. If it be zero, we have by 148),

$$
\alpha_{1}^{\prime 2}=-\frac{1}{\mu} \alpha_{3}^{\prime 2}
$$

Hence $\alpha_{1}^{\prime}=\alpha_{3}^{\prime}=0, \alpha_{2}^{\prime 2}=1$, so that we may take $S=O_{1,2}^{0, \alpha_{2}^{\prime}}$.

19\%. For the case $\left.{ }^{1}\right)-1$ a square in the $G F\left[p^{n}\right]$ and $\mu=1$, it follows from $\S \S 178-179$ that $O_{1}\left(3, p^{n}\right)$ contains a subgroup of order at least $p^{n}\left(p^{2 n}-1\right)$ generated by the substitutions $O_{i, j}^{\alpha, \beta}$, together with $R_{123}$ if $p^{n}=5$, all of determinant +1 . But, by $\S 172$, the order of $O_{1}\left(3, p^{n}\right)$ is $P_{1}\left(3, p^{n}\right) P_{1}\left(2, p^{n}\right)$. Here $P_{1}\left(2, p^{n}\right)=p^{n}-1$, being the number of functions

$$
\alpha_{1} \xi_{1}+\alpha_{2} \xi_{2} \quad\left(\alpha_{1}^{2}+\alpha_{2}^{2}=1\right)
$$

by which the substitutions of $O_{1}\left(2, p^{n}\right)$ can replace $\xi_{1}$. Also

$$
P_{1}\left(3, p^{n}\right) \overline{\overline{<}} p^{2 n}+p^{n}
$$

In fact, if a substitution of $O_{1}\left(3, p^{n}\right)$ replace $\xi_{1}$ by

then

$$
\omega_{1} \equiv \alpha_{1} \xi_{1}+\alpha_{2} \xi_{2}+\alpha_{3} \xi_{3}
$$

$$
\alpha_{1}^{2}+\alpha_{2}^{2}+\alpha_{3}^{2}=1 \text {. }
$$

By $\S 66$, this equation has $p^{2 n}+p^{n}$ sets of solutions in the $G F\left[p^{n}\right]$, -1 being a square. The order of $O_{1}\left(3, p^{n}\right)$ is thus at most $\left(p^{2 n}+p^{n}\right)\left(p^{n}-1\right)$. From the two results it follows that this number equals the order of $O_{1}\left(3, p^{n}\right)$ and that for every set of solutions of 150 ) there exists a substitution of $O_{1}\left(3, p^{n}\right)$, derived from $O_{i, j}^{\alpha, \beta}$ and $R$, which replaces $\xi_{1}$ by $\omega_{1}$.

178. Theorem. - The first orthogonal group $O_{1}\left(3, p^{n}\right)$ contains a subgroup $O_{1}^{\prime}\left(3, p^{n}\right)$ holoedrically isomorphic with the group $L F\left(2, p^{n}\right)$ of linear fractional substitutions of determinant unity.

Let $-1=i^{2}$, so that $i$ belongs to the $G F\left[p^{n}\right]$ if and only if -1 be a square in that field. Introduce in place of $\xi_{1}, \xi_{2}, \xi_{3}$ the new indices

so that

$$
\eta_{1} \equiv-i \xi_{1}, \quad \eta_{2} \equiv \xi_{2}-i \xi_{3}, \quad \eta_{3} \equiv \xi_{2}+i \xi_{3},
$$

$$
-\eta_{1}^{2}+\eta_{2} \eta_{3} \equiv \xi_{1}^{2}+\xi_{2}^{2}+\xi_{3}^{2} \text {. }
$$

1) For a more direct treatment of this case, but one involving considerable calculation, see Amer. Journal, vol. 21; pp. 202-204, in which the proof of Jordan, Traité, pp. 164-166, for $n=1$, is corrected and generalized. 
The following substitution of determinant unity,

$$
\begin{array}{rlll}
Y: & \eta_{1} & \eta_{2} & \eta_{3} \\
& \eta_{1}^{\prime}=\sqrt{\alpha \delta+\beta \gamma} & \alpha \gamma & \beta \delta \\
2 \alpha \beta & \alpha^{2} & \beta^{2} \\
2 \gamma \delta & \gamma^{2} & \delta^{2}
\end{array} \quad(\alpha \delta-\beta \gamma)=1
$$

leaves $\eta_{2} \eta_{3}-\eta_{1}^{2}$ absolutely invariant. Written in terms of the indices $\xi_{1}, \xi_{2}, \xi_{3}$, it takes the form

$$
X \equiv\left(\begin{array}{clc}
\alpha \delta+\beta \gamma & i(\alpha \gamma+\beta \delta) & \alpha \gamma-\beta \delta \\
-i(\alpha \beta+\gamma \delta) & \frac{1}{2}\left(\alpha^{2}+\beta^{2}+\gamma^{2}+\delta^{2}\right) & \frac{i}{2}\left(-\alpha^{2}+\beta^{2}-\gamma^{2}+\delta^{2}\right) \\
\alpha \beta-\gamma \delta & \frac{i}{2}\left(\alpha^{2}+\beta^{2}-\gamma^{2}-\delta^{2}\right) & \frac{1}{2}\left(\alpha^{2}-\beta^{2}-\gamma^{2}+\delta^{2}\right)
\end{array}\right) .
$$

It follows that $X$ has determinant unity $(\S 101)$ and leaves $\xi_{1}^{2}+\xi_{2}^{2}+\xi_{3}^{2}$ absolutely invariant. Giving to the substitution $Y$ the notation

$$
\left[\begin{array}{ll}
\alpha & \beta \\
\gamma & \delta
\end{array}\right] \equiv\left[\begin{array}{ll}
-\alpha & -\beta \\
-\gamma & -\delta
\end{array}\right] \quad(\alpha \delta-\beta \gamma=1)
$$

we readily verify the formula of composition

$$
\left[\begin{array}{ll}
\alpha^{\prime} & \beta^{\prime} \\
\gamma^{\prime} & \delta^{\prime}
\end{array}\right]\left[\begin{array}{ll}
\alpha & \beta \\
\gamma & \delta
\end{array}\right]=\left[\begin{array}{ll}
\alpha \alpha^{\prime}+\beta \gamma^{\prime}, & \alpha \beta^{\prime}+\beta \delta^{\prime} \\
\gamma \alpha^{\prime}+\delta \gamma^{\prime} & \gamma \beta^{\prime}+\delta \delta^{\prime}
\end{array}\right]
$$

The group of the substitutions $X$, being isomorphic with the group of the substitutions $Y$, is isomorphic with the group of the linear fractional substitutions 151). But $Y$ and therefore $X$ is the identity if and only if $\alpha=\delta= \pm 1, \beta=\gamma=0$. Hence the isomorphism is holoedric.

If -1 be a square in the $G F\left[p^{n}\right]$, so that the coefficients of $X$ belong to that field, the substitutions $X$ form a group $O_{1}^{\prime}\left(3, p^{n}\right)$, a subgroup of $O_{1}\left(3, p^{n}\right)$, which is holoedrically isomorphic with $L F\left(2, p^{n}\right)$.

If -1 be a not-square in the $G F\left[p^{n}\right]$, the coefficients of $X$ will belong to the $G F\left[p^{n}\right]$ if we choose $\alpha, \beta, \gamma, \delta$ in the $G F\left[p^{2 n}\right]$ such that $\alpha$ is conjugate ( $(73)$ with $\delta, \beta$ with $-\gamma$, with respect to the $G F\left[p^{n}\right]$. By $\S 144$, the resulting substitutions 151) of determinant unity form a group holoedrically isomorphic with $L F\left(2, p^{n}\right)$. The corresponding substitutions $X$ form a subgroup $O_{1}^{\prime}\left(3, p^{n}\right)$ of $O_{1}\left(3, p^{n}\right)$.

In each case, the subgroup $O_{1}^{\prime}\left(3 ; p^{n}\right)$ has the order $\frac{1}{2} p^{n}\left(p^{2 n}-1\right)$, since it is holoedrically isomorphic with $\operatorname{LF}\left(2, p^{n}\right)$. We proceed to prove that this subgroup does not coincide with $O_{1}\left(3, p^{n}\right)$. In order that $O_{2,3}^{a, b}$ shall be of the form $X$, it is necessary and sufficient that

$$
\beta=\gamma=0, \quad \alpha \delta=1, \quad \alpha^{2}=a+b i, \quad \delta^{2}=a-b i .
$$


According to the definition of $\alpha, \beta, \gamma, \delta$ in the above cases, the expressions

$$
A \equiv \frac{1}{2}(\delta+\alpha), \quad B \equiv \frac{i}{2}(\delta-\alpha)
$$

belong to the $G F\left[p^{n}\right]$. The above conditions then give

$$
a=2 A^{2}-1, \quad b=2 A B, \quad A^{2}+B^{2}=1,
$$

so that $O_{2,3}^{a, b}$ must be the special substitution $Q_{2,3}^{A, B}$ defined in $\S 181$ Any orthogonal substitution $O_{2,3}$ not of the form $Q_{2,3}$, and therefore not of the form $X$, will extend $O_{1}^{\prime}\left(3, p^{n}\right)$ to a larger subgroup $G$ of $O_{1}\left(3, p^{n}\right)$. The order of $G$ is therefore at least $p^{n}\left(p^{2 n}-1\right)$. From the remarks at the end of $\S 177$, it follows that $G$ has-exactly this order and hence coincides with $O_{1}\left(3, p^{n}\right)$.

179. We proceed to the proof that, if -1 be a square $i^{2}$ in the $G F\left[p^{n}\right]$, the group $O_{1}\left(3, p^{n}\right)$ is generated by the substitutions $O_{i, j}^{\alpha, \beta}$ together with $R_{123}$ if $p^{n}=5$. If $p^{n}>5$, there exist ( $\left.\$ 64\right)$ marks $\beta$ and $\tau$ in the $G F\left[p^{n}\right]$ such that

Then the product

$$
1+\beta^{2}=\tau^{2} \quad(\beta \neq 0, \tau \neq 0) .
$$

$$
\begin{aligned}
O_{1,2}^{0,1} O_{2,3}^{\frac{1}{\tau}, \frac{\beta}{\tau}} O_{1,2}^{i \beta, \tau} O_{2,3}^{\frac{1}{2}\left(\frac{1}{\tau}+\tau\right), \frac{i}{2}\left(\frac{1}{\tau}-\tau\right)} \\
\equiv\left(\begin{array}{ccc}
1 & i \beta & -\beta \\
-i \beta & 1+\frac{1}{2} \beta^{2} & \frac{i}{2} \beta^{2} \\
\beta & \frac{i}{2} \beta^{2} & 1-\frac{1}{2} \beta^{2}
\end{array}\right) \equiv\left[\begin{array}{ll}
1 & \beta \\
0 & 1
\end{array}\right] .
\end{aligned}
$$

But $\left[\begin{array}{ll}\alpha & 0 \\ 0 & \alpha-1\end{array}\right]$, which is an $O_{2,3}$, transforms $\left[\begin{array}{ll}1 & \beta \\ 0 & 1\end{array}\right]$ into $\left[\begin{array}{ll}1 & \beta \alpha^{2} \\ 0 & 1\end{array}\right]$. Furthermore,

$$
\left[\begin{array}{ll}
1 & \beta \alpha_{1}^{2} \\
0 & 1
\end{array}\right]\left[\begin{array}{ll}
1 & \beta \alpha_{2}^{2} \\
0 & 1
\end{array}\right]=\left[\begin{array}{ll}
1 & \beta\left(\alpha_{1}^{2}+\alpha_{2}^{2}\right) \\
0 & 1
\end{array}\right]
$$

Since $\beta \neq 0$, we can $(\S, 64)$ find marks $\alpha_{1}$ and $\alpha_{2}$ in the field such that $\beta\left(\alpha_{1}^{2}+\alpha_{2}^{2}\right)=x$, where $x$ is an arbitrary mark $\neq 0$. Also

$$
\left[\begin{array}{rr}
0 & 1 \\
-1 & 0
\end{array}\right]^{-1}\left[\begin{array}{rr}
1 & -x \\
0 & 1
\end{array}\right]\left[\begin{array}{rr}
0 & 1 \\
-1 & 0
\end{array}\right]=\left[\begin{array}{ll}
1 & 0 \\
\varkappa & 1
\end{array}\right], \quad\left[\begin{array}{rr}
0 & 1 \\
-1 & 0
\end{array}\right] \equiv O_{1,3}^{-1,0} \text {. }
$$

Hence, if $p^{n}>5$, we have reached from the $O_{i, j}^{\alpha, \beta}$ the substitutions

$$
\left[\begin{array}{ll}
1 & x \\
0 & 1
\end{array}\right],\left[\begin{array}{ll}
1 & 0 \\
x & 1
\end{array}\right]
$$

( $x$ arbitrary).

By $\S \S 100$ and 108, these substitutions generate the group $L F\left(2, p^{n}\right)$. Hence the $O_{i, j}^{\alpha, \beta}$ from which they were derived generate the isomorphic 
group $O_{1}^{\prime}\left(3, p^{n}\right)$. Then, by the last section, . all the $O_{i, j}^{\alpha, \beta}$ generate $O_{1}\left(3, p^{n}\right)$. For $p^{n}=5, i=2$, we have

$$
R_{123} \equiv\left(\begin{array}{lll}
1 & 1 & 2 \\
1 & 2 & 1 \\
2 & 1 & 1
\end{array}\right)=\left[\begin{array}{ll}
2 & 1 \\
0 & 3
\end{array}\right]=\left[\begin{array}{ll}
1 & 3 \\
0 & 1
\end{array}\right]\left[\begin{array}{ll}
2 & 0 \\
0 & 2-1
\end{array}\right]
$$

Hence from $R_{123}$ and $\left[\begin{array}{ll}2 & 0 \\ 0 & 2-1\end{array}\right] \equiv O_{2,3}^{-1,0}$ we reach $\left[\begin{array}{ll}1 & 3 \\ 0 & 1\end{array}\right]$. It follows as above that $R_{123}$ and $O_{2,3}^{-1,0} \equiv C_{2} C_{3}$ and $C_{1} C_{3}$ generate $O_{1}^{\prime}(3,5)$. The latter is extended to $O_{1}(3,5)$ by any $O_{i, j}^{0, \pm 1}$.

180. Theorem. - If $\alpha_{1}, \alpha_{2}, \ldots, \alpha_{m}$ be any set of solutions in the $G F\left[p^{n}\right]$ of

$$
\alpha_{1}^{2}+\alpha_{2}^{2}+\cdots+\alpha_{m-1}^{2}+\frac{1}{\mu} \alpha_{m}^{2}=1,
$$

there exists a substitution $S$ derived from the generators ${ }^{1}$ ) of $\S 173$ which replaces $\xi_{1}^{\circ}$ by $\omega_{1} \equiv \alpha_{1} \xi_{1}+\alpha_{2} \xi_{2}+\cdots+\alpha_{m} \xi_{m}$.

The proposition having been proven for $m=2$ and $m=3$, we will give a proof by induction from $m-1$ to $m$, supposing $m>3$.

Consider first the case in which every sum of three of the terms $\alpha_{1}^{2}, \alpha_{2}^{2}, \ldots, \alpha_{m-1}^{2}, \frac{1}{\mu} \alpha_{m}^{2}$ is zero. These terms must all be equal and therefore

$$
m \alpha_{1}^{2}=1, \quad 3 \alpha_{1}^{2}=0, \quad \mu=\text { square. }
$$

Hence $p=3$, while $m$ is of the form $3 k+2$ or $3 k+1$.

If $m=3 k+2$, we have $1-\alpha_{1}^{2}=\alpha_{1}^{2} \neq 0$, so that the theorem is reduced by $\S 174$ to the case of $m-1$ indices.

If $m=3 k+1$, we must have $\alpha_{1}^{2}=1$. But the product $O_{1,2}^{\alpha, \beta} S$ will replace $\xi_{1}$ by $\alpha_{1}^{\prime} \xi_{1}+\cdots+\alpha_{m}^{\prime} \xi_{m}$, where

$$
\alpha_{1}^{\prime} \equiv \alpha \alpha_{1}-\beta \alpha_{2}, \quad \alpha_{2}^{\prime} \equiv \beta \alpha_{1}+\alpha \alpha_{2}, \quad \alpha_{j}^{\prime} \equiv \alpha_{j} \quad(j=3, \ldots, m) .
$$

Of the $3^{n} \pm 1$ sets of values in the $G F\left[3^{n}\right]$ satisfying

$$
\alpha^{2}+\beta^{2}=1
$$

at most two give the same value to $\alpha_{1}^{\prime}$ and hence at most four make $\alpha_{1}^{\prime}=1$. Hence, if $n>1$, we can avoid the case $\alpha_{1}^{2}=1$. For $p^{n}=3$, we may take

1) For the case $p^{n}=5, m \overline{>}, \mu=$ not-square, it would appear that the generator $R_{123}$ were necessary in addition to the $O_{i, j}^{\alpha, \beta}$. We can, however, express $R_{123}$ in terms of the generators

$$
O_{i, m}^{2,1}:\left\{\begin{array}{l}
\xi_{i}^{\prime}=2 \xi_{i}+\xi_{m} \\
\xi_{m}^{\prime}=3 \xi_{i}+2 \xi_{m}
\end{array}\right.
$$

leaving invariant $\xi_{1}^{2}+\xi_{2}^{2}+\cdots+\xi_{m-1}^{2}+3 \xi_{m}^{2}$. Indeed,

$$
R_{123}=O_{1 m} O_{2 m} O_{3 m}^{-1} O_{1 m} O_{2 m}^{-1} O_{3 m}^{-1} .
$$




$$
S=C W_{1234} W_{1567} \ldots W_{13 k-13 k 3 k+1} K
$$

where $C$ and $K$ denote products of an even number of the $C_{i}$ [compare the end of $\S 175]$.

Suppose next that the above sums are not all zero, for example ${ }^{1}$ )

$$
\alpha_{1}^{2}+\alpha_{2}^{2}+\frac{1}{\mu} \alpha_{i n}^{2} \neq 0 .
$$

We have proven that, for every set of solutions of

$$
\alpha^{2}+\beta^{2}+\frac{1}{\mu} \gamma^{2}=1
$$

there exists a substitution $\Sigma$ of the group, $\xi_{1}^{\prime}=\alpha \xi_{1}+\beta \xi_{2}+\gamma \xi_{m}, \quad \xi_{2}^{\prime}=\alpha^{\prime} \xi_{1}+\beta^{\prime} \xi_{2}+\gamma^{\prime} \xi_{m}, \quad \xi_{m}^{\prime}=\alpha^{\prime \prime} \xi_{1}+\beta^{\prime \prime} \xi_{2}+\gamma^{\prime \prime} \xi_{m}$ which therefore satisfies the relation 152) and the following: $\stackrel{2}{\alpha}^{\prime}+\hat{\beta}^{\prime}+\frac{1}{\mu} \stackrel{2}{\gamma}^{\prime}=1, \quad \alpha^{2}+\stackrel{2}{\alpha}^{\prime}+\mu \alpha^{\prime \prime}=1, \quad \alpha \beta+\alpha^{\prime} \beta^{\prime}+\mu \alpha^{\prime \prime} \beta_{\bullet}^{\prime \prime}=0$, etc. If there be a substitution $S^{\prime}$ in our group which replaces $\xi_{1}$ by

where

$$
\alpha_{1}^{\prime} \xi_{1}+\alpha_{2}^{\prime} \xi_{2}+\alpha_{m}^{\prime} \xi_{m}+\sum_{j=3}^{m-1} \alpha_{j} \xi_{j},
$$

$$
\begin{aligned}
& \alpha_{1}^{\prime}=\alpha \alpha_{1}+\beta \alpha_{2}+\frac{\gamma}{\mu} \alpha_{m}, \\
& \alpha_{2}^{\prime}=\alpha^{\prime} \alpha_{1}+\beta^{\prime} \alpha_{2}+\frac{\gamma^{\prime}}{\mu} \alpha_{m}, \\
& \alpha_{m}^{\prime}=\mu \alpha^{\prime \prime} \alpha_{1}+\mu \beta^{\prime \prime} \alpha_{2}+\gamma^{\prime \prime} \alpha_{m},
\end{aligned}
$$

then the group will contain $\Sigma S^{\prime}$ which replaces $\xi_{1}$ by $\omega_{1}$. The proposition is therefore true for the quantities $\alpha_{j}$ if true for $\alpha_{1}^{\prime}, \alpha_{2}^{\prime}, \alpha_{m}^{\prime}$, $\alpha_{3}, \alpha_{4}, \ldots, \alpha_{m-1}$. We may thus make our proof by induction from $m-1$ to $m$ by showing that it is possible to choose $\alpha, \beta, \gamma$ among the sets of solutions of 152 ) in such a way that $\alpha_{1}^{\prime}=0$. We may suppose that $\alpha_{1} \neq 0$, since otherwise the proposition is already proven.

If $\alpha_{1}^{2}+\alpha_{2}^{2}=0$, then $\alpha_{2} \neq 0$. From $\frac{1}{\mu} \alpha_{m}^{2}=1$, it follows that $\mu$ is a square, say $\mu=1$. Then the values

$$
\alpha=\frac{-\alpha_{m}}{2 \alpha_{1}}, \quad \beta=\frac{-\alpha_{m}}{2 \alpha_{2}}, \quad \gamma=1
$$

satisfy 152) and make $\alpha_{1}^{\prime}=0$.

If $\alpha_{1}^{2}+\alpha_{2}^{2} \neq 0$, the condition 152) combines with $\dot{\alpha}_{1}^{\prime}=0$ to give a single condition for $\beta$ and $\gamma$ :

$$
\left(\beta \alpha_{2}+\frac{\gamma}{\mu} \alpha_{m}\right)^{2}+\alpha_{1}^{2}\left(\beta^{2}+\frac{1}{\mu} \gamma^{2}\right)=\alpha_{1}^{2} .
$$

1) The treatment for a case like $\alpha_{1}^{2}+\alpha_{2}^{2}+\alpha_{3}^{2}=\mid=0$ is quite similar, taking $\mu=1$. 
Multiplying this by $\alpha_{1}^{2}+\alpha_{2}^{2}$, it may be given the form

$$
\left\{\beta\left(\alpha_{1}^{2}+\alpha_{2}^{2}\right)+\frac{\alpha_{2} \alpha_{m}}{\mu} \gamma\right\}^{2}+\frac{\gamma^{2} \alpha_{1}^{2}}{\mu}\left(\alpha_{1}^{2}+\alpha_{2}^{2}+\frac{\alpha_{m}^{2}}{\mu}\right)=\alpha_{1}^{2}\left(\alpha_{1}^{2}+\alpha_{2}^{2}\right) .
$$

Since the coefficient of $\gamma^{2}$ is not zero, this equation has in the $G F\left[p^{n}\right]$. (by $\left.\S 64\right) p^{n} \pm 1$ sets of solutions for $\gamma$ and

$$
\left\{\beta\left(\alpha_{1}^{2}+\alpha_{2}^{2}\right)+\frac{\alpha_{2} \alpha_{m}}{\mu} \gamma\right\}
$$

and hence as many sets of solutions $\beta, \gamma$.

The structure of the first and second orthogonal groups, §§ 181-198. 181. The group $O_{\mu}\left(m, p^{n}\right)$ contains the substitutions

$$
O_{i, j}^{\rho, \sigma}: \quad\left\{\begin{array}{l}
\xi_{i}^{\prime}=\rho \xi_{i}+\sigma \xi_{j} \\
\xi_{j}^{\prime}=-\frac{\sigma}{\lambda} \xi_{i}+\varrho \xi_{j}
\end{array} \quad\left(\varrho^{2}+\frac{1}{\lambda} \sigma^{2}=1\right)\right.
$$

leaving $\xi_{i}^{2}+\lambda \xi_{j}^{2}$ invariant, where $\lambda=1$ if $i, j<m$, but $\lambda=\mu$ if $i<j=m$. For $i$ and $j$ fixed, while $\varrho, \sigma$ take all possible values in the field such that $\rho^{2}+\frac{1}{\lambda} \sigma^{2}=1$, the substitutions $O_{i, j}^{o, \sigma}$ form $\cdot$ a subgroup denoted by $O_{i, j}$. Its substitutions are commutative since the following product is unaltered if we interchange $\rho$ with $\varrho^{\prime}$ and $\sigma$ with $\sigma^{\prime}$ :

$$
O_{i, j}^{\rho^{\prime}, \sigma^{\prime}} O_{i, j}^{o, \sigma}:\left\{\begin{array}{l}
\xi_{i}^{\prime}=\left(\rho \varrho^{\prime}-\frac{\sigma \sigma^{\prime}}{\lambda}\right) \xi_{i}+\left(\rho \sigma^{\prime}+\varrho^{\prime} \sigma\right) \xi_{j}, \\
\xi_{j}^{\prime}=-\left(\frac{\rho \sigma^{\prime}+\varrho^{\prime} \sigma}{\lambda}\right) \xi_{i}+\left(\rho \varrho^{\prime}-\frac{\sigma \sigma^{\prime}}{\lambda}\right) \xi_{j} .
\end{array}\right.
$$

By $\S 64$, the order of $O_{i j}$ is $p^{n}-\varepsilon_{i j}$, where $\varepsilon_{i j}=+1$ or -1 according as $-1 / \lambda$ is. a square or a not-square in the GF[ $\left[p^{n}\right]$.

The squares of the substitutions of $O_{i, j}$ form a commutative group $Q_{i, j}$, composed of the substitutions,

$$
Q_{i, j}^{\rho, \sigma} \equiv\left(O_{i, j}^{\rho, \sigma}\right)^{2}: \quad\left\{\begin{array}{l}
\xi_{i}^{\prime}=\left(2 \varrho^{2}-1\right) \xi_{i}+2 \rho \sigma \xi_{j}, \\
\xi_{j}^{\prime}=-\frac{2 \varrho \sigma}{\lambda} \xi_{i}+\left(2 \varrho^{2}-1\right) \xi_{j} .
\end{array}\right.
$$

The order of $Q_{i j}$ is $\frac{1}{2}\left(p^{n}-\varepsilon_{i j}\right)$. Indeed, the identity

$$
Q_{i, j}^{o, \sigma} \equiv Q_{i, j}^{\ell^{\prime}, \sigma^{\prime}}
$$

holds if and only if $\varrho^{\prime}= \pm \rho, \sigma^{\prime}= \pm \sigma$. 
Let $O_{i, j}^{\rho, \sigma}$ be a particular substitution which extends $Q_{i j}$ to $O_{i j}$, the values $\varrho$, $\sigma$ depending on $\lambda$. Consider the subgroup $O_{\mu}^{\prime}\left(m, p^{n}\right)$ of $O_{\mu}\left(m, p^{n}\right)$ generated by the substitutions

$$
Q_{i, j}^{\alpha, \beta}, \quad O_{i, j}^{\rho, \sigma} \cdot O_{k l}^{\rho, \sigma} \quad(i, j, k, l=1, \ldots, m ; i \neq j, k \neq l)
$$

where $\alpha, \beta$ take all the values in the $G F\left[p^{n}\right]$ satisfying $\alpha^{2}+\frac{1}{\lambda} \beta^{2}=1$, the generator $R, W$ or $V$ being added in the exceptional cases of $\S 173$.

182. Theorem. - The order of $O_{\mu}^{\prime}\left(m, p^{n}\right)$ is at least half of the order of $O_{\mu}\left(m, p^{n}\right)$.

By the theorem of $\S 173$, every substitution of $O_{\mu}\left(m, p^{n}\right)$ has the form

$$
S \equiv h_{1} O_{i, j}^{o, \sigma} h_{2} O_{\ddot{k}, l}^{o, \sigma} h_{3} \ldots
$$

where the $h_{i}$ (and the $h_{i}^{\prime}, h^{\prime \prime}, h, \ldots$ below) are derived from the generators of $O_{\mu}^{\prime}\left(m, p^{n}\right)$. For $m>2, O_{1,2}^{\gamma, \delta}$ and $O_{1,2}^{\gamma,-\delta} \equiv O_{2,1}^{\gamma, \delta}$ are reciprocal. Hence

Hence

$$
O_{i, j}^{o, \sigma}=O_{i, j}^{\rho, \sigma} \cdot O_{2,1}^{o, \sigma} O_{i, 2}^{o, \sigma}=h_{4} O_{i, 2}^{\rho, \sigma} .
$$

$$
S=h_{1}^{\prime} O_{1,2}^{o, \sigma} h_{2}^{\prime} O_{1,2}^{o, j} h_{3}^{\prime} \ldots
$$

Furthermore, $O_{i, 2}^{\rho, \sigma}$ is commutative with every $Q_{1,2}^{\alpha, \beta}$ and every $Q_{i, j}^{\alpha, \beta}$, $i$ and $j>2$. Since the square of $O_{i, k}^{o, \sigma}$ is $Q_{i, k}^{o, \sigma}$, whose reciprocal is $Q, \frac{\rho,-\sigma}{j}$.

$$
\begin{aligned}
& O_{1,2}^{\rho, \sigma} Q_{1, k}^{\alpha, \beta}=O_{1,2}^{o, \sigma}\left(O_{1, k}^{\rho, \sigma}\right)^{2} Q_{1, k}^{o,-\sigma} \cdot Q_{1, k}^{\alpha, \beta} \\
= & O_{1,2}^{\rho, \sigma} O_{i, k}^{o, \sigma} \cdot Q_{1, k}^{\rho, \sigma} Q_{1, k}^{\alpha, \beta} \cdot O_{1, k}^{\rho, \sigma}=h^{\prime} O_{1, k}^{\rho, \sigma}=h^{\prime \prime} O_{i, 2}^{o, \sigma} .
\end{aligned}
$$

Aside from the above exceptional cases, we may conclude that $S$ is of the form $h$ or else $h \cdot O_{1,2}^{o, \sigma}$. We treat next the exceptional cases.

$1^{0}$. For $p^{n}=5, m>3, \mu=1$, the additional generator is $R_{123}$, and the only $Q_{i, j}^{\alpha, \beta}$ are $Q_{i, j}^{0, \pm 1} \equiv C_{i} C_{j}$ and $Q_{i, j}^{ \pm 1,0}=I$. Since

$$
O_{i, j}^{0,-1} \equiv T_{i j} C_{i}
$$

where $\dot{T}_{i j} \equiv\left(\xi_{i} \xi_{j}\right)$, is not in $Q_{i j}$, it may be taken for $O_{i, j}^{o, \sigma}$. To omplete the proof. that $S=h$ or $h O_{1,2}^{o, \sigma}$, we note that

$$
I_{12} C_{1} \cdot R_{123}=C_{1} C_{3} R_{123} \cdot T_{23} C_{2} \cdot T_{12} C_{1} \cdot C_{1} C_{3} \cdot T_{12} C_{1} \text {. }
$$

$2^{0}$. For $p^{n}=3, m>4, \mu=1$, the additional generator is $W_{1234}$. The remarks of $1^{0}$ apply here, if we replace the last formula by

$$
T_{12} C_{1} \cdot W_{1234}=C_{1} C_{2} W_{1234}^{2} T_{12} C_{1} \text {. }
$$

$3^{0}$. For $p^{n}=3, m>3, \mu=\nu=-1$, the additional generator is $V_{12 m}$ and the only $O_{i, m}^{\alpha, \beta}\left(\alpha^{2}-\beta^{2}=1\right)$ are $C_{i} C_{m}$ and $I$, the only $Q_{i, m}^{\alpha, \beta}$ being $I$. We may take $O_{i, j}^{\rho, \sigma} \equiv T_{i j} C_{i}(i, j<m)$ and $O_{i, m}^{o, \sigma} \equiv C_{i} C_{m}$. To complete our proof, we use the formulae

$$
I_{12} C_{1} V_{12 m}=C_{1} C_{2} V_{12 m}^{2} T_{12} C_{1}, \quad C_{1} C_{m} V_{12 m}=V_{12 m}^{2} T_{12} C_{2} \text {. }
$$


183. Theorem. - The group $O_{1}^{\prime}\left(3, p^{n}\right)$ just defined is identical with the subgroup of $O_{1}\left(3, p^{n}\right)$ of index two defined in $\S 178$.

It is only necessary to show that every $Q_{i, j}^{a, b}$ and every $O_{i, j}^{\alpha, \sigma} O_{i, k}^{\alpha, \sigma}$ are of the form $X$ or, if we prefer, 151). We have

$$
\begin{gathered}
O_{1,2}^{*, \sigma} O_{2,3}^{\alpha, \sigma} \equiv\left(\begin{array}{ccc}
\varkappa & \sigma & 0 \\
-\varkappa \sigma & x^{2} & \sigma \\
\sigma^{2} & -\varkappa \sigma & x
\end{array}\right) \equiv\left[\begin{array}{cc}
\frac{x+1+i \sigma}{2} & -\frac{x-1+i \sigma}{2} \\
\frac{x-1-i \sigma}{2} & \frac{x+1-i \sigma}{2}
\end{array}\right], \\
T_{12} T_{13} \equiv\left[\begin{array}{cc}
\frac{1}{2}(1-i) & -\frac{1}{2}(1-i) \\
\frac{1}{2}(1+i) & \frac{1}{2}(1+i)
\end{array}\right] .
\end{gathered}
$$

In particular, we reach $T_{12} T_{13}$ and $T_{12} T_{23}$. These suffice to transform $O_{1,2}^{*, \sigma} O_{2}^{\psi, \sigma}$ into $O_{2,3}^{\psi, \sigma} O_{3,1}^{\psi, \sigma}$ and $O_{3,1}^{\psi, \sigma} O_{1,2}^{\psi, \sigma}$. Transforming these products by $C_{1} C_{2}, C_{1} C_{3}$ and $C_{2} C_{3}$, we obtain every $O_{i, j}^{*, \sigma} O_{k, l}^{*, \sigma}$, since $C_{i}$ transforms $O_{i, j}^{\alpha, \sigma}$ into $O_{j, i}^{\alpha, \sigma}$. Transforming $Q_{2,3}^{a, b}$ (which is of the form $X$ by $\S 178)$ by $T_{12} T_{13}$ and $T_{12} T_{23}$, we obtain every $Q_{i, j}^{a, b}$.

184. Theorem. - The group $O_{v}^{\prime}\left(3, p^{n}\right)$ is of index two under the second orthogonal group $O_{v}\left(3, p^{n}\right)$.

Consider the substitutions, in which $\alpha^{2}+\beta^{2}=\nu$,

$$
O:\left\{\begin{array}{l}
\xi_{1}^{\prime}=\alpha \xi_{1}-\beta \xi_{2} \\
\xi_{2}^{\prime}=\beta \xi_{1}+\alpha \xi_{2}
\end{array}, \quad O^{-1}:\left\{\begin{array}{l}
\xi_{1}^{\prime}=\frac{\alpha}{\nu} \xi_{1}+\frac{\beta}{\nu} \xi_{2} \\
\xi_{2}^{\prime}=-\frac{\beta}{\nu} \xi_{1}+\frac{\alpha}{v} \xi_{2}:
\end{array}\right.\right.
$$

Since $O$ transforms $\xi_{1}^{2}+\xi_{2}^{2}+\nu \xi_{3}^{2}$ into $\nu\left(\xi_{1}^{2}+\xi_{2}^{2}+\xi_{3}^{2}\right)$, it transforms the group $O_{1}\left(3, p^{n}\right)$ of the latter into the group $O_{v}\left(3, p^{n}\right)$ of the former. But $O$ is commutative with $O_{1,2}^{o, \sigma}$. Hence if $O_{1,2}^{2, \tau}$ serves to extend the subgroup $O_{1}^{\prime}\left(3, p^{n}\right)$ to $O_{1}\left(3, p^{n}\right)$, there exists a subgroup $G$ of $O_{v}\left(3, p^{n}\right)$ which $O_{1,2}^{\alpha, \tau}$ extends to the latter. We readily prove that $G$ is identical with the $O_{v}^{\prime}\left(3, p^{n}\right)$ defined in $\S 181$. For example, $O$ transform $O_{1,2}^{*, \tau} O_{1,2}^{o, \sigma} C_{1} C_{3}$ into $O_{1,2}^{*, \tau} C_{1} C_{3}$, where

$$
\varrho \equiv\left(\alpha^{2}-\beta^{2}\right) / \nu, \quad \sigma \equiv-2 \alpha \beta / \nu, \quad \varrho^{2}+\sigma^{2}=1 .
$$

Here $O_{1,2}^{o, \sigma}$ is not a $Q_{1,2}$ since $(1+\varrho) / 2 \equiv \alpha^{2} / \nu$ is a not-square. But $C_{1} C_{3}$ is a $Q_{1,3}$ in $O_{1}\left(3, p^{n}\right)$, but not in $O_{v}\left(3, p^{n}\right)$. It follows that $G$ contains the product $O_{1,2}^{2, \tau} C_{1} C_{3}$, neither factor being a $Q$.

185. It will be shown in the following sections that $O_{\mu}^{\prime}\left(m, p^{n}\right)$ is not identical with $O_{\mu}\left(m, p^{n}\right)$ in the cases $m=4,5,6$ and therefore, by $\S 182$, that its index under $O_{\mu}\left(m, p^{n}\right)$ is exactly two. By $\S \S 181-184$, the same result is true for $m=2$ and $m=3$. For 
various reasons it would seem that the same result holds true when $m>6$, but no explicit investigation has yet been made. The developments in $\S \S 191-193$ are made on the assumption that this index is 2. Moreover, if this conjecture prove false, very simple alterations would be necessary in the treatment.

186. We continue the investigations begun in $\S 163$ on the senary group $G_{4,2}^{\prime}$, whose substitutions leave absolutely invariant the Pfaffian [ $\left[\begin{array}{llll}1 & 2 & 3 & 4\end{array}\right]$, viz.,

$$
F_{4} \equiv Y_{12} Y_{34}-Y_{13} Y_{24}+Y_{14} Y_{23} .
$$

Denote by $G_{6}$ the group of all substitutions of determinant unity in the $G F\left[p^{n}\right], p>2$, which leave $F_{4}$ absolutely invariant. We will prove that $G_{6}$ is holoedrically isomorphic with $O_{\mu}\left(6, p^{n}\right)$, where $\mu=1$ or $\nu$ according as $p^{n}=4 l+1$ or $4 l+3$. Hence $G_{6}$ has the order (§ 172)

$$
\left(p^{4 n}-1\right) p^{3 n}\left(p^{3 n}-1\right) p^{2 n}\left(p^{2 n}-1\right) p^{n}
$$

It will therefore follow from the theorem of $\S 163$ that $G_{4,2}$ is a subgroup of index two under the group $G_{6}$.

18\%. Let $p^{n}=4 l+1$, so that -1 is the square of a mark $i$ belonging to the $G F\left[p^{n}\right]$. We make the following transformation of indices:

$$
\begin{array}{lrr}
Y_{12} \equiv \xi_{1}+i \xi_{2}, & -Y_{13} \equiv \xi_{3}+i \xi_{4}, & Y_{14} \equiv \xi_{5}+i \xi_{6}, \\
Y_{34} \equiv \xi_{1}-i \xi_{2}, & Y_{24} \equiv \xi_{3}-i \xi_{4}, & Y_{23} \equiv \xi_{5}-i \xi_{6} .
\end{array}
$$

Then $F_{4}$ takes the form

$$
\Psi \equiv \sum_{i=1}^{6} \xi_{i}^{2} .
$$

Hence $G_{6}$ is holoedrically isomorphic with $O_{1}\left(6, p^{n}\right)$. By $\S 164$, the following substitution of $G_{6}$ (leaving four of the indices fixed):

$$
Y_{13}^{\prime}=\tau Y_{13}, \quad Y_{24}^{\prime}=\tau^{-1} Y_{24}
$$

belongs to the subgroup $G_{4,2}^{\prime}$ if and only if $\tau$ be a square in the field. Expressed in the new indices, it has the form

$$
\begin{aligned}
& \xi_{3}^{\prime}=\frac{1}{2}\left(\tau+\tau^{-1}\right) \xi_{3}+\frac{i}{2}\left(\tau-\tau^{-1}\right) \xi_{4}, \\
& \xi_{4}^{\prime}=-\frac{i}{2}\left(\tau-\tau^{-1}\right) \xi_{3}+\frac{1}{2}\left(\tau+\tau^{-1}\right) \xi_{4} .
\end{aligned}
$$

For $\tau$ an arbitrary mark $\neq 0$ of the field, 155) may be written

$$
O_{3,4}^{0, \sigma} \quad \varrho \equiv \frac{1}{2}\left(\tau+\tau^{-1}\right), \sigma \equiv \frac{i}{2}\left(\tau-\tau^{-1}\right), \rho^{2}+\sigma^{2}=1
$$


For $\left.\tau=t^{2}, 155\right)$ may be expressed in the form

$$
\text { 157) } Q_{3,4}^{\gamma, \delta} \quad \gamma \equiv \frac{1}{2}\left(t+t^{-1}\right), \delta \equiv \frac{i}{2}\left(t-t^{-1}\right), \gamma^{2}+\delta^{2}=1 .
$$

For $\tau$ a not-square, 155) is not of the form 157), since that would require $\gamma^{2}=(\tau+1)^{2} / 4 \tau$. It follows that to the subgroup $G_{4,2}^{\prime}$ of $G_{6}$ of index two there corresponds a subgroup $O$ of $O_{1}\left(6, p^{n}\right)$ of index two, where $O$ is extended to $O_{1}\left(6, p^{n}\right)$ by any substitution $O_{3,4}^{o, \sigma}$ not of the form $Q_{3,4}$. We proceed to prove that $O$ is identical with the group $O_{1}^{\prime}\left(6,-p^{n}\right)$ defined in $\S 181$. We first show that $O$ contains all even substitutions on the six letters $\xi_{1}, \ldots, \xi_{6}$. Expressing the substitution

$$
\left(\xi_{1} \xi_{2} \xi_{3}\right): \quad \xi_{1}^{\prime}=\xi_{2}, \quad \xi_{2}^{\prime}=\xi_{3}, \quad \xi_{3}^{\prime}=\xi_{1}, \quad \xi_{i}^{\prime}=\xi_{i} \quad(i=4,5,6)
$$

in terms of the indices $Y_{i j}$, it takes the form

$$
\begin{aligned}
& Y_{12}^{\prime}=\begin{array}{cccccc}
Y_{12} & Y_{13} & Y_{14} & Y_{23} & Y_{24} & Y_{34} \\
-i / 2 & -i / 2 & 0 & 0 & i / 2 & i / 2
\end{array} \\
& Y_{13}^{\prime}=\begin{array}{llllll}
-1 / 2 & 1 / 2 & 0 & 0 & 1 / 2 & -1 / 2
\end{array} \\
& Y_{14}^{\prime}=\begin{array}{llllll}
0 & 0 & 1 & 0 & 0 & 0
\end{array} \\
& Y_{23}^{\prime}=\begin{array}{llllll}
0 & 0 & 0 & 1 & 0 & 0
\end{array} \\
& Y_{24}^{\prime}=\begin{array}{llllll}
1 / 2 & 1 / 2 & 0 & 0 & 1 / 2 & 1 / 2
\end{array} \\
& Y_{34}^{\prime}=\mid \begin{array}{llllll}
-i / 2 & i / 2 & 0 & 0 & -i / 2 & i / 2
\end{array}
\end{aligned}
$$

By inspection this substitution is the second compound of

$$
\left(\begin{array}{cccc}
\frac{1}{2}(1-i) & 0 & 0 & \frac{1}{2}(1-i) \\
0 & \frac{1}{2}(1-i) & \frac{1}{2}(1-i) & 0 \\
0 & -\frac{1}{2}(1+i) & \frac{1}{2}(1+i) & 0 \\
-\frac{1}{2}(1+i) & 0 & 0 & \frac{1}{2}(1+i)
\end{array}\right)
$$

having determinant unity. Hence $O$ contains the substitution $\left(\xi_{1} \xi_{2} \xi_{3}\right)$. In the transformation of indices 154), the pairs $\xi_{1}$ and $\xi_{2}, \xi_{3}$ and $\xi_{4}$, $\xi_{5}$ and $\xi_{6}$ enter symmetrically. Hence $O$ contains the substitution $\left(\xi_{i} \xi_{j} \xi_{k}\right)$, two of the distinct integers $i, j, k$, each $\overline{<} 6$, being chosen from one of the above pairs. But the linear substitution denoted by $\left(\xi_{1} \xi_{2} \xi_{3}\right)$ transforms $\left(\xi_{1} \xi_{2} \xi_{5}\right)$ into $\left(\xi_{1} \xi_{5} \xi_{3}\right)$. Hence $O$ contains every cyclic substitution $\left(\xi_{r} \xi_{s} \xi_{t}\right)$ on the six indices and therefore every even permutation of the six indices. ${ }^{1}$ )

1) Netto-Cole, The theory of substitutions, p. 35 . 
Having every $Q_{3,4}^{\gamma, \delta}, O$ contains their transformed $Q_{i, j}^{\gamma, \delta}(i \neq j)$ by the even substitution $\left(\xi_{3} \xi_{i}\right)\left(\xi_{4} \xi_{j}\right)$. By $\S 164$, Note, $O$ contains the product $O_{i, 2}^{o, \sigma} O_{3,4}^{o, \sigma}$ and therefore also every $O_{i, j}^{o, \sigma} O_{k, l}^{o, \sigma}$, where $i, j, k, l$ are distinct. Hence $O$ contains

$$
O_{i, 2}^{o, \sigma} O_{3,4}^{\rho, \sigma} \cdot O_{4,3}^{\rho, \sigma} O_{i, 5}^{\rho, \sigma} \equiv O_{1,2}^{\rho, \sigma} O_{i, 5}^{o, \sigma}
$$

and therefore every $O_{i, j}^{o, \sigma} O_{k, l}^{\rho, \sigma}$ in which two of the subscripts are alike. For the case $p^{n}=5, i=2$, there is an additional generator, viz., $R_{123}$. Expressing $R_{123}$ in the indices $Y_{i j}$, we obtain the substitution

$$
\left(\begin{array}{llllll}
4 & 3 & 0 & 0 & 2 & 4 \\
0 & 1 & 0 & 0 & 0 & 3 \\
0 & 0 & 1 & 0 & 0 & 0 \\
0 & 0 & 0 & 1 & 0 & 0 \\
0 & 0 & 0 & 0 & 1 & 2 \\
0 & 0 & 0 & 0 & 0 & 4
\end{array}\right) .
$$

By inspection, this is the second compound of the following substitution of determinant unity with coefficients $\bmod 5$ :

$$
\left(\begin{array}{llll}
2 & 0 & 0 & 4 \\
0 & 2 & 4 & 0 \\
0 & 0 & 3 & 0 \\
0 & 0 & 0 & 3
\end{array}\right) .
$$

The group $O$ therefore contains all the generators of $O_{1}^{\prime}\left(6, p^{n}\right)$. Since $O$ is of index 2 under $O_{1}\left(6, p^{n}\right)$ and $O_{1}^{\prime}\left(6, p^{n}\right)$ of index at most 2 under $O_{1}\left(6, p^{n}\right)(\S 182)$, it follows that $O \equiv O_{1}^{\prime}\left(6, p^{n}\right)$. We have therefore, by $\S 163$, the theorem:

For $p^{n}=4 l+1$, the group $O_{1}^{\prime}\left(6, p^{n}\right)$ has a maximal invariant subgroup $\left\{I, T \equiv C_{1} C_{2} \ldots C_{6}\right\}$ of order two, the quotient-group being holoedrically isomorphic with the simple group $\operatorname{LF}\left(4, p^{n}\right) . \quad O_{1}^{\prime}\left(6, p^{n}\right)$ is of index two under the first orthogonal group $O_{1}\left(6, p^{n}\right)$ and is extended to it by any $O_{i, j}^{o, \sigma}$ not a $Q_{i j}$.

188. Let $p^{n}=4 l+3$, so that -1 is a not-square in the $G F\left[p^{n}\right]$. We make the following transformation of indices:

$$
\begin{aligned}
& Y_{12} \equiv \xi_{1}-\alpha \xi_{2}+\beta \xi_{4},-Y_{13} \equiv \xi_{3}-\beta \xi_{2}-\alpha \xi_{4}, \quad Y_{14} \equiv \xi_{5}-\xi_{6}, \\
& Y_{34} \equiv \xi_{1}+\alpha \xi_{2}-\beta \xi_{4}, \quad Y_{24} \equiv \xi_{3}+\beta \xi_{2}+\alpha \xi_{4}, \quad Y_{23} \equiv \xi_{5}+\xi_{6} \text {, }
\end{aligned}
$$

where $\alpha$ and $\beta$ is a suitable set of solutions in the field of

$$
\alpha^{2}+\beta^{2}=-1 \text {. }
$$


Under this transformation, $F_{4}$ takes the form

$$
\psi^{\prime} \equiv \xi_{1}^{2}+\xi_{2}^{2}+\xi_{3}^{2}+\xi_{4}^{2}+\xi_{5}^{2}-\xi_{6}^{2} .
$$

Hence $G_{6}$ is holoedrically isomorphic with the second orthogonal group $O_{-1}\left(6, p^{n}\right)$. Reversing equations 158$)$, we find

$$
\text { 160) } \begin{aligned}
& 2 \xi_{1} \equiv Y_{12}+Y_{34}, 2 \xi_{2} \equiv \alpha Y_{12}-\alpha Y_{34}-\beta Y_{13}-\beta Y_{24}, 2 \xi_{3} \equiv Y_{24}-Y_{13}, \\
& 2 \xi_{5} \equiv Y_{14}+Y_{23}, 2 \xi_{4} \equiv-\beta Y_{12}+\beta Y_{34}-\alpha Y_{13}-\alpha Y_{24}, 2 \xi_{6} \equiv Y_{23}-Y_{14}
\end{aligned}
$$

The following substitution leaving $F_{4}$ invariant,

$$
T_{\tau}: \quad Y_{14}^{\prime}=\tau Y_{14}, \quad Y_{23}^{\prime}=\tau^{-1} Y_{23},
$$

becomes, when expressed in the new indices 160),

$$
\begin{aligned}
& \xi_{5}^{\prime}=\frac{1}{2}\left(\tau+\tau^{-1}\right) \xi_{5}-\frac{1}{2}\left(\tau-\tau^{-1}\right) \xi_{6}, \\
& \xi_{6}^{\prime}=-\frac{1}{2}\left(\tau-\tau^{-1}\right) \xi_{5}+\frac{1}{2}\left(\tau+\tau^{-1}\right) \xi_{6} .
\end{aligned}
$$

It is always an $O_{5,6}$, but is of the form $Q_{5,6}^{\gamma, \delta}$ if and only if $\tau$ be a square. It follows that $O_{-1}\left(6, p^{n}\right)$ contains a subgroup $O^{\prime}$ of index 2 , which is the form taken by $G_{4,2}$ when expressed in terms of the $\xi_{i}$. The subgroup $O^{\prime}$ may be extended to $O_{-1}\left(6, p^{n}\right)$ by the substitution $C_{5} C_{6}$, the new form of $T_{-1}$.

We proceed to prove that $O^{\prime}$ is identical with the subgroup $O_{-1}^{\prime}\left(6, p^{n}\right)$ defined in $\S 181$. Expressing the orthogonal substitution $O_{2,4}^{o, \sigma}$ in the indices $Y_{i j}$, we obtain the substitution, denoted for the moment $\bar{O}_{2,4}^{\rho, \sigma}$ :

$$
\begin{aligned}
& Y_{12}^{\prime}=\begin{array}{cccccc}
Y_{12} & Y_{13} & Y_{14} & Y_{23} & Y_{24} & Y_{34} \\
\hline \frac{1}{2}(1+\varrho) & -\frac{1}{2} \sigma & 0 & 0 & -\frac{1}{2} \sigma & \frac{1}{2}(1-\varrho)
\end{array} \\
& Y_{13}^{\prime}=\quad \begin{array}{lllllll}
\frac{1}{2} \sigma & \frac{1}{2}(1+\varrho) & 0 & 0 & -\frac{1}{2}(1-\varrho) & -\frac{1}{2} \sigma
\end{array} \\
& Y_{14}^{\prime}=\begin{array}{lllllll}
0 & 0 & 1 & 0 & 0 & 0
\end{array} \\
& Y_{23}^{\prime}=\quad \begin{array}{llllll}
0 & 0 & 0 & 1 & 0 & 0
\end{array} \\
& Y_{24}^{\prime}=\quad \begin{array}{llllll}
\frac{1}{2} \sigma & -\frac{1}{2}(1-\varrho) & 0 & 0 & \frac{1}{2}(1+\varrho) & -\frac{1}{2} \sigma
\end{array} \\
& Y_{34}^{\prime}=\mid \begin{array}{llllll}
\frac{1}{2}(1-\varrho) & \frac{1}{2} \sigma & 0 & 0 & \frac{1}{2} \sigma & \frac{1}{2}(1+\varrho)
\end{array}
\end{aligned}
$$

For $\varrho \equiv 2 \gamma^{2}-1, \sigma \equiv 2 \gamma \delta$, whence $O_{2,4}^{\rho, \sigma} \equiv Q_{2,4}^{\gamma, \delta}$, we see that $\bar{O}_{2,4}^{\circ, \sigma}$ is the second compound of the substitution of determinant $\left(\gamma_{1}^{2}+\delta^{2}\right)^{2}=1$

$$
\left(\begin{array}{rrrr}
\gamma & 0 & 0 & \delta \\
0 & \gamma & -\delta & 0 \\
0 & \delta & \gamma & 0 \\
-\delta & 0 & 0 & \gamma
\end{array}\right) .
$$


As shown above, $T_{-1}$ corresponds to $C_{5} C_{6}$. The product $\bar{O}_{2,4}^{\circ, \sigma} T_{-1}$ is seen to be the second compound of

$$
\left(\begin{array}{cccc}
x & 0 & 0 & \frac{-\sigma}{2 x} \\
0 & -x & \frac{-\sigma}{2 x} & 0 \\
0 & \frac{\sigma}{2 x} & -x & 0 \\
\frac{\sigma}{2 x} & 0 & 0 & x
\end{array}\right) \quad\left[x^{2} \equiv-\frac{1}{2}(1+\varrho)\right]
$$

But $x$ belongs to the $G F\left[p^{n}\right]$, in which -1 is a not-square, if and only if $\frac{1}{2}(1+\varrho)$ is a not-square, which occurs if and only if $O_{2,4}^{o, \sigma}$ is not of the form $Q_{2,4}^{o, \sigma}$. Hence $O^{\prime}$ contains $O_{2,4}^{o, \sigma} C_{5} C_{6}$ if $O_{2,4}^{o, \sigma}$ is not a $Q_{2,4}$, but not in the contrary case. As shown above, $O^{\prime}$ contains every $Q_{2,4}^{\gamma, \delta}$. To prove that $O^{\prime}$ contains all the generators of $O_{-1}^{\prime}\left(6, p^{n}\right)$, it evidently remains only to prove that $O^{\prime}$ contains all even substitutions on $\xi_{1}, \xi_{2}, \xi_{3}, \xi_{4}, \xi_{5}$, and, if $p^{n}=3$, also $V_{1,2,6}$.

Expressing the linear substitution $\left(\xi_{1} \xi_{2} \xi_{3}\right)$ in the indices $Y_{i j}$, we get

$$
\left(\begin{array}{cccccc}
\frac{1}{2}\left(\alpha-\beta^{2}\right) & \frac{1}{2}(\alpha-\beta-\alpha \beta) & 0 & 0 & -\frac{1}{2}(\alpha+\beta+\alpha \beta) & -\frac{1}{2}\left(\alpha-\beta^{2}\right) \\
-\frac{1}{2}(1+\alpha \beta) & -\frac{1}{2}\left(\beta+\alpha^{2}\right) & 0 & 0 & \frac{1}{2}\left(\beta-\alpha^{2}\right) & -\frac{1}{2}(1-\alpha \beta) \\
0 & 0 & 1 & 0 & 0 & 0 \\
0 & 0 & 0 & 1 & 0 & 0 \\
\frac{1}{2}(1-\alpha \beta) & -\frac{1}{2}\left(\beta+\alpha^{2}\right) & 0 & 0 & \frac{1}{2}\left(\beta-\alpha^{2}\right) & \frac{1}{2}(1+\alpha \beta) \\
\frac{1}{2}\left(\alpha+\beta^{2}\right) & -\frac{1}{2}(\alpha+\beta-\alpha \beta) & 0 & 0 & \frac{1}{2}(\alpha-\beta+\alpha \beta) & -\frac{1}{2}\left(\alpha+\beta^{2}\right)
\end{array}\right) .
$$

This substitution is the second compound of

$$
\left(\begin{array}{cccc}
x & 0 & 0 & y \\
0 & z & w & 0 \\
0 & -W & Z & 0 \\
-Y & 0 & 0 & X
\end{array}\right)
$$

having determinant unity, where

$$
\begin{array}{llll}
x^{2}=\frac{\alpha-\beta-\alpha \beta}{2}, & z=\frac{\alpha-\beta^{2}}{2 x}, & w=\frac{\alpha-\beta-\alpha \beta}{2 x}, & y=\frac{x\left(\alpha-\beta^{2}\right)}{\alpha-\beta-\alpha \beta}, \\
X=\frac{x(1+\alpha \beta)}{\alpha-\beta-\alpha \beta}, & Z=\frac{-\beta-\alpha^{2}}{2 x}, & W=\frac{1+\alpha \beta}{2 x}, & Y=\frac{-x\left(\beta+\alpha^{2}\right)}{\alpha-\beta-\alpha \beta} .
\end{array}
$$

In order that 161 ) shall belong to the $G F\left[p^{n}\right]$, it is necessary and sufficient that $x$ be a mark $\neq 0$ of the field. We proceed to prove that, for every set of solutions in the field of $\alpha^{2}+\beta^{2}=-1$, the expression 
LINEAR GROUP WITH QUADRATIC INVARIANT.

$$
s \equiv \frac{1}{2}(\alpha-\beta-\alpha \beta)
$$

is a square in the field or else zero. ${ }^{1}$ ) Eliminating $\beta$ between the two equations, we find

or

$$
1+\alpha^{2}+\left(\frac{\alpha-2 s}{1+\alpha}\right)^{2}=0
$$

or

$$
\left(1+\alpha+\alpha^{2}\right)^{2}-4 \alpha s+4 s^{2}=0
$$

$$
\left(1+\alpha+\alpha^{2}+2 s\right)^{2}=4 s(1+\alpha)^{2} \text {. }
$$

Hence will $s$ be a square. ${ }^{2}$ ) Solving, we find

$$
x \equiv s^{\frac{1}{2}}=\frac{1}{2}(1+\alpha-\beta) .
$$

The linear substitution $\left(\xi_{2} \xi_{4} \xi_{5}\right)$ expressed in the indices $Y_{i j}$ takes the following form, say $V$ :

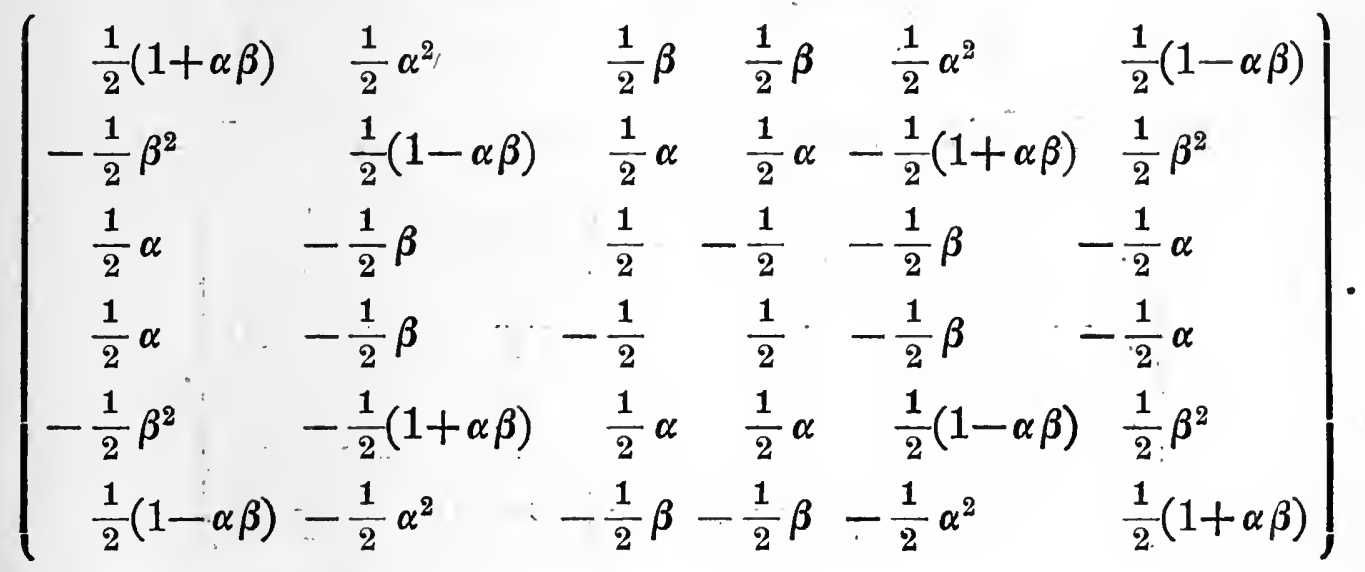

The product $V E$ will be simpler than $V$, if we take as $E$ :

$Y_{12}^{\prime}=Y_{12}-\beta Y_{23}, \quad Y_{13}^{\prime}=Y_{13}-\alpha Y_{23}, \quad Y_{14}^{\prime}=Y_{14}-Y_{23}-\beta Y_{12}+\beta Y_{34}-\alpha Y_{13}-\alpha Y_{24}$ $Y_{34}^{\prime}=Y_{34}+\beta Y_{23}, \quad Y_{24}^{\prime}=Y_{24}-\alpha Y_{23}, \quad Y_{23}^{\prime}=Y_{23}$,

which is recognized as the second compound of

$$
E^{\prime}=\left(\begin{array}{rrrr}
1 & -\alpha & \beta & 0 \\
0 & 1 & 0 & 0 \\
0 & 0 & 1 & 0 \\
0 & -\beta & -\alpha & 1
\end{array}\right)
$$

1) The case $s=0$ requires $1+\alpha+\alpha^{2}=0$, and may thus be avoided.

2) For $p^{n}=3,7$ or 11 , there exist solutions of $\alpha^{2}+\beta^{2}=-1$ for which is an arbitrary square in the field. Is this always true?

DICKSON, Linear Groups. 
The product $V E$ has indeed the simple form $U \equiv$

$$
\left(\begin{array}{rrrcrr}
\frac{1}{2} & -\frac{1}{2} & \beta & 0 & -\frac{1}{2} & \frac{1}{2} \\
\frac{1}{2} & \frac{1}{2} & \alpha & 0 & -\frac{1}{2} & -\frac{1}{2} \\
0 & 0 & 2 & 0 & 0 & 0 \\
\alpha / 2 & -\beta / 2 & -\frac{1}{2} & 1 / 2 & -\beta / 2 & -\alpha / 2 \\
1 / 2 & -1 / 2 & \alpha & 0 & 1 / 2 & -1 / 2 \\
1 / 2 & 1 / 2 & -\beta & 0 & 1 / 2 & 1 / 2
\end{array}\right)
$$

which is seen by inspection to be the second compound of

$$
U^{\prime} \equiv\left(\begin{array}{cccc}
1 & 0 & 0 & 1 \\
\frac{1}{2}(\alpha-\beta) & 1 / 2 & -1 / 2 & \frac{1}{2}(\alpha+\beta) \\
-\frac{1}{2}(\alpha+\beta) & 1 / 2 & 1 / 2 & \frac{1}{2}(\alpha-\beta) \\
-1 & 0 & 0 & 1
\end{array}\right) .
$$

Hence $V \equiv U E^{-1}$ is the second compound of $\left.V^{\prime} \equiv U^{\prime} E^{\prime-1}:^{1}\right)$

$$
\left(\begin{array}{cccc}
\frac{1}{2} & \frac{1}{2}(\alpha-\beta) & -\frac{1}{2}(\alpha+\beta) & \frac{1}{2} \\
\frac{1}{2}(\alpha-\beta) & \frac{1}{2} & -\frac{1}{2} & \frac{1}{2}(\alpha+\beta) \\
-\frac{1}{2}(\alpha+\beta) & \frac{1}{2} & \frac{1}{2} & \frac{1}{2}(\alpha-\beta) \\
-\frac{1}{2} & \frac{1}{2}(\alpha+\beta) & \frac{1}{2}(\alpha-\beta) & \frac{1}{2}
\end{array}\right)
$$

Having the linear substitutions $\left(\xi_{1} \xi_{2} \xi_{3}\right)$ and $\left(\xi_{2} \xi_{4} \xi_{5}\right), O^{\prime}$ contains every even substitution on $\xi_{1}, \ldots, \xi_{5}$. It will suffice to prove this for literal substitutions (123), etc. Transforming (245) by (123) and by $(123)^{2}$, we reach (345) and (145). We then get

$$
\begin{aligned}
& (124)=(154)(245), \quad(314)=(132)^{-1}(124)(132), \\
& (12)(34)=(124)(134),(12)(45)=(12)(34) \cdot(354) .
\end{aligned}
$$

But (123), (12)(34) and (12)(45) generate the alternating group on five letters (Cf. $\S \S 265-266)$.

For $p^{n}=3, O_{-1}^{\prime}\left(6, p^{n}\right)$ requires an additional generator $V_{126}$. Expressing the latter in terms of the indices $Y_{i j}$ defined by 158), where we may now take $\alpha=\beta=+1$, it becomes $\bar{V}$ :

1) The reciprocal of $E^{\prime}$ is given by changing the signs of $\alpha$ and $\beta$. 


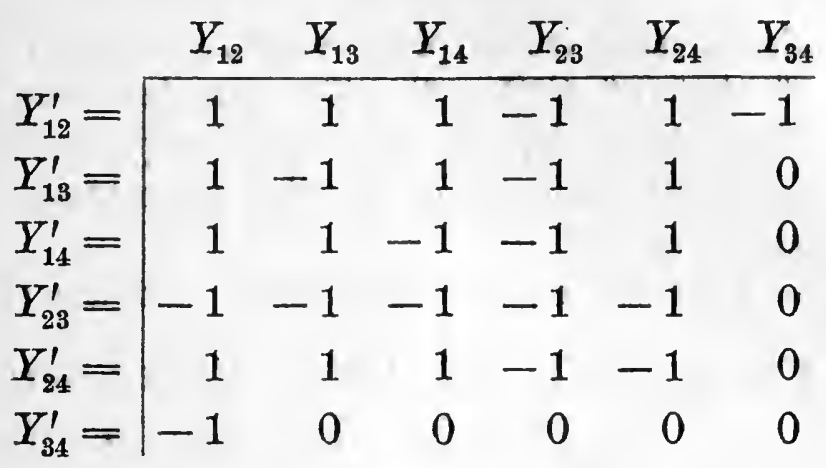

having determinant unity. $\bar{V}$ is seen to be the second compound of

$$
\left(\begin{array}{rrrr}
-1 & 0 & -1 & 1 \\
0 & -1 & -1 & -1 \\
-1 & -1 & 0 & 0 \\
1 & -1 & 0 & 0
\end{array}\right)
$$

of determinant +1 . Hence $O^{\prime}$ contains $V_{126}$ when $p^{n}=3$.

Since $O^{\prime}$ contains the group $O_{-1}^{\prime}\left(6, p^{n}\right)$ but is of index 2 under $O_{-1}\left(6, p^{n}\right)$, it follows from $\S 182$ that $O^{\prime} \equiv O_{-1}^{\prime}\left(6, p^{n}\right)$. Applying $\S 163$, we have the theorem:

For $p^{n}=4 l+3$, the group $O_{-1}^{\prime}\left(6, p^{n}\right)$ is holoedrically isomorphic with the simple group $\operatorname{LF}\left(4, p^{n}\right)$ and is of index two under the second orthogonal group $O_{-1}\left(6, p^{n}\right)$, being extended to it by $C_{5} C_{6}$.

189. Theorem. - The subgroup $O_{1}^{\prime}\left(5, p^{n}\right)$ is of index two under $O_{1}\left(5, p^{n}\right)$ and is holoedrically isomorphic with the simple Abelian group $A\left(4, p^{n}\right)$.

By $\S 161, A\left(4, p^{n}\right), p>2$ is holoedrically isomorphic with the second compound $A_{4,2}$ of the quaternary special Abelian group. $A_{4,2}$ leaves absolutely invariant the Pfaffian [1234] and $Y_{12}+Y_{34}$. By the introduction of the new indices ( $\$ 162$ )

$Y \equiv \frac{1}{2}\left(Y_{12}-Y_{34}\right), \quad Z_{1} \equiv \frac{1}{2}\left(Y_{12}+Y_{34}\right) ; \quad Y_{12} \equiv Z_{1}+Y, \quad Y_{34} \equiv Z_{1}-Y$,

$A_{4,2}$ takes a form not involving $Z_{1}$ and so becomes a quinary group $Q$ leaving absolutely invariant the quadratic function

$$
\Phi \equiv Y^{2}+Y_{13} Y_{24}-Y_{14} Y_{23} \text {. }
$$

The group $G$ of all quinary linear substitutions of determinant unity which leave $\Phi$ absolutely invariant will be proven holoedrically isomorphic with $O_{1}\left(5, p^{n}\right)$ and therefore $(\S 172)$ of order

$$
\left(p^{4 n}-1\right) p^{3 n}\left(p^{2 n}-1\right) p^{n}
$$


Since $Q$ is holoedrically isomorphic with $A\left(4, p^{n}\right)$, its order is, by $\S 115$, half of that of $O_{1}\left(5, p^{n}\right)$. To complete the proof of the theorem, we then show that $Q$ is holoedrically isomorphic with a subgroup of $O_{1}\left(5, p^{n}\right)$ containing all the generators of the group $O_{1}^{\prime}\left(5, p^{n}\right)$ defined in $\S 181$.

Let first -1 be the square of a mark $i$ of the $G F\left[p^{n}\right]$. Set.

whence

$$
\begin{aligned}
& Y \equiv i \xi_{2}, \quad-Y_{13} \equiv \xi_{3}+i \xi_{4}, \quad Y_{14} \equiv \xi_{5}+i \xi_{6}, \\
& Y_{24} \equiv \xi_{3}-i \xi_{4}, \quad Y_{23} \equiv \xi_{5}-i \xi_{6} \text {, }
\end{aligned}
$$

$$
-\Phi \equiv \xi_{2}^{2}+\xi_{3}^{2}+\xi_{4}^{2}+\xi_{5}^{2}+\xi_{6}^{2} \text {. }
$$

Hence $G$ is holoedrically isomorphic with $O_{1}\left(5, p^{n}\right)$. Proceeding $\left.{ }^{1}\right)$ as in $\S 187$, we find that $Q^{\prime}\left(Q\right.$ expressed in the indices $\left.\xi_{i}\right)$ contains the substitution $O_{3,4}^{o, \sigma}$ if and only if it be a $Q_{3,4}$, also that $Q^{\prime}$ contains $\left(\xi_{3} \xi_{5} \xi_{6}\right)$. The latter with $\left(\xi_{2} \xi_{3} \xi_{4}\right)$ will generate all even substitutions on $\xi_{2}, \ldots, \xi_{6}$ by the preceding section. But $\left(\xi_{2} \xi_{3} \xi_{4}\right)$ expressed in the indices $Y_{i j}$ is

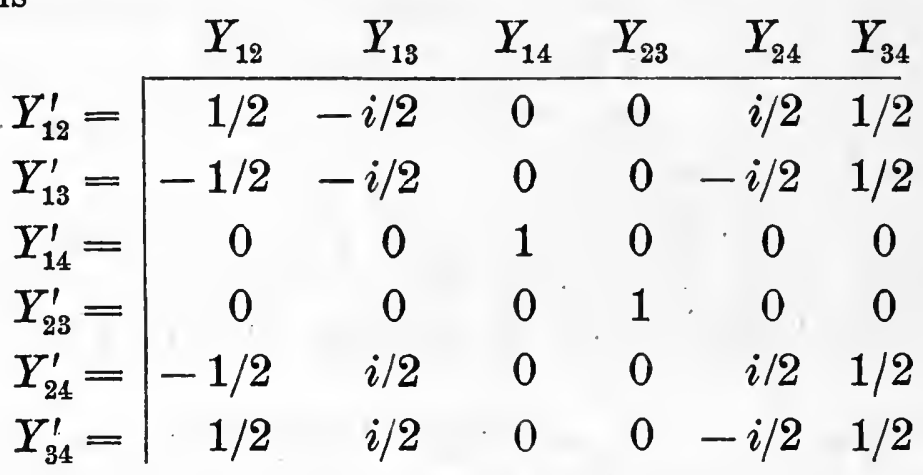

This is seen to be the second compound of the following special Abelian substitution:

$$
\left(\begin{array}{cccc}
\frac{1}{2}(1-i) & 0 & 0 & -\frac{1}{2}(1+i) \\
0 & \frac{1}{2}(1+i) & \frac{1}{2}(1-i) & 0 \\
0 & -\frac{1}{2}(1+i) & \frac{1}{2}(1-i) & 0 \\
\frac{1}{2}(1-i) & 0 & 0 & \frac{1}{2}(1+i)
\end{array}\right)
$$

It follows that $Q^{\prime}$ contains every $Q_{i, j}^{\gamma, \delta}(i, j=2, \ldots 6 ; i \neq j)$. Also $Q^{\prime}$ contains $O_{3,4}^{o, \sigma} O_{5,6}^{o, \sigma}$ and hence every $O_{i, j}^{\rho, \sigma} O_{k, l}^{o, \sigma}$. For $p^{n}=5$, we take $i=2$. Expressing the additional generator $R_{345}$ in the indices $Y_{i j}$, we reach the substitution $(\bmod 5)$.

1) Comparing the transformations of indices 154) and 162), we note that they are identical as far as $\xi_{5}, \xi_{4}, \xi_{5}$ and $\xi_{6}$ are concerned. 
LINEAR GROUP WITH QUADRATIC INVARIANT.

$$
\left(\begin{array}{rrrrrr}
1 & 0 & 0 & 0 & 0 & 0 \\
0 & -1 & 3 & 3 & 1 & 0 \\
0 & 0 & 1 & 0 & 2 & 0 \\
0 & 0 & 0 & 1 & 2 & 0 \\
0 & 0 & 0 & 0 & -1 & 0 \\
0 & 0 & 0 & 0 & 0 & 1
\end{array}\right) .
$$

which is the second compound of the special Abelian substitution

$$
\left(\begin{array}{llll}
3 & 1 & 0 & 0 \\
0 & 2 & 0 & 0 \\
0 & 0 & 3 & 1 \\
0 & 0 & 0 & 2
\end{array}\right) .
$$

Hence $Q^{\prime}$ coincides with $O_{1}^{\prime}\left(5, p^{n}\right)$.

Consider next the case -1 a not-square in the $G F\left[p^{n}\right]$. Set

$$
\begin{array}{rrr}
Y \equiv \xi_{5}, & Y_{13} \equiv \xi_{1}-\alpha \xi_{2}+\beta \xi_{4}, & -Y_{14} \equiv \xi_{3}-\beta \xi_{2}-\alpha \xi_{4}, \\
& Y_{24} \equiv \xi_{1}+\alpha \xi_{2}-\beta \xi_{4}, & Y_{23} \equiv \xi_{3}+\beta \xi_{2}+\alpha \xi_{4},
\end{array}
$$

where $\alpha^{2}+\beta^{2}=-1$. Then $\Phi$ becomes $\xi_{1}^{2}+\xi_{2}^{2}+\xi_{3}^{2}+\xi_{4}^{2}+\xi_{5}^{2}$. Hence $G_{6}$ is holoedrically isomorphic with $O_{1}\left(5, p^{n}\right)$. Reversing equations 163$)$, we get

164)

$$
\begin{array}{ll}
2 \xi_{1}=Y_{13}+Y_{24}, & 2 \xi_{2}=\alpha Y_{13}-\alpha Y_{24}-\beta Y_{14}-\beta Y_{23}, \quad \xi_{5}=Y, \\
2 \xi_{3}=Y_{23}-Y_{14}, & 2 \xi_{4}=-\beta Y_{13}+\beta Y_{24}-\alpha Y_{14}-\alpha Y_{23} .
\end{array}
$$

As in $\S 188$, we find that $Q_{1}\left(Q\right.$ expressed in the indices $\left.\xi_{i}\right)$ contains every $Q_{2,4}^{\gamma, \delta}$ and the linear substitution $\left(\xi_{1} \xi_{2} \xi_{3}\right)$ and consequently also $Q_{1,4}^{\gamma, \delta}$, the transformed of the former by the latter.

Expressing in the indices $\xi_{i}$ the following substitution of $G_{6}$,

$$
K: \quad Y_{13}^{\prime}=-Y_{13}, \quad Y_{24}^{\prime}=-Y_{24},
$$

we get $C_{1} C_{4} O_{2,4}^{\alpha^{2}-\beta^{2}, 2 \alpha \beta}$. This $O_{2,4}$ is not a $Q_{2,4}^{x, y}$ since $2 x^{2}-1=\alpha^{2}-\beta^{2}$ requires $x^{2}=-\beta^{2}$. But $C_{1} C_{4}=Q_{1,4}^{0,1}$ belongs to $Q_{1}$. Since $K$ does not belong to $Q$ (§ 164), it follows that $O_{2,4}^{0, \sigma}$

$$
\varrho \equiv \alpha^{2}-\beta^{2}, \quad \sigma \equiv 2 \alpha \beta,
$$

extends $Q_{1}$ to $O_{1}\left(5, p^{n}\right)$. If $\bar{O}_{1,3}^{o, \sigma}$ denotes $O_{1,3}^{o, \sigma}$ when expressed in the indices $Y_{i j}$, we find that the product $K \bar{O}_{1,3}^{o, \sigma}$ has the form 


$$
\begin{aligned}
& Y_{13}^{\prime}=\begin{array}{llll}
Y_{13} & Y_{14} & Y_{23} & Y_{24} \\
\hline \beta^{2} & -\alpha \beta & \alpha \beta & -\alpha^{2}
\end{array} \\
& Y_{14}^{\prime}=\begin{array}{llll}
-\alpha \beta & -\beta^{2} & -\alpha^{2} & -\alpha \beta
\end{array} \\
& Y_{23}^{\prime}=\quad \begin{array}{ccc}
\alpha \beta & -\alpha^{2} & -\beta^{2}
\end{array} \quad \alpha \beta \\
& Y_{24}^{\prime}=\mid \begin{array}{llll}
-\alpha^{2} & -\alpha \beta & \alpha \beta & \beta^{2}
\end{array}
\end{aligned}
$$

and is the second compound of the special Abelian substitution

$$
\left(\begin{array}{rrrr}
\beta & \alpha & 0 & 0 \\
\alpha & -\beta & 0 & 0 \\
0 & 0 & \beta & -\alpha \\
0 & 0 & -\alpha & -\beta
\end{array}\right) .
$$

Hence $Q_{1}$ contains $O_{2,4}^{0, \sigma} O_{1,3}^{0, \sigma}$. We next show that $Q_{1}$ contains the linear substitution $\left(\xi_{2} \xi_{4} \xi_{5}\right)$, so that with $\left(\xi_{1} \xi_{2} \xi_{3}\right) Q_{1}$ will contain all even substitutions on $\xi_{1}, \ldots, \xi_{5}$. Expressing $\left(\xi_{2} \xi_{4} \xi_{5}\right)$ in the indices $Y_{i j}$, we get

$$
\left(\begin{array}{cccccc}
\frac{1}{2} & \frac{\alpha}{2} & \frac{-\beta}{2} & \frac{-\beta}{2} & \frac{-\alpha}{2} & \frac{1}{2} \\
\frac{\beta}{2} & \frac{1+\alpha \beta}{2} & \frac{\alpha^{2}}{2} & \frac{\alpha^{2}}{2} & \frac{1-\alpha \beta}{2} & \frac{-\beta}{2} \\
\frac{\alpha}{2} & \frac{-\beta^{2}}{2} & \frac{1-\alpha \beta}{2} & \frac{-1-\alpha \beta}{2} & \frac{\beta^{2}}{2} & \frac{-\alpha}{2} \\
\frac{\alpha}{2} & \frac{-\beta^{2}}{2} & \frac{-1-\alpha \beta}{2} & \frac{1-\alpha \beta}{2} & \frac{\beta^{2}}{2} & \frac{-\alpha}{2} \\
\frac{-\beta}{2} & \frac{1-\alpha \beta}{2} & \frac{-\alpha^{2}}{2} & \frac{-\alpha^{2}}{2} & \frac{1+\alpha \beta}{2} & \frac{\beta}{2} \\
\frac{1}{2} & \frac{-\alpha}{2} & \frac{\beta}{2} & \frac{\beta}{2} & \frac{\alpha}{2} & \frac{1}{2}
\end{array}\right)
$$

which is the second compound of the special Abelian substitution

$$
\left(\begin{array}{cccc}
\frac{1}{2} & \frac{-1}{2} & \frac{-1}{2}(\alpha-\beta) & \frac{1}{2}(\alpha+\beta) \\
\frac{1}{2} & \frac{1}{2} & \frac{1}{2}(\alpha+\beta) & \frac{1}{2}(\alpha-\beta) \\
\frac{-1}{2}(\alpha-\beta) & \frac{1}{2}(\alpha+\beta) & \frac{1}{2} & \frac{-1}{2} \\
\frac{1}{2}(\alpha+\beta) & \frac{1}{2}(\alpha-\beta) & \frac{1}{2} & \frac{1}{2}
\end{array}\right) .
$$

For $p^{n}=3, O_{1}^{\prime}\left(5, p^{n}\right)$ requires an additional generator $W_{1234}$. For $\alpha=\beta=1$, the following substitution 


$$
Y_{13}^{\prime}=Y_{13}+Y_{23}, \quad Y_{14}^{\prime}=Y_{14}+Y_{24}
$$

when expressed in the indices $\xi_{1}, \ldots, \xi_{5}$ becomes $(\bmod 3)$

$$
\left(\begin{array}{lllll}
1 & 2 & 2 & 2 & 0 \\
1 & 1 & 2 & 1 & 0 \\
1 & 1 & 1 & 2 & 0 \\
1 & 2 & 1 & 1 & 0 \\
0 & 0 & 0 & 0 & 1
\end{array}\right) \equiv W_{1234}\left(\xi_{2} \xi_{3} \xi_{4}\right)
$$

In every case it follows that $Q_{1}$ coincides with $O_{1}^{\prime}\left(5, p^{n}\right)$.

190. Theorem. $\left.{ }^{1}\right)$ - If $p^{n}=4 l+1, O_{v}^{\prime}\left(6, p^{n}\right)$ is holoedrically isomorphic with the simple group $H A\left(4, p^{2 n}\right)$. If $p^{n}=4 l+3, O_{1}^{\prime}\left(6, p^{n}\right)$ has the maximal invariant subgroup $\left\{I, C_{1} C_{2} C_{3} C_{4} C_{5} C_{6}\right\}$ of order 2, the quotient-group being holoedrically isomorphic with $H A\left(4, p^{2 n}\right)$. In either case, $O_{\mu}^{\prime}\left(6, p^{n}\right)$ is of index 2 under $O_{\mu}\left(6, p^{n}\right)$ and is extended to the latter by any substitution $O_{i j}$ not $a Q_{i j}$.

Consider the group $H^{\prime}$ of quaternary hyperabelian substitutions in the $G F\left[p^{2 n}\right]$ of determinant unity. It has the order

$$
h^{\prime} \equiv\left(p^{4 n}-1\right) p^{3 n}\left(p^{3 n}+1\right) p^{2 n}\left(p^{2 n}-1\right) p^{n} .
$$

The special Abelian group $S A\left(4, p^{n}\right)$ is a subgroup of $H^{\prime}$. Denote their second compound groups by $A_{4,2}$ and $H_{4,2}$ respectively. By $\S 161, A_{4,2}$ leaves absolutely invariant the functions

$$
F_{4} \equiv Y_{12} Y_{34}-Y_{13} Y_{24}+Y_{14} Y_{23}, \quad Z \equiv Y_{12}+Y_{34} \text {. }
$$

For an arbitrary mark $\omega \neq 0$ in the $G F\left[p^{2 n}\right]$, the substitution

$$
\Omega \equiv\left(\begin{array}{llll}
\omega & 0 & 0 & 0 \\
0 & \omega^{-p^{n}} & 0 & 0 \\
0 & 0 & \omega^{-1} & 0 \\
0 & 0 & 0 & \omega^{p^{n}}
\end{array}\right)
$$

is hyperabelian and of determinant unity. Its second compound is

$$
\Omega^{\prime}:\left\{\begin{array}{lll}
Y_{12}^{\prime}=\omega^{-p^{n}+1} Y_{1 q}, & Y_{13}^{\prime}=Y_{13}, & Y_{14}^{\prime}=\omega^{p^{n}+1} Y_{14}, \\
Y_{34}^{\prime}=\omega^{p^{n}-1} Y_{34}, & Y_{24}^{\prime}=Y_{24}, & Y_{23}^{\prime}=\omega^{-p^{n}-1} Y_{23} .
\end{array}\right.
$$

Taking $p>2$, we introduce in place of $Y_{12}, Y_{34}$ the new indices

$$
\xi_{1} \equiv \frac{1}{2}\left(Y_{12}-Y_{34}\right), \quad \xi_{6} \equiv \frac{-1}{2 J}\left(Y_{12}+Y_{34}\right)
$$

where $J$ is a mark of the $G F\left[p^{2 n}\right]$ satisfying the equation

$$
J^{p^{n}-1}=-1 \text {. }
$$

1) Bulletin Amer. Math. Soc., May, 1900; Transactions, July, 1900. 
Reversing relations 165), we find

$$
Y_{12} \equiv \xi_{1}-J \xi_{6}, \ldots Y_{34} \equiv-\xi_{1}-J \xi_{6} \text {. }
$$

Written in the new indices, the substitution $\Omega^{\prime}$ becomes

where

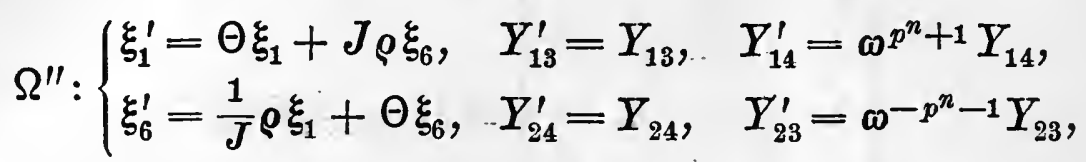

$$
\Theta \equiv \frac{1}{2}\left(\omega^{p^{n}-1}+\omega^{-p^{n}+1}\right) ; \quad \varrho \equiv \frac{1}{2}\left(\omega^{p^{n}-1}-\omega^{-p^{n}+1}\right) \text {. }
$$

The coefficients $\Theta, J \varrho, \varrho / J$ belong to the $G F\left[p^{n}\right]$ since

$$
\Theta^{p^{n}} \equiv \Theta, \quad J^{p^{n}}=-J, \quad \varrho^{p^{n}}=-\varrho .
$$

Hence $\Omega^{\prime \prime}$ belongs to the $G F\left[p^{n}\right]$ and has determinant unity.

If, in $\S 162$, we set $Y \equiv \xi_{1}, Z_{1} \equiv-J \xi_{6}$, we obtain the present transformation of indices 165). Hence, if we express any substitution $[\alpha]_{2}$ of $A_{4,2}$ in terms of the new indices, we obtain a substitution, not involving $\xi_{6}$, the matrix of whose coefficients is given in $\S 162$. Hence $A_{4,2}$ is transformed into a group $A^{\prime \prime}$ of substitutions belonging to the $G F\left[p^{n}\right]$ which do not involve $\xi_{6}$ and which leave absolutely invariant

$$
\xi_{1}^{2}+Y_{13} Y_{24}-Y_{14} Y_{23} \text {. }
$$

In order that $A^{\prime \prime}$ shall contain the substitution

$$
\text { A: } \quad Y_{14}^{\prime}=\omega^{p^{n}+1} Y_{14}, \quad Y_{23}^{\prime}=\omega^{-p^{n}-1} Y_{23},
$$

it is necessary and sufficient (by $\S 164$ ) that $\omega^{p^{n}+1}$ be a square in the $G F\left[p^{n}\right]$ and hence that $\omega$ be a square in the $G F\left[p^{2 n}\right]$,

$$
\omega^{\left(p^{n}+1\right)\left(p^{n}-1\right) / 2}=+1 \text {. }
$$

Hence the group $G^{\prime \prime}$, given by the extension of $A^{\prime \prime}$ by $\Omega^{\prime \prime}$, will contain

$$
K \equiv \Omega^{\prime \prime} A^{-1}: \quad \xi_{1}^{\prime}=\Theta \xi_{1}+J \varrho \xi_{6}, \quad \xi_{6}^{\prime}=\frac{1}{J} \varrho \xi_{1}+\Theta \xi_{6},
$$

if and only if $\omega$ be a square in the $G F\left[p^{2 n}\right]$. Now $K$ leaves $-\xi_{1}^{2}+J^{2} \xi_{6}^{2}$ invariant and is therefore an $O_{16}$. We proceed to prove that, if $\omega$ be a square in the $G F\left[p^{2 n}\right]$, every $K$ is a $Q_{1,6}^{\alpha, \beta}$ and every $Q_{1,6}^{\alpha, \beta}$ is a $K$, where $\alpha, \beta$ belong to the $G F\left[p^{n}\right]$. Let, in fact,

$$
\text { 168) } \alpha \equiv \frac{1}{2}\left(\omega^{\left(p^{n}-1\right) / 2}+\omega^{\left(-p^{n}+1\right) / 2}\right), \quad \beta \equiv \frac{1}{2} J\left(\omega^{\left(p^{n}-1\right) / 2}-\omega^{\left(-p^{n}+1\right) / 2}\right) \text {. }
$$

Since $\omega^{\left(p^{2} n-1\right) / 2}=1$, we see that $\alpha$ and $\beta$ belong to the $G F\left[p^{n}\right]$. Also

$$
\alpha^{2}-\beta^{2} / J^{2}=1
$$

170)

$$
2 \alpha \beta=\frac{1}{2} J\left(\omega^{p^{n}-1}-\omega^{-p^{n}+1}\right), 2 \alpha^{2}-1=\frac{1}{2}\left(\omega^{p^{n}-1}+\omega^{-p^{n}+1}\right) .
$$

Hence $K$ has the form $Q_{1,6}^{\alpha, \beta}$, where $\alpha, \beta$ are defined by 168). 
Given, inversely, a $Q_{1,6}^{\alpha, \beta}$, where $\alpha, \beta$ are marks of the $G F\left[p^{n}\right]$ satisfying 169 ), we can determine a square $\omega$ in the $G F\left[p^{2 n}\right]$ which satisfies 170). In fact, 170) may be written in the solved form

$$
\omega^{p^{n}-1}=2 \alpha^{2}-1+2 \alpha \beta / J=\left(\alpha+\frac{\beta}{J}\right)^{2}, \quad \omega^{-p^{n}+1}=\left(\alpha-\frac{\beta}{J}\right)^{2},
$$

of which the second follows from the first in virtue of 169). That the first can be satisfied by a square $\omega$ in the $G F\left[p^{2 n}\right]$ follows from the relation

$$
\left(\alpha+\frac{\beta}{J}\right)^{p^{n}+1}=\left(\alpha+\frac{\beta}{J^{p^{n}}}\right)\left(\alpha+\frac{\dot{\beta}}{J}\right)=\alpha^{2}-\frac{\beta^{2}}{J^{2}}=1 .
$$

For $\omega$ a not-square in the $G F\left[p^{2 n}\right], \Omega^{\prime \prime}$ is the product of an $O_{16}$, not a $Q_{16}$ in the $G F\left[p^{n}\right]$, by the substitution $A$, neither factor belonging separately to $G^{\prime \prime}$.

Under the transformation of indices 165), $F_{4}$ becomes

$$
\Psi \equiv-\xi_{1}^{2}+J^{2} \xi_{6}^{2}-Y_{13} Y_{24}+Y_{14} Y_{23},
$$

where, by 166), $J^{2}$ belongs to the $G F\left[p^{n}\right]$ but is a not-square in it.

We introduce in place of the $Y_{i j}$ new indices such that

$$
Y_{13} Y_{24}-Y_{14} Y_{23} \equiv \xi_{2}^{2}+\xi_{3}^{2}+\xi_{4}^{2}+\xi_{5}^{2} \text {. }
$$

Then $\Psi$ becomes $J^{2} \xi_{6}^{2}-\sum_{i=1}^{5} \xi_{i}^{2}$. Therefore, by $\S 189, A^{\prime \prime}$ will be transformed into $O_{1}^{\prime}\left(5, p^{n}\right)$.

For -1 the square of a mark $i$ in the $G F\left[p^{n}\right]$, we may take

$$
Y_{13} \equiv \xi_{2}+i \xi_{3}, \quad Y_{24} \equiv \xi_{2}-i \xi_{3}, \quad-Y_{14} \equiv \xi_{4}+i \xi_{5}, \quad Y_{23} \equiv \xi_{4}-i \xi_{5} \text {. }
$$

As in $\S 187, A$ becomes an $O_{4,5}$, which is a $Q_{4,5}$ if and only if $\omega$ be a square in the $G F\left[p^{2 n}\right]$. Hence $G^{\prime \prime}$ is isomorphic with a subgroup of $O_{v}\left(6, p^{n}\right)$. The subgroup contains every $Q_{1,6}$ and every $O_{16} O_{45}$, neither factor a $Q$, but does not contain the separate factors.

For -1 a not-square, so that $p^{n}=4 l+3$, we may take $\omega$ so that

$$
\omega^{p^{n}+1}=-1, \quad \omega^{\left(p^{2} n-1\right) / 2}=-1 .
$$

Then $A$ multiplies $Y_{14}$ and $Y_{23}$ by -1 . The required transformation of indices, transforming $G^{\prime \prime}$ into a subgroup of $O_{1}\left(6, p^{n}\right)$, is the following:

$$
\begin{array}{lrl}
Y_{13} \equiv \xi_{2}-\alpha \xi_{4}+\beta \xi_{5}, & -Y_{14} \equiv \xi_{3}-\beta \xi_{4}-\alpha \xi_{5}, & \\
Y_{24} \equiv \xi_{2}+\alpha \xi_{4}-\beta \xi_{5}, & Y_{23} \equiv \xi_{3}+\beta \xi_{4}+\alpha \xi_{5} . & \left(\alpha^{2}+\beta^{2}=-1\right)
\end{array}
$$

As in $\S 189, A$ becomes in the new indices $C_{3} C_{4} O_{4,5}^{\alpha^{2}-\beta^{2}, 2 \alpha \beta}$, the last factor being not of the form $Q_{4,5}$, while $C_{3} C_{4} \equiv Q_{3,4}^{0,1}$ belongs to $O_{1}^{\prime}\left(5, p^{n}\right)$. Hence $G^{\prime \prime}$ is isomorphic with a subgroup of $O_{1}\left(6, p^{n}\right)$. The subgroup contains every $Q_{16}$ and every $O_{16} O_{45}$, neither factor being a $Q$, but does not contain the factors separately. 
It follows that $G^{\prime \prime}$ is holoedrically isomorphic with $O_{v}^{\prime}\left(6, p^{n}\right)$ or $O_{1}^{\prime}\left(6, p^{n}\right)$ according as $p^{n}=4 l+1$ or $4 l+3$. But, for $p>2$, the order of the second compound $H_{4,2}$ of $H^{\prime}$ is $\frac{1}{2} h^{\prime}$ and therefore equals that of $O_{\mu}^{\prime}\left(6, p^{n}\right)$. Hence $G^{\prime \prime}, H_{4,2}$ and $O_{\mu}^{\prime}\left(6, p^{n}\right)$ are holoedrically isomorphic.

By $\S 132$, we pass from $H^{\prime}$ to the quotient-group $H A\left(4, p^{2 n}\right)$ by making the substitutions $T_{x}(106)$ correspond to the identity. The corresponding substitutions of $H_{4,2}$ are the identity $I$ if $p^{n}=4 l+1$, but are $I$ and the substitution $T$ changing the signs of the six indices if $p^{n}=4 l+3$. Hence $O_{v}^{\prime}\left(6, p^{n}\right)$ is holoedrically isomorphic with $H A\left(4, p^{2 n}\right)$ if $p^{n}=4 l+1$; while, for $p^{n}=4 l+3, O_{1}^{\prime}\left(6, p^{n}\right)$ has the maximal invariant subgroup $\left\{I, C_{1} C_{2} C_{3} C_{4} C_{5} C_{6}\right\}$ of order 2 , the quotientgroup being isomorphic with $H A\left(4, p^{2 n}\right)$.

191. We proceed to determine the structure of the orthogonal subgroups $O_{\mu}^{\prime}\left(m, p^{n}\right), m>7$. Every $m$-ary linear homogeneous substitution is commutative with

$$
C \equiv C_{1} C_{2} \ldots C_{m}: \quad \xi_{i}^{\prime}=-\xi_{i} \quad(i=1, \ldots, m) .
$$

$C$ belongs to the group $O_{\mu}^{\prime}\left(m, p^{n}\right)$ only when $m$ is even and $\mu=1$ (see $\S 185$ ). Suppose that $O_{\mu}^{\prime}\left(m, p^{n}\right)$ has a self-conjugate subgroup $G$ containing a substitution $S$ neither the identity $I$ nor $C$ :

$$
S: \quad \xi_{i}^{\prime}=\sum_{j=1}^{m} \alpha_{i j} \xi_{j} \quad(i=1, \ldots, m) .
$$

Suppose first that $S$ reduces to the form

$$
\xi_{i}^{\prime}=\alpha_{i i} \xi_{i} \quad(i=1, \ldots, m)
$$

where $\alpha_{i i}^{2}=1$. Then $S$ is merely a product of an even number of the $C_{i}$, in which certain ones as $C_{k}$ are lacking since $S \neq C$. If $\mu=\nu$ and therefore $m$ even by hypothesis, we may suppose that both $C_{m}$ and $C_{k}(k<m)$ are lacking, since $C_{i} C_{m}$ does not belong to $O_{v}^{\prime}\left(m, p^{n}\right)$. But if $S \equiv C_{i} C_{j} C_{r} C_{s} \ldots$, its transformed by $T_{i j} T_{i k}$ (always in the main group) gives $S^{\prime} \equiv C_{k} C_{j} C_{r} C_{s} \ldots$, so that $G$ contains the product

$$
S^{\prime} S^{-1} \equiv C_{k} C_{i} \text {. }
$$

From it we obtain in $G$ the substitution $C_{1} C_{2}$ and are thus led to the case treated in $\S 193$.

Suppose, on the contrary, that $S$ is not of the form 171). We may assume that $\alpha_{12}, \alpha_{13}, \ldots, \alpha_{1 m}$ are not all zero. In fact, either $S$ or its reciprocal will have at least one $\alpha_{i j} \neq 0$ in which $i<j$. Transforming the one or the other by $T_{1 j} T_{1 i}$, if $j<m$, we obtain a substitution in $G$ which replaces $\xi_{1}$ by

$$
\alpha_{i i} \xi_{1}+\alpha_{i j} \xi_{i}+\cdots \text {. }
$$


If $j=m$, we transform $S$ by $T_{1 k} T_{1 i}(k$ not $1, i$ or $m$ ) and obtain a substitution in $G$ which replaces $\xi_{1}$ by

$$
\alpha_{i i} \xi_{1}+\cdots+\alpha_{i m} \xi_{m} \text {. }
$$

From the resulting substitution $S$ in which $\alpha_{12}, \alpha_{13}, \ldots, \alpha_{1 m}$ are not all zero, we derive a substitution $S_{1}$ belonging to $G$ and having $\alpha_{11}^{2}+\alpha_{12}^{2} \neq 1$. We get $S_{1}$ immediately if $\alpha_{11}^{2}+\alpha_{1 j}^{2} \neq 1$ for $j=2,3, \ldots$, or $m-1$. In the contrary case, we have

$$
\alpha_{11}^{2}+\alpha_{12}^{2}=1, \quad \alpha_{12}^{2}=\alpha_{13}^{2}=\cdots=\alpha_{1 m-1}^{2} .
$$

If $\alpha_{12}=0$, then $\alpha_{11}^{2}=1$ and therefore $\alpha_{1 m}=0$ by 147), contrary to the assumption that $\alpha_{12}, \alpha_{13}, \ldots, \alpha_{1 m}$ are not all zero. Hence $\alpha_{12} \neq 0$. Transforming $S$ by a suitable product of the $C_{i}$, we can take

$$
\alpha_{12}=\alpha_{13}=\cdots=\alpha_{1 m-1} \neq 0 .
$$

Transforming ${ }^{1}$ ) the resulting substitution by $O_{2,3}^{\alpha, \beta}$, we obtain a substitution which replaces $\xi_{1}$ by

$$
\alpha_{11} \xi_{1}+\left(\alpha \alpha_{12}+\beta \alpha_{13}\right) \xi_{2}+\left(-\beta \alpha_{12}+\alpha \alpha_{13}\right) \xi_{3}+\alpha_{14} \xi_{4}+\cdots+\alpha_{1 m} \xi_{m} .
$$

If $p^{n}>5$, we can determine $\alpha$ and $\beta$ in the $G F\left[p^{n}\right]$ such that

$$
\alpha^{2}+\beta^{2}=1, \quad \alpha_{11}^{2}+\left(\alpha \alpha_{12}+\beta \alpha_{13}\right)^{2} \neq 1 .
$$

Indeed, since $\alpha_{12}=\alpha_{13} \neq 0$, and $\alpha_{11}^{2}+\alpha_{12}^{2}=1$, the second condition becomes $2 \alpha \beta \neq 0$. But, of the $p^{n}-\varepsilon$ sets of solutions in the $G F\left[p^{n}\right]$ of the first condition, where $\varepsilon= \pm 1$ according as -1 is a square or a not-square in the field, only four sets of solutions have either $\alpha$ or $\beta$ equal zero. Hence, if $p^{n}>5$, there exist other solutions.

For $p^{n}=3$, we transform $S$, in which

$$
\alpha_{11}=0, \alpha_{12}=\alpha_{13}=\alpha_{14}=\alpha_{15}= \pm 1,
$$

by $W_{3245}^{2}$ and obtain a substitution in $G$ which replaces $\xi_{1}$ by

$$
\pm \xi_{3}+\alpha_{16} \xi_{6}+\cdots+\alpha_{1 m} \xi_{m}
$$

for which therefore $\alpha_{11}^{2}+\alpha_{12}^{2}=0$.

For $p^{n}=5, S$ has $\alpha_{11}=0, \alpha_{12}^{2}=\alpha_{13}^{2}=\alpha_{14}^{2}=1$ in virtue of 172). Transforming $S$ by a product of the $C_{i}$, we may take

$$
-\alpha_{12}=\alpha_{13}=\alpha_{14}=1 \text {. }
$$

The resulting substitution is transformed by $R_{234}$ into a substitution of $G$ which replaces $\xi_{1}$ by $2 \xi_{2}+2 \xi_{3}+\alpha_{15} \xi_{5}+\cdots$, for which

$$
\alpha_{11}^{2}+\alpha_{12}^{2}=4 \text {. }
$$

1) If the transformer does not belong to $O_{\mu}^{\prime}\left(m, p^{n}\right)$, we afterwards transform by $O_{4,5}^{\alpha, \beta}$. Since the product $O_{2,3}^{\alpha, \beta} O_{4,5}^{\alpha, \beta}$ belongs to the main group, the transformed substitution will belong to $G$. A like remark is to be understood throughout this section. 
Taking the reciprocal of the substitution of $G$ which has

$$
\alpha_{11}^{2}+\alpha_{12}^{2} \neq 1 \text {, }
$$

we obtain in $G$ a substitution $S$ in which $\alpha_{11}^{2}+\alpha_{21}^{2} \neq 1$. Then $G$ contains the product

$$
S_{1} \equiv S^{-1} C_{1} C_{2} S \cdot C_{1} C_{2} \equiv S_{\alpha} C_{1} C_{2}
$$

where $S_{\alpha} \equiv S^{-1} C_{1} C_{2} S$ is of period two and has the form

$$
\xi_{i}^{\prime}=\xi_{i}-2 \alpha_{i 1}\left(\sum_{j=1}^{m-1} \alpha_{j 1} \xi_{j}+\mu \alpha_{m 1} \xi_{m}\right)-2 \alpha_{i 2}\left(\sum_{j=1}^{m-1} \alpha_{j 2} \xi_{j}+\mu \alpha_{m 2} \xi_{m}\right)
$$

$$
(i=1,2, \ldots, m) \text {. }
$$

$S_{1}$ is not the identity since $S$ would then be commutative with $C_{1} C_{2}$ and would therefore break up into the product of

$$
\xi_{1}^{\prime}=\alpha_{11} \xi_{1}+\alpha_{12} \xi_{2}, \quad \xi_{2}^{\prime}=\alpha_{21} \xi_{1}+\alpha_{22} \xi_{2} \quad\left(\alpha_{11}^{2}+\alpha_{21}^{2}=1\right)
$$

by a substitution on $\xi_{3}, \ldots, \xi_{m}$.

We readily obtain the transformed $S_{\alpha^{\prime}}$ of $S_{\alpha}$ by an orthogonal substitution $O$, in which $i, j<k$ :

$\xi_{i}^{\prime}=\alpha \xi_{i}+\beta \xi_{j}+\gamma \xi_{k}, \quad \xi_{j}^{\prime}=\alpha^{\prime} \xi_{i}+\beta^{\prime} \xi_{j}+\gamma^{\prime} \xi_{k}, \quad \xi_{k}^{\prime}=\alpha^{\prime \prime} \xi_{i}+\beta^{\prime \prime} \xi_{j}+\gamma^{\prime \prime} \xi_{k}$, where by 145 )

$$
\alpha^{2}+\beta^{2}+\frac{1}{\lambda} \gamma^{2}=1 \quad(\lambda=1 \text { if } k<m ; \lambda=\mu \text { if } k=m) .
$$

We have $S_{\alpha^{\prime}} \equiv(S O)^{-1} C_{1} C_{2}(S O)$. But $S^{\prime} \equiv S O$ has the coefficients

$$
\begin{aligned}
& \left\{\begin{array}{l}
\alpha_{i l}^{\prime} \equiv \alpha \alpha_{i l}+\beta \alpha_{j l}+\gamma \alpha_{k l} \\
\alpha_{j l}^{\prime} \equiv \alpha^{\prime} \alpha_{i l}+\beta^{\prime} \alpha_{j l}+\gamma^{\prime} \alpha_{k l} \quad(l=1,2, \ldots, m) \\
\alpha_{k l}^{\prime} \equiv \alpha^{\prime \prime} \alpha_{i l}+\beta^{\prime \prime} \alpha_{j l}+\gamma^{\prime \prime} \alpha_{k l}
\end{array}\right. \\
& \alpha_{s l}^{\prime} \equiv \alpha_{s l} \quad(s=1, \ldots, m ; s \neq i, j, k) .
\end{aligned}
$$

If $\alpha_{i 1}^{2}+\alpha_{j 1}^{2}+\lambda \alpha_{k 1}^{2} \neq 0$, we can find solutions in the $G F\left[p^{n}\right]$ of 173), which make $\alpha_{i 1}^{\prime}=0$. We suppose $\alpha_{i 1} \neq 0$, the transformation of $S_{\alpha}$ being unnecessary if $\alpha_{i 1}$ be already zero. Eliminating $\alpha$ from 173) and

$$
\alpha \alpha_{i 1}+\beta \alpha_{j 1}+\gamma \alpha_{k 1}=0
$$

we find the single condition on $\beta$ and $\gamma$,

$$
\beta^{2}\left(\alpha_{i 1}^{2}+\alpha_{j 1}^{2}\right)+2 \beta \gamma \alpha_{j 1} \alpha_{k 1}+\gamma^{2}\left(\alpha_{k 1}^{2}+\frac{1}{\lambda} \alpha_{i 1}^{2}\right)=\alpha_{i 1}^{2} .
$$

If $\alpha_{i 1}^{2}+\alpha_{j 1}^{2}=0$, so that $\alpha_{j 1} \neq 0$ and $\alpha_{k 1} \neq 0$, this equation determines $\beta$, when $\gamma$ is assigned any value $\neq 0$ in the field. Then 174) determines $\alpha$ in the field. But, if $\alpha_{i 1}^{2}+\alpha_{j 1}^{2}$ be $\neq 0$, we multiply it into 175), which then takes the form

$$
\left\{\beta\left(\alpha_{i 1}^{2}+\alpha_{j 1}^{2}\right)+\gamma \alpha_{j 1} \alpha_{k 1}\right\}^{2}+\frac{1}{\lambda} \alpha_{i 1}^{2}\left(\alpha_{i 1}^{2}+\alpha_{j 1}^{2}+\lambda \alpha_{k 1}^{2}\right) \gamma^{2}=\alpha_{i 1}^{2}\left(\alpha_{i 1}^{2}+\alpha_{j 1}^{2}\right) .
$$


The coefficient of $\gamma^{2}$ being not zero, this equation has solutions for

$$
\left\{\beta\left(\alpha_{i 1}^{2}+\alpha_{j 1}^{2}\right)+\gamma \alpha_{j 1} \alpha_{k 1}\right\}, \gamma
$$

in the field and hence solutions $\beta, \gamma$.

Transforming $S_{\alpha}$ by the orthogonal substitution

$$
\xi_{i}^{\prime}=\alpha \xi_{i}+\beta \xi_{j}+\gamma \xi_{l}+\delta \xi_{k}, \quad \xi_{j}^{\prime}=\alpha^{\prime} \xi_{i}+\beta^{\prime} \xi_{j}+\gamma^{\prime} \xi_{l}+\delta^{\prime} \xi_{k}, \ldots
$$

$$
\alpha^{2}+\beta^{2}+\gamma^{2}+\frac{1}{\lambda} \delta^{2}=1,
$$

we obtain as above a substitution ${ }^{-} S_{\bar{\alpha}}$ in which

$$
\bar{\alpha}_{i 1} \equiv \alpha \alpha_{i 1}+\beta \alpha_{j 1}+\gamma \alpha_{l 1}+\delta \alpha_{k 1} \text {. }
$$

We proceed to show that solutions of 176) exist in the $G F\left[p^{n}\right]$ which make $\bar{\alpha}_{i 1}=0$. We may suppose that $\alpha_{i 1}, \alpha_{j 1}, \alpha_{l 1}$ are not zero, since otherwise the result follows "by inspection. If either of the sums

$$
\alpha_{i 1}^{2}+\alpha_{j 1}^{2}+\alpha_{l 1}^{2}, \quad \alpha_{i 1}^{2}+\alpha_{j 1}^{2}+\lambda \alpha_{k 1}^{2}
$$

be not zero, the problem is solved as above. If both sums be zero, then

$$
\alpha_{l 1}^{2}=\lambda \alpha_{k 1}^{2}, \quad \lambda=\text { square }=1, \quad \alpha_{i 1}^{2}+\alpha_{j 1}^{2}+\alpha_{k 1}^{2}=0 .
$$

Then the following set of solutions of 176) will make $\bar{\alpha}_{i 1}$ zero:

$$
\alpha=-\alpha_{j 1} / \alpha_{l 1}, \quad \beta=\alpha_{i 1} / \alpha_{l 1}, \quad \gamma=-\alpha_{k 1} / \alpha_{l 1}, \quad \delta=1 .
$$

192. Transforming $S_{1} \equiv S_{\alpha} C_{1} C_{2}$ by $O_{534 m}, O_{634 m}, \ldots, O_{m-134 m}$ in succession, we obtain in $G$ a substitution $S^{\prime}$ in which $\alpha_{51}^{\prime}, \alpha_{61}^{\prime}, \ldots$, $\alpha_{m-11}^{\prime}$, are all zero. Then by 143 ),

Also

$$
\stackrel{2}{\alpha_{11}^{\prime}}+\stackrel{2}{\alpha_{21}^{\prime}}+\stackrel{2}{\alpha_{31}^{\prime}}+\stackrel{2}{\alpha_{41}^{\prime}}+\mu \stackrel{\mu}{\alpha_{m 1}^{\prime}}=1 \text {. }
$$

$$
\alpha_{11}^{\prime}+\alpha_{21}^{2} \equiv \alpha_{11}^{2}+\alpha_{21}^{2} \neq 1 \text {. }
$$

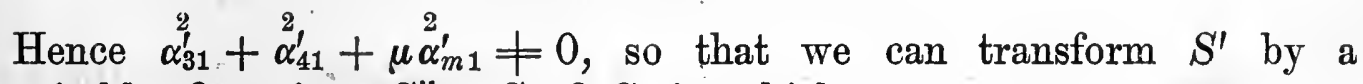
suitable $O_{34 m}$ into $S^{\prime \prime} \equiv S_{\alpha^{\prime \prime}} C_{1} C_{2}$ in which

$$
\alpha_{41}^{\prime \prime}=\alpha_{51}^{\prime \prime}=\alpha_{61}^{\prime \prime}=\cdots=\alpha_{m-11}^{\prime \prime}=0 .
$$

Transforming $S^{\prime \prime}$ by $O_{j 456}(j=7,8, \ldots, m-1)$ in succession, we can obtain a substitution $S_{2} \equiv S_{\beta} C_{1} C_{2}$ which leaves $\xi_{7}, \xi_{8}, \ldots, \xi_{m-1}$ fixed and has $\beta_{41}=\beta_{51}=\beta_{61}=0$. If $\beta_{42}, \beta_{52}, \beta_{62}$ are all not zero, we transform $S_{2}$ by $O_{456}$ and obtain a substitution $S_{2}^{\prime}$ in which we can make $\beta_{62}^{\prime}=0$ except in the case ${ }^{1}$ )

$$
\beta_{42}^{2}+\beta_{52}^{2}+\beta_{62}^{2}=0, \quad \beta_{31} \neq 0, \quad \beta_{m 1} \neq 0 .
$$
$\beta_{62}^{\prime}=0$.

1) If $\beta_{31}=0$ or $\beta_{m 1}=0$, we transform $S_{2}$ by $O_{6345}$ or $O_{6 m 45}$ and make 
In the latter case, we transform $S_{2}$ by $O_{3, m, 4,5,6}^{a, b, c, d_{2} e}$ and require that

Taking

$$
\begin{gathered}
\beta_{62}^{\prime} \equiv a \beta_{32}+b \beta_{m 2}+c \beta_{42}+d \beta_{52}+e \beta_{62}=0 \\
\beta_{61}^{\prime} \equiv a \beta_{31}+b \beta_{m 1}=0 . \\
a=-b \beta_{m 1} / \beta_{31}, \quad c=-d \beta_{52} / \beta_{42} .
\end{gathered}
$$

the second condition becomes an identity and the first takes the form

$$
e \beta_{62}=b \beta, \quad \beta \equiv \frac{\beta_{m 1}}{\beta_{31}} \beta_{32}-\beta_{m 2} .
$$

The further condition $a^{2}+\frac{1}{\mu} b^{2}+c^{2}+d^{2}+e^{2}=1$ then becomes

$$
d^{2}\left(1+\frac{\beta_{52}^{2}}{\beta_{42}^{2}}\right)+b^{2}\left(\frac{1}{\mu}+\frac{\beta_{m 1}^{2}}{\beta_{31}^{2}}+\frac{\beta^{2}}{\beta_{62}^{2}}\right)=1 \text {. }
$$

Since $\beta_{11}^{2}+\beta_{21}^{2} \neq 1, \beta_{11}^{2}+\beta_{21}^{2}+\beta_{31}^{2}+\mu \beta_{m 1}^{2}=1$, it follows that

$$
\frac{1}{\mu}+\frac{\beta_{m 1}^{2}}{\beta_{31}^{2}} \neq 0
$$

The coefficient of $b^{2}$ is zero for at most two values of $\beta_{62}$. In view of 178), these two values of $\beta_{62}$ can be avoided, if $p^{n}>3$, by an earlier transformation of $S_{2}$ by $O_{456}$, an operation not affecting the previous argument. Also the coefficient of $d^{2}$ is not zero. Hence 177) has solutions $d, b$ in the field. The conditions $\beta_{62}^{\prime}=\beta_{61}^{\prime}=0$ can thus be satisfied.

For $p^{n}=3,178$ ) requires $\mu=1$. The coefficient of $b^{2}$ in 177) is then zero only when $\beta \neq 0$. If $\beta \neq 0$, we can determine $a$ and $b$ $($ each $\neq 0)$ such that

$$
a \beta_{32}+b \beta_{m 2}=1, \quad a \beta_{31}+b \beta_{m 1}=0 .
$$

Since $a^{2}=b^{2}=1, \mu=1$, the remaining conditions become

$$
c \beta_{42}+d \beta_{52}+e \beta_{62}=-1, \quad c^{2}+d^{2}+e^{2}=-1 .
$$

These are satisfied modulo 3 by taking $c=\beta_{42}, d=\beta_{52}, e=0$.

193. We have thus reached in $G$ a substitution $\Sigma$ which leaves fixed $\xi_{6}, \xi_{7}, \ldots, \xi_{m-1}$ and which is not the identity. If

$$
\Sigma \equiv C_{1} C_{2} C_{3} C_{4} C_{5} C_{m} \text {, }
$$

we obtain from it the substitution $C_{1} C_{2}$ as at the beginning of $\S 191$. From the known structure of the subgroup $O_{\mu}^{\prime}\left(6, p^{n}\right)$, it follows that $G$ contains all the substitutions of this subgroup. Transforming these by suitable even substitutions on the $\xi_{i}$, we obtain all the generators of $O_{\mu}^{\prime}\left(m, p^{n}\right)$, with which $G$ therefore coincides.

194. In stating our results concerning the structure of the orthogonal groups on $m \neq 4$ indices, we introduce permanent notations for the simple groups reached. For the first orthogonal 
group the case $m=4$ is shown in $\S \S 195-196$ to be quite exceptional. We denote by $F O\left(m, p^{n}\right)$ the first orthogonal subgroup $O_{1}^{\prime}\left(m, p^{n}\right)$, when $m$ is odd, and the quotient-group of $O_{1}^{\prime}\left(m, p^{n}\right)$ by its maximal self-conjugate subgroup $\{I, C\}$, when $m$ is even and $>4$. By $\S 72, F O\left(m, p^{n}\right)$ has the order

$F O\left[m, p^{n}\right] \equiv \frac{1}{2}\left(p^{n(m-1)}-1\right) p^{n(m-2)}\left(p^{n(m-3)}-1\right) p^{n(m-4)} \ldots\left(p^{2 n}-1\right) p^{n}$ for $m$ odd; while, for $m$ even, $m>4$,

$F O\left[m, p^{n}\right] \equiv \frac{1}{4}\left[p^{n(m-1)}-\varepsilon^{\frac{m}{2}} p^{n\left(\frac{m}{2}-1\right)}\right]\left(p^{n(m-2)}-1\right) p^{n(m-3)} \ldots\left(p^{2 n}-1\right) p^{n}$.

The second orthogonal group on an even number $m>4$ of indices has a simple subgroup $\left.{ }^{1}\right) S O\left(m, p^{n}\right)$, previously denoted by $O_{v}^{\prime}\left(m, p^{n}\right)$ of order

$S O\left[m, p^{n}\right] \equiv \frac{1}{2}\left[p^{n(m-1)}+\varepsilon^{\frac{m}{2}} p^{n}\left(\frac{m}{2}-1\right)\right]\left(p^{n(m-2)}-1\right) p^{n(m-3)} \ldots\left(p^{2 n}-1\right) p^{n}$.

It will be shown in $\S \S 197-198$ that this result holds true for $m=4^{2}$ ). In both places, $\varepsilon$ equals \pm 1 according to the form $4 l \pm 1$ of $p^{n}$.

Theorem. - The first orthogonal group $O_{1}\left(m, p^{n}\right)$ has for $m$ even and $>4$ the factors of composition $2, F O\left[m, p^{n}\right], 2$ and for $m$ odd the factors of composition $2, F O\left[m, p^{n}\right]$, the case $m=3, p^{n}=3$ being exceptional. The second orthogonal group $O_{v}\left(m, p^{n}\right)$ on an even number $m>2$ of indices has the factors of composition 2, SO $\left[m, p^{n}\right]$. The orthogonal groups on 2 indices are commutative groups.

195. In virtue of the identity

$\xi_{1}^{2}+\xi_{2}^{2}+\cdots+\xi_{s}^{2}-\xi_{8+1}^{2}-\xi_{s+2}^{2}-\cdots-\xi_{2 s}^{2} \equiv \sum_{i=1}^{s}\left(\xi_{i}-\xi_{s+i}\right)\left(\xi_{i}+\xi_{s+i}\right)$,

it follows from $\S 169$ that the group $\left.{ }^{3}\right) L_{s, p^{n}}$ of $2 s$-ary linear homogeneous substitutions of determinant unity in the $G F\left[p^{n}\right], p>2$, which leave $\sum_{i=1}^{s} X_{i} Y_{i}$ invariant is holoedrically isomorphic with $O_{1}\left(2 s, p^{n}\right)$ if -1 be a square in the $G F\left[p^{n}\right], p>2$, or if -1 be a not-square while $s$ is even, but is isomorphic with $O_{v}\left(2 s, p^{n}\right)$ if -1 be a notsquare while $s$ is odd. In particular, $L_{2, p^{n}}$ is, for $p>2$, holoedrically isomorphic with $O_{1}\left(4, p^{n}\right)$. In determining the structure of $L_{2, p^{n}}$ we do not exclude the case $p=2$.

1) In view of the not-square factor in its invariant, it first appeared in the literature with the notation $N S\left(m, p^{n}\right)$.

2) This result is readily verified for the case $p^{n}=3$ not treated in $\$ \S 197-198$.

3) The structure of this group was first determined by the author without making use of its isomorphism with orthogonal groups, Proc. Lond. Math. Soc., vol. 30, pp. $70-98$. 
196. Theorem. - The factors of composition of $L_{2, p^{n}}$ are

$$
\begin{array}{ll}
\text { if } p>2) & 2, \frac{1}{2}\left(p^{2 n}-1\right) p^{n}, \frac{1}{2}\left(p^{2 n}-1\right) p^{n}, \quad 2, \\
\text { (if } p=2) & 2,\left(2^{2 n}-1\right) 2^{n},\left(2^{2 n}-1\right) 2^{n},
\end{array}
$$

except when $p^{n}=2$ or 3 , when the composite numbers 6 and 12 respectively are to be replaced by their prime factors.

To determine the quaternary substitutions leaving $\xi_{1} \eta_{1}+\xi_{2} \eta_{2}$ absolutely invariant, consider the two pairs of equations ${ }^{1}$ ),

$$
\begin{aligned}
& \xi_{1}+x \xi_{2}=0, \quad \eta_{2}-x \eta_{1}=0, \\
& \xi_{1}+x \eta_{2}=0, \quad \xi_{2}-x \eta_{1}=0 .
\end{aligned}
$$

The most general quaternary linear homogeneous substitution, leaving invariant the pair of equations 179), for every value of $x$ in the field, is readily seen to be

$$
\begin{cases}\xi_{1}^{\prime}=\alpha \xi_{1}+\gamma \eta_{2}, & \xi_{2}^{\prime}=-\gamma \eta_{1}+\alpha \xi_{2}, \\ \eta_{1}^{\prime}=\delta \eta_{1}-\beta \xi_{2}, & \eta_{2}^{\prime}=\beta \xi_{1}+\delta \eta_{2},\end{cases}
$$

having the determinant $(\alpha \delta-\beta \gamma)^{2}$. For it we have

$$
\begin{aligned}
& \xi_{1}^{\prime}+x \xi_{2}^{\prime}=\alpha\left(\xi_{1}+x \xi_{2}\right)+\gamma\left(\eta_{2}-x \eta_{1}\right), \\
& \eta_{2}^{\prime}-x \eta_{1}^{\prime}=\beta\left(\xi_{1}+x \xi_{2}\right)+\delta\left(\eta_{2}-x \eta_{1}\right) .
\end{aligned}
$$

The group of the substitutions 181) is therefore simply isomorphic with the binary group on the variables $\xi_{1}+x \xi_{2}$ and $\eta_{2}-x \eta_{1}$. Since the transposition $\left(\xi_{2} \eta_{2}\right)$ transforms the pair of equations 179) into the pair 180), we obtain the most general linear homogeneous substitution, leaving invariant the pair of equations 180), for every $x$, if we transform the set of substitutions 181) by $\left(\xi_{2} \eta_{2}\right)$, giving the set

$$
\begin{cases}\xi_{1}^{\prime}=A \xi_{1}+C \xi_{2}, & \xi_{2}^{\prime}=B \xi_{1}+D \xi_{2} \\ \eta_{1}^{\prime}=D \eta_{1}-B \eta_{2}, & \eta_{2}^{\prime}=-C \eta_{1}+A \eta_{2} .\end{cases}
$$

The product of any substitution 181) by any substitution 182) gives

183)

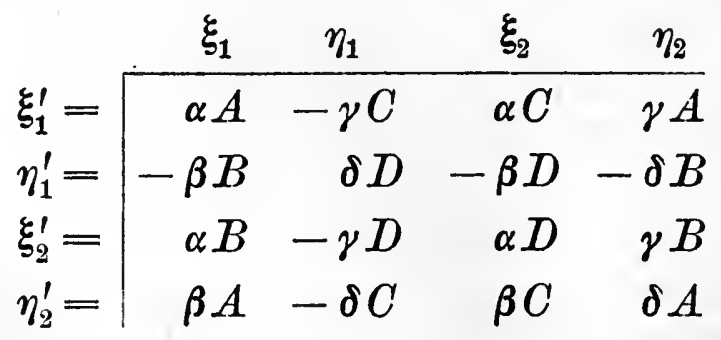

1) They give the two sets of generators on the ruled surface $\xi_{1} \eta_{1}+\xi_{2} \eta_{2}=0$. 
The same result holds if the substitutions be compounded in reverse order, so that the substitutions are commutative. Further, the only substitutions belonging to both of the sets 181) and 182) are seen to be

$$
\xi_{1}^{\prime}=\alpha \xi_{1}, \quad \eta_{1}^{\prime}=\alpha \eta_{1}, \quad \xi_{2}^{\prime}=\alpha \xi_{2}, \quad \eta_{2}^{\prime}=\alpha \eta_{2} .
$$

The substitution 181) leaves $\xi_{1} \eta_{1}+\xi_{2} \eta_{2}$ absolutely invariant if and only if $\alpha \delta-\beta \gamma=1$. Hence there are $\left(p^{2 n}-1\right) p^{n}$ such substitutions. It follows that there are

$$
\begin{array}{r}
\left\{\left(p^{2 n}-1\right) p^{n}\right\}^{2}, \\
\frac{1}{2}\left\{\left(p^{2 n}-1\right) p^{n}\right\}^{2}
\end{array}
$$

distinct substitutions 183) for which

$$
\alpha \delta-\beta \gamma=1, A D-B C=1 .
$$

The substitution $T_{2, x}$, defined in $\S 114$, will be of the form 183) only if

$$
\alpha A=\delta D=1, \quad \alpha D=\varkappa, \quad \delta A=x^{-1}, \quad \beta=\gamma=B=C=0 .
$$

Therefore $A=\alpha^{-1}, D=\varkappa \alpha^{-1}, \delta=\varkappa^{-1} \alpha$, so that

$$
\alpha \delta-\beta \gamma=x^{-1} \alpha^{2} \quad A D-B C=x \alpha^{-2} \text {. }
$$

It will thus satisfy the relations 185) only when $x$ is a square in the $G F\left[p^{n}\right]$. Hence there are at least $\left\{\left(p^{2 n}-1\right) p^{n}\right\}^{2}$ substitutions 183) which satisfy the single relation

$$
(\alpha \delta-\beta \gamma)(A D-B C)=1 \text {. }
$$

For $p>2, L_{2, p^{n}}$ is holoedrically isomorphic with $O_{1}\left(4, p^{n}\right)$ and therefore, by $\S 172$, has the order $\left(p^{3 n}-p^{n}\right)\left(p^{2 n}-1\right) p^{n}$. Hence $L_{2, p^{n}}$ is composed of the substitutions 183) alone. Those of these substitutions which satisfy 185) form a subgroup $L_{2, p^{n}}^{\prime}$ of index two. It is extended to the main group $L_{2, p^{n}}$ by a substitution $T_{2, x}$.

For $p=2$, the substitutions 183) which satisfy 185) form a subgroup $L_{2,2^{n}}^{\prime}$ of index two under $L_{2,2^{n}}$. In fact, by $\S 204$, the order of $L_{2,2^{n}}$ is $2\left(2^{2 n}-1\right)^{2} 2^{2 n}$, which is double the order of $L_{2,2^{n}}^{\prime}$. The transposition $\left(\xi_{1} \eta_{1}\right)$ serves to extend $L_{2,2^{n}}$ to $L_{2,2^{n}}$; for, if 183) reduces to the form $\left(\xi_{1} \eta_{1}\right)$, then $\alpha A=\alpha C=\alpha B=0, \alpha D=1$, whence $A=C=B=0$.

For either $p>2$ or $p=2$, the group $L_{2, p^{n}}$ of the substitutions 183) satisfying 185) has an invariant subgroup formed of the substitutions 181) which satisfy the relation $\alpha \delta-\beta \gamma=1$. The quotientgroup is holoedrically isomorphic with the simple group $L F\left(2, p^{n}\right)$. Indeed, it is clearly the quotient-group of the group of substitutions 182) satisfying $A D-B C=1$ by the group of the substitutions 184), $\alpha^{2}=1$, common to the two sets 181) and 182) under the conditions 185).

DICKsON, Linear Groups. 
197. Theorem. - For $p^{n}>3$, the second orthogonal group $O_{v}\left(4, p^{n}\right)$ is holoedrically isomorphic with the group $E_{4, p^{n}}$ of quaternary linear substitutions in the $G F\left[p^{n}\right]$ of determinant unity which leave absolutely invariant the function

$$
f \equiv \xi_{1} \eta_{1}+\xi_{2} \eta_{2}+\xi_{1}^{2}+\lambda^{2} \eta_{1}^{2}
$$

in which $q \equiv \xi_{1} \eta_{1}+\xi_{1}^{2}+\lambda^{2} \eta_{1}^{2}$ is irreducible in the field.

For $p^{n}=3$, the theorem necessarily fails, since $q$ then becomes $\left(\xi_{1}-\eta_{1}\right)^{2}$. For $p^{n}>3$, there exists a quaternary substitution in the $G F\left[p^{n}\right]$ which transforms the invariant of the orthogonal group,

$$
\Phi \equiv \xi_{1}^{2}+\xi_{2}^{2}+\xi_{3}^{2}+\nu \xi_{4}^{2} \quad(\nu=\text { not-square })
$$

into the function $f_{1} \equiv \xi_{1} \eta_{1}+\xi_{2} \eta_{2}+\lambda \xi_{1}^{2}+\lambda \eta_{1}^{2}$. But, for any $p^{n}, f_{1}$ is transformed into $\lambda^{-1} f$ by the substitution $\xi_{1}^{\prime}=\lambda^{-1} \xi_{1}, \xi_{2}^{\prime}=\lambda^{-1} \xi_{2}$.

If -1 be a not-square in the $G F\left[p^{n}\right]$, we may take $v=-1$. Then the substitution of determinant $\alpha \beta$,

$\xi_{1}=\alpha\left(\xi_{1}-\eta_{1}\right), \quad \xi_{2}=\beta\left(\xi_{1}+\eta_{1}\right), \quad \xi_{3}=\frac{1}{2}\left(\eta_{2}+\xi_{2}\right), \quad \xi_{4}=\frac{1}{2}\left(\eta_{2}-\xi_{2}\right)$ converts $\Phi$ into the function

$$
\left(2 \beta^{2}-2 \alpha^{2}\right) \xi_{1} \eta_{1}+\xi_{2} \eta_{2}+\left(\alpha^{2}+\beta^{2}\right)\left(\xi_{1}^{2}+\eta_{1}^{2}\right) .
$$

Of the $p^{n}-1$ sets of solutions in the $G F\left[p^{n}\right], p>2$, of $2 \beta^{2}-2 \alpha^{2}=1$, two sets make $\alpha \beta=0 .{ }^{1}$ ) Hence there are $p^{n}-3$ substitutions which reduce $\Phi$ to $f_{1}$. The irreducibility of $q$ follows from that of $\xi_{1}^{2}+\xi_{2}^{2}$. If $-1=I^{2}$, where $I$ belongs to the $G F\left[p^{n}\right]$, the substitution $\xi_{1}=\frac{1}{2}\left(\xi_{2}+\eta_{2}\right), \quad \xi_{2}=\frac{I}{2}\left(-\xi_{2}+\eta_{2}\right), \quad \xi_{3}=\alpha\left(\xi_{1}-\eta_{1}\right), \quad \xi_{4}=\beta\left(\xi_{1}+\eta_{1}\right)$ of determinant $I \alpha \beta$ transforms $\Phi$ into the function

$$
\xi_{2} \eta_{2}+\left(2 \nu \beta^{2}-2 \alpha^{2}\right) \xi_{1} \eta_{1}+\left(\alpha^{2}+\nu \beta^{2}\right)\left(\xi_{1}^{2}+\eta_{1}^{2}\right) .
$$

Of the $p^{n}+1$ sets of solutions in the $G F\left[p^{n}\right], p>2$, of $2 v \beta^{2}-2 \alpha^{2}=1$, two sets make $\alpha \beta=0$. Hence there are $p^{n}-1$ substitutions which transform $\Phi$ into $f_{1}$. The irreducibility of $q$ now follows from that of $\xi_{3}^{2}+\nu \xi_{4}^{2}$.

198. Theorem. - Whether $p=2$ or $p>2$, the group $E_{4, p^{n}}$ contains a subgroup $E_{4, p^{n}}^{\prime}$ of index two which is holoedrically isomorphic with $L F\left(2, p^{2 n}\right)$. . According as $p=2$ or $p>2, E_{4, p^{n}}^{\prime}$ is extended to $E_{4, p^{n}}$ by $\left(\xi_{1} \eta_{1}\right)$ or $T_{2, v}$.

1) According as 2 is a square or a not-square, the solutions are given by $\alpha=0$ or $\beta=0$ respectively. 
Let $\sigma$ be a root of the equation

$$
\sigma^{2}+\sigma+\lambda^{2}=0
$$

which is irreducible in the $G F\left[p^{n}\right]$ in virtue of the irreducibility of $q$. The second root is therefore $\sigma^{p^{n}} \equiv \bar{\sigma}$, so that $\sigma \bar{\sigma}=\lambda^{2}$. The substitution

$$
Z: \quad X=\xi_{1}-\sigma \eta_{1}, \quad Y=\xi_{1}-\bar{\sigma} \eta_{1}
$$

transforms the function $F \equiv X Y+\xi_{2} \eta_{2}$ into $f$. Let $\alpha, \beta, \gamma, \delta$ be any set of marks in the $G F\left[p^{2 n}\right]$ subject to the condition $\alpha \delta-\beta \gamma=1$. Then $F$ is absolutely invariant under the substitution [see 181)]

$$
U: X^{\prime}=\alpha X+\gamma \eta_{2}, Y^{\prime}=\delta Y-\beta \xi_{2}, \xi_{2}^{\prime}=-\gamma Y+\alpha \xi_{2}, \eta_{2}^{\prime}=\beta X+\delta \eta_{2} .
$$

If we regard $\left.{ }^{1}\right) \xi_{1}, \eta_{1}, \xi_{2}, \eta_{2}$ to be arbitrary marks of the $G F\left[p^{n}\right]$, $Y$ will be conjugate to $X$ with respect to the $G F\left[p^{n}\right]$, while $F$ will be absolutely invariant under the following substitution conjugate to $U$ [see 182)]

$$
\bar{U}: X^{\prime}=\bar{\delta} X-\bar{\beta} \xi_{2}, \quad Y^{\prime}=\bar{\alpha} Y+\bar{\gamma} \eta_{2}, \quad \xi_{2}^{\prime}=-\bar{\gamma} X+\bar{\alpha} \xi_{2}, \quad \eta_{2}^{\prime}=\bar{\beta} Y+\bar{\delta} \eta_{2} .
$$

If therefore the product $U \bar{U}$ be expressed in terms of the indices $\xi_{1}, \eta_{1}, \xi_{2}, \eta_{2}$, the resulting substitution $W$ will leave $f$ absolutely invariant and have its coefficients in the $G F\left[p^{n}\right]$. To give the explicit form of $W$, let $U$ and $\bar{U}$ become $U_{1}$ and $\bar{U}_{1}$ when written in the indices $\xi_{i}, \eta_{i}$. Since the reciprocal of $Z$ is

$$
Z^{-1}:(\bar{\sigma}-\sigma) \xi_{1}=\bar{\sigma} X-\sigma Y, \quad(\bar{\sigma}-\sigma) \eta_{1}=X-Y,
$$

we find for $U_{1}$ the substitution

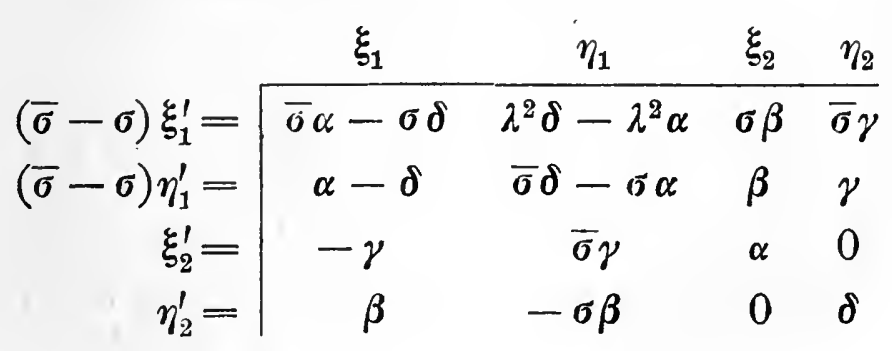

The coefficients of $\bar{U}_{1}$ are conjugate to the corresponding coefficients of $U_{1}$. The product $W \equiv U_{1} \bar{U}_{1}$ is readily found to be the substitution

1) This interpretation is not a necessary one in view of the later explicit calculations. 


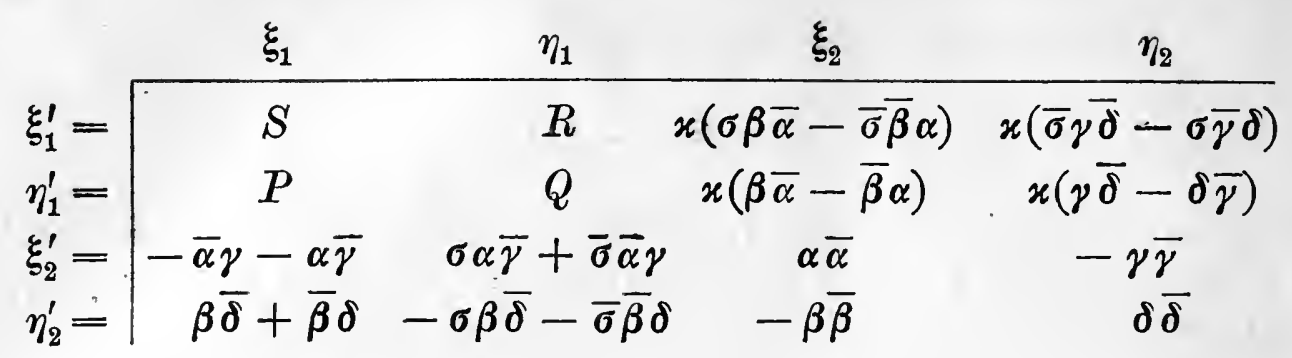

where $x^{-1} \equiv \bar{\sigma}-\sigma$ and

$$
\begin{array}{ll}
S \equiv \varkappa(\bar{\sigma} \alpha \bar{\delta}-\sigma \delta \bar{\alpha}+\bar{\sigma} \gamma \bar{\beta}-\sigma \beta \bar{\gamma}), & R \equiv x\left(\lambda^{2} \delta \bar{\alpha}-\lambda^{2} \alpha \bar{\delta}+\sigma^{2} \beta \bar{\gamma}-\bar{\sigma}^{2} \bar{\beta} \gamma\right), \\
Q \equiv x(\overline{\sigma \alpha} \delta-\sigma \alpha \bar{\delta}-\bar{\sigma} \gamma \bar{\beta}+\sigma \beta \bar{\gamma}), & P \equiv x(\alpha \bar{\delta}-\delta \bar{\alpha}+\bar{\beta} \gamma-\beta \bar{\gamma}) .
\end{array}
$$

Since every coefficient of $W$ equals its own conjugate with respect to the $G F\left[p^{n}\right], W$ belongs to that field.

As in $\S 196$, the substitutions $U$ form a group $\{U\}$ holoedrically isomorphic with the group of binary linear substitutions of determinant unity in the $G F\left[p^{2 n}\right.$. The substitutions $W$ form an isomorphic group $\{W\}$ leaving $f$ absolutely invariant and therefore a subgroup of $E_{4, p^{n}}$. Indeed, if we take $U \sim W$ and $U^{\prime} \sim W^{\prime}$, then to $U U^{\prime}$ will correspond

$$
U_{1} U_{1}^{\prime} \cdot \overline{U_{1} U_{1}^{\prime}} \equiv U_{1} U_{1}^{\prime} \bar{U}_{1} \bar{U}_{1}^{\prime}=U_{1} \bar{U}_{1} \cdot U_{1}^{\prime} \bar{U}_{1}^{\prime} \equiv W W^{\prime},
$$

since the set of substitutions $U$ is commutative with the set $\bar{U}$ by § 196. Moreover, an identity $U \bar{U}=U^{\prime} \bar{U}^{\prime}$ or $U^{\prime-1} U=\bar{U}^{\prime} \bar{U}^{-1}$ requires $U^{\prime}=U$ or $C U$, where $C$ merely changes the signs of the four indices. In fact, the groups $\{U\}$ and $\{\bar{U}\}$ have in common only the identity and $C$. Hence $C U$ is the only substitution in addition to $U$ which corresponds to the product $W \equiv U_{1} \overline{U_{1}} \equiv C U_{1} \cdot C \overline{U_{1}}$. It follows that the quotient-group of $\{U\}$ by $\{I, C\}$ is holoedrically isomorphic both with the simple linear fractional group $L F\left(2, p^{2 n}\right)$ and with the group $\{W\}$. In particular, the order of $\{W\}$ is $\frac{1}{2}\left(p^{4 n}-1\right) p^{2 n}$ or $\left(2^{4 n}-1\right) 2^{2 n}$ according as $p>2$ or $p=2$. For $p>2, p^{n}>3, E_{4, p^{n}}$ has the order $\left(p^{3 n}+p^{n}\right)\left(p^{2 n}-1\right) p^{n}$, being holoedrically isomorphic with $O_{v}\left(4, p^{n}\right)$, whose order is given in $\S 172$. For $p=2, E_{4,2^{n}}$ is holoedrically isomorphic with the group leaving $\xi_{1} \eta_{1}+\xi_{2} \eta_{2}+\lambda \xi_{1}^{2}+\lambda \eta_{1}^{2}$ absolutely invariant, whose order is shown in $\S 204$ to be $2\left(2^{4 n}-1\right) 2^{2 n}$. Hence $\{W\}$ is of index 2 under $E_{4, p^{n}}$. According as $p>2$ or $p=2,\{W\}$ is extended to $E_{4, p^{n}}$ by $T_{2, *}$ or $\left(\xi_{1} \eta_{1}\right)$, where $\varkappa$ is any not-square in the $G F\left[p^{n}\right]$. It is only necessary to show that these substitutions are not of the form $W$. If $\left(\xi_{1} \eta_{1}\right)$ were of the form $W$, then $\gamma \bar{\gamma}=\beta \bar{\beta}=0, S=Q=0$. Hence $\beta=\gamma=0, \bar{\sigma} \alpha \bar{\delta}=\dot{\sigma} \delta \bar{\alpha}, \bar{\sigma} \bar{\alpha} \delta=\sigma \alpha \bar{\delta}$. Hence would $\sigma \delta \bar{\alpha}$ and $\sigma \alpha \bar{\delta}$ 
and consequently also their product belong to the $G F\left[p^{n}\right]$. But $\sigma^{2} \alpha \bar{\alpha} \delta \bar{\delta}$ belongs to that field only when $\alpha$ or $\delta$ vanishes, so that $\alpha \delta-\beta \gamma=0$.

If $W$ reduce to the form $T_{2, x}$, then $\alpha \bar{\alpha}=x, \delta \bar{\delta}=x^{-1}, \beta=\gamma=0$, $S=Q=1, R=P=0$. By the latter, $\bar{\alpha} \delta=\alpha \bar{\delta}$. Then $S=I$ gives $\delta \bar{\alpha}=1$. But $\alpha \delta=\alpha \delta-\beta \gamma=1$. Hence $\alpha=\bar{\alpha}$, so that $x=\alpha^{2}$, $\alpha$ belonging to the $G F\left[p^{n}\right]$.

\section{CHAPTER VIII.}

\section{LINEAR HOMOGENEOUS GROUP IN THE $G F\left[2^{n}\right]$. DEFINED BY A QUADRATIC INVARIANT.}

199. Theorem. - If a quadratic form with coefficients in the $G F\left[2^{n}\right]$

$$
f \equiv \sum_{i=\sum^{j}}^{i, j=1, \ldots, m} \alpha_{i j} \xi_{i} \xi_{j}
$$

can not be expressed in the field as a quadratic form in fewer than $m$ linear homogeneous functions of $\xi_{1}, \ldots, \xi_{m}$, it can be reduced by a linear homogeneous substitution belonging to the field to one of the canonical forms

$$
\begin{array}{ll}
F \equiv \xi_{1} \xi_{2}+\xi_{3} \xi_{4}+\cdots+\xi_{m-2} \xi_{m-1}+\xi_{m}^{2} & \text { (m odd) } \\
F_{\lambda} \equiv \xi_{1} \xi_{2}+\xi_{3} \xi_{4}+\cdots+\xi_{m-3} \xi_{m-2}+\xi_{m-1} \xi_{m}+\lambda \xi_{m-1}^{2}+\lambda \xi_{m}^{2} & \text { (m even) }
\end{array}
$$

where $\lambda$ is zero or is a particular one of the values $\lambda^{\prime}$ for which

$$
Q \equiv \xi_{m-1} \xi_{m}+\lambda^{\prime} \xi_{m-1}^{2}+\lambda^{\prime} \xi_{m}^{2}
$$

is irreducible in the $G F\left[2^{n}\right]$.

We first prove that, if $m \sum 3, f$ can be transformed into a quadratic form having $\alpha_{11}=0$. If every $\alpha_{i j}(i, j=1, \ldots, m ; i<j)$ were zero, $f$ would reduce modulo 2 to the form

$$
\left(\sqrt{\alpha_{11}} \xi_{1}+\sqrt{\alpha_{22}} \xi_{2}+\cdots+\sqrt{\alpha_{m m}} \xi_{m}\right)^{2} .
$$

This being contrary to our hypothesis, we may assume that $\alpha_{23} \neq 0$, for example. We may also suppose that $\alpha_{22} \neq 0$, since otherwise the transformed of $f$ by $\left(\xi_{1} \xi_{2}\right)$ would have $\alpha_{11}=0$. The terms of $f$ which involve $\xi_{2}$ may be written thus,

$$
\alpha_{22} \xi_{2}^{2}+\xi_{2}\left(\alpha_{12} \xi_{1}+\alpha_{23} \xi_{3}+\alpha_{24} \xi_{4}+\cdots+\alpha_{2 m} \xi_{m}\right) .
$$


Hence the inverse of the following substitution,

$$
\begin{aligned}
& \xi_{3}^{\prime}=\alpha_{12} \xi_{1}+\alpha_{23} \xi_{3}+\alpha_{24} \xi_{4}+\cdots+\alpha_{2 m} \xi_{m}, \\
& \xi_{i}^{\prime}=\xi_{i} \quad(i=1, \ldots, m ; i \neq 3),
\end{aligned}
$$

will transform $f$ into

$$
\alpha_{22} \xi_{2}^{2}+\xi_{2} \xi_{3}+\Sigma \beta_{i j} \xi_{i} \xi_{j}
$$

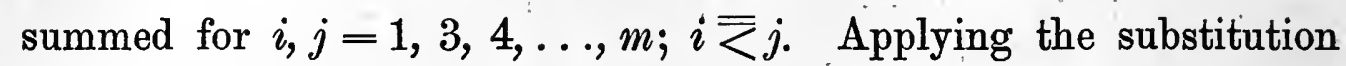

$$
\xi_{2}^{\prime}=\xi_{2}+\lambda \xi_{1}, \quad \xi_{i}^{\prime}=\xi_{i}, \quad(i=1,3,4, \ldots, m)
$$

we obtain as the new coefficient of $\xi_{1}^{2}$ the function $\alpha_{22} \lambda^{2}+\beta_{11}$, which may be made to vanish by determining $\lambda$.

We may therefore suppose that $\alpha_{11}=0$ in our original function $f$. Since the $\alpha_{1 j}$, are not all zero, we may assume that $\alpha_{12} \neq 0$. Applying to $f$ the inverse of the substitution

$$
\xi_{2}^{\prime}=\alpha_{12} \xi_{2}+\alpha_{13} \xi_{3}+\cdots+\alpha_{1 m} \xi_{m}, \quad \xi_{i}^{\prime}=\xi_{i} \quad(i=1,3,4, \ldots, m)
$$

we obtain the function

$$
\xi_{1} \xi_{2}+\sum_{i \overline{\overline{<}}^{j}}^{i=2 \ldots, m} \gamma_{i j} \xi_{i} \xi_{j} .
$$

Replacing $\xi_{1}+\gamma_{22} \xi_{2}+\gamma_{23} \xi_{3}+\cdots+\gamma_{2 m} \xi_{m}$, by $\xi_{1}$, we get

$$
f^{\prime} \equiv \xi_{1} \xi_{2}+\sum_{i=\bar{\Sigma}^{j}}^{i, j=3 \ldots, m} \gamma_{i j} \xi_{i} \xi_{j} .
$$

Similarly, if $m>5$, we can transform $f^{\prime}$ into

$$
\xi_{1} \xi_{2}+\xi_{3} \xi_{4}+\sum_{i, j=5, \ldots, m}^{j} \varepsilon_{i j} \xi_{i} \xi_{j} .
$$

If $m$ be odd, we reach ultimately the form

$$
\xi_{1} \xi_{2}+\xi_{3} \xi_{4}+\cdots+\xi_{m-2} \xi_{m-1}+x \xi_{m}^{2} \text {. }
$$

Applying to it the substitution which replaces $\xi_{m}$ by $x^{-1 / 2} \xi_{m}$, we obtain $F$.

If $m$ be even, we reach ultimately the form

$$
\Theta \equiv \xi_{1} \xi_{2}+\xi_{3} \xi_{4}+\cdots+\xi_{m-3} \xi_{m-2}+\alpha \xi_{m-1}^{2}+\beta \xi_{m-1} \xi_{m}+\gamma \xi_{m}^{2} .
$$

If $\alpha \xi_{m-1}+\beta \xi_{m-1} \xi_{m}+\gamma \xi_{m}^{2}$ be reducible in the $G F\left[2^{n}\right]$, i. e., be the product of two linear homogeneous functions of $\xi_{m-1}$ and $\xi_{m}$, an evident substitution will reduce $\Theta$ to $F_{0}$. In the contrary case, $\alpha, \beta, \gamma$ are certainly distinct from zero, so that the substitution 


$$
\xi_{m-1}^{\prime}=\alpha^{-1 / 2} \xi_{m-1}, \quad \xi_{m}^{\prime}=\alpha^{1 / 2} \beta^{-1} \xi_{m}, \quad \xi_{i}^{\prime}=\xi_{i} \quad(i=1, \ldots, m-2)
$$

will belong to the $G F\left[2^{n}\right]$. It transforms $\Theta$ into

187) $\xi_{1} \xi_{2}+\xi_{3} \xi_{4}+\cdots+\xi_{m-3} \xi_{m-2}+\xi_{m-1}^{2}+\xi_{m-1} \xi_{m}+\delta \xi_{m}^{2}$

$\delta$ being such a mark that the equation

$$
\xi^{2}+\xi+\delta=0
$$

is irreducible in the $G F\left[2^{n}\right]$. It follows from 188) that

$$
\xi^{2^{n}}=\xi+\delta+\delta^{2}+\delta^{4}+\cdots+\delta^{2^{n-1}} \text {. }
$$

Hence 188) has a root $\xi$ in the $G F\left[2^{n}\right]$ if and only if

$$
\delta+\delta^{2}+\cdots+\delta^{2^{n-1}}=0 .
$$

The left member being its own square in the $G F\left[2^{n}\right]$ and hence either 0 or 1 , it follows that 188) is irreducible in that field if and only if

$$
\delta+\delta^{2}+\delta^{4}+\cdots+\delta^{2^{n-1}}=1 .
$$

Applying to the quadratic form 187) the transformation

$$
\xi_{m-1}^{\prime}=\xi_{m-1}+\lambda \xi_{m}, \quad \xi_{i}^{\prime}=\xi_{i}, \quad(i=1, \ldots, m ; i \neq m-1)
$$

the constant $\delta$ is replaced by

$$
\delta^{\prime} \equiv \delta+\lambda+\lambda^{2},
$$

which is therefore a root of 189). Giving to $\lambda$ all possible values in the $G F\left[2^{n}\right]$, we obtain the $2^{n-1}$ roots of 189 ). Indeed, if in the $G F\left[2^{n}\right]$,

$$
\delta+\lambda+\lambda^{2}=\delta+\lambda_{1}+\lambda_{1}^{2}
$$

we must have $\lambda_{1}=\lambda$ or $\lambda+1$. Hence all irreducible quadratic forms in two variables of the $G F\left[2^{n}\right]$ can be transformed linearly into each other. Applying, finally, the transformation

$$
\xi_{m-1}^{\prime}=\delta^{\frac{1}{4}} \xi_{m-1}, \quad \xi_{m}^{\prime}=\delta^{-\frac{1}{4}} \xi_{m}^{\prime}, \quad \xi_{i}^{\prime}=\xi_{i}(i=1, \ldots, m-2)
$$

187) becomes $F_{\delta}^{\frac{1}{2}}$.

200. Changing the notation used in exhibiting $F$, the canonical quadratic form for an odd number $2 m+1$ of indices may be written

$$
\Psi \equiv \xi_{0}^{2}+\xi_{1} \eta_{1}+\xi_{2} \eta_{2}+\cdots+\xi_{m} \eta_{m}
$$

The conditions upon the coefficients of the substitution $S$ : 


$$
\left\{\begin{array}{lr}
\xi_{i}^{\prime}=x_{i} \xi_{0}+\sum_{j=1}^{m}\left(\alpha_{i j} \xi_{j}+\gamma_{i j} \eta_{j}\right) & (i=0,1, \ldots, m) \\
\eta_{i}^{\prime}=\sigma_{i} \xi_{0}+\sum_{j=1}^{m}\left(\beta_{i j} \xi_{j}+\delta_{i j} \eta_{j}\right) & (i=1, \ldots, m)
\end{array}\right.
$$

in order that it leave $\Psi$ absolutely invariant are seen to be the special Abelian relations ${ }^{1}$ ) 76) for $\mu=1$ together with the following:

$$
\begin{gathered}
\sum_{i=1}^{m}\left(x_{i} \beta_{i k}+\sigma_{i} \alpha_{i k}\right)=0, \quad \sum_{i=1}^{m}\left(\varkappa_{i} \delta_{i k}+\sigma_{i} \gamma_{i k}\right)=0, \quad(k=1,2, \ldots, m) \\
\alpha_{0 j}^{2}=\sum_{i=1}^{m} \alpha_{i j} \beta_{i j}, \quad \gamma_{0 j}^{2}=\sum_{i=1}^{m} \gamma_{i j} \delta_{i j}, \quad \varkappa_{0}^{2}+\sum_{i=1}^{m} \varkappa_{i} \sigma_{i}=1 .
\end{gathered}
$$

It follows from $\S 114$ that every set of solutions $\alpha_{i j}, \beta_{i j}, \gamma_{i j}, \delta_{i j}$ in the $G F\left[2^{n}\right]$ of the relations 76$)_{\mu=1}$ leads to a special Abelian substitution

$$
\Sigma: \quad \xi_{i}^{\prime}=\sum_{j=1}^{m}\left(\alpha_{i j} \xi_{j}+\gamma_{i j} \eta_{j}\right), \quad \eta_{i}^{\prime}=\sum_{j=1}^{m}\left(\beta_{i j} \xi_{j}+\delta_{i j} \eta_{j}\right) \quad(i=1, \ldots, m)
$$

whose determinant $\Delta$ is unity. ${ }^{2}$ )

The determinant of the coefficients of the $2 m$ quantities $x_{i}, \sigma_{i}$ in the $2 m$ equations 190) is seen to equal $\Delta$. Hence, since $\Delta \neq 0$,

$$
x_{i}=\sigma_{i}=0
$$$$
(i=1, \ldots, m) \text {. }
$$

It follows that $S$ takes the form

$$
S^{\prime}:\left\{\begin{array}{l}
\left.\xi_{i}^{\prime}=\sum_{j=1}^{m}\left(\alpha_{i j} \xi_{j}+\gamma_{i j} \eta_{j}\right), \quad \eta_{i}^{\prime}=\sum_{j=1}^{m}\left(\beta_{i j} \xi_{j}+\delta_{i j} \eta_{j}\right) \quad i=1, \ldots, m\right) \\
\xi_{0}^{\prime}=\xi_{0}+\sum_{j=1}^{m}\left\{\left(\sum_{i=1}^{m} \alpha_{i j} \beta_{i j}\right)^{1 / 2} \xi_{j}+\left(\sum_{i=1}^{m} \gamma_{i j} \delta_{i j}\right)^{1 / 2} \eta_{j}\right\},
\end{array}\right.
$$

the coefficients of $S^{\prime}$ being subject to the Abelian conditions 76) only. The group of the substitutions $S$ is therefore holoedrically isomorphic with the special Abelian group $S A\left(2 m, 2^{n}\right)$ of the substitutions $\Sigma$. The structure of the latter group is given in $\S 11$.

201. Changing the notation employed in exhibiting the function $F_{\lambda}$, the canonical quadratic form for $2 m$ indices may be written

$$
f_{\lambda} \equiv \sum_{i=1}^{m} \xi_{i} \eta_{i}+\lambda \xi_{m}^{2}+\lambda \eta_{m}^{2}
$$

1) Since $p=2$, we have $-1=+1$ in the field.

2) For a direct proof that $\Delta=0$, see American Journal, vol. 21, p. 244. 
We study the group $G_{2}$ of $2 m$-ary linear substitutions in the $G F\left[2^{n}\right]$,

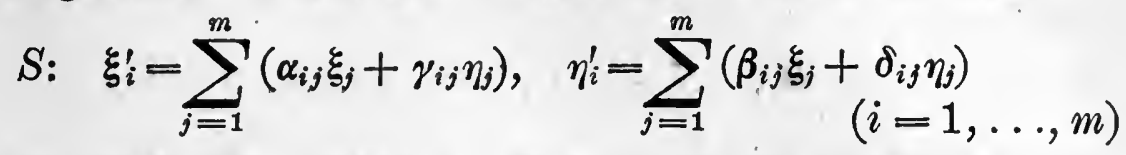

which leave $f_{2}$ absolutely invariant. The conditions upon the coefficients of $S$ are the Abelian relations ${ }^{1}$ ) 76), for $\mu=1$, together with

$$
\left\{\begin{array}{lll}
\sum_{i=1}^{m} \alpha_{i j} \beta_{i j}+\lambda \alpha_{m j}^{2}+\lambda \beta_{m j}^{2}=0 & \\
\lambda & (j<m) \\
\sum_{i=1}^{m} \gamma_{i j} \delta_{i j}+\lambda \gamma_{m j}^{2}+\lambda \delta_{m j}^{2}=\frac{0}{\lambda} & (j=m), \\
\lambda & (j=m)
\end{array}\right.
$$

Since $S$ must be an Abelian substitution in the $G F\left[2^{n}\right]$, its reciprocal is obtained by replacing $\alpha_{i j}, \beta_{i j}, \gamma_{i j}, \delta_{i j}$ by respectively $\delta_{j i}, \beta_{j i}, \gamma_{j i}$, $\alpha_{j i}$. Writing for $S^{-1}$ the conditions 76 ) and 193), we obtain the equivalent set of conditions 78 ), for $\mu=1$, and

$$
\left\{\begin{array}{lll}
\sum_{i=1}^{m} \delta_{j i} \beta_{j i}+\lambda \delta_{j m}^{2}+\lambda \beta_{j m}^{2}= & 0 \\
\lambda & (j<m) \\
\sum_{i=1}^{m} \gamma_{j i} \alpha_{j i}+\lambda \gamma_{j m}^{2}+\lambda \alpha_{j m}^{2}=\begin{array}{ll}
0 \\
\lambda
\end{array} & (j=m), \\
& (j<m) \\
& (j=m) .
\end{array}\right.
$$

Among the simplest substitutions leaving $f_{\lambda}$ invariant occur

$$
\begin{array}{rll}
M_{i} \equiv\left(\xi_{i} \eta_{i}\right) & (i=1, \ldots, m) ; \quad N_{i, j, x}, \quad R_{i, j, x}, \quad Q_{i, j, x}, T_{i, x}, \quad P_{i j} \\
L: & \left.\xi_{m}^{\prime}=\eta_{m}, \quad \eta_{m}^{\prime}=\xi_{m}+\lambda^{-1} \eta_{m} \quad \text { (if } \lambda=\lambda^{\prime}\right) ; \\
N_{m, j, x}: & \xi_{m}^{\prime}=\xi_{m}+x \eta_{j}, \quad \xi_{j}^{\prime}=\xi_{j}+\lambda x^{2} \eta_{j}+x \eta_{m} \\
R_{m, j, x}: & \eta_{m}^{\prime}=\eta_{m}+x \xi_{j}, \quad \eta_{j}^{\prime}=\eta_{j}+\lambda x^{2} \xi_{j}+x \xi_{m} \\
Q_{m, j, x}: & \xi_{m}^{\prime}=\xi_{m}+x \xi_{j}, \quad \eta_{j}^{\prime}=\eta_{j}+\lambda x^{2} \xi_{j}+x \eta_{m} \\
Q_{j, m, x}: & \eta_{m}^{\prime}=\eta_{m}+x \eta_{j}, \quad \xi_{j}^{\prime}=\xi_{j}+\lambda x^{2} \eta_{j}+x \xi_{m}
\end{array}
$$

which reduce, when $\lambda=0$, to the $N_{m . j, x}, R_{m, j, x}$, etc., defined in $\S 114$.

According as $\lambda=0$ or $\lambda=\lambda^{\prime}, G_{\lambda}$ is called the first or the second hypoabelian group ${ }^{2}$ ). The name arises from the fact that $G_{\lambda}$ is a subgroup of the special Abelian group $S A\left(2 m, 2^{n}\right)$.

1) This also follows from the fact that the invariance of $f_{\lambda}$ implies that of its polar. Hence, if $p=2, G_{\lambda}$ leaves invariant $\sum_{i=1}^{m}\left(\xi_{i 1} \eta_{i 2}+\xi_{i 2} \eta_{i 1}\right)$, where $\xi_{i 1}$, $\eta_{i 1}$ and $\xi_{i 2}, \eta_{i 2}$ are sets of cogredient variables.

2) For the case $n=1$, these groups were studied at length by Jordan, Traité des substitutions, pp. 195-213 and p. 440. For general $n$, they were set up and investigated by the author in the papers, Quarterly Journal, 1898, pp.1-16; Bulletin of the Amer. Math. Soc., 1898, pp.495-510; Proceed. Lond. Math. Soc., vol. 30, pp. 70-98; American Journal, 1899, pp. 222-243. 
202. Theorem. - If $m>1 ; G_{2}$ may be generated by the substitutions $^{1}$ )

195) $M_{i}, \quad N_{i, j, x} \quad(i, j=1, \ldots, m ; \varkappa$ arbitrary in the field $)$.

We note that $M_{i}$ transforms $N_{j, i, x}$ into $Q_{j, i, x}$, and $Q_{i, j, x}$ into $R_{i, j, x}$. Further, for $i, j<m$ if $\lambda=\lambda^{\prime}$, we have

$$
\begin{aligned}
P_{i j} & \equiv Q_{j, i, 1}^{-1} Q_{i, j, 1} Q_{j, i, 1} \\
T_{i, \mu} & T_{j, \mu} \equiv M_{i} M_{j} P_{i j} R_{i, j, \mu}-1 N_{i, j, \mu} R_{i, j, \mu}-1 \\
T_{i, \mu^{2}} & \equiv T_{i, \mu} T_{j, \mu} \cdot M_{j}^{-1} T_{i, \mu} T_{j, \mu} M_{j} .
\end{aligned}
$$

But every mark of the $G F\left[2^{n}\right]$ may be expressed as a square $\mu^{2}$. Except in the case $m=2, \lambda=\lambda^{\prime}$, we thus reach every $T_{i, x}$. In the latter case, we derive every $T_{1, x}$ from the formula

$$
N_{m, 1, x} Q_{m, 1, x}-1_{\lambda-1} N_{m, 1, x}=L M_{1} M_{m} T_{1, \lambda x^{2}} .
$$

Taking first $x=\lambda^{-1 / 2}$, we find that $L$ may be derived from the substitutions 195). Applying 196) again, we reach every $T_{1, \lambda x^{2}}$.

To prove that every substitution $S$ satisfying the relations 78$)_{\mu=1}$ and 194) can be derived from the substitutions 195), we first set up a substitution $T$ derived from them which, like $S$, replaces $\xi_{1}$ by

where by 194),

$$
f_{1} \equiv \sum_{j=1}^{m}\left(\alpha_{1 j} \xi_{j}+\gamma_{1 j} \eta_{j}\right)
$$

$$
\sum_{j=1}^{m} \alpha_{1 j} \gamma_{1 j}+\lambda \alpha_{1 m}^{2}+\lambda \gamma_{1 m}^{2}=0 .
$$

a) If $\alpha_{11} \neq 0$, we may take as $T$ the product

$$
T_{1, \alpha_{11}} Q_{1,2, \alpha_{12}} N_{2,1, \gamma_{12}} \ldots Q_{1, m, \alpha_{1 m}} N_{m, 1, \gamma_{1 m}}
$$

since it replaces $\xi_{1}$ by

$\alpha_{11} \xi_{1}+\alpha_{11}^{-1}\left(\alpha_{12} \gamma_{12}+\cdots+\alpha_{1 m} \gamma_{1 m}+\lambda \alpha_{1 m}^{2}+\lambda \gamma_{1 m}^{2}\right) \eta_{1}+\sum_{j=2}^{m}\left(\alpha_{1 j} \xi_{j}+\gamma_{1 j} \eta_{j}\right) \equiv f_{1}$.

b) If $\alpha_{11}=0, \gamma_{11} \neq 0$, we may take for $T$ the product

$$
T_{1, \gamma_{11}}{ }^{-1} Q_{2,1, \gamma_{12}} R_{2,1, \alpha_{12}} \ldots Q_{m, 1, \gamma_{1 m}} R_{m, 1, \alpha_{1 m}} \cdot M_{1} M_{m}
$$

which replaces $\xi_{1}$ by

$\gamma_{11} \eta_{1}+\gamma_{11}^{-1}\left(\alpha_{12} \gamma_{12}+\cdots+\alpha_{1 m} \gamma_{1 m}+\lambda \alpha_{1 m}^{2}+\lambda \gamma_{1 m}^{2}\right) \xi_{1}+\sum_{j=2}^{m}\left(\alpha_{1 j} \xi_{j}+\hat{\gamma}_{1 j} \eta_{j}\right) \equiv f_{\cdot 1}$

1) The structure of $G_{\lambda}$ being evident from $\S 203$ if $m=1$, we exclude this case henceforth. 
c) If $\alpha_{1 j}=\gamma_{1 j}=0,(j=1, \ldots, k-1)$, but $\alpha_{1 k}$ and $\gamma_{1 k}$ not both zero, we may, for $k<m$, proceed as in case a) or b) and obtain a substitution $T^{\prime}$ which replaces $\xi_{k}$ by $f_{1}$ and is derived from the substitutions 195). We then take $T=T^{\prime} P_{1 k}$.

d) If $\alpha_{1 j}=\gamma_{1 j}=0(j=1, \ldots, m-1)$, the proof given in c) applies if $\lambda=0$, since then $P_{1 m}$ is generated by the substitutions 195) of $G_{\lambda}$. For $\lambda=\lambda^{\prime}$, this case cannot exist, since the equation

$$
\alpha_{1 m} \gamma_{1 m}+\lambda^{\prime} \alpha_{1 m}^{2}+\lambda^{\prime} \gamma_{1 m}^{2}=0
$$

requires $\alpha_{1 m}=\gamma_{1 m}=0$ on account of the irreducibility of $Q$. Then would $f_{1} \equiv 0$.

It follows that $S=T S_{1}$, where $S_{1}$ leaves $\xi_{1}$ fixed but is a substitution belonging to $G_{\lambda}$. Let $S_{1}$ replace $\eta_{1}$ by

$$
f^{\prime} \equiv \sum_{j=1}^{m}\left(\beta_{1 j} \xi_{j}+\delta_{1 j} \eta_{j}\right)
$$

where, by 78$), \mu=1$, and 194),

The product

$$
\delta_{11}=1, \sum_{j=1}^{m} \beta_{1 j} \delta_{1 j}+\lambda \beta_{1 m}^{2}+\lambda \delta_{1 m}^{2}=0 .
$$

$$
S^{\prime} \equiv R_{2,1, \beta_{12}} Q_{2,1, \delta_{12}} \ldots R_{m, 1, \beta_{1 m}} Q_{m, 1, \delta_{1 m}}
$$

replaces $\xi_{1}$ by $\xi_{1}$ and $\eta_{1}$ by

$$
\left(\beta_{12} \delta_{12}+\cdots+\beta_{1 m} \delta_{1 m}+\lambda \beta_{1 m}^{2}+\lambda \delta_{1 m}^{2}\right) \xi_{1}+\eta_{1}+\sum_{j=2}^{m}\left(\beta_{1 j} \xi_{j}+\delta_{1 j} \eta_{j}\right),
$$

which equals $f^{\prime}$ since the coefficient of $\xi_{1}$ equals $\beta_{11}$ by 198).

We may therefore set $S_{1}=S^{\prime} S_{2}$, where $S_{2}$ is a substitution of $G_{\lambda}$ which leaves $\xi_{1}$ and $\eta_{1}$ fixed. Then by 78 ),

$$
\alpha_{i 1}=\beta_{i 1}=\gamma_{i 1}=\delta_{i 1}=0 \quad(i=2, \ldots, m) .
$$

The relations holding between $\alpha_{i j}, \beta_{i j}, \gamma_{i j}, \delta_{i j}(i, j=2, \ldots, m)$ are seen to be the relations 78) and 194) when $m-1$ is written for $m$. Proceeding with $S_{2}$ as we did with $S$, etc., we find ultimately that $S=T^{\prime} \Sigma$, where $T^{\prime}$ is derived from the substitutions 195), while $\Sigma$ is a substitution of $G_{\lambda}$ which affects only $\xi_{m}$ and $\eta_{m}$,

$$
\Sigma: \quad \xi_{m}^{\prime}=\alpha \xi_{m}+\gamma \eta_{m}, \quad \eta_{m}^{\prime}=\beta \xi_{m}+\delta \eta_{m} .
$$

The conditions 78), 193) and 194) become, for $m=1$,

$$
\begin{array}{ll}
\text { 199) } \alpha \delta+\beta \gamma=1, & \alpha \beta+\lambda \alpha^{2}+\lambda \beta^{2}=\lambda, \quad \gamma \delta+\lambda \gamma^{2}+\lambda \delta^{2}=\lambda, \\
\text { 200) } & \delta \beta+\lambda \delta^{2}+\lambda \beta^{2}=\lambda, \quad \gamma \alpha+\lambda \gamma^{2}+\lambda \alpha^{2}=\lambda .
\end{array}
$$

Combining 199) with 200), we may replace 200) by

$$
\beta(\alpha+\delta)=\gamma(\alpha+\delta)=\lambda(\alpha+\delta)^{2} \text {. }
$$


Suppose first that $\alpha+\delta \neq 0$. By 201), $\Sigma$ becomes

$$
O_{m}^{\alpha, \delta}:\left\{\begin{array}{l}
\xi_{m}^{\prime}=\alpha \xi_{m}+\lambda(\alpha+\delta) \eta_{m} \\
\eta_{m}^{\prime}=\lambda(\alpha+\delta) \xi_{m}+\delta \eta_{m}
\end{array} \quad\left(\alpha \delta+\lambda^{2} \alpha^{2}+\lambda^{2} \delta^{2}=1\right)\right.
$$

Suppose next that $\beta+\gamma \neq 0$. Applying the above procedure to

$$
\Sigma_{1} \equiv \Sigma M_{m}: \quad \xi_{m}^{\prime}=\beta \xi_{m}+\delta \eta_{m}, \quad \eta_{m}^{\prime}=\alpha \xi_{m}+\gamma \eta_{m},
$$

it follows that $\Sigma_{1}=O_{m}^{\beta, \gamma}$. Hence $\Sigma=O_{m}^{\beta, \gamma} M_{m}$.

Suppose finally that $\alpha+\delta=\beta+\gamma=0$. Conditions 199) and 201) become

so that $\Sigma=I$ or $\cdot M_{m}$.

$$
\alpha^{2}+\beta^{2}=1, \quad \alpha \beta=0,
$$

In every case, $\Sigma=O_{m}^{\alpha, \delta}$ or $\Sigma=O_{m}^{\beta, \gamma} M_{m}$. If $\lambda=0, O_{m}^{\alpha, \delta} \equiv T_{m, \alpha}$ and the theorem is proven. If $\lambda=\lambda^{\prime}, \lambda^{\prime}$ being suitably chosen, we prove in the next section that every $O_{m}^{\alpha, \delta}$ is a power of $L \equiv O_{m}^{0,2} z^{-1}$ and may therefore be derived from the substitutions 195).

203. Let $\varrho$ be a primitive root of $\rho^{9^{n}+1}=1$. It will satisfy an equation belonging to and irreducible in the $G F\left[2^{n}\right]$,

$$
\varrho^{2}+\Theta_{\rho}+1=0 \text {. }
$$

If we set $\Theta=\lambda^{-1}, \varrho=\xi_{m} / \eta_{m}$, we find that $\lambda \xi_{m}^{2}+\lambda \eta_{m}^{2}+\xi_{m} \eta_{m}$ is irreducible in and belongs to the $G F\left[2^{n}\right]$. Changing the variable from $\varrho$ to $\sigma \equiv \lambda \varrho$, we obtain for $\sigma$ the irreducible equation

$$
\sigma^{2}+\sigma+\lambda^{2}=0 \text {. }
$$

Since the roots of 203) are $\sigma$ and $\sigma^{2^{n}}$, we have $\sigma+\sigma^{2^{n}}=1$.

We make the transformation of indices:

$$
\text { 204) } \xi_{m} \equiv \lambda^{3 / 2} \sigma^{-1} Y_{12}+\sigma \lambda^{-1 / 2} Y_{34}, \quad \eta_{m} \equiv \lambda^{1 / 2}\left(Y_{12}+Y_{34}\right) \text {. }
$$

Solving, we find, for $p=2$,

205) $\quad Y_{12} \equiv \lambda^{1 / 2} \xi_{m}+\sigma \lambda^{-1 / 2} \eta_{m}, \quad Y_{34} \equiv \lambda^{1 / 2} \xi_{m}+\lambda^{3 / 2} \sigma^{-1} \eta_{m}$.

Then

$$
\xi_{m} \eta_{m}+\lambda \xi_{m}^{2}+\lambda \eta_{m}^{2} \equiv Y_{12} Y_{34}
$$

The substitution 202) takes the form

where

$$
\begin{aligned}
Y_{12}^{\prime} & =\tau Y_{12}, \quad Y_{34}^{\prime}=\tau^{-1} Y_{34} \\
\tau & \equiv \alpha+(\alpha+\delta) \sigma, \quad \tau^{-1} \equiv \delta+(\alpha+\delta) \sigma \\
\tau \tau^{-1} & \equiv \alpha \delta+\sigma(\alpha+\delta)^{2}+\sigma^{2}(\alpha+\delta)^{2} \equiv \alpha \delta+\lambda^{2}\left(\alpha^{2}+\delta^{2}\right)=1
\end{aligned}
$$

We have $\tau^{2^{n}+1}=1$ since $(\bmod 2)$,

$$
\tau^{2^{n}}=\alpha+(\alpha+\delta) \sigma^{2^{n}}=\alpha+(\alpha+\delta)(\sigma+1)=\delta+(\alpha+\delta) \sigma=\tau^{-1} .
$$

In particular, $L \equiv O_{m}^{0,2-1}$ takes the form

$$
Y_{12}^{\prime}=\varrho Y_{12}, \quad Y_{34}^{\prime}=\varrho^{-1} Y_{34}
$$


The substitutions 206) are evidently powers of 207), $\varrho$ being a primitive root of $x^{2^{n}+1}=1$. Hence the substitutions 202) are powers of $L$.

Inversely, every substitution 206) for which $\tau^{2^{n}+1}=1$ may be transformed by 205) into a substitution 202) of the $G F\left[2^{n}\right]$. In fact,

$$
\alpha+\delta=\tau+\tau^{-1}, \quad \alpha=\tau+\left(\tau+\tau^{-1}\right) \sigma, \quad \delta=\tau^{-1}+\left(\tau+\tau^{-1}\right) \sigma,
$$

so that $\alpha+\delta$ belongs to the $G F\left[2^{n}\right]$ and likewise $\alpha$ since

$$
\alpha^{2^{n}}=\tau^{2^{n}}+\left(\tau+\tau^{-1}\right) \sigma^{2^{n}}=\tau^{-1}+\left(\tau+\tau^{-1}\right)(\sigma+1)=\alpha .
$$

The number of substitutions 206) is $2^{n}+1$. The number of substitutions 202) is therefore $2^{n}+1$. Furthermore $M_{m} \equiv\left(\xi_{m} \eta_{m}\right)$ takes the form

$$
Y_{12}^{\prime}=\varrho Y_{34}, \quad Y_{34}^{\prime}=\varrho^{-1} Y_{12} \quad\left(\varrho \equiv \sigma \lambda^{-1}\right) .
$$

We have therefore a new proof of the results at the end of $\S 202$.

It is worth while to verify independently that the number of substitutions 202) is $2^{n} \pm 1$ according as $\lambda=\lambda^{\prime}$ or $\lambda=0$. We have only to determine the number of sets of solutions in the $G F\left[2^{n}\right]$ of

$$
\alpha \delta+\lambda^{2} \dot{\alpha}^{2}+\lambda^{2} \delta^{2}=1 \text {. }
$$

The result for the case $\lambda=0$ being evident, we suppose that $\lambda=\lambda^{\prime}$. The left member of 208) vanishes only when $\alpha=\delta=0$; for, otherwise,

$$
\left(1+\omega+\lambda^{2} \omega^{-1}\right)^{2}=0, \quad \omega^{2} \equiv \lambda^{2} \alpha / \delta
$$

would be reducible in the field, contrary to the irreducibility of 203). Hence each of the $2^{2 n}-1$ sets of marks $\alpha_{1}, \delta_{1}$, not both zero, in the $G F\left[2^{n}\right]$ will make

$$
\alpha_{1} \delta_{1}+\lambda^{2} \alpha_{1}^{2}+\lambda^{2} \delta_{1}^{2}=\varkappa^{2} \neq 0 .
$$

Then will $\alpha_{1} / \varkappa, \delta_{1} / \varkappa$ be a set of solutions of 208), and inversely every set of solutions of 208) may be so obtained. Hence, if $\lambda=\lambda^{\prime}$, the number of distinct sets of solutions is $\left(2^{2 n}-1\right) /\left(2^{n}-1\right)$.

204. We can now readily determine the order $\Omega_{m, n}^{(\lambda)}$ of $G_{\lambda \text {. The }}$ number of distinct linear functions $f_{1}$ by which the substitutions of $G_{\lambda}$ can replace $\xi_{1}$ is $P_{m, n}^{(\lambda)}-1$, if $P_{m, n}^{(\lambda)}$ denotes the number of sets of solutions in the $G F\left[2^{n}\right]$ of 197 ). For $m>1$, the pair of equations

$$
\alpha_{11} \gamma_{11}=\tau, \quad \sum_{j=2}^{m} \alpha_{1 j} \gamma_{1 j}+\lambda \alpha_{1 m}^{2}+\lambda \gamma_{1 m}^{2}=\tau
$$

has $\left(2^{n+1}-1\right) P_{m-1, n}^{(\lambda)}$ sets of solutions when $\tau=0$ and has

$$
\left(2^{n}-1\right)\left(2^{n(2 m-2)}-P_{m-1, n}^{(\lambda)}\right)
$$

sets of solutions when $\tau$ runs through the series of marks $\neq 0$ of the $G F\left[2^{n}\right]$. We have therefore the recursion formula $(m>1)$

$$
P_{m, n}^{(2)}=2^{n} P_{m-1, n}^{(\lambda)}+\left(2^{n}-1\right) 2^{n(2 m-2)} \text {. }
$$


According as $\lambda=0$ or $\lambda=\lambda^{\prime}$, the number of sets of solutions of

$$
\alpha_{11} \gamma_{11}+\lambda \alpha_{11}^{2}+\lambda \gamma_{11}^{2} \doteq 0
$$

is $P_{1, n}^{(0)} \equiv 2^{n+1}-1$ or $P_{1, n}^{\left(\lambda^{\prime}\right)} \equiv 1$. We find by simple induction,

$$
P_{s, n}^{(0)}-1=\left(2^{n s}-1\right)\left(2^{n(s-1)}+1\right), \quad P_{s, n}^{\left(\lambda^{\prime}\right)}-1=\left(2^{n s}+1\right)\left(2^{n(s-1)}-1\right) .
$$

The number of distinct linear functions $f^{\prime}$ is $2^{n(2 m-2)}$. In fact, 198) determines $\beta_{11}$ in terms of $\beta_{1 j}, \delta_{1 j}(j=2, \ldots, m)$, so that the latter may be chosen arbitrarily in the $G F\left[2^{n}\right]$.

It follows therefore, from $\S 202$, that

$$
\Omega_{m, n}^{(\lambda)}=\left(P_{m, n}^{(\lambda)}-1\right) 2^{2 n(m-1)} \Omega_{m-1, n}^{(\lambda)} \quad(m>1) .
$$

By $\S 203$, we have the initial values

$$
\Omega_{1, n}^{(0)}=2\left(2^{n}-1\right), \quad \Omega_{1, n}^{\left(2^{\prime}\right)}=2\left(2^{n}+1\right) .
$$

We now readily obtain the formulae

$$
\begin{aligned}
& \Omega_{m, n}^{(0)}=2\left(2^{n m}-1\right)\left(2^{2 n(m-1)}-1\right) 2^{2 n(m-1)}\left(2^{2 n(m-2)}-1\right) 2^{2 n(m-2)} \ldots\left(2^{2 n}-1\right) 2^{2 n} \\
& \Omega_{m, n}^{\left(\lambda^{\prime}\right)}=2\left(2^{n m}+1\right)\left(2^{2 n(m-1)}-1\right) 2^{2 n(m-1)}\left(2^{2 n(m-2)}-1\right) 2^{2 n(m-2)} \ldots\left(2^{2 n}-1\right) 2^{2 n}
\end{aligned}
$$

205. Theorem. - Those substitutions of $G_{2}$ which satisfy the further relation

$$
\text { 209) } I(\alpha, \beta, \gamma, \delta) \equiv \sum_{i, j}^{1, \ldots{ }^{n}} \alpha_{i j} \delta_{i j}+\lambda^{2}\left(\alpha_{m m}^{2}+\beta_{m m}^{2}+\gamma_{m m}^{2}+\delta_{m m}^{2}\right)=m
$$

form a subgroup of index 2 which any $M_{i}$ extends to $G_{\lambda}$. If $m>2$, this subgroup is identical with the group generated as follows:

$$
J_{\lambda} \equiv\left\{M_{i} M_{j}, N_{i, j, \varkappa}\right\} \quad(i, j=1, \ldots, m ; \varkappa \text { arbitrary in field }) .
$$

If $m=2$, it is identical with the group

$$
J_{\lambda} \equiv\left\{M_{1} M_{2}, N_{2,1,1}, T_{1, x}, Q_{2,1,1}\right\}
$$

We first prove that every substitution of $J_{\lambda}$ satisfies 209). To do this, it suffices to show that, if $\Sigma$ be any substitution of $G_{\lambda}$ which satisfies 209), the products $M_{i} M_{j} \Sigma, N_{i, j, x} \Sigma$ will also satisfy 209), the case $m=2$, being treated later. Let $\Sigma$ have the form 192).

a) If the product $M_{j} \Sigma$ be expressed in the form

$$
\text { 210) } \xi_{i}^{\prime}=\sum_{j=1}^{m}\left(\alpha_{i j}^{\prime} \xi_{j}+\gamma_{i j}^{\prime} \eta_{j}\right), \quad \eta_{i}^{\prime}=\sum_{j=1}^{m}\left(\beta_{i j}^{\prime} \xi_{j}+\delta_{i j}^{\prime} \eta_{j}\right) \quad(i=1, \ldots, m)
$$

we have

$$
\begin{aligned}
& \alpha_{i j}^{\prime}=\gamma_{i j}, \quad \gamma_{i j}^{\prime}=\alpha_{i j}, \quad \beta_{i j}^{\prime}=\delta_{i j}, \quad \delta_{i j}^{\prime}=\beta_{i j} \quad(i=1, \ldots, m) \\
& \alpha_{i k}^{\prime}=\alpha_{i k}, \quad \beta_{i k}^{\prime}=\beta_{i k}, \quad \gamma_{i k}^{\prime}=\gamma_{i k}, \quad \delta_{i k}^{\prime}=\delta_{i k} \quad\left(\begin{array}{l}
i=1, \ldots, m \\
k=1, \ldots, m ; k \neq j
\end{array}\right) .
\end{aligned}
$$


Hence

$$
\begin{gathered}
I\left(\alpha^{\prime}, \beta^{\prime}, \gamma^{\prime}, \delta^{\prime}\right) \equiv \sum_{k=1}^{i, k=1, \ldots, m} \alpha_{i k} \delta_{i k}+\sum_{i=1}^{m} \gamma_{i j} \beta_{i j}+\lambda^{2}\left(\alpha_{m m}^{2}+\beta_{m m}^{2}+\gamma_{m m}^{2}+\delta_{m m}^{2}\right) \\
=I(\alpha, \beta, \gamma, \delta)+\sum_{i=1}^{m}\left(\gamma_{i j} \beta_{i j}-\alpha_{i j} \delta_{i j}\right)=m+1
\end{gathered}
$$

upon applying 209) and 76). Hence $M_{j} \Sigma$ does not satisfy 209), while $M_{i} M_{j} \Sigma$ does.

b) If the product $N_{m, j, x} \Sigma$ be expressed in the form 210), we have

$$
\begin{array}{llll}
\alpha_{r s}^{\prime}=\alpha_{r s}, & \beta_{r s}^{\prime}=\beta_{r s} & (r, s=1, \ldots, m) \\
\gamma_{r s}^{\prime}=\gamma_{r s}, & \delta_{r s}^{\prime}=\delta_{r s} & (r, s=1, \ldots, m ; s \neq m, j) \\
\gamma_{r m}^{\prime}=\gamma_{r m}+x \alpha_{r j}, & \gamma_{r j}^{\prime}=\gamma_{r j}+x \alpha_{r m}+\lambda x^{2} \alpha_{r j} & (r=1, \ldots, m) \\
\delta_{r m}^{\prime}=\delta_{r m}+x \beta_{r j}, & \delta_{r j}^{\prime}=\delta_{r j}+x \beta_{r m}+\lambda x^{2} \beta_{r j} & (r=1, \ldots, m) .
\end{array}
$$

Hence $I\left(\alpha^{\prime}, \beta^{\prime}, \gamma^{\prime}, \delta^{\prime}\right)$ equals

$$
\begin{gathered}
\sum_{s=1=m, j}^{r, s=1, \ldots, m} \alpha_{r s} \delta_{r s} \\
+\sum_{r=1}^{m} \alpha_{r m}\left(\delta_{r m}+\varkappa \beta_{r j}\right)+\sum_{r=1}^{m} \alpha_{r j}\left(\delta_{r j}+\varkappa \beta_{r m}+\lambda \varkappa^{2} \beta_{r j}\right) \\
+\lambda^{2}\left\{\alpha_{m m}^{2}+\beta_{m m}^{2}+\left(\gamma_{m m}+\varkappa \alpha_{m j}\right)^{2}+\left(\delta_{m m}+\varkappa \beta_{m j}\right)^{2}\right\} \\
=I(\alpha, \beta, \gamma, \delta)+x \sum_{r=1}^{m}\left(\alpha_{r m} \beta_{r j}+\alpha_{r j} \beta_{r m}\right)+\lambda \varkappa^{2}\left(\sum_{r=1}^{m} \alpha_{r j} \beta_{r j}+\lambda \alpha_{m j}^{2}+\lambda \beta_{m j}^{2}\right) .
\end{gathered}
$$

But the last two sums are zero by 76) and 193).

c) An analogous proof holds for $N_{i, j, x} .(i, j<m)$, the above terms involving $\lambda x^{2}$ not being present.

d) Since the substitutions $Q_{i, j, x}, R_{i, j, x}(i, j=1, \ldots, m)$ and $P_{i j}$, $T_{i, \varkappa}\left(i, j<m\right.$, if $\left.\lambda=\lambda^{\prime}\right)$ may be expressed as a product of the $N_{i, j, \varkappa}$ and an even number of the $M_{i}(\S 202)$, the products $T_{i, r} \Sigma, Q_{i, j, r} \Sigma$, etc., will satisfy 209) if $\Sigma$ does.

Inversely, every substitution $S$ of $G_{\lambda}$ which satisfies 209) belongs to $J_{\lambda}$. In fact, by the proof given in $\S 202, S$ is of one of the two forms $K, K M_{m}$, where $K$ is derived from $\left.{ }^{1}\right) M_{i} M_{j}, Q_{i, j, x}, N_{i, j, x}$, $R_{i, j, x}(i, j=1, \ldots, m) ; P_{i j}, T_{i, x}\left(i, j<m\right.$ if $\left.\lambda=\lambda^{\prime}\right)$. Since $S$ shall satisfy 209), it is not of the form $K M_{m}$. It remains to show that these substitutions $M_{i} M_{j}, Q_{i, j, \varkappa}, \ldots, T_{i, \%}$ belong to $J_{\lambda}$.

For $m>2, J_{\lambda}$ contains $Q_{i, j, x}$, the transformed of $N_{i, j, x}$ by $M_{j} M_{k}(k \neq i, j)$; also $R_{i, j, x}$ and $Q_{j, i, \varkappa}$, the transformed of $N_{i, j, x}$ and $Q_{i, j, x}$ respectively by $M_{i} M_{j}$. Applying the formulae at the beginning of $\S 202$, we reach $P_{i j}$ and $T_{i, \mu} T_{j, \mu}\left(i, j<m\right.$, if $\left.\lambda=\lambda^{\prime}\right)$. Then $J_{\lambda}$ contains $T_{i, \mu} T_{j, \mu}-1$, the transformed of the latter by $M_{m} M_{j}$. The product of the two gives $T_{i, \mu^{2}}$. $N_{2,1, \varkappa}$.

1) By 196), $L$ and therefore every $O_{m}^{\alpha, \delta}$ is derived from $M_{1}^{\prime} M_{2}, Q_{2,1, \varkappa}$ and 
For $m=2, J_{2}$ contains $M_{1} M_{2}, T_{1, x}, N_{2,1, x}, R_{2,1, x}, Q_{2,1, x}, Q_{1,2, x}$ If $\lambda=0, J_{\lambda}$ contains $P_{12} \equiv Q_{2,1,1}^{-1} Q_{1,2,1} Q_{2,1,1}$.

The fact that $M_{1} M_{2}$ and $N_{1,2, x}$ do not generate $J_{0}$, for $m=2$, follows readily from $\S 196$. Since $M_{1} M_{2}$ transforms $N_{1,2, x}$ into $R_{1,2, \%}$, every substitution derived from the two former may be given the form $V$ or $V M_{1} M_{2}$, where $V$ is derived from $N_{1.2, x}$ and $R_{1,2, x}$. The latter two are of the form 181). Hence the group of the substitutions $V$ is a subgroup of the group of the substitutions 181) having the order

$$
v \equiv\left(2^{2 n}-1\right) 2^{n} \text {. }
$$

Hence $M_{1} M_{2}$ and the $N_{1,2, x}$ generate a group whose order is at most $2 v$. But $2 v<\left(2^{2 n}-1\right)^{2} 2^{2 n}$, the order of $J_{0}$ for $m=2$.

It follows similarly from $\S \S 197-198$ that $M_{1} M_{2}$ and $N_{2,1, x}$ do not generate $J_{\lambda^{\prime}}$ for $m=2$. This result may be shown directly for the case $n=1$, when $J_{\lambda^{\prime}}$ has the order 60 (\$204). In fact, setting $M \equiv M_{1} M_{2}, N \equiv N_{2,1,1}, R \equiv R_{2,1,1}=M^{-1} N M$, the group generated by $M$ and $N$ contains only ten distinct substitutions:

$$
I, M, N, R, N M, R M, R N, N R, N R M, R N M \text {. }
$$

For $m=2$, the structure of $J_{0}$ was determined in $\S 196$ and that of $J_{\lambda^{\prime}}$ in $\S \S 197-198$.

206. Theorem. - The senary first hypoabelian group $J_{0}$ in the $G F\left[2^{n}\right]$ is a simple group holoedrically isomorphic with $\operatorname{LF}\left(4,2^{n}\right)$.

We obtained in $\S 163$ a senary group $G_{4,2}^{\prime}$, leaving absolutely invariant

$$
Y_{12} Y_{34}-Y_{13} Y_{24}+Y_{14} Y_{23}
$$

which is holoedrically isomorphic with the simple group $\operatorname{LF}\left(4,2^{n}\right)$. To identify $G_{4,2}^{\prime}$ with $J_{0}(m=3)$, we set

$$
Y_{12}=\xi_{1}, \quad Y_{13}=\xi_{2}, \quad Y_{14}=\xi_{3}, \quad Y_{23}=\eta_{3}, \quad Y_{24}=\eta_{2}, \quad Y_{34}=\eta_{1} .
$$

The general substitution $[\alpha]_{2}$ of $G_{4,2}^{\prime}$, given in $\S 164$, may be written

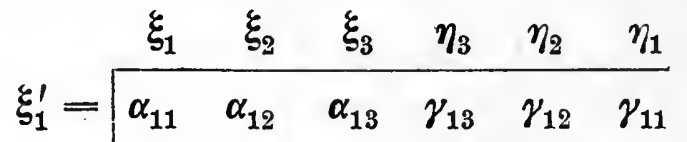

$$
\begin{aligned}
& \xi_{2}^{\prime}=\begin{array}{lllllll}
\alpha_{21} & \alpha_{22} & \alpha_{23} & \gamma_{23} & \gamma_{22} & \gamma_{21}
\end{array} \\
& \xi_{3}^{\prime}=\begin{array}{lllllll}
\alpha_{31} & \alpha_{32} & \alpha_{33} & \gamma_{33} & \gamma_{32} & \gamma_{31}
\end{array}
\end{aligned}
$$

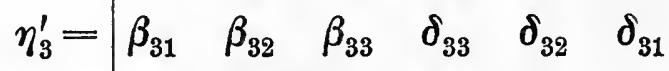

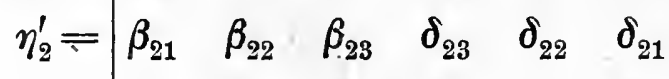

$$
\begin{aligned}
& \eta_{1}^{\prime}=\mid \begin{array}{llllll}
\beta_{11} & \beta_{12} & \beta_{13} & \delta_{13} & \delta_{12} & \delta_{11}
\end{array}
\end{aligned}
$$

In this form the notation agrees with that employed in $\S 201$ for the substitutions of $J_{0}$. In view of $\S 165$, the above general substitution of $G_{4,2}^{\prime}$ must satisfy the relation $(\bmod 2)$ $\alpha_{11} \delta_{11}+\alpha_{12} \delta_{12}+\alpha_{13} \delta_{13}+\alpha_{21} \delta_{21}+\alpha_{22} \delta_{22}+\alpha_{23} \delta_{23}+\alpha_{31} \delta_{31}+\alpha_{32} \delta_{32}+\alpha_{33} \delta_{33} \equiv 1$. But this is relation 209) for $\lambda=0, m=3$, which defines the subgroup $J_{0}$ of the first hypoabelian group. Hence $G_{4,2}^{\prime} \equiv J_{0}$. 
20\%. Theorem. - The senary second hyperabelian group $J_{\lambda^{\prime}}$ in the $G F\left[2^{n}\right]$ is a simple group holoedrically isomorphic with $H A\left(4,2^{2 n}\right)$.

We begin as in $\S 190$, but make the following transformation of indices, including the transformation 204) for $m=3$ :

$$
\begin{aligned}
& \xi_{1}=Y_{14}, \quad \xi_{2}=Y_{13}, \quad \eta_{1}=Y_{23}, \quad \eta_{2}=Y_{24}, \\
& \xi_{3} \equiv \lambda^{3 / 2} \sigma^{-1} Y_{12}+\sigma \lambda^{-1 / 2} Y_{34}, \quad \eta_{3}=\lambda^{1 / 2}\left(Y_{12}+Y_{34}\right) .
\end{aligned}
$$

The invariant of the second compound group is transformed thus:

$$
Y_{12} Y_{34}-Y_{13} Y_{24}+Y_{14} Y_{23}=\xi_{1} \eta_{1}+\xi_{2} \eta_{2}+\xi_{3} \eta_{3}+\lambda \xi_{3}^{2}+\lambda \eta_{3}^{2} \text {. }
$$

If we take

$$
\tau \equiv \omega^{-2^{n}+1}, \quad \tau^{2^{n}+1} \equiv \omega^{-2^{2 n}+1}=1,
$$

the substitution 206) becomes in the new indices a substitution 202) with coefficients in the $G F\left[2^{n}\right]$. In particular, if $\omega$ be a suitable primitive root of the $G F\left[2^{2 n}\right]$, $\tau$ will be the primitive root $\varrho$ of $x^{2^{n}+1}=1$. We thus reach, by 207), the substitution $L$.

We next express in the new indices the general substitution $[\alpha]_{2}$, given in $\S 164$, of the second compound $A_{4,2}$ of the group of quaternary Abelian substitutions of determinant unity in the $G F\left[2^{n}\right]$. For example, it will replace $\xi_{2} \equiv Y_{13}$ by

$$
\begin{gathered}
\left|\begin{array}{ll}
1 & 3 \\
1 & 2
\end{array}\right|\left(\lambda^{1 / 2} \xi_{3}+\sigma \lambda^{-1 / 2} \eta_{3}\right) \\
+\left|\begin{array}{ll}
1 & 3 \\
1 & 3
\end{array}\right| \xi_{2}+\left|\begin{array}{ll}
1 & 3 \\
1 & 4
\end{array}\right| \xi_{1}+\left|\begin{array}{ll}
1 & 3 \\
2 & 3
\end{array}\right| \eta_{1}+\left|\begin{array}{ll}
1 & 3 \\
2 & 4
\end{array}\right| \eta_{2} \\
+\left|\begin{array}{ll}
1 & 3 \\
3 & 4
\end{array}\right|\left(\lambda^{1 / 2} \xi_{3}+\lambda^{3 / 2} \sigma^{-1} \eta_{3}\right) .
\end{gathered}
$$

\begin{tabular}{|c|c|c|c|c|c|c|c|c|}
\hline & $\xi_{1}$ & & $\xi_{2}$ & $\xi_{3}$ & $\eta_{3}$ & $\eta_{2}$ & & $\eta_{1}$ \\
\hline$\xi_{1}^{\prime}=$ & \begin{tabular}{|l|}
14 \\
14
\end{tabular} \mid & & $\left|\begin{array}{l}14 \\
13\end{array}\right|$ & $0 \lambda^{-\frac{1}{2}}$ & $\left|\begin{array}{ll}1 & 4 \\
1 & 2\end{array}\right|$ & $\left|\begin{array}{l}14 \\
24\end{array}\right|$ & & $\left|\begin{array}{ll}1 & 4 \\
2 & 3\end{array}\right|$ \\
\hline$\xi_{2}^{\prime}=$ & $\left|\begin{array}{l}13 \\
14\end{array}\right|$ & & $\left|\begin{array}{l}13 \\
13\end{array}\right|$ & $0 \lambda^{-\frac{1}{2}}$ & $\left|\begin{array}{ll}1 & 3 \\
1 & 2\end{array}\right|$ & $\left|\begin{array}{l}13 \\
24\end{array}\right|$ & & $\left|\begin{array}{ll}1 & 3 \\
2 & 3\end{array}\right|$ \\
\hline 1) $\xi_{3}^{\prime}=$ & $\lambda^{-\frac{1}{2}} \mid \begin{array}{l}12 \\
14\end{array}$ & $\lambda^{-\frac{1}{2}}$ & $\mid \begin{array}{l}12 \\
13\end{array}$ & $1 \lambda^{-1}$ & $\left|\begin{array}{ll}1 & 2 \\
1 & 2\end{array}\right|+\lambda^{-1} \lambda^{-\frac{1}{2}}$ & $\left|\begin{array}{l}12 \\
24\end{array}\right|$ & $\lambda^{-\frac{1}{2}}$ & $\left|\begin{array}{ll}1 & 2 \\
2 & 3\end{array}\right|$ \\
\hline$\eta_{3}^{\prime}=$ & 0 & & 0 & 0 & 1 & 0 & & 0 \\
\hline$\eta_{\dot{z}}^{\prime}=$ & $\mid \begin{array}{l}24 \\
14\end{array}$ & & $\mid \begin{array}{l}24 \\
13\end{array}$ & $0 \lambda^{-\frac{1}{2}}$ & $\left|\begin{array}{l}24 \\
12\end{array}\right|$ & $\left|\begin{array}{l}24 \\
24\end{array}\right|$ & & $\begin{array}{ll}2 & 4 \\
2 & 3\end{array} \mid$ \\
\hline$\eta_{1}^{\prime}=$ & $\left|\begin{array}{l}23 \\
14\end{array}\right|$ & & $\left|\begin{array}{l}23 \\
13\end{array}\right|$ & $0 \lambda^{-\frac{1}{2}}$ & $\left|\begin{array}{ll}2 & 3 \\
12 & 2\end{array}\right|$ & $\left|\begin{array}{l}23 \\
24\end{array}\right|$ & & $\begin{array}{l}23 \\
23\end{array}$ \\
\hline
\end{tabular}

Here the coefficient of $\xi_{3}$ is $\equiv 0(\bmod 2)$ and that of $\eta_{3}$ is $\lambda^{-1 / 2}\left|\begin{array}{l}13 \\ 12\end{array}\right|$, since

$$
\left|\begin{array}{ll}
1 & 3 \\
1 & 2
\end{array}\right|+\left|\begin{array}{ll}
1 & 3 \\
3 & 4
\end{array}\right| \equiv\left|\begin{array}{ll}
\alpha_{11} & \alpha_{12} \\
\alpha_{31} & \alpha_{32}
\end{array}\right|+\left|\begin{array}{ll}
\alpha_{13} & \alpha_{14} \\
\alpha_{33} & \alpha_{34}
\end{array}\right|=0
$$

by one of the Abelian conditions, while $\lambda^{2} \sigma^{-1}+\sigma+1 \equiv 0$ by 203). Proceeding in this manner, we find that $[\alpha]_{2}$ takes the form

DlCKsoN Linear Groups. 
To prove that this substitution satisfies relation 209) for $m=3$, consider it to be expressed in the notation used for the general substitution $[\alpha]_{2}$ of $\S 206$. The condition 209) built for the substitution 211) therefore becomes

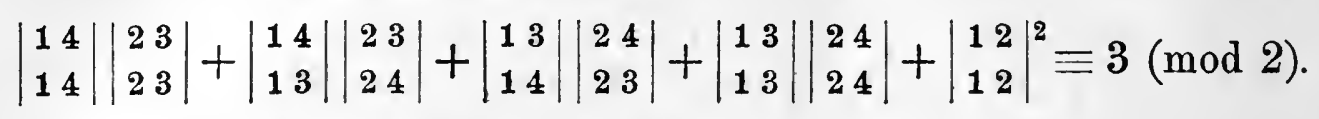

The left member may be written $(\bmod 2)$ :

$$
\alpha_{11}\left|\begin{array}{lll}
0 & \alpha_{23} & \alpha_{24} \\
\alpha_{32} & \alpha_{33} & \alpha_{34} \\
\alpha_{42} & \alpha_{43} & \alpha_{44}
\end{array}\right|+\alpha_{31}\left|\begin{array}{lll}
0 & \alpha_{13} & \alpha_{14} \\
\alpha_{22} & \alpha_{23} & \alpha_{24} \\
\alpha_{42} & \alpha_{43} & \alpha_{44}
\end{array}\right|+\alpha_{41}\left|\begin{array}{lll}
0 & \alpha_{13} & \alpha_{14} \\
\alpha_{22} & \alpha_{23} & \alpha_{24} \\
\alpha_{32} & \alpha_{33} & \alpha_{34}
\end{array}\right|+\alpha_{11}^{2} \alpha_{22}^{2}+\alpha_{12}^{2} \alpha_{21}^{2}
$$

Upon expanding according to the elements of the first column the determinant on the left of the following identity

$$
\left|\begin{array}{llll}
\alpha_{11} & \alpha_{12} & \alpha_{13} & \alpha_{14} \\
\alpha_{21} & \alpha_{22} & \alpha_{23} & \alpha_{24} \\
\alpha_{31} & \alpha_{32} & \alpha_{33} & \alpha_{34} \\
\alpha_{41} & \alpha_{42} & \alpha_{43} & \alpha_{44}
\end{array}\right| \equiv 1
$$

we obtain the first three terms in the above expression together with

$$
\alpha_{21}\left|\begin{array}{lll}
\alpha_{12} & \alpha_{13} & \alpha_{14} \\
\alpha_{32} & \alpha_{33} & \alpha_{34} \\
\alpha_{42} & \alpha_{43} & \alpha_{44}
\end{array}\right|+\alpha_{11} \alpha_{22}\left|\begin{array}{ll}
\alpha_{33} & \alpha_{34} \\
\alpha_{43} & \alpha_{44}
\end{array}\right|+\alpha_{31} \alpha_{12}\left|\begin{array}{ll}
\alpha_{23} & \alpha_{24} \\
\alpha_{43} & \alpha_{44}
\end{array}\right|+\alpha_{41} \alpha_{12}\left|\begin{array}{ll}
\alpha_{23} & \alpha_{24} \\
\alpha_{33} & \alpha_{34}
\end{array}\right| .
$$

It remains to show that the sum of these terms together with $\alpha_{11}^{2} \alpha_{22}^{2}+\alpha_{12}^{2} \alpha_{21}^{2}$ is zero. Upon applying the Abelian relations ( $\left.\bmod 2\right)$,

$$
\begin{aligned}
& \left|\begin{array}{ll}
\alpha_{33} & \alpha_{34} \\
\alpha_{43} & \alpha_{44}
\end{array}\right|=\left|\begin{array}{ll}
\alpha_{11} & \alpha_{12} \\
\alpha_{21} & \alpha_{22}
\end{array}\right|,\left|\begin{array}{ll}
\alpha_{32} & \alpha_{34} \\
\alpha_{42} & \alpha_{44}
\end{array}\right|=\left|\begin{array}{ll}
\alpha_{12} & \alpha_{14} \\
\alpha_{22} & \alpha_{24}
\end{array}\right|,\left|\begin{array}{ll}
\alpha_{32} & \alpha_{33} \\
\alpha_{42} & \alpha_{43}
\end{array}\right|=\left|\begin{array}{ll}
\alpha_{12} & \alpha_{13} \\
\alpha_{22} & \alpha_{23}
\end{array}\right|, \\
& \left|\begin{array}{ll}
\alpha_{23} & \alpha_{24} \\
\alpha_{43} & \alpha_{44}
\end{array}\right|=\left|\begin{array}{ll}
\alpha_{21} & \alpha_{22} \\
\alpha_{41} & \alpha_{42}
\end{array}\right|, \quad\left|\begin{array}{ll}
\alpha_{23} & \alpha_{24} \\
\alpha_{33} & \alpha_{34}
\end{array}\right|=\left|\begin{array}{ll}
\alpha_{21} & \alpha_{22} \\
\alpha_{31} & \alpha_{32}
\end{array}\right|,\left|\begin{array}{ll}
\alpha_{13} & \alpha_{14} \\
\alpha_{23} & \alpha_{24}
\end{array}\right|=\left|\begin{array}{ll}
\alpha_{31} & \alpha_{32} \\
\alpha_{41} & \alpha_{42}
\end{array}\right|,
\end{aligned}
$$

the sum is seen to be congruent to zero $(\bmod 2)$. The substitutions 211) therefore belong to $J_{\lambda^{\prime}}$. Their number equals the order

$$
\left(2^{4 n}-1\right) 2^{3 n}\left(2^{2 n}-1\right) 2^{n}
$$

of the quaternary Abelian group $S A\left(4,2^{n}\right)$ (§ 115), which was shown above to be holoedrically isomorphic with the group of the substitutions 211) leaving $\eta_{3}$ fixed. We prove in the next section that this number equals the total number of substitutions belonging to $J_{\lambda^{\prime}}(m=3)$ and leaving $\eta_{3}$ fixed. It follows that the substitutions 211) include the following substitutions of $J_{\lambda^{\prime}}$ not altering $\eta_{m}$ :

$$
M_{1} M_{2}, \quad N_{1,2, \varkappa}, \quad N_{m, 1, x}, \quad N_{m, 2, \varkappa}, \quad Q_{m, 1, \varkappa} .
$$


These substitutions must therefore belong to the group $C$, the second compound of $H A\left(4,2^{2 n}\right)$ when expressed in the indices $\xi_{i}, \eta_{i}$. Also $C$ contains $L$ and therefore also $M_{1} M_{m}$ by formula 196). Hence $C$ contains all the generators of $J_{\lambda^{\prime}}(m=3)$. But the order of $C$, being equal to that of $H A\left(4,2^{2 n}\right)$, is

$$
\left(2^{4 n}-1\right) 2^{3 n}\left(2^{3 n}+1\right) 2^{2 n}\left(2^{2 n}-1\right) 2^{n},
$$

which equals the order of $J_{\lambda^{\prime}}(m=3)$. Hence $J_{\lambda^{\prime}} \equiv C$.

208. Theorem. - If $m=3$, the number of substitutions of $J_{\lambda^{\prime}}$ which leave $\xi_{m}$ fixed is

$$
\left(2^{2 n}+1\right) 2^{2 n}\left(2^{2 n}-1\right)^{2} 2^{2 n}
$$

If a substitution $S$ of $J_{\lambda^{\prime}}$ does not alter $\xi_{m}$ and replaces $\eta_{m}$ by

$$
f_{m} \equiv \sum_{j=1}^{m}\left(\beta_{m j} \xi_{j}+\delta_{m j} \eta_{j}\right)
$$

we must have, in virtue of the relations 78) and 194),

$$
\delta_{m m}=1, \sum_{j=1}^{m} \beta_{m j} \delta_{m j}+\lambda \beta_{m m}^{2}=0 \text {. }
$$

We proceed to prove, inversely, that if $\beta_{m j}, \delta_{m j}$ be any set of solutions in the $G F\left[2^{n}\right]$ of 212) there exists a substitution $\Sigma$ in $J_{\lambda^{\prime}}$ which leaves $\xi_{m}$ fixed and replaces $\eta_{m}$ by $f_{m}$.

If $\beta_{m j}=\delta_{m j}=0(j=1, \ldots, m-1)$, then $\beta_{m m}=0$ or $\lambda^{-1}$. Hence we may take as $\Sigma$ the identity or $M_{2} M_{m} L$ respectively.

In the contrary case, let $\beta_{m 2} \neq 0$, for example. Then $J_{\lambda^{\prime}}$ contains a first hypoabelian substitution $T$ leaving $\xi_{m}$ and $\eta_{m}$ fixed and replacing $\eta_{2}$ by

$$
\beta_{m 1} \xi_{1}+\delta_{m 1} \eta_{1}+\beta_{m 2} \xi_{2}+\delta \eta_{2}, \quad \delta \equiv \delta_{m 2}+\frac{1}{\beta_{m 2}}\left(\beta_{m m}+\lambda \beta_{m m}^{2}\right),
$$

since $\beta_{m 1} \delta_{m 1}+\beta_{m 2} \delta=0$ in virtue of 212). Then we may take

$$
\Sigma=Q_{2, m, x} T Q_{2, m, 1} \quad \boldsymbol{x} \equiv \boldsymbol{\beta}_{m m} / \boldsymbol{\beta}_{m 2} .
$$

For $m=3$, the number of sets of solutions in the $G F\left[2^{n}\right]$ of 212):

$$
\beta_{m 1} \delta_{m 1}+\beta_{m 2} \delta_{m 2}+\beta_{m m}+\lambda \beta_{m m}^{2}=0
$$

is $\left(2^{2 n}+1\right) 2^{2 n}$. Indeed, there are $2^{n-1}$ distinct values in the $G F\left[2^{n}\right]$ of

$$
\tau \equiv \beta_{m m}+\lambda \beta_{m m}^{2} \text {. }
$$

By $\S 204, \beta_{m 1} \delta_{m 1}+\beta_{m 2} \delta_{m 2}=\tau$ has $2^{3 n}+2^{2 n}-2^{n}$ sets of solutions if $\tau=0$; while, if $\tau$ have any one of the $2^{n-1}-1$ possible values $\neq 0$, it has 


$$
\frac{2^{4 n}-\left(2^{3 n}+2^{2 n}-2^{n}\right)}{2^{n}-1} \equiv 2^{n}\left(2^{2 n}-1\right)
$$

sets of solutions, and therefore in all

$$
2^{3 n}+2^{2 n}-2^{n}+\left(2^{n-1}-1\right) 2^{n}\left(2^{2 n}-1\right) \equiv 2^{4 n-1}+2^{2 n-1}
$$

sets of solutions. But each value of $\boldsymbol{\tau}$ furnishes two values of $\beta_{m m}$.

209. Theorem. - The hypoabetian groups $J_{\lambda}$ on $2 m>6$ indices are simple.

Let $K$ be a self-conjugate subgroup of $J_{\lambda}$ containing a substitution

$S: \quad \xi_{i}^{\prime}=\sum_{j=1}^{m}\left(\alpha_{i j} \xi_{j}+\gamma_{i j} \eta_{j}\right), \quad \eta_{i}^{\prime}=\sum_{j=1}^{m}\left(\beta_{i j} \xi_{j}+\delta_{i j} \eta_{j}\right) \quad(i=1, \ldots, m)$

not the identity $I$. We first prove that $K$ contains a substitution $\neq I$ which multiplies $\xi_{1}$ by a constant. Let $S$ replace $\xi_{1}$ by

where by 194),

$$
f_{1} \equiv \sum_{j=1}^{m}\left(\alpha_{1 j} \xi_{j}+\gamma_{1 j} \eta_{j}\right)
$$

$$
\sum_{j=1}^{m} \alpha_{1 j} \gamma_{1 j}+\lambda \alpha_{1 m}^{2}+\lambda \gamma_{1 m}^{2}=0
$$

If $f_{1} \neq \alpha_{11} \xi_{1}$, we have one of the following three sub-cases.

a) $\gamma_{11} \neq 0$. Then $J_{2}$ contains the product

$$
T \equiv T_{1, \gamma_{11}}-1 R_{2,1, \alpha_{12}} Q_{2,1, \gamma_{12}} \ldots R_{m, 1, \alpha_{1 m}} Q_{m, 1, \gamma_{1 m}}
$$

which replaces $\xi_{1}$ by $\gamma_{11}^{-1} \xi_{1}$ and $\eta_{1}$ by the function

$\gamma_{11}^{-1}\left(\alpha_{12} \gamma_{12}+\cdots+\alpha_{1 m} \gamma_{1 m}+\lambda \alpha_{1 m}^{2}+\lambda \gamma_{1 m}^{2}\right) \xi_{1}+\gamma_{11} \eta_{1}+\sum_{j=2}^{m}\left(\alpha_{1 j} \xi_{j}+\gamma_{1 j} \eta_{j}\right)$.

This equals $t_{1}$, since the coefficient of $\xi_{1}$ is congruent to $\alpha_{11}$ modulo 2, in virtue of 213). Hence $K$ contains $S_{1} \equiv T^{-1} S T$, which replaces $\xi_{1}$ by $\gamma_{11}^{-1} \eta_{1}$.

If $J_{\lambda}$ contains a substitution $T_{1}$ which leaves $\xi_{1}$ and $\eta_{1}$ fixed and is not commutative with $S_{1}, K$ will contain the product

$$
S_{1}^{-1} \cdot T_{1}^{-1} S_{1} T_{1} \neq I
$$

which leaves $\xi_{1}$ fixed. Suppose on the contrary that $S_{1}$ is commutative with every substitution of $J_{\lambda}$ which leaves $\xi_{1}$ and $\eta_{1}$ fixed. Among the latter are $R_{2,3, x}$ and $Q_{3,2, x}$. If we equate the two expressions by which $S_{1} R_{2,3, \%}$ and $R_{2,3, \%} S_{1}$ replace $\eta_{3}$, we find

$$
\eta_{3}^{\prime}+x \xi_{2}^{\prime}=\eta_{3}^{\prime}+() \xi_{2}+() \xi_{3} \text {. }
$$

Similarly, if $S_{1}$ be commutative with $Q_{3,2, \varkappa}$, we have

$$
\xi_{3}^{\prime}+x \xi_{2}^{\prime}=\xi_{3}^{\prime}+() \xi_{2}+() \eta_{3} \text {. }
$$


Hence $\xi_{2}^{\prime}=() \xi_{2}$. Transforming $S_{1}$ by $P_{12}$ we obtain a substitution $\neq I$ which multiplies $\xi_{1}$ by a constant and belongs to $K$.

b) Let $\gamma_{11}=0, \alpha_{12}=\alpha_{13}=\cdots=\alpha_{1 m-1}=0$ and, if $\lambda=0$, also $\alpha_{1 m}=0$. If $\lambda=\lambda^{\prime}$, we must have $\alpha_{1 m}=\gamma_{1 m}=0$, since 213) reduces to

$$
\alpha_{1 m} \gamma_{1 m}+\lambda^{\prime} \alpha_{1 m}^{2}+\lambda^{\prime} \gamma_{1 m}^{2}=0
$$

whereas $Q(\S 199)$ is irreducible in the field. Since $f_{1} \neq \alpha_{11} \xi_{1}$, we cannot have $\gamma_{12}=\gamma_{13}=\cdots=\gamma_{1 m-1}$ together with $\gamma_{1 m}=0$, if $\lambda=0$. Transforming $S$ by a suitable $P_{2 j}\left(j<m\right.$, if $\left.\lambda=\lambda^{\prime}\right)$, we reach a substitution $S^{\prime}$ having $\gamma_{12} \neq 0$ and belonging to $K$. Transforming $S^{\prime}$ by $M_{2} M_{3}$, we reach a substitution of $K$ in which $\gamma_{11}=0, \alpha_{12} \neq 0$ [case c)].

c) Let $\gamma_{11}=0, \alpha_{12}, \ldots, \alpha_{1 m-1}, \alpha_{1 m}$ be not all zero if $\lambda=0$; let $\gamma_{11}=0, \alpha_{12}, \ldots, \alpha_{1 m-1}$ be not all zero if $\lambda=\lambda^{\prime}$. Transforming $S$ by a suitable $P_{2 j}$, we reach a substitution $S^{\prime}$ of $K$ having $\alpha_{12} \neq 0$. Then $J_{\lambda}$ contains

$$
T \equiv T_{2, \alpha_{12}} Q_{2,1, \alpha_{11}} \cdot Q_{2,3, \alpha_{13}} N_{2,3, \gamma_{13}} \ldots Q_{2, m, \alpha_{1 m}} N_{2, m, \gamma_{1 m}}
$$

which does not alter $\xi_{1}$ but replaces $\xi_{2}$ by

$$
\begin{aligned}
& \alpha_{11} \xi_{1}+\alpha_{12} \xi_{2}+\alpha_{12}^{-1}\left(\alpha_{13} \gamma_{13}+\cdots+\alpha_{1 m} \gamma_{1 m}+\lambda \alpha_{1 m}^{2}+\lambda \gamma_{1 m}^{2}\right) \eta_{2} \\
& +\sum_{j=3}^{m}\left(\alpha_{1 j} \xi_{j}+\gamma_{1 j} \eta_{j}\right) .
\end{aligned}
$$

Since $\gamma_{11}=0$, this reduces to $f_{1}$ in virtue of 213). Hence $K$ contains $S_{1}$, the transformed of $S^{\prime}$ by $T$, which replaces $\xi_{1}$ by $\xi_{2}$.

If $S_{1}$ be commutative with both $R_{3,1, x}$ and $R_{3,2, x}$, it merely multiplies $\xi_{3}$ by a constant, so that its transform by $P_{13}$ gives the required substitution. In fact, $S_{1} R_{3, j, x}$ and $R_{3, j, x} S_{1}$ replace $\eta_{j}$ by respectively

$$
\eta_{j}^{\prime}+\lambda \xi_{3}^{\prime}, \quad \eta_{j}^{\prime}+() \xi_{3}+() \xi_{j}
$$

In the contrary case, $K$ contains the two products

$$
S_{1}^{-1} \cdot R_{3, j, \varkappa}^{-1} S_{1} R_{3, j, \%} \quad(j=1,2)
$$

which leave $\xi_{1}$ fixed and do not both reduce to the identity.

Next, $K$ contains a substitution $\neq I$ leaving $\xi_{1}$ and $\eta_{1}$ fixed. We have previously reached in $K$ a substitution $S \neq I$ which replaces $\xi_{1}$ by $\alpha \xi_{1}$. Let it replace $\eta_{1}$ by $\sum_{j=1}^{m}\left(\beta_{1 j} \xi_{j}+\delta_{1 j} \eta_{j}\right)$. By an Abelian relation 78), $\delta_{11}=\alpha^{-1}$. By 194), we have

$$
\sum_{j=1}^{m} \beta_{1 j} \delta_{1 j}+\lambda \beta_{1 m}^{2}+\lambda \delta_{1 m}^{2}=0
$$


a) Let $\beta_{11}=0, \beta_{1 j}=\delta_{1 j}=0(j=2, \ldots, m-1)$, and, if $\lambda=0$, also $\beta_{1 m}=\delta_{1 m}=0$. If $\lambda=\lambda^{\prime}$, then must $\beta_{1 m}=\delta_{1 m}=0$ by 214). Evidently $S=T_{1, \alpha} S_{1}$, where $S_{1}$ leaves $\xi_{1}$ and $\eta_{1}$ fixed. By the Abelian relations 78$), S_{1}$ involves only the indices $\xi_{i}, \eta_{i}(i=2, \ldots, m)$. If $S_{1}$ be not commutative with every substitution $\Sigma_{1}$ of $J_{\lambda}$ which does not involve $\xi_{1}, \eta_{1}$, then $K$ will contain a product

$$
S^{-1} \Sigma_{1}^{-1} S \Sigma_{1} \equiv S_{1}^{-1} \Sigma_{1}^{-1} S_{1} \Sigma_{1} \neq I,
$$

which leaves $\xi_{1}$ and $\eta_{1}$ fixed. In the contrary case, $S_{1}$ is commutative with $R_{2,3, x}$ and $Q_{3,2, x}$, so that, as shown above, $S_{1}$ will replace $\xi_{2}$ by $\varrho \xi_{2}$. Since $S_{1}$ is to be commutative with $M_{2} M_{3}$ also, it will replace $\eta_{2}$ by $\varrho \eta_{2}$. Hence, by an Abelian relation, $\varrho^{2}=1$; whence $\varrho=1$. Transforming $S$ by $P_{12}$, we obtain a substitution $\neq I$ which leaves $\xi_{1}$ and $\eta_{1}$ fixed and belongs to $K$.

b) Let $\beta_{11}=0, \beta_{1 j}, \delta_{1 j}(j=2, \ldots, m)$ be not all zero if $\lambda=0$, but let $\beta_{11}=0, \beta_{1 j}, \delta_{1 j}(j=2, \ldots, m-1)$ be not all zero if $\lambda=\lambda^{\prime}$. Then by $\S 202, J_{\lambda}$ contains a substitution $T$, affecting only

which replaces $\xi_{2}$ by

$$
\xi_{i}, \eta_{i} \quad(i=2, \ldots, m),
$$

$$
\sum_{j=2}^{m}\left(\beta_{1 j} \xi_{j}+\delta_{1 j} \eta_{j}\right), \quad \sum_{j=2}^{m} \beta_{1 j} \delta_{1 j}+\lambda \beta_{1 m}^{2}+\lambda \delta_{1 m}^{2}=0 .
$$

Hence $K$ contains $S_{1}$, the transformed of $S$ by $T$. $S_{1}$ replaces $\xi_{1}$ by $\alpha \xi_{1}$ and $\eta_{1}$ by $\alpha^{-1} \eta_{1}+\xi_{2}$.

If $J_{\lambda}$ contains a substitution $V$, leaving $\xi_{1}, \eta_{1}, \xi_{2}$ fixed, which is not commutative with $S_{1}, K$ will contain

$$
S_{1}^{-1} \cdot V^{-1} S_{1} V \neq I
$$

which leaves $\xi_{1}$ and $\eta_{1}$ fixed.

In the contrary case, $S_{1}$ will be commutative with $R_{2,3, \lambda}$ and $R_{m, 3, \lambda}$ and $M_{3} M_{m}$. Equating the two functions by which $S_{1} R_{2,3, x}$ and $R_{2,3, x} S_{1}$ replace $\eta_{2}$, we find $\xi_{3}^{\prime}=() \xi_{3}+() \xi_{2}$. Equating the two functions by which $S_{1} R_{m, 3, *}$ and $R_{m, 3, x} S_{1}$ replace $\eta_{m}$, we find that $\xi_{3}^{\prime}=() \xi_{3}+() \xi_{m}$. Hence $\xi_{3}^{\prime}=\varrho \xi_{3}$. Since $S_{1}$ is to be commutative with $M_{3} M_{m}, \eta_{3}^{\prime}=\varrho \eta_{3}$. Then $\varrho=1$. Transforming $S_{1}$ by $P_{13}$, we have a substitution $\neq I$ in $K$ which leaves $\xi_{1}$ and $\eta_{1}$ fixed.

c) Let $\beta_{11} \neq 0$. We can determine a substitution $S^{\prime}$ of $K$ of form similar to that of $S$ but having also $\delta_{12} \neq 0$. In fact, if $\lambda=0$, the products $\beta_{1 j} \delta_{1 j}(j=2, \ldots, m)$ are not all zero by 214). Transforming by a suitable $P_{2 i}$, we have $\beta_{12} \delta_{12} \neq 0$. If $\lambda=\lambda^{\prime}$, the same result follows unless $\beta_{1 j}=\delta_{1 j}=0(j=2, \ldots, m-1)$, in which case either $\beta_{1 m} \neq 0$ or $\delta_{1 m} \neq 0$ by 214). In the latter case, we can take 
$\delta_{1 m} \neq 0$, transforming by $M_{2} M_{m}$ if necessary. Transforming the resulting substitution of the form

$\xi_{1}^{\prime}=\alpha \xi_{1}, \quad \eta_{1}^{\prime}=\beta_{11} \xi_{1}+\alpha^{-1} \eta_{1}+\beta_{1 m} \xi_{m}+\delta_{1 m} \eta_{m}, \ldots\left(\beta_{11} \neq 0, \delta_{1 m} \neq 0\right)$

by the substitution $R_{m, 2, \varkappa}$, we obtain a similar substitution having in $\eta_{1}^{\prime}$ the additional term $\varkappa \delta_{1 m} \xi_{2}$.

Recurring to $S^{\prime}$, in which $\delta_{12} \neq 0$, we transform it by $T_{2}, \delta_{12}^{-1}$ and obtain a substitution $S_{1}$ of $K$ having the form

$$
\xi_{1}^{\prime}=\alpha \xi_{1}, \quad \eta_{1}^{\prime}=\beta_{11} \xi_{1}+\alpha^{-1} \eta_{1}+\beta_{12} \xi_{2}+\eta_{2}+\sum_{j=3}^{m}\left(\beta_{1 j} \xi_{j}+\delta_{1 j} \eta_{j}\right), \ldots
$$

Consider the following product, leaving $\xi_{1}, \eta_{1}$, $\xi_{2}$ fixed,

$$
W \equiv Q_{3,2, \delta_{13}} R_{3,2, \beta_{13}} \ldots Q_{m, 2, \delta_{1 m}} R_{m, 2, \beta_{1 m}} .
$$

It replaces $\eta_{2}$ by the function

$$
\eta_{2}+\left(\beta_{13} \delta_{13}+\cdots+\beta_{1 m} \delta_{1 m}+\lambda \beta_{1 m}^{2}+\lambda \delta_{1 m}^{2}\right) \xi_{2}+\sum_{j=3}^{m}\left(\beta_{1 j} \xi_{j}+\delta_{1 j} \eta_{j}\right),
$$

in which the coefficient of $\xi_{2}$ equals $\beta_{11} \alpha^{-1}+\beta_{12}$ by 214), since $\delta_{12}=1$ and $\delta_{11}=\alpha^{-1}$. Hence $W$ transforms $S_{1}$ into the substitution $S_{2}$ :

$$
\xi_{1}^{\prime}=\alpha \xi_{1}, \quad \eta_{1}^{\prime}=\beta_{11} \xi_{1}+\alpha^{-1} \eta_{1}+\eta_{2}+\beta_{11} \alpha^{-1} \xi_{2}, \ldots
$$

Let $\mu \equiv \beta_{11} \alpha^{-1} \neq 0$. If among the substitutions $Q_{3,2, \mu} N_{2,3,1}$, $T_{2, \mu} M_{2} M_{3}$, etc., of $J_{\lambda}$, leaving $\xi_{1}, \eta_{1}$ and $\mu \xi_{2}+\eta_{2}$ invariant, there exists one, say $V$, which is not commutative with $S_{2}$, then $K$ contains

$$
S_{2}^{-1} \cdot V^{-1} S_{2} V \neq I
$$

which leaves $\xi_{1}$ and $\eta_{1}$ fixed. In the contrary case, we find, on equating the functions by which $S_{2} Q_{3,2, \mu} N_{2,3,1}$ and $Q_{3,2, \mu} N_{2,3,1} S_{2}$ replace $\xi_{2}$, that

$$
\eta_{3}^{\prime}=\left(\alpha_{22}+\mu \alpha_{23}+\mu \gamma_{22}\right) \eta_{3}+\alpha_{23} \eta_{2}+\mu \alpha_{23} \xi_{2} .
$$

By one of the relations 194), we find $\alpha_{23}=0$. Then, if $S_{2}$ be also commutative with $T_{2, \mu} M_{2} M_{3}$, we must have $\xi_{3}^{\prime}=\xi_{3}, \eta_{3}^{\prime}=\eta_{3}$.

In proving that $K$ contains a substitution $S \neq I$ which leaves $\xi_{1}$ and $\eta_{1}$ fixed, we assumed the existence of the indices

$$
\xi_{i}, \quad \eta_{i} \quad(i=1,2,3, m)
$$

only. But, by the relations 78) and 194) $S$ is a hypoabelian substitution on the indices $\xi_{i}, \eta_{i}(i=2, \ldots, m)$. Hence, if $m>4$, a repetition of the previous argument shows that $K$ contains a substitution $\neq I$ involving only the indices $\xi_{i}, \eta_{i}(i=3, \ldots, m)$. After $m-3$ such steps, we reach in $K$ a substitution $\neq I$ and affecting only six indices $\xi_{i}, \eta_{i}(i=m-2, m-1, m)$. In view of the simplicity of the senary hypoabelian groups, $K$ will contain all the sub- 
stitutions of $J_{2}$ will affect only the last six indices, and, in particular, $M_{i} M_{j}, N_{i, j, x}(i, j=m-2, m-1, m)$. Transforming the latter by suitable substitutions $P_{r s}\left(r, s<m\right.$, if $\left.\lambda=\lambda^{\prime}\right)$, we reach all the generators of $J_{\lambda}$. Hence $K \equiv J_{\lambda}$, so that $J_{\lambda}$ is a simple group.

In view of the importance of the subgroups $J_{0}$ and $J_{\lambda^{\prime}}$ of the first and second hypoabelian groups respectively, they will be designated by the more explicit notation $F H\left(2 m, 2^{n}\right)$ and $S H\left(2 m, 2^{n}\right)$. They are both simple when $m>3$. The second is simple and the first is composite for $m=2$ ( $\$ \S 196-198)$.

\section{MISCELLANEOUS EXERCISES UPON CHAPTERS I-VIII.}

1. Every $m$-ary linear homogeneous substitution in the $G F$ [2] leaves invariant the function $s_{1}+s_{2}+\cdots+s_{m}$, where $s_{r}$ denotes the sum of the products of the $m$ indices taken $r$ at a time.

2. An $m$-ary linear homogeneous substitution in the $G F\left[p^{n}\right]$ of determinant $D$ multiplies by $D$ the function of the indices

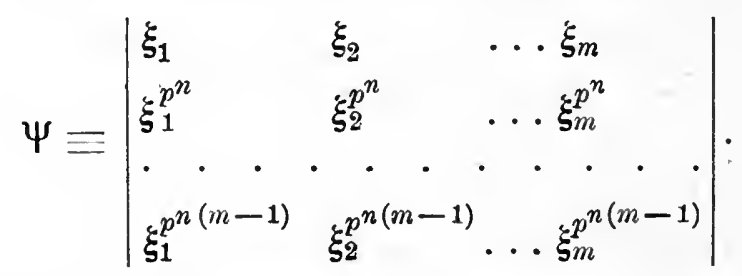

Hence $\Psi$ is a relative invariant of the group $G L H\left(m, p^{n}\right)$.

3 . The structure of the $m$-ary linear homogeneous group in the $G F\left[2^{n}\right]$ which leaves $\xi_{1}^{2}+\xi_{2}^{2}+\cdots+\xi_{m}^{2}$ absolutely invariant may be derived from that of the special linear group $S L H\left(m-1,2^{n}\right)$.

[Take as new indices $X \equiv \xi_{1}+\xi_{2}+\cdots+\xi_{m}$ and $\xi_{2}, \xi_{3}, \ldots, \xi_{m}$ ].

4. Those substitutions of the hyperorthogonal group $G_{m, 2, n}(\S 143)$ whose coefficients all belong to the $G F\left[2^{n}\right]$ form a group $G$, a subgroup of the group of Ex. 3. Prove that $G$ is generated by the binary substitutions

$$
\xi_{i}^{\prime}=\alpha \xi_{i}+(\alpha+1) \xi_{j}, \quad \xi_{j}^{\prime}=(\alpha+1) \xi_{i}+\alpha \xi_{j},
$$

and that $G$ is a solvable group of order $2^{n m(m-1) / 2}$.

5 . Consider the group $C$ of $2 m$-ary substitutions in the $G F\left[p^{n}\right], p>2$,

$$
S: \quad \xi_{i}^{\prime}=\sum_{j=1}^{m}\left(\alpha_{i j} \xi_{j}+\gamma_{i j} \eta_{j}\right), \quad \eta_{i}^{\prime}=\sum_{j=1}^{m}\left(\beta_{i j} \xi_{j}+\delta_{i j} \eta_{j}\right) \quad(i=1, \ldots, m)
$$

common to the special Abelian and orthogonal groups. Being Abelian its reciprocal is obtained by replacing $\alpha_{i j}, \gamma_{i j}, \beta_{i j}, \delta_{i j}$ by $\delta_{j i},-\gamma_{j i}$, $-\beta_{j i}, \alpha_{j i}$ respectively. Being orthogonal, its reciprocal is obtained by replacing the former by $\alpha_{j i}, \beta_{j i}, \gamma_{j i}, \delta_{j i}$. Hence must

c)

$$
\alpha_{j i}=\delta_{j i}, \quad \beta_{j i}=-\gamma_{j i}
$$$$
(i, j=1, \ldots, m) \text {. }
$$ 
The conditions that an arbitrary substitution $S$, for which c) hold, shall be orthogonal are the same as the conditions that it shall be a special Abelian substitution.

6. The most general $2 m$-ary substitution commutative with the special Abelian substitution $M \equiv M_{1} M_{2} \ldots M_{m}$ has the form

$$
S: \quad \xi_{i}^{\prime}=\sum_{j=1}^{m}\left(\alpha_{i j} \xi_{j}+\gamma_{i j} \eta_{j}\right), \quad \eta_{i}^{\prime}=\sum_{j=1}^{m}\left(-\gamma_{i j} \xi_{j}+\alpha_{i j} \eta_{j}\right) \quad(i=1, \ldots, m) .
$$

The group in the $G F\left[p^{n}\right], p>2$, commutative with $M$ is identical with $C$ of Ex. 5. becomes

7. Setting $-1=I^{2}, \quad X_{i} \equiv \xi_{i}+I \eta_{i}, A_{i j} \equiv \alpha_{i j}-I \gamma_{i j}, S$ of Ex. 6

$$
\Sigma: \quad X_{i}^{\prime}=\sum_{j=1}^{m} A_{i j} X_{j} \quad(i=1, \ldots, m) .
$$

If -1 be a not-square in the $G F\left[p^{n}\right]$, we may pass, inversely, from an arbitrary substitution $\Sigma$ in the $G F\left[p^{2 n}\right]$ to a substitution $S$ in the $G F\left[p^{n}\right]$ by equating the coefficients of $I^{0}$ and $I . \quad \Sigma$ leaves invariant the function

$$
\sum_{i=1}^{m} X_{i}^{p^{n}+1}=\sum_{i=1}^{m}\left(\xi_{i}+I \eta_{i}\right)\left(\xi_{i}-I \eta_{i}\right)=\sum_{i=1}^{m}\left(\xi_{i}^{2}+\eta_{i}^{2}\right)
$$

Hence, if $p^{n}$ be of the form $4 l+1$, the group $C$ is simply isomorphic with the hyperorthogonal group $G_{m, p, n}$. If -1 be a square in the $G F\left[p^{n}\right]$, we introduce the further indices $Y_{i} \equiv \xi_{i}-I \eta_{i}, B_{i j} \equiv \alpha_{i j}+I \gamma_{i j}$, when $S$ becomes

$$
\Sigma_{1}: \quad X_{i}^{\prime}=\sum_{j=1}^{m} A_{i j} X_{j}, \quad Y_{i}^{\prime}=\sum_{j=1}^{m} B_{i j} Y_{j} \quad(i=1, \ldots, m)
$$

leaving invariant $\sum_{i=1}^{m} X_{i} Y_{i}$. Inversely, from every substitution $\Sigma_{1}$ we derive a substitution of the form $S$. The group of "dualistic" substitutions is simply isomorphic with $G L H\left(m, p^{n}\right)$, since the $B_{i j}$ are determined in terms of the $A$ 's.

8. The simple group $A\left(4, p^{n}\right), p>2$, of order

$$
\frac{1}{2}\left(p^{4 n}-1\right)\left(p^{2 n}-1\right) p^{4 n}
$$

contains just two sets of conjugate substitutions of period 2. The one set contains $\frac{1}{2}\left(p^{2 n}+1\right) p^{2 n}$ substitutions conjugate with $T_{1,-1}$. Those of the other set are conjugate with $M_{1} M_{2}$ and are in number $\frac{1}{2} p^{3 n}\left(p^{2 n}+1\right)\left(p^{n} \mp 1\right)$ according to the form $4 l \mp 1$ of $p^{n}$. 
9. The group of all quaternary linear homogeneous substitutions in the $G F\left[p^{n}\right]$ which leave absolutely invariant the functions $\xi_{1} \eta_{1}+\xi_{2} \eta_{2}$ and $\xi_{1}+\eta_{1}$ has a subgroup of index 4 holoedrically isomorphic with $L F\left(2, p^{n}\right)$.

10. The squares of the substitutions of the first orthogonal group $O_{1}\left(m, p^{n}\right)$ generate the subgroup $O_{1}^{\prime}\left(m, p^{n}\right)$ of $\S 181$.

11. To the subgroup $E_{4, p^{n}}^{\prime}$ of $E_{4, p^{n}}$ corresponds, for $p>2$, the subgroup $O_{v}^{\prime}\left(4, p^{n}\right)$ of $O_{v}\left(4, p^{n}\right)$ defined in $\S 181$.

12. In order that $\lambda_{1} \xi_{1}^{p^{s}+1}+\lambda_{2} \xi_{2}^{p^{s}+1}$ shall be capable of transformation into $\mu\left(\xi_{1}^{p^{s}}+1+\xi_{2}^{p^{s}+1}\right)$ by a binary linear substitution with coefficients in the $G F\left[p^{2 s}\right]$, it is necessary and sufficient that the ratio $\lambda_{1} / \lambda_{2}$ shall belong to the $G F\left[p^{s}\right]$.

\section{CHAPTER IX.}

\section{LINEAR GROUPS WITH CERTAIN INVARIANTS \\ OF DEGREE $q>2$.}

211. Consider the group $G_{3}$ of substitutions in an arbitrary field

$$
S\left\{\begin{array}{l}
x_{i}^{\prime}=\sum_{j=1}^{r}\left(L_{i j} x_{j}+M_{i j} y_{j}+N_{i j} z_{j}\right) \\
y_{i}^{\prime}=\sum_{j=1}^{r}\left(\lambda_{i j} x_{j}+\mu_{i j} y_{j}+\nu_{i j} z_{j}\right) \\
z_{i}^{\prime}=\sum_{j=1}^{r}\left(l_{i j} x_{j}+m_{i j} y_{j}+n_{i j} z_{j}\right)
\end{array}\right.
$$

which leave absolutely invariant the function of degree $q=3$

$$
\Phi_{3} \equiv \sum_{i=1}^{r} x_{i} y_{i} z_{i}
$$

It will be convenient to employ a symbol, analogous to a determinant,

$$
\left\{\begin{array}{lll}
A & B & C \\
\alpha & \beta & \gamma \\
a & b & c
\end{array}\right\} \equiv A \beta c+A \gamma b+B \alpha c+B \gamma a+C \alpha b+C \beta a \text {. }
$$

The conditions that $S$ shall leave $\Phi_{3}$ absolutely invariant are then

$$
\sum_{i=1}^{r} L_{i j} \lambda_{i j} l_{i j}=0, \quad \sum_{i=1}^{r} M_{i j} \mu_{i j} m_{i j}=0, \quad \sum_{i=1}^{r} N_{i j} \nu_{i j} n_{i j}=0,
$$


LINEAR GROUPS WITH CERTAIN INVARIANTS OF DEGREE $q>2.219$

$$
\begin{aligned}
& \text { 216) } \sum_{i=1}^{r}\left(L_{i j} \lambda_{i j} l_{i k}+\dot{L}_{i j} l_{i j} \lambda_{i k}+\lambda_{i j} l_{i j} L_{i k}\right)=0 \text {, } \\
& \text { 217) } \sum_{i=1}^{r}\left(L_{i j} \lambda_{i j} m_{i k}+L_{i j} l_{i j} \mu_{i k}+\lambda_{i j} l_{i j} M_{i k}\right)=0 \text {, } \\
& \text { 218) } \sum_{i=1}^{r}\left(L_{i j} \lambda_{i j} n_{i k}+L_{i j} l_{i j} \nu_{i k}+\lambda_{i j} l_{i j} N_{i k}\right)=0 \text {, } \\
& \sum_{i=1}^{r}\left\{\begin{array}{ccc}
L_{i j} & L_{i k} & L_{i t} \\
\lambda_{i j} & \lambda_{i k} & \lambda_{i t} \\
l_{i j} & l_{i k} & l_{i t}
\end{array}\right\}=0, \sum_{i=1}^{r}\left\{\begin{array}{ccc}
L_{i j} & L_{i k} & M_{i t} \\
\lambda_{i j} & \lambda_{i k} & \mu_{i t} \\
l_{i j} & l_{i k} & m_{i t}
\end{array}\right\}=0, \sum_{i=1}^{r}\left\{\begin{array}{ccc}
L_{i j} & L_{i k} & N_{i t} \\
\lambda_{i j} & \lambda_{i k} & \nu_{i t} \\
l_{i j} & l_{i k} & n_{i t}
\end{array}\right\}=0, \\
& \text { 220) } \sum_{i=1}^{r}\left\{\begin{array}{ccc}
L_{i j} & M_{i k} & N_{i t} \\
\lambda_{i j} & \mu_{i k} & \nu_{i t} \\
l_{i j} & m_{i k} & n_{i t}
\end{array}\right\}=\begin{array}{l}
1 \\
0
\end{array} \\
& \text { (if } j=k=t \text { ) } \\
& \text { (unless } j=k=t \text { ), }
\end{aligned}
$$

where, throughout, $i, j, k=1, \ldots, r$, while $k \neq j$ in 216) and 219), and $t \neq j, k$ in the first of the relations 219); together with relations derived from 216), 217), 218) and 219) upon interchanging $L, \lambda, l$ with $M, \mu, m$ or with $N, v, n$. But relation 216) must also hold for $k=j$, being then derived from the first one of set 215) upon multiplying the latter by 3 . Similarly 219) must hold for $k=j$, being then derived from 216), 217), 218). Lastly, the first of relations 219) must hold for $t=k=j$, being then derived from the first of the set 215) upon multiplying by 6 . Hence the above conditions must hold for $i, j, k=1, \ldots, r$ independently.

Let $j$ be any fixed integer $\overline{\overline{<}} r$ and consider the $3 r$ equations $216), 217), 218)$ for $k=1, \ldots, r$. Taking as unknowns the $3 r$ products

$$
L_{i j} \lambda_{i j}, \quad L_{i j} l_{i j}, \quad \lambda_{i j} l_{i j} \quad(i=1, \ldots, r),
$$

the determinant of their coefficients is seen to equal the determinant of $S$ and is therefore not zero by hypothesis. Hence the products 221) are all zero. From the analogous conditions,

$$
\begin{array}{cl}
M_{i j} \mu_{i j}=M_{i j} m_{i j}=\mu_{i j} m_{i j}=0 & (i, j=1, \ldots, r), \\
N_{i j} \nu_{i j}=N_{i j} n_{i j}=v_{i j} n_{i j}=0 & (i, j=1, \ldots, r) .
\end{array}
$$

Expanding the symbols in 219) according to the last columns and applying a similar reasoning to the resulting equations, we find

$$
L_{i j} \lambda_{i k}+L_{i k} \lambda_{i j}=L_{i j} l_{i k}+L_{i k} l_{i j}=\lambda_{i j} l_{i k}+\lambda_{i k} l_{i j}=0 .
$$

We obtain similar identities 225) and 226) between the $M, \mu, m$ and the $N, v, n$. From 220) for $j \neq k$ and the following of type 219), 
220 CHAPTER IX. LINEAR GROUPS WITH CERTAIN INVARIANTS etc.

$$
\sum_{i=1}^{r}\left\{\begin{array}{ccc}
L_{i j} M_{i k} & M_{i t} \\
\lambda_{i j} & \mu_{i k} & \mu_{i t} \\
l_{i j} & m_{i k} & m_{i t}
\end{array}\right\}=0, \quad \sum_{i=1}^{r}\left\{\begin{array}{ccc}
L_{i j} M_{i k} & L_{i t} \\
\lambda_{i j} & \mu_{i k} & \lambda_{i t} \\
l_{i j} & m_{i k} & l_{i t}
\end{array}\right\}=0
$$

each set holding for $t=1, \ldots ; r$, we derive as above

227) $L_{i j} \mu_{i k}+M_{i k} \lambda_{i j}=L_{i j} m_{i k}+M_{i k} l_{i j}=\lambda_{i j} m_{i k}+\mu_{i k} l_{i j}=0$.

By a similar process, we get, for $k \neq j$,

228) $\quad L_{i j} z_{i k}+N_{i k} \lambda_{i j}=L_{i j} n_{i k}+N_{i k} l_{i j}=\lambda_{i j} n_{i k}+\nu_{i k} l_{i j}=0$,

229) $N_{i j} \mu_{i k}+M_{i k} \nu_{i j}=N_{i j} m_{i k}+M_{i k} n_{i j}=\nu_{i j} m_{i k}+\mu_{i k} n_{i j}=0$.

212. Theorem. - The group $G_{3}$ is generated by the substitutions 230)

$$
\left(x_{i} y_{i}\right), \quad\left(x_{i} z_{i}\right), \quad P_{i j} \equiv\left(x_{i} x_{j}\right)\left(y_{i} y_{j}\right)\left(z_{i} z_{j}\right) .
$$

together with the substitutions of the type

$$
\text { 231) } \quad x_{i}^{\prime}=L_{i} x_{i}, \quad y_{i}^{\prime}=\mu_{i} y_{i}, \quad z_{i}^{\prime}=n_{i} z_{i}, \quad L_{i} \mu_{i} n_{i}=1, \quad(i=1, \ldots, r) .
$$

Let $S$ denote any given substitution of $G_{3}$. We can determine a suitable product $\Sigma$ of the substitutions 230 ) such that $\Sigma S \equiv S_{1}$ will have the coefficient $L_{11} \neq 0$. Then by 221), 224), 227), 228), we find $\lambda_{11}=l_{11}=0, \lambda_{1 k}=l_{1 k}=0, \mu_{1 k}=m_{1 k}=0, \nu_{1 k}=n_{1 k}=0 \quad\left(k_{k}=2, \ldots, r\right)$. Hence $S_{1}$ replaces $y_{1}$ and $z_{1}$ by the respective functions

$$
\mu_{11} y_{1}+\nu_{11} z_{1}, \quad m_{11} y_{1}+n_{11} z_{1} \text {. }
$$

The product $\Sigma_{1} S_{1} \equiv S_{2}$, where $\Sigma_{1}$ is the identity if $\mu_{11} \neq 0$ but $\Sigma_{1} \equiv\left(y_{1} z_{1}\right)$ if $\mu_{11}=0$, will be of the form $S_{1}$ with the new coefficient $\mu_{11} \neq 0$. Then by 222), 225), 227) and 229), we find

$$
M_{11}=m_{11}=0, \quad M_{1 k}=0, \quad L_{1 j}=0, \quad N_{1 j}=0 \quad\left(j, l_{i}=2, \ldots, r\right) .
$$

Hence must $n_{11} \neq 0$ and therefore $N_{11}=\nu_{11}=0$ by 223). Hence $S_{2}$ replaces $x_{1}, y_{1}, z_{1}$ by $L_{11} x_{1}, \mu_{11} y_{1}, n_{11} z_{1}$. respectively. Also

$$
S=\Sigma^{-1} \Sigma_{1}^{-1} S_{2} \text {. }
$$

Since the determinant of $S_{2}$ is not zero, the coefficients $L_{2 j}$, $M_{2 j}, N_{2 j}(j=2, \ldots, r)$ are not all zero. We may therefore determine a suitable product $\Sigma^{\prime}$ of the substitutions 230 ), in which $i, j>1$, such that $\Sigma^{\prime} S_{2} \equiv S_{3}$ will have $L_{22} \neq 0$. Proceeding as above, we find that $S=\Sigma^{\prime \prime} S_{4}$, where $\Sigma^{\prime \prime}$ is derived from the substitutions 230), while $S_{4}$ merely multiplies $x_{1}, y_{1}, z_{1}, x_{2}, y_{2}, z_{2}$ by constants. After $r$ such steps, we reach a substitution of the form 231).

Corollary. - Any substitution leaving $\Phi_{3}$ invariant may be expressed as a product $A B$, where $A$ is of the form 231) and $B$ is derived from the substitutions 230). 
213. The preceding methods may be employed ${ }^{1}$ ) to investigate the group $G_{q}$ of linear substitutions $S$ on $r q$ indices with coefficients in an arbitrary field which leave absolutely invariant the function

$$
\Phi_{q} \equiv \sum_{i=1}^{r} \xi_{i 1} \xi_{i 2} \ldots \xi_{i q} .
$$

For $q>2$, it is seen that $S=A B$, where $A$ merely multiplies each index $\xi_{i j}$ by a constant, while $B$ is a permutation on the indices $\xi_{i j}$ having the imprimitive systems ${ }^{2}$ )

232) $\xi_{11}, \xi_{12}, \ldots, \xi_{1 q} ; \quad \xi_{21}, \xi_{22}, \ldots, \xi_{2 q} ; \ldots ; \quad \xi_{r 1}, \xi_{r 2}, \ldots, \xi_{r q}$.

The substitutions $A$ form a commutative group which is transformed into itself by every substitution $B$ and is therefore self-conjugate under $G_{q}$. The quotient-group is the group of the substitutions $B$. The latter has a self-conjugate subgroup $R$ formed by the direct product of $r$ symmetric groups, the general one being on the $q$ letters $\xi_{i 1}$, $\xi_{i 2}, \ldots, \xi_{i q}$; the quotient-group $\{L\} / R$ is a symmetric group on $r$ letters, viz., the $r$ sets 232). The structure of the group $G_{q}, q>2$, is therefore completely determined. The result is essentially different from that for the case $q=2$ (see $\S 195$ ).

\section{CHAPTER X.}

\section{CANONICAL FORM AND CLASSIFICATION OF LINEAR SUBSTITUTIONS.}

Canonical form of linear homogeneous substitutions ${ }^{3}$ ), $\$$ 214-216. 214. Consider a substitution with coefficients in the $G F\left[p^{n}\right]$,

$$
S: \quad \xi_{i}^{\prime}=\sum_{j=1}^{m} \alpha_{i j} \xi_{j} \quad(i=1, \ldots, m) .
$$

In order that $S$ shall multiply by a constant $K$ the linear function

we must have

$$
\eta \equiv \sum_{i=1}^{m} \lambda_{i} \xi_{i}
$$


Hence $K$ must be a root of the characteristic equation

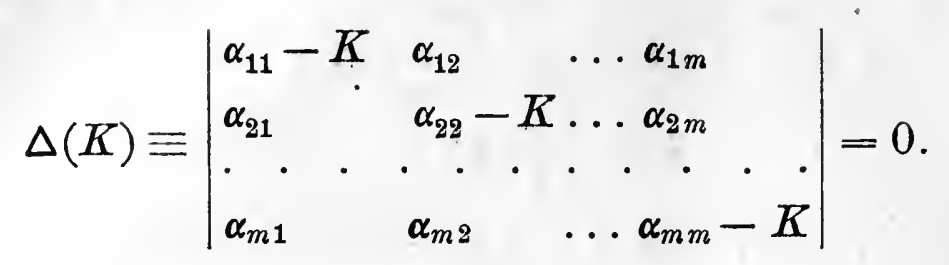

Corresponding to each root $K$, we may determine at least one set of solutions $\lambda_{i}$ of the above linear equations and hence one invariant function $\eta$.

If $\Delta(K)=0$ has $m$ distinct roots $K_{1}, K_{2}, \ldots, K_{m}$ (not necessarily in the initial $G F\left[p^{n}\right]$, we reach $m$ linear functions $\eta_{1}, \eta_{2}, \ldots, \eta_{m}$, which $S$ multiplies by) $K_{1}, K_{2}, \ldots, K_{m}$ respectively. These functions are linearly independent with respect to the variables $\xi_{i}$. For, if constants $\mu_{i}$ exist such that

$$
\mu_{1} \eta_{1}+\mu_{2} \eta_{2}+\cdots+\mu_{m} \eta_{m} \equiv 0,
$$

we have on applying the substitutions $S, S^{2}, \ldots, S^{m-1}$ the further identities

$$
\begin{aligned}
& K_{1} \mu_{1} \eta_{1}+K_{2} \mu_{2} \eta_{2}+\cdots+K_{m} \mu_{m} \eta_{m} \equiv 0, \\
& K_{1}^{2} \mu_{1} \eta_{1}+K_{2}^{2} \mu_{2} \eta_{2}+\cdots+K_{m}^{2} \mu_{m} \eta_{m} \equiv 0 \text {, } \\
& K_{1}^{m-1} \mu_{1} \eta_{1}+K_{2}^{m-1} \mu_{2} \eta_{2}+\cdots+K_{m}^{m-1} \mu_{m} \eta_{m} \equiv 0 .
\end{aligned}
$$

But the determinant

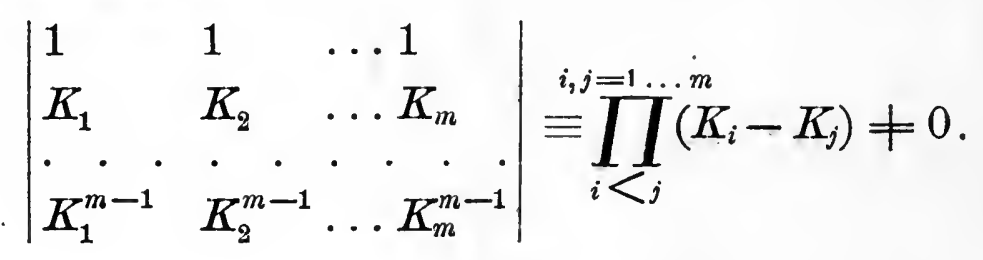

Hence

$$
\mu_{1}=\mu_{2}=\cdots=\mu_{m}=0 .
$$

Introducing the linear functions $\eta_{i}$ as new indices in place of the $\xi_{i}$, the substitution $S$ takes the canonical form

$$
S^{\prime}: \quad \eta_{i}^{\prime}=K_{i} \eta_{i} \quad(i=1, \ldots, m) .
$$

If we take in place of $\eta_{1}$ a suitable multiple of $\eta_{1}$, we may suppose the reduction of $S$ to $S^{\prime}$ to be accomplished by a transformation of indices of determinant unity.

Suppose, however, that the roots of $\Delta(K)=0$ are not all distinct. Let 


$$
\Delta(K) \equiv\left[F_{k}(K)\right]^{\alpha}\left[F_{l}(K)\right]^{\beta} \ldots(k \alpha+l \beta+\cdots \equiv m)
$$

where $F_{k}(K), F_{l}(K), \ldots$ are the distinct factors of $\Delta(K)$ which belong to and are irreducible in the $G F\left[p^{n}\right]$. Designate the roots of $F_{k}(K)=0$, and of $F_{l}(L)=0$, etc., by the notations

$$
\begin{array}{lll}
K_{0}, & K_{1} \equiv K_{0}^{p^{n}}, & K_{2} \equiv K_{0}^{p^{2 n}}, \ldots, K_{k-1} \equiv K_{0}^{p^{n(k-1)}} ; \\
L_{0}, & L_{1} \equiv L_{0}^{p^{n}}, \quad L_{2} \equiv L_{0}^{p^{2 n}}, \ldots, L_{l-1} \equiv L_{0}^{p^{n(l-1)}} ; \ldots
\end{array}
$$

Theorem. - By a suitable transformation of indices, $S$ can be reduced to a canonical form of the following type:

$$
\begin{aligned}
& \eta_{i 1}^{\prime}=K_{i} \eta_{i 1} \quad, \quad \eta_{i j}^{\prime}=K_{i}\left(\eta_{i j}+\eta_{i j-1}\right) \\
& \eta_{i a_{1}+1}^{\prime}=K_{i} \eta_{i a_{2}+1} \quad, \quad \eta_{i a_{1}+j}^{\prime}=K_{i}\left(\eta_{i a_{1}+j}+\eta_{i a_{1}+j-1}\right) \quad\left(j=2, \ldots, a_{2}\right) \\
& \eta_{i a_{2}+a_{1}+1}^{\prime}=K_{i} \eta_{i a_{2}+a_{1}+1}, \quad \eta_{i a_{2}+a_{1}+j}^{\prime}=K_{i}\left(\eta_{i a_{2}+a_{1}+j}+\eta_{i \alpha_{2}+a_{1}+j-1}\right)\left(j=2, \ldots, a_{3}\right) \\
& \xi_{i 1}=L_{i} \xi_{i 1} \quad, \quad \xi_{i j}=L_{i}\left(\xi_{i j}+\xi_{i j-1}\right) \\
& \xi_{i b_{1}+1}^{\prime}=L_{i} \xi_{i b_{1}+1} \quad, \quad \xi_{i b_{1}+j}^{\prime}=L_{i}\left(\xi_{i b_{1}+j}+\xi_{i b_{1}+j-1}\right) \\
& \left(j=2, \ldots, b_{1}\right) \\
& \left(j=2, \ldots, b_{2}\right) \\
& (i=0,1, \ldots, l-1)
\end{aligned}
$$

where $a_{1}+a_{2}+a_{3}+\cdots=\alpha, b_{1}+b_{2}+\cdots=\beta, \ldots$; and where the indices have the properties:

1) The indices $\eta_{0 s}(s=1, \ldots, \alpha)$ are linear homogeneous functions of the initial indices $\xi_{i}$ having as coefficients polynomials in $K_{0}$ with coefficients in the $G F\left[p^{n}\right]$;

2) The indices $\eta_{i s}$ are conjugate to the $\eta_{0 s}$, being obtained by replacing $K_{0}$ by $K_{i}$ in the coefficients of $\eta_{0}$;

3) The indices $\xi_{0 s}(s=1, \ldots, \beta)$ are linear homogeneous functions of the indices $\xi_{i}$ whose coefficients are polynomials in $L_{0}$ with coefficients in the $G F\left[p^{n}\right]$;

4) The indices $\xi_{\text {is }}$ are obtained from the $\xi_{0 \text { s }}$ by replacing $L_{0}$ by $L_{i}$; etc.

5) The $k \alpha$ indices $\eta_{i s}(i=0,1, \ldots, k-1 ; s=1, \ldots, \alpha)$ may be replaced by $k$ a linear homogeneous functions $y_{i s}$ of the initial indices $\xi_{i}$ with coefficients in the $G F\left[p^{n}\right]$, such that $S$ replaces each $y_{i s}$ by a linear homogeneous function of the $y_{i s}$ with coefficients in the $G F\left[p^{n}\right]$;

6) The $l \beta$ indices $\xi_{i s}$ may be replaced by an equal number of linear homogeneous functions $z_{i s}$ of the $\xi_{i}$ with coefficients in the $G F\left[p^{n}\right]$, such that $S$ replaces each by a linear homogeneous function of the $z_{i s}$ with coefficients in the field; etc. 
For the case $\alpha=\beta=\cdots=1$, we obtained above the canonical form

$$
\begin{array}{ll}
\eta_{i 1}^{\prime}=K_{i} \eta_{i 1} & (i=0,1, \ldots, k-1) \\
\xi_{i 1}^{\prime}=L_{i} \xi_{i 1} & (i=0,1, \ldots, l-1)
\end{array}
$$

where $\eta_{01}=f\left(\xi_{1}, \ldots, \xi_{m} ; K_{0}\right)$ and $\eta_{i 1}=f\left(\xi_{1}, \ldots, \xi_{m} ; K_{i}\right)$, and, similarly, $\xi_{i 1}$ are conjugate with $\xi_{01}$. The new indices therefore have the properties 1)-4).

We will prove the general theorem by induction, supposing it true for every substitution belonging to the $G F\left[p^{n}\right]$ whose characteristic determinant has no irreducible factors other than $F_{k}(K)$, $F_{l}(K), \ldots$, and has these to a degree at most $\alpha-1, \beta, \ldots$ respectively. We will prove that the theorem is true for any substitution $S$ for which these factors occur to the degree $\alpha, \beta, \ldots$ respectively, where $\alpha>1$.

Corresponding to the distinct roots $K_{0}, K_{1}, \ldots, K_{k-1}$ of $F_{k}(K)=0$, we obtain as above a set of linearly independent conjugate functions $\lambda_{0}, \lambda_{1}, \ldots, \lambda_{k-1}$ which $S$ multiplies by $K_{0}, K_{0}, \ldots, K_{k-1}$ respectively. We may introduce these in place of an equal number of the original indices, e. g., $\xi_{m-k+1}, \ldots, \xi_{m}$. The substitution $S$ then takes the form

$$
S^{\prime} \begin{cases}\lambda_{i}^{\prime}=K_{i} \lambda_{i} & (i=0,1, \ldots, k-1) . \\ \xi_{i}^{\prime}=\sum_{j=1}^{m} \beta_{i j} \xi_{j}+\sum_{j=0}^{k-1} \gamma_{i j} \lambda_{j} & (i=1,2, \ldots, m-k) .\end{cases}
$$

The coefficients $\beta_{i j}$ belong to the $G F\left[p^{n}\right]$. Indeed, we may set

$$
\begin{gathered}
\lambda_{i} \equiv X_{0}+K_{i} X_{1}+K_{i}^{2} X_{2}+\cdots+K_{i}^{k-1} X_{k-1}, \\
(i=0,1, \ldots, k-1)
\end{gathered}
$$

where the $X_{i}$ are linear functions of the $\xi_{i}$ with coefficients in the $G F\left[p^{n}\right]$. Since the $\lambda_{i}$ are linearly independent, the $X_{i}$ must be linearly independent functions of the $\xi_{i}$. Since

$$
\left|K_{i}^{j}\right| \equiv \prod_{r<s}^{0, \ldots, k-1}\left(K_{r}-K_{s}\right) \neq 0
$$

the $X_{j}$ can be expressed as linear functions of the $\lambda_{i}$. Taking the $X_{j}$ as new indices in place of the $\lambda_{i}, S^{\prime}$ takes the form $S^{\prime \prime}$, a substitution on the indices $X_{j}$ and $\xi_{i}$ with coefficients in the $G F\left[p^{n}\right]$. But $S^{\prime \prime}$ replaces $\xi_{i}$ by

$$
\sum_{j=1}^{m-k} \beta_{i j} \xi_{j}+\sum_{j=0}^{k-1} \delta_{i j} X_{j}
$$

for $i=1, \ldots, m-k$. Since these functions belong to the field for arbitrary $\xi_{j}$ and $X_{j}$, the coefficients $\beta_{i j}, \delta_{i j}$ must belong to the field. 
Since the determinant of a linear substitution is not altered by a linear transformation of indices ( $\S 101$ ), the determinant of $S^{\prime}$ equals the determinant of $S$ :

$$
K_{0} K_{1} \ldots K_{k-1} \cdot\left|\beta_{i j}\right|=D \text {. }
$$

We may, therefore, consider the following substitution in the $G F\left[p^{n}\right]$ :

$$
S_{1}: \quad \xi_{i}^{\prime}=\sum_{j=1}^{m-k} \beta_{i j} \xi_{j} \quad(i=1, \ldots, m-k)
$$

of determinant $\neq 0$. Also, the characteristic determinant $\Delta(K)$ of $S$ equals that of the transformed substitution $S^{\prime}$, viz.:

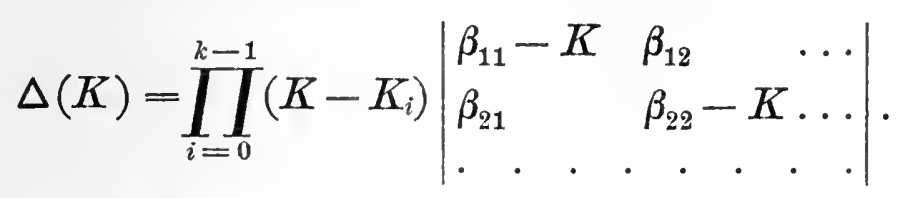

Hence, the characteristic determinant of $S_{1}$ is

$$
\frac{\Delta(K)}{F_{k}(K)} \equiv\left[F_{k}(K)^{\alpha-1}\left[F_{l}(K)\right]^{\beta} \ldots\right.
$$

Hence, by hypothesis, $S_{1}$ can be reduced to a canonical form of the above type. Applying the same transformation of indices to $S^{\prime}$, it takes the form $\bar{S}$ :

$$
\begin{aligned}
& \lambda_{i}=K_{i} \lambda_{i} \\
& \eta_{01}^{\prime}=K_{0} \eta_{01}+\sum_{i=0}^{k-1} \alpha_{1 i} \lambda_{i}, \eta_{0 j}^{\prime}=K_{0}\left(\eta_{0 j}+\eta_{0 j-1}\right)+\sum_{i=0}^{k-1} \alpha_{j i} \lambda_{i} \quad\left(j=2, \ldots, a_{1}\right) \\
& \eta_{0 a_{1}+1}^{\prime}=K_{0} \eta_{0 a_{1}+1}+\Sigma \beta_{1 i} \lambda_{i}, \eta_{0 \alpha_{1}+j}^{\prime}=K_{0}\left(\eta_{0 a_{1}+j}+\eta_{0 a_{1}+j-1}\right)+\Sigma \beta_{j i} \lambda_{i}\left(j=2, \ldots, a_{2}\right) \\
& \xi_{01}^{\prime}=L_{0} \xi_{01}+\Sigma \alpha_{1 i}^{\prime} \lambda_{i}, \quad \xi_{0 j}^{\prime}=L_{0}\left(\xi_{0 j}+\xi_{0 j-1}\right)+\Sigma \alpha_{j i}^{\prime} \lambda_{i} \quad\left(j=2, \ldots, b_{1}\right)
\end{aligned}
$$

the expression for $\eta_{i s}^{\prime}$ being derived from that for $\eta_{0_{s}}^{\prime}$ by replacing $K_{0}$ by $K_{i}$; the expression for $\xi_{i s}^{\prime}$ from $\xi_{0 s}$ upon replacing $L_{0}$ by $L_{i}$, etc.

To simplify the form of $\bar{S}$, introduce as new indices

$$
\begin{aligned}
Y_{0 s} \equiv \eta_{0 s}+\sum_{i=0}^{k-1} A_{s i} \lambda_{i} & (s=1, \ldots, \alpha), \\
Z_{0 s} \equiv \xi_{0 s}+\sum_{i=0}^{k-1} B_{s i} \lambda_{i} & (s=1, \ldots, \beta), \ldots,
\end{aligned}
$$

and their conjugate functions $Y_{i s}, Z_{i s}, \ldots$ Then $\bar{S}$ replaces $Y_{01}$. $Y_{02}, Y_{03}$ by 


$$
\begin{aligned}
& K_{0} Y_{01}+\alpha_{10} \lambda_{0}+\sum_{i=1}^{k-1}\left[\alpha_{1 i}+\left(K_{i}-K_{0}\right) A_{1 i}\right] \lambda_{i}, \\
& K_{0}\left(Y_{02}+Y_{01}\right)+\sum_{i=0}^{k-1}\left[\alpha_{2 i}-K_{0} A_{1 i}+\left(K_{i}-K_{0}\right) A_{2 i}\right] \lambda_{i}, \\
& K_{0}\left(Y_{03}+Y_{02}\right)+\sum_{i=0}^{k-1}\left[\alpha_{3 i}-K_{0} A_{2 i}+\left(K_{i}-K_{0}\right) A_{3 i}\right] \lambda_{i}
\end{aligned}
$$

respectively. By choice of the $A_{j i}$, we can make the terms in brackets all zero; those of the first sum by choice of $A_{11}, \ldots, A_{1 k-1}$, those of the second by choice of $A_{10}, A_{21}, \ldots, A_{2 k-1}$, those of the third by choice of $A_{20}, A_{31}, \ldots, A_{3 k-1}$. A like result holds for the remaining $Y_{0 s}(s=1, \ldots, \alpha)$.

$\bar{S}$ replaces $Z_{01}, Z_{02}, \ldots$ by respectively

$$
\begin{gathered}
L_{0} Z_{01}+\sum_{i=0}^{k-1}\left[\alpha_{1 i}^{\prime}+\left(K_{i}-L_{0}\right) B_{1 i}\right] \lambda_{i}, \\
L_{0}\left(Z_{02}+Z_{01}\right)+\sum_{i=0}^{k-1}\left[\alpha_{2 i}^{\prime}-L_{0} B_{1 i}+\left(K_{i}-L_{0}\right) B_{2 i}\right) \lambda_{i}, \ldots
\end{gathered}
$$

Since $K_{i}-L_{0} \neq 0$, the coefficients of $\lambda_{i}$ may be made to vanish by choice of the $B_{1 i}$. Hence, $\bar{S}$ takes the form $S_{2}$ :

$$
\begin{aligned}
& \lambda_{i}^{\prime}=K_{i} \lambda_{i}, \\
& Y_{i 1}^{\prime}=K_{i} Y_{i 1}+\varphi\left(K_{i}\right) \cdot \lambda_{i}, \quad Y_{i j}^{\prime}=K_{i}\left(Y_{i j}+Y_{i j-1}\right)\left(j=2, \ldots, a_{1}\right) \\
& Y_{i a_{1}+1}^{\prime}=K_{i} Y_{i a_{1}+1}+\Psi\left(K_{i}\right) \cdot \lambda_{i}, \quad Y_{i a_{1}+j}^{\prime}=K_{i}\left(Y_{i a_{1}+j}+Y_{i a_{1}+j-1}\right) \\
& \left(j=2, \ldots, a_{2}\right) \\
& Z_{i 1}^{\prime}=L_{i} Z_{i 1} \\
& Z_{i b_{1}+1}^{\prime}=L_{i} Z_{i b_{1}+1} \\
& (i=0,1, \ldots, k-1) \\
& \begin{aligned}
Z_{i j}^{\prime} & =L_{i}\left(Z_{i j}+Z_{i j-1}\right) \quad\left(j=2, \ldots, b_{1}\right) \\
Z_{i b_{1}+j}^{\prime} & =L_{i}\left(Z_{i b_{1}+j}+Z_{i b_{1}+j-1}\right)
\end{aligned} \\
& \left(j=2, \ldots, b_{2}\right) \\
& (i=0,1, \ldots, l-1)
\end{aligned}
$$

If the constants $\varphi\left(K_{i}\right), \psi\left(K_{i}\right), \chi\left(K_{i}\right), \ldots$ are all zero, no further reduction is necessary. If any two are not zero, as $\varphi$ and $\psi$, suppose for definiteness that $a_{1} \overline{\overline{<}} a_{2}$, and introduce in place of $Y_{i 1}, \ldots, Y_{i a_{1}}$ the new indices

$$
\bar{Y}_{i j} \equiv Y_{i j}-\frac{\varphi}{\psi} Y_{i a_{1}+j} . \quad\left(j=1, \ldots, a_{1}\right)
$$


The substitution $S_{2}$ replaces $\bar{Y}_{i 1}, \bar{Y}_{i j}\left(j=2, \ldots, a_{1}\right)$ by respectively

$$
K_{i} \bar{Y}_{i 1}, \quad K_{i}\left(\bar{Y}_{i j}+\bar{Y}_{i j-1}\right) \quad\left(j=2, \ldots, a_{1}\right) .
$$

Hence, the introduction of the $\bar{Y}_{i j}$ has the effect of setting $\varphi=0$ in $S_{2}$. Proceeding similarly, we can suppose that $\varphi, \psi, \chi, \ldots$ are all zero but one, say $\psi \neq 0$. In the latter case, we set

$$
\psi\left(K_{i}\right) \cdot \lambda_{i} \equiv K_{i} y_{i a_{1}}
$$

and find for $S_{2}$ the canonical form

$$
\begin{aligned}
& Y_{i 1}^{\prime}=K_{i} Y_{i 1}, \quad Y_{i j}^{\prime}=K_{i}\left(Y_{i j}+Y_{i j-1}\right),\left(j=2, \ldots, a_{1}\right) \\
& y_{i a_{1}}^{\prime}=K_{i} y_{i a_{1}}, \quad Y_{i a_{1}+1}^{\prime}=K_{i}\left(Y_{i a_{1}+1}+y_{i a_{1}}\right) \text {, } \\
& Y_{i a_{1}+j}^{\prime}=K_{i}\left(Y_{i a_{1}+j}+Y_{i a_{1}+j-1}\right) \text {, } \\
& \left(j=2, \ldots, a_{2}\right) \\
& Y_{i a_{2}+a_{2}+1}^{\prime}=K_{i} Y_{i a_{2}+a_{2}+1}, \quad Y_{i a_{1}+a_{2}+j}^{\prime}=K_{i}\left(Y_{i a_{1}+a_{2}+j}+Y_{i a_{1}+a_{2}+j-1}\right), \\
& \left(j=2, \ldots, a_{3}\right) \\
& Z_{i 1}^{\prime} \quad=L_{i} Z_{i 1}, \quad Z_{i j}^{\prime} \quad=L_{i}\left(Z_{i j}+Z_{i j-1}\right), \quad\left(j=2, \ldots, b_{1}\right)
\end{aligned}
$$

In every case we reach a canonical form of the type given in the theorem, for which the indices $Y_{i s}$ have the properties 1) and 2). But the indices $Z_{i j}$ are linear functions of the $\xi_{i}$ with coefficients which certainly involve $L_{i}$ and apparently ${ }^{1}$ ) also $K_{i}$. If the $K_{i}$ be involved, we proceed as follows. From the canonical form actually reached, $S=Y S_{1}$, where $Y$ is the partial substitution on the indices $Y_{i j}$, not altering the indices $Z_{i j}$, etc., while $S_{1}$ does not involve the indices $Y_{i j}$, but affects the $Z_{i j}$, etc. Setting

$$
\begin{aligned}
Y_{i s} \equiv y_{s}+y_{s}^{\prime} K_{i}+y_{s}^{\prime \prime} K_{i}^{2}+y_{s}^{(k-1)} K_{i}^{k-1}, & (s=1, \ldots, \alpha ; i=0, \ldots, k-1)
\end{aligned}
$$

where the $y$ 's are linear functions of the $\xi_{i}$ with coefficients in the $G F\left[p^{n}\right]$, we can evidently introduce the $y^{\prime}$ s as new indices in place of the $Y_{i s}$, so that $Y$ takes the form of a substitution belonging to the $G F\left[p^{n}\right]$ and affecting only $k \alpha$ indices. Likewise, by introducing in place of the $Z_{i j}$, etc., an equal number of linear functions $z_{i j}$, etc., belonging to the $G F\left[p^{n}\right]$, it is possible to give to $S_{1}$ the form of a substitution in the field and affecting only $m-k \alpha$ indices. Its characteristic determinant is $\left[F_{l}(K)\right]^{\beta} \ldots$ Hence, by the hypothesis made for the induction, $S_{1}$ can be reduced by a linear transformation $T$ to a canonical form

1) By the considerations in the text, we may dispense with the difficult proof, analogous to that of Jordan, Traité, pp. 121-122, that the $Z_{i j}$ do not involve $K_{i}$, but the single imaginary $L_{i}$. 


$$
\xi_{i 1}^{\prime}=L_{i} \xi_{i 1}, \quad \xi_{i j}^{\prime}=L_{i}\left(\xi_{i 1}+\xi_{i j-1}\right), \quad\left(j=2, \ldots, b_{1}\right)
$$

where the $\xi_{i j}$ are linear functions of the $\xi_{i}$ with coefficients involving the imaginary $L_{i}$ only. As the transformation $I$ does not alter the indices which $Y$ affects, we obtain the desired canonical form.

215. Consider as an example the substitution in the $G F\left[p^{n}\right], p^{n}$ of the form $4 l-1$,

$$
S: \quad \xi_{1}^{\prime}=-2 \xi_{2}-\xi_{4}, \quad \xi_{2}^{\prime}=\xi_{1}, \quad \xi_{3}^{\prime}=\xi_{2}, \quad \xi_{4}^{\prime}=\xi_{3},
$$

having the characteristic determinant

$$
\Delta(K) \equiv\left(K^{2}+1\right)^{2},
$$

where $K^{2}+1$ is irreducible in the field. A root of $i^{2}=-1$ belongs to the $G F\left[p^{2 n}\right]$ but not to the $G F\left[p^{n}\right]$. The functions which $S$ multiplies by $i$ and $-i$ are readily found to be respectively

$$
\lambda_{1} \equiv-i x_{1}+x_{2}-i x_{3}+x_{4}, \quad \lambda_{2} \equiv i x_{1}+x_{2}+i x_{3}+x_{4} .
$$

Introducing $\lambda_{1}, \lambda_{2}$ in place of the indices $x_{2}, x_{3}, S$ takes the form $x_{1}^{\prime}=x_{4}-\lambda_{1}-\lambda_{2}, \quad x_{4}^{\prime}=-x_{1}+i / 2 \lambda_{1}-i / 2 \lambda_{2}, \quad \lambda_{1}^{\prime}=i \lambda_{1}, \quad \lambda_{2}^{\prime}=-i \lambda_{2}$. The partial substitution of determinant unity,

$$
x_{1}^{\prime}=x_{4}, \quad x_{4}^{\prime}=-\dot{x}_{1}
$$

multiplies $y_{1} \equiv x_{1}-i x_{4}$ by $i$ and multiplies $y_{2} \equiv x_{1}+i x_{4}$ by $-i$. Introducing $y_{1}$ and $y_{2}$ as new indices in place of $x_{1}$ and $x_{4}, S$ takes the form

$$
\begin{aligned}
& y_{1}^{\prime}=i y_{1}-\frac{1}{2} \lambda_{1}-\frac{3}{2} \lambda_{2}, \quad y_{2}^{\prime}=-i y_{2}-\frac{1}{2} \lambda_{2}-\frac{3}{2} \lambda_{1}, \\
& \lambda_{1}^{\prime}=i \lambda_{1}, \quad \lambda_{2}^{\prime}=-i \lambda_{2} .
\end{aligned}
$$

Introducing as new indices,

$$
\bar{\lambda}_{1} \equiv i / 2 \lambda_{1}, \quad \bar{\lambda}_{2} \equiv-i / 2 \lambda_{2}, \quad \bar{y}_{1} \equiv y_{1}+\frac{3 i}{4} \lambda_{2}, \quad \bar{y}_{2} \equiv y_{2}-\frac{3 i}{4} \lambda_{1},
$$

$S$ takes the canonical form

$$
\left.\bar{\lambda}_{1}^{\prime}=i \bar{\lambda}_{1}, \quad \bar{y}_{1}^{\prime}=i\left(\bar{y}_{1}+\bar{\lambda}_{1}\right), \quad \bar{\lambda}_{2}^{\prime}=-i \bar{\lambda}_{2}, \quad \bar{y}_{2}^{\prime}=-i \overline{(y}_{2}+\bar{\lambda}_{2}\right),
$$

where $\bar{\lambda}_{1}$ and $\bar{\lambda}_{2}$ are conjugate linear functions of $\xi_{1}, \xi_{2}, \xi_{3}, \xi_{4}$, and likewise for $\bar{y}_{1}, \bar{y}_{2}$.

216. Theorem. - Two linear homogeneous substitutions $\mathbb{S}$ and $T$ in the $G F\left[p^{n}\right]$ on the indices $\xi_{1}, \xi_{2}, \ldots, \xi_{m}$ have the same canonical form $C$ if, and only if, $T$ is the transformed of $S$ by a linear homogeneous substitution $W$ in the $G F\left[p^{n}\right]$ on the same indices.

If $T=W^{-1} S W$, then $S$ can be reduced to $T$ by the introduction of new indices defined by the transformation $W$ and therefore $S$ and $T$ have the same canonical form. 
Suppose, inversely, that two substitutions $S$ and $T$ in the $G F\left[p^{n}\right]$ on the indices $\xi_{i}$ can be reduced to the same canonical form by the respective transformations $S^{\prime}$ and $T^{\prime}$. Let $I^{\prime}$ denote the transformation from the indices $\xi_{1}, \ldots, \xi_{m}$ to the indices $\eta_{i s}, \xi_{i s}, \ldots$, where

$$
\begin{aligned}
\eta_{i s} \equiv Y_{s}+Y_{s}^{\prime} K_{i}+Y_{s}^{\prime \prime} K_{i}^{2}+\cdots+Y_{s}^{(k-1)} K_{i}^{k-1} \\
(s=1, \ldots, \alpha ; i=0,1, \ldots, k-1) \\
\xi_{i s} \equiv Z_{s}+Z_{s}^{\prime} L_{i}+Z_{s}^{\prime \prime} L_{i}^{2}+\cdots+Z_{s}^{(l-1)} L_{i}^{l-1} \\
(s=1, \ldots, \beta ; i=0,1, \ldots, l-1),
\end{aligned}
$$

$Y_{s}, Y_{s}^{\prime}, \ldots, Z_{s}, Z_{s}^{\prime}, \ldots$ being linearly independent linear functions of the $\xi_{i}$ with coefficients in the $G F\left[p^{n}\right]$. Denote by $\tau$ the transformation of indices from $\eta_{i s}, \xi_{i s}, \ldots$ to $Y_{s}, Y_{s}^{\prime}, \ldots, Z_{s}, \ldots$ By hypothesis, $I^{\prime}$ transforms $T$ into the canonical form $C$. Let $\tau$ transform $C$ into $C_{\tau}$. Then $I^{\prime} \tau$ is a substitution in the $G F\left[p^{n}\right]$ which transforms $T$ into $C_{\tau}$, likewise in the $G F\left[p^{n}\right]$. Similarly, let $S^{\prime}$ denote the transformation from the indices $\xi_{1}, \ldots, \xi_{m}$ to the indices $\bar{\eta}_{i s}, \bar{\xi}_{i s}, \ldots$, where

$$
\bar{\eta}_{i s} \equiv \bar{Y}_{s}+\bar{Y}_{s}^{\prime} K_{i}+\cdots, \quad \bar{\zeta}_{i s} \equiv \bar{Z}_{s}+\bar{Z}_{s}^{\prime} L_{i}+\cdots, \quad \cdots
$$

Denote by $\sigma$ the transformation of indices from $\bar{\eta}_{i s}, \bar{\xi}_{i s}, \ldots$ to $\bar{Y}_{s}, \ldots, \bar{Z}_{s}, \ldots$ By hypothesis, $S^{\prime}$ transforms $S$ into the canonical form $\bar{C}$, which in the same substitution on the indices $\bar{\eta}_{i s}, \bar{\xi}_{i s}, \ldots$ that $C$ is on the indices $\eta_{i s}, \xi_{i s}, \ldots$ Let $\sigma$ transform $\bar{C}$ into $\bar{C}_{\sigma \text {. }}$ Then, if $R$ be the substitution in the $G F\left[p^{n}\right]$ which transforms $Y_{s}, \ldots, Z_{s}, \ldots$ into $\bar{Y}_{s}, \ldots, \bar{Z}_{s}, \ldots$ respectively, then

$$
\overline{C_{\sigma}}=R^{-1} C_{\imath} R \text {. }
$$

It follows that the product $I^{\prime} \tau R\left(S^{\prime} \sigma\right)^{-1}$ is a substitution on the indices $\xi_{i}$ with coefficients in the $G F\left[p^{n}\right]$ which transforms $T$ into $S$.

$$
\S \S 217-220 \text {. }
$$

Substitutions commutative with a given linear substitution ${ }^{1}$ ).

21\%. Let the given linear homogeneous substitution $S$ on $m$ indices $\xi_{i}$ with coefficients in the $G F\left[p^{n}\right]$ be brought to its canonical form $S_{1}$. For definiteness, suppose there are three sets of new indices, $\begin{aligned} \eta_{i j}(i=0,1, \ldots, k-1 ; j=1, \ldots, \alpha) ; \quad & \xi_{i j}(i=0, \ldots, l-1 ; j=1, \ldots, \beta) ; \\ & \psi_{i j}(i=0, \ldots, q-1 ; j=1, \ldots, \gamma) ;\end{aligned}$

where

$\alpha \equiv a_{1}+a_{2}+\cdots+a_{r+1}, \quad \beta \equiv b_{1}+b_{2}+\cdots+b_{s+1}, \quad \gamma \equiv c_{1}+c_{2}+\cdots+c_{t+1}$.

1) Amer. Journ., vol. 22, pp. 121-137; Proceed. Lond. Math. Soc., vol. 32, pp. $165-170$. 
In order to express more compactly the canonical form $S_{1}$, we let $a, b, c$ denote an arbitrary one of the respective sets of integers
a) $1, a_{1}+1, a_{1}+a_{2}+1, \ldots, a_{1}+a_{2}+\cdots+a_{r}+1$;
b) $1, b_{1}+1, b_{1}+b_{2}+1, \ldots, b_{1}+b_{2}+\cdots+b_{s}+1$;
c) $1, c_{1}+1, c_{1}+c_{2}+1, \ldots, c_{1}+c_{2}+\cdots+c_{t}+1$.

Also let $A$ denote any integer $\overline{<} \alpha$ not an $a, B$ any integer $\overline{<} \beta$ not a $b, C$ any integer $\overline{<} \gamma$ not a $c$. The canonical form $S_{1}$ may now be written as follows:

$$
\begin{array}{lll}
\eta_{i a}^{\prime}=K_{i} \eta_{i a}, & \eta_{i A}^{\prime}=K_{i} \eta_{i A}+K_{i} \eta_{i A-1} & (i=0,1, \ldots, l-1) \\
\xi_{i b}^{\prime}=L_{i} \xi_{i b}, & \xi_{i B}^{\prime}=L_{i} \xi_{i B}+L_{i} \xi_{i B-1} & (i=0,1, \ldots, l-1) \\
\psi_{i c}^{\prime}=Q_{i} \psi_{i c}, & \psi_{i C}^{\prime}=Q_{i} \psi_{c C}+Q_{i} \psi_{i C-1} & (i=0,1, \ldots, q-1) .
\end{array}
$$

An arbitrary linear homogeneous substitution $I_{1}$ on these indices replaces $\eta_{i j}$ by a linear function

$$
\Sigma D_{\gamma_{u} u}^{i j} \eta_{x_{u}}+\Sigma E_{\lambda_{v}}^{i j} \xi_{\lambda_{v}}+\Sigma F_{\mu w}^{i j} \psi_{\mu w}
$$

where (as henceforth) the summation indices have the series of values

$$
\begin{array}{lll}
x=0,1, \ldots, k-1 ; & \lambda=0,1, \ldots, l-1 ; & \mu=0,1, \ldots, q-1 ; \\
u=1, \ldots, \alpha ; & v=1, \ldots, \beta ; & w=1, \ldots, \gamma .
\end{array}
$$

In order that $T_{1}$ be commutative with $S_{1}$ it is necessary that 233) involve only the indices $\eta_{i u}(u=1, \ldots, \alpha)$. Equating the functions by which $T_{1} S_{1}$ and $S_{1} T_{1}$ replace $\eta_{i a}$, we get

$$
\begin{aligned}
& K_{i}\left(\Sigma D_{* u}^{i a} \eta_{\psi_{u}}+\Sigma E_{\lambda_{v}}^{i a} \xi_{\lambda v}+\Sigma F_{\mu w}^{i a} \psi_{\mu w}\right) \\
& \equiv \Sigma D_{x, u}^{i a} K_{x_{1}} \eta_{\psi_{u} u}+\Sigma E_{\lambda v}^{i a} L_{\lambda} \xi_{\lambda v}+\Sigma F_{\mu w}^{i a} Q_{\mu} \psi_{\mu w} \\
& +\sum_{\varkappa, A} D_{\psi A}^{i a} K_{\varkappa} \eta_{* A-1}+\sum_{\lambda, B} E_{\lambda B}^{i a} L_{\lambda} \xi_{\lambda B-1}+\sum_{\mu, C} F_{\mu C}^{i a} Q_{\mu} \psi_{\mu C-1}
\end{aligned}
$$

Equating the coefficients of the $\eta$ 's and $\xi$ 's in this identity, we get

$$
\begin{array}{ll}
K_{i} D_{\varkappa u}^{i a}=K_{\varkappa} D_{\varkappa u}^{i a} & (\imath \neq A-1) \\
K_{i} D_{\varkappa A-1}^{i a}=K_{\varkappa} D_{\varkappa A-1}^{i a}+K_{\varkappa} D_{\varkappa A}^{i a} & \\
K_{i} E_{\lambda v}^{i a}=L_{\lambda} E_{\lambda v}^{i a} & (v \neq B-1) \\
K_{i} E_{\lambda B-1}^{i a}=L_{\lambda} E_{\lambda B-1}^{i a}+L_{\lambda} E_{\lambda B}^{i a} . &
\end{array}
$$

Since $K_{i} \neq L_{\lambda}$, the third equation gives $E_{\lambda b-1}^{i a}=0$, where $b$ is any integer $>1$ of the set $b$ ). If $b-1$ is a $B$, the fourth equation gives $E_{\lambda b-2}^{i a}=0$. In the contrary case, $b-\dot{2} \neq B-1$, and the third equation gives $E_{\lambda b-2}^{i a}=0$. Similarly, according as $b-2$ is or is not. a $B$, the fourth or third equation gives $E_{\lambda b-3}^{i a}=0$. Proceeding in this manner, we find that every $E_{\lambda_{v}}^{i a}=0(\lambda=0, \ldots, l-1 ; v=1, \ldots, \beta)$. 
By a similar argument, the first and second equations give

$$
D_{\varkappa u}^{i a}=0 \quad(x \neq i, u=1, \ldots, \alpha), \quad D_{i A}^{i a}=0 .
$$

Equating the coefficients of the $\psi$ 's in the above identity, we find analogously that every $F_{\mu w}^{i a}=0$. Hence $T_{1}$ replaces $\eta_{i a}$ by

$$
\sum_{a^{\prime}} D_{i a^{\prime}}^{i a} \eta_{i a^{\prime}} \quad\left(a^{\prime}=1, a_{1}+1, a_{1}+a_{2}+1, \ldots\right) .
$$

Consider any $a$ such that $a+1$ is an $A$ and equate the functions by which $T_{1} S_{1}$ and $S_{1} T_{1}$ replace $\eta_{i a+1}$. Among the relations occur

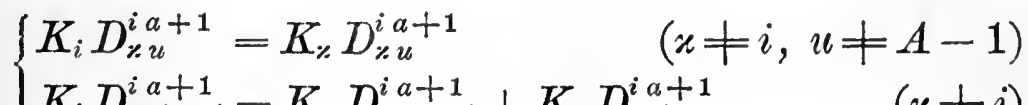

$$
\begin{aligned}
& \left\{K_{i} D_{* A-1}^{i a+1}=K_{\%} D_{\% A-1}^{i a+1}+K_{\%} D_{\% A}^{i a+1} \quad(\varkappa \neq i)\right. \\
& \left\{K_{i} E_{\lambda v}^{i a+1}=L_{\lambda} E_{\lambda v}^{i a+1} \quad(v \neq B-1)\right. \\
& \left\{K_{i} E_{\lambda B-1}^{i a+1}=L_{\lambda} E_{\lambda B-1}^{i a+1}+L_{\lambda} E_{\lambda B}^{i a+1}\right. \\
& \left\{K_{i} F_{\mu w}^{i a+1}=Q_{\mu} F_{\mu \omega}^{i a+1} \quad(w \neq C-1)\right. \\
& \left\{K_{i} F_{\mu C-1}^{i a+1}=Q_{\mu} F_{\mu C-1}^{i a+1}+Q_{\mu} F_{\mu C}^{i a+1}\right. \text {. }
\end{aligned}
$$

From these three pairs of equations we find (as above) respectively

$$
D_{\varkappa u}^{i a+1}=0(\varkappa \neq i), \quad E_{\lambda_{v}}^{i a+1}=0, \quad F_{\mu w}^{i a+1}=0 .
$$

Hence $T_{1}$. replaces $\eta_{i a+1}$ by a function of the $\eta_{i u}$ only.

Considering any $a$ such that $a+1$ and $a+2$ are of the set $A$, we find by the same method that $T_{1}$ replaces $\eta_{i a+2}$ by a function of the $\eta_{i u}$ only. We readily verify that, if $T_{1}$ replaces $\eta_{i a+d}$ by a function of the $\eta_{i u}$ only, the same will hold for $\eta_{i a+d+1}$. Since the series $a, a+1, a+2, a+3, \ldots$ yields every integer, we have proven that $T_{1}$ must replace each $\eta_{i j}$ by a function of the $\eta_{i u}$ only, if $T_{1}$ shall be commutative with $S_{1}$.

Similarly, $T_{1}$ must replace each $\xi_{i j}$ by a function of the $\xi_{i v}$ only and each $\psi_{i j}$ by a function of the $\psi_{i w}$ only.

When we return from the indices $\eta_{i j}, \xi_{i j}, \psi_{i j}$ to the initial indices $\xi_{1}, \ldots, \xi_{m}, S_{1}$ becomes, by hypothesis, a substitution $S$ having its coefficients in the $G F\left[p^{n}\right]$. Under what conditions will $T, T_{1}$ in the indices $\xi_{i}$, have its coefficients in the $G F\left[p^{n}\right]$ ? We have shown that $T_{1}$ replaces $\eta_{i j}$ by a function of the form $\sum_{u=1}^{\alpha} D_{i u}^{i j} \eta_{i u}$. Recurring to the properties 1) and 2), $\S 214$, of the indices $\eta_{i j}$, we must have as the $D_{i u}^{i j}$ certain polynomials in the quantity $K_{i}$ with coefficients in the $G F\left[p^{n}\right]$, such that

$$
D_{i u}^{i j} \equiv D_{0 u}^{0 j}\left(K_{i}\right)=\left[D_{0 u}^{0 j}\left(K_{0}\right)\right]^{p^{n i}} \equiv\left(D_{0 u}^{0 j}\right)^{p^{n i}} .
$$


Similar remarks hold for the indices $\xi_{i j}$ and $\psi_{i j}$. We may now state our results in the following form:

Theorem. - To determine the most general linear homogeneous substitution $T$ on $m$ indices with coefficients in the $G F\left[p^{n}\right]$ which shall be commutative with a particular one $S$, we apply the transformation of indices which reduces $S$ to its canonical form $S_{1}$ and $T$ to some form $T_{1}$. Then $S_{1}$ may be expressed as a product

$$
S_{1} \equiv \eta_{0} \eta_{1} \ldots \eta_{k-1} \xi_{0} \xi_{1} \ldots \xi_{l-1} \psi_{0} \psi_{1} \ldots \psi_{q-1}
$$

where each substitution $\eta_{i}, \xi_{i}, \psi_{i}$ is defined thus:

$$
\begin{array}{rlll}
\eta_{i}: & \eta_{i a}^{\prime}=K_{i} \eta_{i a}, & \eta_{i A}^{\prime}=K_{i} \eta_{i A}+K_{i} \eta_{i A-1} & (\text { for every } a, A) \\
\xi_{i}: & \xi_{i b}^{\prime}=L_{i} \xi_{i b}, \quad \xi_{i B}^{\prime}=L_{i} \xi_{i B}+L_{i} \xi_{i B-1} & (\text { for every } b, B) \\
\psi_{i}: & \psi_{i c}^{\prime}=Q_{i} \psi_{i c}, \quad \psi_{i c}^{\prime}=Q_{i} \psi_{i C}+Q_{i} \psi_{i c-1} & (\text { for every } c, C) .
\end{array}
$$

The most general $T_{1}$ must be expressible as a product

$$
I_{1} \equiv \mathrm{H}_{0} \mathrm{H}_{1} \ldots \mathrm{H}_{k-1} \mathrm{Z}_{0} \mathrm{Z}_{1} \ldots \mathrm{Z}_{l-1} \Psi_{0} \Psi_{1} \ldots \Psi_{q-1} \text {, }
$$

where the individual substitutions have the forms:

$$
\begin{array}{lll}
\mathrm{H}_{i}: & \eta_{i j}^{\prime}=\sum_{u=1}^{\alpha} \delta_{j u}^{p^{n i} \eta_{i u}} & (j=1, \ldots, \alpha) \\
Z_{i}: & \xi_{i j}^{\prime}=\sum_{v=1}^{\beta} \varrho_{j v}^{p^{n i} \xi_{i v}} & (j=1, \ldots, \beta) \\
\Psi_{i}: & \psi_{i j}^{\prime}=\sum_{w=1}^{\gamma} \sigma_{j w}^{p^{n i}} \psi_{i w} & (j=1, \ldots, \gamma),
\end{array}
$$

the coefficients $\delta_{j u}, \varrho_{j v}, \sigma_{j w}$ being polynomials in $K_{0}, L_{0}, Q_{0}$, respectively, with coefficients in the $G F\left[p^{n}\right]$. Furthermore, $\mathrm{H}_{0}$ must be commutative with $\eta_{0}, Z_{0}$ with $\xi_{0}, \Psi_{0}$ with $\psi_{0}$.

Inversely, if these conditions on $\mathrm{H}_{i}, Z_{i}, \Psi_{i}$ be satisfied, then the substitution $T$ corresponding to the product $T_{1}$ will be commutative with $S$ and will have its coefficients in the $G F\left[p^{n}\right]$.

218. In order that the substitutions $H_{0}$ and $\eta_{0}$ be commutative, it is necessary and sufficient that, for every $a, A$ and $A^{\prime}$,

234) $\delta_{a A}=0, \quad \delta_{A-1 a-1}=0(a>1), \quad \delta_{A-1 a}=0, \quad \delta_{A-1 A^{\prime}-1}=\delta_{A A^{\prime}}$. Indeed, $\eta_{0} \mathrm{H}_{0}$ and $\mathrm{H}_{0} \eta_{0}$ replace $\eta_{0 a}$ by the same function only if every $\delta_{a A}=0$. In order that they shall replace $\eta_{0 A}$ by the same function, we must have

$$
\sum_{A^{\prime}} \delta_{A A^{\prime}} \eta_{0 A^{\prime}-1}=\sum_{u=1}^{\alpha} \delta_{A-1 u} \eta_{0 u} .
$$

If $u$ is not of the form $A^{\prime}-1$, it must be of the form $a-1$ or else $\alpha$. 
CANONICAL FORM AND CLASSIFICATION OF LINEAR SUBSTITUTIONS. 233

To take an example, let $r=2$ and $a_{1}=3, a_{2}=3, a_{3}=2$. Then $\delta_{1 A}=\delta_{4 A}=\delta_{7 A}=0(A=2,3,5,6,8) ; \quad \delta_{2 u}=\delta_{5 u}=0 \quad(u=3,6,8)$ $\delta_{A A^{\prime}}=\delta_{A-1 A^{\prime}-1} \quad\left(A, A^{\prime}=2,3,5,6,8\right)$.

Setting $\eta_{0 u} \equiv \eta_{u}$, we find that $H_{0}$ has the following form ${ }^{1}$ ):

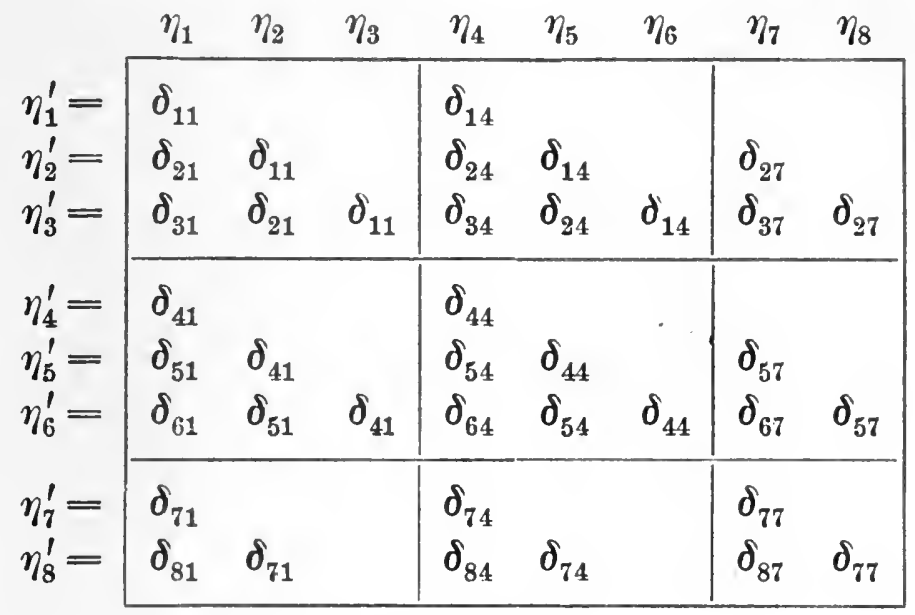

Its determinant is readily seen to equal

$$
\delta_{77}^{2}\left|\begin{array}{ll}
\delta_{11} & \delta_{14} \\
\delta_{41} & \delta_{44}
\end{array}\right|^{3}
$$

In the general case, $H_{0}$ is seen to take the form:

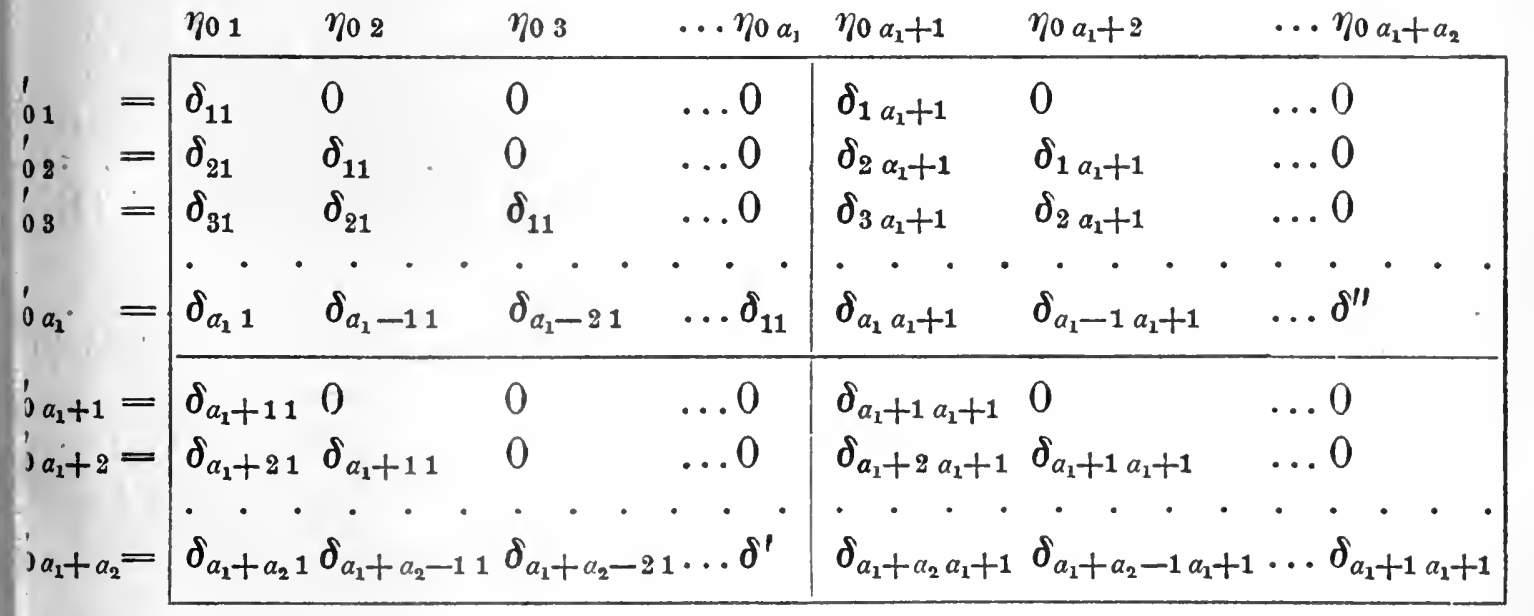

If $a_{1}=a_{2}, \delta^{\prime}=\delta_{a_{1}+11}$ and $\delta^{\prime \prime}=\delta_{1 a_{1}+1}$. If $a_{1}>a_{2}$, we have

$$
\delta^{\prime} \equiv \delta_{a_{1}+a_{2} a_{3}}=\delta_{a_{1}+1 a_{1}-a_{2}+1}=0, \quad \delta^{\prime \prime} \equiv \delta_{a_{1} a_{1}+a_{2}}=\delta_{a_{1}-a_{2}+1 a_{1}+1},
$$

and $\delta_{1 a_{1}+1}=\delta_{2 a_{1}+1}=\cdots=\delta_{a_{1}-a_{2} a_{1}+1}=0$. Finally, if $a_{1}<a_{2}$, we have

$$
\delta^{\prime \prime}=0, \quad \delta^{\prime}=\delta_{a_{2}+11}, \quad \delta_{a_{1}+11}=\cdots=\delta_{a_{2} 1}=0 .
$$

1) $\delta_{17}, \delta_{47}, \delta_{83}, \delta_{86}$ are zero, being equal to $\delta_{28}, \delta_{58}, \delta_{72}, \delta_{75}$ respectively. 
The matrix of the coefficients of $\mathrm{H}_{0}$ is made up of $(r+1)^{2}$ rectangles, of which the general one $R_{i j}$ is of height $a_{i}$ and of base $a_{j}$. Let $t$ be the smaller of the integers $i, j$ or their common value if $i=j$. Then $R_{i j}$ includes at its left or bottom a square array $S_{t}$ of coefficients $a_{t}$ to a side. The coefficients in its diagonal are all equal; likewise those in any parallel to the diagonal. All the coefficients in $R_{i j}$ which lie above or to the right of the diagonal of the square $S_{t}$ are zeros.

219. The results of $\S 218$ will be applied only in such simple cases that the determinant $D$ of $H_{0}$ can be simplified by inspection. It will therefore be sufficient to state without proof ${ }^{1}$ ) the simplest expression which can be given to $D$. Our notations may be fixed so that $a_{1} \overline{>} a_{2} \overline{>} a_{3} \overline{>} \cdots \overline{>} a_{r+1}$. Let

where

$$
\begin{gathered}
a_{1}=a_{2}=\cdots=a_{\lambda_{1}} \equiv A_{1}, \quad a_{\lambda_{1}+1}=\cdots=a_{\lambda_{1}+\lambda_{2}} \equiv A_{2}, \ldots, \\
a_{\lambda_{1}+\lambda_{2}+\cdots+\lambda_{\tau-1}+1}=\cdots=a_{\lambda_{1}+\cdots+\lambda_{\tau}} \equiv A_{\tau},
\end{gathered}
$$

$$
\lambda_{1}+\lambda_{2}+\cdots+\lambda_{\tau} \equiv r+1 \text {. }
$$

The determinant $D$ equals $D_{\lambda_{1}}^{A_{1}} D_{\lambda_{2}}^{A_{2}} \ldots D_{\lambda_{\tau}}^{A_{\tau}}$, where, if $(i, j) \equiv \delta_{i j}$,

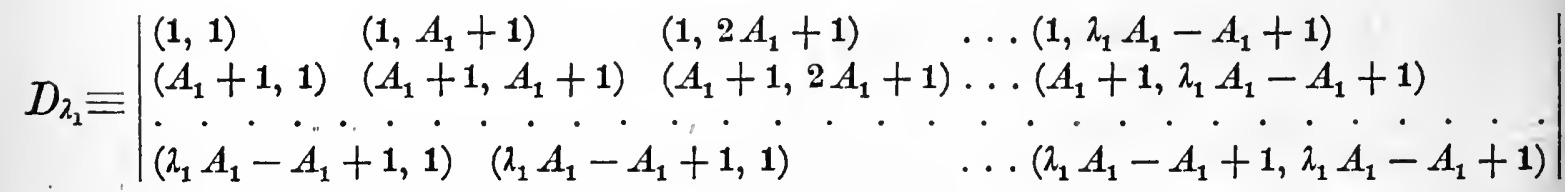

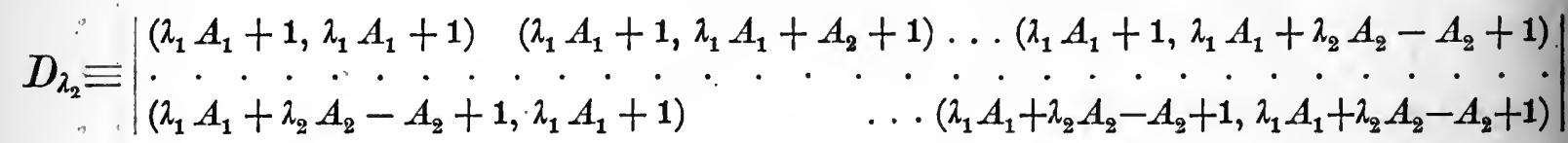

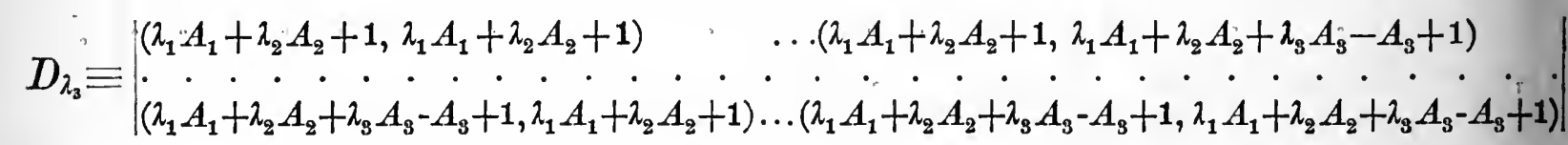

Since the coefficients $\delta_{i j}$ are functions of $K_{0}$, a root of an equation of degree $k$ belonging to and irreducible in the $G F\left[p^{n}\right]$, the number of sets of values for the $\lambda_{\sigma}^{2}$ coefficients entering $D_{\lambda_{\sigma}}$ for which this determinant is not zero is $(\$ 99)$

$$
\Omega\left(\lambda_{\sigma}, p^{n k}\right) \equiv\left(p^{n k \lambda_{\sigma}}-1\right)\left(p^{n k \lambda_{\sigma}}-p^{n k}\right) \ldots\left(p^{n k \lambda_{\sigma}}-p^{n k \lambda_{\sigma}-n k}\right) .
$$

Excluding the coefficients of $H_{0}$ which are always zero, there remains the following number of distinct coefficients $\delta_{i j}$ :

1) A method of proof is given by the author in the American Journal, vol. 22, pp. $133-134$. 


$$
\begin{aligned}
\omega & \equiv\left(a_{1}+a_{2}+a_{3}+\cdots+a_{r+1}\right)+\left(2 a_{2}+a_{3}+\cdots+a_{r+1}\right) \\
& +\left(3 a_{3}+a_{4}+\cdots+a_{r+1}\right)+\cdots+\left(\overline{r+1} a_{r+1}\right)
\end{aligned}
$$

the $q^{\text {th }}$ parenthesis giving the number of such $\delta_{i j}$ in the $q^{\text {th }}$ row of rectangles. On account of the equalities among the $a$ 's, we find

$$
\begin{gathered}
\omega \equiv A_{1} \lambda_{1}^{2}+A_{2} \lambda_{2}\left(\lambda_{2}+2 \lambda_{1}\right)+A_{3} \lambda_{3}\left(\lambda_{3}+2 \lambda_{1}+2 \lambda_{2}\right)+\cdots \\
+A_{\tau} \lambda_{\tau}\left(\lambda_{\tau}+2 \lambda_{1}+\cdots+2 \lambda_{\tau-1}\right) .
\end{gathered}
$$

Excluding also the $\lambda_{1}^{2}+\lambda_{2}^{2}+\cdots+\lambda_{\tau}^{2}$ coefficients in the determinants $D_{\lambda_{\sigma}}$, there remains the following number of wholly arbitrary $\delta_{i j}$ :

$$
\begin{aligned}
\Omega \equiv \sum_{\sigma=1}^{\tau} \lambda_{\sigma}^{2}\left(A_{\sigma}-1\right) & +2 A_{2} \lambda_{2} \lambda_{1}+2 A_{3} \lambda_{3}\left(\lambda_{2}+\lambda_{1}\right)+\cdots \\
& +2 A_{\tau} \lambda_{\tau}\left(\lambda_{\tau-1}+\cdots+\lambda_{2}+\lambda_{1}\right) .
\end{aligned}
$$

Each one of these $\Omega$ coefficients may take $p^{n k}$ values. The total number of substitutions $H_{0}$ is therefore

$$
f\left(a_{1}, \ldots, a_{r+1}, k, p^{n}\right) \equiv \Omega\left(\lambda_{1}, p^{n k}\right) \Omega\left(\lambda_{2}, p^{n k}\right) \ldots \Omega\left(\lambda_{\tau}, p^{n k}\right) \cdot p^{n k \Omega} .
$$

The total number of $m$-ary linear homogeneous substitutions $T$ in the $G F\left[p^{n}\right]$ commutative with a particular one $S$, whose canonical form is expressed in the notations of $\S 217$, is given by the product ${ }^{1}$ )

$$
f\left(a_{1}, \ldots, a_{r+1}, k, p^{n}\right) \cdot f\left(b_{1}, \ldots, b_{s+1}, l, p^{n}\right) \cdot f\left(c_{1}, \ldots, c_{t+1}, q, p^{n}\right) \ldots
$$

Recurring to the above example, $a_{1}=3, a_{2}=3, a_{3}=2$, we have

$$
f\left(a_{1}, a_{2}, a_{3}, k, p^{n}\right) \equiv\left(p^{2 n k}-1\right)\left(p^{2 n k}-p^{n k}\right) \cdot\left(p^{n k}-1\right) \cdot p^{17 n k},
$$

as is directly evident from the form of $H_{0}$ and its determinant.

220. As an important example, suppose that $S$ has the canonical form

$$
\begin{aligned}
& \eta_{i}^{\prime}=K_{i} \eta_{i} \\
& (i=0,1, \ldots, k-1) \\
& \xi_{i}^{\prime}=L_{i} \xi_{i} \\
& (i=0,1, \ldots, l-1) \\
& \psi_{i}^{\prime}=Q_{i} \psi_{i} \\
& (i=0,1, \ldots, q-1) \text {. }
\end{aligned}
$$

The most general substitution $T_{1}$ commutative with $S$ replaces $\eta_{0}$, $\xi_{0}, \ldots, \psi_{0}$ by $x\left(K_{0}\right) \eta_{0}, \lambda\left(L_{0}\right) \xi_{0}, \ldots, \varrho\left(Q_{0}\right) \psi_{0}$ respectively, in which the coefficients of the functions $x, \lambda, \ldots, \varrho$ belong to the $G F\left[p^{n}\right]$. If $K, L, \ldots, Q$ be primitive roots of the Galois fields of orders $p^{n k}$, $p^{n l}, \ldots, p^{n q}$ respectively, we may set

$$
\varkappa\left(K_{0}\right) \equiv K^{d}, \lambda\left(L_{0}\right) \equiv L^{e}, \ldots, \varrho\left(Q_{0}\right) \equiv Q^{f} .
$$

1) This result is in accord with that of Jordan, who treats the case $n=1$. His method of proof is merely illustrated by the consideration of a particular example, Traité, pp. 128-136. Moreover, it does not give the explicit form of the commutative substitutions. 
If, upon returning to the initial indices $\xi_{i}$ upon which $S$ is a substitution with coefficients in the $G F\left[p^{n}\right], I_{1}$ shall become a substitution with coefficients in that field, $T_{1}$ must have the form

$$
\begin{aligned}
& \eta_{i}^{\prime}=K^{d p^{n i}} \eta_{i} \quad(i=0,1, \ldots, k-1) \\
& \xi_{i}^{\prime}=L^{e p^{n i}} \xi_{i} \quad(i=0,1, \ldots, l-1) \\
& \psi_{i}^{\prime}=Q^{f p^{n i}} \psi_{i} \quad(i=0,1, \ldots, q-1) .
\end{aligned}
$$

Distribution of the substitutions of the general linear homogeneous group into complete sets of conjugate substitutions, §§ 221-223.

221. The substitutions of the group $G_{m} \equiv G L H\left(m, p^{n}\right)$ are to be classified into complete sets of conjugate substitutions and the number of substitutions in each set determined. Although a complete solution of this problem is furnished by the preceding general theorems, their generality and complexity make it desirable to consider in detail the special cases $m=3$ and $m=4$.

The classification employed is based upon the canonical forms of the substitutions of $G_{m}$. These in turn depend upon the characteristic determinants of the substitutions $\left(\alpha_{i j}\right)$, viz.,

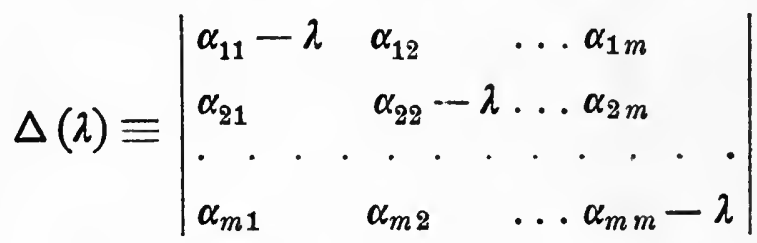

$$
\begin{aligned}
& \equiv(-1)^{m}\left\{\lambda^{m}-\alpha_{1} \lambda^{m-1}-\alpha_{2} \lambda^{m-2}-\cdots-\alpha_{m-1} \lambda-\alpha_{m}\right\} \text {. }
\end{aligned}
$$

Furthermore, $G_{m}$ contains a substitution in whose characteristic determinant the coefficients $\alpha_{1}, \alpha_{2}, \ldots, \alpha_{m}$ are any preassigned marks of the $G F\left[p^{n}\right]$ such that $\alpha_{m} \neq 0$. The required substitution is

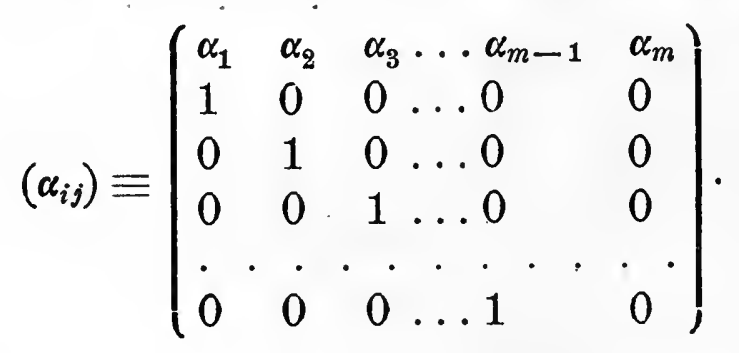

222. Consider first the group $G_{3}$ of order

$$
N \equiv\left(p^{3 n}-1\right)\left(p^{3 n}-p^{n}\right)\left(p^{3 n}-p^{2 n}\right) \text {. }
$$

By $\S \S 214-215$, every linear homogeneous substitution in the $G F\left[p^{n}\right]$ on $m=3$ indices can be reduced by a linear ternary transformation 
(not necessarily in the $G F\left[p^{n}\right]$ ) to one of the following five types of canonical forms:

$$
\begin{array}{lll}
A: & x^{\prime}=\lambda x, \quad y^{\prime}=\lambda p^{n} y, & z^{\prime}=\lambda^{2 n} z \\
B: & x^{\prime}=\mu x, \quad y^{\prime}=\mu^{p^{n} y,} & z^{\prime}=\alpha z \\
C: & x^{\prime}=\alpha x, \quad y^{\prime}=\beta y, & z^{\prime}=\gamma z \\
D: & x^{\prime}=\alpha x, \quad y^{\prime}=\beta y, & z^{\prime}=\beta(z+y) \\
E: & x^{\prime}=\alpha x, \quad y^{\prime}=\alpha(y+x), & z^{\prime}=\alpha(z+y),
\end{array}
$$

where $\lambda$ satisfies a cubic equation and $\mu$ a quadratic equation each belonging to and irreducible in the $G F\left[p^{n}\right]$, while $\alpha, \beta, \gamma$ denote marks $\neq 0$ of the $G F\left[p^{n}\right]$.

Upon replacing $\lambda$ by $\lambda^{p^{n}}$ or by $\lambda^{2^{2 n}}$, we obtain from $A$ a substitution conjugate with $A$. Any other replacement of $\lambda$ leads to a substitution not conjugate with $A$ ( $\S 102$, Corollary), since its characteristic determinant differs from that of $A$. Hence the type $A$ includes $\frac{1}{3}\left(p^{3 n}-p^{n}\right)$ distinct sets of conjugate substitutions, those in different sets being not conjugate under $G_{3}$.

Let $S$ be a substitution of $G_{3}$ having the canonical form $A$, where $\lambda$ is a definite mark of the $G F\left[p^{3 n}\right]$ not in the $G F\left[p^{n}\right]$. If a substitution $T$ of $G_{3}$ be commutative with $S$ and if we apply to $T$ the same transformation of indices which reduces $S$ to the form $A$, then $(\S 220) T$ will take the form

$$
x^{\prime}=\sigma^{r} x, \quad y^{\prime}=\sigma^{r p^{n}} y, \quad z^{\prime}=\sigma^{r x^{2 n}} z,
$$

where $\sigma$ is a primitive root of the $G F\left[p^{3 n}\right]$ and $r$ is some positive integer $\bar{\sum} p^{3 n}-1$. Hence $S$ is commutative with exactly $p^{3 n}-1$ substitutions of $G_{3}$, so that $S$ is one of $N \div\left(p^{3 n}-1\right)$ conjugate substitutions within $G_{3}$. The total number of substitutions of $G_{3}$ reducible to the canonical forms $A$ is therefore

a)

$$
\frac{1}{3}\left(p^{3 n}-p^{n}\right)\left(p^{3 n}-p^{n}\right)\left(p^{3 n}-p^{2 n}\right) .
$$

Type $B$ includes $\frac{1}{2}\left(p^{2 n}-p^{n}\right)\left(p^{n}-1\right)$ distinct sets of conjugate substitutions. In fact, the replacement of $\mu$ by $\mu^{p^{n}}$ leads to a substitution conjugate with $B$, while any other replacement of $\mu$ or any change in $\alpha$ leads to a substitution not conjugate with $B$. A substitution of $G_{3}$ commutative with a particular substitution reducible to a type $B$ has the canonical form 


$$
x^{\prime}=\varrho^{r} x, \quad y^{\prime}=\varrho^{r p^{n}} y, \quad z^{\prime}=\delta z,
$$

where $\varrho$ is a primitive root of the $G F\left[p^{2 n}\right]$ and $\delta$ belongs to the $G F\left[p^{n}\right], r$ being an integer $\overline{<} p^{2 n}-1$. The number of such substitutions is $\left(p^{2 n}-1\right)\left(p^{n}-1\right)$. Hence the total number of substitutions of $G_{3}$ reducible to the canonical forms $B$ is

$$
\frac{1}{2}\left(p^{2 n}-p^{n}\right)\left(p^{n}-1\right)\left(p^{3 n}-1\right) p^{3 n} .
$$

Type $C$ includes $p^{n}-1$ canonical forms with $\alpha=\beta=\gamma$; $\left(p^{n}-1\right)\left(p^{n}-2\right)$ canonical forms with $\alpha=\beta \neq \gamma$; a like number with $\alpha=\gamma \neq \beta$; a like number with $\beta=\gamma \neq \alpha$; and $\left(p^{n}-1\right)\left(p^{n}-2\right)\left(p^{n}-3\right)$ with $\alpha, \beta, \gamma$ all distinct. By a suitable transformation of indices the multipliers $\alpha, \beta, \gamma$ in $C$ are permuted in an arbitrary manner. We have therefore the following numbers of distinct sets of conjugate canonical substitutions $C$ :

$$
\begin{aligned}
& p^{n}-1 \text { of type } C_{1} \text { with } \alpha=\beta=\gamma ; \\
& \left(p^{n}-1\right)\left(p^{n}-2\right) \text { of type } C_{2} \text { with only two equal multipliers, } \\
& \quad \text { say } \alpha=\beta \neq \gamma ; \\
& \frac{1}{6}\left(p^{n}-1\right)\left(p^{n}-2\right)\left(p^{n}-3\right) \text { of type } C_{3} \text { with all three mul- } \\
& \text { tipliers distinct. }
\end{aligned}
$$

The most general substitution of $G_{3}$ commutative with $C_{3}$ is

$$
x^{\prime}=a x, \quad y^{\prime}=b y, \quad z^{\prime}=c z \quad\left(a, b, c \text { in the } G F\left[p^{n}\right]\right) .
$$

Hence $C_{3}$ is one of $N \div\left(p^{n}-1\right)^{3}$ conjugate substitutions within $G_{3}$ The most general substitution of $G_{3}$ commutative with $C_{2}$ is

$$
x^{\prime}=a x+b y, \quad y^{\prime}=c x+d y, \quad z^{\prime}=e z .
$$

Hence $C_{2}$ is one of $N \div\left(p^{2 n}-1\right)\left(p^{2 n}-p^{n}\right)\left(p^{n}-1\right)$ conjugate substitutions. Finally, $C_{1}$ is commutative with every substitution of $G_{3}$ and thus is conjugate only with itself. The total number of substitutions of $G_{3}$ reducible to the canonical forms $C$ is thus

c)

$$
\begin{aligned}
\left(p^{n}-1\right) & +\left(p^{3 n}-1\right)\left(p^{n}-2\right) p^{2 n} \\
& +\frac{1}{6}\left(p^{n}-2\right)\left(p^{n}-3\right)\left(p^{3 n}-1\right)\left(p^{n}+1\right) p^{3 n}
\end{aligned}
$$

Of the substitutions of type $D$, there are $p^{n}-1$ with $\alpha=\beta$ and $\left(p^{n}-1\right)\left(p^{n}-2\right)$ with $\alpha \neq \beta$, no two being conjugate under $G_{3}$. A 
substitution $D$ with $\alpha=\beta$ is commutative only with the $p^{3 n}\left(p^{n}-1\right)^{2}$ substitutions of $G_{3}$

$x^{\prime}=d y+e x, y^{\prime}=a y, z^{\prime}=b y+a z+c x \quad\left(a, b, c, d, e\right.$ in the $\left.G F\left[p^{n}\right]\right)$.

A substitution $D$ with $\alpha \neq \beta$ is commutative only with the $p^{n}\left(p^{n}-1\right)^{2}$ substitutions of $G_{3}$

$$
x^{\prime}=e x, \quad y^{\prime}=a y, \quad z^{\prime}=b y+a z .
$$

The total number of substitutions of $G_{3}$ reducible to the types $D$ is thus

d) $\left(p^{n}-1\right)\left(p^{3 n}-1\right)\left(p^{n}+1\right)+\left(p^{n}-1\right)\left(p^{n}-2\right)\left(p^{3 n}-1\right)\left(p^{n}+1\right) p^{2 n}$.

No two of the $p^{n}-1$ substitutions of type $E$ are conjugate under $G_{3}$. Each is commutative only with the $p^{2 n}\left(p^{n}-1\right)$ substitutions of $G_{3}$

$$
x^{\prime}=a x, \quad y^{\prime}=b x+a y, \quad z^{\prime}=c x+b y+a z .
$$

The number of substitutions reducible to the canonical forms $E$ is

e)

$$
\left(p^{n}-1\right)\left(p^{3 n}-1\right)\left(p^{2 n}-1\right) p^{n}
$$

A check on the above enumeration of the substitutions of $G_{3}$ consists is verifying that the sum of the numbers a), b), c), d), e) equals the order $N$ of $G_{3}$.

223. Consider next the group $\left.{ }^{1}\right) G_{4}$ of order

$$
N \equiv\left(p^{4 n}-1\right)\left(p^{4 n}-p^{n}\right)\left(p^{4 n}-p^{2 n}\right)\left(p^{4 n}-p^{3 n}\right) .
$$

By $\S 221, G_{4}$ contains a substitution in whose characteristic determinant $\Delta(\lambda) \equiv \lambda^{4}-\alpha_{1} \lambda^{3}-\alpha_{2} \lambda^{2}-\alpha_{3} \lambda-\alpha_{4}$ the coefficients $\alpha_{1}, \alpha_{2}, \alpha_{3}$, $\alpha_{4}$ are arbitrary marks of the $G F\left[p^{n}\right], \alpha_{4} \neq 0$. According to the possible factorizations of $\Delta(\lambda)$ in the $G F\left[p^{n}\right]$, we distinguish the cases: I) irreducible; II) linear factor and irreducible cubic; III) two distinct irreducible quadratic factors; IV) equal irreducible quadratic factors; V) irreducible quadratic and two distinct linear factors; VI) irreducible quadratic and two equal linear factors; VII) - XI) four linear factors, according to the number of equal factors. Denote by $\lambda_{t}, \mu_{t}$ marks of the $G F\left[p^{n t}\right]$ not in the $G F\left[p^{n} \tau\right], \tau<t$. For simplicity, the subscript unity is omitted from the marks $\alpha, \beta, \gamma, \delta$ of the $G F\left[p^{n}\right]$. The types of canonical forms of the substitutions of $G_{4}$ may be exhibited in the following complete list:

1) Cf. T. M. Putnam, Amer. Journ. Math., vol. XXIII, pp. 41-48. For the author's treatment of the case $n=3$, ibid, pp. 37-40. 


\begin{tabular}{|c|c|c|c|c|c|}
\hline \multirow{2}{*}{$\frac{\text { Type }}{\text { I }}$} & \multicolumn{4}{|c|}{ Canonical s } & $\begin{array}{l}\text { Number } M \text { of distinct } \\
\text { canonical forms }\end{array}$ \\
\hline & $\lambda_{4} x$ & $\lambda_{4}^{p^{n}} y$ & $\lambda_{4}^{p^{2} n} z$ & $\lambda_{4}^{p^{3 n}} w$ & $\frac{1}{4}\left(p^{4 n}-p^{2 n}\right)$ \\
\hline II & $\lambda_{3} x$ & $\lambda_{3}^{p^{n}} y$ & $\lambda_{3}^{p^{2 n}} z$ & $\lambda_{1} w$ & $\frac{1}{3}\left(p^{3 n}-p^{n}\right)\left(p^{n}-1\right)$ \\
\hline III & $\lambda_{2} x$ & $\lambda_{2}^{p^{n}} y$ & $\mu_{2} z$ & $\mu_{2}^{p^{n}} w$ & $\frac{1}{8}\left(p^{2 n}-p^{n}\right)\left(p^{2 n}-p^{n}-2\right)$ \\
\hline $\mathrm{IV}_{1}$ & $\lambda_{2} x$ & $\lambda_{2}(y+x)$ & $\lambda_{2}^{p^{n}} z$ & $\lambda_{2}^{p^{n}}(w+z)$ & $\frac{1}{2}\left(p^{2 n}-p^{n}\right)$ \\
\hline $\mathrm{IV}_{2}$ & $\lambda_{2} x$ & $\lambda_{2} y$ & $\lambda_{2}^{p^{n}} z$ & $\lambda_{z}^{p^{n}} w$ & $\frac{1}{2}\left(p^{2 n}-p^{n}\right)$ \\
\hline $\mathrm{V}$ & $\lambda_{1} x$ & $\mu_{1} y$ & $\lambda_{2} z$ & $\lambda_{2}^{p^{n}} w$ & $\frac{1}{4}\left(p^{2 n}-p^{n}\right)\left(p^{n}-1\right)\left(p^{n}-2\right)$ \\
\hline $\mathrm{VI}_{1}$ & $\lambda_{1} x$ & $\lambda_{1}(y+x)$ & $\lambda_{2} z$ & $\lambda_{2}^{p^{n}} w$ & $\frac{1}{2}\left(p^{2 n}-p^{n}\right)\left(p^{n}-1\right)$ \\
\hline $\mathrm{VI}_{2}$ & $\lambda_{1} x$ & $\lambda_{1} y$ & $\lambda_{2} z$ & $\lambda_{2}^{p^{n}} w$ & $\frac{1}{2}\left(p^{2 n}-p^{n}\right)\left(p^{n}-1\right)$ \\
\hline VII & $\alpha x$ & $\beta y$ & $\gamma z$ & $\delta w$ & $\frac{1}{24}\left(p^{n}-1\right)\left(p^{n}-2\right)\left(p^{n}-3\right)\left(p^{n}-4\right)$ \\
\hline $\mathrm{VIII}_{1}$ & $\alpha x$ & $\beta y$ & $\gamma z$ & $\gamma(w+z)$ & $\frac{1}{2}\left(p^{n}-1\right)\left(p^{n}-2\right)\left(p^{n}-3\right)$ \\
\hline $\mathrm{VIII}_{2}$ & $\alpha x$ & $\beta y$ & $\gamma z$ & $\gamma w$ & $\frac{1}{2}\left(p^{n}-1\right)\left(p^{n}-2\right)\left(p^{n}-3\right)$ \\
\hline $\mathrm{IX}_{1}$ & $\alpha x$ & $\beta y$ & $\beta(z+y)$ & $\beta(w+z)$ & $\left(p^{n}-1\right)\left(p^{n}-2\right)$ \\
\hline $\mathrm{IX}_{2}$ & $\alpha x$ & $\beta y$ & $\beta(z+y)$ & $\beta w$ & $\left(p^{n}-1\right)\left(p^{n}-2\right)$ \\
\hline $\mathrm{IX}_{3}$ & $\alpha x$ & $\beta y$ & $\beta z$ & $\beta w$ & $\left(p^{n}-1\right)\left(p^{n}-2\right)$ \\
\hline $\mathrm{X}_{1}$ & $\alpha x$ & $\alpha(y+x)$ & $\alpha(z+y)$ & $\alpha(w+z)$ & $p^{n}-1$ \\
\hline & $\alpha x$ & $\alpha(y+x)$ & $\alpha(z+y)$ & $\alpha 2 v$ & $p^{n}-1$ \\
\hline $\mathrm{X}_{3}$ & $\alpha x$ & $\alpha(y+x)$ & $\alpha z$ & $\alpha(w+z)$ & $p^{n}-1$ \\
\hline & $\alpha x$ & $\alpha(y+x)$ & $\alpha z$ & $\alpha w$ & $p^{n}-1$ \\
\hline$X$ & $\alpha x$ & $\alpha y$ & $\alpha z$ & $\alpha w$ & $p^{n}-1$ \\
\hline $\mathrm{XI}_{1}$ & $\alpha x$ & $\alpha(y+x)$ & $\gamma z$ & $\gamma(w+z)$ & $\frac{1}{2}\left(p^{n}-1\right)\left(p^{n}-2\right)$ \\
\hline $\mathrm{XI}_{2}$ & $\alpha x$ & $\alpha(y+x)$ & $\gamma z$ & $\gamma w$ & $\left(p^{n}-1\right)\left(p^{n}-2\right)$ \\
\hline $\mathrm{XI}_{3}$ & $\alpha x$ & $\alpha y$ & $\gamma z$ & $\gamma w$ & $\frac{1}{2}\left(p^{n}-1\right)\left(p^{n}-2\right)$ \\
\hline
\end{tabular}

1) The notation $\alpha x, \beta y, \gamma z, \gamma(w+z)$, for example, is used for the substitution

$$
x^{\prime}=\alpha x, \quad y^{\prime}=\beta y, \quad z^{\prime}=\gamma z, \quad w^{\prime}=\gamma(w+z)
$$


Table giving the form and number $C$ of the substitutions of the group $G_{4}$ commutative with the various types of canonical forms:

\begin{tabular}{|c|c|c|c|c|c|}
\hline I & $\lambda x$ & $\lambda^{p^{n}} y$ & $\lambda^{p^{2 n}} z$ & $\lambda^{p^{3 n}} w$ & $p^{4 n}-1$ \\
\hline II & $\mu x$ & $\mu^{p^{n}} y$ & $\mu^{p^{2 n} z}$ & $a w$ & $\left(p^{3} n-1\right)\left(p^{n}-1\right)$ \\
\hline III & $\varrho x$ & $\varrho^{p^{n}} y$ & $\sigma z$ & $\sigma^{p^{n}} w$ & $\left(p^{2 n-1}\right)^{2}$ \\
\hline $\mathrm{IV}_{1}$ & $\varrho x$ & $\sigma x+\varrho y$ & . $\varrho^{p^{n}} z$ & $\sigma^{p^{n}} z+\varrho^{p^{n}} a v$ & $\left(p^{2 n}-1\right) p^{2 n}$ \\
\hline $\mathrm{IV}_{2}$ & $\sigma x+\varrho y$ & $x x+\tau y$ & $\sigma^{p^{n}} z+\varrho^{p^{n}} w$ & $x^{p^{n}} z+\tau^{p^{n}} w$ & $\left(p^{4 n}-1\right)\left(p^{4 n}-p^{2 n}\right)$ \\
\hline V & $a x$ & $\dot{b} y$ & $\varrho z$ & $\varrho^{p^{n}} w$ & $\left(p^{2} n-1\right)\left(p^{n}-1\right)^{2}$ \\
\hline $\mathrm{VI}_{1}$ & $a x$ & $b x+a y$ & $\varrho z$ & $\varrho^{p^{n}} w$ & $\left(p^{2 n}-1\right)\left(p^{2 n}-p^{n}\right)$ \\
\hline $\mathrm{VI}_{2}$ & $a x+b y$ & $c x+d y$ & $\varrho z$ & $\varrho^{p^{n}} w$ & $\left(p^{2 n}-1\right)^{2}\left(p^{2 n}-p^{n}\right)$ \\
\hline VII & $a x$ & $b y$ & $c z$ & $d w$ & $\left(p^{n}-1\right)^{4}$ \\
\hline VIII $_{1}$ & $a x$ & $b y$ & $c z$ & $d z+c w$ & $\left(p^{n}-1\right)^{3} p^{n}$ \\
\hline $\mathrm{VIII}_{2}$ & $a x$ & by & $c z+d w$ & $e z+f w$ & $\left(p^{2 n}-p^{n}\right)\left(p^{2} n-1\right)\left(p^{n}-1\right)^{2}$ \\
\hline $\mathrm{IX}_{1}$ & $a x$ & by & $c y+b z$ & $d y+c z+b w$ & $\left(p^{n}-1\right)^{2} p^{2 n}$ \\
\hline $\mathrm{IX}_{2}$ & $a x$ & $b y$ & $c y+b z+e w$ & $f y+d w$ & $\left(p^{n}-1\right)^{3} p^{3} n$ \\
\hline $\mathrm{IX}_{3}$ & $a x$ & $b y+c z+d w$ & $e y+f z+g w$ & $h y+i z+j w$ & $\left(p^{3 n}-1\right)\left(p^{2} n-1\right)\left(p^{n}-1\right)^{2} p^{3} n$ \\
\hline$X_{1}$ & $a x$ & $b x+a y$ & $c x+b y+a z$ & $a x+c y+b z+a w$ & $\left(p^{n}-1\right) p^{3 n}$ \\
\hline $\mathrm{X}_{2}$ & $a x$ & $b x+a y$ & $c x+b y+a z+e w$ & $f x+d w$ & $\left(p^{n}-1\right)^{2} p^{4 n}$ \\
\hline $\mathrm{X}_{3}$ & $|a x+e z|$ & $b x+a y+f z+e w$ & $g x+c z$ & $h x+g y+d z+c w$ & $\left(p^{2} n-1\right)\left(p^{2} n-p^{n}\right) p^{4 n}$ \\
\hline $\mathrm{X}_{4}$ & $a \ddot{x}$ & $b x+a y+f z+e w$ & $g x+c z+k w$ & $h x+d z+l w$ & $\left(p^{2 n}-1\right)\left(p^{2 n}-p^{n}\right)\left(p^{n}-1\right) p^{5 n}$ \\
\hline$X_{5}$ & & & arbitrary & . & $N$ \\
\hline $\mathrm{XI}_{1}$ & $a x$ & $b x+a y$ & $c z$ & $d z+c w$ & $\left(p^{n}-1\right)^{2} p^{2 n}$ \\
\hline$X I_{y}$ & $a x$ & $b x+a y$ & $c z+d w$ & $e z+f w$ & $\left(p^{2 n}-1\right)\left(p^{2} n-p^{n}\right)\left(p^{n}-1\right) p^{n}$ \\
\hline $\mathrm{XI}_{3}$ & $a x+b y$ & $g x+\dot{h} y$ & $c z+d w$ & $e z+f w$ & $\left(p^{2 n}-1\right)^{2}\left(p^{2} n-p^{n}\right)^{2}$ \\
\hline
\end{tabular}

Here $\lambda$ belongs to the $G F\left[p^{4 n}\right], \mu$ to the $G F\left[p^{3 n}\right], \varrho, \sigma, \varkappa, \tau$ to the $G F\left[p^{2 n}\right]$, and $a, b, c, \ldots, j$ belong to the $G F\left[p^{n}\right]$. If $M$ denote the number of distinct canonical forms in a general type, and $C$ the number of substitutions of $G_{4}$ commutative with each, the number of substitutions of $G_{4}$ reducible to that type is $M N / C$. The sum of these numbers is found to equal $N$, the total number of the substitutions of $G_{4}$. 


\section{CHAPTER XI.}

\section{OPERATORS AND CYCLIC SUBGROUPS OF THE SIMPLE GROUP $\left.L F\left(3, p^{n}\right)^{1}\right)$}

224. By $\& 108$ the group $G \equiv L F\left(3, p^{n}\right)$ of all substitutions of determinant 1 ,

$$
S: \quad x^{\prime}=\frac{\alpha_{11} x+\alpha_{12} y+\alpha_{18}}{\alpha_{31} x+\alpha_{32} y+\alpha_{38}}, \quad y^{\prime}=\frac{\alpha_{21} x+\alpha_{29} y+\alpha_{28}}{\alpha_{31} x+\alpha_{32} y+\alpha_{38}}, \quad\left|\alpha_{i j}\right|=1,
$$

in which the coefficients $\alpha_{i j}$ belong to the $G F\left[p^{n}\right]$, is a simple group of order

$$
N \equiv \frac{1}{d}\left(p^{3 n}-1\right)\left(p^{2 n}-1\right) p^{3 n},
$$

where $d$ is the greatest common divisor of 3 and $p^{n}-1$, so that

$$
d=1 \text {, if } p^{n}=3^{n} \text { or } 3 l-1 ; d=3 \text {, if } p^{n}=3 l+1 \text {. }
$$

The equation $\tau^{3}=1$ has in the $G F\left[p^{n}\right]$ a single root $\theta=1$, if $d=1$; but has three roots $\theta, \theta^{2}, \theta^{3} \equiv 1$, if $d=3$. Hence, if $d=1$, there is a single homogeneous substitution of determinant unity

$$
\Sigma: \quad \xi_{i}^{\prime}=\alpha_{i 1} \xi_{1}+\alpha_{i 2} \xi_{2}+\alpha_{i 3} \xi_{3} \quad(i=1,2,3)
$$

which, when taken fractionally, leads to the non-homogeneous substitution $S$. If $d=3$, let $\theta$ denote the homogeneous substitution of determinant unity which multiplies each index by $\theta$. Then there are exactly the three homogeneous substitutions of determinant unity, $\Sigma, \Theta \Sigma \equiv \Sigma \Theta, \Theta^{2} \Sigma \equiv \Sigma \Theta^{2}$ :

$$
\theta^{r} \Sigma: \quad \xi_{i}^{\prime}=\theta^{r}\left(\alpha_{i 1} \xi_{1}+\alpha_{i 2} \xi_{2}+\alpha_{i 3} \xi_{3}\right) \quad(i=1,2,3),
$$

which, when taken fractionally, lead to the non-homogeneous substitution $S$. Combining the two cases, we may employ the group of ternary linear homogeneous substitutions of determinant unity in place of the group $G$ provided we consider to be identical the $d$ substitutions $\Sigma, \Theta \Sigma$ and $\Theta^{2} \Sigma$. Under this convention concerning the homogeneous substitutions, we employ henceforth the homogeneous notation for the substitutions of the group $G$.

225. Any substitution of $G$ can be reduced by a linear ternary transformation of indices (not necessarily in the $G F\left[p^{n}\right]$ and not necessarily of determinant unity) to one of the canonical forms $A$, $B, C, D, E$ of $\S 222$. In the present case, the determinants of $A, \ldots, E$ must be unity.

1) For $n=1$, Burnside, Proceed. Lond. Math. Soc., vol. 26, pp. 58-106; for general $n$, Dickson, Amer. Journ., vol. 22, pp. 231-252, where certain errors in Burnside's paper are pointed out. 
If two substitutions $S$ and $T$ of the group $G$ have the same canonical form, there exists ( $\S 216)$ a ternary homogeneous substitution $W$ belonging to the $G F\left[p^{n}\right]$ such that $T=W^{-1} S W$. It remains to consider whether or not there exists a ternary homogeneous substitution $W_{1}$ belonging to the $G F\left[p^{n}\right]$ and having determinant unity such that $W_{1}$ transforms $S$ into $I$. If the canonical form be $A, B, C$ or $D$, such a $W_{1}$ will be shown to exist; while for the canonical form $E$ such a $W_{1}$ does not always exist.

It is first shown that any one of the types $A, B, C, D$ can be transformed into itself by a substitution $V$ of determinant equal to an arbitrary mark $\neq 0$ of the $G F\left[p^{n}\right]$ and obeying the same laws in regard to the conjugacy of its indices as does the canonical form in question. For type $A$ we may take as $V$ the substitution

$$
x^{\prime}=\sigma^{r} x, \quad y^{\prime}=\sigma^{r p^{n}} y, \quad z^{\prime}=\sigma^{r p^{2 n}} z,
$$

where $\sigma$ is a primitive root of the $G F\left[p^{3 n}\right]$ so that $\tau \equiv \sigma^{1+p^{n}+p^{n 2}}$ is a primitive root of the $G F\left[p^{n}\right]$. The determinant of $V$ is thus $\tau^{r}$, which by suitable choice of $r$ may be made equal to an arbitrary mark $\neq 0$ of the $G F\left[p^{n}\right]$. For types $B$ and $C$ we may take $V$ to be

$$
x^{\prime}=x, \quad y^{\prime}=y, \quad z^{\prime}=\tau^{r} z .
$$

For type $D$ we may take as $V$ the substitution

$$
x^{\prime}=\tau^{r} x, \quad y^{\prime}=y, \quad z^{\prime}=z .
$$

Let $W$ have the determinant $w$ and choose $V$ so that its determinant is $w^{-1}$. We may take as the required substitution $W_{1}$ the product $V_{1} W$, where $V_{1}$ is the form taken by $V$ when expressed in the initial indices. In fact $V_{1}$ and $W$ have their coefficients in the $G F\left[p^{n}\right]$, while the product $V_{1} W$ transforms $S$ into $T$ and has the determinant $w^{-1} \cdot w=1$. Hence, if two substitutions of $G$ have the same canonical form $A, B, C$, or $D$, they are conjugate within the group $G$.

For type $E$ there arise two cases. If $d=1$, so that 3 is prime to $p^{n}-1$, every mark of the $G F\left[p^{n}\right]$ is a cube ( $\$ 63$, Corollary). Hence an integer $r$ may be determined so that $\tau^{3 r}$ shall be an arbitrary mark $\neq 0$ in the field. Hence the above argument holds if we choose as $V$ the substitution

$$
x^{\prime}=\tau^{r} x, \quad y^{\prime}=\tau^{r} y, \quad z^{\prime}=\tau^{r} z .
$$

For $d=3$, only $\frac{1}{3}\left(p^{n}-1\right)$ of the marks $\neq 0$ of the $G F\left[p^{n}\right]$ are cubes. Their products by $\beta$ and $\beta^{2}$ will be not-cubes, if $\beta$ be any particular not-cube. We can therefore determine $V^{\prime}$, of determinant a cube, such that $I$ is the transformed of $S$ by the sub- 
stitution $V_{1}^{\prime} W \equiv W^{\prime}$ belonging to the $G F\left[p^{n}\right]$ and having as determinant one of the three marks $1, \beta, \beta^{2}$. Consider the three substitutions of $G$

$$
E_{r}: \quad x^{\prime}=x, \quad y^{\prime}=y+\beta^{r} x, \quad z^{\prime}=z+y \quad(r=0,1,2) .
$$

The following substitution of determinant $\beta$ :

$$
R: \quad x^{\prime}=\beta x, \quad y^{\prime}=y, \quad z^{\prime}=z
$$

transforms $E_{1}$ into $E_{0}$ and $E_{2}$ into $E_{1}$. If $E$ has determinant unity, it is identical with $E_{0}$ in the group $G$. It follows from the proof above that any substitution $T$ of $G$, which can be transformed into $E_{0}$ by a linear substitution $W$ belonging to the $G E\left[p^{n}\right]$, can be transformed into $E_{0}$ by a similar substitution $W^{\prime}$ of determinant $\beta^{t}(t=0,1$ or 2$)$. Also $R^{-t}$ transforms $E_{0}$ into $E_{t}$. Hence $T$ is transformed into $E_{t}$ by the product $W^{\prime} R^{-t}$. which belongs to the $G F\left[p^{n}\right]$ and has determinant unity. Hence every substitution of $G$ of canonical form $E$ is conjugate within $G$ to one of the types $E_{0}, E_{1}, E_{2}$.

We next prove that no two of the types $E_{0}, E_{1}, E_{2}$ are conjugate within $G$, i. e., by means of a substitution of determinant unity. The most general ternary homogeneous substitution which transforms $E_{0}$ into $E_{1}$ is seen to be

$$
x^{\prime}=\beta^{-1} c x, \quad y^{\prime}=c y+b x, \quad z^{\prime}=c z+b y+a x,
$$

of determinant $\beta^{-1} c^{3}$; which can not be made unity. Transforming the latter by $R^{-1}$, we obtain the most general substitution which transforms $E_{1}$ into $\dot{E}_{2}$, viz.,

$$
x^{\prime}=\beta^{-1} c x, \quad y^{\prime}=c y+\beta b x, \quad z^{\prime}=c z+b y+\beta a x,
$$

of determinant $\beta^{-1} c^{3} \neq 1$. Finally, by $\S 102, E_{0}$ can not be transformed into $\Theta E_{1}$, nor $E_{1}$ into $\Theta E_{2}$, by a linear substitution. The results now proven may be stated in the explicit form:

Every substitution of $G$ can be reduced by a ternary linear homogeneous transformation to one of the canonical forms

$$
\begin{array}{llll}
A: & x^{\prime}=\lambda x, \quad y^{\prime}=\lambda^{p^{n}} y, & z^{\prime}=\lambda^{p^{2 n}} z & \left(\lambda^{p^{2 n}+p^{n}+1}=1\right) \\
B: & x^{\prime}=\mu x, \quad y^{\prime}=\mu^{p^{n}} y, \quad z^{\prime}=\mu^{-p^{n}-1} z & \\
C: & x^{\prime}=\alpha x, \quad y^{\prime}=\beta y, & z^{\prime}=\gamma^{2} & \\
D: & x^{\prime}=\alpha-2 x, y^{\prime}=\alpha y, & z^{\prime}=\alpha(z+y) \\
E_{0}: & x^{\prime}=x, \quad y^{\prime}=y+x, & \left.z^{\prime}=z+y=1\right) \\
E_{1}: & x^{\prime}=x, & y^{\prime}=y+\beta x, z^{\prime}=z+y\left(\beta \text { not-cube in } G F\left[p^{n}\right]\right) \\
E_{2}: & x^{\prime}=x, & y^{\prime}=y+\beta^{2} x, z^{\prime}=z+y, &
\end{array}
$$

in which $\lambda$ satisfies a cubic and $\mu$ a quadratic equation each belonging: to and irreducible in the $G F\left[p^{n}\right]$, while $\alpha, \beta, \gamma$ belong to the $G F\left[p^{n}\right]$. 
Of the substitutions of $G$ reducible to the forms $A$ and $B$, those and only those are conjugate within $G$ which are reducible to the same form $A$ or to the same form $B$. Every other substitution of $G$ is conjugate within $G$ to one of the types $C, D, E_{0}, E_{1}, E_{2}$ and no two of the latter types are conjugate within $G$.

226. Type $A$. The substitution of determinant unity

$$
x^{\prime}=\alpha_{1} x+\alpha_{2} y+z, \quad y^{\prime}=x, \quad z^{\prime}=y
$$

has the characteristic determinant

$$
\Delta(\lambda) \equiv-\lambda^{3}+\alpha_{1} \lambda^{2}+\alpha_{2} \lambda+1 .
$$

Hence $\alpha_{1}$ and $\alpha_{2}$ may be chosen in the $G F\left[p^{n}\right]$ so that a root $\lambda$ of $\Delta(\lambda)=0$ is a primitive root of the equation

$$
\lambda^{p^{2 n}+p^{n}+1}=1 \text {. }
$$

The order of the corresponding substitution $A$ is the least integer $m$ for which

$$
\lambda^{m}=\lambda^{m p^{n}}=\lambda^{m p^{2 n}},
$$

i. e., for which $m\left(p^{n}-1\right)$ is a multiple of $p^{2 n}+p^{n}+1$. But the greatest common divisor of $p^{n}-1$ and $p^{2 n}+p^{n}+1$ is also that of $p^{n}-1$ and 3 and therefore equals $d$. The order $m$ is consequently $\frac{1}{d}\left(p^{2 n}+p^{n}+1\right)$.

Moreover, the roots of any irreducible cubic of the form $\Delta(\lambda)=0$ may be written $\lambda^{s}, \lambda^{s p^{n}}, \lambda^{s p^{2} n}$, so that the corresponding substitution is the $s^{\text {th }}$ power of the substitution just considered. Hence the orders of all substitutions having irreducible characteristic determinants are factors of $\frac{1}{d}\left(p^{2 n}+p^{n}+1\right)$.

Consider a substitution $S$ of $G$ of canonical form $A$ for which $\lambda$ is a primitive root of equation 235). By $\S 220$, the only substitutions of $G$ which are commutative with $S$ have, simultaneously with the canonical form $A$ of $S$, the canonical form

$$
x^{\prime}=\sigma^{r} x, \quad y^{\prime}=\sigma^{r p^{n}} y, \quad z^{\prime}=\sigma^{r p^{2 n}} z \quad\left(\sigma^{r\left(1+p^{n}+p^{2 n}\right)}=1\right)
$$

where $\sigma$ is a primitive root of the $G F\left[p^{3 n}\right]$. Hence $r\left(1+p^{n}+p^{2 n}\right)$ must be divisible by $p^{3 n}-1$ and therefore $r$ divisible by $p^{n}-1$. Setting $r=\varrho\left(p^{n}-1\right)$,

$$
\sigma^{r}=\left(\sigma^{p^{n}-1}\right) \varrho=\lambda^{t} \varrho,
$$

since $\sigma^{p^{n}-1}$ is a primitive root of 235) and hence equal to some power $t$ of $\lambda$. The only substitutions of $G$ which are commutative with $S$ are therefore the powers of $S$. It follows that $S$ is one of a set of 


$$
s \equiv \frac{N}{1 / d\left(p^{2 n}+p^{n}+1\right)}
$$

distinct conjugate substitutions, $N$ being the order of $G$.

The only distinct powers of $S$ which have the same characteristic determinant as $S$ are evidently $S, S^{p^{n}}$ and $S^{p^{2 n}}$. To each set of three substitutions such as $S^{r}, S^{r p^{n}}, S^{r p^{2 n}}$ contained in the cyclic group generated by $S$ and all belonging to the same characteristic determinant, there corresponds a set of $s$ distinct conjugate substitutions. Hence there exist in $G$

$$
\frac{1}{3}\left[\frac{1}{d}\left(p^{2 n}+p^{n}+1\right)-1\right]
$$

such sets of $s$ conjugate substitutions. It follows that $G$ contains in all

$$
\frac{d N}{3\left(p^{2 n}+p^{n}+1\right)}\left[\frac{1}{d}\left(p^{2 n}+p^{n}+1\right)-1\right]
$$

substitutions not the identity whose orders are factors of

$$
\frac{1}{d}\left(p^{2 n}+p^{n}+1\right) \text {. }
$$

Hence $G$ contains $\frac{d N}{3\left(p^{2 n}+p^{n}+1\right)}$ distinct conjugate cyclic subgroups of order

$$
\frac{1}{d}\left(p^{2 n}+p^{n}+1\right) \text {. }
$$

22\%. Type $B$. Since $G$ contains substitutions in whose characteristic determinant $-\lambda^{3}+\alpha_{1} \lambda^{2}+\alpha_{2} \lambda+1$ both $\alpha_{1}$ and $\alpha_{2}$ are arbitrary in the $G F\left[p^{n}\right]$, we can choose

so that

$$
\alpha_{1} \equiv \gamma+1 / \delta,-\alpha_{2} \equiv \delta+\gamma / \delta,
$$

$$
\Delta(\lambda) \equiv-(\lambda-1 / \delta)\left(\lambda^{2}-\gamma \lambda+\delta\right),
$$

where $\gamma$ and $\delta$ are arbitrary in the $G E\left[p^{n}\right]$. In particular, $G$ contains a substitution $T$ whose characteristic determinant has an irreducible quadratic factor which vanishes for a primitive root $\mu$ of the $G F\left[p^{2 n}\right]$. The canonical form of $T$ is then $B$. The order of $T$ is therefore the least integer $t$ for which

$$
\mu^{t}=\mu^{t p^{n}}=\mu^{-t\left(p^{n}+1\right)}
$$

i. e., for which both $t\left(p^{n}-1\right)$ and $t\left(p^{n}+2\right)$ are divisible by $p^{2 n}-1$. But $3 t$ and $t\left(p^{n}-1\right)$ are both divisible by $p^{2 n}-1$, for $t$ a minimum, if and only if

$t=p^{2 n}-1$, when $p^{n}=3^{n}$ or $3 l-1 ; \quad t=\frac{1}{3}\left(p^{2 n}-1\right)$, when $p^{n}=3 l+1$.

Hence the order of $T$ is $\frac{1}{d}\left(p^{2 n}-1\right)$. 
By $\S 220$, the most general substitution of $G$ commutative with $T$ has the canonical form

$$
x^{\prime}=\mu^{r} x, \quad y^{\prime}=\mu^{r p^{n}} y, \quad z^{\prime}=\mu^{-r\left(p^{n}+1\right)} z
$$

and hence is $T^{r}$. Hence $T$ is one of a set of $d N \div\left(p^{2 n}-1\right)$ distinct conjugate substitutions. The only distinct powers of $S$ which have the same multipliers as $S$ are $S$ and $S^{p^{n}}$. Hence $G$ contains $\frac{1}{2} \frac{d N}{p^{2 n}-1}$ distinct conjugate cyclic subgroups of order $\frac{1}{d}\left(p^{2 n}-1\right)$.

The number of substitutions of $G$ whose orders are factors of $\frac{1}{d}\left(p^{2 n}-1\right)$ without being factors of $\frac{1}{d}\left(p^{n}-1\right)$, and hence not of $p^{n}-1$, is

$$
\frac{1}{2} N p^{n} /\left(p^{n}+1\right)
$$

In fact, such substitutions form in all

$$
\frac{1}{2 d}\left[\left(p^{2 n}-1\right)-\left(p^{n}-1\right)\right] \equiv \frac{1}{2 d}\left(p^{n}-1\right) p^{n}
$$

different sets; those in each set having the same characteristic determinant. Each set contains $d N \div\left(p^{2 n}-1\right)$ distinct conjugate substitutions. The product of the two numbers gives formula 237).

228. We can exhibit $G$ as a permutation-group on $p^{2 n}+p^{n}+1$ letters. Every linear function $A \xi_{1}+B \xi_{2}+C \xi_{3}$, in which $A, B, C$ are marks not all zero of the $G F\left[p^{n}\right]$, can be put into one of the forms,

$$
\mu\left(\xi_{3}+\varrho \xi_{2}+\sigma \xi_{1}\right), \mu\left(\xi_{2}+\varrho \xi_{1}\right), \mu \xi_{1},
$$

where $\mu, \varrho, \sigma$ are marks of the $G F\left[p^{n}\right]$ and $\mu \neq 0$. Combining into one system $\left\{A \xi_{1}+B \xi_{2}+C \xi_{3}\right\}$ the $p^{n}-1$ linear functions

$$
\mu\left(A \xi_{1}+B \xi_{2}+C \xi_{3}\right),
$$

$\mu$ denoting in succession the $p^{n}-1$ marks $\neq 0$ of the field, we obtain $p^{2 n}+p^{n}+1$ distinct systems,

$$
\left\{\xi_{3}+\varrho \xi_{2}+\sigma \xi_{1}\right\}, \quad\left\{\xi_{2}+\varrho \xi_{1}\right\}, \quad\left\{\xi_{1}\right\} \quad[\rho, \sigma \text { arbitrary marks]. }
$$

Any ternary homogeneous linear substitution replaces the functions $\mu\left(A \xi_{1}+B \xi_{2}+C \xi_{3}\right)$, comprising one system, by linear functions

$$
\mu\left(A \xi_{1}^{\prime}+B \xi_{2}^{\prime}+C \xi_{3}^{\prime}\right) \equiv \mu\left(\alpha \xi_{1}+\beta \xi_{2}+\gamma \xi_{3}\right)
$$

all belonging to a single system. Hence it permutes the above $p^{2 n}+p^{n}+1$ symbols amongst themselves. It follows that $G$ is isomorphic with a permutation-group $G^{\prime}$ on these symbols. But a homogeneous substitution altering none of the symbols must have the form

$$
\xi_{1}^{\prime}=\alpha \xi_{1}, \quad \xi_{2}^{\prime}=\alpha \xi_{2}, \quad \xi_{3}^{\prime}=\alpha \xi_{3} .
$$


If it have determinant unity, it corresponds in $G$ to the identity. Hence $G$ is simply isomorphic with $G^{\prime}$.

The permutation-group $G^{\prime}$ is doubly-transitive. We need only prove that $G^{\prime}$ contains a permutation converting $\left\{\xi_{1}\right\},\left\{\xi_{2}+\xi_{1}\right\}$ into respectively

$$
\left\{A \xi_{1}+B \xi_{2}+C \xi_{3}\right\}, \quad\left\{A^{\prime} \xi_{1}+B^{\prime} \xi_{2}+C^{\prime} \xi_{3}\right\}
$$

the latter being any two distinct symbols, viz.,

$$
A: B: C \neq A^{\prime}: B^{\prime}: C^{\prime} \text {. }
$$

For the corresponding homogeneous substitution, we may take

$$
\begin{aligned}
& \xi_{1}^{\prime}=A \xi_{1}+B \xi_{2}+C \xi_{3}, \quad \xi_{2}^{\prime}=\left(A^{\prime}-A\right) \xi_{1}+\left(B^{\prime}-B\right) \xi_{2}+\left(C^{\prime}-C\right) \xi_{3}, \\
& \xi_{3}^{\prime}=\alpha \xi_{1}+\beta \xi_{2}+\gamma \xi_{3},
\end{aligned}
$$

where $\alpha, \beta, \gamma$ are chosen in any manner such that the determinant of the substitution is unity, viz.,

$$
\alpha\left|\begin{array}{ll}
B & C \\
B^{\prime} & C^{\prime}
\end{array}\right|+\beta\left|\begin{array}{ll}
C & A \\
C^{\prime} & A^{\prime}
\end{array}\right|+\gamma\left|\begin{array}{cc}
A & B \\
A^{\prime} & B^{\prime}
\end{array}\right|=1 .
$$

By hypothesis the determinants are not all zero, so that solutions $\alpha, \beta, \gamma$ in the $G F\left[p^{n}\right]$ certainly exist.

229. Type $D$ for $\alpha^{3} \neq 1$. Let $\alpha$ be a primitive root in the $G F\left[p^{n}\right]$, the cases $p^{n}=2$ and $p^{n}=2^{2}$ being necessarily excluded. For such an $\alpha$, substitution $D$ generates a cyclic group of order $\frac{1}{d} p\left(p^{n}-1\right)$.

Considered as an operation of the isomorphic permutation-group, $D$ belongs to a subgroup of $G$ which leaves fixed the symbols $\{x\}$ and $\{y\}$. The general substitution of $G$ possessing this property has the form

$$
R: \quad x^{\prime}=\gamma x, \quad y^{\prime}=\beta y, \quad z^{\prime}=\alpha z+\alpha^{\prime} y+\alpha^{\prime \prime} x \quad(\alpha \beta \gamma=1) .
$$

In order that $R$ shall have the order $\frac{1}{d} p\left(p^{n}-1\right)$, it is necessary and sufficient that $\alpha$ be a primitive root in the $G F\left[p^{n}\right]$ and that either

(i) $\alpha^{\prime} \neq 0, \quad \alpha=\beta \neq \gamma$; or (ii) $\quad \alpha^{\prime \prime} \neq 0, \quad \alpha=\gamma \neq \beta$.

In fact, if both $\beta$ and $\gamma$ differ from $\alpha, R$ may be given the form

$$
x^{\prime}=\gamma x, \quad y^{\prime}=\beta y, \quad Z^{\prime}=\alpha Z,
$$

whose $\left(p^{n}-1\right)^{\text {st }}$ power is unity, by introducing in place of $z$ the index

$$
Z \equiv z+\frac{\alpha^{\prime}}{\alpha-\beta} y+\frac{\alpha^{\prime \prime}}{\alpha-\gamma} x
$$

Hence, if $\alpha \neq \beta$, we may take $\alpha=\gamma$. Then $\alpha^{\prime \prime} \neq 0$; for, if $\alpha^{\prime \prime}=0$, $R$ multiplies $z+\frac{\alpha^{\prime}}{\alpha-\beta} y$ by $\alpha$, so that $R$ would have as order a factor 
of $p^{n}-1$. Similarly, if $\alpha \neq \gamma$, then must $\alpha=\beta, \alpha^{\prime} \neq 0$. Finally, if $\alpha=\beta=\gamma$, each may be taken equal to unity. Then, by induction,

$R^{r}: \quad x^{\prime}=x, \quad y^{\prime}=y, \quad z^{\prime}=z+r \alpha^{\prime} y+r \alpha^{\prime \prime} x$,

so that $R$ would have the period $p$. Hence either (i) or (ii) must be satisfied.

Suppose, inversely, that relations ( $i)$ are satisfied. Setting

$R$ takes the form

$$
Y \equiv \frac{\alpha^{\prime}}{\alpha} y, \quad Z \equiv z+\frac{\alpha^{\prime \prime} x}{\beta-\gamma},
$$

$$
x^{\prime}=\alpha^{-2} x, \quad Y^{\prime}=\alpha Y, \quad Z^{\prime}=\alpha(Z+Y),
$$

and is thus of period $\frac{1}{d} p\left(p^{n}-1\right)$ if, and only if, $\alpha$ be a primitive root of the $G F\left[p^{n}\right]$. Interchanging $x$ with $y$, the proof follows for case (ii).

Using the theorem just proved, we proceed to determine the number and conjugacy of the cyclic subgroups of order $\frac{1}{d} p\left(p^{n}-1\right)$ which leave the symbols $\{x\}$ and $\{y\}$ fixed. For case (i),

$R: \quad x^{\prime}=\alpha^{-2} x, \quad y^{\prime}=\alpha y, \quad z^{\prime}=\alpha z+\alpha^{\prime} y+\alpha^{\prime \prime} x \quad\left(\alpha^{\prime} \neq 0, \alpha^{3} \neq 1\right)$, where $\alpha$ is a primitive root of the GF[ $\left.p^{n}\right]$. By induction we find

$$
R^{t}: x^{\prime}=\alpha^{-2 t} x, y^{\prime}=\alpha^{t} y, \quad z^{\prime}=\alpha^{t} z+t \alpha^{\prime} \alpha^{t-1} y+\alpha^{\prime \prime} \alpha^{t-1}\left(\frac{\alpha^{-3 t}-1}{\alpha^{-3}-1}\right) x .
$$

In order that $\Theta^{r} R^{t}$ shall be identical with the substitution

$$
x^{\prime}=\alpha^{-2} x, \quad y^{\prime}=\alpha y, \quad z^{\prime}=\alpha z+\varrho^{\prime} y+\varrho^{\prime \prime} z,
$$

it is necessary and sufficient that

$$
\theta^{r} \alpha^{t-1}=1, \quad t \alpha^{\prime}=\varrho^{\prime}, \quad \alpha^{\prime \prime}=\varrho^{\prime \prime} .
$$

Let $M_{i}$ denote any one of the $\left(p^{n}-1\right) /(p-1)$ distinct marks $M_{1}$, $M_{2}, \ldots$ such that no two have as their ratio an integral mark $\left.{ }^{1}\right)$. If $\alpha$ be a fixed mark $\neq 0$ and $M$ an arbitrary mark, the $p^{n}\left(p^{n}-1\right) /(p-1)$ substitutions

238)

$$
x^{\prime}=\alpha^{-2} x, \quad y^{\prime}=\alpha y, \quad z^{\prime}=\alpha z+M_{i} y+M x
$$

have the property that no power of any one of them reduces to one of the set. We therefore obtain that number of cyclic subgroups of order $\frac{1}{d} p\left(p^{n}-1\right)$.

Furthermore, every substitution $V$ of the subgroup leaving $\{x\}$ and $\{y\}$ fixed, and having $\alpha=\beta$, and of order a divisor of $\frac{1}{d} p\left(p^{n}-1\right)$

1) The marks $M_{1}, M_{2}, \ldots$ are evidently the multipliers in a rectangular array of the marks $=0$ of the $G F\left[p^{n}\right]$, the first row being formed by the integral marks $1,2, \ldots, p-1$. 
without being a factor of $p$ or $p^{n}-1$, is contained in one of the above cyclic subgroups. In fact, by the earlier argument, we may set

$V: \quad x^{\prime}=\alpha^{-2 s} x, \quad y^{\prime}=\alpha^{s} y, \quad z^{\prime}=\alpha^{s} z+\alpha^{\prime} y+\alpha^{\prime \prime} x \quad\left(\alpha^{\prime} \neq 0, \alpha^{3 s} \neq 1\right)$.

Let $M_{i}$ be a mark $\neq 0$ such that its ratio to $\alpha^{\prime} \alpha^{1-s}$ is an integral mark. The power $s+k\left(p^{n}-1\right)$ of 238$)$ gives

$$
\begin{aligned}
& x^{\prime}=\alpha^{-2 s} x, \quad y^{\prime}=\alpha^{s} y, \\
& z^{\prime}=\alpha^{s} z+\left[s+k\left(p^{n}-1\right)\right] \alpha^{s-1} M_{i} y+M \alpha^{s-1}\left(\frac{\alpha^{-3 s}-1}{\alpha^{-3}-1}\right) x .
\end{aligned}
$$

By choice of $k$ and $M$, we can make the coefficient of $y$ in $z^{\prime}$ equal $\alpha^{\prime}$ and that of $x$ equal $\alpha^{\prime \prime}$.

Hence there are $p^{n}\left(p^{n}-1\right) /(p-1)$ cyclic subgroups of $G$ of order $\frac{1}{d} p\left(p^{n}-1\right)$ for which $\alpha=\beta$, and as many more for which $\alpha=\gamma$, each leaving the symbols $\{x\}$ and $\{y\}$ fixed, and together containing all substitutions having the last property and having an order not $p$ nor a factor of $p^{n}-1$.

These cyclic subgroups are all conjugate within $G$ and, indeed, within the subgroup which leaves fixed $\{x\}$ and $\{y\}$ or merely permutes them. First, the substitution

$$
x^{\prime}=x, \quad y^{\prime}=y, \quad z^{\prime}=z+\frac{M^{\prime}-M}{\alpha^{-2}-\alpha} x
$$

transforms 238) into a like substitution with $M^{\prime}$ in place of $M$. Also

$$
x^{\prime}=\lambda^{-1} \varrho^{-2} x, \quad y^{\prime}=\varrho y, \quad z^{\prime}=\lambda \varrho z
$$

transforms 238) into the substitution

$$
x^{\prime}=\alpha^{-2} x, \quad y^{\prime}=\alpha y, \quad z^{\prime}=\alpha z+\lambda M_{i} y+\lambda^{2} \varrho^{3} M x .
$$

Hence the cyclic subgroups given by $\alpha=\beta$ are all conjugate within the group leaving fixed $\{x\}$ and $\{y\}$. These symbols are interchanged by

$$
x^{\prime}=y, \quad y^{\prime}=-x, \quad z^{\prime}=z,
$$

which transforms 238) into the substitution

$$
x^{\prime}=\alpha x, \quad y^{\prime}=\alpha^{-2} y, \quad z^{\prime}=\alpha z-M y+M_{i} x .
$$

Hence the set of cyclic subgroups given by $\alpha=\beta$ are conjugate to the set given by $\alpha=\gamma$ within the group leaving fixed the symbols $\{x\}$ and $\{y\}$ or permuting them. The latter group consequently contains $2 p^{n}\left(p^{n}-1\right) /(p-1)$ conjugate cyclic groups of order $\frac{1}{d} p\left(p^{n}-1\right)$ and those substitutions of these groups whose orders are not divisors of $p$ or $p^{n}-1$ are all distinct. Since the permutationgroup isomorphic with $G$ is doubly transitive, it contains 


$$
\frac{1}{2}\left(p^{2 n}+p^{n}+1\right)\left(p^{2 n}+p^{n}\right)
$$

conjugate subgroups leaving fixed or permuting the two symbols. Hence there are altogether

$$
2 p^{n}\left(\frac{p^{n}-1}{p-1}\right) \cdot \frac{1}{2}\left(p^{2 n}+p^{n}+1\right)\left(p^{2 n}+p^{n}\right) \equiv \frac{d N}{p^{n}\left(p^{n}-1\right)(p-1)}
$$

conjugate cyclic subgroups of order $\frac{1}{d} p\left(p^{n}-1\right)$. Each contains $p+\frac{1}{d}\left(p^{n}-1\right)-1$ substitutions of period $p$ or a divisor of $\frac{1}{d}\left(p^{n}-1\right)$. There remain in each cyclic group $(p-1)\left[\frac{1}{d}\left(p^{n}-1\right)-1\right]$ substitutions. Hence $G$ contains

$$
N\left(p^{n}-1-d\right) \div p^{n}\left(p^{n}-1\right)
$$

substitutions whose orders divide $\frac{1}{d} p\left(p^{n}-1\right)$ but not $p$ or $p^{n}-1$.

For the cases $p^{n}=2$ and $p^{n}=2^{2}$ above excluded, formula 239) reduces to zero. Hence the result is always true.

230. Type $D$ when $\alpha^{3}=1$. We are to consider substitutions of period $p$ having the canonical form:

$$
D^{\prime}: \quad x^{\prime}=x, \quad y^{\prime}=y, \quad z^{\prime}=z+y .
$$

From the investigation at the beginning of $\S 229$ it follows that the only substitutions of period $p$ which leave fixed the symbols $\{x\}$ and $\{y\}$ have the form

240) $x^{\prime}=x, y^{\prime}=y, \quad z^{\prime}=z+\alpha x+\beta y \quad$ ( $\alpha$ and $\beta$ not both zero).

There are $p^{2 n}-1$ distinct substitutions of this form. They are all conjugate to $D^{\prime}$ within $G$. In fact, if $\beta \neq 0$, the substitution

$$
x^{\prime}=x, \quad y^{\prime}=y+\varrho x, \quad z^{\prime}=z
$$

transforms 240) into

$$
x^{\prime}=x, \quad y^{\prime}=y, \quad z^{\prime}=z+(\alpha-\beta \varrho) x+\beta y .
$$

By choice of $\varrho$, we can make $\alpha-\beta \varrho=0$. If $\beta=0$, we transform 240) by

and get

$$
\begin{aligned}
& x^{\prime}=y, \quad y^{\prime}=x, \quad z^{\prime}=-z, \\
& x^{\prime}=x, \quad y^{\prime}=y, \quad z^{\prime}=z-\alpha y .
\end{aligned}
$$

In either case we reach a substitution of the form 230). but having $\alpha=0, \beta \neq 0$. It is transformed into $D^{\prime}$ by the substitution of $G$

$$
x^{\prime}=\beta^{-1} x, \quad y^{\prime}=\beta y, \quad z^{\prime}=z .
$$


The $p^{2 n}-1$ substitutions 230$)$ determine $\left(p^{2 n}-1\right) /(p-1)$ conjugate cyclic subgroups of order $p$ contained in the subgroup of $G$ which leaves fixed the symbols $\{x\}$ and $\{y\}$ and hence also $\{x+\varrho y\}$, $\varrho$ being an arbitrary mark of the $G F\left[p^{n}\right]$.

Each such group therefore leaves fixed $p^{n}+1$ (and no more) symbols. But the $p^{2 n}+p^{n}+1$ symbols furnish

$$
\frac{\frac{1}{2}\left(p^{2 n}+p^{n}+1\right)\left(p^{2 n}+p^{n}\right)}{\frac{1}{2}\left(p^{n}+1\right) p^{n}} \equiv p^{2 n}+p^{n}+1
$$

such sets of symbols. Hence $G$ contains

$$
\left(p^{2 n}+p^{n}+1\right) \frac{\left(p^{2 n}-1\right)}{(p-1)} \equiv \frac{d N}{p^{3 n}\left(p^{n}-1\right)(p-1)}
$$

such conjugate cyclic'subgroups, all of whose substitutions are conjugate under $G$. Each such subgroup is therefore contained selfconjugately within a subgroup of order $\frac{1}{d} p^{3 n}\left(p^{n}-1\right)(p-1)$. The total number of distinct substitutions of $G$ of order $p$ of the type considered has thus been shown to be

$$
\frac{d N}{p^{3 n}\left(p^{n}-1\right)} \text {. }
$$

231. Types $E_{i}$. By induction we find that

$$
E_{0}^{t}: \quad x^{\prime}=x, \quad y^{\prime}=y+t x, \quad z^{\prime}=z+t y+\frac{1}{2} t(t-1) x .
$$

Hence $E_{0}$ is of period $p$ or 4 according as $p>2$ or $p=2$. The most general substitution of $G$ transforming $E_{0}$ into itself is

$$
x^{\prime}=a x, \quad y^{\prime}=a y+b x, \quad z^{\prime}=a z+b y+c x \quad\left(a^{3}=1\right) .
$$

Exactly $p^{2 n}$ of these substitutions are distinct in the group $G$.

Suppose first that $p>2$. For any positive integer $t<p$, the substitution

$$
x^{\prime}=\frac{1}{t} x, \quad y^{\prime}=y-\frac{t-1}{2 t} x, \quad z^{\prime}=t z
$$

is of determinant unity and transforms $E_{0}$ into $E_{0}^{t}$. Taking

$$
t=1,2, \ldots, p-1 \text {, }
$$

we see that $G$ contains exactly $p^{2 n}(p-1)$ distinct substitutions which transform into itself the cyclic group generated by $E_{0}$. The cyclic group $\left\{E_{0}\right\}$ is, for $p>2$, one of $N / p^{2 n}(p-1)$ distinct conjugate subgroups of $G$. In particular, $G$ contains $N / p^{2 n}$ distinct conjugate substitutions of the type $E_{0}$.

Suppose next that $p=2$. Then $E_{0}$ is of period 4 . Since

$$
E_{0}^{2}: \quad x^{\prime}=x, \quad y^{\prime}=y, \quad z^{\prime}=z+x
$$

leaves fixed the $2^{n}+1$ symbols $\{x\},\{y+\lambda x\}, \lambda$ any mark of the $G F\left[2^{n}\right]$, while $E_{0}$ leaves fixed but one symbol $\{x\}$, the two sub- 
stitutions are not conjugate under $G$. But $E_{0}$ is transformed into $E_{0}^{3}$ by the substitution 242) for $t=3$, viz.,

$$
x^{\prime}=x, \quad y^{\prime}=y+x, \quad z^{\prime}=z .
$$

The cyclic group generated by $E_{0}$ is therefore transformed into itself by exactly $2 \cdot 2^{2 n}$ substitutions of $G$. For $p=2,\left\{E_{0}\right\}$ is one of a complete set of $N / 2^{2 n+1}$ conjugate cyclic subgroups of $G$. Just two of the four substitutions of every such cyclic group are of type $E_{0}$, while the remaining one not the identity is of type $D$ with $\alpha^{3}=1$. Hence, for $p=2, G$ contains $N / 2^{2 n}$ distinct substitutions conjugate with $E_{0}$.

Since $E_{1}$ and $E_{2}$ are conjugate to $E_{0}$ within the general ternary linear homogeneous group in the $G F\left[p^{n}\right]$, the number of substitutions of $G$ conjugate to $E_{0}$ within $G$ equals the number conjugate to $E_{1}$ or the number conjugate to $E_{2}$. Hence $G$ contains altogether

$$
3 N / p^{2 n}
$$

distinct substitutions of the canonical forms $E_{i}$; they form three distinct sets of conjugate substitutions under $G$. Also, $E_{0}, E_{1}, E_{2}$ each lead to the same number of conjugate cyclic subgroups of $G$.

232. Type $C$. The substitutions of canonical form $C$ are of order a divisor of $p^{n}-1$. Of the $\left(p^{n}-1\right)^{2}$ sets of solutions in the $G F\left[p^{n}\right]$ of $\alpha \beta \gamma=1, \alpha$ sets have $\alpha=\beta=\gamma$ and hence each equal to $\theta^{r}(r=0,1$, or 2$)$. If $\alpha$ be any mark different from $0,1, \theta, \theta^{2}$, and if $\beta=\alpha$, then $\gamma=\alpha^{-2} \neq \alpha$. Hence there are $3\left(p^{n}-d-1\right)$ sets of solutions in which two and only two of the quantities $\alpha, \beta, \gamma$ are equal. There remain

$$
\left(p^{n}-1\right)^{2}-3\left(p^{n}-d-1\right)-d \equiv p^{2 n}-5 p^{n}+4+2 d
$$

sets of solutions in which $\alpha, \beta, \gamma$ are all distinct. Dividing this number by 6 to allow for permutations, we obtain the number of distinct sets of unequal multipliers of ternary homogeneous substitutions $C$.

If, for $d=3, \alpha, \beta, \gamma$ do not form a permutation of $1, \theta, \theta^{2}$, the three sets

$$
\alpha, \beta, \gamma ; \theta \alpha, \theta \beta, \theta \gamma ; \theta^{2} \alpha, \theta^{2} \beta, \theta^{2} \gamma,
$$

are not equivalent sets of multipliers in the homogeneous group, but are equivalent in the non-homogeneous group $G$. The number of sets of unequal multipliers in $G$ is therefore

$1+\frac{1}{3}\left(\frac{p^{2} n-5 p^{n}+4+2 d}{6}-1\right)$, for $d=3 ; \frac{p^{2} n-5 p^{n}+4+2 d}{6}$, for $d=1$. 
We proceed to prove that the total number of substitutions of $G$ of canonical form $C$ with $\alpha, \beta, \gamma$ distinct is, for $d=1$ or 3 ,

$$
\frac{N}{\left(p^{n}-1\right)^{2}} \cdot \frac{p^{2 n}-5 p^{n}+4+2 d}{6} \text {. }
$$

By $\S 220$, the only ternary homogeneous substitutions commutative with $C$ with $\alpha, \beta, \gamma$ distinct are the $\left(p^{n}-1\right)^{2}$ substitutions

$$
T: \quad x^{\prime}=a x, \quad y^{\prime}=b y, \quad z^{\prime}=c z \quad(a b c=1) .
$$

For $d=1$, each set of unequal multipliers therefore leads to $N /\left(p^{n}-1\right)^{2}$ conjugate substitutions, so that we obtain the number 244). For $d=3$, the substitutions $T$ give only $\frac{1}{d}\left(p^{n}-1\right)^{2}$ distinct substitutions in $G$. Furthermore, by $\S 102, C$ can be transformed into $\theta C$ if, and only if, the multipliers $\alpha, \beta, \gamma$ form a permutation of $1, \theta, \theta^{2}$. The special substitution $C$,

$$
x^{\prime}=x, \quad y^{\prime}=\theta y, \quad z^{\prime}=\theta^{2} z
$$

is transformed into $C, \Theta C$ or $\Theta^{2} C$ by exactly the $3\left(p^{n}-1\right)^{2}$ products $T,(x y z) T,(x z y) T$. The corresponding substitution is therefore one of $N /\left(p^{n}-1\right)^{2}$ distinct conjugate substitutions under $G$. Each of the remaining substitutions $C$ with unequal multipliers is one of a set of $N \div \frac{1}{3}\left(p^{n}-1\right)^{2}$ conjugate substitutions under $G$.

Corresponding to the $p^{n}-d-1$ sets of multipliers $\alpha, \beta, \gamma$ of which two are equal, there are $\frac{1}{d}\left(p^{n}-d-1\right)$ substitutions $C^{\prime}$ of $G$, no two of which are conjugate. Such a substitution

$$
C^{\prime}: \quad x^{\prime}=\alpha x, \quad y^{\prime}=\alpha y, \quad z^{\prime}=\gamma z \quad\left(\alpha^{2} \gamma=1, \gamma \neq \alpha\right)
$$

cannot be transformed into $\Theta C^{\prime}$. By $\S 218$, the most general ternary linear homogeneous substitution which transforms $C^{\prime}$ into itself is

$$
x^{\prime}=a x+b y, \quad y^{\prime}=a^{\prime} x+b^{\prime} y, \quad z^{\prime}=c^{\prime \prime} z .
$$

The number of such substitutions in the GF[p $\left.p^{n}\right]$ of determinant unity is

$$
\left(p^{2 n}-1\right)\left(p^{2 n}-p^{n}\right) \text {. }
$$

Hence the total number of substitutions in $G$ of the canonical form $C^{\prime}$ is

$$
\frac{1}{d}\left(p^{n}-d-1\right) \cdot \frac{N}{\frac{1}{d}\left(p^{2 n}-1\right)\left(p^{2} n-p^{n}\right)} .
$$

233. As a check upon the accuracy of our enumeration of the substitutions of $G$, we may verify that the numbers given by the formulae 236), 237), 239), 241), 243), 244) and 245), together with unity, to count the identical substitution, give as total sum the order $N$ of the group $G$. 
234. To complete the enumeration of the cyclic subgroups of $G$, it remains to determine those generated by substitutions of the canonical forms $C$. The method will be sufficiently illustrated if we confine the investigation to the case $d=1 .^{1}$ ) If $\alpha$ be a primitive root of the $G F\left[p^{n}\right]$, we may set

$$
C: \quad x^{\prime}=\alpha^{r} x, \quad y^{\prime}=\alpha^{s} y, \quad z^{\prime}=\alpha^{-r-s} z,
$$

where $r$ and $s$ are integers chosen from the series $0,1, \ldots, p^{n}-2$. Let $g$ denote the greatest common divisor of $r$ and $s$. The period of $C$ is the least positive integer $l$ for which $l r$ and $l s$, and therefore also $l g$, are multiples of $p^{n}-1$. Hence $C$ is of period $p^{n}-1$ if, and only if, $g$ be relatively prime to $p^{n}-1$. In general, $C$ is the $g^{\text {th }}$ power of a similar substitution with the multipliers $\alpha^{r / g}, \alpha^{s / g}, \alpha^{(-r-s) / g}$, the latter of period $p^{n}-1$. Hence, for $d=1$, the substitutions of type $C$ are all included in the cyclic groups generated by those substitutions of type $C$ which have the period $p^{n}-1$. We may therefore confine our attention to these largest cyclic groups. The exponents $r, s$ in the expression of any substitution $C$ of period $p^{n}-1$ must occur among the sets of two positive integers less than $p^{n}-1$ and having their greatest common divisor prime to $p^{n}-1$. Denote by $F\left(p^{n}-1\right)$ the number of such sets. A similar remark holds for the couples $s, r ; r,-r-s ;-r-s, r ; s,-r-s ;-r-s, s ;$ provided $-r-s$ be replaced by its least positive residue modulo $p^{n}-1$. If $r, s,-r-s$ be distinct, the above couples form six of the $F\left(p^{n}-1\right)$ sets, but lead to the same set of three multipliers in $C$. If two of the exponents be equal and therefore different from the third, we may take them to be $r, r,-2 r$. Then the couples $r, r ; r,-2 r ;-2 r, r$ form three of the $F\left(p^{n}-1\right)$ sets, but lead to the same set of multipliers in $C$. Here $r$ may be any one of the $\Phi\left(p^{n}-1\right)$ integers less than and prime to $p^{n}-1$. Hence there are $3 \Phi\left(p^{n}-1\right)$ sets leading to $\Phi\left(p^{n}-1\right)$ distinct sets of multipliers two of which are equal, while the remaining sets lead to $\frac{1}{6}\left[F\left(p^{n}-1\right)-3 \Phi\left(p^{n}-1\right)\right]$ distinct sets of three unequal multipliers, together yielding all the substitutions $C$ of period $p^{n}-1$. The value of $F\left(p^{n}-1\right)$ is given by the following theorem. ${ }^{2}$ )

The number of sets of two integers, not both zero, chosen from the series $0,1, \ldots, k-1$ so that their greatest common divisor is prime to $k$ is $F(k) \equiv k^{2}-\sum_{i=1}^{*} \frac{k^{2}}{q_{i}^{2}}+\sum_{i, j}^{1, \ldots,{ }^{2}} \frac{k^{2}}{q_{i}^{2} q_{j}^{2}}-\cdots \equiv k^{2}\left(1-\frac{1}{q_{1}^{2}}\right)\left(1-\frac{1}{q_{2}^{2}}\right) \cdots\left(1-\frac{1}{q_{*}^{2}}\right)$ where $q_{1}, q_{2}, \ldots, q_{k}$ are the distinct prime factors of $k$.

1) The case $d=3$ is more intricate and the results quite complicated. The results are given in the Amer. Journ., vol. XXII, p. 251; the proofs in vol. XXIV.

2) Jordan, Traité, p. 96. 
Of the $k^{2}$ sets of two integers each $<k, k^{2} / q_{i}^{2}$ have their integers chosen from the $k / q_{i}$ multiples of $q_{i}$ and are to be excluded. We thereby exclude, in particular, the sets of integers each of which is one of the $k / q_{i} q_{j}$ multiples of $q_{i} q_{j}$. Hence, in afterwards excluding the sets of integers each of . which is a multiple of $q_{j}$, we subtract the number $k^{2} / q_{j}^{2}-k^{2} / q_{i}^{2} q_{j}^{2}$. After the required exclusions have all been made, there evidently remains the number of sets indicated by $F(k)$. Among the latter sets, the couple 0,0 does not occur since

$$
1-x+\frac{x(x-1)}{1 \cdot 2}-\frac{x(x-1)(x-2)}{1 \cdot 2 \cdot 3}+\cdots \equiv(1-1)^{x}=0 .
$$

235. A cyclic group generated by a substitution $C$ of period $p^{n}-1$.will be called special if two of its substitutions $C^{a}, C^{b}$ of period $p^{n}-1$ are conjugate within $G$, i. e., have the same. set of multipliers. Since $a$ and $b$ must be prime to $p^{n}-1$, the condition requires that $C$ and $C^{b a_{1}}$ shall have the same set of multipliers, where $a_{1}$ is determined from $a a_{1} \equiv 1\left(\bmod p^{n}-1\right)$. It thus suffices to investigate when $C$ and $C^{m}$ have the same multipliers, $m$ being prime to $p^{n}-1$ and $1<m<p^{n}-1$. The three distinct ways in which the two sets

$$
\alpha^{r}, \alpha^{s}, \alpha^{t} ; \alpha^{m r}, \alpha^{m s}, \alpha^{m t} \quad r+s+t \equiv 0\left(\bmod p^{n}-1\right)
$$

may be identical in some order will be considered in turn.

i) If $\alpha^{m r}=\alpha^{r}, \alpha^{m s}=\alpha^{s}, \alpha^{m t}=\alpha^{t}$, then $r(m-1), s(m-1)$, and therefore also $g(m-1)$, are divisible by $p^{n}-1$. Since $g$ is prime to $p^{n}-1, m-1$ must be divisible by $p^{n}-1$, contrary to hypothesis.

ii) If $\alpha^{m r}=\alpha^{s}, \alpha^{m s}=\alpha^{r}, \alpha^{m t}=\alpha^{t}$, then must

$$
m r \equiv s, \quad m s \equiv r, \quad m^{2} r \equiv r\left(\bmod p^{n}-1\right) .
$$

Then $r$ must be prime to $p^{n}-1$; for a common factor would divide $s$ in virtue of the first congruence, whereas the greatest common divisor of $r$ and $s$ is prime to $p^{n}-1$. Hence, by the last congruence,

$$
m^{2} \equiv 1 \quad\left(\bmod p^{n}-1\right)
$$

Inversely, if $m$ be any solution of 246) and if $r$ be any integer less than and prime to $p^{n}-1$ and if $s$ be determined by

$$
s \equiv m r \quad\left(\bmod p^{n}-1\right)
$$

then $C$ and $C^{m}$ have the same multipliers. Moreover, $C$ is the $r^{\text {th }}$ power of a substitution with the multipliers $\alpha, \alpha^{m} ; \alpha^{-m-1}$, which may therefore be taken in place of $C$ as generator of the special cyclic group.

If $2^{k}$ be the highest power of 2 contained in $p^{n}-1$ and if $\dot{x}=0$ when $k=0$ or $1, x=1$ when $k=2, x=2$ when $k>3$, and if $\mu$ be 
the number of distinct odd prime factors of $p^{n}-1$, then the congruence 246) has exactly $2^{x+\mu}$ solutions $m .{ }^{1}$ ) The solution $m \equiv 1$ is to be excluded. Consider the $2^{x+\mu}-1$ substitutions with the multipliers $\alpha, \alpha^{m}, \alpha^{-m-1}, m>1$. They generate as many cyclic groups. In fact, $\left(\alpha^{m}\right)^{x}=\alpha$ requires $x \equiv m\left(\bmod p^{n}-1\right)$; while $\left(\alpha^{-m-1}\right)^{y}=\alpha$ is impossible since $m+1$ has a factor $>1$ in common with $p^{n}-1$. Moreover, the sets of multipliers of the substitutions of period $p^{n}-1$ in each cyclic group are the same in pairs. Hence these special cyclic groups contain altogether $\frac{1}{2} \Phi\left(p^{n}-1\right)\left(2^{x+\mu}-1\right)$ distinct sets of unequal multipliers.

(iii) If $\alpha^{m r}=\alpha^{s}, \alpha^{m s}=\alpha^{t}$, $\alpha^{m t}=\alpha^{r}$, we find that $r\left(m^{2}+m+1\right) \equiv t+s+r \equiv 0, \quad s\left(m^{2}+m+1\right) \equiv r+t+s \equiv 0 \quad\left(\bmod p^{n}-1\right)$. Hence $M \equiv m^{2}+m+1$ must be divisible by $p^{n}-1$. Since $m(m+1)$ is even, $M$ is an odd number. Hence $p^{n}-1$ must be odd and therefore $p^{n}=2^{n}$. Since $d=1,3$ is not a factor of $p^{n}-1$. Hence each prime factor $q$ of $p^{n}-1$ is of one of the forms $6 k+5,6 k+1$. Now $M$ and hence also $m^{3}-1$ must be divisible by $q$. If $q=6 k+5$, Fermat's theorem gives $m^{6 k+4} \equiv 1(\bmod q)$. Since $m^{3} \equiv 1$, we have $m \equiv 1(\bmod q)$ and therefore $M \equiv 3 \equiv 0(\bmod q)$, which is impossible. Hence must $q=6 k+1$. Inversely, if $q=6 k+1, m^{6 k}-1 \equiv 0(\bmod q)$ has $6 k$ distinct integral solutions. But the left member is divisible by $m^{3}-1$ and therefore by $M$. Hence $M \equiv 0(\bmod q)$ has two distinct solutions. Each of these solutions leads to one, and but one, solution of $M \equiv 0\left(\bmod q^{\tau}\right)$. To give a proof by induction from $\tau=e$ to $\tau=e+1$, let $m^{3}-1=Q q^{e}$. Then

$$
\left(m+x q^{e}\right)^{3}-1 \equiv Q q^{e}+3 m^{2} x q^{e} \quad\left(\bmod q^{2 e}\right)
$$

and will therefore be divisible by $q^{e+1}$ if, and only if,

$$
Q+3 m^{2} x \equiv 0 \quad(\bmod q) \text {. }
$$

Since 3 and $m$ are prime to $q, x$ is uniquely determined $\bmod q$. Hence each $m$ determines one solution $y \equiv m+x q^{e}$ of

$$
y^{3}-1 \equiv 0 \quad\left(\bmod q^{\circ+1}\right) .
$$

Hence, if $m^{2}+m+1$ be divisible by $q^{2}, y-1$ will be prime to $q$ and hence $y^{2}+y+1$ will be divisible by $q^{e+1}$. Supposing that the prime factors of $2^{n}-1$ are all of the form $6 k+1$ and that the number of distinct ones is $\gamma$, it follows that $M \equiv 0\left(\bmod 2^{n}-1\right)$ has $2^{\gamma}$ solutions $m$. But, if $m$ be a solution, then $-m-1$ will be

1) Dirichlet, Zahlentheorie, § 37 .

DICKsoN, Linear Groups. 
a second solution. Hence $C$ is the $r^{\text {th }}$ power of one of the $2^{\gamma-1}$ substitutions with the multipliers $\alpha, \alpha^{m}, \alpha^{-m-1}$. These generate distinct cyclic groups, since $\left(\alpha^{m}\right)^{x}=\alpha$ requires $x=-m-1$. Hence there are $2^{\gamma-1}$ of these special cyclic groups and the substitutions of period $p^{n}-1$ in each give just $\frac{1}{3} \Phi\left(p^{n}-1\right)$ distinct sets of multipliers.

Excluding the special sets of multipliers of types (ii) and (iii), there remain

$\frac{1}{6}\left[F\left(p^{n}-1\right)-3 \Phi\left(p^{n}-1\right)\right]-\frac{1}{2} \Phi\left(p^{n}-1\right)\left(2^{x+\mu}-1\right)-\frac{1}{3} \Phi\left(p^{n}-1\right) 2^{\gamma-1}$

sets of unequal multipliers, the last term occurring only for certain values of $p^{n}$. The corresponding substitutions $C$ lie in sets of $\Phi\left(p^{n}-1\right)$ in cyclic subgroups not conjugate under $G$. Noting that $F\left(p^{n}-1\right)$ is divisible by $\Phi\left(p^{n}-1\right)$, giving the quotient

$$
\Psi\left(p^{n}-1\right) \equiv\left(p^{n}-1\right)\left(1+\frac{1}{q_{1}}\right)\left(1+\frac{1}{q_{2}}\right) \cdots\left(1+\frac{1}{q_{\gamma}}\right),
$$

where $q_{1}, q_{2}, \ldots, q_{\gamma}$ are the distinct prime factors of $p^{n}-1$, we may combine our results in the theorem:

- If $p^{n}-1$ be not divisible by 3 , the substitutions $C$ generate the following types of cyclic groups of order $p^{n}-1$ not conjugate under $G$ : $\alpha, \alpha, \alpha^{-2}$

a) one group generated by the substitution with multipliers

b) $2^{x+\mu}-1$ generated by substitutions with multipliers $\alpha, \alpha^{m}$, $\alpha^{-m-1}$, where $m^{2} \equiv 1\left(\bmod p^{n}-1\right), x$ and $\mu$ defined in (ii);

c) $2^{\gamma-1}$ generated by similar substitutions with

$$
m^{2}+m+1 \equiv 0 \quad\left(\bmod p^{n}-1\right),
$$

occurring only when $p^{n}-1 \equiv 2^{n}-1$ has only prime factors ( $\gamma$ distinct ones) of the form $6 j+1$;

d) $\frac{1}{6}\left[\Psi\left(p^{n}-1\right)-3\right]-\frac{1}{2}\left(2^{x+\mu}-1\right)-\frac{1}{3} \cdot 2^{\gamma-1}$ further groups.

236. As a first example, let $p^{n}=8$, so that $\mu=1, \varkappa=0, \gamma=1$. There is just one cyclic group of each of the first three types. The generators have the sets of multipliers $\alpha, \alpha, \alpha^{-2} ; \alpha, \alpha^{-1}, 1 ; \alpha, \alpha^{2}$, $\alpha^{-3}$ respectively.

As second example, let $p^{n}=17$, so that $\mu=0, x=2$, while the third type of group does not occur. There are three cyclic groups of the second type determined by the sets of multipliers $\alpha, \alpha^{-1}, 1$; $\alpha, \alpha^{7}, \alpha^{8} ; \alpha, \alpha^{9}, \alpha^{6}$. The two cyclic groups of the fourth type may be determined by the sets of multipliers $\alpha, \alpha^{2}, \alpha^{13} ; \alpha, \alpha^{3}, \alpha^{12}$. 
23\%. It remains to determine the number of cyclic subgroups of $G$ conjugate with each group of the types a), b), c), d). Type a) is generated by the substitution

$$
x^{\prime}=\alpha x, \quad y^{\prime}=\alpha y, \quad z^{\prime}=\alpha^{-2} z \quad\left(\alpha^{-2} \neq \alpha\right)
$$

and is commutative with exactly $\left(p^{2 n}-1\right)\left(p^{2 n}-p^{n}\right)$ substitutions of $G$, viz.,

$$
x^{\prime}=a x+b y, \quad y^{\prime}=c x+d y, \quad z^{\prime}=e z .
$$

The cyclic group of order $p^{n}-1$ generated by the substitution

$$
x^{\prime}=\alpha x, \quad y^{\prime}=\alpha^{m} y, \quad z^{\prime}=\alpha^{-m-1} z, \quad m^{2} \equiv 1\left(\bmod p^{n}-1\right)
$$

is transformed into itself by $2\left(p^{n}-1\right)^{2}$ substitutions, viz.,

$$
S: \quad x^{\prime}=a x, \quad y^{\prime}=b y, \quad z^{\prime}=c z \quad(a b c=1)
$$

and the products $T S$, where $T$ replaces $x$ by $y$ and $y$ by $-x$. When cyclic groups of the third type exist, each is transformed into itself by the $3\left(p^{n}-1\right)^{2}$ substitutions $S,(x y z) S,(x z y) S$. Each cyclic group of the fourth type is transformed into itself by exactly the $\left(p^{n}-1\right)^{2}$ substitutions $S$.

238. For $p^{n}=2^{2}$, the simple group $G$ has the order $N \equiv 20160$. There is, by 244), a single canonical form $C$, not the identity, its multipliers being $1, \theta, \theta^{2}$. The $N /\left(p^{n}-1\right)^{2} \equiv 2240$ substitutions of $G$ of period 3 are therefore all conjugate and generate a single set of conjugate cyclic groups. Applying the results of $\S \S 226-231$ to the case $p^{n}=2^{2}$, we see that $G$ contains

960 conjugate cyclic groups of order 7 with 5760 substitutions of period 7 2016

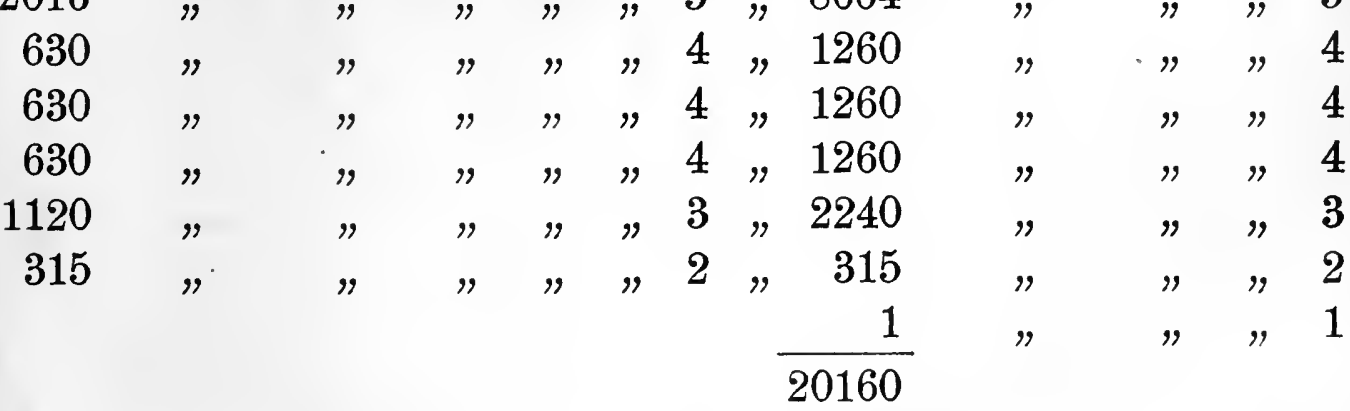

The substitutions of period 2 are all contained in the cyclic groups of order 4.

The group $G$ differs in structure from the alternating group on 8 letters, likewise of order 20,160. Indeed, the latter contains 5760 substitutions of type (1234567), 3360 of type (123456)(78), 1344 of type (12345), 2688 of type (12345)(678), 2520 of type $(1234)(56)$, 1260 of type (1234)(5678), 112 of type (123), 1120 of type (123)(456), 
1680 of type $(123)(45)(67), 210$ of type (12)(34), 105 of type $(12)(34)(56)(78)$, and the identity. The alternating group has substitutions of periods 6 and 15, while $G$ does not. Both groups contain the same number of substitutions of period 7, the same number of period 4, the same number of period 2. But the distribution into sets of conjugates of the substitutions of period 2, or of period 3, or of period 4, differs in the two groups. In particular, $G$ is not isomorphic with the alternating group on 8 letters, each group being simple and of order 20:160.1)

\section{CHAPTER XII.}

\section{SUBGROUPS OF THE LINEAR FRACTIONAL GROUP $\left.L F\left(2, p^{n}\right)^{2}{ }^{2}\right)$}

239. In $\S 108$ was defined the group of linear fractional substitutions

$$
S: \quad z^{\prime}=\frac{\alpha z+\beta}{\gamma z+\delta} \quad(\Delta \equiv \alpha \delta-\beta \gamma \neq 0)
$$

on an arbitrary variable $z$ with coefficients in the $G F\left[p^{n}\right]$. We proceed to represent it as a permutation-group on $p^{n}+1$ letters. Suppose $z$ runs through the series of marks of the $G F\left[p^{n}\right]$. For $\gamma=0, z^{\prime}$ will also run through the series of marks. For $\gamma \neq 0$, the value $z=-\delta / \gamma$ gives $z^{\prime}=\frac{-\Delta / \gamma}{0}$, so that $z^{\prime}$ can not be determined as a mark of the field. We may, however, obtain a set of elements which are merely permuted by $S$ by adjoining to the series of marks a new element $\infty \equiv \frac{\mu}{0}$, necessarily the same for every mark $\mu \neq 0$, since $\frac{1}{0} \equiv \frac{\mu \cdot 1}{\mu \cdot 0}=\frac{\mu}{0}$, and assumed to combine with the marks $\lambda \neq 0$ of the field according to the laws

$$
\infty+\lambda=\lambda+\infty=\infty, \quad \lambda \infty=\infty \lambda=\infty, \quad \lambda / \infty=0, \quad \infty / \lambda=\infty,
$$

while the indeterminate fraction $\frac{\alpha \infty+\beta}{\gamma \infty+\delta}$ is assumed to equal $\alpha / \gamma$. Setting henceforth $s \equiv p^{n}$, the group $L F(2, s)$ of linear fractional substitutions of determinant unity in the $G F[s]$ may therefore be

1) Miss Schottenfels established this theorem by direct calculations, Annals of Mathematics, (2) vol. 1, pp. 147-152.

2) Moore, Mathematical Papers Chicago Congress of 1893, pp. 208-242, Math. Ann., vol. 55 (56?); Wiman, Sweedish Acad., vol. 25 (1899), pp. 1-47; Burnside, Proc. Lond. Math. Soc., vol. 25 (1894), p. 132. The work of Galois, Mathieu and Gierster is cited in the exposition for $n=1$ in Klein-Fricke, Modulfunctionen I, p. 411 and pp. $419-491$. 
represented concretely as a permutation-group $G_{M(s)}^{s+1}$ on $s+1$ letters and having the order

$$
M(s) \equiv \frac{s\left(s^{2}-1\right)}{2 ; 1} \quad(2 ; 1 \text { according as } p>2 ; p=2) .
$$

The group of all substitutions $S$ has the order $(2 ; 1) M(s)$. For $p>2$, it may be represented as a permutation-group $G_{2 M(s)}^{s+1}$. For $p=2$, it is the former group.

The group $G_{M(s)}^{s+1}$ is doubly transitive. It is only necessary to prove that a substitution $T$ with coefficients in the field and of determinant unity may be found which will replace two arbitrary distinct elements $\rho, \sigma$ by the elements $0, \infty$. If both $\rho$ and $\sigma$ are marks of the field, we may take as $T$

$$
z^{\prime}=\frac{x(z-\varrho)}{z-\sigma}, \quad x \equiv \frac{1}{\rho-\sigma} .
$$

If $\rho$ is a mark and $\sigma=\infty$, we may take $T$ to be $z^{\prime}=z-\varrho$.

The inverse of $S \equiv\left(\frac{\alpha, \beta}{\gamma, \delta}\right)$ of determinant unity is $S^{-1} \equiv\left(\frac{\delta,-\beta}{-\gamma, \alpha}\right)$, so that $S$ is of period two if and only if $\alpha+\delta=0$.

240. A substitution $S$, not the identity, of the group $G_{M(s)}^{8+1}$ leaves fixed at most two elements. The fixed elements are given by the equation

$$
\gamma z^{2}+(\delta-\alpha) z-\beta=0 \text {. }
$$

By $\S 15$, it has at most two roots in the field $G F[s]$ unless $\gamma=\beta=0$, $\alpha=\delta$, when $S$ is the identity. Now $S$ leaves $\infty$ fixed only when $\infty=\alpha / \gamma$, whence $\gamma=0$. The other fixed elements are given by $(\delta-\alpha) z-\beta=0$, which, for $S \neq I$, is satisfied only by $z=\infty$ or $z=$ mark according as $\delta-\alpha=0$ or $\neq 0$.

If $S$ leaves fixed two distinct elements $z_{1}$ and $z_{2}$, it can be transformed by a suitably chosen substitution $T$ of the group into a substitution with the fixed elements 0 and $\infty$, having therefore the form

$$
\Sigma: \quad z^{\prime}=\frac{a z}{b} \quad(a b=1) .
$$

Its period is a divisor of $\frac{1}{2}\left(p^{n}-1\right)$ or $p^{n}-1$ according as $p>2$ or $p=2$. into

If $S$ leaves fixed a single element $z_{1} \equiv z_{2}$, it can be transformed

$$
z^{\prime}=z+\beta
$$

leaving fixed the single element $\infty$. Its period is therefore $p$. But the condition for a double root of 248$)$ is $(\alpha+\delta)^{2}=4$

If $S$ leaves no element fixed, the quadratic 248) is irreducible in the $G F\left[p^{n}\right]$. By the corollary of $\S 31$, its roots $z_{1}$ and $z_{2}$ are 
marks of the $G F\left[p^{2 n}\right]$ conjugate with respect to the $G F\left[p^{n}\right]$. Now $S$ multiplies the function $\left(z-z_{1}\right) \div\left(z-z_{2}\right)$ by the constant $a / b$, where

$$
a \equiv \alpha-\gamma z_{1}, \quad b \equiv \alpha-\gamma z_{2} .
$$

The product $a b$ reduces to $\alpha \delta-\beta \gamma=1$. Also $a$ and $b$ are conjugate (\$ 73). Hence

$$
a^{-1}=b=a^{p^{n}}, \quad a^{p^{n}+1}=1 .
$$

Hence $S$ can be transformed into a substitution of the form $\Sigma$, whose period is a divisor of $\frac{1}{2}\left(p^{n}+1\right)$ or $p^{n}+1$ according as $p>2$ or $p=2$.

In particular, the substitutions of period $p$ are characterized by the invariant $(\alpha+\delta)^{2}=4$.

241. Commutative subgroups of order $p^{n}$. The substitutions

$$
S_{\mu} \equiv\left(\frac{1, \mu}{0,1}\right), \quad \quad \mu \text { in the } G F\left[p^{n}\right],
$$

form a commutative subgroup $G_{s}^{(\infty)}$ of order $s \equiv p^{n}$, containing all the substitutions of $G_{M(s)}$ leaving the single element $\infty$ fixed and containing no other substitutions. Each of its substitutions except the identity is of period $p$. Hence there are $\left(p^{n}-1\right) /(p-1)$ cyclic subgroups $G_{p}$ of order $p$ in the $G_{s}^{(\infty)}$. To determine the conjugacy of these substitutions and subgroups under $G_{M(s)}$, we transform $S_{\mu}(\mu \neq 0)$ by $V=\left(\frac{\alpha, \beta}{\gamma, \delta}\right)$ and (see formula of composition at end of $\S 108$ ) obtain the substitution ${ }^{1}$ )

$$
V^{-1} S_{\mu} V=\left(\frac{1-\alpha \gamma \mu, \quad \alpha^{2} \mu}{-\gamma^{2} \mu, \quad 1+\alpha \gamma \mu}\right)
$$

This substitution belongs to $G_{s}^{(\infty)}$ if, and only if, $\gamma=0$, when it becomes $S_{\alpha^{2} \mu}$. In particular, $S_{\mu}$ is transformed into itself only by the substitutions $\left(\frac{1, \beta}{0,1}\right)$. Within $G_{M(s)}$ any substitution $S_{\mu}(\mu \neq 0)$ is self-conjugate in exactly the $G_{s}^{(\infty)}$, while the $G_{s}^{(\infty)}$ is self-conjugate in

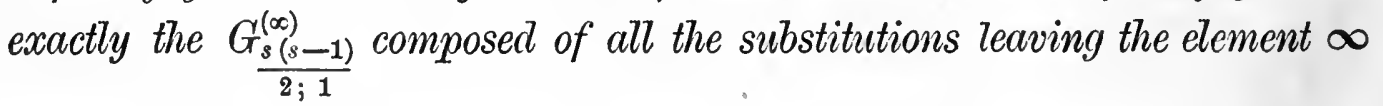
invariant, viz., $\left(\frac{\alpha, \beta}{0, \alpha^{-1}}\right)$. As to the order of the latter group, $\beta$ may be any mark of the $G F\left[p^{n}\right]$ and $\alpha$ any mark $\neq 0$; but $-\alpha,-\beta$ gives the same substitution as $+\alpha,+\beta$.

1) This order of the factors of a product is employed by Wiman, the reverse order by Moore. 
Within $G_{M(s)}, S_{\mu}$ is conjugate only with the substitutions $S_{\alpha^{2} \mu}$. Hence the $s-1$ substitutions, not the identity, of $G_{s}^{(\infty)}$ are all conjugate if $p=2$, but separate into two sets of $\frac{1}{2}(s-1)$ conjugate substitutions if $p>2$. The $p-1$ substitutions of a cyclic group $G_{p}$ generated by $S_{\mu}$ belong half to one and half to the other set if $p>2$ and $n$ be odd, but all belong to the same set if $n$ be even ( $\$ 62)$.

In place of $\infty$ the fixed element may be any one of the $p^{n}$ marks of the $G F\left[p^{n}\right]$. Since $G_{M(s)}$ permutes the $p^{n}+1$ elements $x$ transitively, it contains $p^{n}+1$ conjugate commutative groups $G_{s}^{(x)}$. This result also follows from the numerical identity

$$
\frac{s\left(s^{2}-1\right)}{2 ; 1} \div \frac{s(s-1)}{2 ; 1}=s+1 \equiv p^{n}+1 \text {. }
$$

Each $G_{s}^{(x)}$ is defined by any one of its substitutions not the identity as the group in which that substitution is self-conjugate. These $p^{n}+1$ groups have therefore no substitution in common except the identity and contain in all $p^{2 n}-1$ distinct substitutions of period $p$.

242. Cyclic subgroups of order $\frac{s-1}{2 ; 1}$. If $\varrho$ be a primitive root of the $G F\left[p^{n}\right]$, the substitution

$$
P=\left(\frac{\rho, 0}{0, \rho^{-1}}\right)
$$

generates a cyclic group of order $\frac{1}{2}\left(p^{n}-1\right)$ if $p>2$, but of order $p^{n}-1$ if $p=2$. It contains all the substitutions

$$
\Sigma=\left(\frac{a, 0}{0, a^{-1}}\right) . \quad\left(a \text { in the } G F\left[p^{n}\right]\right) .
$$

Since it contains all the substitutions which leave fixed the elements

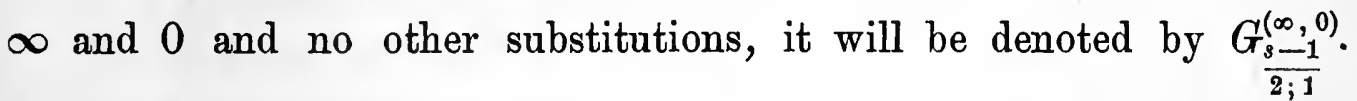
Any new substitution transforming this cyclic group into itself must interchange the elements $\infty$ and 0 and hence have the form

$$
B=\left(\frac{0, \beta}{-\beta^{-1}, 0}\right) \text {. }
$$

Inversely, every $B$ transforms $\Sigma$ into its reciprocal $\Sigma^{-1}$. These $\frac{s-1}{2 ; 1}$ substitutions $B$ of period two together with the substitutions $\Sigma$ form a dihedron-group $\left.{ }^{1}\right) G_{\frac{2}{2 ; 1}}^{(\infty, 0)}$, which is the largest subgroup of $G_{M(s)}$ within which the above cyclic group is self-conjugate.

1) See the definition given in $\S 245$. 
Since $\infty, 0$ form only one of the $\frac{1}{2} p^{n}\left(p^{n}+1\right)$ pairs of the $p^{n}+1$ elements, $G_{M(s)}$ contains exactly $\frac{1}{2} p^{n}\left(p^{n}+1\right)$ conjugate cyclic. groups $G_{\frac{s-1}{2 ; 1}}^{(x, \lambda)}$, each self-conjugate in exactly a dihedron $G_{\frac{2-1}{2 ; 1}}^{(x, \lambda)}$ Each. of these cyclic groups is defined by any one of its substitutions not the identity as the largest cyclic group containing that substitution. These $\frac{1}{2} p^{n}\left(p^{n}+1\right)$ groups have therefore no substitution in common except the identity and contain in all $\frac{1}{4} s(s+1)(s-3)$ or $\frac{1}{2} s(s+1)(s-2)$ substitutions (not the identity) according as $p>2$ or $p=2$.

243. Cyclic subgroups of order $\frac{s+1}{2 ; 1}$. By $\S 144, \operatorname{LF}\left(2, p^{n}\right)$ is holoedrically isomorphic with the group $H \equiv H O\left(2, p^{2 n}\right)$ of binary hyperorthogonal substitutions of determinant unity in the $G F\left[p^{2 n}\right]$ when taken fractionally, viz.,

$$
V=\left(\frac{A, B}{-\bar{B}, \bar{A}}\right) \quad(A \bar{A}+B \bar{B}=1)
$$

where $\bar{A} \equiv A^{p^{n}}$ is the conjugate of $A$ with respect to the $G F\left[p^{n}\right]$. The reciprocal of $V$ is, by $\S 142$,

$$
V^{-1}=\left(\frac{\bar{A},-B}{\bar{B}, \quad A}\right)
$$

If $J$ be a primitive root of $J^{p^{n}+1}=1$, so that $\bar{J}=J^{-1}$, the following substitution of $H$,

$$
Q=\left(\frac{J, 0}{0, \bar{J}}\right)
$$

generates a cyclic group $\frac{G_{\frac{s+1}{2}}}{2 ; 1}$. composed of the substitutions

$$
Q^{g}=\left(\frac{J^{g}, 0}{0, \bar{J}^{g}}\right)
$$

Any substitution $V$ of $H$ transforms $Q^{g}$ into

$$
V^{-1} Q^{g} V=\left(\frac{A \bar{A} J^{g}+B \bar{B} \bar{J}^{g},-A B\left(J^{g}-\bar{J}^{g}\right)}{-\bar{A} \bar{B}\left(J^{g}-\bar{J}^{g}\right), A \bar{A} \bar{J}^{g}+B \bar{B} J^{g}}\right) .
$$

This substitution belongs to the cyclic group generated by $Q$ if and only if $A B=0$. Two cases arise.

If $B=0$, then $A \bar{A}=1$ so that $V=\left(\frac{A, 0}{0, \bar{A}}\right)$ belongs to the cyclic group and evidently transforms every $Q^{g}$ into itself. 
If $A=0$, then $B \bar{B}=1$, so that $V=\left(\frac{0, B}{-\bar{B}, 0}\right)$. The latter transforms $Q^{g}$ into $\left(\frac{\bar{J}^{g}, 0}{0, J^{g}}\right) \equiv Q^{-g}$, which is distinct from $Q^{g}$ unless the latter be of period two.

The largest subgroup of $H$ within which the cyclic group $\frac{G_{s+1}}{2 ; 1}$ is self-conjugate is therefore a dihedron-group of order $2 \cdot \frac{s+1}{2 ; 1}$. Hence $H$, and consequently also $G_{M(s)}$, contains

$$
\frac{s\left(s^{2}-1\right)}{2 ; 1} \div 2 \frac{s+1}{2 ; 1}=\frac{1}{2} s(s-1)
$$

cyclic groups conjugate with $\frac{G_{s+1}}{2 ; 1}$. Each of these is defined by any substitution lying in it (the identity excepted) as the largest cyclic group containing that substitution. The $\frac{1}{2} s(s-1)$ groups have therefore only the identity in common and contain in all $\frac{1}{4} s(s-1)^{2}$ or $\frac{1}{2} s^{2}(s-1)$ further substitutions according as $p>2$ or $p=2$.

244. To verify that we have now enumerated all the individual operators of $G_{M(s)}$ and consequently all the largest cyclic subgroups, we note that

$$
\begin{array}{ll}
1+\left(s^{2}-1\right)+\frac{1}{4} s(s+1)(s-3)+\frac{1}{4} s(s-1)^{2}=\frac{1}{2} s\left(s^{2}-1\right), & p>2 ; \\
1+\left(s^{2}-1\right)+\frac{1}{2} s(s+1)(s-2)+\frac{1}{2} s^{2}(s-1)=s\left(s^{2}-1\right), & p=2 .
\end{array}
$$

It was shown that if any substitution $S$ of a cyclic $G_{\frac{s \mp 1}{2 ; 1}}$ be of period $>2$ (viz., $S \neq S^{-1}$ ), then $S$ is transformed into itself by no substitutions of $G_{M(s)}$ other than those of the cyclic $G_{\frac{s \mp 1}{2}}$. Hence the latter is the largest commutative subgroup of $G_{M(s)}$ which contains the substitution $S$. A commutative subgroup containing an operator of period $>2$ and different from $p$ is therefore a cyclic group. A commutative group containing an operator of period $p$ contains only operators of period $p$ ( $\$ 241)$. Hence if a commutative subgroup of $G_{M(s)}, p>2$, contains an operator of period $>2$, it contains at most one operator of period 2 .

245. Cyclic and dihedron groups and their subgroups. The abstract dihedron-group $G_{2 k}$ may be generated by operators $A, B$ subject only to the generational relations

$$
A^{k}=I, \quad B^{2}=I, \quad A B=B A^{-1} .
$$


From the latter two follow the relations (holding for any integer $r$ )

$$
B^{-1} A^{\mathrm{r}} B=A^{-r}, \quad A^{-r} B A^{r}=B A^{2 r} \text {. }
$$

The cyclic subgroup $G_{k}$ generated by $A$ is therefore self-conjugate under $G_{2 k}$. The latter is said to have the cyclic base $G_{k}$. The $k$ operators

$$
B A^{i} \quad(i=0,1, \ldots, k-1)
$$

are of period two. For $k$ odd, they are all conjugate under $G_{2 k}$ since $B$ transforms $B A$ into $B A^{-1} \equiv B A^{k-1}$, which belongs to the series $B, B A^{2}, B A^{4}, \ldots$ For $k$ even, they form two sets of conjugate operators

$$
\begin{array}{ccc}
B, & B A^{2}, & B A^{4}, \ldots, B A^{k-2} \\
B A, & B A^{3}, & B A^{5}, \ldots, B A^{k-1}
\end{array}
$$

According as $k$ is odd or even, they generate cyclic groups $G_{2}$ forming one set or two sets of conjugate subgroups.

For every divisor $d$ of $k, G_{k}$ contains a single cyclic subgroup $G_{d}$, which is formed by the operators

$$
A^{\delta}, \quad A^{2 \delta}, \quad A^{3 \delta}, \ldots, A^{d \delta} \equiv I
$$

If $\mu$ be a given one of the integers $1,2, \ldots, \delta$, the following $d$ operators

$$
B A^{\mu+\delta}, B A^{\mu+2 \delta}, B A^{\mu+3 \delta}, \ldots, B A^{\mu+d \delta} \equiv B A^{\mu}
$$

extend the cyclic group $G_{d}$ to the same dihedron $G_{2 d}$. There are exactly $\delta$ such dihedron-groups. If $k$ be odd, these $G_{2 d}$ are all conjugate under $G_{2 k}$. If $\delta$ be odd, but $k$ be even, the exponents $\mu$, $\mu+\delta, \mu+2 \delta, \ldots$ are alternately even and odd, so that each $G_{2 d}$ contains operators of both of the sets 249); the groups $G_{2_{d}}$ are therefore all conjugate under $G_{2 k}$. If $\delta$ be even and hence $k$ even, the exponents are all even or all odd, so that the operators all belong to a single one of the two sets 249 ); the groups $G_{2 d}$ thus belong to two distinct systems of conjugate subgroups of $G_{2 k}$.

If $d>2, G_{2 d}$ has a single cyclic $G_{d}$ and $G_{2 k}$ a single cyclic $G_{k}$, so that the above process furnishes every dihedron subgroup $G_{2 d}$ of $G_{2 k}$. The theorem stated below therefore follows if $d>2$.

We consider next the case $d=2, k$ even and $>2$. The only operators of period two in $G_{2 k}$ are then $A^{k / 2}$ and

$$
B A^{i} \quad(i=0,1, \ldots, k-1) .
$$

Hence any dihedron $G_{4}$ must contain two operators $B A^{r}, B A^{s}(r \neq s)$ and therefore their product $B A^{r} B A^{s} \equiv A^{s-r}$. Hence every $G_{4}$ must contain $A^{k / 2}$ and may therefore be based on the subgroup $G_{2}$ of $G_{k}$. The theorem then follows as before. The $k / 2$ possible groups $G_{4}$ in $G_{2 k}$ are given by the formula

$$
\left\{I, \quad A^{k / 2}, \quad B A^{r}, \quad B A^{r+k / 2}\right\} \quad\left(r=0,1, \ldots, \frac{k}{2}-1\right) .
$$


Theorem. - For every divisor $d$ of $k$ the dihedron $G_{2 k}$ contains exactly $k / d$ dihedrons $G_{2 d}$ forming one system or two systems of conjugate subgroups according as $k / d$ is odd or even.

246. Cyclic and dihedron subgroups of $G_{M(s)}$ whose cyclic bases are subgroups of the cyclic $G_{8 \mp 1}$. By $\S \S 242-243, G_{M(s)}$ contains $\frac{1}{2} s(s \pm 1)$ conjugate cyclic subgroups $G_{\frac{s \mp 1}{2 ; 1}}$ each self-conjugate in a dihedron subgroup $G_{2 \frac{s \mp 1}{2 ; 1}}$, but self-conjugate in no larger subgroup of $G_{M(s)}$. Hence these dihedrons are all conjugate under the main group. $\left.{ }^{1}\right)$ Let $d_{\mp}$ be any divisor of $\frac{s \mp 1}{2 ; 1}$ and denote the quotient by $\delta_{\mp} . \quad G_{M(s)}$ contains $\frac{1}{2} s(s \pm 1)$ conjugate cyclic groups $G_{\delta_{\mp}}$, each of which is $(\S 245)$ the cyclic base for $\delta_{\mp}$ dihedron subgroups $G_{2 d_{\mp}}$. Under $G_{2 \frac{s \mp 1}{2 ; 1}}$ they form one system or two systems of conjugate subgroups according as $\delta_{\mp}$ is odd or even.

For $d_{\mp}>2$, two subgroups $G_{2 d_{\mp}}$ of $G_{\frac{2 \frac{s \mp 1}{2 ; 1}}{}}$ are conjugate within the latter if conjugate within $G_{M(s)}$; indeed, the transforming substitution must be commutative with $G_{d_{\mp}}$, the only cyclic group of order $d_{\mp}$ in either $G_{2_{\mp}}$, and therefore commutative with the cyclic $G_{\frac{s \mp 1}{2 ; 1}}$ determined by it. Hence if $d_{\mp}$ be any divisor $>2$ of $\frac{s \mp 1}{2 ; 1}$ and the quotient be $\delta_{\mp}, G_{M(s)}$ contains in all $M(s) / 2 d_{\mp}$ dihedron $G_{2 d_{\mp}}$ forming one system or two systems of conjugate groups according as $\delta_{\mp}$ is odd or even. In the former case, a $G_{2 d_{\mp}}$ is self-conjugate only under itself; in the latter case, self-conjugate under a dihedron $G_{2 \cdot 2} d_{\mp}$. These, $G_{2_{\mp}}$ are all conjugate within $G_{(2 ; 1) M(s)}$.

For $d \mp=2$, we have $p>2$ since $s-1$ is not divisible by 2 for $p=2$. Then $s \equiv p^{n}$ is of the form $4 h \pm 1$ according as the Jacobi-Legendre symbol $\left(\frac{-1}{s}\right)$ is \pm 1 ; hence $\frac{1}{2}\left[s-\left(\frac{-1}{s}\right)\right]$ is even, say $=2 \sigma$. Then all the substitutions $V_{2}$ of period two of $G_{M(s)}$ belong to the conjugate cyclic $G_{2 \sigma}$. It remains to study the fourgroups $G_{4}$, each a dihedron $G_{2.2}$ containing three cyclic $G_{2}$. Now $G_{M(s)}$ contains $\frac{1}{2} s\left[s+\left(\frac{-1}{s}\right)\right]$ conjugate cyclic $G_{2}$. Each $G_{2}$ lies in $\frac{1}{4}\left[s-\left(\frac{-1}{s}\right)\right]$ four-groups $G_{4}$. Hence, if $p>2, G_{M(s)}$ contains in

1) For every operator commutative with a group $G$ is transformed into an operator commutative with $G^{\prime}$ by the operator which transforms $G$ into $G^{\prime}$. 
all $M(s) / 12$ four-groups. Also the $\sigma$ four-groups contained in a dihedron $G_{4 \sigma}$ form (under the latter) one system or two systems of conjugate subgroups according as $\sigma$ is odd or even, viz., according as $s \equiv p^{n}$ has the form $8 h \pm 3$ or $8 h \pm 1$. Since the $G_{4 \sigma}$ are all conjugate within $G_{M(s)}$, it follows, for $\sigma$ odd, that all the four-groups of $G_{M(s)}$ are conjugate; while, for $\sigma$ even, they form at most two systems of conjugate subgroups under $G_{M(s)}$. For $\sigma$ even, each $G_{4}$ is one of $\sigma / 2$ conjugate subgroups of a certain $G_{4 \sigma}$ and is therefore self-conjugate under a subgroup of order 8 of $G_{4 \sigma}$. Suppose that, for $\sigma$ even, the subgroups $G_{4}$ of $G_{M(s)}$ form a single system of conjugate subgroups. Then each $G_{4}$ would be one of $M(s) / 12$ conjugate subgroups and consequently commutative with exactly the 12 operators of a subgroup $G_{12}$. By an earlier remark, the $G_{4}$ is commutative with a subgroup $G_{8}$. Since 8 is not a divisor of 12 , our hypothesis is untenable. Hence, for $\sigma$ even, the $G_{4}$ form exactly two systems of conjugate subgroups of $G_{M(s)}$. For $\left.{ }^{1}\right) p>2$, the $M(s) / 12$ fourgroups $G_{4}$ contained in $G_{M(s)}$ form one system or two systems of conjugate subgroups according as $s \equiv p^{n}$ has the form $8 h \pm 3$ or $8 h \pm 1$. In the former case, $a G_{4}$ is self-conjugate under $a G_{12}$; in the latter case, under $a G_{24}$. In the $G_{2 M(s)}$ the $G_{4}$ form a single system of conjugate subgroups and each is self-conjugate under $a G_{24}$. Each $G_{12}$ is not a commutative group by $\S 244$ and so is of the tetrahedral type ( $\$ 247$ ). Likewise, each $G_{24}$ contains a tetrahedral subgroup $G_{12}$. The latter is of index 2 and consequently self-conjugate under $G_{24}$. Since $G_{12}$ contains a set of 4 conjugate $G_{3}$, the $G_{24}$ will contain a complete system of 4 conjugate $G_{3}$. Each is self-conjugate under a $G_{6}$, which is a dihedron since it is not commutative (§ 244). Finally, no operator of period 2 is self-conjugate under $G_{24}$; for it is self-conjugate only under a dihedron $G_{s} \mp 1$ which contains no tetrahedral subgroup and hence none of the present $G_{24}$. Then by $\S 248$ each $G_{24}$ is an octahedral group.

24\%. A non-commutative group of order 12 having a self-conjugate four-group is of the tetrahedral type.

Let the operators of the four-group be $I, V_{2}, V_{2}^{\prime}, V_{2}^{\prime \prime}$, so that they are commutative and the product of any two $V$ 's gives the third $V$. The $G_{12}$ contains at least one operator $V_{3}$ of period 3 . The products

$$
V_{3}^{i}, \quad V_{2} V_{3}^{i}, \quad V_{2}^{\prime} V_{3}^{i}, \quad V_{2}^{\prime \prime} V_{3}^{i} \quad(i=0,1,2)
$$

are all distinct and so give all the operators of $G_{12}$. The $G_{12}$ would be a commutative group if $V_{3}$ were commutative with $V_{2}, V_{2}^{\prime}, V_{2}^{\prime \prime}$.

1) For $p=2$, the four-groups are determined in $\S 249$. There are $\frac{1}{6}\left(2^{n}-1\right)\left(2^{n}-2\right)$ sets. 
Since therefore $V_{3}$ does not transform each $V$ into itself and since it does not permute two of them, its period being $\neq 2$, it must permute them in a cycle. Fixing the notation, we thus have

$$
\begin{aligned}
& V_{3}^{-1} V_{2} V_{3}=V_{2}^{\prime}, \quad V_{3}^{-1} V_{2}^{\prime} V_{3}=V_{2}^{\prime \prime} \quad V_{3}^{-1} V_{2}^{\prime \prime} V_{3}=V_{2}, \\
& \left(V_{3} V_{2}\right)^{3} \equiv V_{3} V_{2} V_{3}^{-1} \cdot V_{3}^{-1} V_{2} V_{3} \cdot V_{2}=V_{2}^{\prime \prime} V_{2}^{\prime} V_{2}=I
\end{aligned}
$$

Hence $V_{3}, V_{2}$ generate $G_{12}$ and satisfy the generational relations

$$
V_{3}^{3}=I, \quad V_{2}^{2}=I, \quad\left(V_{3} V_{2}\right)^{3}=I .
$$

of the tetrahedral group, an abstract group of order 12 holoedrically isomorphic with the alternating group on 4 letters ( $\$ 265$ ).

248. A group of order 24 having no self-conjugate operator of period 2 and having a set of 4 conjugate $G_{3}$ each self-conjugate in a dihedron $G_{6}$ is of the octahedral type.

The 4 conjugate $G_{3}$ are transformed into each other by the operators of $G_{24}$. Hence $G_{24}$ is isomorphic with a substitution-group on 4 letters. The isomorphism will be holoedric and consequently the latter the symmetric group $G_{24}^{(4)}$, if the identity be the only operator of $G_{24}$ which transforms each $G_{3}$ into itself, i. e., if the four $G_{6}$ have only the identity in common. But if a substitution of period 3 were common to the dihedron $G_{6}$, it would be common to the $G_{3}$, and these would be identical contrary to hypothesis. If the $G_{6}$ contain in common two substitutions of period 2, they would contain in common the product of the two which is a substitution not the identity of the cyclic bases $G_{3}$ (§ 245). Finally, if the conjugate $G_{6}$ contain in common a single substitution of period 2, it would be self-conjugate under $G_{24}$ contrary to hypothesis. Now the $G_{24}^{(4)}$ is of the octahedral type

249. Subgroups of the $s+1$ commutative $G_{s}^{(*)}$. Since these groups are all conjugate under $G_{M(s)}$, it suffices to determine the subgroups of $G_{s}^{(\infty)}$ formed of the commutative substitutions $S_{\mu}$ of period $p$. If a subgroup contain $S_{\mu_{1}}, S_{\mu_{2}}, \ldots, S_{\mu_{t}}$, it will contain $S_{\mu}$, where $\mu=c_{1} \mu_{1}+c_{2} \mu_{2}+\cdots+c_{t} \mu_{t}$, the $c_{i}$ running independently through the series $0,1, \ldots, p-1$. Hence to every subgroup $G_{p^{m}}$ of order $p^{m} \overline{<} p^{n}$, there corresponds an additive-group in the $G F\left[p^{n}\right]$ of rank $m$ with respect to the $G F[p]$ and inversely. Hence, by $\S 69$, the number of distinct subgroups $G_{p^{m}}$ of $G_{p^{n}}$ is

$$
\frac{\left(p^{n}-1\right)\left(p^{n}-p\right)\left(p^{n}-p^{2}\right) \ldots\left(p^{n}-p^{m-1}\right)}{\left(p^{m}-1\right)\left(p^{m}-p\right)\left(p^{m}-p^{2}\right) \ldots\left(p^{m}-p^{m-1}\right)} \text {. }
$$

Let $G_{p^{m}}$ be one such group composed of the substitutions $S_{\lambda \text {, }}$ where $\lambda$ ranges over an additive-group $\left[\lambda_{1}, \ldots, \lambda_{m}\right]$ of rank $m$ with 
respect to the $G F[p]$. By $\S 241, G_{p^{m}}$ is transformed into itself only by substitutions of the form $V=\left(\frac{\alpha, \beta}{0, \alpha^{-1}}\right)$. Since $V$ transforms $S_{\lambda}$ into $S_{\alpha^{2} \lambda}$, a further condition is that $\alpha^{2} \lambda$ and $\lambda$ should run simultaneously through the series of marks of the $\left[\lambda_{1}, \ldots, \lambda_{m}\right]$. Suppose that there are in the $G F\left[p^{n}\right]$ exactly $e$ marks $\varepsilon_{1}, \ldots, \varepsilon_{e}$ such that $\left[\lambda_{1}, \ldots, \lambda_{m}\right]=\left[\varepsilon_{i}^{2} \lambda_{1}, \ldots, \varepsilon_{i}^{2} \lambda_{m}\right]$. Then, according as $p>2$; $p=2$, the $\frac{s e}{2 ; 1}$ substitutions

$$
V=\left(\frac{\varepsilon_{i},}{\varepsilon_{i} \beta}\right) \equiv\left(\frac{1, \beta}{0,1}\right)\left(\frac{\varepsilon_{i},}{0,1} \varepsilon_{i}^{-1}\right),
$$

where $\beta$ ranges over the $G F[s]$, constitute the largest subgroup $H$ of $G_{M(s)}$ under which $G_{p^{m}}$ is self-conjugate. But the multipliers $x$ of the additive-group $\left[\lambda_{1}, \ldots, \lambda_{m}\right]$ are $(\S 70)$ the marks $x \neq 0$ of the multiplier $G F\left[p^{k}\right], k$ being a divisor of $m$ and $n$. It remains to distinguish which of them are squares of marks $\varepsilon_{i}$ of the $G F\left[p^{n}\right]$. For the respective cases

$$
p>2 \text { with } n / k \text { even, } p>2 \text { with } n / k \text { odd; } p=2 \text {, }
$$

there are $(\S 62)$ exactly $e=(2,1 ; 1)\left(p^{k}-1\right)$ marks $\varepsilon_{i}$, so that $H$ is of order $\frac{s e}{2 ; 1} \equiv \frac{s\left(p^{k}-1\right)}{1,2 ; 1}$. Hence $G_{p^{m}}$ is one of a system of

$$
\left(p^{2 n}-1\right) \div(2,1 ; 1)\left(p^{k}-1\right)
$$

conjugate subgroups of $G_{M(s)}$. Here the value of $k$ depends on the individual $G_{p^{m}}$ chosen. Given $k$, the number of the corresponding sets of $G_{p^{m}}$ follows from $\S 71$.

250. Non-commutative subgroups of the $s+1$ conjugate $\frac{G_{\frac{s(s-1)}{(x)}}^{(; 1}}{\text {. }}$. It suffices to study the group $G$ given by $\varkappa=\infty$. It is composed of the substitutions

$$
\left(\frac{\alpha, \beta}{0, \alpha^{-1}}\right)=\left(\begin{array}{ll}
1, & \mu \\
0, & 1
\end{array}\right)\left(\begin{array}{ll}
\alpha, & 0 \\
0, & \alpha^{-1}
\end{array}\right)
$$

For a given mark $\alpha \neq 0, \mu$ and $\beta$ run simultaneously through the series of marks of the $G F[s]$. A rectangular array of the substitutions of $G$ may be formed by taking as the first row the substitutions $S_{\mu}$, which form the self-conjugate subgroup $G_{s}^{(\infty)}$, and as righthand multipliers the substitutions $P_{\alpha} \equiv\left(\frac{\alpha, 0}{0, \alpha^{-1}}\right)$ of the cyclic $G_{\frac{s-1}{2 ; 1}}^{(\infty, 0)}$. In any subgroup $G^{\prime}$ of $G$ the totality of substitutions of period $p$ give rise to a commutative group $G_{p^{m}}$ of substitutions $S_{\lambda}$, where $\lambda$ ranges over an additive-group $\left[\lambda_{1}, \ldots, \lambda_{m}\right]$. Hence $G_{p^{m}}$ is selfconjugate under $G^{\prime}$. A rectangular array of the substitutions of $G^{\prime}$ 
with those of $G_{p^{m}}$ in the first row has the property that the substitutions in each row are all found in a corresponding row of the rectangular array for $G$. In fact, two operators $A, B$ of $G^{\prime}$ lie in the same or in different rows of the array for $G^{\prime}$ according as $A B^{-1}$ is or is not in $G_{p}{ }^{m}$. But $A B^{-1}$ belongs to $G^{\prime}$ and hence belongs to $G_{p}$ if, and only if, it occurs among the substitutions in the first row of the array for $G$. Hence each row for $G^{\prime}$ lies wholly in a row for $G$. The quotient-group $G^{\prime} / G_{p^{m}}$ is therefore a subgroup $G_{d-}$ of the quotient-group $G / G_{s}^{(\infty)}$, the latter being a cyclic $G_{s-1}$.

Indeed, these quotient-groups may be obtained concretely as groups of the permutations of the rows of $G$ induced by applying as righthand multipliers the substitutions of $G$ or $G^{\prime}$. But all the substitutions in the same row of $G$ (and, à fortiori, all in the same row of $G^{\prime}$ ) give rise to the same permutation. Hence $G_{d-}$ is an abstract

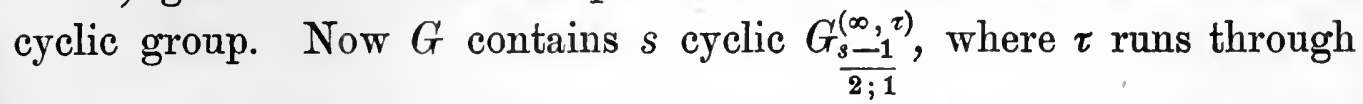
the series of marks of the $G F[s]$, all conjugate under the transformers $S_{\mu}$. Leaving different elements $\tau$ fixed, they have no substitution other than the identity in common. Counting also the $s-1$ substitutions of period $p$, we have accounted for all the substitutions of $G$. Besides the cyclic subgroups of $G_{s}^{(\infty)}, G$ therefore contains no cyclic subgroups other than the $G_{d}^{(\infty, \tau)}$, for the various divisors $d$ of $\frac{s-1}{2 ; 1}$. Among these cyclic groups occurs one whose substitutions may be chosen as the right-hand multipliers in forming the above array for $G^{\prime}$. In fact, within the row of $G^{\prime}$ corresponding to the generator of the quotient cyclic $G_{d}$ there must exist a substitution $A$ such that $A^{d}$, and no lower power, belongs to the group $G_{p^{m}}$ whose substitutions form the first row. The right-hand multipliers for the array may thus be chosen to be $I, A, A^{2}, \ldots, A^{d-1}$. Hence $G^{\prime}$ is given by the extension of the $G_{p^{m}}$ by a certain $G_{d}^{(\infty, \tau)}$, within which $G_{p^{m}}$ is self-conjugate. But the largest subgroup of $G_{M(s)}$ within which $G_{p^{m}}$ is self-conjugate is ( $\left.\$ 249\right)$ the group $H$ of order $s K$, $K \equiv \frac{p^{k}-1}{1,2 ; 1}$, given by the extension of $G_{s}^{(\infty)}$ by a cyclic $G_{K}^{(\infty, 0)}$. In particular, $d$ must be a divisor of $K$, so that $d$ depends upon the $G_{p^{m}}$. The cyclic $G_{K}^{(\infty, 0)}$ contains a single cyclic $G_{d}^{(\infty, 0)}$. Hence, by transforming $G^{\prime}$ by a suitably chosen $S_{\mu}$, we obtain a group $G_{p}^{\prime m_{d}}$ (conjugate with $G^{\prime}$ under $G$ ) given by the extension of $G_{p} m$ by the subgroup $G_{d}^{(\infty, 0)}$ of $G_{K}^{(\infty, 0)}$. The substitutions $S_{\lambda}$ of $G_{p^{m}}$ transform that subgroup into $p^{m}$ conjugate cyclic $G_{d}^{(\infty, 0)}$, since $S_{\lambda}$ replaces the fixed elements $\infty, 0$ by elements $\infty, \lambda$. These $p^{m}$ groups together with $G_{p^{m}}$ contain all the substitutions of $G_{p}^{\prime} m_{d}$, as shown by simple 
enumeration. The largest subgroup of $G_{M(s)}$ transforming $G_{p}^{\prime} m_{d}$ into itself must therefore transform $G_{p}$ into itself (and thus be a subgroup of $H$ ) and transform the groups of the single set of conjugate $G_{d}^{(\infty, \lambda)}$ amongst themselves. Of substitutions of period $p$, it must therefore contain only the $S_{\lambda}$. The required group is thus a subgroup of the group $H^{\prime}$ of order $p^{m} K$ given by the extension of $G_{p^{m}}$ by $G_{K}^{(\infty), 0)}$. Moreover, it is $H^{\prime}$ itself since any substitution $\left(\frac{\alpha, 0}{0, \alpha^{-1}}\right)$ of $G_{K}^{(\infty, 0)}, \alpha$ such that $\alpha^{2} \lambda=\lambda^{\prime}$ is of the $\left[\lambda_{1}, \ldots, \lambda_{m}\right]$, replaces the elements $\infty, \lambda$ by elements $\infty, \lambda^{\prime}$ and consequently transforms $G_{d}^{(\infty, \lambda)}$ into $G_{d}^{\left(\infty, \lambda^{\prime}\right)}$. Hence the group $G_{p}^{\prime} m_{d}$ is one of a system of

$$
\frac{\left(p^{n}+1\right)\left(p^{n}-1\right) p^{n-m}}{(2,1 ; 1)\left(p^{k}-1\right)}
$$

conjugate groups. Finally, if the subgroup $G^{\prime}$ contains no substitution of period $p$, it is a cyclic subgroup $G_{d}^{(\infty, x)}$ of one of the cyclic $\frac{G_{8-1}^{(\infty, x)}}{2 ; 1}$

251. Subgroups of $G_{M(s)}$ containing operators of period $p$. - The substitutions of period $p$ of a subgroup $G_{\Omega}$ of the $G_{M(s)}$ distribute themselves over certain $s+1$ subgroups $G_{p m_{\mu}}^{(\mu)}$ of the $s+1$ conjugate $G_{s}^{(\mu)}(\S 241)$. By hypothesis at least one of the orders $p^{m^{\prime}}{ }_{\mu}$ is $>1$. By suitable transformation within $G_{M(s)}$, we arrange it so that $p^{m}>1, m \equiv m_{\infty}>0$. Under the $p^{m}$ transformers $S_{\beta}$ of the $G_{p^{m}}^{(\infty)}$, the remaining $G_{p^{m_{\mu}}}^{(\mu)}$ with $m_{\mu}>0(\mu \neq \infty)$, if any, arrange themselves in sets each consisting of $p^{m}$ conjugate groups. Under the $G_{\Omega}$ the $G_{p^{m}}^{(\infty)}$ is then one of a set of $1+f p^{m}$ conjugate groups, $f$ being a positive integer or zero. The $G_{\Omega}$ contains no group $G_{p}^{(\mu)}\left(m_{\mu}>0\right)$ other than the $1+f p^{m}$ groups of this set. For, any such group would be one of a set of $n_{\mu}$ conjugate groups, where $n_{\mu}$ would necessarily have at the same time the forms $1+f_{\mu} p^{m_{\mu}}$ and $f_{\mu}^{\prime} p^{m}$. Hence: Every $G_{\Omega}$ which contains operators of period $p$ contains these operators in $1+f p^{m}$ groups $G_{p}^{(\mu)}$ conjugate under $G_{\Omega}$, where for each $G_{\Omega}, f$ and $m$ are properly determined integers $f \overline{>}, m>0$.

The groups $G_{\Omega}$ with $f=0$ have been enumerated in $\S \S 249-250$. Consider the group $G_{\Omega}$ with $f>1, m>0$. It contains $1+f p^{m}$ groups conjugate with a certain $G_{p}^{(\infty)}$ formed of the substitutions $S_{\lambda}$, where $\lambda$ ranges over the an additive-group $\left[\lambda_{1}, \ldots, \lambda_{m}\right]$. The $G_{p^{m}}^{(\infty)}$ is $(\S 250)$ self-conjugate within $G_{\Omega}$ under a certain largest subgroup $G_{p}^{(\infty)} m_{d}$. Hence

$$
\Omega=\left(1+f p^{m}\right) p^{m} d .
$$


As in $\S 250$, we transform ${ }^{1}$ ) by a suitable $S_{\mu}$ and obtain a $G_{p}^{(\infty)}$ given by the extension of the group $G_{p} m$ of the $S_{\lambda}$ by the cyclic group $G_{d}^{(\infty, 0)}$ of the substitutions $P_{\eta}$ contained within the cyclic group $G_{e /(2 ; 1)}^{(\infty, 0)}$ of substitutions $P_{e_{i}}$. The group $G_{p m_{d}}^{(\infty)}$ is thus composed of the substitutions ${ }^{2}$ )

$$
V_{\eta, \lambda} \equiv\left(\frac{\eta, \eta \lambda}{0, \eta^{-1}}\right) \equiv\left(\frac{1, \lambda}{0,1}\right)\left(\frac{\eta, 0}{0, \eta^{-1}}\right)=S_{\lambda} P_{\eta}
$$

Since $-\eta$ and $+\eta$ lead to the same $P_{\eta}$, there are $(2 ; 1) d$ marks $\eta$, the distinct powers of a primitive root of $\eta_{0}^{d}=-1$. Since each $\eta$ is an $\varepsilon_{i}$, each $\eta^{2}$ is a multiplier of the additive-group $\left[\lambda_{1}, \ldots, \lambda_{m}\right]$.

To normalize $G_{\Omega}$ we transform by $P_{\sigma}$ :

$$
P_{\sigma}^{-1}\left(\frac{\alpha, \beta}{\gamma, \delta}\right) P_{\sigma}=\left(\frac{\alpha, \sigma^{2} \beta}{\sigma^{-2} \gamma, \delta}\right), \quad P_{\sigma}^{-1} V_{\eta, \lambda} P_{\sigma}=V_{\eta, \sigma^{2} \lambda} .
$$

Taking $\sigma=\sqrt{\lambda_{0}^{-1}}$, the transformer $P_{\sigma}$ is a substitution $z^{\prime}=\lambda_{0}^{-1} z$ of the $G_{(2 ; 1) M(s)} ; G_{M(s)}$ is transformed into itself and $G_{\Omega}$ into $G_{\Omega}^{\prime}$. The new additive-group $\left[\lambda_{1}^{\prime}, \ldots, \lambda_{m}^{\prime}\right]$ contains the mark $\sigma^{2} \lambda_{0} \equiv 1$ and hence all the marks $\neq 0$ of its multiplier $G F\left[p^{k}\right]$. We suppose this transformation to have been made and the primes dropped from $G^{\prime}, \lambda_{i}^{\prime}$.

The $G_{\Omega}$ of order 250) is obtained by extending the $G_{p}^{(\infty)} m_{d}$, formed of the $V_{\eta, \lambda}$, by certain $f p^{m}$ extenders $V_{j}\left(j=1, \ldots, f p^{m}\right)$,

$$
V_{j}=\left(\frac{\alpha_{j}, \beta_{j}}{\gamma_{j}, \delta_{j}}\right), \quad V_{\eta_{1} \lambda} V_{j}=\left(\frac{\alpha_{j} \eta, \alpha_{j} \eta \lambda+\beta_{j} \eta-1}{\gamma_{j} \eta, \gamma_{j} \eta \lambda+\delta_{j}^{\prime} \eta-1}\right) \quad\left(\gamma_{j} \neq 0\right) .
$$

It was shown above that $G_{\Omega}$ contains $\left(1+f p^{m}\right)\left(p^{m}-1\right)$ substitutions of period $p$. Of these $p^{m}-1$ are the $S_{\lambda}$ lying in $G_{p^{m}}^{(\infty)}$. The remaining $f p^{m}\left(p^{m}-1\right)$ are substitutions $V_{\eta, \lambda} V_{j}$ satisfying the necessary and sufficient conditions for period $p$ (§240),

$$
\alpha_{j} \eta+\delta_{j} \eta^{-1}+\gamma_{j} \eta \lambda= \pm 2 .
$$

Given $V_{j}\left(\gamma_{j} \neq 0\right)$ and $\eta(\eta \neq 0)$, there are at most $(2 ; 1)$ values $\lambda$ satisfying 251). For a given $V_{j}\left(\gamma_{j} \neq 0\right)$ there are consequently at most $(2 ; 1) d$ substitutions $V_{\eta, \lambda} \equiv V_{-\eta, \lambda}$ such that $V_{\eta, \lambda} V_{j}$ is of period $p$ Hence the various $V_{j}$ lead to at most $f p^{m}(2 ; 1) d$. such substitutions.

1) For $p^{k}=3, n / k$ odd, we have $d=1$, so that this transformation is here unnecessary and is reserved for use in $\S 252$.

2) The non-fractional substitutions (viz., with $\gamma=0$ ) of $G_{\Omega}$ are all of the form $V_{\eta}, 2$. Indeed, they form a group $G^{\prime}$ leaving the element $\infty$ invariant. Its substitutions of period $p$ form the subgroup $G_{p^{m}}^{(\infty)}$ which must be selfconjugate under $G^{\prime}$. Hence $G^{\prime} \equiv G_{p}^{(\infty)} m_{d}$.

DrCKsoN, Linear Groups. 
Comparing this maximum with the actual number $f p^{m}\left(p^{m}-1\right)$, we have $p^{m}-1 \overline{\overline{<}}(2 ; 1) d$. Since each of the corresponding $(2 ; 1) d$ marks $\eta$ must be one of the $e$ marks $\varepsilon_{i}$ of $\S 249$, then $(2 ; 1) d \overline{<} e$, where $e=(2,1 ; 1)\left(p^{k}-1\right)$ ' Finally $(\S 70), k$ is a divisor of $m$. Hence

$$
\text { 252) } \quad p^{m}-1 \overline{\overline{<}}(2 ; 1) d \overline{\overline{<}}(2,1 ; 1)\left(p^{k}-1\right) \overline{\overline{<}}(2,1 ; 1)\left(p^{m}-1\right) .
$$

Since the third number is always $\overline{<} 2\left(p^{k}-1\right)<2 p^{k}-1$, we have $p^{m}<2 p^{k}$, so that $m=k, m$ being a divisor of $k$. The additivegroup $\left[\lambda_{1}, \ldots, \lambda_{m}\right]$ is therefore its own multiplier $G F\left[p^{k}\right]$ and every $\lambda$ is zero or a multiplier $x$.

There are in all two cases:

[A] $\quad m=k, \quad p^{k}-1=(2 ; 1) d, \quad \Omega=\left(1+f p^{k}\right) p^{k}\left(p^{k}-1\right) /(2 ; 1)$

[B] $m=k, p>2, n / k$ even, $p^{k}-1=d, \Omega=\left(1+f p^{k}\right) p^{k}\left(p^{k}-1\right)$,

where for $(2 ; 1)$ we read 2 or 1 according as $p>2$ with $n / k$ odd or $p=2$.

The following lemma finds repeated application below:

If $V_{j}\left(\gamma_{j} \neq 0\right)$ be of period 2 , the ratio $\alpha_{j} / \gamma_{j}$ differs from the $\alpha_{i} / \gamma_{i}$ of every other $V_{i}$ and so is a characteristic invariant of the $\nabla_{\eta_{2}, \lambda} V_{j}$.

For $i \neq j, V_{i} V_{j}$ is not of the form $V_{\eta, \lambda}$, since otherwise

$$
V_{i}=V_{\eta, \lambda} V_{j}
$$

contrary to the choice of the extenders $V_{i}$. Hence in $V_{i} V_{j}$ the term corresponding to $\gamma$ is $\neq 0$, viz., $\alpha_{i} \gamma_{j}+\gamma_{i} \delta_{j} \neq 0$. Dividing by $\gamma_{i} \gamma_{i}$ and applying $\delta_{j}=-\alpha_{j}\left(V_{j}\right.$ being of period 2$)$ we find that

$$
\alpha_{i} / \gamma_{i}-\alpha_{j} / \gamma_{j} \neq 0 \text {. }
$$

252. For case [A] with $p^{k}>2$, the group $G_{\Omega}$ is the group $G_{M\left(p^{k}\right)}$ of all linear fractional substitutions of determinant unity in the $G F\left[p^{k}\right]$. For $p^{k}=2, G_{\Omega}$ is a dihedron $G_{2(1+2 f)}$, which for $f=1$ is the $G_{M(2)}$.

For $p^{k}>2$, it is shown that every $V_{j}$ may be chosen so that $\alpha_{j}, \beta_{j}, \gamma_{j}, \delta_{j}$ all belong to the $G F\left[p^{k}\right]$. Hence $G_{\Omega}$ is a subgroup of $G_{M\left(p^{k}\right)}$. But, if $f>1, \Omega>M\left(p^{k}\right)$. Hence must $f=1, \Omega=M\left(\dot{p}^{k}\right)$, so that $G_{\Omega} \equiv G_{M\left(p^{k}\right)}$.

For case [A], relations 252) become equalities, so that the earlier argument shows that, for $V_{j}$ and $\eta$ given $\left(\gamma_{j} \neq 0, \eta \neq 0\right)$, there exist exactly $(2 ; 1)$ marks $\lambda$ of the $\left[\lambda_{1}, \ldots, \lambda_{m}\right]$ which satisfy 251$)$. The given $\eta$ may be any one of the multipliers $x$, since the number $(2 ; 1) d$ of $\eta$ 's equals the number $p^{k}-1$ of $x$ 's.

The extender $V_{j}$ may be replaced by any one of the products $V_{\eta_{3} \lambda} V_{j}$ and in particular by one of period $p$, having therefore $\alpha_{j}+\delta_{j}= \pm 2$. Changing if necessary the signs of all four coefficients 
of $V_{j}$, we may take $\alpha_{j}+\delta_{j}=2$. 'With this normalized $V_{j}$, the condition 251) becomes (upon setting $\eta=x$ )

$$
u_{j}\left(x-x^{-1}\right)+\gamma_{j} \dot{x} \lambda= \pm 2-2 x^{-1} .
$$

For any given $j$ and any given mark $x \neq 0$ of the $G F\left[p^{k}\right]$ and for each sign \pm , this equation must determine a mark $\lambda \equiv \lambda_{j, x, \pm}$ of the $G F\left[p^{k}\right]$. If $p>2,253$ ) for $x=1$ gives

$$
\gamma_{j} \lambda_{j, 1,-}=-4 \neq 0,
$$

so that $\gamma_{j}$ belongs to the $G F\left[p^{k}\right]$. For $p^{k}>3, x$ has a value different from \pm 1 and from zero; for such a $\varkappa 253$ ) requires that $\alpha_{j}$ belong to the $G F\left[p^{k}\right]$ : Then $\delta_{j}=2 \div \alpha_{j}$ belongs to the field. The determinant being unity, $\beta_{j}$ also belongs to the field.

For $p^{k}=3$, the non-vanishing marks $\gamma_{j}, \eta$ may be restricted to the value +1 . Since $\alpha_{j}+\delta_{j}=2$ in $V_{j}$, the $\alpha+\delta$ of $V_{1,1} V_{j} \equiv V_{j}^{\prime}$ has the value $\alpha_{j}+\delta_{j}+1=0$ in the field. Hence $V_{j}^{\prime}$ takes the form

$$
\left(\frac{\alpha,-1-\alpha^{2}}{1,-\alpha}\right) \equiv W_{\alpha}
$$

The $W_{\alpha}$ may be taken as extenders in place of the $V_{j}$. The subgroup $G_{p} m_{d}$ is here composed of three substitutions $V_{1, \lambda}, \lambda=0, \pm 1$. Hence every substitution of $G_{\Omega}$ has as its $\gamma$ and $\alpha+\delta$ marks of the $G F\left[p^{k}\right]$. Transforming the group by $S_{-} \alpha_{0}$, where $\alpha_{0}$ is a particular $\alpha$, each $V_{\eta, \lambda} \equiv S_{\lambda}$ is transformed into itself and each $W_{\alpha}$ into $W_{\alpha-\alpha_{0}}$. Hence, in the transformed group each $\gamma$ and $\alpha+\delta$ belong to the $G F\left[p^{k}\right]$. Among the new extenders $W_{a} \equiv W_{\alpha-\alpha_{0}}$ occurs $W_{0}$. Hence $G_{\Omega}$ contains

$$
W_{0} \equiv\left(\frac{0,-1}{1,-0}\right), \quad W_{a} W_{0}=\left(\frac{-1, a}{a,-1-a^{2}}\right)
$$

so that the mark $a$, being in the position of a $\gamma$, belongs to the $G F\left[p^{k}\right]$.

For $p=2, k>1$, there exist marks $x$ different from 0 and $1(+1=-1)$; for such a $x, 253)$ shows that $\alpha_{j} / \gamma_{j}$ is a mark $\lambda_{j}$ of the $G F\left[2^{k}\right]$. Since $p=2, \alpha_{j}+\delta_{j}=2$ gives $\alpha_{j}=\delta_{j}$, and $\delta_{j} / \gamma_{j}=\lambda^{j}$. There are $f p^{k}$ substitutions $V_{j}$ and $f p^{k}>2$. The product $V_{i} V_{j}(i \neq j)$ belongs to $G_{\Omega}$ and is not of the form $V_{x, \lambda}$ since $V_{i} \neq V_{j} V_{x, \lambda}$ and since $V_{j}$ is of period 2. Hence we may set $V_{i} V_{j}=V_{\varkappa, \lambda} V_{l}$. Since $i \neq j, \lambda_{i}+\lambda_{j} \neq 0$ (end of $\S 251$ ). We find that

$$
\lambda_{l}=\frac{\alpha_{i} \alpha_{j}+\gamma_{i} \beta_{j}}{\alpha_{i} \gamma_{j}+\gamma_{i} \alpha_{j}}=\frac{\lambda_{i} \lambda_{j}}{\lambda_{i}+\lambda_{j}}+\frac{1}{\lambda_{i}+\lambda_{j}} \cdot \frac{\beta_{j}}{\gamma_{j}} .
$$

Hence every $\beta_{j} / \gamma_{j}$ belongs to the $G F\left[p^{k}\right]$. Then $\alpha_{j} \delta_{j}-\beta_{j} \gamma_{j}=1$ requires that $\gamma_{j}^{2}$ belong to that field and hence also $\gamma_{j}, p$ being 2. Then $\alpha_{j}, \beta_{j}, \delta_{j}$ belong to the field since their ratios to $\gamma_{j}$ do. 
For $p=2 ; k=1$, the group $G_{\Omega}$ of order $2(1+2 f)$ is given by the extension of $G_{2}^{(\infty)}$, formed of the substitutions $S_{0} \equiv I$ and $S_{1}$, by certain $2 f$ extenders $V_{j}(j=1, \ldots, 2 f)$ each of period 2. By $\S 251$, all the substitutions of period 2 in $G_{\Omega}$ form one set of $1+2 f$ conjugate substitutions. Setting $V_{0} \equiv S_{1}$, the substitutions of period 2 in $G_{\Omega}$ are $V_{j}(j=0,1, \ldots, 2 f)$ and the remaining substitutions $V_{0} V_{j} \equiv U_{j}$ are of period $\neq 2$. Hence no $U$ is conjugate with a $V$. The product $V_{j^{\prime}} U_{j}$ cannot be a $U$; for the substitution of $G_{\Omega}$ which transforms $V_{j^{\prime}}$ into $V_{0}$ transforms the product into $V_{0} U_{i} \equiv V_{i}$, but transforms the $U$ into some $U$. Hence $V_{j^{\prime}} U_{j}$ is of the form $V_{j^{\prime \prime}}$ so that $V_{0} U_{j^{\prime}} U_{j}=V_{0} U_{j^{\prime \prime}}$. Hence every product $U_{j^{\prime}} U_{j}$ is a $U_{j^{\prime \prime}}$. The substitutions $U$ form a group $G_{\frac{1}{2} \Omega}$. Since $U_{j}=V_{0} V_{j}$, we have, for every $j$,

2j4)

$$
V_{0}^{-1} U_{j} V_{0}=U_{j}^{-1}
$$

For $U_{j}$ and $U_{j^{\prime}}$ arbitrary, there exists in the $G_{\frac{1}{2} \Omega}$ a $U_{i}$ such that

$$
U_{j} U_{j^{\prime}}=\left(U_{j^{\prime}}^{-1} U_{j}^{-1}\right)^{-1}=\left(U_{i}\right)^{-1}=\nabla_{0}^{-1} U_{i} V_{0}=V_{0}^{-1}\left(U_{j^{\prime}}^{-1} U_{j}^{-1}\right) V_{0}=U_{j^{\prime}} U_{j} .
$$

The group $G_{1+2 f}$ of the $U$ 's is, therefore commutative and contains substitutions of period $>2$, By $\S 244$, it is a cyclic subgroup of $G_{s \mp 1}$. In view of 254) the group $G_{\Omega}$ is a dihedron $G_{2(1+2 f)}$ based on the cyclic $G_{1+2 f}(\S 245)$. These groups $G_{\Omega}$ have therefore been enumerated in $\S 246$ and may be dropped from further consideration.

253. For case $[\mathrm{B}], p>2, n / k$ is even and $p^{k}-1=d$. The $2 d$ marks $\eta$ are the square roots of the $p^{k}-1$ marks $x$ and hence are the distinct powers of $\eta_{0}=\sqrt{x_{0}}$, where $x_{0}$ is a primitive root of the $G F\left[p^{k}\right]$. In particular, there is a mark $\eta=\sqrt{-1}$.

Within $G_{\Omega}$ there are exactly $1+f p^{k}$ groups conjugate with the $G_{p^{k}\left(p^{k}-1\right)}^{(\infty)}$. The latter contains $p^{k}$ conjugate cyclic $G_{p^{k}-1}^{(\infty, \lambda)}$ and hence in all $p^{k}$ substitutions $T$ of period 2 , each conjugate with

$$
I_{0}=\left(\frac{\sqrt{-1}, 0}{0,-\sqrt{-1}}\right) \text {. }
$$

Under $G_{\Omega}$ of order $\Omega=\left(1+f p^{k}\right) p^{k}\left(p^{k}-1\right)$, this $T_{0}$ is one of a system of $\left(1+f p^{k}\right) p^{k}$ or $\frac{1}{2}\left(1+f p^{k}\right) p^{k}$ conjugate substitutions $T$ according as $T_{0}$ is within $G_{\Omega}$ self-conjugate under the cyclic $G_{p k-1}^{(\infty, 0)}$ or under a dihedron obtained by extending the former by a substitution $T_{0}^{\prime}$ which interchanges the elements $\infty, 0$ (§§ 242, 246). In the respective cases there would be at least $f p^{2 k}$ or $\frac{1}{2}\left(f p^{k}-1\right) p^{k}$ substitutions $\nabla_{\eta, \lambda} V_{j}(j>0)$ of period 2 , necessarily satisfying the relation 251 ),

$$
\alpha_{j} \eta+\delta_{j} \eta^{-1}+\gamma_{j} \eta \lambda=0 \text {. }
$$


For each of the $f p^{k}$ extenders $V_{j}\left(j>0, \gamma_{j} \neq 0\right)$, each value of $\eta$ gives a single value of $\lambda$, which may or may not belong to the $G F\left[p^{k}\right]$. Hence there are at most $f p^{k}\left(p^{k}-1\right)$ substitutions $V_{\eta, \lambda} V_{j}$ of period 2. The second alternative therefore holds, so that $G_{\Omega}$ contains a substitution of the form

$$
T_{0}^{\prime}=\left(\frac{0,-\tau^{-1}}{\tau, \quad 0}\right) \text {. }
$$

Also $\frac{1}{2}\left(1+f p^{k}\right)$ is an integer so that $f$ is odd.

In case a $V_{j}(j>0)$ gives rise to one or more substitutions $T=V_{\eta, \lambda} V_{j}$, we replace $V_{j}$ by one such $T$, so that the new $V_{j}$ has $\alpha_{j}+\delta_{j}=0$. Let $N$ denote the number of these $V_{j}$ for which there exists a product $V_{\eta_{2},} V_{j}$ distinct from $V_{j}$ and of period 2. For such a $V_{j}$ the equation

$$
\alpha_{j}\left(\eta-\eta^{-1}\right)+\gamma_{j} \eta \lambda=0
$$

will be satisfied by a pair $\eta, \lambda \neq \pm 1,0$, such that $\eta^{2}$ and $\lambda$ belong to the $G F\left[p^{k}\right]$. Hence will

$$
\alpha_{j} / \eta_{j}=\eta^{2} \lambda /\left(1-\eta^{2}\right)
$$

belong to that field. Inversely, if $\alpha_{j} / \gamma_{j}$ belong to that field, and $\eta^{2}$ be an arbitrary mark $\neq 0$ of that field, there exists an unique solution $\lambda$ in the field, so that there will be $p^{k}-1$ substitutions $V_{\eta, \lambda} V_{j}$ of period 2. By the lemma at the end of $\S 251$, the $N$ substitutions $V_{j}$ have distinct values for $\alpha_{j} / \gamma_{j}$, here shown to belong to the $G F\left[p^{k}\right]$. Hence $N \overline{<} p^{k}$. Let $M$ denote the number of the $V_{j}$ leading to a single $V_{\eta, \lambda} V_{j}$ of period 2. Then $M \overline{<} f p^{k}-N$. The total number of the $V_{\eta, 2} V_{j}(j>0)$ of period 2 is therefore

$$
N\left(p^{k}-1\right)+M \overline{<} N\left(p^{k}-1\right)+f p^{k}-N \text {. }
$$

The second member is greatest when $N$ has its maximum value $p^{k}$. By comparing the minimum and maximum numbers for the

of period 2 in $G_{\Omega}$, we have

$$
V_{\eta, 2} V_{j}
$$

$$
\frac{1}{2}\left(f p^{k}-1\right) p^{k} \overline{<} p^{k}\left(p^{k}-1\right)+(f-1) p^{k} .
$$

Hence must $f=1$ or 3 , leading to the two cases:

$$
\begin{array}{lll}
(f=1) & p>2, \quad n / k \text { even, } \quad \Omega=\left(p^{k}+1\right) p^{k}\left(p^{k}-1\right) \equiv 2 M\left(p^{k}\right) \\
(f=3) & p=3, \quad k=1, \quad n \text { even, } \quad \Omega=60 .
\end{array}
$$

Consider first the case $f=1 . G_{\Omega}$ contains the transformed of $T_{o}^{\prime}$ by $S_{\lambda}$,

$$
T_{\lambda}^{\prime}=\left(\frac{\lambda \tau,-\lambda^{2} \tau-\tau^{-1}}{\tau,-\lambda \tau}\right)
$$


Létting $\lambda$ run through the series of marks of the $G F\left[p^{k}\right]$, the ratio $\alpha / \gamma \equiv \lambda \tau / \tau \equiv \lambda$ takes $p^{n}$ distinct values. By the lemma at the end of $\S 251$, the $T_{\lambda}^{\prime}$ may be chosen as the $p^{k} \equiv f p^{k}$ extenders $V_{j}$. For each $V_{j}$. the ratios $\alpha_{j} / \gamma_{j}, \delta_{j} / \gamma_{j}$ are marks $\lambda_{j}, \lambda_{j}^{\prime \prime}$ of the $G F\left[p^{k}\right]$. As in case [A] for $p=2, k>1$, the ratio $\beta_{j} / \gamma_{j}$ is a mark $\lambda_{j}^{\prime}$ of the field. The determinant being unity," $\gamma_{j}^{2}$ belongs to the field, so that $\gamma_{j}$ is some $\eta_{j}$. Hence

$$
\dot{V_{j}}=\left(\frac{\lambda_{j} \eta_{j}, \quad \lambda_{j}^{\prime} \eta_{j}}{\eta_{j}, \quad \lambda_{j}^{\prime \prime} \eta_{j}}\right)
$$

According as $\eta_{j}$ is an even or an odd power of $\eta_{0} \equiv \sqrt{x_{0}}, V_{j}$ or $V_{\eta_{0}, 0} V_{j}$ has its coefficients in the $G F\left[p^{k}\right]$. The one having this property is denoted by $V_{j}^{\prime}$. These $p^{k}$ substitutions $V_{j}^{\prime}$ serve to extend the group $G_{\frac{1}{2} p^{k}\left(p^{k}-1\right)}^{(\infty)}$ of the $V_{x, \lambda}$ to the group $G_{M\left(p^{k}\right)}$ of all linear fractional substitutions of determinant unity in the $G F\left[p^{k}\right]$. It - is transformed into itself by

$$
P_{\eta_{0}}=\left(\frac{\eta_{0}, 0}{0, \eta_{\overline{0}^{-1}}}\right) \equiv\left(\frac{x_{0},}{0,}\right)
$$

whose square $P_{\eta^{2} \mathrm{o}} \equiv P_{\psi_{0}}$ belongs to $G_{M\left(p^{k}\right)}$. Hence $P_{\eta_{0}}$ extends the latter to the group $G_{2 M\left(p^{k}\right)}$ of all linear fractional substitutions in the $G F\left[p^{k}\right]$. The latter is a subgroup of $G_{\Omega}$ and is of order $\Omega$. $G_{\Omega}$ is therefore identical with the linear fractional group $G_{2 M\left(x^{k}\right)}$.

For the case $f=3, p^{k}=3$, the relation 255) becomes an equality, so that there are exactly $12+3=15$ substitutions $T$ of period 2 in $G_{60}$. At the beginning of the section, each $T$ was shown to be self-conjugate within $G_{60}$ under exactly a dihedron $G_{4}$. The 15 substitutions $T$ are therefore all conjugate under $G_{60}$ and form 5 conjugate four-groups $G_{4}$. By $\S 251, G_{60}$ contains one set of $1+f p^{k}=10$ conjugate $G_{3}$. Hence, if the $G_{60}$ exists, it is of the icosahedral type (§ 254). For $n$ even, $5 \equiv \frac{1}{2}\left(3^{2}+1\right)$ divides $\frac{1}{2}\left(3^{2 n}-1\right)$, so that the existence of icosahedral subgroups of $G_{M\left(3^{n}\right)}$ follows from $§ 259$ The question of the conjugacy of the icosahedral subgroups is answered in that section.

254. A group of order 60 is of the icosahedral type if it contains exactly ten conjugate $G_{3}$ and exactly 15 operators of period 2 lying in 5 conjugate four-groups.

Since there is a complete set of 5 conjugate $G_{4}$ within the $G_{60}$, each $G_{4}$ is self-conjugate under exactly a subgroup $G_{12}$. The latter is of the tetrahedral type by $\S 247$; for if cqmmutative it would contain a self-conjugaté $G_{3}$ which would be one of a set of at most 
5 conjugate subgroups of $G_{60}$. Hence $G_{60}$ contains a set of 5 conjugate tetrahedral $G_{12}$. No two of them are identical since each contains a single four-group. They have only the identity in common. Indeed, their common operators form a self-conjugate subgroup of $G_{60}$ and hence a self-conjugate subgroup of each $G_{12}$. Aside from the identity and $G_{12}$ itself (cases requiring no further discussion), the .only self-conjugate subgroup of a tetrahedral $G_{12}$ is its four-group. But the 5 four-groups are all distinct. Hence the identity is the only operator of $G_{60}$ which transforms each $G_{4}$ into itself. Applied as transformers, the operators of $G_{60}$ permute the 5 conjugate $G_{4}$, so that $G_{60}$ is holoedrically isomorphic with a substitution-group on 5 letters. Being of order 60 , the latter is necessarily the alternating group on 5 letters. ${ }^{1}$ ) Hence the $G_{60}$ is of the icosahedral type ( $\$ 267$ ).

255. It remains to study the conjugacy of the linear fractional subgroups $G_{M\left(p^{k}\right)}$ and $G_{2 M\left(p^{k}\right)}$ of $G_{M(s)}$. Within $G_{M(s)}$ the $G_{M\left(p^{k}\right)}$ is self-conjugate exactly under $G_{2 M\left(p^{k}\right)}, G_{M\left(p^{k}\right)} ; G_{M\left(p^{k}\right)}$ according as $p>2$ with $n / k$ even, $p>2$ with $n / k$ odd; or $p=2$, and hence is one of $a$ system of $M(s) /(2,1 ; 1) M\left(p^{k}\right)$ conjugate groups. In proof, we note that a substitution $V=\left(\frac{\alpha, \beta}{\gamma, \delta}\right)$ of $G_{M(s)}$ transforms (\$240) the substitutions $\left(\frac{1, \sigma}{0,1}\right)$ and $\left(\frac{1,0}{\sigma, 1}\right)$ into respectively

$$
\left(\frac{1-\alpha \gamma \sigma, \alpha^{2} \sigma}{-\gamma^{2} \sigma, 1+\alpha \gamma \sigma}\right), \quad\left(\frac{1+\beta \delta \sigma,-\beta^{2} Q}{\delta^{2} \sigma, 1-\beta \delta \sigma}\right) .
$$

If $\sigma$ belongs to the $G F\left[p^{n}\right]$, these substitutions belong to that field if, and only if, $\alpha$ and $\gamma$ are each marks $\mu$ of the $G F\left[p^{k}\right]$ or are each of the form $\mu \sqrt{\nu}$, where $\nu$ is a not-square in the $G F\left[p^{k}\right]$, and $\beta, \delta$ are each marks $\mu$ or are each of the form $\mu \sqrt{\nu}$. Since $\alpha \delta-\beta \gamma=1, \alpha, \beta, \gamma, \delta$ are all of the form $\mu$ or all of the form $\mu \sqrt{\nu}$. Hence $V$ is either a substitution $S$ of $G_{M\left(\gamma^{k}\right)}$ or else a product $S P_{\sqrt{v}}$. The latter alternative does not occur if $p=2$. Also, if $p>2, \sqrt{v}$ belongs to the $G F\left[p^{n}\right]$ if; and only, if $n / k$ is even. Hence $G_{M\left(p^{k}\right)}$ is self-conjugate within $G_{M(s)}$ in a larger group, viz., $G_{2 M\left(\gamma^{k}\right)}$, if and only if $p>2$ with $n / k$ even.

Within $G_{M(s)}$ the $G_{2 M\left(p^{k}\right)}$, when existent, is self-conjugate only under itself. For any substitution of the former which transforms the latter into itself must transform its self-conjugate subgroup $G_{M\left(p^{k}\right)}$

1) If a $G_{60}^{(5)}$ contained odd substitutions, it would have a subgroup $G_{30}^{(5)}$ of even substitutions. The latter would be of index two under the alternating group $G_{60}^{(5)}$ and hence self-conjugate under it, whereas it is simple. 
into itself and hence belong to $G_{2 M\left(p^{k}\right)}$. The latter thus forms one of a system of $M(s) / 2 M\left(p^{k}\right)$ conjugate subgroups.

It remains to determine the number of systems of conjugate subgroups of these two types; indeed, in $\S 251$, there entered the transformer $P_{\sqrt{\lambda_{0}-1}}$ which belongs to $G_{M(s)}$ if and only if $\lambda_{0}$ is a square in the $G F\left[p^{n}\right]$. For $p=2, \lambda_{0}$ is necessarily a square; for $p>2, n / k$ odd, $\lambda_{0}$ may be chosen as a square, since every additivegroup $\left[\lambda_{1}, \ldots, \lambda_{k}\right]$ with the multiplier $G F\left[p^{k}\right]$ has half of its nonvanishing marks squares in the $G F\left[p^{n}\right]$. In these two cases there is evidently but one system of conjugate subgroups $G_{M\left(p^{k}\right)}$ of $G_{M(s)}$. For $p>2, n / k$ even, all the marks of $\left[\lambda_{1}, \ldots, \lambda_{k}\right]$ are squares or all are not-squares in the $G F\left[p^{n}\right]$; indeed, they are all obtained from a single one by multiplication by the $p^{n}$ marks of the multiplier $G F\left[p^{k}\right]$ and the latter are all squares in the $G F\left[p^{n}\right]$. In this case there are consequently two systems of conjugate subgroups $G_{M\left(p^{k}\right)}$ and two systems of conjugate $G_{2 M\left(p^{k}\right)}$, the systems of each type being interchanged upon transformation by $P_{\sqrt{v}}$, belonging to $G_{2 s(s)}$, where $v$ is any not-square in the $G F\left[p^{n}\right]$. Hence there are $(2,1 ; 1)$ systems of conjugate $G_{M\left(p^{k}\right)}$ and $(2,0 ; 0)$ systems of conjugate $G_{2 M\left(p^{k}\right)}$ within $G_{M(s)}$.

256. Subgroups of $G_{M(s)}$ containing no operators of period $p$. Every substitution of such a subgroup $G_{\Omega}$ lies in and determines a largest cyclic subgroup $G_{d}$ of $G_{\Omega}$ (§§ 242-243). Two such groups $G_{d}$ have only the identity in common. According as $G_{d}$ is selfconjugate within $G_{\Omega}$ only under itself or under a dihedron $G_{2 d}$ based on $G_{d}$, it is one of a system of $\Omega / d$ or $\Omega / 2 d$ conjugate subgroups of $G_{\Omega}$. Let $r$ denote the number of such systems. The enumeration of the substitutions of $G_{\Omega}$ leads to the relations

$$
\begin{array}{lr}
\Omega=1+\sum_{i=1}^{r}\left(d_{i}-1\right) \frac{\Omega}{f_{i} d_{i}} & \left(f_{i}=1 \text { or } 2\right) \\
\Omega>f_{i} d_{i} & (i=1,2, \ldots, r) .
\end{array}
$$

If two non-conjugate cyclic $G_{d_{i}}, G_{d_{j}}$ of odd order are present in $G_{\Omega}$, there are at least $d_{j}$ groups in the system determined by $G_{d_{i}}$, viz., the transformed of the latter by the operators of $G_{d_{j}}$, and vice versa, so that

$$
\Omega \equiv d_{i}\left(d_{j}-1\right)+d_{j}\left(d_{i}-1\right)+1 .
$$

Solving 256) for $1 / \Omega$, we get

$$
1-\sum_{i=1}^{r} \frac{\left(d_{i}-1\right)}{f_{i} d_{i}}=\frac{1}{\Omega} .
$$


Since $f_{i}=1$ or 2 , the least value of $\left(d_{i}-1\right) / f_{i} d_{i}$ is $1 / 4$. Since 259) must be positive, there can be at most three terms in the sum, whence $r \overline{\overline{<}} 3$.

For $r=1$, the reciprocal of 259) is not an integer if $f_{1}=2$. For $f_{1}=1, \Omega=d_{1}$, and the $G_{\Omega}$ is a cyclic group considered in $\S \S 242-243$.

For $r=2$, we have

$$
1-\frac{1}{\Omega}=\frac{1}{f_{1}}\left(1-\frac{1}{d_{1}}\right)+\frac{1}{f_{2}}\left(1-\frac{1}{d_{2}}\right) .
$$

If $f_{1}=f_{2}=1$, the left member is $<1$ and the right member is $\overline{>}$. If $f_{1}=f_{2}=2$,

$$
\frac{2}{\Omega}=\frac{1}{d_{1}}+\frac{1}{d_{2}}, \quad \frac{2}{\Omega} \overline{<} \frac{1}{d_{1}}
$$

Hence these two cases are to be excluded. The case $f_{1}=2, f_{2}=1$ differs only in notation from the case $f_{1}=1, f_{2}=2$. In the latter case,

$$
\frac{1}{\Omega}=\frac{1}{d_{1}}+\frac{1}{2 d_{2}}-\frac{1}{2} \overline{<} \frac{1}{d_{1}}-\frac{1}{4},
$$

so that $d_{1}<4$. For $d_{1}=2, \Omega=2 d_{2}$, so that $G_{\Omega}$ is a dihedron $G_{2 d_{2}}$ with $d_{2}$ odd $(\S 245)$ yielding a group considered in $\S 246$. For $d_{1}=3$, $d_{2}$ must be 2 , whence $\Omega=12$. The operator of period $d_{2}=2$ is self-conjugate within $G_{12}$ under exactly a dihedron $G_{4}$, so that $G_{12}$ is not a commutative group. Since the operators of period 2 fall into a single set of 3 conjugate operators, there is a single subgroup $G_{4}$, so that it is self-conjugate under $G_{12}$. By $\S 247$, the $G_{12}$ is a tetrahedral group.

For $r=3$, then $f_{1}=f_{2}=f_{3}=2$. For if $f_{1}=1$, for example, 259) becomes

$$
\frac{1}{d_{1}}-\frac{\left(d_{2}-1\right)}{f_{2} d_{2}}-\frac{\left(d_{3}-1\right)}{f_{3} d_{3}} \overline{<} \frac{1}{d_{1}}-\frac{1}{4}-\frac{1}{4} \overline{<} 0 .
$$

Setting each $f_{i}=2$, equation 259) may be written

$$
1+\frac{2}{\Omega}=\frac{1}{d_{1}}+\frac{1}{d_{2}}+\frac{1}{d_{s}} \text {. }
$$

If every $d_{i} \bar{\Sigma} 3$, the right member would be $\overline{<}$. Setting $d_{3}=2$,

$$
\frac{1}{2}+\frac{2}{\Omega}=\frac{1}{d_{1}}+\frac{1}{d_{2}} \text {. }
$$

If either $d_{1}$ or $d_{2}$ is 2 , we may take $d_{2}=2$, whence $\Omega=2 d_{1}$ and $G_{\Omega}$ is a dihedron $G_{2 d_{2}}$ with $d_{1}$ even $(\S 245)$ yielding a group considered in $\S 246$. In the contrary case, $d_{1}>2, d_{2}>2$. Then both do not exceed 3 , since otherwise the right member would be at most $\frac{1}{4}+\frac{1}{4}=\frac{1}{2}$. Taking $d_{2}=3$, we have

$$
\frac{1}{6}+\frac{2}{\Omega}=\frac{1}{d_{1}}
$$


Hence $d_{1}<6$. For $d_{1}=3,4,5$ we find $\Omega=12,24,60$ respectively. But $d_{1}=3, d_{2}=3, d_{3}=2, \Omega=12$ is excluded by 258 ). For $d_{1}=4$, $d_{2}=3, d_{3}=2$, the $G_{24}$ is of the octahedral type ( $(248)$. For $d_{1}=5$, $d_{2}=3, d_{3}=2$, the $G_{60}$ is of the icosahedral type ( $\left.\$ 254\right)$.

25\%. The tetrahedral and octahedral subgroups of the $G_{M(s)}$. A group of either type must contain a self-conjugate four-group. For $p>2$, the desired groups are therefore given by the theorem at the end of $\S 246$. For $p=2$, they contain operators of period 2 and are therefore to be sought among the subgroups determined in $\S \S 250-253$. But for $p=2$, the dihedron $G_{2(1+2 f)}$ and the $G_{M\left(p^{k}\right)}$ are neither of the tetrahedral and neither of the octahedral type. There remain for consideration only the subgroups of the $G_{s(s-1)}^{(x)}$ of $\$ 250$. There is no octahedral subgroup of $G_{s(s-1)}^{(\infty)}$ since the substitutions of period $p=2$ in the latter are all commutative. In a tetrahedral group the three substitutions, of period 2 are all commutative. Hence if there be a tetrahedral subgroup of $G_{s(s-1)}^{(o)}, p=2$, then must $2^{m}=4, d=3$ and $n$ even (since 3 must divide $2^{n}-1$ ). Inversely, if $m=2, p=2, n$ even, there exists a subgroup $G_{2} m_{d} \equiv G_{12}$ of $G_{s(s-1)}^{(\infty)}$. The $G_{12}$ is not commutative, since it would then contain only operators of period $p=2(\S 241)$, and therefore $G_{12}$ has the tetrahedral type ( $\$ 247)$. We may state the complete theorems:

For $s \equiv p^{n}=8 h \pm 1$, the $G_{M(s)}$ contains two systems each of $M(s) / 24$ conjugate octahedral groups $G_{24}$ and two systems each of $M(s) / 24$ conjugate tetrahedral groups $G_{12}$. Every $G_{12}$ is self-conjugate under a $G_{24}$. The two systems are conjugate under $G_{2 M(s)}$.

For $s=8 h \pm 3$ or $s=2^{n}$, n even, the $G_{M(s)}$ contains no octahedral $G_{24}$ but contains one system of $M(s) / 12$ conjugate tetrahtdral $G_{12}$.

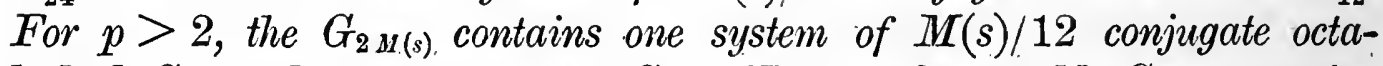
hedral $G_{24}$ each containing one $G_{12}$. For $s=2^{n}, n$ odd, $G_{M(s)}$ contains no octahedral and no tetrahedral groups.

258. Icosahedral subgroups of $G_{M(s)}$ for $p=5$. An icosahedral $G_{60}$ is generated by two operators $V_{5}, V_{2}$ different from the identity and subject to the generational relations ( $\$ 267)$

$$
V_{5}^{5}=V_{2}^{2}=I, \quad\left(V_{5} V_{2}\right)^{3}=I .
$$

$G_{M(5)}$ contains $4(5+1)=24$ substitutions of period 5 and each is conjugate within $G_{M(5)}$ with one of the substitutions ( $\$ 241$ ).

$$
\text { - } V_{5}=\left(\frac{1, \mu}{0,1}\right) . \quad \mu \equiv \equiv(\bmod 5) .
$$


The only substitutions $V_{2}$ of period 2 of $G_{M(5)}$ which satisfy the condition $\left.^{1}\right)\left(V_{5} V_{2}\right)^{3}=I$ are seen to be the following five

$$
\dot{V}_{2}=\left(\frac{\alpha,-\mu\left(1+\alpha^{2}\right)}{\mu-1,-\alpha}\right) . \quad(\alpha \equiv 0,1,2,3,4) .
$$

Hence $G_{M(5)}$ is an icosahedral group ${ }^{2}$ ) and contains $24 \cdot 5=120$ pairs of generators $V_{5}, V_{2}$. By $\S 255, G_{M\left(5^{n}\right)}$ contains $M\left(5^{n}\right) / 60$ icosahedral subgroups forming two systems or one system of conjugate groups according as $n$ is even or odd.

259. Icosahedral subgroups of $G_{M(s)}$ for $p \neq 5$. The order $p^{n}\left(p^{2 n}-1\right) /(2 ; 1)$ of $G_{M(s)}$ is divisible by 60 if, and only if, $p^{2 n}-1$ be divisible by 5 and hence either $p^{n}+1$ or $p^{n}-1$ divisible by 5 . In either case $G_{M(s)}$ contains cyclic subgroups $G_{5}$ all of which are conjugate ( $\$ \S 242,243$ ).

(i) Let $p^{n}-1$ be divisible by 5 and set $\lambda \equiv\left(p^{n}-1\right) / 5$. Let $\varrho$ be a primitive root of the $G F\left[p^{n}\right]$, so that $\varrho^{\lambda}$ is of period 5 . Setting

$$
\begin{gathered}
\quad V_{5}=\left(\frac{\rho^{2}, 0}{0, \varrho^{-\lambda}}\right), \quad V_{2}=\left(\frac{\alpha, \beta}{\gamma,-\alpha}\right) \\
-\alpha^{2}-\beta \gamma=1,
\end{gathered}
$$

we seek the conditions under which the product

$$
V_{5} V_{2}=\left(\frac{\alpha \rho^{\lambda}, \quad \beta \rho^{\lambda}}{\gamma \varrho^{-\lambda},-\alpha \rho^{-\lambda}}\right)
$$

shall be of period 3. The necessary and sufficient condition is

$$
\alpha\left(\rho^{\lambda}-\rho^{-\lambda}\right)= \pm 1 \text {. }
$$

The upper sign may be chosen, changing if necessary the signs of $\alpha$, $\beta, \gamma$ in $V_{2}$. Hence $\alpha$ is determined uniquely. Combining with 260),

$$
\beta \gamma=-1-\left(\varrho^{\lambda}-\varrho^{-\lambda}\right)^{-2} \neq 0 .
$$

Indeed, if the second member vanish, $\varrho^{4 \lambda}-\varrho^{2 \lambda}+1=0$, so that $\varrho^{6 \lambda}+1=0$ and therefore $\varrho^{2 \lambda}=+1$, whereas $\varrho^{\lambda}$ is of period 5 . Hence to each of the $p^{n}-1$ values $\neq 0$ of $\beta$ corresponds a single value of $\gamma$. But $G_{M 1(s)}$ contains $(\S 242)$ exactly $\frac{1}{2} p^{n}\left(p^{n}+1\right)$ distinct cyclic $G_{5}$. Hence there are $2 p^{n}\left(p^{2}-1\right)$ pairs of generators $V_{5},-V_{2}$ of icosahedral subgroups. 1) It is readily verified that a substitution $\left(\frac{\alpha, \beta}{\gamma, \delta^{\prime}}\right)$ of determinant unity
is of period 3 if, and only if, $\alpha+\delta= \pm 1$.

2) Cf. $\S 280$. 
(ii) For $p^{n}+1$ divisible by 5 , let $g=\left(p^{n}+1\right) / 5$ and set $(\S 243)$

$$
\begin{gathered}
V_{5}=\left(\frac{J^{g}, 0}{0, \bar{J}^{g}}\right), \quad V_{2}=\left(\frac{A, \quad B}{-\bar{B},-A}\right), \\
J \bar{J}=1, \quad-A^{2}+B \bar{B}=1, \quad \bar{A}=-A .
\end{gathered}
$$

The condition $\left(V_{5} \nabla_{2}\right)^{3}=I$ is satisfied if, and only if,

$$
A\left(J^{g}-\bar{J}^{g}\right)=1 .
$$

The $A$ thus determined satisfies the condition $\bar{A}=-A$. Then must

$$
B \bar{B} \equiv B^{p^{n}+1}=1+A^{2}=1+\left(J^{g}-\bar{J}^{g}\right)^{-2} .
$$

The last term is a mark $\mu \neq 0$ of the $G F\left[p^{n}\right]$. Hence $B^{p^{n}+1}=\mu$ has a solution $B_{0}$ in the $G F\left[p^{2 n}\right]$ and consequently $p^{n}+1$ distinct solutions $B_{0}, B_{0} J, B_{0} J^{2}, \ldots, B_{0} J^{p^{n}}$. But $G_{M(s)}$ contains exactly $\frac{1}{2} p^{n}\left(p^{n}-1\right)$ conjugate cyclic $G_{5}(\S 243)$. Hence there are $2 p^{n}\left(p^{2} n-1\right)$ pairs of generators $V_{5}, V_{2}$ of icosahedral subgroups.

Since each icosahedral group contains (\$258) exactly 120 pairs of generators $V_{5}, V_{2}$, it follows that, for $p^{2 n}-1$ divisible by 5 , $G_{M\left(p^{n}\right)}$ contains in all $p^{n}\left(p^{2 n}-1\right) / 60$ icosahedral subgroups.

For $p=2,2^{2 n}-1$ is divisible by $5 \equiv 2^{2}+1$ if and only if $n$ be even. If $n$ be even, $G_{M\left(2^{n}\right)}$ contains a single system of $M(s) / 60$ subgroups $G_{M\left(2^{2}\right)}(\S 255)$, the latter being icosahedral by case (ii). Hence $G_{M\left(2^{n}\right)}$ contains no icosahedral groups if $n$ be odd, but, for $n$ even, contains $2^{n}\left(2^{2 n}-1\right) / 60$ icosahedral groups forming a single system of conjugate groups.

To determine, for $p>2$, the distribution of the icosahedral subgroups into sets of conjugates within $G_{M Y(s)}$ and within $G_{2 M(s)}$, consider first the case (i) and set $\varepsilon^{2}=\rho$, so that only the even powers of $\varepsilon$ belong to the $G F\left[p^{n}\right]$. Then will

$$
z^{\prime}=\varepsilon^{e} z / \varepsilon^{-e} \equiv \varrho^{e} z
$$

transform $V_{5}$ into itself, but transforms $V_{2}$ into

$$
\left(\frac{\alpha, \beta \varrho^{e}}{\gamma \varrho^{-e},-\alpha}\right) \text {. }
$$

Hence the groups $G_{60}$ are all conjugate under $G_{2 M(s)}$ and form at most two systems of conjugate subgroups under $G_{M(s)}$. But if there were a single system, their number would be at most $M(s) / 60$, whereas it is $M(s) / 30$. Hence there are two systems each of $M(s) / 60$ conjugate icosahedral groups within $G_{M(s)}$ and each is self-conjugate only under itself. 
For case $^{1}$ ) (ii), let $E^{2}=J$, so that $E \bar{E}=J^{\left(p^{n}+1\right) / 2}=-1$. Then

$$
z^{\prime}=E^{e} z / E^{-e}
$$

transforms $V_{5}$ into itself and transforms $V_{2}$ into

$$
\left(\frac{A, \quad B J^{e}}{-\bar{B} J^{-e},-A}\right)
$$

Taking $e=0,1, \ldots, p^{n}$, we reach the various $p^{n}+1$ substitutions $V_{2}$. If $e$ be even, the transformer belongs to the hyperorthogonal group since $\bar{J}=J^{-1}$. For $e$ odd, it may be given the hyperorthogonal form with determinant a not-square. In fact, there exist in the $G F\left[p^{2 n}\right]$ solutions of $X^{p^{n}-1}=-1$, so that $\bar{X}=-X$. Then

$$
\left(\frac{E, 0}{0, E^{-1}}\right)=\left(\frac{E, 0}{0,-\bar{E}}\right)=\left(\frac{X E, \quad 0}{0,-X \bar{E}}\right)=\left(\frac{X E, 0}{0, \bar{X} \bar{E}}\right)
$$

of determinant $X^{2}$. Hence the groups $G_{60}$ are all conjugate within $G_{2 M(s)}$ but form two systems of conjugates within $G_{M(s)}$.

260. Summary of the subgroups of $G_{M(s)}, s \equiv p^{n}$ :

$s+1$ conjugate commutative groups of order $s$

$\frac{1}{2} s(s \pm 1)$ conjugate cyclic groups of order $\frac{s \mp 1}{2 ; 1}, 2 ; 1$ according as $p>2 ; p=2$;

$\frac{1}{2} s(s \pm 1)$ conjugate cyclic $G_{d_{\mp}}$ for every divisor $d_{\mp}$ of $\frac{s \mp 1}{2 ; 1} ;$ $M(s) / 2 d_{\mp}$ conjugate dihedron $G_{2 d_{\mp}}$, for $d_{\mp}$ odd;

two systems each of $M(s) / 4 d_{\mp}$ conjugate dihedron $G_{2 d_{\mp}}$, for $d_{\mp}$ even and $>2$;

for $p^{n}=8 h \pm 3$, one set of $M(s) / 12$ conjugate four-groups;

for $p^{n}=8 h \pm 1$, two sets each of $M(s) / 24$ conjugate four-groups $\left.{ }^{2}\right)$; $\frac{\left(p^{n}-1\right)\left(p^{n}-p\right) \ldots\left(p^{n}-p^{m-1}\right)}{\left(p^{m}-1\right)\left(p^{m}-p\right) \ldots\left(p^{m}-p^{m-1}\right)}$ sets each of $\frac{p^{2 n}-1}{(2,1 ; 1) \cdot\left(p^{k}-1\right)}$ conjugate commutative groups of order $p^{m}$, where $(2,1 ; 1)$ is read 2,1 , or 1 according as $p>2$ with $n / k$ an even integer, $p>2$ with $n / k$ an odd integer, or $p=2$ with $n / k$ an integer, and where $k$ is a divisor of $m$ depending on the particular $G_{p} m$;

1) This case may be made to depend on (i) since 5 divides $p^{2 n}-1$. Hence each $G_{60}$ is self-conjugate only under itself within the group $G_{M\left(s^{2}\right)}$ and so within its subgroup $G_{2 M(s)}$. Hence each $G_{60}$ is one of a system of $2 M(s) / 60$ conjugate groups within $G_{2} M(8)$, so that the icosahedral subgroups all form a single system of conjugates within $G_{2 M(s)}$. They fall into two systems in $G_{M(s) \text {. }}$

2) For $p=2$, the four-groups occur among the groups of order $p^{m=2^{2}}$ given later. 
286 CHAPTER XII. SUBGROUPS OF THE LINEAR FRACTIONAL etc.

certain sets of $\frac{\left(p^{2} n-1\right) p^{n-m}}{(2,1 ; 1)\left(p^{k}-1\right)}$ conjugate $G_{p^{m_{d}}}$, where $k$ and $d_{-}$ depend on $m$;

$(2,1 ; 1)$ sets each of $M(s) /(2,1 ; 1) M\left(p^{k}\right)$ conjugate $G_{M\left(p^{k}\right)}, k$ a divisor of $n$, each group being isomorphic with the group of linear fractional substitutions of determinant unity in the $G F\left[p^{k}\right]$

two systems each of $M(s) / 2 M\left(p^{k}\right)$ conjugate $G_{2 M\left(p^{k}\right)}, p>2, n / k$ an even integer, each group isomorphic with the linear fractional group in the $G F\left[p^{k}\right]$;

for $s=8 h \pm 1$, two sets each of $M(s) / 24$ conjugate octahedral $G_{24}$; for $s=8 h \pm 1$, two sets each of $M(s) / 24$ conjugate tetrahedral $G_{12}$; for $s=8 h \pm 3$ or $s=2^{n}, n$ even, $M(s) / 12$ conjugate tetrahedral $G_{12} ;$ for $s=10 l \pm 1$, two sets each of $M(s) / 60$ conjugate icosahedral $G_{60}{ }^{1}$ ) simple.

261. Theorem. - If $p^{n}>3$, the linear fractional group $G_{M(s)}$ is

Indeed, the only cases in which the number of groups in a set of conjugate subgroups is unity are the following two:

$p^{n}=2, d_{+}=3, M(s) / 2 d_{+}=1$, when the $G_{6}$ has a self-conjugate $G_{3} ;$ $p^{n}=3, M(s) / 12=1$, when the $G_{12}$ has a self-conjugate four-group:

262. Theorem..$\left.^{2}\right)$ - The group $G_{M(s)}$ always has subgroups "of index $s+1$, but has subgroups of lower index only when

$$
s=2,3,5,7,3^{2}, 11 \text {. }
$$

Every subgroup of $G_{M(s)}$ is contained in one of the following: $G_{\frac{s(s-1)}{2 ; 1}}$, dihedron $G_{s \mp 1}(p>2), G_{M\left(p^{k}\right)}(n / k$ an odd integer if $p>2)$, $G_{2 M\left(p^{k}\right)}(p>2, n / k$ an even integer $), G_{12}(s=8 h \pm 3), G_{24}(s=8 h \pm 1)$, $G_{60}(s=10 l \pm 1)$. The first group is always of order greater than the $G_{M\left(p^{k}\right)}$ and $G_{2 M\left(p^{k}\right)}$; indeed, since $k \overline{\overline{<}} n / 2$,

$$
p^{k}\left(p^{2 k}-1\right)<p^{k}\left(p^{n}-1\right)<\frac{s(s-1)}{2 ; 1} \text {. }
$$

Also $s(s-1) /(2 ; 1)>s+1>s-1$ if $s>3$ and $s(s-1) /(2 ; 1)>60$

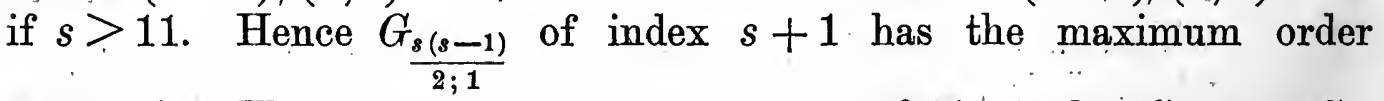
if $s>11$. The same result holds for $s=2^{3}$ since the $G_{M\left(2^{e}\right)} \equiv G_{60}$. is then not a subgroup; likewise for $s=2^{2}$ since. it is ( $\left.\$ 257\right)$ then

1) For $p=2$ or $p=5$ the icosahedral subgroups are of the type $G_{M}\left(2^{2}\right)$ or $G_{M(5)}$ given earlier.

2) For $n=1$, this is the celebrated theorem stated without proof by Galois in the letter to his friend Auguste Chevalier written before, the fatal duel:: For references to the proofs by Betti, Gierster, etc., see Klein, Math. Ann., vol. 14. 
the tetrahedral $G_{12}$. For $s=11,3^{2}, 7,5$, the subgroups of maximum order are $G_{60}, G_{60}, G_{24}, G_{12}$ respectively, the index under $G_{M(s)}$ being $11,6,7,5$ and hence $<s+1$. For $s=2,3$ the $G_{M(s)}$ is a dihedron $G_{6}$, a tetrahedron $G_{12}$, respectively, and has a subgroup of maximum order $G_{3}, G_{4}$ respectively.

263. A simple group can be represented as a transitive substitution-group on $N$ letters if, and only if, it contains a complete system of $N$ conjugate subgroups. ${ }^{1}$ ) For $s>3, G_{M(s)}$ is simple (\$261). Hence $G_{M(s)}$ can be represented as a transitive group on $<s+1$ letters only when $s=5,7,3^{2}, 11$. For $s=2,3$ it can be represented as a transitive group on 3, 4 letters respectively, but on no fewer, being of order $G_{6}, G_{12}$. If a simple group be represented as an intransitive substitution-group on $D$ letters, $D$ must equal the sum of the degrees of two or more transitive representations; for $G_{J 1(s)}$ we have always $D>s+1$. Hence the linear fractional group $G_{M(s)}$ may be represented as a substitution-group on $s+1$ letters but on no feiver number except when $s=5,7,9,11$, for which the minimum number of letters is 5, 7, 6,11 respectively.

\section{CHAPTER XIII.}

\section{AUXILIARY THEOREMS ON ABSTRACT GROUPS. ABSTRACT FORMS OF VARIOUS LINEAR GROUPS. ${ }^{2}$ )}

264. Theorem. - The symmetric substitution-group on $k$ letters is holoedrically isomorphic with the abstract group $G(k)$ generated by the operators $B_{1}, B_{2}, \ldots, B_{k-1}$ with the generational relations

261) $B_{1}^{2}=B_{2}^{2}=\cdots=B_{k-1}^{2}=I$,

262) $\quad B_{i} B_{j}=B_{j} B_{i} \quad(i=1,2, \ldots, k-3 ; j=i+2, i+3, \ldots, k-1)$,

263) $B_{j} B_{j+1} B_{j}=B_{j+1} B_{j} B_{j+1} \quad(j=1,2, \ldots, k-2)$.

The symmetric group $G_{k !}^{(k)}$ on the letters $l_{1}, l_{2}, \ldots, l_{k}$ may be generated by the transpositions

$$
S_{d} \equiv\left(l_{d} l_{d+1}\right) \quad(d=1,2, \ldots, k-1),
$$

which satisfy the relations 261), 262), 263) prescribed for the generators $B_{d}$ of the abstract group $G(k)$ and conceivably also other

1) For a proof of this theorem due to Dyck see Burnside, The Theory of Groups, § 123.

2) The theorem's of $\S \S 264,265$ are due to Professor Moore, Proceed. Lond. Math. Soc., vol. XXVIII, pp. 357-366. The proofs given in $\S \S 264,266$ are due to the author; for that in $\S 264$ see Proceed. Lond. Math. Soc., vol. XXXI, $351-353$; for that in $\S 266$ see Math. Ann., vol..54, pp. 564-569. 
relations not derivable therefrom. The order $O(k)$ of $G(k)$ is therefore $\sum k$ !

Denote by $G$ the subgroup $G(k-1)$ generated by $B_{1}, B_{2}, \ldots$, $B_{k-2}$ and consider the following sets of operators ${ }^{1}$ ) of $G(k)$ :

$O_{k} \equiv G, O_{k-1} \equiv G B_{k-1}, O_{k-2} \equiv G B_{k-1} B_{k-2}, \ldots, O_{1} \equiv G B_{k-1} B_{k-2} \ldots B_{1}$.

It will be shown that these sets of operators are merely permuted amongst themselves upon applying as right-hand multipliers the generators $B_{r}(r=1, \ldots, k-1)$. Since $B_{r}^{2}=I$, we have

$$
\begin{aligned}
& O_{r+1} B_{r} \equiv G B_{k-1} \ldots B_{r+1} B_{r} \equiv O_{r} \\
& O_{r} B_{r} \equiv G B_{k-1} \ldots B_{r} B_{r} \equiv O_{r+1} .
\end{aligned}
$$

If $i>r+1$, we find, on applying 262) to move $B_{r}$ to the left of $B_{i}, \ldots, B_{k-1}$,

$$
O_{i} B_{r} \equiv G B_{k-1} \ldots B_{i} B_{r}=G B_{r} B_{k-1} \ldots B_{i}=G B_{k-1} \ldots B_{i} \equiv O_{i} .
$$

If $i<r$, we find, on moving $B_{r}$ to the left of $B_{i}, B_{i+1}, \ldots, B_{r-2}$,

$$
O_{i} B_{r} \equiv G B_{k-1} \ldots B_{i} B_{r}=G B_{k-1} \ldots B_{r+1} B_{r} B_{r-1} B_{r} B_{r-2} \ldots B_{i} \text {. }
$$

By 263), we may replace $B_{r} B_{r-1} B_{r}$ by $B_{r-1} B_{r} B_{r-1}$. We then move the first $B_{r-1}$ to the left of $B_{r+1}, \ldots, B_{k-1}$ and merge it into $G$ and get

$$
O_{i} B_{r}=G B_{k-1} \ldots B_{r+1} B_{r} B_{r-1} B_{r-2} \ldots B_{i} \equiv O_{i} .
$$

Hence the right-hand multiplier $B_{r}$ gives rise to the transposition $\left(O_{r} O_{r+1}\right)$ on the $k$ sets $O_{1}, \ldots, O_{k}$. It follows that the product of any operator of these $k$ sets by an arbitrary operator of $G(k)$ is an operator belonging to these sets. Taking for the former operator the identity, we see that these $k$ sets include all the operators of the group $G(k)$. The number of operators in $G(k)$ is therefore at most $k$ times the number in $G(k-1)$. Hence

$$
O(k) \overline{<} k \cdot O(k-1) \overline{<} \cdots \overline{\overline{<}} k !
$$

Combining this result with the earlier one, we have $O(k)=k$ ! The proof of the holoedric isomorphism of $G(k)$ and $G_{k !}^{(k)}$ is therefore complete.

The relations 261), 262), 263) may be combined into the formulae

$$
\text { 264) } I=B_{i}^{2}=\left(B_{i} B_{i+1}\right)^{3}=\left(B_{i} B_{j}\right)^{2} \quad(i, j=1, \ldots, k-1 ; j>i+1) \text {. }
$$

1) It turns out that these sets form a rectangular table for $G(k)$ with the operators of $G$ in the first line. 
265. Theorem. - The alternating group on $k$ letters is holoedrically isomorphic with the abstract group $G\{k\}$ generated by the operators $E_{1}, E_{2}, \ldots, E_{k-2}$ subject to the generational relations

265) $I=E_{1}^{3}=E_{i+1}^{2}=\left(E_{i} E_{i+1}\right)^{3}=\left(E_{i} E_{j}\right)^{2} \quad(i, j=1, \ldots, k-2 ; j>i+1)$.

The abstract symmetric group $G(k)$ may be generated by $B_{1}$ and 266)

$E_{d} \equiv B_{d+1} B_{1}$

$(d=1,2, \ldots, k-2)$.

From the relations 264) we readily derive 265) together with

$$
B_{1}^{2}=I, \quad E_{d} B_{1}=B_{1} E_{d}^{-1}
$$$$
(d=1,2, \ldots, k-2) \text {. }
$$

Inversely, from 265) and 267), we can easily get relations 264). Hence $B_{1}, E_{1}, E_{2}, \ldots, E_{k-2}$, subject only to the relations 265$)$ and 267), generate the abstract group $G(k)$. Upon extending $G\{k\}$ by the operator $B_{1}$ subject to the relations 267), we obtain a group whose operators are of the form $E$ or $E B_{1}, E$ being derived from $E_{1}, E_{2}, \ldots, E_{k-2}$, and hence of order $2 O\{k\}$. But the extended group was shown to be $G(k)$. Hence $G\{k\}$ is a subgroup of $G(k)$ of order $\frac{1}{2} k$ ! It is readily shown to be the abstract alternating group $G_{\frac{1}{2} k i}$. Since the generational relations 264) involve the generators $B_{i}$ evenly, the various expressions for an operator of $G(k)$ in terms of its generators involve all an even or all an odd number of the generators, so that its operators may be classed into even and odd operators. By 266), the operators of the subgroup $G\{k\}$ are all even, so that it is a subgroup of $G_{\frac{1}{2} k i}$. Since its order is $\frac{1}{2} k !$, it is identical with the latter.

266. The last theorem may be readily proved by the direct method of $\& 264$. The generational relations 265) are seen to be satisfied by the substitutions

$$
A_{d} \equiv\left(l_{d+1} l_{d+2}\right)\left(l_{1} l_{2}\right) \equiv S_{d+1} S_{1} \quad\left(d=1, \ldots, l_{c}-2\right)
$$

whịch generate the alternating group on $l_{1}, l_{2}, \ldots, l_{k}$. Hence

$$
O\{k\}>\frac{1}{2} k !
$$

The theorem being evident if $k=3$, we take $k>4$. Denote by $\Gamma$ the subgroup $G\{k-1\}$ generated by $E_{1}, E_{2}, \ldots, E_{k-3}$ and consider the following sets of operators of $G\{k\}$ :

$$
\begin{aligned}
R_{k-1} \equiv\left\ulcorner, R_{k-2} \equiv\left\ulcorner E_{k-2}, R_{k-3} \equiv\left\ulcorner E_{k-2} E_{k-3}, \ldots, R_{2} \equiv\left\ulcorner E_{k-2} E_{k-3} \ldots E_{2},\right.\right.\right.\right. \\
R_{1} \equiv\left\ulcorner E_{k-2} \ldots E_{2} E_{1}, \quad R_{k} \equiv\left\ulcorner E_{k-2} \ldots E_{2} E_{1}^{2} .\right.\right.
\end{aligned}
$$

The reader may readily verify, as in $\S 264$, that $E_{1}$ and $E_{r}(r>1)$, when applied as right-hand multipliers to the above sets, give rise

DICKSON, Linear Groups. 
to the permutations $\left(R_{1} R_{k} R_{2}\right)$ and $\left(R_{r} R_{r+1}\right)\left(R_{1} R_{k}\right)$ respectively. The sets $R_{1}, \ldots, R_{k}$ therefore include all the operators of $G\{k\}$, so that

$$
O\{k\} \overline{<} k \cdot O\{k-1\} \overline{<} \cdots \overline{<} k(k-1) \cdots 4 \cdot O\{3\} \equiv \frac{1}{2} k !
$$

Combining this result with the earlier one, $O\{k\}=\frac{1}{2} k !$

26\%. Theorem. - The abstract alternating group $G_{\frac{1}{2} 5 !}$ may be generated by two operators $V$ and $W$ subject to the generational relations

$$
V^{5}=W^{2}=I, \quad(V W)^{3}=I .
$$

For $k=5$, the relations 265 ) defining $G_{\frac{1}{2} 5 t}$ may be written

$$
\text { 269) } \quad E_{1}^{3}=E_{2}^{2}=E_{3}^{2}=\left(E_{1} E_{2}\right)^{3}=\left(E_{2} E_{3}\right)^{3}=\left(E_{1} E_{3}\right)^{2}=I \text {. }
$$

The group contains two operators $V \equiv E_{1} E_{2} E_{3}, W \equiv E_{3}$ such that $W^{2}=I,(V W)^{3} \equiv\left(E_{1} E_{2}\right)^{3}=I$. To prove that $V^{5}=I$, we apply 269) and find that

$$
\begin{aligned}
& V^{2}=E_{1} E_{2} E_{1}^{2} E_{3} E_{2} E_{3}=E_{1} E_{2} E_{1}^{2} E_{2} E_{3} E_{2}=E_{1}^{2} E_{2} E_{3} E_{1}^{2} E_{2}, \\
& V^{4}=E_{1}^{2} E_{2} E_{3} E_{2} E_{1} E_{3} E_{1}^{2} E_{2}=E_{1}^{2} E_{3} E_{2} E_{1} E_{2}=E_{3} E_{2} E_{1}=V^{-1}
\end{aligned}
$$

Inversely, if $V, W$ satisfy 268) and we set ${ }^{1}$ )

$$
E_{3}=W, \quad E_{2}=V^{2} W V^{-2}, \quad E_{1}=V W E_{2}=W V^{-2} W V^{2},
$$

the relations 269) will follow. We have at once $E_{3}^{2}=I, E_{2}^{2}=I$, $\left(E_{1} E_{3}\right)^{2}=I,\left(E_{1} E_{2}\right)^{3}=I$. Also $\left(E_{2} E_{3}\right)^{3}=I$ and $E_{1}^{3}=I$. In fact

$$
\begin{aligned}
\left(E_{2} E_{3}\right)^{2} & =\left(V^{2} W V^{-2} W\right)^{2}=V^{2} W V^{2} W V^{-2} W V^{2} W \\
& =V W V^{-2} W V^{-1} \cdot V^{2} W V^{-2} W V^{-1}=V W V^{2} W V^{2} W V^{-1} \\
& =W V^{-2} W V^{-1} \cdot V^{2} W V^{-1}=W V^{2} W V^{-2}=\left(E_{2} E_{3}\right)^{-1} .
\end{aligned}
$$

$E_{1}^{2}=V W V^{2} W V^{3} \cdot W V^{-2} W V^{2}=V W V^{2} \cdot V W V^{2} W V \cdot V^{-2} W V^{2}$

$=V \cdot V W V^{2} W V \cdot V^{2} W V^{-1} W V^{2}=V^{2} W V^{2} W V^{3} \cdot V W V \cdot V^{2}$

$=V^{2} W V^{2} \cdot V W V \cdot V^{3}=V^{2} W V^{-2} W V^{-1}=V^{-2} W V^{2} W=E_{1}^{-1}$.

268. Theorem.2) - The general linear homogeneous group $G L H(4,2)$ is holoedrically isomorphic with the alternating group on 8 letters.

1) The later reductions depend upon the formulae $V W V=W V^{-1} W, \quad W V^{2} W=V^{-1} W V^{-2} W V^{-1}, \quad W V^{-2} W=V W V^{2} W V$.

2) Jordan, Traité des substitutions, No.516; Moore, Math. Annalen, vol. 51, pp. 417-444; Dickson, ibid vol. 54, pp. 564-569. 
The following substitutions of $G L H(4,2)$

$$
\begin{array}{ll}
E_{1}=\left(\begin{array}{llll}
1 & 1 & 1 & 1 \\
0 & 0 & 0 & 1 \\
1 & 1 & 0 & 0 \\
0 & 1 & 0 & 1
\end{array}\right), & E_{2}=\left(\begin{array}{llll}
0 & 1 & 0 & 1 \\
0 & 0 & 1 & 0 \\
0 & 1 & 0 & 0 \\
1 & 0 & 1 & 0
\end{array}\right),
\end{array}
$$

satisfy the relations 265) for $k=8$ and therefore generate a subgroup $L$ which is isomorphic with the alternating group on the letters $1,2, \ldots, 8$. The latter group being simple, the isomorphism is holoedric. Since the order of $\operatorname{GLH}(4,2)$ equals $\frac{1}{2} 8$ ! by $\S 99$, it coincides with its subgroup $L$. The correspondence of generators of $L \equiv G L H(4,2)$ and $G_{\frac{1}{2} s !}$ is as follows:

$$
\begin{aligned}
& \text { 270) } \quad E_{1} \sim(23)(12), \quad E_{2} \sim(34)(12), \quad E_{3} \sim(45)(12) \text {, } \\
& E_{4} \sim(56)(12), \quad E_{5} \sim(67)(12), \quad E_{6} \sim(78)(12) .
\end{aligned}
$$

269. To effect the inversion of 270), so that we shall be able to pass readily from an arbitrary substitution of $L$ to the corresponding substitution of $G_{\frac{1}{2} s !}$, we begin with the simple identities,

$$
\begin{array}{lll}
\left(\xi_{1} \xi_{3}\right) B_{24}=E_{5}, & \left(\xi_{2} \xi_{4}\right) B_{31}=E_{5} E_{4} E_{5}, & \left(\xi_{1} \xi_{4}\right)\left(\xi_{2} \xi_{3}\right)=E_{2} E_{4}, \\
B_{12} B_{43}=E_{2} E_{6}, & \left(\xi_{3} \xi_{4}\right) B_{21}=E_{1} E_{6}, & \left(\xi_{1} \xi_{2}\right)\left(\xi_{3} \xi_{4}\right) B_{32}=E_{2} E_{3} E_{1} E_{5} .
\end{array}
$$

Since these relations can be solved for $E_{5}, E_{4}, E_{2}, E_{6}, E_{1}, E_{3}$ in order, their left members may be chosen as generators of $L$. By 270), we have

$$
\begin{aligned}
& \left(\xi_{1} \xi_{3}\right) B_{24} \sim(67)(12), \quad\left(\xi_{2} \xi_{4}\right) B_{31} \sim(57)(12), \quad\left(\xi_{1} \xi_{4}\right)\left(\xi_{2} \xi_{3}\right) \sim(34)(56), \\
& B_{12} B_{43} \sim(34)(78), \quad\left(\xi_{3} \xi_{4}\right) B_{21} \sim(23)(78), \\
& \alpha \equiv\left(\xi_{1} \xi_{2}\right)\left(\xi_{3} \xi_{4}\right) B_{32} \sim(67)(2354) .
\end{aligned}
$$

From these generators of $L$, we obtain in succession the substitutions

$$
\begin{aligned}
& \left(\xi_{2} \xi_{3}\right) B_{32} \equiv B_{32} B_{23}=\alpha^{-1}\left(\xi_{1} \xi_{4}\right)\left(\xi_{2} \xi_{3}\right) \alpha\left(\xi_{1} \xi_{4}\right)\left(\xi_{2} \xi_{3}\right), \\
& \left(\xi_{1} \xi_{2} \xi_{4} \xi_{3}\right)=\alpha\left[\left(\xi_{2} \xi_{3}\right) B_{32}\right]^{-1} \\
& \left(\xi_{3} \xi_{4}\right) B_{12}=\left(\xi_{1} \xi_{2} \xi_{4} \xi_{3}\right) \cdot\left(\xi_{2} \xi_{4}\right) B_{31} \cdot\left(\xi_{1} \xi_{2} \xi_{4} \xi_{3}\right)^{-1}, \\
& \left(\xi_{1} \xi_{2}\right) B_{12} \equiv B_{12} B_{21}=\left(\xi_{3} \xi_{4}\right) B_{12} \cdot\left(\xi_{3} \xi_{4}\right) B_{21}, \\
& \left(\xi_{1} \xi_{2}\right)\left(\xi_{3} \xi_{4}\right)=\left(\xi_{1} \xi_{2}\right) B_{12} \cdot\left(\xi_{3} \xi_{4}\right) B_{12}, \\
& B_{34}\left(\xi_{3} \xi_{4}\right) \equiv\left(\xi_{3} \xi_{4}\right) B_{43}=\left(\xi_{3} \xi_{4}\right) B_{12} \cdot B_{12} B_{43}, \\
& B_{42}=\left[B_{34}\left(\xi_{3} \xi_{4}\right)\right]^{-1} \cdot B_{32} \cdot B_{34}\left(\xi_{3} \xi_{4}\right) .
\end{aligned}
$$


These results lead at once to the following correspondences: $\left(\xi_{2} \xi_{3}\right) B_{32} \sim(265)(347),\left(\xi_{1} \xi_{2} \xi_{4} \xi_{3}\right) \sim(27)(3645),\left(\xi_{3} \xi_{4}\right) B_{12} \sim(24)(17)$, $\left(\xi_{1} \xi_{2}\right) B_{12} \sim(187)(243),\left(\xi_{1} \xi_{2}\right)\left(\xi_{3} \xi_{4}\right) \sim(18)(34),\left(\xi_{3} \xi_{4}\right) B_{43} \sim(187)(234)$, $B_{42} \sim(16)(25)(34)(78), B_{32} \sim(23)(45)(67)(18),\left(\xi_{2} \xi_{3}\right) \sim(18)(27)(35)(46)$.

By simple transformations, we complete the proof of the

Theorem. - The correspondences 270) give reciprocally

$$
\begin{gathered}
\left(\xi_{1} \xi_{2}\right) \sim(13)(27)(48)(56), \quad\left(\xi_{1} \xi_{3}\right) \sim(16)(27)(34)(58), \\
\left(\xi_{1} \xi_{4}\right) \sim(18)(27)(36)(45), \\
\left(\xi_{2} \xi_{3}\right) \sim(18)(27)(35)(46), \quad\left(\xi_{2} \xi_{4}\right) \sim(15)(27)(34)(68), \\
\left(\xi_{3} \xi_{4}\right) \sim(14)(27)(38)(56), \\
B_{12} \sim(12)(38)(47)(56), \quad B_{31} \sim(17)(25)(34)(68), \\
B_{32} \sim(18)(23)(45)(67), \\
B_{14} \sim(18)(23)(46)(57), \quad B_{24} \sim(17)(26)(34)(58), \\
B_{43} \sim(12)(37)(48)(56) .
\end{gathered}
$$

By $\S 100$, these relations enable us to pass from an arbitrary substitution of the linear group on 4 indices modulo 2 to the corresponding even substitution on 8 letters.

Abstract form of the simple group $\left.F O(5,3)^{1}\right)$, §§ $270-274$.

$2 \%$. By the notation of $\S 194, F O(5,3)$ denotes the group $O_{1}^{\prime}(5,3)$. By $\S 189$ and 181, it is of order 25920 and is generated by the substitutions ${ }^{2}$ )

$$
C_{i} C_{j}, \quad\left(\xi_{i} \xi_{j}\right)\left(\xi_{k} \xi_{l}\right), \quad w \equiv W_{1234} \quad(i, j, k, l=1, \ldots, 5) .
$$

It has a commutative subgroup $L_{16}$ composed of the substitutions $I, C_{1} C_{2}, C_{1} C_{3}, C_{1} C_{4}, C_{1} C_{5}, C_{2} C_{3}, C_{2} C_{4}, C_{2} C_{5}, C_{3} C_{4}, C_{3} C_{5}, C_{4} C_{5}$, $C_{1} C_{2} C_{3} C_{4}, C_{1} C_{2} C_{3} C_{5}, C_{1} C_{3} C_{4} C_{5}$ and $C_{2} C_{3} C_{4} C_{5}$. The $\left(\xi_{i} \xi_{j}\right)\left(\xi_{k} \xi_{l}\right)$ generate a subgroup $L_{60}$ of the even linear substitutions on $\xi_{1}, \ldots, \xi_{5}$. The groups $L_{16}$ and $L_{60}$ are commutative with each other and have only the identity in common; hence they generate a subgroup $A_{960}$ of $F O(5,3)$. We readily determine the abstract forms of these subgroups. By $\S 265$, we have the theorem:

1) Taken from the author's papers, Comptes Rendus, vol. 128, pp. 873-875; Proceed. Lond. Math. Soc., vol. 32, pp. 3-10. In the earlier paper, Proceed. Lond. Math. Soc., vol. 31, pp. 30-68, another set of generators was determined by a more complicated analysis.

2) For $p^{n}=3, O_{i, j}^{\alpha, \beta}$ is either the identity, $C_{i} C_{j},\left(\xi_{i} \xi_{j}\right) C_{i}$ or $\left(\xi_{i} \xi_{j}\right) C_{j}$, the first two alone being of the form $Q_{i j}$. Here $\left(\xi_{i} \xi_{j}\right)$ denotes the linear substitution $\xi_{i}^{\prime}=\xi_{j}, \xi_{j}^{\prime}=\xi_{i}$. They are to be compounded as linear substitutions; for example, $\left(\xi_{1} \xi_{3}\right)\left(\xi_{1} \xi_{2}\right)=\left(\xi_{1} \xi_{2} \xi_{3}\right)$. Also $C_{i}$ denotes the substitution changing the sign of the index $\xi_{i}$. 
The abstract group $G_{60}$ generated by $E_{1}, E_{2}, E_{3}$ subject to the relations 271) $E_{1}^{3}=E_{2}^{2}=E_{3}^{2}=I, \quad\left(E_{1} E_{2}\right)^{3}=\left(E_{2} E_{3}\right)^{3}=\left(E_{1} E_{3}\right)^{2}=I$

is put into holoedric isomorphism with $L_{60}$ by the correspondences

$$
\text { 272) } E_{1} \sim\left(\xi_{1} \xi_{2} \xi_{3}\right), \quad E_{2} \sim\left(\xi_{3} \xi_{4}\right)\left(\xi_{1} \xi_{2}\right), \quad E_{3} \sim\left(\xi_{4} \xi_{5}\right)\left(\xi_{1} \xi_{2}\right) .
$$

The following theorem is quite evident:

The abstract group $G_{16}$ generated by $B_{1}, B_{2}, B_{3}, B_{4}$ subject to the relations

$$
B_{i}^{2}=I, \quad B_{i} B_{j}=B_{j} B_{i} \quad(i, j=1,2,3,4)
$$

is put into holoedric isomorphism with $L_{16}$ by the correspondences

$$
\text { 274) } B_{1} \sim C_{1} C_{2}, \quad B_{2} \sim C_{2} C_{3}, \quad B_{3} \sim C_{3} C_{4}, \quad B_{4} \sim C_{4} C_{5} \text {. }
$$

If we impose the relations 275 ) below, the two groups $G_{60}$ and $G_{16}$ will be permutable. Writing the analogous relations between the corresponding orthogonal substitutions 272), 274), we readily see that they are satisfied. We have therefore the theorem:

The abstract group generated by $E_{1}, E_{2}, E_{3}, B_{1}, B_{2}, B_{3}, B_{4}$ subject to the generational relations 271$), 273$ ), and

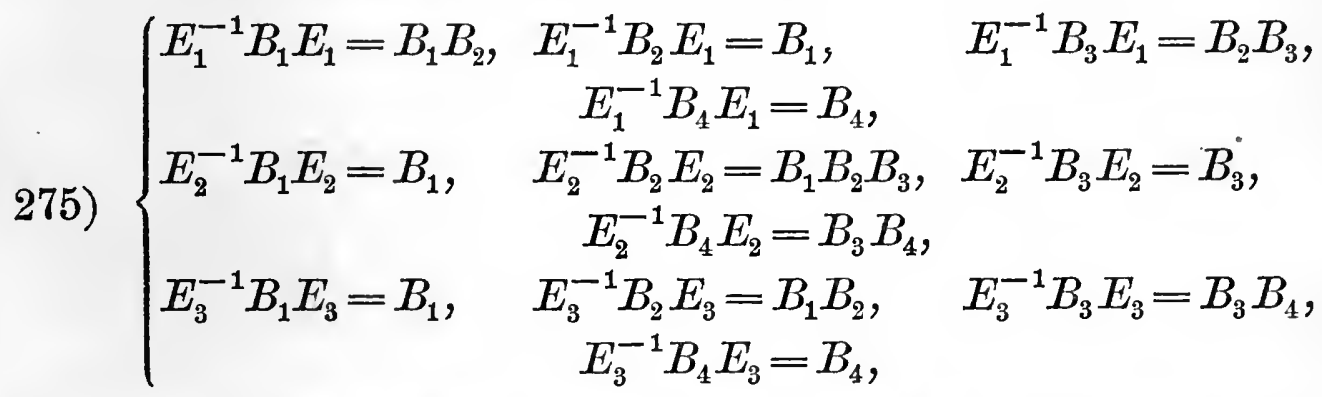

is of order 960 and is holoedrically isomorphic with the linear group $A_{960}$.

2\%1. Theorem. - The abstract group $G_{960}$ of $\S 270$ may be generated by the operators $E_{1}, E_{2}, E_{3}, B_{1}$ subject to the generational relations

$$
\begin{array}{ll}
E_{1}^{3}=E_{2}^{2}=E_{3}^{2}=B_{1}^{2}=I, & \left(E_{1} E_{2}\right)^{3}=\left(E_{2} E_{3}\right)^{3}=\left(B_{1} E_{1}\right)^{3}=I \\
& \left(E_{1} E_{3}\right)^{2}=\left(B_{1} E_{2}\right)^{2}=\left(B_{1} E_{3}\right)^{2}=I
\end{array}
$$

These relations follow immediately from 271), 273), 275), with the exception of $\left(B_{1} E_{1}\right)^{3}=I$, which is derived from the first two of 275$)$ :

$$
E_{1}^{-1} B_{1} E_{1} B_{1}=B_{2}=E_{1} B_{1} E_{1}^{-1}
$$

together with $E_{1}^{3}=B_{1}^{2}=I$. Furthermore, we have by 275),

277) $B_{2}=E_{1} B_{1} E_{1}^{2}, \quad B_{3}=E_{1} E_{2} E_{1}^{2} B_{1} E_{1} E_{2} E_{1}^{2}, \quad B_{4}=E_{2} E_{3} B_{3} E_{3} E_{2}$. 
Inversely, if $B_{2}, B_{3}, B_{4}$ be defined by 277), the relations 271), 273), 275) all follow from 276). Since $B_{1} E_{1}$ is of period 3 ,

$$
\begin{aligned}
B_{1} B_{2} & =B_{1} E_{1} B_{1} E_{1}^{2}=E_{1}^{2} B_{1} E_{1}, \\
B_{1} B_{2} B_{3} & =E_{1}^{2} B_{1} E_{1} \cdot B_{3}=E_{1}^{2} B_{1} E_{1}^{2} E_{2} E_{1}^{2} B_{1} E_{1} E_{2} E_{1}^{2} \\
& =E_{1}^{2} B_{1} \cdot E_{2} E_{1} E_{2} \cdot B_{1} E_{1} E_{2} E_{1}^{2} \\
& \left.=E_{1}^{2} E_{2} B_{1} E_{1} B_{1} E_{2} E_{1} E_{2} E_{1}^{2} \text { (interchanging } B_{1} E_{2} \text { with } E_{2} B_{1}\right) \\
& =E_{1}^{2} E_{2} \cdot E_{1}^{2} B_{1} E_{1}^{2} \cdot E_{1}^{2} E_{2} E_{1}^{2} \cdot E_{1}^{2}=E_{2} E_{1} E_{2} B_{1} E_{1} E_{2} E_{1} \\
& =E_{2} E_{1} E_{2} B_{1} \cdot E_{2} E_{1}^{2} E_{2}=E_{2} E_{1} B_{1} E_{1}^{2} E_{2} \equiv E_{2}^{-1} B_{2} E_{2} . \\
E_{3}^{-1} B_{2} E_{3} & =E_{3} E_{1} B_{1} E_{1}^{2} E_{3}=E_{1}^{2} E_{3} B_{1} E_{3} E_{1}=E_{1}^{2} B_{1} E_{1}=B_{1} B_{2} .
\end{aligned}
$$

Upon setting $B_{1} E_{2}=E_{2} B_{1}, B_{1} E_{1}^{2} B_{1} E_{1}=E_{1} B_{1} E_{1}^{2}$, we find that

$$
\begin{aligned}
B_{2} B_{3} & =E_{1} B_{1} E_{2} E_{1}^{2} B_{1} E_{1} E_{2} E_{1}^{2}=E_{1} E_{2} \cdot E_{1} B_{1} E_{1}^{2} \cdot E_{2} E_{1}^{2} \\
& =E_{2} E_{1}^{2} E_{2} B_{1} E_{2} E_{1} E_{2}=E_{2} E_{1}^{2} B_{1} E_{1} E_{2} \equiv E_{1}^{-1} B_{3} E_{1} .
\end{aligned}
$$

Since $E_{2} E_{1} E_{2} E_{1}^{2}=E_{1} E_{2} E_{1}^{2} E_{2}$, we get .

$$
\begin{aligned}
E_{2}^{-1} B_{3} E_{2} & =E_{2} E_{1} E_{2} E_{1}^{2} B_{1} E_{1} E_{2} E_{1}^{2} E_{2} \\
& =E_{1} E_{2} E_{1}^{2}\left(E_{2} B_{1} E_{2}\right) E_{1} E_{2} E_{1}^{2}=B_{3} .
\end{aligned}
$$

Since $\quad E_{1} E_{2} E_{1}^{2} E_{2} \cdot E_{3} E_{1} E_{2} E_{1}^{2}=E_{2} E_{1} E_{2} E_{1}^{2} \cdot E_{3} E_{1} E_{2} E_{1}^{2}$

$$
\begin{aligned}
& =E_{2} E_{1} E_{2} E_{1} E_{3} E_{2} E_{1}^{2}=E_{1}^{2} E_{2} E_{3} E_{2} E_{1}^{2}=E_{1}^{2} E_{3} E_{2} E_{3} E_{1}^{2} \\
& =E_{3} E_{1} E_{2} E_{1} E_{3}=E_{3} E_{2} E_{1}^{2} E_{2} E_{3},
\end{aligned}
$$

we find by 277) that

$$
\begin{aligned}
B_{3} B_{4} & =E_{1} E_{2} E_{1}^{2} B_{1} \cdot E_{3} E_{2} E_{1}^{2} E_{2} E_{3} \cdot B_{1} E_{1} E_{2} E_{1}^{2} E_{3} E_{2} \\
& =E_{1} E_{2} E_{1}^{2} E_{3} E_{2} B_{1} E_{1}^{2} B_{1} E_{2} E_{3} E_{1} E_{2} E_{3} E_{1} E_{2} \\
& =E_{1} E_{2} E_{3} E_{1} E_{2} \cdot E_{1} B_{1} E_{1} \cdot E_{2} E_{1}^{2} E_{3} E_{2} E_{3} E_{1} E_{2} \\
& =E_{1} E_{2} E_{3} E_{2} E_{1}^{2}\left(E_{2} B_{1} E_{2}\right) E_{1} E_{2} E_{1}^{2} E_{3} E_{1}^{2} E_{2} E_{1}^{2} \\
& =E_{1} E_{3} E_{2} E_{1}\left(E_{3} B_{1} E_{3}\right) E_{1}^{2} E_{2} E_{3} E_{1}^{2} \\
& =E_{3} \cdot E_{1} E_{2} E_{1}^{2} B_{1} E_{1} E_{2} E_{1}^{2} \cdot E_{3} \equiv E_{3}^{-1} B_{3} E_{3}
\end{aligned}
$$

upon setting $E_{3} B_{1} E_{3}=B_{1}=E_{2} B_{1} E_{2}, \quad E_{1}^{2} E_{2} E_{1} E_{2}=E_{1} E_{2} E_{1}^{2}$ and applying also the equation given by taking the reciprocals of the last substitutions. Using 277) and the last result,

$$
E_{2}^{-1} B_{4} E_{2}=E_{3} B_{3} E_{3}=B_{3} B_{4} \text {. }
$$

In order to prove that $E_{1}^{-1} B_{4} E_{1}=B_{4}$, we note that

or

$$
E_{1}^{-1}\left(E_{3} B_{3} E_{3}\right) E_{1}=E_{1}^{-1}\left(B_{3} B_{4}\right) E_{1} \text {, }
$$

$$
E_{3} E_{1} B_{3} E_{1}^{2} E_{3}=B_{2} B_{3} \cdot E^{-1} B_{4} E_{1} \text {. }
$$

But the left member equals $B_{2} B_{3} B_{4}$. Indeed, by the earlier results,

Hence

$$
E_{1}^{-1} B_{1} B_{2} B_{3} E_{1}=B_{1} B_{2} \cdot B_{1} \cdot B_{2} B_{3}=\left(E_{1}^{2} B_{1} E_{1}\right)^{2} B_{3}=B_{3} \text {. }
$$

$$
E_{1} B_{3} E_{1}^{2}=B_{1} B_{2} B_{3}, \quad E_{3} B_{1} B_{2} B_{3} E_{3}=B_{1} \cdot B_{1} B_{2} \cdot B_{3} B_{4} \text {. }
$$


Finally,

$$
E_{3}^{-1} B_{4} E_{3}=E_{3} E_{2} E_{3} B_{3} E_{3} E_{2} E_{3}=E_{2} E_{3}\left(E_{2} B_{3} E_{2}\right) E_{3} E_{2}=B_{4} \text {. }
$$

We have now derived from 276) all of the relations 275). It remains to derive 273). Since $B_{2}, B_{3}, B_{4}$ are conjugate with $B_{1}$ by 277), they are of period 2. By 275), $B_{1} B_{2}$ is conjugate with $B_{1}, B_{2} B_{3}$ with $B_{3}, B_{3} B_{4}$ with $B_{3}$. Hence they are of period 2 and therefore $B_{3}$ is commutative with $B_{2}$ and $B_{4}, B_{1}$ with $B_{2}$. Since $E_{2}^{-1} B_{2} E_{2}$ is its own reciprocal, we have

$$
B_{1} B_{3} B_{2}=B_{1} B_{2} B_{3}=\left(B_{1} B_{2} B_{3}\right)^{-1}=B_{3} B_{2} B_{1}=B_{3} B_{1} B_{2} \text {, }
$$

so that $B_{1} B_{3}=B_{3} B_{1}$. Since $B_{2} B_{3} B_{4}$ was shown to be the transformed of $B_{3}$ by $E_{1}^{2} E_{3}$, we have

$$
B_{3} B_{2} B_{4}=B_{2} B_{3} B_{4}=\left(B_{2} B_{3} B_{4}\right)^{-1}=B_{4} B_{3} B_{2}=B_{3} B_{4} B_{2} \text {. }
$$

Hence $B_{2}$ is commutative with $B_{4}$. Since $B_{1}$ is commutative with $B_{3}, E_{2}$ and $E_{3}$, it is commutative with $B_{4}$ by 277 ).

2\%2. Theorem. - Every substitution of $F O(5,3)$ is given once and but once by the following 27 sets, in which $A$ denotes the subgroup $A_{960}$ :

$$
\begin{array}{rlrl}
R_{t} & \equiv A w^{t} & & (t=0,1,2) \\
R_{s i t} & \equiv A w^{s}\left(\xi_{1} \xi_{2}\right)\left(\xi_{i} \xi_{5}\right) w^{*} & \left(\begin{array}{l}
s=1,2 ; t=0,1,2 \\
i=1,2,3,4
\end{array}\right) .
\end{array}
$$

Since $w$ is not in $A$, a substitution of $R_{t}$ belongs to $R_{\tau}$ if and only if $t=\tau$. If a substitution of $R_{t}$ belong to $R_{s i \tau}$, the product

$$
w^{s}\left(\xi_{1} \xi_{2}\right)\left(\xi_{i} \xi_{5}\right) w^{\tau-t}
$$

must belong to $A$, whereas it replaces $\xi_{5}$ by a linear function of $\xi_{1}, \xi_{2}, \xi_{3}, \xi_{4}$, every coefficient being \pm 1 .

If a substitution of $R_{s i t}$ belong to $R_{\sigma j \tau}$, the product

$$
S \equiv w^{s}\left(\xi_{1} \xi_{2}\right)\left(\xi_{i} \xi_{5}\right) w^{t-\tau}\left(\xi_{j} \xi_{5}\right)\left(\xi_{1} \xi_{2}\right) w^{-\sigma}
$$

must belong to $A$. Supposing first that $t-\tau \neq 0$, we show that $S$ replaces $\xi_{5}$ by a function involving more than one index and therefore does not belong to $A$. In fact, $w^{-s} S$ replaces $\xi_{5}$ by a function of the form

$$
f \equiv \pm \xi_{a} \pm \xi_{b} \pm \xi_{c} \pm \xi_{5},
$$

where $a, b, c$ are three of the integers $1,2,3,4$. Then $w^{s}$ replaces $f$ by $f_{1} \pm \xi_{5}$, where $f_{1}$ is a linear function of $\xi_{1}, \xi_{2}, \xi_{3}, \xi_{4}$ with coefficients not all $\equiv 0(\bmod 3)$. Hence $S$ replaces $\xi_{5}$ by $f_{1} \pm \xi_{5}$, involving two or more indices. Suppose, however, that $t=\tau$. If then $i \neq j$, $S$ replaces $\xi_{5}$ by a linear function of $\xi_{1}, \xi_{2}, \xi_{3}, \xi_{4}$ with coefficients \pm 1 . If $i=j, S \equiv w^{s-\sigma}$, which belongs to $A$ only if $s=\sigma$. But in the latter case, the two sets $R_{s i t}$ and $R_{\sigma j \tau}$ are themselves identical. 
273. Theorem. - The abstract group $O$ generated by the operators ${ }^{1}$ ) $E_{1}, E_{2}, E_{3}, B_{1}, W$ subject to the generational relations 271) and

$$
W^{3}=I, \quad W^{-1} E_{1} W=B_{1} E_{1}, \quad W^{-1} E_{2} W=B_{1} E_{2},
$$

$$
W^{-1} B_{1} W=B_{2} E_{2} B_{2}
$$

$$
W B_{4} W=B_{4} E_{1} B_{1} E_{2} E_{1}^{2}
$$

$$
\left(W E_{3} E_{2} E_{1} W\right) E_{3}=E_{1}^{2} E_{2} E_{3} E_{2} E_{1}\left(W E_{3} E_{2} E_{1} W\right) \text {, }
$$

$B_{2}$ and $B_{4}$ being defined by 277), is holoedrically isomorphic with FO $(5,3)$.

Writing these relations for the corresponding orthogonal substitutions as defined by 272$), 274$ ) and $W \sim w$, we obtain relations which reduce to identities modulo 3 . The order $\Omega$ of $O$ is therefore $\sum 25920$. The holoedric isomorphism will be established when it is shown that $\Omega \overline{<} 25920$. To prove this statement, consider the following 27 sets $^{2}$ ) of operators of $O$, those of the first set being the operators of $G \equiv G_{960}$ :

$$
\begin{gathered}
R_{t} \equiv G W^{t}, R_{s 4 t} \equiv G W^{s} E_{3} W^{t}, R_{s 3 t} \equiv G W^{s} E_{3} E_{2} W^{t} \\
\quad R_{s 2 t} \equiv G W^{s} E_{3} E_{2} E_{1} W^{t}, \quad R_{s 1 t} \equiv G W^{s} E_{3} E_{2} E_{1}^{2} W^{t}
\end{gathered} \quad\left(\begin{array}{l}
t=0,1,2 \\
s=1,2
\end{array}\right) .
$$

It is shown in the next section that the generators $E_{1}, E_{2}, E_{3}, W$, and therefore an arbitrary operator $\alpha$ of the group $O$, gives rise to a mere interchange of the above 27 sets when applied as a righthand multipliers. Since the first set $G$ contains the identity 1 , the product $I \alpha \equiv \alpha$ lies in one of the 27 sets. Hence $O$ contains at most $27 \cdot 960 \equiv 25920$ operators. In particular, it follows that the 27 sets form a rectangular table for $O$ with the operators $G_{960}$ in the first row.

We make use of the formulae derived' from 271), 278), 279), 280), 277):

$$
\begin{array}{ll} 
& E_{3} E_{2} E_{1}^{2} B_{3}=B_{2} E_{3} E_{2} E_{1}^{2}, \quad E_{3} E_{2} E_{1} B_{3}=B_{1} B_{2} E_{3} E_{2} E_{1}, \\
& W E_{2}=B_{3} W, \quad W^{2} E_{2}=B_{1} E_{2} W^{2}, \quad W E_{1}=B_{3} E_{2} E_{1} W \\
& W^{2} E_{1}=B_{1} E_{1} W^{2}, \\
& W B_{1}=B_{3} E_{2} W, \quad W^{2} B_{1}=B_{3} B_{1} E_{2} W^{2}, \quad W B_{3}=E_{2} B_{1} W \\
& E_{1} W=W B_{1} E_{1}, \\
& E_{1}^{2} W=W E_{1}^{2} B_{1}, \quad E_{2} W=W E_{2} B_{1}, \quad E_{2} W^{2}=W^{2} B_{3}, \\
& E_{2} E_{1} W=W E_{2} E_{1} .
\end{array}
$$

1) For simplicity $B_{1}$ is retained. It may be dropped since

$$
B_{1}=W^{-1} E_{1} W E_{1}^{-1}=W^{-1} E_{2} W E_{2}^{-1} \text {. }
$$

2) They correspond in $F O(5,3)$ with the 27 rows of the rectangular table. 
2\%4. Theorem. - When applied as right-hand multipliers to the above 27 sets, the generators $W, E_{1}, E_{2}, E_{3}$ give rise to the respective permutations:

[W]: $\left(R_{0} R_{1} R_{2}\right)\left(R_{s i 0} R_{s i 1} R_{s i 2}\right)$,

[E] : $\left(R_{s 10} R_{s 30} R_{s 20}\right)\left(R_{s 21} R_{s 31} R_{2 s 41}\right)\left(R_{s 22} R_{2 s 12} R_{s 32}\right)$,

[E $E_{2}$ : $\left(R_{s 10} R_{s 20}\right)\left(R_{s 30} R_{s 40}\right)\left(R_{s 22} R_{2 s 12}\right)\left(R_{s 32} R_{s 42}\right)\left(R_{131} R_{231}\right)\left(R_{141} R_{241}\right)$,

$\left[E_{3}\right]:\left(R_{s} R_{s 40}\right)\left(R_{s 10} R_{s 20}\right) \cdot\left(R_{211} R_{242}\right)\left(R_{221} R_{132}\right)\left(R_{112} R_{222}\right)\left(R_{122} R_{141}\right)$ $\left(R_{212} R_{231}\right)\left(R_{131} R_{241}\right)$,

where $i=1,2,3,4$ and $s=1,2$, while the first subscript $2 s$ is to be reduced modulo 3 .

The form of $[W]$ is evident. Consider the multiplier $E_{2}$.

$$
\begin{array}{r}
R_{0} E_{2}=R_{0}, \quad R_{1} E_{2}=G W E_{2}=G B_{3} W=G W=\dot{R}_{1} . \\
R_{2} E_{2}=G W^{2} E_{2}=G B_{1} E_{2} W^{2}=G W^{2}=R_{2} . \\
R_{s 11} E_{2}=G W^{s} E_{3} E_{2} E_{1}^{2} W E_{2}=G W^{s} E_{3} E_{2} E_{1}^{2} B_{3} W=R_{s 11}
\end{array}
$$

[by 281)].

$R_{s 21} E_{2}=G W^{s} E_{3} E_{2} E_{1} W E_{2}=G W^{s} E_{3} E_{2} E_{1} B_{3} W=R_{s 21}$

$$
\begin{aligned}
R_{s 10} E_{2} & =G W^{s} E_{3} E_{2} E_{1}^{2} E_{2}=G W^{s} E_{3} E_{1} E_{2} E_{1} \\
& =G W^{s} E_{1}^{2} E_{3} E_{2} E_{1}=R_{s 20} . \\
R_{s 30} E_{2} & =G W^{s} E_{3} E_{2} \cdot E_{2}=R_{s 40} . \\
R_{s 22} E_{2} & =G W^{s} E_{3} E_{2} E_{1} \cdot B_{1} E_{2} W^{2}=G W^{s} B_{4} E_{1} E_{3} E_{2} E_{1}^{2} W^{2} \\
& =R_{2 s 12},
\end{aligned}
$$

by 279$)$, since $E_{3} E_{2} E_{1} B_{1} E_{2}=B_{2} B_{3} B_{4} E_{1} E_{3} E_{2} E_{1}^{2}$.

$$
\begin{aligned}
R_{s 32} E_{2} & =G W^{s} E_{3} E_{2} W^{2} E_{2}=G W^{s} E_{3} E_{2} \cdot B_{1} E_{2} W^{2} \\
& =G W^{s} B_{1} E_{3} W^{2}=R_{s 42} \cdot \\
R_{131} E_{2} & =G W E_{3} E_{2} \cdot B_{3} W=G W B_{3} B_{4} E_{3} E_{2} W \\
& =G W^{2} E_{3} E_{2} W=R_{231} . \\
R_{141} E_{2} & =G W E_{3} \cdot B_{3} W=G W^{2} E_{3} W=R_{241} .
\end{aligned}
$$

Next, $\quad R_{s 21} E_{1}=G W^{s} E_{3} E_{2} E_{1} \cdot B_{3} E_{2} E_{1} W=G W^{s} E_{3}\left(E_{2} E_{1}\right)^{2} W$

$$
\begin{gathered}
=G W^{s} E_{1} E_{3} E_{2} W=R_{s 31}, \text { upon applying 281). } \\
R_{s 22} E_{1}=G W^{s} E_{3} E_{2} E_{1} \cdot B_{1} E_{1} W^{2}=G W^{s} B_{2} B_{3} B_{4} E_{3} E_{2} E_{1}^{2} W^{2}
\end{gathered}
$$

$$
=R_{2 s 12} \text {. }
$$

$R_{2 s 12} E_{1}=G W^{2 s} E_{3} E_{2} E_{1}^{2} \cdot B_{1} E_{1} W^{2}$

$$
=G W^{2 s} B_{1} B_{2} B_{3} B_{4} E_{3} E_{2} W^{2}=R_{s 32} \text {. }
$$$$
R_{s 11} E_{1}=G W^{s} E_{3} E_{2} E_{1}^{2} \cdot B_{3} E_{2} E_{1} W=G W^{s} E_{3} E_{2} E_{1}^{2} \cdot E_{2} E_{1} W
$$

$$
=G W^{s} E_{3} E_{1} E_{2} E_{1}^{2} W=G W^{s} E_{1}^{2} E_{3} E_{2} E_{1}^{2} W=R_{s 11} \text {. }
$$

The remaining cases follow immediately. 
For the right-hand multiplier $E_{3}$, the calculations are not so simple.

$$
\begin{aligned}
R_{121} E_{3} & =G W E_{3} E_{2} E_{1} W E_{3}=G W E_{3} E_{2} E_{1} W=R_{121} \quad \text { [by 280)] } \\
R_{221} E_{3} & =G W\left(E_{1}^{2} E_{2} E_{3} E_{2} E_{1} W E_{3} E_{2} E_{1} W\right) \\
& =G W E_{3} E_{2} E_{1} W E_{3} \cdot E_{2} E_{1} W \\
& =G W E_{3} E_{2} E_{1} W \cdot E_{2} E_{1} W=G W E_{3} E_{2} E_{1} \cdot E_{2} E_{1} W^{2}=R_{132} \\
R_{211} E_{3} & =G W^{2} E_{3} E_{2} E_{1} \cdot W B_{1} E_{1} \cdot E_{3}=G W^{2} E_{3} E_{2} E_{1} W E_{3} B_{1} E_{1}^{2} \\
& =R_{221} E_{3} B_{1} E_{1}^{2}=R_{132} B_{1} E_{1}^{2}=G W E_{3} E_{2} \cdot B_{1} B_{2} E_{2} E_{1}^{2} W^{2} \\
& =G W B_{1} B_{2} B_{3} B_{4} E_{3} E_{1}^{2} W^{2}=G W^{2} E_{3} W^{2}=R_{242} \\
R_{231} E_{3} & =G W^{2} E_{3} E_{2} \cdot E_{1} W E_{1}^{2} B_{1} \cdot E_{3}=R_{221} E_{3} E_{1} B_{1}=R_{132} E_{1} B_{1} \\
& =G W E_{3} E_{2} \cdot B_{1} E_{1} B_{3} B_{1} E_{2} W^{2}=G W B_{3} B_{4} E_{1} \cdot E_{3} E_{2} E_{1}^{2} W^{2} \\
& =R_{212} .
\end{aligned}
$$$$
R_{131} E_{3}=G W E_{3} E_{2} \cdot E_{1} W E_{1}^{2} B_{1} \cdot E_{3}=G W E_{3} E_{2} E_{1} W E_{1} B_{1} \text { [by 280)] }
$$$$
=G W E_{3} E_{2} E_{1} \cdot B_{3} E_{2} E_{1} B_{3} E_{2} W=G W^{2} E_{3} W=R_{241}
$$$$
R_{232} E_{3}=G W^{2} E_{3} \cdot W^{2} B_{3} \cdot E_{3}=G W^{2} E_{3} W^{2} E_{3} B_{3} B_{4}
$$$$
\text { [by 281)]. }
$$$$
=R_{242} E_{3} B_{3} B_{4}=R_{211} B_{3} B_{4}
$$$$
=G W^{2} E_{3} E_{2} E_{1}^{2} \cdot E_{2} B_{1} \cdot B_{4} E_{1} B_{1} E_{2} E_{1}^{2} W^{2}
$$$$
=G W^{2} B_{1} B_{3} E_{3} E_{1}^{2} E_{2} W^{2}=R_{232} \text {. }
$$

$$
\begin{aligned}
R_{122} E_{3} & =G W E_{3} E_{2} \cdot B_{1} W^{2} E_{1} \cdot E_{3}=G W E_{3} E_{2} W^{2} E_{3} E_{1}^{2} \\
& =R_{132} E_{3} E_{1}^{2}=R_{221} E_{1}^{2}=R_{141} .
\end{aligned}
$$$$
R_{222} E_{3}=G W^{2} E_{3} E_{2} \cdot B_{1} W^{2} E_{1} \cdot E_{3}=G W^{2} E_{3} E_{2} W^{2} E_{3} E_{1}^{2}
$$$$
=R_{232} E_{3} E_{1}^{2}=R_{232} E_{1}^{2}=R_{112} \text {. }
$$$$
R_{111} E_{3}=G W E_{3} E_{2} \cdot W E_{1}^{2} B_{1} \cdot E_{3}=R_{131} E_{3} E_{1} B_{1}=R_{241} E_{1} B_{1}=R_{121} B_{1}
$$$$
=G W E_{3} E_{2} E_{1} \cdot B_{3} E_{2} \cdot W=G W E_{3} E_{2} E_{1} E_{2} W=R_{111}[\text { by 281)]. }
$$

$$
\begin{aligned}
R_{142} E_{3} & \equiv G W E_{3} W^{2} E_{3}=G W E_{3} E_{2} W^{2} B_{3} E_{3} \\
& =G W E_{3} E_{2} W^{2} E_{3} B_{3} B_{4}=R_{132} E_{3} B_{3} B_{4} \\
& =R_{221} B_{3} B_{4}=G W^{2} E_{3} E_{2} E_{1} \cdot E_{2} B_{1} \cdot B_{4} E_{1} B_{1} E_{2} E_{1}^{2} W^{2} \\
& =G W^{2} B_{2} B_{4} E_{3} E_{1}^{2} W^{2}=G W E_{3} W^{2}=R_{142} .
\end{aligned}
$$

275. Theorem. - The simple group $H A\left(4,2^{2}\right)$ is put into holoedric isomorphism with the abstract group $O$ by the correspondences of generators

$$
W \sim\left(\begin{array}{llll}
0 & 1 & 1 & 0 \\
1 & 1 & 1 & 0 \\
0 & 0 & 1 & 0 \\
1 & 0 & 1 & 1
\end{array}\right), \quad E_{1} \sim\left(\begin{array}{llll}
1 & 0 & I^{2} & 0 \\
0 & 0 & I^{2} & I^{2} \\
I & 0 & 0 & 0 \\
I & I & 1 & 1
\end{array}\right), \quad E_{2} \sim\left(\begin{array}{llll}
0 & 0 & 1 & 0 \\
0 & 0 & 1 & 1 \\
1 & 0 & 0 & 0 \\
1 & 1 & 0 & 0
\end{array}\right)
$$




$$
\begin{aligned}
& E_{3} \sim\left(\begin{array}{llll}
0 & 1 & I^{2} & 0 \\
0 & 0 & I^{2} & I^{2} \\
I & 0 & 1 & 1 \\
I & I & 1 & 1
\end{array}\right), B_{1} \sim\left(\begin{array}{llll}
1 & 1 & 0 & 0 \\
0 & 1 & 0 & 0 \\
0 & 0 & 0 & 1 \\
0 & 0 & 1 & 0
\end{array}\right), \quad B_{2} \sim\left(\begin{array}{llll}
1 & 1 & I^{2} & I^{2} \\
0 & 1 & 0 & 0 \\
0 & I & 1 & 0 \\
0 & I & 0 & 1
\end{array}\right), \\
& B_{3} \sim\left(\begin{array}{llll}
1 & 0 & 1 & 1 \\
0 & 1 & 0 & 0 \\
0 & 1 & 1 & 0 \\
0 & 1 & 0 & 1
\end{array}\right), \quad B_{4} \sim\left(\begin{array}{llll}
1 & 0 & I^{2} & I^{2} \\
0 & 1 & 0 & 0 \\
0 & I & 1 & 0 \\
0 & I & 0 & 1
\end{array}\right)
\end{aligned}
$$

where $I$ is a root of the irreducible congruence $x^{2} \equiv x+1(\bmod 2)$.

Indeed, it may be verified that these correspondences preserve the generational relations ( $\$ 273$ ) prescribed for the generators of $O$. Furthermore, by $\S 132$ the order of $H A\left(4,2^{2}\right)$ is 25920 , so that the isomorphism is holoedric.

2\%6. The correspondences established in the last section enable us to pass readily from any orthogonal substitution $S$ to the corresponding substitution of $H A\left(4,2^{2}\right)$. In fact, we have only to express $S$ in terms of the simple generators $w,\left(\xi_{1} \xi_{2} \xi_{3}\right),\left(\xi_{3} \xi_{4}\right)\left(\xi_{1} \xi_{2}\right),\left(\xi_{4} \xi_{5}\right)\left(\xi_{1} \xi_{2}\right)$, $C_{1} C_{2}, C_{2} C_{3}, C_{3} C_{4}, C_{4} C_{5}$ of $F O(5,3)$.

It is not difficult to invert these correspondences and obtain the orthogonal substitutions which correspond to the simplest set of generators of $H A\left(4,2^{2}\right)$, viz.: -

$$
\begin{gathered}
L_{1,1} \sim C_{2} C_{3} C_{4} C_{5}, \quad M_{2} \sim C_{1} C_{3} C_{4} C_{5}, J \sim\left(\xi_{3} \xi_{5} \xi_{4}\right) C_{3} C_{5}, \\
M_{1} \sim\left(\begin{array}{lllll}
1 & 0 & 1 & 1 & 2 \\
0 & 2 & 0 & 0 & 0 \\
1 & 0 & 1 & 2 & 1 \\
1 & 0 & 2 & 1 & 1 \\
2 & 0 & 1 & 1 & 1
\end{array}\right), L_{2,1} \sim\left(\begin{array}{lllll}
2 & 0 & 0 & 0 & 0 \\
0 & 1 & 1 & 1 & 2 \\
0 & 1 & 1 & 2 & 1 \\
0 & 1 & 2 & 1 & 1 \\
0 & 2 & 1 & 1 & 1
\end{array}\right), R_{2,1,1} \sim\left(\begin{array}{lllll}
0 & 2 & 1 & 1 & 1 \\
2 & 0 & 1 & 1 & 1 \\
1 & 1 & 2 & 0 & 2 \\
1 & 1 & 0 & 2 & 2 \\
1 & 1 & 2 & 2 & 0
\end{array}\right) .
\end{gathered}
$$

Here $J$ denotes the hyperabelian substitution of period 3:

$$
\xi_{1}^{\prime}=I \xi_{1}, \quad \eta_{1}^{\prime}=I \eta_{1}, \quad \xi_{2}^{\prime}=I^{2} \xi_{2}, \quad \eta_{2}^{\prime}=I^{2} \eta_{2} .
$$

2\%\%. By § 189, the orthogonal group $F O(5,3)$ is holoedrically isomorphic with the Abelian group $A(4,3)$. Given an arbitrary Abelian substitution, the process of forming the second compound and a subsequent transformation of indices ( $\$ 189$ ) enables us to find quite readily the corresponding orthogonal substitution. The inverse problem is solved by employing the set ${ }^{1}$ ) of Abelian substitutions which correspond to the simplest orthogonal generators $w,\left(\xi_{1} \xi_{2} \xi_{3}\right)$, etc.

1) Transact. Amer. Math. Soc., July, 1900, p. 366. 
278. Theorem. $\left.{ }^{1}\right)$ - The special linear homogeneous group $S L H\left(2, p^{n}\right)$ of binary linear substitutions of determinant unity in the $G F\left[p^{n}\right]$ is holoedrically isomorphic with the abstract group $L$ generated by the operators $T$ and $S_{\lambda}$, where $\lambda$ runs through the series of $p^{n}$ marks of the field, subject to the generational relations

a) $\quad S_{0}=I, \quad S_{\lambda} S_{\mu}=S_{\lambda+\mu}$

b) $T^{4}=I, \quad S_{\lambda} T^{2}=T^{2} S_{i}$,

$(\lambda, \mu$ any marks $)$

c) $S_{\lambda} T S_{\mu} T S_{\frac{\lambda-1}{\lambda \mu-1}} T S_{-(\lambda \mu-1)} T S_{\frac{\mu-1}{2 \mu-1}} T=I \quad(\lambda, \mu$ any marks, $\lambda \mu \neq 1)$.

Since the relations a), b), c) are satisfied by the substitutions

$$
T=\left(\begin{array}{rr}
0,-1 \\
1, & 0
\end{array}\right), \quad S_{\lambda}=\left(\begin{array}{l}
1, \lambda \\
0,1
\end{array}\right)
$$

which ( $\$ 100$, Cor. II) serve to generate $S L H\left(2, p^{n}\right)$, the order $l$ of the abstract group is at least $p^{n}\left(p^{2 n}-1\right)$. We proceed to prove that $l$ is at most $p^{n}\left(p^{2}-1\right)$. Then will $S L H\left(2, p^{n}\right)$ and $L$ be of equal order and so holoedrically isomorphic.

Consider the following sets of operators of $L$

$$
S_{\sigma} T S_{\alpha} T S_{\alpha}^{-1}, \quad S_{\sigma} T S_{\alpha} T S_{\tau} T \quad(\sigma, \alpha, \tau \text { arbitrary, } \alpha \neq 0) .
$$

At most $p^{n}\left(p^{n}-1\right)+p^{2 n}\left(p^{n}-1\right) \equiv\left(p^{n}-1\right) p^{n}\left(p^{n}+1\right)$ of them are distinct. If it be shown that every operator of $L$ occurs in these sets, it will follow that $l \bar{\sum} p^{n}\left(p^{2 n}-1\right)$. The proof consists in showing that the product of any operator of the sets by $T$ or by any $S_{\lambda}$ equals an operator of the sets. Since an arbitrary operator $\Sigma$ of $L$ is derived from $T$, and $S_{\lambda}$, it will follow that $I \Sigma \equiv \Sigma$ belongs to the sets.

c) gives

In view of a) the reciprocal of $S_{\lambda}$ is $S_{-\lambda}$. For $\lambda=1, \mu \neq 1$, d)

$$
S_{1} T^{3} S_{1} T S_{1} T \equiv\left(S_{1} T^{3}\right)^{3}=I \text {. }
$$

Applying $T$ as a right-hand multiplier, the product of any operator of the first set by $T$ gives one of the second set. We next show that

$$
S_{\sigma} T S_{\alpha} T S_{\alpha^{-1}} T \cdot T=S_{\sigma-2 \alpha}-1 T S_{-\alpha} T S_{-\alpha}^{-1} .
$$

Applying a) and b) the condition for this identity is seen to be

$$
T S_{\alpha} T S_{2 \alpha}-1 T S_{\alpha} T S_{2 \alpha-1} T^{2}=I \text {. }
$$

For $p=2$, it reduces to an identity. For $p>2$, we have by $c$ )

$$
T S_{\alpha} T S_{2 \alpha^{-1}} T S_{\alpha-1} T S_{-1} T S_{2 \alpha^{-1}-1}=I \text {. }
$$

From this e) follows upon replacing $S_{-1} T S_{-1} T S_{-1}$ by $T^{3}$ as allowed by d).

1) Due to Professor Moore, who gave a different proof. 
For operators of the second set with $\alpha \neq 0, \tau \neq \alpha^{-1}$, we prove that

$$
S_{\sigma} T S_{\alpha} T S_{\tau} T \cdot T=S_{\sigma_{1}} T S_{\alpha_{1}} T S_{\tau_{1}} T,
$$

where $\sigma_{1}, \alpha_{1}, \tau_{1}$ are suitably chosen marks, $\alpha_{1} \neq 0$. The equivalent condition

$$
S_{\alpha} I S_{\tau} T S_{-\tau_{1}} T S_{-\alpha_{1}} I S_{-\sigma_{1}+\sigma} T=I
$$

may be satisfied by c) by proper choice of $\tau_{1}, \alpha_{1}, \sigma_{1}$, with

$$
\alpha_{1} \equiv \alpha \tau-1 \neq 0 \text {. }
$$

We next apply $S_{\varrho}$ as a right-hand multiplier. $S_{\sigma} T S_{\alpha} T S_{\alpha}-1 S_{\varrho}$ will be of the form $S_{\sigma_{1}} T S_{\alpha_{1}} I S_{\tau_{1}} T^{3}$, and consequently belong to the sets by the previous proof, if we have

$$
S_{\alpha} T S_{\alpha-1}+\varrho T S_{-\tau_{1}} T S_{-\alpha_{1}} T S_{-\sigma_{1}+\sigma} T=I .
$$

Since $\alpha\left(\alpha^{-1}+\varrho\right) \equiv 1+\alpha \varrho \neq 1$, this condition is of the form $\left.c\right)$ if $\alpha_{1}, \sigma_{1}, \tau_{1}$ be suitably chosen. If $\varrho=\alpha /(\alpha \tau-1)$, so that $\alpha \tau \neq 1$, we have, by $c)$,

$$
S_{\sigma} T S_{\alpha} T S_{\tau} T \cdot S_{Q}=S_{\sigma+\frac{1-\tau}{\alpha \tau-1}} T S_{\alpha \tau-1} T S_{\frac{1}{\alpha \tau-1}} .
$$

For the case $A \equiv \alpha-\rho(\alpha \tau-1) \neq 0$, we prove that

$$
\text { f) } \quad S_{\sigma} T S_{\alpha} T S_{\tau} T \cdot S_{\varrho}=S_{\sigma+\tau \varrho A^{-1}} T S_{A} T S_{\alpha \tau A^{-1}} T \text {. }
$$

If $\alpha \tau \neq 1$, we replace $T S_{\alpha} T S_{\tau} T$ by its equivalent derived from c) and find that condition $f$ ) becomes

$$
S_{A} T S_{\alpha \tau A^{-1}} T S_{\frac{A-1}{\alpha \tau-1}} I S_{-(\alpha \tau-1)} T S_{\frac{\alpha \tau A^{-1}-1}{\alpha \tau-1}} T=I,
$$

and hence is satisfied from c). If, however, $\alpha \tau=1$, so that $A=\alpha$, then $f$ ) takes the simpler form

$$
T S_{\alpha} T S_{\alpha-1} T S_{\varrho}=S_{\alpha-2}{ }_{\varrho} T S_{\alpha} T S_{\alpha-1} T .
$$

If also $\varrho \neq \alpha$, we replace $T S_{\alpha}-1 T S_{\varrho}$ by its equivalent derived from c) and find the condition, where $\nu \equiv \alpha^{-1} \varrho-1$

$$
T S_{(1-\alpha) / \nu} T^{-1} S_{\nu} T S_{\left(1-\alpha^{-1}\right) / \nu} T=S_{\alpha-2}{ }_{\varrho} T S_{\alpha} T S_{\alpha-1} T .
$$

This reduces to the identity c) for $\lambda=\alpha^{-2} \varrho, \mu=\alpha$, whence $\lambda \mu \neq 1$. In particular, $\left.f^{\prime}\right)$ is true if $\varrho=\alpha+x, \varkappa \neq 0$, so that

$$
\left(T S_{\alpha} T S_{\alpha-1} T S_{\alpha}\right) S_{\varkappa}=S_{\alpha}-2_{\varkappa}\left(S_{\alpha-1} T S_{\alpha} T S_{\alpha-1} T\right) .
$$

The products in the parentheses are identical and so $f^{\prime}$ ) is true for $\varrho=\alpha$, if the following condition be true for any particular mark $x \neq 0$,

$$
S_{-\alpha-2}\left(T S_{\alpha} T S_{\alpha-1} T S_{\alpha}\right) S_{\varkappa}=\left(T S_{\alpha} T S_{\alpha-1} T S_{\alpha}\right) \text {. }
$$

The latter is of the form $f^{\prime}$ ) for $\varrho \equiv-x$ and hence is true if $-\varkappa \neq \alpha$. But marks $x \neq 0,-\alpha$ exist if $p^{n}>2$. For $p^{n}=2, \alpha=1$, so that $f^{\prime}$ ) is true for any $\rho$ by $d$ ). 
302 CHAPTER XIII. AUXILIARY THEOREMS ON ABSTRACT GROUPS. etc.

Corollary. - The quotient-group $L F\left(2, p^{n}\right)$ is holoedrically isomorphic with the abstract group $F$ generated by the operators $T$ and $S_{2}$. subject to the relations $T^{2}=I$ together with a) and c).

279. For $\lambda=0$ or 1 or for $\mu=0$ or 1 , relations c) always reduce to d) upon applying a) and b). For the group $L F\left(2, \hat{p}^{n}\right)$, d) becomes

D)

$$
\left(S_{1} T\right)^{3}=I \text {. }
$$

If neither $\lambda$ nor $\mu$ is 0 or 1 , the product of any two consecutive subscripts in c) is not unity, the first subscript $\lambda$ being regarded as consecutive with the last subscript $(\mu-1) /(\lambda \mu-1)$. Using any two consecutive subscripts as the initial $\lambda, \mu$, the resulting identity $c)$ is seen to be an immediate consequence of the given identity c). Taking for $\lambda$ any one of the $p^{n}-2$ marks $\neq 0,1$ and for $\mu$ any of the $p^{n}-3$ marks $\neq 0,1, \lambda^{-1}$, the remaining subscripts in c) are different from 0 and 1. Hence those identities c) which do not reduce to D) are equivalent in sets of five, an exception being those with all subscripts equal to $\lambda$, where $\lambda^{2}+\lambda=1$. If the latter has $\sigma$ solutions in the $G F\left[p^{n}\right]$, it follows that there are exactly

$$
N \equiv \sigma+\frac{1}{5}\left\{\left(p^{n}-2\right)\left(p^{n}-3\right)-\sigma\right\}
$$

distinct identities c) not immediately reducible to $\mathrm{D}$ ). For $p=2$, $\sigma=0$ or 2 according as $n$ is odd or even; for $p=5, \sigma=1$; for $p \neq 2, \neq 5, \sigma=0$ or 2 according as $p^{n}=5 k \pm 2$ or $p^{n}=5 k \pm 1$.

280. For the group $L F(2,5)$ of order 60 , the $N=2$ relations c) are

$$
\left(S_{2} T\right)^{5}=I, \quad S_{2} T S_{4} T S_{3} T S_{3} T S_{4} T=I \text {. }
$$

These may both be derived from a), D) and $T^{2}=I$, so that $\operatorname{LF}(2,5)$ is generated by $A \equiv S_{1}, B \equiv T$ subject to the relations

$$
A^{5}=I, \quad B^{2}=I, \quad(A B)^{3}=I .
$$

In proof, we apply $D)$ repeatedly and find that

$$
\begin{aligned}
\left(S_{2} T\right)^{5} & =\left(S_{1} T S_{-1} T S_{1} T\right)^{2} S_{2} T=S_{1} T S_{-3} T S_{1} T S_{2} T \\
& =S_{1} T S_{1} \cdot S_{1} T S_{1} T S_{1} \cdot S_{1} T=S_{1} T S_{1} \cdot T \cdot S_{1} T=I .
\end{aligned}
$$

Hence also $\left(T S_{3}\right)^{5}=I$, so that the second relation becomes

$$
S_{2} T S_{1}\left(S_{3} T\right)^{4} T S_{1} T=S_{2} T S_{1} T S_{2} T S_{1} T=S_{1}\left(S_{1} T\right)^{2} S_{1}\left(S_{1} T\right)^{2}=I .
$$

281. The group $\operatorname{LF}\left(2,2^{2}\right)$ of order 60 may be generated by $A=T S_{i}$ and $B=S_{i^{2}}$ subject to the relations 282), where $i$ and $i^{2}$ are the roots of $x^{2}+x \equiv 1(\bmod 2)$. Indeed, the $N=\sigma=2$ 'relations c) to be considered in addition to D) are

$$
\left(S_{i} T\right)^{5}=I, \quad\left(S_{i^{2}} T\right)^{5}=I .
$$


The latter only serves to define the operator $I$ in terms of $A$ and $B$ :

$$
T=B A B A^{-1} B A B A^{-1} B .
$$

The resulting expressions for $S_{i}, S_{1}, S_{i^{2}}$ are seen to be commutative and of period 2, so that relations a) follow from 282),

282. The group $\operatorname{LF}(2,7)$ of order 168 is defined by relations a), D), $I^{2}=I$, together with the following $N=4$ relations

$$
\begin{array}{ll}
S_{2} T S_{2} T S_{5} T S_{4} T S_{5} T=I, & S_{3} T S_{3} T S_{2} T S_{6} T S_{2} T=I, \\
S_{4} T S_{4} T S_{3} T S_{6} T S_{3} T=I, & S_{5} T S_{5} T S_{6} T S_{4} T S_{6} T=I .
\end{array}
$$

Applying a), D) and $T^{2}=I$, the second and third relations become

$$
\begin{aligned}
& S_{3} T S_{3} T S_{3} \cdot S_{-1} T S_{6} T S_{-1} \cdot S_{3} T=\left(S_{3} T\right)^{4}=I, \\
& S_{4} T S_{4} T=T S_{4} T S_{1} T S_{4}=T S_{3} \cdot S_{1} T S_{1} T S_{1} \cdot S_{3}=T S_{3} T S_{3} .
\end{aligned}
$$

The first relation may be written $S_{2} T S_{2} T S_{1} \cdot S_{4} T S_{4} T S_{4} \cdot S_{1} I=I$ or $S_{4} T S_{4} T S_{4}=S_{-1} T S_{-2} T S_{-2} T S_{-1}=\left(S_{-1} T S_{-1}\right)^{3}=\left(I S_{1} I\right)^{3}=I S_{3} T$. The fourth relation becomes an identity if we replace $S_{5} T S_{5} T$ by $T S_{5} T S_{4} T S_{5}$ as derived from the first relation. Hence the $G_{168}$ may be generated by $S_{1}$ and $T$ subject only to the generational relations ${ }^{1}$ )

$$
\text { 283) } \quad T^{2}=I, \quad S_{1}^{7}=I, \quad\left(S_{1} T\right)^{3}=I, \quad\left(S_{1}^{4} T\right)^{4}=I .
$$

Corollary. - The group $\operatorname{LF}(3,2)$ of order 168 is isomorphic with $L F(2,7)$. In fact, the relations 283$)$ are satisfied by the substitutions

$$
T=\left(\begin{array}{lll}
1 & 0 & 0 \\
0 & 1 & 0 \\
0 & 1 & 1
\end{array}\right), \quad S_{1}=\left(\begin{array}{lll}
1 & 1 & 1 \\
1 & 0 & 1 \\
1 & 0 & 0
\end{array}\right)
$$

\section{CHAPTER XIV.}

\section{GROUP OF THE EQUATION FOR THE 27 STRAIGHT LINES} ON A GENERAL SURFACE OF THE THIRD ORDER. $\left.{ }^{2}\right)$

283. A general cubic surface contains 27 straight lines such that ${ }^{3}$ ) $1^{0}$. Any one of the lines $A$ meets ten others which intersect two by two, forming with $A$ five triangles. The total number of -such triangles on the cubic surface is $5 \cdot 27 / 3 \equiv 45$.

1) Dyck, Math. Ann., vol. 20, p. 41; Burnside, The Theory of Groups, p. 305.

2) Compare Jordan, 'Traité, pp. 316-329, 365-369; Dickson, Comptes Rendus, vol. 128 , pp. $873--875$.

3) Steiner, Crelle, vol. 53. 
$2^{0}$. Any two triangles $A B C$ and $A^{\prime} B^{\prime} C^{\prime}$ having no side in common determine uniquely a third triangle $A^{\prime \prime} B^{\prime \prime} C^{\prime \prime}$ such that the corresponding sides of the three triangles intersect and form three new triangles $A A^{\prime} A^{\prime \prime}, B B^{\prime} B^{\prime \prime}, C C^{\prime} C^{\prime \prime}$. The former set of three triangles is said to constitute a trieder, which will be designated $\left[A B C, A^{\prime} B^{\prime} C^{\prime}, A^{\prime \prime} B^{\prime \prime} C^{\prime \prime}\right]$.

These two properties completely define the configuration of the 45 triangles formed by the 27 lines on the cubic surface.

Denoting the lines by $R_{t}, R_{s i t}(s=1,2 ; i=1,2,3,4 ; t=0,1,2)$, it will be shown that the 45 triangles are given by the notation ${ }^{1}$ )

$$
\begin{array}{ll}
R_{0} R_{1} R_{2}, \quad R_{s 10} R_{s 11} R_{s 12} & {[s=1,2]} \\
R_{t} R_{1 i t} R_{2 i t} & {[t=0,1,2 ; i=1,2,3,4]} \\
R_{s 2 t} R_{s 3 t \pm 1} R_{s 4 t \mp 1} & {[s=1,2 ; t \equiv 0,1,2(\bmod 3)]} \\
R_{s 1 t} R_{s j t-1} R_{2 s j t+1} & {[s=1,2 ; j=2,3,4 ; t \equiv 0,1,2]}
\end{array}
$$

where the subscript $2 s$ is to be replaced by 1 when $s=2$.

Each element $R$ lies in exactly five of these sets. Thus $R_{t}$ lies in the sets $R_{0} R_{1} R_{2}, R_{t} R_{1 i t} R_{2 i t}(i=1,2,3,4) ; R_{s 1 t}$ lies in the 5 sets

$$
R_{s 10} R_{s 11} R_{s 12}, \quad R_{t} R_{11 t} R_{21 t}, \quad R_{s 1 t} R_{s j t-1} R_{2 s j t+1} \quad(j=2,3,4) ;
$$

finally, $R_{s j t}$ lies in the following 5 sets, in the last two of which $\tau$ is to be suitably chosen modulo 3 :

$$
\begin{aligned}
& R_{t} R_{1 j t} R_{2 j t}, R_{s 1 t+1} R_{s j t} R_{2 s j t+2}, R_{2 s 1 t-1} R_{2 s j t-2} R_{s j t}, \\
& R_{s 2 \tau} R_{s 3 \tau \pm 1} R_{s 4 \tau \mp 1} .
\end{aligned}
$$

Hence each element can be associated with exactly ten other elements to determine a set. Property $1^{0}$ thus holds for the 45 sets.

The set $R_{0} R_{1} R_{2}$ lies in exactly the following sixteen trieders:

$$
\begin{aligned}
& {\left[\begin{array}{lll}
R_{0} R_{1} R_{2}, \quad R_{110} R_{111} R_{112}, \quad R_{210} R_{211} R_{212}
\end{array}\right],} \\
& {\left[R_{t} R_{t \pm 1} R_{t \mp 1}, R_{12 t} R_{13 t \pm 1} R_{14 t \mp 1}, R_{22 t} R_{23 t \pm 1} R_{24 t \mp 1}\right],} \\
& {\left[R_{t} R_{t-1} R_{t+1}, \quad R_{11 t} R_{1 j t-1} R_{2 j t+1}, R_{21 t} R_{2 j t-1} R_{1 j t+1}\right],}
\end{aligned}
$$

where $j=2,3$ or $4, t \equiv 0,1$ or $2(\bmod 3)$. Property $2^{0}$ therefore holds for the set $R_{0} R_{1} R_{2}$ in. conjunction with any set no one of whose elements is $R_{0}, R_{1}$ or $R_{2}$. It is next shown that the property holds for an arbitrary pair of sets $A B C, A^{\prime} B^{\prime} C^{\prime}$ which have no element in common. By the next section the 45 sets are merely permuted by the substitutions $[W],\left[E_{1}\right],\left[E_{2}\right],\left[E_{3}\right]$ given in $\S 274$ : The latter generate a substitution-group [O] holoedrically isomorphic

1) The connection with the 27 sets of orthogonal substitutions exhibited in $\S 272$ will be shown in the sequel. 
with the abstract simple group $O$ of $\S 273$. From its origin [O] is transitive and hence contains a substitution $S$ which replaces $R_{0}$ by an arbitrary element $A$. We proceed to prove that [O] contains a substitution $S_{1}$ which leaves $R_{0}$ fixed and replaces $R_{1}$ by an arbitrary one of the ten elements $R_{1}, R_{2}, R_{1 i 0}, R_{2 i 0}(i=1,2,3,4)$ which lie in sets with $R_{0}$. The substitutions $\left[E_{3}\right],\left[E_{3}\right]\left[E_{2}\right],\left[E_{3}\right]\left[E_{2}\right]\left[E_{1}\right]$, $\left[E_{3}\right]\left[E_{2}\right]\left[E_{1}\right]^{2}$ replace $R_{1}$ by $R_{140}, R_{130}, R_{120}, R_{110}$ respectively, without altering $R_{0}$. The transformed of $\left[E_{1}\right]$ by $[W]$ gives the substitution

$$
\left(R_{s 11} R_{s 31} R_{s 21}\right)\left(R_{s 22} R_{s 32} R_{2 s 42}\right)\left(R_{s 20} R_{2 s 10} R_{s 30}\right)
$$

which replaces $R_{120}$ by $R_{210}, R_{110}$ by $R_{230}$. Then [ $\left.E_{1}\right]$ and $\left[E_{2}\right]$ replace $R_{230}$ by $R_{220}$ and $R_{240}$ respectively. Finally, [ $\left.E_{3}\right]$ replaces $R_{240}$ by $R_{2}$. It follows that [O]. contains a substitution $S_{1} S$ which replaces the set $R_{0} R_{1} R_{2}$ by a set $A B C$ in which $A$ is any one of the 27 elements and $B$ any of the 10 elements which lie in sets with $A$. Hence [O] contains a substitution $\Sigma$ replacing the set $R_{0} R_{1} R_{2}$ by an arbitrary one of the 45 sets. Then $\Sigma^{-1}$ replaces the given pair $A B C, A^{\prime} B^{\prime} C^{\prime}$ by a pair $R_{0} R_{1} R_{2}, A_{1} B_{1} C_{1}$ having no elements in common. The latter sets determine a trieder by the earlier proof. Applying to it the substitution $\Sigma$, which was derived from $[W]$ and $\left[E_{i}\right]$ and therefore replaces sets by sets, we obtain a trieder containing $A B C$, $A^{\prime} B^{\prime} C^{\prime}$ and determined by them. Hence the above distribution of the 27 elements $R$ into 45 sets is a suitable notation for the configuration of the 45 triangles formed by the 27 lines on a general cubic surface.

284. The next step is to verify that the substitutions $[W],\left[E_{1}\right]$, $\left[E_{2}\right]$ and $\left[E_{3}\right]$ of $\S 274$ permute amongst themselves the 45 triangles. $[W]$ gives rise to the following even substitution:

$$
\begin{aligned}
& \left(R_{0} R_{1 i 0} R_{2 i 0}, \quad R_{1} R_{1 i 1} R_{2 i 1}, \quad R_{2} R_{1 i 2} R_{2 i 2}\right) \\
& \left(R_{s 20} R_{s 31} R_{s 42}, \quad R_{s 21} R_{s 32} R_{s 40}, \quad R_{s 22} R_{s 30} R_{s 41}\right) \\
& \left(R_{s 20} R_{s 32} R_{s 41}, \quad R_{s 21} R_{s 30} R_{s 42}, \quad R_{s 22} R_{s 31} R_{s 40}\right) \\
& \left(R_{s 10} R_{s j 2} R_{2 s j 1}, \quad R_{s 11} R_{s j 0} R_{2 s j 2}, \quad R_{s 12} R_{s j 1} R_{2 s j 0}\right.
\end{aligned}
$$

where $i=1,2,3,4 ; j=2,3,4 ; s=1,2$.

$\left[E_{2}\right]$ gives rise to the even substitution on the 45 triangles:

$\left(R_{0} R_{110} R_{210}, R_{0} R_{120} R_{220}\right)\left(R_{s 10} R_{s 11} R_{s 12}, R_{s 20} R_{s 11} R_{2 s 22}\right)$

$\left(R_{0} R_{130} R_{230}, R_{0} R_{140} R_{240}\right)\left(R_{s 10} R_{s 22} R_{2 s 21}, R_{s 20} R_{2 s 12} R_{2 s 21}\right)$

$\left(R_{2} R_{112} R_{212}, R_{2} R_{122} R_{222}\right)\left(R_{s 20} R_{s 31} R_{s 42}, R_{s 10} R_{2 s 31} R_{s 32}\right)$

$\left(R_{2} R_{132} R_{232}, R_{2} R_{142} R_{242}\right)\left(R_{s 20} R_{s 32} R_{s 41}, R_{s 10} R_{s 42} R_{2 s 41}\right)$

$\left(R_{s 11} R_{s 30} R_{2 s 32}, R_{s 11} R_{s 40} R_{2 s 42}\right)\left(R_{s 21} R_{s 32} R_{s 40}, R_{s 21} R_{s 42} R_{s 30}\right)$

$\left(R_{s 22} R_{s 30} R_{s 41}, R_{2 s 12} R_{s 40} R_{2 s 41}\right)\left(R_{s 22} R_{s 31} R_{s 40}, R_{2 s 12} R_{2 s 31} R_{s 30}\right)$.

Similarly $\left[E_{1}\right]$ and $\left[E_{3}\right]$ give rise to even permutations of the 45 triangles. 
285. Theorem. - The group $G$ of the equation for the 27 lines on a general cubic surface is of order 51840 and has a subgroiup of index 2 holoedrically isomorphic with the abstract group 0 .

The group $G$ is formed of the substitutions on the 27 elements $R$ which permute the 45 triangles. These substitutions can replace $R_{0}$ by at most 27 elements. Those leaving $R_{0}$ fixed can replace $R_{1}$ by no element other than the ten lying with $R_{0}$ in some triangle; namely, $R_{1}, R_{2}, R_{1 i 0}, R_{2 i 0}(i=1,2,3,4)$. The substitutions leaving $R_{0}$ and $R_{1}$ fixed and consequently the triangle $R_{0} R_{1} R_{2}$ cannot alter $R_{2}$ and must replace $R_{130}$ by one of the 8 elements

$$
R_{s i 0} \quad(s=1,2 ; i=1,2,3,4)
$$

which enter the four remaining triangles containing $R_{0}$. The substitutions leaving $R_{0}, R_{1}, R_{130}$ fixed cannot alter $R_{2}$ or $R_{230}$, and must permute amongst themselves the triangles which contain $R_{1}$ and likewise the triangles which contain $R_{130}$. Hence they must permute the pairs $R_{111}, R_{211} ; R_{121}, R_{221} ; R_{131}, R_{232} ; R_{141}, R_{241}$; and likewise permute the pairs $R_{111}, R_{232} ; R_{212}, R_{231} ; R_{122}, R_{141} ; R_{121}, R_{142}$. Hence the elements $R_{111}, R_{121}, R_{231}, R_{141}$ common to the two sets must be permuted amongst themselves, which can be done in at most 24 ways. Finally, a substitution of $G$ which leaves fixed $R_{0}, R_{1}, R_{2}, R_{130}, R_{230}$, $R_{111}, R_{121}, R_{231}$ and $R_{141}$ must not alter $R_{211}, R_{221}, R_{131}, R_{241}, R_{232}$, $R_{212}, R_{122}$ and $R_{142}$ and therefore must leave fixed the third element in each of the triangles $R_{211} R_{212} R_{210}, R_{241} R_{232} R_{220}, R_{221} R_{122} R_{110}$, $R_{210} R_{121} R_{222}, R_{230} R_{131} R_{112}, R_{230} R_{221} R_{242}, R_{131} R_{142} R_{120}, R_{s 22} R_{s 31} R_{s 40}$, and $R_{121} R_{140} R_{132}$. Such a substitution therefore leaves fixed. every element and is therefore the identity. The order of $G$ is therefore at most $27 \cdot 10 \cdot 8 \cdot 24=51840$.

But $G$ contains the subgroup $[O]$ of order 25920 whose substitutions permute the 45 triangles evenly. Also $G$ contains

$$
T \equiv \Pi\left(R_{s 2 t} R_{s 4 t}\right) \quad(s=1,2 ; t=0,1,2)
$$

which gives rise to the following odd substitution on the triangles:

$$
\begin{aligned}
& \left(R_{t} R_{12 t} R_{22 t}, R_{t} R_{14 t} R_{24 t}\right) \\
& \left(R_{12 t} R_{11 t+1} R_{22 t-1}, R_{14 t} R_{11 t+1} R_{24 t-1}\right) \\
& \left(R_{22 t} R_{21 t+1} R_{12 t-1}, R_{24 t} R_{21 t+1} R_{14 t-1}\right) \\
& \left(R_{s 2 t} R_{s 3 t+1} R_{s 4 t-1}, R_{s 2 t-1} R_{s 4 t} R_{s 3 t+1}\right)
\end{aligned}
$$

containing $3+3+3+6=15$ transpositions. The order of $G$ is therefore at least $2 \cdot 25920$. The order is consequently 51840 .

286. Certain subgroups of the abstract group $O$ of order 25920 appear at once by considering the various isomorphic linear groups. 
By $\S \S 118$ and 133 , the simple group $H A\left(4,2^{2}\right)$, which is isomorphic with $O$ by $\S 275$, has a complete set of 36 conjugate subgroups $A(4,2)$ holoedrically isomorphic with the symmetric group on 6 letters. By $\S 136, H A\left(4,2^{2}\right)$ has a complete set of 216 conjugate subgroups $L F\left(2,2^{2}\right)$, holoedrically isomorphic with the alternating group on 5 letters. By $\S \S 270-274, O$ has a subgroup $G_{960}$ of index 27. The quotient-group $A(4,3)$ of the special Abelian group $S A(4,3)$ is (§ 189) holoedrically isomorphic with $F O(5,3)$ and therefore with the abstract group $O$. By $\S 114, S A(4,3)$ contains $3^{3}\left(3^{2}-1\right) 3$ substitutions which leave $\xi_{1}$ fixed, so that $A(4,3)$ contains a subgroup of index $25920 \div 8 \cdot 3^{4} \equiv 40$. By $\S 121, S A(4,3)$ contains exactly $\left(3^{2}+1\right) 3^{2}$ substitutions conjugate with $T_{1,-1}$. But the latter is conjugate with $T_{2,-1}$, the two being identical in the quotientgroup $A(4,3)$. Hence $A(4,3)$ has a subgroup of index 45 . Hence the simple group $O$ has subgroups of indices 27, 36, 40, 45, 216. By a lengthy analysis ${ }^{1}$ ), it has been shown that $O$ contains no subgroup of index $<27$. The problem of the determination of the 27 straight lines on a general cubic surface has therefore resolvent equations of degrees $27,36,40,45$ but none of degree $<27$.

Since $O$ is isomorphic with $A(4 ; 3)$, our problem is identical with the problem of the trisection of the periods of hyperelliptic functions with four periods. ${ }^{2}$ )

\section{CHAPTER XV.}

\section{SUMMARY OF THE KNOWN SYSTEMS OF SIMPLE GROUPS.}

28\%. In the preceding chapters were derived the following systems of simple groups, with the specified restrictions upon the prime number $p$ and the positive integers $m$ and $\left.n^{3}\right)$ :

$L F\left(m, p^{n}\right): \frac{1}{d}\left(p^{n m}-1\right) p^{n(m-1)}\left(p^{n(m-1)}-1\right) p^{n(m-2)} \ldots\left(p^{2 n}-1\right) p^{n}$

where $p^{n}>3$ if $m=2$, and $d$ is the greatest common divisor of $m$ and $p^{n}-1$.

$$
\begin{gathered}
H O\left(m, p^{2 n}\right): \frac{1}{g}\left[p^{n m}-(-1)^{m}\right] p^{n(m-1)}\left[p^{n(m-1)}-(-1)^{m-1}\right] p^{n(m-2)} \ldots \\
{\left[p^{2 n}-1\right] p^{n}}
\end{gathered}
$$

where $p^{n}>3$ if $m=2, p^{n}>2$ if $m=3$, and $g$ is the greatest common divisor of $m$ and $p^{n}+1$.

1) Jordan, Traité, pp. $319-329$.

2) Jordan, pp. $354-369$.

3) The notations were introduced in $\S \S 108,119,148,194$ and end of 209. 
$A\left(2 m, p^{n}\right): \frac{1}{a}\left(p^{n(2 m)}-1\right) p^{n(2 m-1)}\left(p^{n(2 m-2)}-1\right) p^{n(2 m-3)} \ldots\left(p^{2 n}-1\right) p^{n}$ where $p^{n}>3$ if $m=1, p^{n}>2$ if $m=2$, and $a=1$ if $p=2, a=2$ if $p>2$.

$$
\begin{gathered}
F O\left(2 m+1, p^{n}\right): \frac{1}{2}\left(p^{n(2 m)}-1\right) p^{n(2 m-1)}\left(p^{n(2 m-2)}-1\right) p^{n(2 m-3)} \ldots \\
\left(p^{2 n}-1\right) p^{n}
\end{gathered}
$$

where $p>2$ and, for $m=1, p^{n}>3$.

$F O\left(2 m, p^{n}\right): \frac{1}{4}\left[p^{n(2 m-1)}-\varepsilon_{\cdot}^{m} p^{n(m-1)}\right]\left(p^{n(2 m-2)}-1\right) p^{n(2 m-3)} \ldots\left(p^{2 n}-1\right) p^{n}$ where $p>2$ and $m>2$, while $\varepsilon= \pm 1$ according as $p^{n}=4 l \pm 1$.

$S O\left(2 m, p^{n}\right): \frac{1}{2}\left[p^{n(2 m-1)}+\varepsilon^{m} p^{n(m-1)}\right]\left(p^{n(2 m-2)}-1\right) p^{n(2 m-3)} \ldots\left(p^{2 n}-1\right) p^{n}$ where $p>2$ and $m>1, \varepsilon= \pm 1$ according as $p^{n}=4 l \pm 1$.

$F H\left(2 m, 2^{n}\right):\left(2^{n m}-1\right)\left(2^{2 n(m-1)}-1\right) 2^{2 n(m-1)}\left(2^{2 n(m-2)}-1\right) 2^{2 n(m-2)} \ldots$ where $m>2$.$$
\left(2^{2 n}-1\right) 2^{2 n}
$$

$S H\left(2 m, 2^{n}\right):\left(2^{n m}+1\right)\left(2^{2 n(m-1)}-1\right) 2^{2 n(m-1)} \ldots\left(2^{2 n}-1\right) 2^{2 n}, m>1$.

In addition to these systems may be added the cyclic groups of prime order and the alternating group on $n>4$ letters.

288. Between certain of the above groups there exists holoedric isomorphism, a relation indicated by the symbol $\sim$. For $p>2$, the following isomorphisms were established in $\S 178$, $\S ~ 187-190$, 197-198:

$F O\left(3, p^{n}\right) \sim L F\left(2, p^{n}\right) ; \quad F O\left(6, p^{n}\right) \sim L F\left(4, p^{n}\right)$, for $p^{n}=4 l+1 ;$ $F O\left(5, p^{n}\right) \sim A\left(4, p^{n}\right) ; \quad S O\left(6, p^{n}\right) \sim L F\left(4, p^{n}\right)$, for $p^{n}=4 l+3 ;$

$\dot{F O}\left(6, p^{n}\right) \sim H O\left(4, p^{2 n}\right), p^{n}=4 l+3 ; S O\left(6, p^{n}\right) \sim H O\left(4, p^{2 n}\right), p^{n}=4 l+1$; $S O\left(4, p^{n}\right) \sim L F\left(2, p^{2 n}\right)$

the latter holding also for $p^{n}=3$, a case not treated in $\S \S 197-198$. For any $p$,

$$
L F\left(2, p^{n}\right) \sim A\left(2, p^{n}\right) \sim H O\left(2, p^{2 n}\right) .
$$

For $p=2$, it was shown in $\S \S 198,206,207$ that $S H\left(4,2^{n}\right) \sim L F\left(2,2^{2 n}\right), F H\left(6,2^{n}\right) \sim L F\left(4,2^{n}\right), S H\left(6,2^{n}\right) \sim H O\left(4,2^{2 n}\right)$. By chapter XIII,

$$
F O(5,3) \sim H O\left(4,2^{2}\right), \quad L F(4,2) \sim G_{\frac{1}{2} 8 !}^{(8)}, \quad L F(3,2) \sim L F(2,7) .
$$


289. Theorem. $\left.{ }^{1}\right)$ - The simple groups $A\left(2 m, p^{n}\right)$ and

$$
F O\left(2 m+1, p^{n}\right), \quad p>2,
$$

of equal order are not isomorphic if $m>2$.

The proof consists in showing that the orthogonal group contains a greater number of sets of conjugate operators of period two than the Abelian group. By $\S 122, A\left(2 m, p^{n}\right), p>2$, has exactly $\frac{1}{2}(m+2)$ or $\frac{1}{2}(m+1)$ distinct sets of conjugate operators of period two according as $m$ is even or odd. But $F O\left(2 m+1, p^{n}\right)$ contains the following $m$ distinct substitutions of period two,

$$
C_{1} C_{2}, \quad C_{1} C_{2} C_{3} C_{4}, \ldots, C_{1} C_{2} C_{3} C_{4} \ldots C_{2 m-1} C_{2 m},
$$

having the respective characteristic determinants,

$$
(1+K)^{2}(1-K)^{2 m-1},(1+K)^{4}(1-K)^{2 m-3}, \ldots,(1+K)^{2 m}(1-K) \text {. }
$$

By $\S 102$, no two of these $m$ substitutions are conjugate under linear transformation.

For $m=1$ or for $m=2$, the corresponding groups are isomorphic (§ 288).

290. The following table gives the 53 known simple groups of composite order less than one million. The alternating group on $n$ letters is designated by its order $\frac{1}{2} n$ ! The isomorphisms indicated in $\S 288$ are not given in the table.

\begin{tabular}{r|l|r|l}
60 & $L F(2,5) \sim L F\left(2,2^{2}\right) \sim \frac{1}{2} 5 !$ & 6072 & $L F(2,23)$ \\
168 & $L F(2,7) \sim L F(3,2)$ & 7800 & $L F\left(2,5^{2}\right)$ \\
360 & $L F\left(2,3^{2}\right) \sim \frac{1}{2} 6 !$ & 7920 & Group on 9 letters $\left.{ }^{2}\right)$ \\
504 & $L F\left(2,2^{3}\right)$ & 9828 & $L F\left(2,3^{3}\right)$ \\
660 & $L F(2,11)$ & 12180 & $L F(2,29)$ \\
1092 & $L F(2,13)$ & 20160 & $L F(2,31)$ \\
2448 & $L F(2,17)$ & 20160 & $L F\left(3,2^{2}\right)$ \\
2520 & $\frac{1}{2} 7 !$ & 25308 & $L F(2,37)$ \\
3420 & $L F(2,19)$ & 25920 & $A(4,3) \sim H O\left(4,2^{2}\right)$ \\
4080 & $L F\left(2,2^{4}\right)$ & 32736 & $L F\left(2,2^{5}\right)$ \\
5616 & $L F(3,3)$ & 34440 & $L F(2,41)$ \\
6048 & $H O\left(3,3^{2}\right)$ & 39732 & $L F(2,43)$ \\
& &
\end{tabular}
in $\S 238$.

1) The existence of two non-isomorphic groups of order $\frac{1}{2} 8$ ! was noted

2) Cole, Quart. Journ. of Math., vol. 27, p. 48, foot-note. 
310 CHAPT.XV. SUMMARY OF THE KNOWN SYSTEMS OF SIMPLE GROUPS.

\begin{tabular}{r|l|l|l}
51888 & $L F(2,47)$ \\
58800 & $L F\left(2,7^{2}\right)$ & 265680 & $L F\left(2,3^{4}\right)$ \\
62400 & $H O\left(3,2^{4}\right)$ & 285852 & $L F(2,83)$ \\
74412 & $L F(2,53)$ & 352440 & $L F(2,89)$ \\
95040 & Group on 12 letters $\left.{ }^{1}\right)$ & 372000 & $L F(3,5)$ \\
102660 & $L F(2,59)$ & 456288 & $L F(2,97)$ \\
113460 & $L F(2,61)$ & 515100 & $L F(2,101)$ \\
126000 & $H O\left(3,5^{2}\right)$ & 546312 & $L F(2,103)$ \\
150348 & $L F(2,67)$ & 612468 & $L F(2,107)$ \\
178920 & $L F(2,71)$ & 647460 & $L F(2,109)$ \\
181440 & $\frac{1}{2} 9 !$ & 721392 & $L F(2,113)$ \\
194472 & $L F(2,73)$ & 885720 & $L F\left(2,11^{2}\right)$ \\
246480 & $L F(2,79)$ & 976500 & $L F\left(2,5^{3}\right)$ \\
262080 & $L F\left(2,2^{6}\right)$ & 979200 & $A\left(4,2^{2}\right)$
\end{tabular}

Aside from the simple groups $L F\left(2, p^{n}\right)$, the known simple groups of composite orders between one million and one billion are the following:

\begin{tabular}{r|l|}
1451520 & $A(6,2)$ \\
1814400 & $\frac{1}{2} 10 !$ \\
1876896 & $L F(3,7)$ \\
3265920 & $H O\left(4,3^{2}\right)$ \\
4680000 & $A(4,5)$ \\
5515776 & $H O\left(3,2^{6}\right)$ \\
5663616 & $H O\left(3,7^{2}\right)$ \\
6065280 & $L F(4,3)$ \\
9999360 & $L F(5,2)$ \\
10200960 & Group on 23 letters $\left.{ }^{2}\right)$ \\
13685760 & $H O\left(5,2^{2}\right)$ \\
16482816 & $L F\left(3,2^{3}\right)$ \\
19958400 & $\frac{1}{2} 11 !$ \\
\hline
\end{tabular}

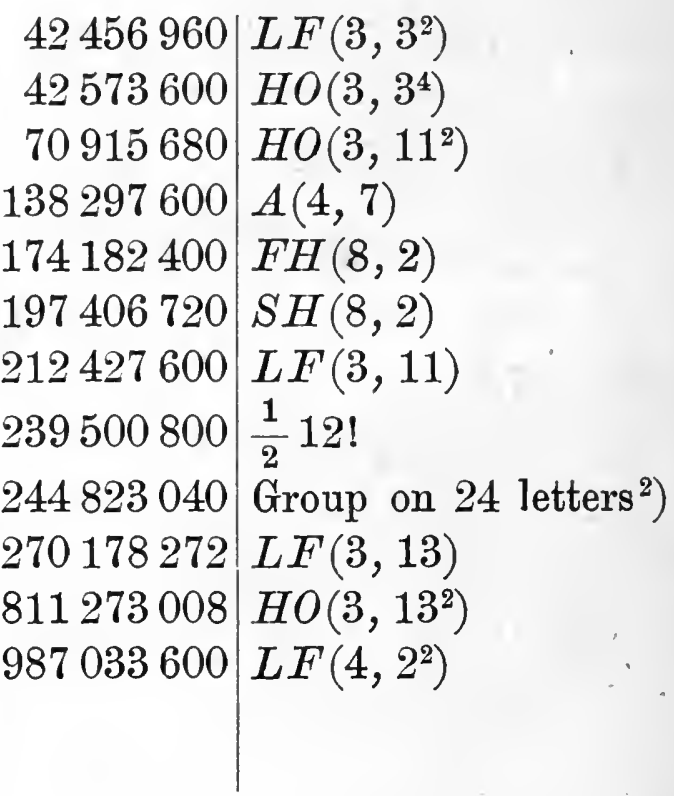

1) Mathieu, Journal de Mathématiques, 1861, p. 270; proof of simplicity by Miller.

2) Miller, Bull. Soc. Math. de France, vol. 28, p. 266 (1900). 


\section{INDEX 0F SUBJECTS.}

(The numbers refer to pages; $g$. or $G$ denotes group.)

Abelian $g, 89,110,115,117,151,179$, 200, 201, 299, 309.

abstract field, 9, 13.

abstract $g, 287,289,292,300$.

additive-field, 5 .

additive- $g, 49,269$.

alternating $g, 4$ letters, 269 .

- 5 letters, 279, 290.

- 8 letters, 259, 290.

$-k$ letters, 289.

basis-system, 49 .

Betti-Mathieu $g, 64,67,69$.

canonical form, 221, 237, 244.

characteristic determinant, 80 . equation, 222.

class of quantic, 29. residue, $3,6,7$.

commutative $g, 262,265$.

compound of $g, \mathbf{1 4 5}$.

configuration 27 lines, 303.

congruent, 3 .

conjugate, 52, 100, 236.

cubic surface, $303,306$.

cyclic base, 266.

dihedron $g, \mathbf{2 6 5}$.

doubly-transitive, 248, 261.

exercises, 19, 42, 70, 216.

existence of Galois $F, 14,19$.

exponent of mark, 11.

- of function, 19.

factors of composition, $81,91,94,191$, 192.

Fermat's theorem, 4, 11.

field, 5.

first hypoabelian, 201, 208.

first orthogonal, 131, 159, 191, 292, $299,309$.

four-group, 267.

Galois Field, 6, 14.

general linear homogeneous $g, 69,75$, 77, 124, 146, 147, 236, 290. group, $65 ; G_{168}, 303 ; G_{20160}, 259$; $G_{25920}, 293,296 ; G_{51840}, 306$; see alternating, icosahedral, dihedron, tetrahedral, octahedral, symmetric, linear, general, special, simple.

Hermite's theorem, 59.

homogeneous, see general, special.

hyperabelian $g, 115,183,209,298$.

hyperelliptic, 307.

hyperorthogonal $g, 131,264$.

hypoabelian, see first, second.

icosahedral, 278, 283, 302.

index of subgroup, 286, 307.

infinity (mark), 260.

invariant, quadratic, 144, 153, 156, 191, 194, 197, 206.

of degree $2,126,218$.

irreducible, 10, 15, 44.

isomorphic, 99, 164, 174, 183, 194, 208, 209, 287, 298, 308.

linear independence, 10, 52.

linear fractional $g, 87,126,132,164$, $174,179,193,194,208,242$, $259,260,286,302,303$.

mark, 9.

modulus, 3,6 .

multiplier Galois $F, 51,270$.

Newton's identities, 53.

non-isomorphic, 260,309 .

not-square, $44,48$.

octahedral $g, 269,282$.

order of field, $5,10$.

orthogonal, see first, second.

period of mark, 11 .

Pfaffian, 147, 172.

primitive root, 13,36 .

irreducible quantic, 21, 35, 44.

quadratic equation, 46 .

-, see invariant.

rank, 49.

reduced quantic, 63. 
representation of substitutions, $\mathbf{5 5}$. residue; $3,6$.

self-conjugate, 82, 117, 279.

second hypoabelian, 201, 209.

- orthogonal, 159, 191, 194.

simple $g, 87,97,100,120,138,152$, 191, 212, 260, 286, 307, 309.

special linear homogeneous $g, 82,125$, $147,151,153,300$.

squares, $44,48$. substitution-quantic, 55,63 . surface third order, 303 . symmetric $g, 6$ letters, 99. $k$ letters, 287. tetrahedral $g, 268,282$. transformation of indices, 80 . transformed subst., 81, 288. transitive, 248, 261. trieder, 304.

\section{ERRATA.}

Page 14, line 12, read $G F\left[p^{m}\right]$ for $G F\left[p^{n}\right]$.

$" \quad 17, \quad, \quad 31$, read $y=x^{p^{n m_{1}}-1}$ for $y=x^{p^{n m_{1}}}-1$.

$" 20, " 21$, read $q_{i}$ for $q i$.

$" 48, \quad, 2$ of $\S 67$, read number of squares.

$" 71, \quad, 5$ of Ex. 6 , read $\lambda^{-1} \lambda_{1}$ for $\lambda^{-1} \lambda$.

$" 78, \quad, 15$, read $B_{r, s, \lambda}$ for $B_{r, s \lambda}$.

$" 93, \quad, 6$, read $\gamma_{12}^{\prime}, \alpha_{1 m}^{\prime}$; line $2, \operatorname{read} M_{1}$ for $M_{j}$.

" 95, " 30, read $\alpha_{21}$ for $a_{21}$.

$" 102, " 17$, read $T_{m-1, \delta_{1 m-1}}^{-1} ;$ line 16, read $T_{m, \alpha_{1 m}}$

$" 113, " 3$, read $j_{1}$ for $j$.

$" 132, " 28$ and line 33 , read for

$" 139, \quad, \quad 8$, read $\bar{\alpha}_{11}^{p^{s}+1} \neq 1$.

"152, , 5, read 139) for 139 ,

$, 172, \quad, 16$, p. $175,1.14$, read $G_{4,2}^{\prime}$ for $G_{4,2}$.

"189, " 3 of $\S 192$, delete comma before "are".

"209, "1, for hyperabelian read hypoabelian.

"221, " 14, for $\{L\} \operatorname{read}\{B\}$.

"227, read $Y_{i s} \equiv y_{s}+y_{s}^{\prime} K_{i}+y_{s}^{\prime \prime} K_{i}^{2}+\cdots+$

"267, line 10, for $G_{\delta_{\mp}}$ read $G_{d \mp}$.

"272, " 3 from bottom, delete "an".

$" 300, \quad, 16$, for $S_{\alpha}^{-1}$ read $S_{\alpha-1}$. 


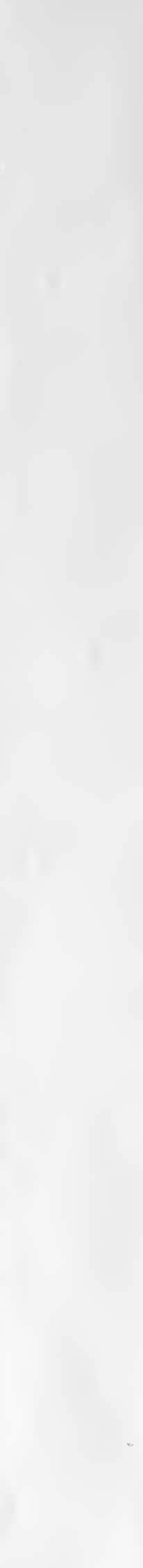



BHDRRC - $=$ W. JUN 3958

QA Dickson, Leonard Eugene

171 Linear groups

D53

Pivitad is

Apoliad sat.

PLEASE DO NOT REMOVE

CARDS OR SLIPS FROM THIS POCKET

UNIVERSITY OF TORONTO LIBRARY 


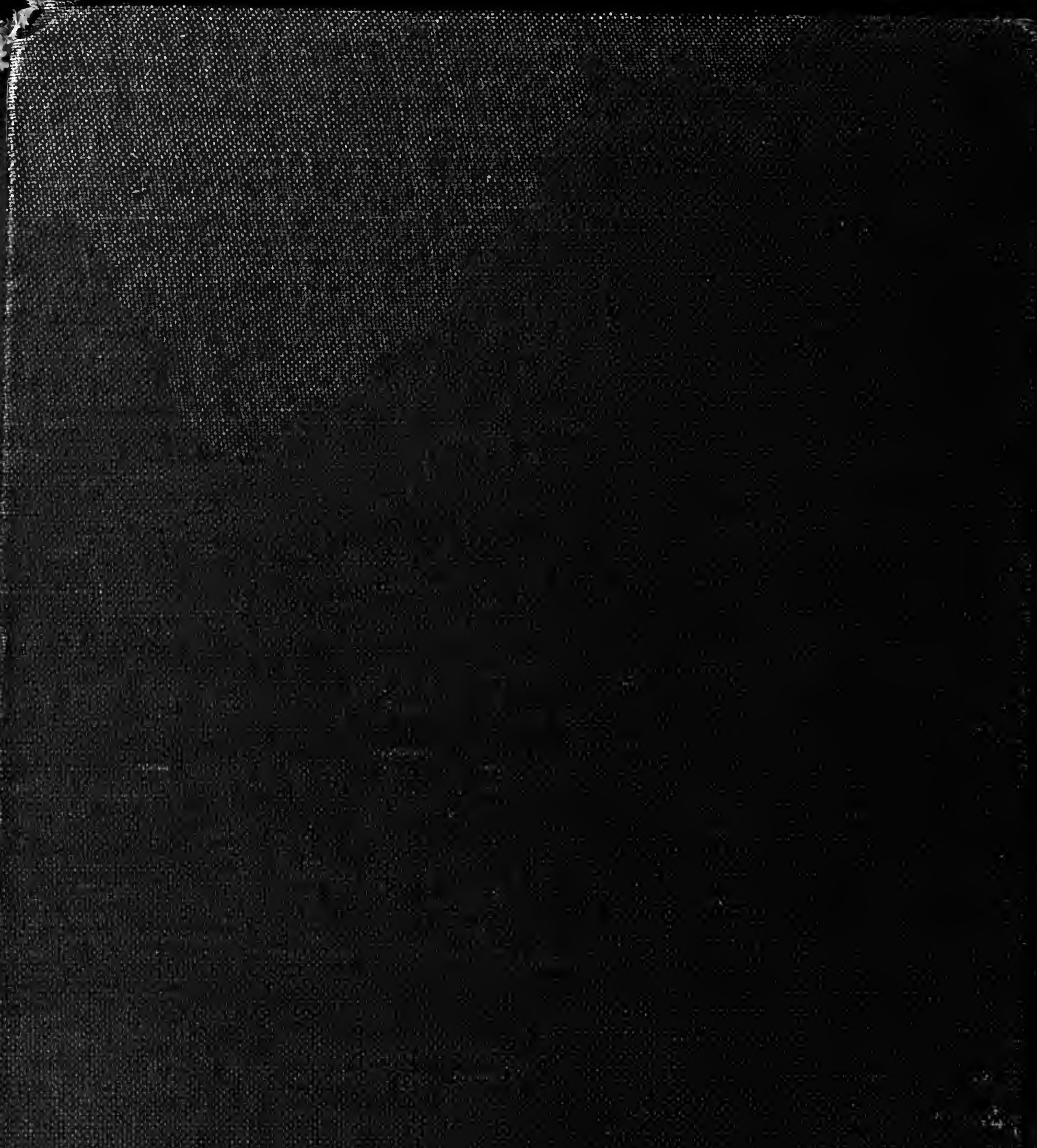

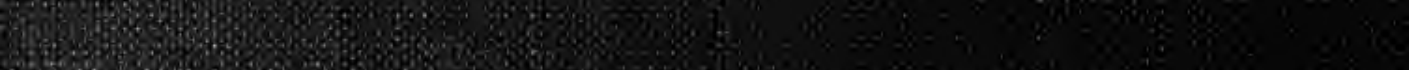

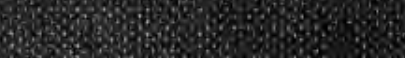

6.8.

(5)

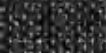

ond

(5)

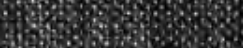

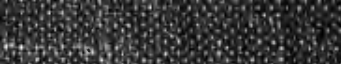

4028

4. $20 \%$

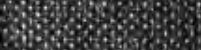

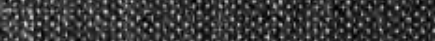

